\title{
Factors and Factorizations of Graphs
}

\author{
Jin Akiyama and Mikio Kano
}

June 2007 Version 1.0 


\section{A list of some notation}

$G$ denotes a graph.

\begin{tabular}{|c|c|}
\hline$X \subseteq Y$ & $X$ is a subset of $Y$. \\
\hline$X \subset \bar{c}$ & $X$ is a proper subset of $Y$. \\
\hline$X-Y$ & $X \backslash Y$, where $Y \subseteq X$ \\
\hline$X-a$ & $X-\{a\}$, where $a \in X$ \\
\hline$X+Y$ & $X \cup Y$, where $X \cap Y=\emptyset$. \\
\hline$X \triangle Y$ & $(X \cup Y)-(X \cap Y)$ \\
\hline$|X|$ & The number of elements in $X$. \\
\hline$V(G)$ & The set of vertices of $G$. \\
\hline$E(G)$ & The set of edges of $G$. \\
\hline$|G|$ & The order of $G=|V(G)|$. \\
\hline$\|G\|$ & The size of $G=|E(G)|$. \\
\hline $\operatorname{deg}_{G}(v)$ & The degree of a vertex $v$ in $G$. \\
\hline$N_{G}(v)$ & The neighborhood of $v$. \\
\hline$N_{G}[v]$ & $N_{G}(v) \cup\{v\}$ \\
\hline$N_{G}(S)$ & $\bigcup_{x \in S} N_{G}(x)$ \\
\hline$E_{G}(X, Y)$ & The set of edges of $G$ joining $X$ to $Y$. \\
\hline$e_{G}(X, Y)$ & The number of edges of $G$ joining $X$ to $Y$. \\
\hline$\Delta(G)$ & The maximum degree of $G$. \\
\hline$\delta(G)$ & The minimum degree of $G$. \\
\hline$\omega(G)$ & The number of components of $G$. \\
\hline $\operatorname{odd}(G)$ & The number of odd components of $G$. \\
\hline$O d d(G)$ & The set of odd components of $G$. \\
\hline$i s o(G)$ & The number of isolated vertices of $G$. \\
\hline $\operatorname{Iso}(G)$ & The set of isolated vertices of $G$. \\
\hline$\kappa(G)$ & The connectivity of $G$. \\
\hline$\lambda(G)$ & The edge connectivity of $G$. \\
\hline$\alpha(G)$ & The independence number of $G$. \\
\hline$\alpha^{\prime}(G)$ & The edge independence number of $G$. \\
\hline $\operatorname{bind}(G)$ & The binding number of $G$. \\
\hline $\operatorname{tough}(G)$ & The toughness of $G$. \\
\hline$K_{n}=K(n)$ & The complete graph of order $n$. \\
\hline$K_{n, m}=K(n, m)$ & The complete bipartite graph of order $n+m$. \\
\hline$P_{n}$ & The path of order $n$. \\
\hline $\mathbb{N}$ & The set of natural numbers $=\{1,2,3, \ldots\}$. \\
\hline $\mathbb{Z}$ & The set of integers. \\
\hline $\mathbb{Z}^{+}$ & The set of non-negative integers $=\{0,1,2, \ldots\}$ \\
\hline
\end{tabular}




\section{A list of some functions}

$S$ and $T$ denote disjoint vertex subsets of a graph $G$.

$$
\begin{aligned}
\delta(S, T) & =\sum_{x \in S} f(x)+\sum_{x \in T}\left(\operatorname{deg}_{G}(x)-f(x)\right)-e_{G}(S, T)-q(S, T) \\
& =\sum_{x \in S} f(x)+\sum_{x \in T}\left(\operatorname{deg}_{G-S}(x)-f(x)\right)-q(S, T),
\end{aligned}
$$

where $q(S, T)$ denotes the number of components $C$ of $G-(S \cup T)$ such that $\sum_{x \in V(C)} f(x)+e_{G}(C, T) \equiv 1(\bmod 2)$.

$\delta^{*}(S, T)=\sum_{x \in S} f(x)+\sum_{x \in T}\left(\operatorname{deg}_{G}(x)-f(x)\right)-e_{G}(S, T)$

$=\sum_{x \in S} f(x)+\sum_{x \in T}\left(\operatorname{deg}_{G-S}(x)-f(x)\right)$

$\gamma(S, T)=\sum_{x \in S} f(x)+\sum_{x \in T}\left(\operatorname{deg}_{G}(x)-g(x)\right)-e_{G}(S, T)-q^{*}(S, T)$

$=\sum_{x \in S} f(x)+\sum_{x \in T}\left(\operatorname{deg}_{G-S}(x)-g(x)\right)-q^{*}(S, T)$,

where $q^{*}(S, T)$ denotes the number of components $C$ of $G-(S \cup T)$ such that $g(x)=f(x)$ for all $x \in V(C)$ and $\sum_{x \in V(C)} f(x)+e_{G}(C, T) \equiv 1(\bmod 2)$.

$\gamma^{*}(S, T)=\sum_{x \in S} f(x)+\sum_{x \in T}\left(\operatorname{deg}_{G}(x)-g(x)\right)-e_{G}(S, T)$

$=\sum_{x \in S} f(x)+\sum_{x \in T}\left(\operatorname{deg}_{G-S}(x)-g(x)\right)$

$\sum_{x \in V(C)} f(x)+e_{G}(C, T) \equiv 1(\bmod 2)$.

$\eta(S, T)=\sum_{x \in S} f(x)+\sum_{x \in T}\left(\operatorname{deg}_{G}(x)-g(x)\right)-e_{G}(S, T)-q(S, T)$, where $q(S, T)$ denotes the number of components $C$ of $G-(S \cup T)$ such that $\sum_{x \in V(C)} f(x)+e_{G}(C, T) \equiv 1(\bmod 2)$. 


\section{Contents}

1 Matchings and 1-Factors 1

1.1 Matchings in Bipartite Graphs . . . . . . . . . . . . . . . 1

1.2 Covers and Transversals . . . . . . . . . . . . . . . . . 12

1.3 Augmenting Paths and Algorithms . . . . . . . . . . . . 19

1.4 1-Factor Theorems . . . . . . . . . . . . . . . 25

1.5 Graphs Having 1-Factors . . . . . . . . . . . . . . . . 34

1.6 Structure Theorem . . . . . . . . . . . . . . . . . . . 47

1.7 Algorithms for Maximum Matchings . . . . . . . . . . . 54

2 Regular Factors and $f$-Factors $\quad 59$

2.1 The $f$-Factor Theorem . . . . . . . . . . . . . . . . . 59

2.2 Regular Factors in Regular Graphs . . . . . . . . . . . . . . . 69

2.3 Regular Factors and $f$-Factors in Graphs . . . . . . . . . . 82

2.4 Regular Factors and $f$-Factors in Bipartite Graphs . . . . . 119

$3(g, f)$-Factors and $[a, b]$-Factors $\quad \mathbf{1 2 9}$

3.1 The $(g, f)$-Factor Theorem . . . . . . . . . . . . . . . 129

3.2 Graphs Having the Odd-Cycle Property . . . . . . . . . . 148

$3.3[a, b]$-Factors and $(g, f)$-Factors $\ldots \ldots \ldots \ldots \ldots$

$4 \quad[a, b]$-Factorizations $\quad \mathbf{1 8 5}$

4.1 Factorizations of Special Graphs . . . . . . . . . . . 185

4.2 Semi-Regular Factorization . . . . . . . . . . . . . . . 190

$4.3[a, b]$-Factorizations of Graphs $\ldots \ldots 195$

5 Parity Factors 213

5.1 Parity $(g, f)$-Factors and $(1, f)$-Odd Factors $\ldots \ldots . . .213$

$5.2(1, f)$-Odd Subgraphs and Structure Theorem . . . . . . . 221

5.3 Partial parity $(g, f)$-factors and coverings . . . . . . . . 238

$5.4 \mathcal{H}$-Factors . . . . . . . . . . . . . . . 247 



\section{Preface}

Among the results in graph theory in the 18th century are Petersen's results on graph factors and factorizations. Petersen's Theorem states: Every even regular graph is 2-factorable. He proved it to approach a problem on Diophantine equations. Then he proved a second theorem. Every 2connected 3-regular graph has a 1-factor. This arose from a counterexample he constructed to Tait's 'theorem': Every 3-regular graph which has no leaf is 1-factorable. This counterexample is the well-known Petersen graph. Petersen's theorems are byproducts of attempts to address other things. Perhaps Petersen himself had no inkling that these theorems would open the door to a very promising area of graph theory.

Later came Hall's Marriage Theorem, a result obtained when he was studying the structure of subsets. Let $G$ be a bipartite graph with bipartition $(A, B)$. Then $G$ has a matching that saturates $A$ if and only if $\left|N_{G}(S)\right| \geq|S|$ for all $S \subseteq A$. König's Theorem followed: Every regular bipartite graph is 1-factorable. And then Tutte's 1-Factor Theorem: A graph $G$ has a 1-factor if and only if odd $(G-S) \leq|S|$ for all $S \subset V(G)$. These five theorems form the foundation of the study of factors and factorizations.

König's theorem was the result of a conscious effort to answer a graph theory problem: Does every bipartite regular graph have a 1-factor? It seems that, at that time, König already had a good idea of how graph factors could be applied. In his paper "On graphs and their applications in determinant theory and set theory", he starts by saying "The following article deals with problems from analysis situs, the theory of determinants and set theory." He then continues to say that in fact, the notion of a graph and its usefulness as a method of representation actually relates these three disparate areas.

A second stage of development is marked by Tutte's $f$-Factor Theorem: Let $G$ be a graph and $f: V(G) \rightarrow \mathbb{Z}^{+}=\{0,1,2,3, \ldots\}$. Then $G$ has an $f$-factor if and only if for all disjoint subsets $S$ and $T$ of $V(G)$,

$$
\sum_{x \in S} f(x)+\sum_{x \in T}\left(\operatorname{deg}_{G-S}(x)-f(x)\right)-q(S, T) \geq 0
$$


where $q(S, T)$ denotes the number of components $C$ of $G-(S \cup T)$ such that $\sum_{x \in V(C)} f(x)+e_{G}(C, T) \equiv 1(\bmod 2)$; and Lovász's $(g, f)$-Factor Theorem: Let $G$ be a graph and $g, f: V(G) \rightarrow \mathbb{Z}$ such that $g(x) \leq f(x)$ for all $x \in V(G)$. Then $G$ has a $(g, f)$-factor if and only if for all disjoint subsets $S$ and $T$ of $V(G)$,

$$
\sum_{x \in S} f(x)+\sum_{x \in T}\left(\operatorname{deg}_{G-S}(x)-g(x)\right)-q^{*}(S, T) \geq 0,
$$

where $q^{*}(S, T)$ denotes the number of components $C$ of $G-(S \cup T)$ such that $g(x)=f(x)$ for all $x \in V(C)$ and $\sum_{x \in V(C)} f(x)+e_{G}(C, T) \equiv 1(\bmod 2)$. Many subsequent theorems were proved based on these results.

A third stage of development began in the 1980's and is marked by the introduction of the notions of semi-regular factors and component factors. Since that time, many substantial results have been obtained and much progress has been made in this area. The results are detailed in this book.

As far as we know, there is no comprehensive book on factors and factorizations. This is one compelling reason for writing this book. Since we wrote our survey paper entitled "Factors and Factorizations of Graphs" published in Journal of Graph Theory, vol. 9 (1985), we collected and analyzed almost all results in the area. In fact we started to write this book ten years ago. On the occassion of KyotoCGGT2007, we made a special effort to complete this version 1 .

This book also chronicles the development of mathematical graph theory in Japan, a development which began with many important results in factors and factorizations of graphs.

This book has a number of desirable features:

1. It is comprehensive and covers almost all the results from 1980 .

2. It is self-contained. One who wants to begin research in graph factors and factorizations can confine himself to this one book to follow the history and development of the area, and to find conjectures and open problems.

3. Proof techniques are standardized. Whenever theorems could be proved in a similar way we did so, although different methods of proof were used when they first appeared.

4. Proofs are presented in their entirety for major theorems, even if they are long. We suggest that the readers refer to various parts as needed.

5. Many detailed illustrations are given to accompany the proofs. 
6. It will be published in electronic form so that it is available to the majority of researchers.

We are planning to publish a more polished version 2 adding two more chapters "Component Factors" and "Spanning Trees". So we will appreciate comments from readers.

Frank Harary predicted that graph theory will grow so much that each chapter of his book Graph Theory will eventually expand to become a book on its own. He was right. This book is an expansion of his Chapter 9, Factorization.

We also predict that the area of factors and factorizations will continue to grow because of many applications to BIBD, Steiner Designs, Matching Theory, OR, etc. that have been found.

The following references were very helpful to us during the preparation of this book:

- Extremal Graph Theory, by Bela Bollobás, Academic Press, (1978)

- Combinatorial Problems and Exercises, by László Lovász, North-Holland, (1979).

- Matching Theory, by László Lovász and Michael D. Plummer, Annals of Discrete Mathematics 29, North-Holland, (1986).

- Lutz Volkmann, Regular graphs, regular factors, and the impact of Petersen's Theorems, Jber. d. Dt. Math.-Verein 97 (1995) 19-42.

- Lutz Volkmann, Graphen und Digraphen: Eine Einführung in die Graphentheorie (Springer-Verlag) (1991).

- Mekkia Kouider and Preben D. Vestergaard, Connected factors in graphs — A survey, Graphs Combinatorics 21 (2005) 1-26.

- Michael D. Plummer, Graph factors and factorization: 1985-2003: A survey, Discrete Mathematics 307 (2007) 791-821.

We would like to thank Haruhide Matsuda, Mari-Jo Ruiz, Lutz Volkmann and our many friends, who work in factor theory, for their assistance in making this book possible.

Jin Akiyama

Mikio Kano 



\section{About the Authors}

Jin Akiyama is Professor of Mathematics and Director of the Research Institute of Educational Development of Tokai University in Tokyo, Japan. His publications are in discrete geometry, graph theory and mathematics education.

Mikio Kano is Professor of Mathematics of Ibaraki University in Hitachi, Japan. His research interests include discrete geometry and graph theory.

Both authors were involved in the founding of the journal "Graphs and Combinatorics" and continue to be actively involved in the publication of the journal, Akiyama as Editor-in-Chief and Kano as Managing Editor. 



\section{Basic Terminology}

We introduce some graph theory definitions and notation which are needed to discuss factor theory in graphs. A graph $G$ consists of a vertex set $V(G)$ and an edge set $E(G)$. Each edge joins two vertices, which are not necessarily distinct. An edge joining a vertex $x$ to a vertex $y$ is denoted by $x y$ or $y x$. An edge $x y$ with $x=y$ is called a loop, that is, a loop is an edge joining a vertex to itself. For two distinct vertices, usually there is at most one edge joining them, but if there are two or more edges joining them, then the graph is said to have multiple edges.

We can define three types of graphs as follows: A graph that may have loops and multiple edges is called a general graph. A graph that has no loops but may have multiple edges is called a multigraph. A graph having neither loops nor multiple edges is called a simple graph (Figure 1). A graph usually means one of these. The meaning can be understood from the content. There are instances when it is not necessary to distinguish them. In this book, we deal with these three types of graphs and each of them plays an important role.

The number of vertices of a graph $G$ is called the order of $G$ and is denoted by $|G|$. On the other hand, the number of edges of $G$ is called the size of $G$ and is denoted by $\|G\|$. For a set $X$, we denote the cardinality of $X$ by $|X|$. Then

$$
\begin{aligned}
|G| & =|V(G)|=\text { the order of } G, \\
|| G|| & =|E(G)|=\text { the size of } G .
\end{aligned}
$$

We consider only graphs of finite order, which are called finite graphs. When there exists an edge $e=x y$ joining $x$ to $y$, we say that $e$ is incident with $x$ and $y$ and vice versa, and $x$ and $y$ are adjacent. Moreover, $x$ and $y$ are called the endvertices of $e$. If two edges have a common endvertex, then they are said to be adjacent. For a vertex $v$ of a graph $G$, the number of edges of $G$ incident with $v$ is called the degree of $v$ in $G$ and is denoted by $\operatorname{deg}_{G}(v)$. Each loop incident with $v$ contributes two to $\operatorname{deg}_{G}(v)$ (Figure 1 (a)).

The maximum degree among all vertices of $G$ is called the maximum 


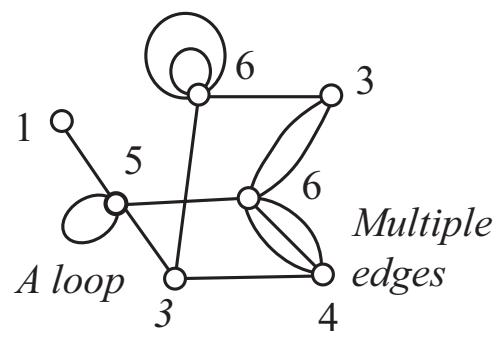

(a)

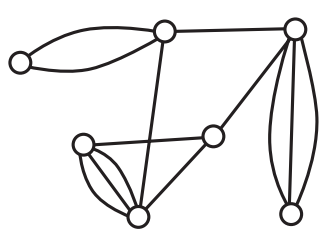

(b)

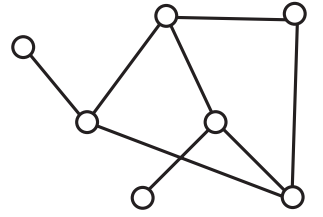

(c)

Figure 1: (a) A general graph with order 7 and size 14, where numbers denote the degrees of the vertices. (b) A multigraph. (c) A simple graph.

degree of $G$ and denoted by $\Delta(G)$. The minimum degree, denoted by $\delta(G)$, of $G$ is defined symmetrically. If every vertex of a graph $G$ has constant degree $r$, then $G$ is called an $r$-regular graph. A 3-regular graph is often called an cubic graph.

A vertex of odd degree is called an odd vertex, and a vertex of even degree is called an even vertex. Moreover a vertex of degree one is called a leaf or pendant vertex, and the edge incident with an end vertex is called pendant edge.

For two given graphs $G$ and $H$, if there exists a bijection $f: V(G) \rightarrow$ $V(H)$ such that $f(x)$ and $f(y)$ are adjacent in $H$ if and only if $x$ and $y$ are adjacent in $G$, then we say that $G$ and $H$ are isomorphic and write $G \cong H$.

The name of the following theorem arises from the fact that if each vertex of a graph represents a person and each edge means that two persons shake hands. Then the sum of degrees is the total number of hands shaken, which is of course equal to twice the number of handshakes.

Theorem 1 (Handshaking Theorem) Let $G$ be a general graph. Then the sum of degrees of vertices of $G$ is equal to twice the number of edges of G. Namely,

$$
\sum_{x \in V(G)} \operatorname{deg}_{G}(x)=2\|G\|
$$

Proof. Let

$$
\Omega=\{(v, e) \mid v \in V(G), e \in E(G), v \text { is incident with } e .\}
$$

which is the set of pairs of vertices and incident edges. Since for every vertex $v$ exactly $\operatorname{deg}_{G}(v)$ edges are incident with $v$, the number of pairs $(v, e)$ in $\Omega$ with fixed $v$ is $\operatorname{deg}_{G}(v)$, and since for each edge $e$ exactly two vertices are 
incident with $e$, the number of pairs $(v, e)$ in $\Omega$ with fixed $e$ is two. Therefore

$$
|\Omega|=\sum_{x \in V(G)} \operatorname{deg}_{G}(x)=2|E(G)|=2\|G\| .
$$

Corollary 2 Every general graph has an even number of odd vertices.

Proof. Let $G$ be a general graph, $U$ the set of odd vertices and $W$ the set of even vertices. Then by the Handshaking Theorem, we have

$$
\begin{aligned}
2\|G\| & =\sum_{x \in U} \operatorname{deg}_{G}(x)+\sum_{x \in W} \operatorname{deg}_{G}(x) \\
0 & \equiv|U| \quad(\bmod 2) .
\end{aligned}
$$

Hence $|U|$ is even.

A graph $H$ is called a subgraph of $G$ if $V(H) \subseteq V(G)$ and $E(H) \subseteq E(G)$. For a vertex subset $X \subseteq V(G)$, the subgraph of $G$ whose vertex set is $X$ and whose edge set consists of the edges of $G$ joining vertices of $X$ is called the subgraph of $G$ induced by $X$ and is denoted by $\langle X\rangle_{G}$. A subgraph $K$ of $G$ is called an induced subgraph of $G$ if there exists a vertex subset $Y$ such that $K=\langle Y\rangle_{G}$. We write $G-X$ for the subgraph of $G$ induced by $V(G)-X$, which is obtained from $G$ by removing all the vertices in $X$ together with all the edges incident with vertices in $X$ (Figure 2). For an edge set $Y \subseteq E(G), G-Y$ denotes the subgraph of $G$ obtained from $G$ by removing all the edges in $Y$. A subgraph $H$ of $G$ called a spanning subgraph of $G$ if $V(H)=V(G)$.
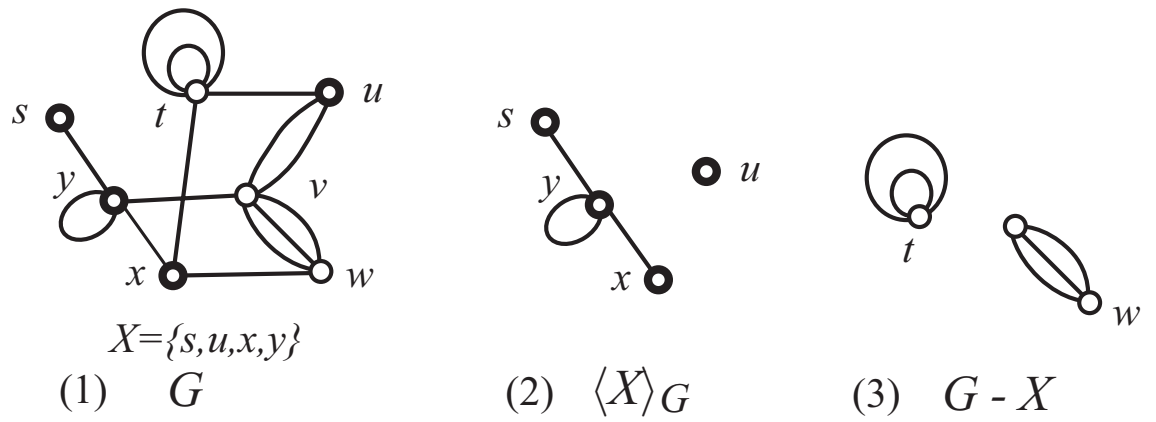

Figure 2: (1) A general graph $G$ and its vertex subset $X=\{s, u, x, y\}$; (2) The induced subgraph $\langle X\rangle_{G}$; (3) The subgraph $G-X$.

For a given graph $H$, if a graph $G$ has no induced subgraph isomorphic to $H$, then we say that $G$ is $H$-free. The star $K_{1,3}$ is often called a claw, and so a $K_{1,3}$-free graph $G$ is often called claw-free. 
xiv

We now give some important classes of simple graphs. Let $G$ be a simple graph. If any two distinct vertices of $G$ are adjacent, then $G$ is called a complete graph. The complete graph of order $n$ is written $K_{n}$ or $K(n)$. If the vertex set of $G$ is partitioned into $m$ disjoint non-empty subsets $V(G)=X_{1} \cup X_{2} \cup \cdots \cup X_{m}$ and any two vertices contained in distinct subsets are adjacent, then $G$ is called a complete $m$-partite graph. If $\left|X_{i}\right|=n_{i}, 1 \leq i \leq m$, then such a complete $m$-partite graph is denoted by $K\left(n_{1}, n_{2}, \ldots, n_{m}\right)$ or $K_{n_{1}, n_{2}, \ldots, n_{m}}$. In particular, $K\left(n_{1}, n_{2}\right)=K_{n_{1}, n_{2}}$ is called a complete bipartite graph. The complete bipartite graph $K(1, n)$ is called the star of order $n+1$. The path $P_{n}$ consists of $n$ vertices $v_{1}, v_{2}, \ldots, v_{n}$ and $n-1$ edges $v_{i} v_{i+1}, 1 \leq i \leq n-1$, where the two vertices $v_{1}$ and $v_{n}$ of degree one are called the endvertices of $P_{n}$. The cycle $C_{n}$ is obtained from the path $P_{n}$ by adding a new edge joining two endvertices of $P_{n}$.

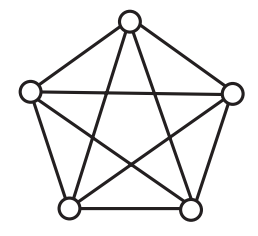

$K_{5}$

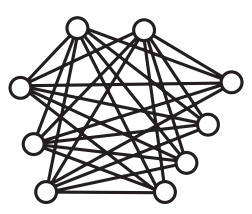

$K(2,3,4)$
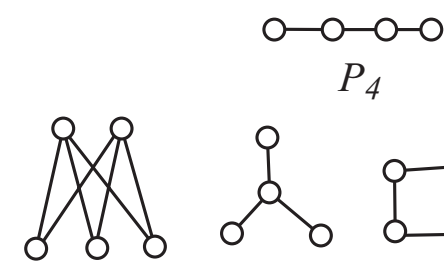

$K_{2,3}=K(2,3)$

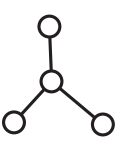

$K_{1,3} \quad C_{4}$

Figure 3: Some graphs.

For two graphs $G$ and $H$, the union $G \cup H$ is the graph with vertex set $V(G) \cup V(H)$ and edge set $E(G) \cup E(H)$, where $V(G) \cap V(H)=\emptyset$. The union $G \cup G$ is often denoted by $2 G$, and for any integer $n \geq 3$, we inductively define $n G$ by $(n-1) G \cup G$. The join $G+H$ denotes the graph with vertex $V(G) \cup V(H)$ and edge set

$$
E(G+H)=E(G) \cup E(H) \cup\{x y \mid x \in V(G) \text { and } y \in V(H)\},
$$

i.e., $G+H$ is obtained from $G \cup H$ by adding all the edges joining a vertex of $G$ to a vertex of $H$ (Figure 4 ). For a simple graph $G$, the complement of $G$, denoted by $\bar{G}$, is the graph with vertex set $V(G)$ such that two vertices are adjacent in $\bar{G}$ if and only if they are non-adjacent in $G$, in particular, $G \cup \bar{G}=K_{|G|}$.

We introduce some notions concerning paths and cycles in a graph. A walk in a graph $G$ is a sequence of vertices and edges

$$
\left(v_{0}, e_{1}, v_{1}, e_{2}, v_{2}, \ldots, v_{n-1}, e_{n}, v_{n}\right)
$$




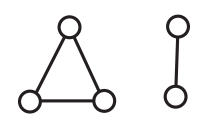

G $\quad H$

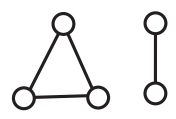

$G \cup H$

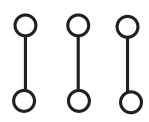

$3 H$

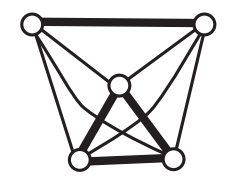

$G+H$

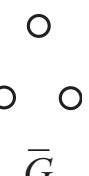

$\bar{G}$

Figure 4: The union, the join, and the complement.

such that every $e_{i}, 1 \leq i \leq n$, is an edge joining vertices $v_{i-1}$ and $v_{i}$. We say that this walk connects two vertices $v_{0}$ and $v_{n}$. Notice that a walk may pass through some edges and some vertices twice or more. The length of a walk is the number of edges appearing in it, and so the walk given above has length $n$. A walk that passes through every its edge exactly once is called a trail, and a walk that passes through every one of its vertices exactly once is called a path. It is easy to see that every path is a trail, but a trail is not always a path. We often denote a trail by a sequence $\left(e_{1}, e_{2}, \ldots, e_{n}\right)$ of edges and a path by a sequence $\left(v_{0}, v_{1}, \ldots, v_{n}\right)$ of vertices. If the initial vertex $v_{0}$ and the terminal vertex $v_{n}$ of a walk are the same, then we say that such a walk is closed. A closed trail is called a circuit, and a closed path is called a cycle. A cycle of even length is called an even cycle. An odd cycle, an even path and an odd path can be defined similarly.

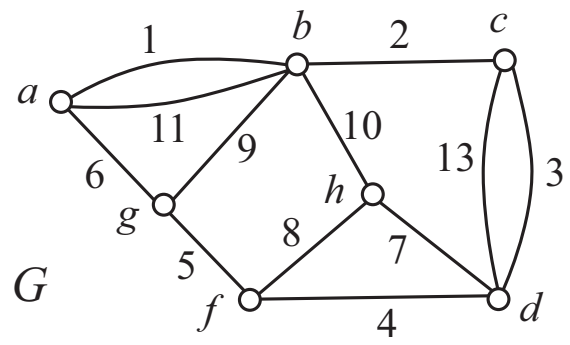

Figure 5: A path $(a, b, h, f, d)$ passing through 1 ; a cycle $(b, h, f, d, c, b)$ passing through 3 ; and a circuit $(9,6,11,10,7,3,2)$.

A graph $G$ is said to be connected if any two vertices are connected by a path in $G$. If $G$ is disconnected, that is, if $G$ is not connected, then $G$ is decomposed into components that are the maximal connected subgraphs of $G$. A connected simple graph having no cycle is called a tree, and a simple graph having no cycle is called a forest. So each component of a forest is a tree. A forest is called a linear forest if its all components are paths, and 
is called a star forest if its all components are stars.

Theorem 3 Every tree $T$ has the following properties (Figure 6).

(i) $\quad\|T\|=|T|-1$.

(ii) For every edge e not contained in $T, T+e$ has a unique cycle, which passes through e.

(iii) Thas at lest two leaves.

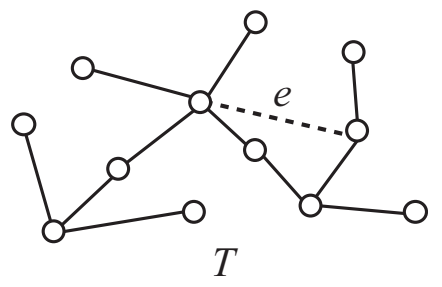

Figure 6: A tree $T$ with 6 leaves and and an edge $e$ not contained in $T$.

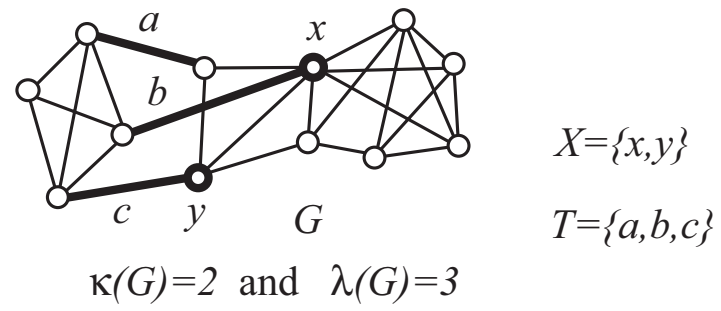

Figure 7: A 2-connected and 3-edge connected graph $G$, for which $G-X$ and $G-T$ are disconnected.

A connected graph $G$, which is not a complete graph, is $k$-connected if for every vertex set $X$ containing at most $k-1$ vertices, $G-X$ is connected. The connectivity of $G$ is defined to be the maximum $k$ for which $G$ is $k$-connected, and is denoted by $\kappa(G)$. Hence if $\kappa(G)=k$, then for every $X \subset V(G)$ with $|X| \leq k-1, G-X$ is connected and there exists a subset $S \subseteq V(G)$ with $|S|=k$ such that $G-S$ is not connected. The connectivity of the complete graph $K_{n}$ is defined to be $n-1$, that is, $\kappa\left(K_{n}\right)=n-1$. We can analogously define edge connectivity as follows. A graph $G$ is said to be $k$-edge connected if for every edge set $X$ containing at most $k-1$ edges, $G-X$ is connected. The edge-connectivity of $G$ is defined to be the maximum $k$ for which $G$ is $k$-edge connected, and is denote by $\lambda(G)$ (see Figure 7). The edge-connectivity of the complete graph $K_{n}$ is $n-1$ by 
the definition. It is easy to see that a connected multigraph $G$ satisfies the following inequality, which was found by Whitney [150].

$$
\kappa(G) \leq \lambda(G) \leq \delta(G)
$$

Equality holds in cubic graphs.

Proposition 4 For a cubic multigraph $G$, it follows that $\kappa(G)=\lambda(G)$.

The proof of the above lemma is left to the reader (see Figure 8).
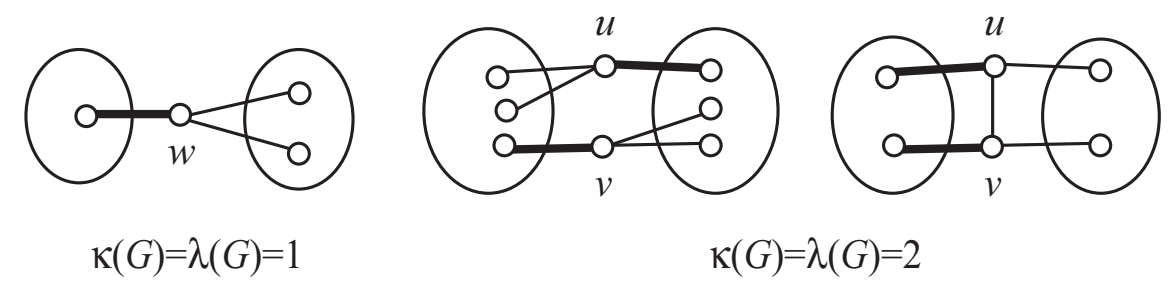

Figure 8: Connected cubic multigraphs.

Let $G$ be a connected graph. Then a vertex $v$ is called a cut vertex if $G-v$ is not connected, and an edge $e$ of $G$ is called a bridge or cut edge if $G-e$ is not connected. For a disconnected graph, we can similarly define a cut vertex and a bridge. More generally, a vertex subset $S \subset V(G)$ of a connected graph $G$ is called a vertex cut if $G-S$ is disconnected, and an edge subset $T \subseteq E(G)$ is called a edge cut if $G-T$ is disconnected.

For two disjoint subsets $X$ and $Y$ of $V(G)$, we denote the set of edges of $G$ joining a vertex of $X$ to a vertex of $Y$ by $E_{G}(X, Y)$, and the number of edges in $E_{G}(X, Y)$ by $e_{G}(X, Y)$, i.e.,

$$
e_{G}(X, Y)=\left|E_{G}(X, Y)\right|=\text { the number of edges of } G \text { joining } X \text { to } Y \text {. }
$$

It is easy to see that a minimal edge cut $T$ is expressed as

$$
T=E_{G}(X, Y) \text {, where } V(G)=X \cup Y, X \cap Y=\emptyset \text {. }
$$

A graph $G$ is called a bipartite graph if $V(G)$ is partitioned into two disjoint sets $A \cup B$ so that every edge of $G$ joins a vertex of $A$ to a vertex of $B$. The two sets $A$ and $B$ are called partite sets and $(A, B)$ is called a bipartition of $G$. Thus a bipartite graph with bipartition $(A, B)$ can be regarded as a subgraph of $K(|A|,|B|)$. Bipartite graphs plays an important role throughout this book, and the following proposition is useful. 
xviii

Proposition 5 (König [89]) A multigraph $G$ is a bipartite graph if and only if every cycle of $G$ has even length.

Proof. Suppose $G$ is a bipartite graph with bipartition $(A, B)$. Then every cycle of $G$ passes alternately through $A$ and $B$, and thus its length must be even.

Conversely, suppose that every cycle of $G$ is of even length. We may assume that $G$ is connected since if $G$ is disconnected, then its components can be considered separately. Assume that $G$ has two distinct vertices such that there are two paths connecting them whose lengths are odd and even, respectively. Choose such a pair $(x, y)$ of vertices so that the sum of lengths of such two paths connecting them is minimum. Let $P_{1}(x, y)$ and $P_{2}(x, y)$ are such paths connecting $x$ and $y$, whose length are odd and even respectively. If $P_{1}(x, y)$ and $P_{2}(x, y)$ meet a vertex $z$ on the way, then either $P_{1}(x, z)$ and $P_{2}(x, z)$ or $P_{1}(z, y)$ and $P_{2}(z, y)$ have distinct parities of lengths, which contradicts the choice of $(x, y)$ since the sum of their lengths is shorter than that of $P_{1}(x, y)$ and $P_{2}(x, y)$ (see Figure 9$)$. Thus $P_{1}(x, y)$ and $P_{2}(x, y)$ do not meet on the way, which implies that $P_{1}(x, y)+P_{2}(x, y)$ forms a cycle of odd length. This contradicts the assumption. Therefore, for any two distinct vertices of $G$, the length of every path connecting them has the same parity.

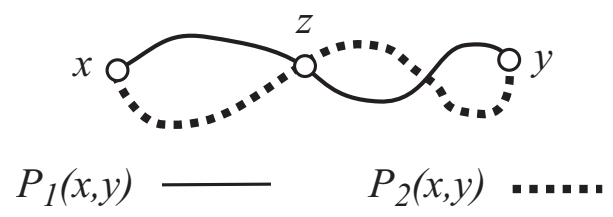

Figure 9: A path $P_{1}(x, y)$ of odd length and another path $P_{2}(x, y)$ of even length.

Choose one vertex $v$ of $G$, and define two vertex subsets of $G$ as follows: $A=\{x \in V(G) \mid$ There exists a path of even length connecting $v$ to $x\}$, $B=\{x \in V(G) \mid$ There exists a path of odd length connecting $v$ to $x\}$.

Then by the above argument, $A \cap B=\emptyset$, and it is easy to see that every edge of $G$ joins $A$ to $B$, and thus $G$ is a bipartite graph with bipartition $(A, B)$.

If a graph $G$ can be drawn in the plane so that no two edges intersect except at their endvertices, then $G$ is called an planar graph, and the graph drawn in the plane is called a plane graph (Figure 10). Note that a planar graph can be drawn in different ways as a plane graph, and a plane graph $G$ means one of such plane graphs. For example the two plane $G_{1}$ and $G_{2}$ in Figure 10 are isomorphic but different plane graphs. 


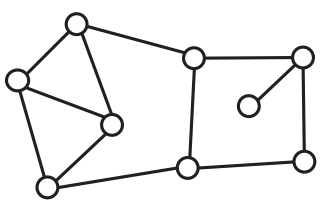

$G_{1}$

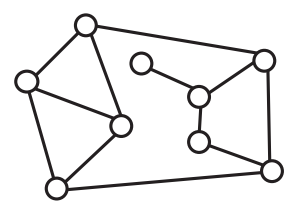

$G_{2}$

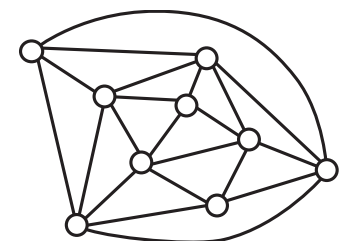

$G_{3}$

Figure 10: A plane graph $G_{1}$ with five regions, whose vertex degrees are 3,3,5,6 and 7; another plane graph representation $G_{2}$ of the same graph; and a maximal planar graph $G_{3}$.

Given a plane graph $G$ in the plane, a region of $G$ is a maximal portion of the plane in which any two points can be joined by a curve that does not cross any edge of $G$. The degree of a region $R$ in $G$, denoted by degree $(R)$, is defined as the number of edges of $G$ in the boundary of $R$. If a region of $G$ is not a triangle, we can add an edge to $G$ so that the resulting graph is still a plane graph. A planar graph is called a maximal planar graph if we cannot add any new edge without violating the planarity (Figure 10). Hence every region of a maximal planar graph is a triangle, and inversely, a plane graph all of whose regions are triangles is a maximal planar graph.

By the same argument as in the proof of Handshaking Theorem, we obtain the following.

Lemma 6 For a connected plane graph $G$, let $\Omega(G)$ be the set of regions of G. Then

$$
\sum_{R \in \Omega(G)} \operatorname{degree}(R)=2\|G\|
$$

The following theorem is very important for planar graphs.

Theorem 7 (Euler's Formula) For a connected plane graph $G$, let $p=|G|$, $q=\|G\|$ and $r$ be the number of regions of $G$. Then

$$
p-q+r=2 \text {. }
$$

Proof. We prove the theorem by induction on $\|G\|$ for all connected plane graphs $G$ of fixed order $n \geq 2$. Since $G$ is connected, a smallest such plane graph is a tree. If $G$ is a tree, then $r=1$ and $q=p-1$ by Theorem 3, and so

$$
p-q+r=p-(p-1)+1=2 \text {. }
$$


Thus we may assume that $G$ is not a tree. Then $G$ has a cycle $C$. Let $e$ be an edge of $C$. Then $G^{\prime}=G-e$ is a connected plane graph, and so by the inductive hypotheses, we have

$$
p^{\prime}-q^{\prime}-r^{\prime}=2,
$$

where $p^{\prime}=\left|G^{\prime}\right|, \quad q^{\prime}=\left\|G^{\prime}\right\|$ and $r^{\prime}=$ the number of regions of $G^{\prime}$. By substituting $p^{\prime}=p, q^{\prime}=q-1$ and $r^{\prime}=r-1$ in the above equation, we have

$$
p-(q-1)+(r-1)=p-q+r=2,
$$

which is the desired equation. Hence the theorem is proved.

Using the above theorem, we can easily show the following theorem.

Theorem 8 Let $G$ be a connected plane simple graph, and let $p=|G|$ and $q=\|G\|$. Then

(i) $q \leq 3 p-6$, where the equality holds if $G$ is a maximal planar graph.

(ii) If $G$ has no triangle, in particular, if $G$ is a bipartite plane graph, then $q \leq 2 p-4$.

Proof. Let $\Omega$ be the set of regions of $G$. By the lemma, we obtain

$$
3 r \leq \sum_{R \in \Omega} \operatorname{degree}(R)=2\|G\|=2 q .
$$

Hence $r \leq(2 q) / 3$. By substituting this into Euler's Formula, we have

$$
p-q+\frac{2 q}{3} \geq 2
$$

Hence $3 p-6 \geq q$. If $G$ has no triangle, then every region has degree at least four, and so

$$
4 r \leq \sum_{R \in \Omega} \operatorname{degree}(R)=2\|G\|=2 q .
$$

This inequality together with Euler's Formula implies $2 p-4 \geq q$.

A vertex coloring of a graph $G$ is an assignment of positive integers $1,2, \ldots, k$ to the vertices of $G$ such that no two adjacent vertices have the same number. Such a vertex coloring is sometimes called a proper vertex coloring. If there exists a vertex coloring with $k$-colors, then we say $G$ is $k$-colorable. The minimum number $k$ for which $G$ is $k$-colorable is called the chromatic number of $G$ and is denoted by $\chi(G)$. Analogous definitions for edges can be introduced as follows: An edge coloring of a graph $G$ is an assignment of positive integers $1,2, \ldots, k$ to the edges of $G$ such that no 
two adjacent edges have the same number. Such an edge coloring is said to be proper. We mainly consider proper edge colorings in this book. If there exists a proper edge coloring with $k$-colors, then we say $G$ is $k$-edge colorable. The minimum number $k$ for which $G$ is $k$-edge colorable is called the chromatic index of $G$ and denoted by $\chi^{\prime}(G)$. For edge coloring, the following famous theorem holds.

Theorem 9 (Vizing [144]) A simple graph $G$ can be properly edge colored with $\Delta(G)+1$ colors. In particular, $\chi^{\prime}(G)=\Delta(G)$ or $\Delta(G)+1$.

We introduced connectivity and edge-connectivity to evaluate the strongness of a graph. There are some other concepts which might evaluate the strongness of a graph in a different point of view. We explain two of them below.

The toughness of a connected non-complete graph $G$, denoted by $\operatorname{tough}(G)$, is defined as

$$
\operatorname{tough}(G)=\min \left\{\frac{|X|}{\omega(G-X)} \mid \omega(G-X) \geq 2, \emptyset \neq X \subseteq V(G)\right\},
$$

where $\omega(G-X)$ denotes the number of components of $G-X$. A graph $G$ is said to be $t$-tough if $\operatorname{tough}(G) \geq t$. Hence, if $G$ is $t$-tough, then for a subset $S \subset V(G)$ with $\omega(G-S) \geq 2$, it follows that

$$
\omega(G-S) \leq \frac{|S|}{\operatorname{tough}(G)} \leq \frac{|S|}{t}
$$

The binding number $\operatorname{bind}(G)$ of a graph $G$, which was introduced by Anderson [12], is defined by

$$
\operatorname{bind}(G)=\min \left\{\frac{\left|N_{G}(X)\right|}{|X|} \mid \emptyset \neq X \subseteq V(G), N_{G}(X) \neq V(G)\right\} .
$$

Thus for every subset $\emptyset \neq S \subset V(G)$ with $N_{G}(S) \neq V(G)$, it follows that $\left|N_{G}(S)\right| \geq \operatorname{bind}(G)|S|$.

We conclude this introduction with two results on circuits and cycles of graphs. For a graph $G$, a circuit that passes through every edge of $G$ precisely once is called an Euler circuit of $G$. A graph having an Euler circuit is called an Eulerian graph. On the other hand, a cycle that passes through every vertex exactly once is called an Hamiltonian cycle. A graph having an Hamiltonian graph is called an Hamiltonian graph. It is easy to see that if a graph $G$ is Hamiltonian, then $\operatorname{tough}(G) \geq 1$. So the non-existence of a Hamiltonian cycle in $G_{4}$ in Figure 11 is shown by $3=\omega\left(G_{4}-X\right)>|X|=2$ for a set $X$, which implies $\operatorname{tough}\left(G_{4}\right)<1$. 
xxii

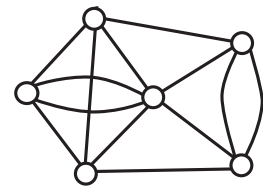

$G_{1}$

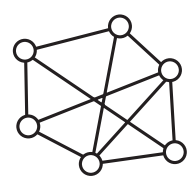

$G_{2}$

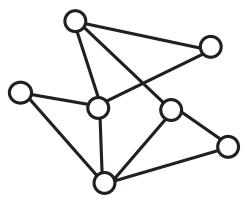

$G_{3}$

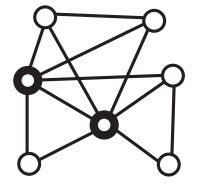

$G_{4}$

Figure 11: An Eulerian multigraph $G_{1}$, a non-Eulerian graph $G_{2}$, a Hamiltonian graph $G_{3}$, and a non-Hamiltonian graph $G_{4}$ with a set $X$ consisting of two bold vertices.

Theorem 10 A connected multigraph is Eulerian if and only if every its vertex has even degree.

Proof. Assume that $G$ has an Eulerian circuit $C$. We move along $C$ and remove an edge when we pass through it. Then for every vertex $v$, when we pass through it, two edges incident with $v$ are removed, and in the final stage, all the edges incident with $v$ are removed, and thus the degree of $v$ is even.

Conversely, assume that every vertex of $G$ has even degree. Then we prove that $G$ has an Eulerian circuit by induction on $\|G\|$. If $\|G\|=2$, then $G$ consists of two vertices and two multiple edges joining them, and so $G$ has an Eulerian circuit. Assume that $\|G\| \geq 3$. Since every vertex has degree at least two, $G$ has a cycle $C$. Every non-trivial component $D$ of $G-E(C)$ has vertices of even degrees, where a non-trivial component means a component having at least one edge. Hence by induction, $D$ has an Eulerian circuit $\operatorname{Ecir}(D)$. We arrange all the non-trivial components of $G-E(C) D_{1}, D_{2}, \ldots, D_{m}$ in the order that $C$ passes through at least one of its vertices. Then we can obtain an Eulerian circuit of $G$ as follows: Start at a vertex $w$ of $C$ and go along $C$ to reach a vertex $v_{1}$ of $D_{1}$, then pass through $\operatorname{Ecir}\left(D_{1}\right)$ and come back to $v_{1}$ and go along $C$ to reach a vertex $v_{2}$ of $D_{2}$, then pass through $E \operatorname{cir}\left(D_{2}\right)$, and continue this procedure until we return to $w$ (see Figure $12(1)$ ). Then we can obtain a circuit that passes through all the edges of $G$.

We now give a well-known theorem about Hamiltonian cycles, where alpha $(G)$ denotes the cardinality of a maximum independent set of a graph $G$.

Theorem 11 (Chvátal and Erdős, (1972)) Every connected simple graph $G$ with $\kappa(G) \geq \alpha(G)$ is Hamiltonian unless $G=K_{2}$.

Proof. Let $k=\kappa(G)$. Then we may assume $k \geq 2$ since $\alpha(G)=1$ implies 
xxiii

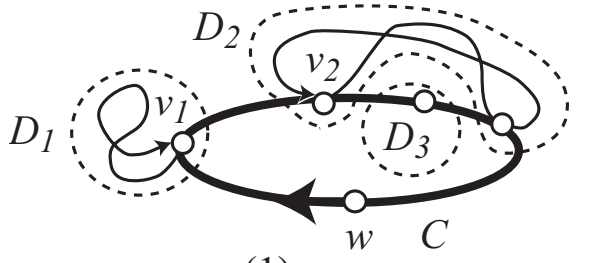

(1)

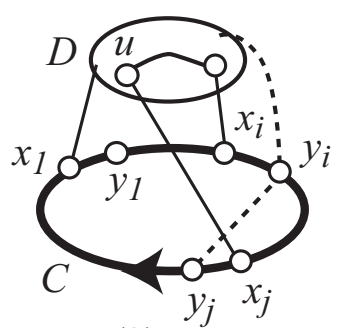

(2)

Figure 12: (1) An Eulerian multigraph $G$; (2) a Hamiltonian graph $G$.

$G=K_{n}$ with $n \geq 3$. Let $C$ be a longest cycle of $G$, which is considered as a directed cycle. We may assume that $C$ is not Hamiltonian. Since $\delta(G) \geq \kappa(G)=k$, the length of $C$ is at least $k+1$ (see Exercise 2). Let $D$ be a component of $G-V(C)$. Since $\kappa(G)=k$, there exist at least $k$ distinct vertices in $C$ that are joined to $D$ by edges of $G$. Denote these vertices by $x_{1}, x_{2}, \ldots, x_{k}$ (see Figure $12(2)$ ). Let $y_{i}$ be the vertex $C$ immediately following $x_{i}$ for all $1 \leq i \leq k$. Then $y_{i}$ is not joined to $D$ by an edge of $G$ since otherwise we can easily find a cycle longer than $C$, which is a contradiction. Moreover, if any two distinct vertices $y_{i}$ and $y_{j}$ are adjacent in $G$, then we can find a cycle longer than $C$ which starts with a vertex $u$ of $D$ adjacent to $x_{j}$. It goes to $x_{j}$, proceeds along $C$ to $y_{i}$, goes to $y_{j}$, proceeds along $C$ to $x_{i}$, goes to a vertex of $D$ adjacent to $x_{i}$, and then goes to $u$ in $D$. This contradicts the choice of $C$. Hence $y_{i}$ and $y_{j}$ are not adjacent in $G$. Therefore $\left\{y_{1}, y_{2}, \ldots, y_{k}\right\} \cup\{v\}, \quad v \in V(D)$ is an independent set of $G$ with cardinality $k+1$, which contradicts $\alpha(G) \leq \kappa(G)=k$.

\section{Exercises}

Exercise 1 Prove that if $G$ is a connected cubic multigraph, then $\kappa(G)=$ $\lambda(G)$.

Exercise 2 (i) Show that every simple graph with $\delta(G) \geq 2$ has a cycle.

(ii) Prove that every simple graph with $\delta(G) \geq 2$ has a cycle with length at least $\delta(G)+1$.

Exercise 3 Prove Theorem 3.

Exercise 4 Let $G$ be a connected graph and $F$ be a spanning forest of $G$. Show that the number of components of $F$ is equal to $|G|-\|F\|$.

Exercise 5 Let $G$ be a connected simple graph. Show that $\kappa(G) \leq \lambda(G) \leq$ $\delta(G)$. 
xxiv

Exercise 6 Find a 4-regular simple graph $G$ with $\kappa(G)=2$ and $\lambda(G)=4$.

Exercise 7 Let $r \geq 2$ be an integer. Prove that every connected $r$-regular bipartite multigraph is 2-edge connected.

Exercise 8 Show that Lemma 6 holds.

Exercise 9 Prove that every connected graph $G$ satisfies $\kappa(G) \geq 2 \cdot \operatorname{tough}(G)$.

Exercise 10 Let $G$ be a connected simple graph. Show that if $\operatorname{bind}(G)>0$, then $\left|N_{G}(X)\right| \geq|G|-(|G|-|X|) / \operatorname{bind}(G)$ for all $\emptyset \neq X \subseteq V(G)$. 


\section{Chapter 1}

\section{Matchings and 1-Factors}

\subsection{Matchings in Bipartite Graphs}

In this section we investigate matchings in bipartite graphs and consider results which will play an important role throughout this book. The reader is referred to the book [108] by Lovász and Plummer for a more detailed treatment of matchings.

Two edges of a general graph are said to be independent if they have no common endvertex and none of them is a loop. A matching in a general graph $G$ is a set of pairwise independent edges of $G$ (Figure 1.1).

In this chapter a bipartite simple graph is briefly called a bipartite graph, and a bipartite graph which may have multiple edges is called a bipartite multigraph. If $M$ is a matching in a general graph $G$, then the subgraph of $G$ induced by $M$, denoted by $\langle M\rangle_{G}$ or $\langle M\rangle$, is the subgraph of $G$ whose edge set is $M$ and whose vertex set consists of the vertices incident with some edge in $M$. In particular, every vertex of $\langle M\rangle$ has degree one. Thus it is possible to define a matching as a subgraph whose vertices all have degree one, and we often regard a matching $M$ as its induced subgraph $\langle M\rangle$.

Let $M$ be a matching in a general graph $G$. Then a vertex of $G$ is said to be saturated or covered by $M$ if it is incident with an edge of $M$; otherwise, it is said to be unsaturated or not covered by $M$. If every vertex of a vertex subset $U$ of $G$ is saturated by $M$, then we say that $U$ is saturated by $M$. A matching with maximum cardinality is called a maximum matching. A matching that saturates all the vertices of $G$ is called a perfect matching or a 1-factor of $G$ (Figure 1.1). It is easy to see that a maximal matching, which is a maximal set of independent edges, is not a maximum matching, and a maximum matching is not a perfect matching. On the other hand, if a matching in a bipartite graph saturates one of its partite sets, then it is 


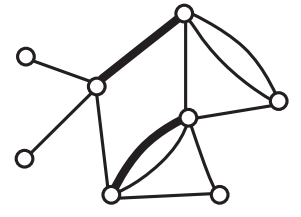

$M_{1}$

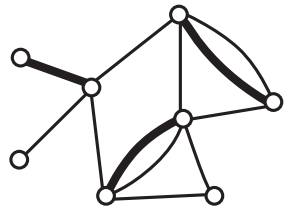

$M_{2}$

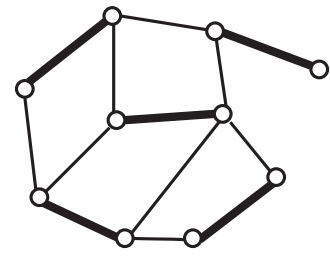

$M_{3}$

Figure 1.1: A maximal matching $M_{1}$; a maximum matching $M_{2}$; and a perfect matching $M_{3}$.

clearly a maximum matching.

For a matching $M$, we write

$\|M\|=$ the size of $\langle M\rangle=$ the number of edges in $M$,

$|M|=$ the order of $\langle M\rangle=$ the number of vertices saturated by $M$.

We first give a criterion for a bipartite multigraph to have a matching that saturates one of its partite sets. Then we apply this criterion to some problems on matchings in bipartite graphs.

The criterion mentioned above is given in the following theorem, which was independently found by Hall (1935) and König (1931) and is called the Marriage Theorem. This theorem plays an important role throughout this book, and the proof given here is due to Halmos and Vaughan [54]. An algorithm for finding a maximum matching in a bipartite graph will be given in Algorithm 1.3.7.

Theorem 1.1.1 (Marriage Theorem, Hall [55], König, [90]) Let $G$ be a bipartite multigraph with bipartition $(A, B)$. Then $G$ has a matching that saturates $A$ if and only if

$$
\left|N_{G}(S)\right| \geq|S| \quad \text { for all } S \subseteq A
$$

Proof. We first construct a bipartite graph $H$ from the given bipartite multigraph $G$ by replacing all the multiple edges of $G$ by single edges. Then it is obvious that $G$ has the desired matching if and only if $H$ has such a matching, and that $G$ satisfies (1.1) if and only if $H$ satisfies it. Therefore we may assume that $G$ itself has no multiple edges by considering $H$ as the given bipartite graph. 

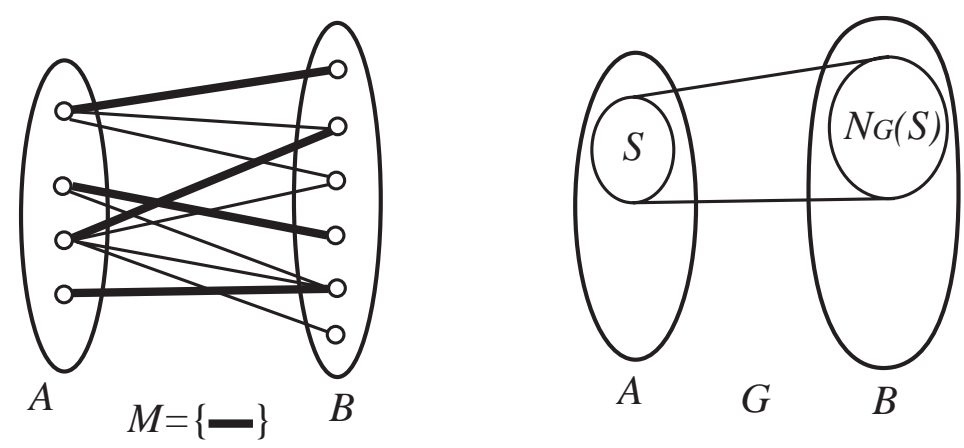

Figure 1.2: A matching $M$ that saturates $A ; \quad S$ and $N_{G}(S)$.

Suppose that $G$ has a matching $M$ that saturates $A$ (Figure 1.2). Then for every subset $S \subseteq A$, we have

$$
\left|N_{G}(S)\right| \geq\left|N_{M}(S)\right|=|S| .
$$

We next prove sufficiency by induction on $|G|$. It is clear that we may assume $|A| \geq 2$. We consider the following two cases.

Case 1. There exists $\emptyset \neq S \subset A$ such that $\left|N_{G}(S)\right|=|S|$.

Let $H=\left\langle S \cup N_{G}(S)\right\rangle_{G}$ and $K=\left\langle(A-S) \cup\left(B-N_{G}(S)\right)\right\rangle_{G}$ be induced subgraphs of $G$ (Figure 1.3). It is clear that $H$ satisfies condition (1.1), and so $H$ has a matching $M_{H}$ that saturates $S$ by induction. For every subset $X \subseteq A-S$, we have

$$
\left|N_{K}(X)\right|=\left|N_{G}(X \cup S)\right|-\left|N_{G}(S)\right| \geq|X \cup S|-|S|=|X| .
$$

Hence, by induction, $K$ also has a matching $M_{K}$ that saturates $A-S$. Therefore $M_{H} \cup M_{K}$ is the desired matching in $G$ which saturates $A$.

Case 2. $\left|N_{G}(S)\right|>|S|$ for all $\emptyset \neq S \subset A$.

Let $e=a b \quad(a \in A, b \in B)$ be an edge of $G$, and let $H=G-\{a, b\}$. Then for every subset $\emptyset \neq X \subseteq A-\{a\}$, by the assumption of this case, we have

$$
\left|N_{H}(X)\right| \geq\left|N_{G}(X) \backslash\{b\}\right|>|X|-1,
$$

which implies $\left|N_{H}(X)\right| \geq|X|$. Therefore $H$ has a matching $M^{\prime}$ that saturates $A-\{a\}$ by induction. Then $M^{\prime}+e$ is the desired matching in $G$. Consequently the theorem is proved.

If the edge set $E(G)$ of a multigraph $G$ is partitioned into its disjoint 1-factors $E(G)=F_{1} \cup F_{2} \cup \cdots \cup F_{r}$, where each $F_{i}$ is a 1-factor of $G$, then 

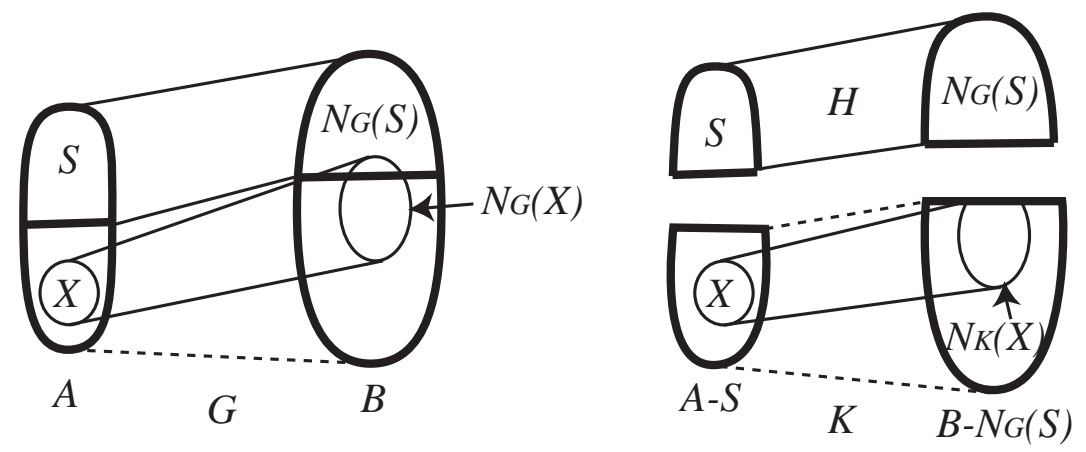

Figure 1.3: The induced subgraphs $H$ and $K$.

we say that $G$ is $\mathbf{1 - f a c t o r a b l e , ~ a n d ~ c a l l ~ t h i s ~ p a r t i t i o n ~ a ~} \mathbf{1}$-factorization of $G$. It is trivial that if $G$ is 1-factorable, then $G$ is regular.

We now give some results on matchings in bipartite graphs, most of which can be proved by making use of the Marriage Theorem. We begin with the following famous theorem, which was obtained by König in 1916 before the Marriage Theorem. However our proof depends on the Marriage Theorem.

Theorem 1.1.2 (König [89]) Every regular bipartite multigraph is 1-factorable, in particular, it has a 1-factor (Figure 1.4).
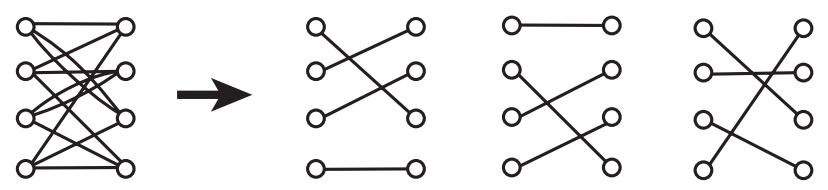

Figure 1.4: A 3-regular bipartite multigraph and its 1-factorization.

Proof. Let $G$ be an $r$-regular bipartite multigraph with bipartition $(A, B)$. Then $|A|=|B|$ since $r|A|=e_{G}(A, B)=r|B|$. For every subset $X \subseteq A$, we have

$$
r|X|=e_{G}\left(X, N_{G}(X)\right) \leq r\left|N_{G}(X)\right|,
$$

and so $|X| \leq\left|N_{G}(X)\right|$. Hence by the Marriage Theorem, $G$ has a matching $M$ saturating $A$, which must saturate $B$ since $|A|=|B|$. Thus $M$ is a 1-factor of $G$.

It is obvious that $G-M$ is a $(r-1)$-regular bipartite multigraph, and so it has a 1-factor by the same argument as above. By repeating this procedure, we can obtain a 1-factorization of $G$. 
Lemma 1.1.3 (König [89]) Let $G$ be a bipartite multigraph with maximum degree $\Delta$ and let $(A, B)$ be the bipartition of $G$ such that $|A| \geq|B|$. Then there exists a $\Delta$-regular bipartite multigraph which contains $G$ as a subgraph and one of whose bipartite sets is equal to $A$.
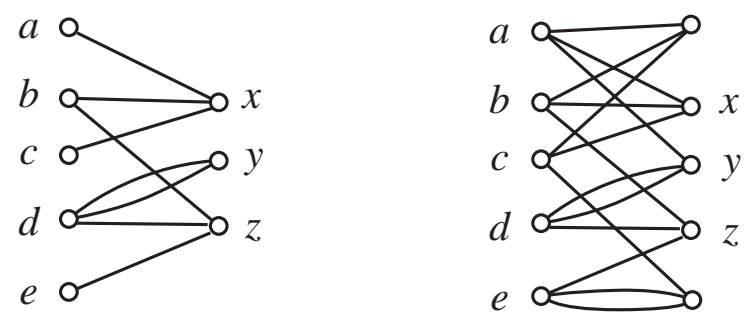

Figure 1.5: A bipartite multigraph and a regular bipartite graph containing it.

Proof. By adding $|A|-|B|$ new vertices to $B$ if $|A|>|B|$, we obtain a bipartite graph with bipartition $\left(A, B^{\prime}\right)$ such that $|A|=\left|B^{\prime}\right|$. Add new edges joining vertices in $A$ to vertices in $B^{\prime}$ whose degrees are less than $\Delta$, one at a time until no new edge can be added. We show that by this procedure, we get the desired $\Delta$-regular bipartite multigraph.

Suppose that the bipartite multigraph $H$ obtained in this way has a vertex $a \in A$ such that $\operatorname{deg}_{H}(a)<\Delta$. Then there exists a vertex $b \in B^{\prime}$ such that $\operatorname{deg}_{H}(b)<\Delta$ because

$$
\sum_{x \in B^{\prime}} \operatorname{deg}_{H}(x)=e_{H}\left(B^{\prime}, A\right)=\sum_{x \in A} \operatorname{deg}_{H}(x)<\Delta|A|=\Delta\left|B^{\prime}\right| .
$$

Hence we can add a new edge $a b$ to $H$, which is a contradiction. Therefore every vertex of $A$ has degree $\Delta$ in $H$, which implies that every vertex of $B^{\prime}$ has degree $\Delta$ as $|A|=\left|B^{\prime}\right|$. Consequently, $H$ is the desired $\Delta$-regular bipartite multigraph.

The next corollary says that the chromatic index of a bipartite multigraph $G$ is equal to the maximum degree of $G$.

Corollary 1.1.4 (König [89]) Let $G$ be a bipartite multigraph with maximum degree $\Delta$. Then $E(G)$ can be partitioned into $E(G)=E_{1} \cup E_{2} \cup \cdots \cup E_{\Delta}$ such that each $E_{i}(1 \leq i \leq \Delta)$ is a matching in $G$.

Proof. By Lemma 1.1.3, there exists a $\Delta$-regular bipartite multigraph $H$ which contains $G$ as a subgraph. Then by Theorem 1.1.2, $E(H)$ can be 
partitioned into 1-factors $F_{1} \cup F_{2} \cup \cdots \cup F_{\Delta}$. It is obvious that $F_{i} \cap E(G)(1 \leq$ $i \leq \Delta)$ is a matching in $G$, and their union is equal to $E(G)$. Therefore the corollary holds.

We now show some various properties of matchings in bipartite graphs. Some of them are generalizations of the Marriage Theorem and some of them give properties that only bipartite graphs possess.

Theorem 1.1.5 A bipartite multigraph $G$ has a matching that saturates all the vertices of degree $\Delta(G)$.

Proof. Let $\Delta=\Delta(G)$. By Lemma 1.1.3, there exists a $\Delta$-regular bipartite multigraph $H$ which contains $G$ as a subgraph. Then by Theorem 1.1.2, $H$ has a 1-factor $F$. It is easy to see that $F \cap E(G)$ is a matching of $G$ that saturates all the vertices $v$ of $G$ with degree $\Delta$ since every edge of $H$ incident with $v$ is an edge of $G$.

Theorem 1.1.6 Let $G$ be a bipartite multigraph with bipartition $(A, B)$. If $\left|N_{G}(S)\right|>|S|$ for all $\emptyset \neq S \subset A$, then for each edge e of $G, G$ has a matching that saturates $A$ and contains $e$.

Proof. Let $e=a b(a \in A, b \in B)$ be any edge of $G$, and $H=G-\{a, b\}$. Then for every subset $\emptyset \neq X \subseteq A-\{a\}$, we have

$$
\left|N_{H}(X)\right| \geq\left|N_{G}(X) \backslash\{b\}\right|>|X|-1,
$$

which implies $\left|N_{H}(X)\right| \geq|X|$. Hence by the Marriage Theorem, $H$ has a matching $M$ saturating $A-\{a\}$. Thus $M+e$ is the desired matching of $G$ which saturates $A$ and contains $e$.

Theorem 1.1.7 Let $G$ be a bipartite graph with bipartition $(A, B)$ such that $|A|=|B|$. Then the following three statements are equivalent.

(i) $G$ is connected, and for each edge $e, G$ has a 1-factor containing e.

(ii) For every subset $\emptyset \neq X \subset A,\left|N_{G}(X)\right|>|X|$.

(iii) For every two vertices $a \in A$ and $b \in B, G-\{a, b\}$ has a 1-factor.

Proof. (i) $\Rightarrow$ (ii) Suppose that $\left|N_{G}(Y)\right| \leq|Y|$ for some subset $\emptyset \neq Y \subset A$. Since $G$ has a 1-factor $F$, we have $\left|N_{G}(Y)\right|=|Y|$ as $\left|N_{G}(Y)\right| \geq\left|N_{F}(Y)\right|=$ $|Y|$. Since $G$ is connected, $G$ has an edge $e$ joining a vertex in $A-Y$ to a vertex in $N_{G}(Y)$. However there exists no 1-factor containing $e$ since $|Y|=\left|N_{G}(Y)\right|$. This contradicts (i). Thus (ii) holds.

(ii) $\Rightarrow$ (iii) Let $H=G-\{a, b\}$ and $X \subseteq A-\{a\}$. Then

$$
\left|N_{H}(X)\right|=\left|N_{G}(X) \backslash\{b\}\right|>|X|-1,
$$


which implies $\left|N_{H}(X)\right| \geq|X|$. Hence $H$ has a matching saturating $A-\{a\}$, which is obviously a 1 -factor of $H$ as $|A-\{a\}|=|B-\{b\}|$.

(iii) $\Rightarrow$ (i) Let $e=a b(a \in A, b \in B)$ be an edge of $G$. Since $G-\{a, b\}$ has a 1 -factor $F, G$ has a 1 -factor $F+e$, which contains $e$. The proof of connectivity will be left to the reader.

Theorem 1.1.8 Suppose that a bipartite multigraph $G$ with bipartition $(A, B)$ has a matching saturating $A$. Then there exists a vertex $v \in A$ possessing the property that every edge incident with $v$ is contained in a matching in $G$ saturating $A$.

Proof. We prove the theorem by induction on $|A|$. It is clear that we may assume $|A| \geq 2$.

If $\left|N_{G}(X)\right|>|X|$ for all $\emptyset \neq X \subset A$, then each vertex in $A$ has the required property by Theorem 1.1.6. Hence we may assume that $\left|N_{G}(S)\right| \leq$ $|S|$ for some $\emptyset \neq S \subset A$. Since $G$ has a matching saturating $A$, it follows from the Marriage Theorem that $\left|N_{G}(S)\right|=|S|$.

By the inductive hypothesis, the induced subgraph $H=\left\langle S \cup N_{G}(S)\right\rangle_{G}$ has a vertex $v \in S$ such that every edge of $H$ incident with $v$ is contained in a matching in $H$ saturating $S$ (Figure 1.6). Note that every edge of $G$ incident with $v$ is contained in $H$.
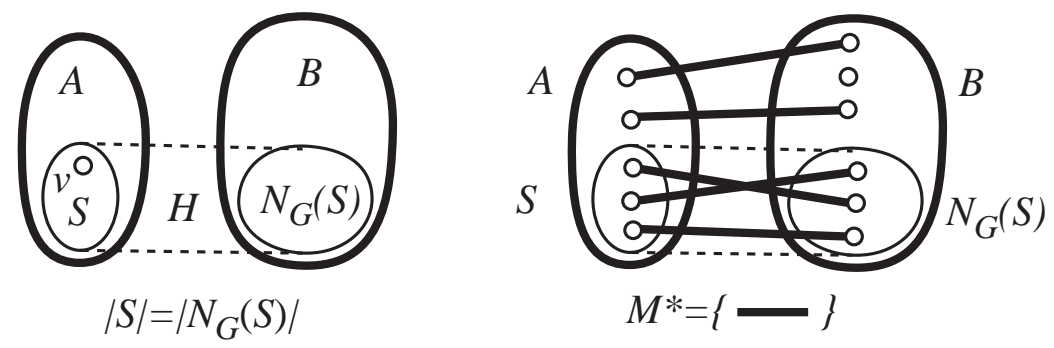

Figure 1.6: A bipartite multigraph $G$ and its subgraph $H$; A matching $M$ in $G$ saturating $A$.

Let $M$ be a matching in $G$ saturating $A$, and $M_{H}$ be a matching in $H$ saturating $S$. Then it is easy to see that $(M \backslash E(H)) \cup M_{H}$ is a matching in $G$ that saturates $A$. Therefore the vertex $v$ in $S$ has the desired property.

Proposition 1.1.9 Let $G$ be a bipartite multigraph with bipartition $(A, B)$ such that $\left|N_{G}(X)\right| \geq|X|$ for every $X \subseteq A$. If two subsets $S, T \subseteq A$ satisfy 


$$
\begin{aligned}
\left|N_{G}(S)\right|= & |S| \text { and }\left|N_{G}(T)\right|=|T| \text {, then } \\
& \left|N_{G}(S \cup T)\right|=|S \cup T| \text { and }\left|N_{G}(S \cap T)\right|=|S \cap T| .
\end{aligned}
$$

In particular, if such subsets exist, there exists a unique maximum subset $A_{0} \subseteq$ A such that $\left|N_{G}\left(A_{0}\right)\right|=\left|A_{0}\right|$

Proof. Since $N_{G}(S \cup T)=N_{G}(S) \cup N_{G}(T)$ and $N_{G}(S) \cap N_{G}(T) \supseteq N_{G}(S \cap T)$, we have

$$
\begin{aligned}
\left|N_{G}(S \cup T)\right| & =\left|N_{G}(S)\right|+\left|N_{G}(T)\right|-\left|N_{G}(S) \cap N_{G}(T)\right| \\
& \leq|S|+|T|-\left|N_{G}(S \cap T)\right| \\
& \leq|S|+|T|-|S \cap T|=|S \cup T| .
\end{aligned}
$$

On the other hand, $\left|N_{G}(S \cup T)\right| \geq|S \cup T|$ by the assumption. Hence $\mid N_{G}(S \cup$ $T)|=| S \cup T \mid$, and also $\left|N_{G}(S \cap T)\right|=|S \cap T|$ by the above inequality.

The following theorem is a generalization of the Marriage Theorem since the subgraph $H$ with $f(x)=1$ given in the following theorem is nothing but a matching.

Theorem 1.1.10 (Generalized Marriage Theorem ) Let $G$ be a bipartite graph with bipartition $(A, B)$, and $f: A \rightarrow \mathbb{N}$ be a function. Then $G$ has a subgraph $H$ such that

$$
\begin{array}{ll}
\operatorname{deg}_{H}(a)=f(a) & \text { for all } a \in A, \text { and } \\
\operatorname{deg}_{H}(b) \leq 1 & \text { for all } b \in B
\end{array}
$$

if and only if

$$
\left|N_{G}(S)\right| \geq \sum_{x \in S} f(x) \quad \text { for all } S \subseteq A
$$

Proof. We first assume that $G$ has a subgraph $H$ that satisfies (1.3) and (1.4) (Figure 1.7). Then for every $S \subseteq A$, we have

$$
\left|N_{G}(S)\right| \geq\left|N_{H}(S)\right|=\sum_{x \in S} \operatorname{deg}_{H}(x)=\sum_{x \in S} f(x) .
$$

Hence (1.5) holds.

In order to prove the sufficiency, we construct a new bipartite graph $G^{*}$ with bipartition $A^{*} \cup B$ as follows: For every vertex $v \in A$, define $f(v)$ 

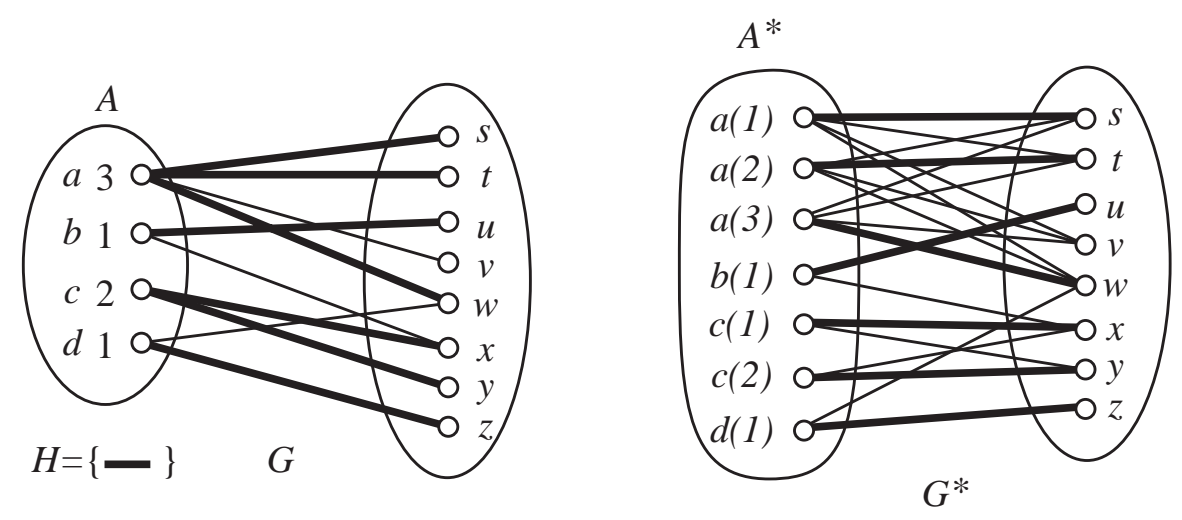

Figure 1.7: A bipartite graph $G$ and its subgraph $H$, where numbers denote $f(v)$; and $G^{*}$.

vertices $v(1), v(2), \ldots, v(f(v))$ of $A^{*}$, and connect them to all the vertices of $N_{G}(v)$ by edges (Figure 1.7). Then

$$
\left|A^{*}\right|=\sum_{x \in A} f(x) \quad \text { and } \quad N_{G^{*}}(v(i))=N_{G}(v) \quad \text { for all } v(i) \in A^{*} .
$$

For every subset $\emptyset \neq S^{*} \subseteq A^{*}$, let $S=\left\{x \in A \mid x(i) \in S^{*}\right.$ for some $\left.i\right\}$. Then we have by $(1.5)$

$$
\left|N_{G^{*}}\left(S^{*}\right)\right|=\left|N_{G}(S)\right| \geq \sum_{x \in S} f(x) \geq\left|S^{*}\right|
$$

Hence by the Marriage Theorem, $G^{*}$ has a matching $M^{*}$ that saturates $A^{*}$. It is clear that the subgraph $H$ of $G$ induced by $M^{*}$ satisfies

$$
\operatorname{deg}_{H}(a)=f(a) \text { for all } a \in A, \text { and } \operatorname{deg}_{H}(b) \leq 1 \text { for all } b \in B .
$$

Hence the theorem is proved.

The next theorem gives a formula for the size of maximum matching of a bipartite graph. Moreover, this formula includes the Marriage Theorem as its corollary since $\left|N_{G}(S)\right| \geq|S|$ for all $S \subseteq A$ implies $\|M\|=|A|$ as $|\emptyset|-\left|N_{G}(\emptyset)\right|=0$

Theorem 1.1.11 (Ore [123]) Let $G$ be a bipartite multigraph with bipartition $(A, B)$, and $M$ a maximum matching in $G$. Then the size of $M$ is given by

$$
\|M\|=|A|-\max _{S \subseteq A}\left\{|S|-\left|N_{G}(S)\right|\right\} .
$$


Proof. Let $M$ be a maximum matching in $G, d=\max _{S \subseteq A}\left\{|S|-\left|N_{G}(S)\right|\right\}$, and $X \subseteq A$ such that $|X|-\left|N_{G}(X)\right|=d$. Then

$$
\begin{aligned}
\|M\| & =\left|N_{M}(A-X)\right|+\left|N_{M}(X)\right| \\
& \leq|A-X|+\left|N_{G}(X)\right|=|A|-\left(|X|-\left|N_{G}(X)\right|\right) \\
& =|A|-d .
\end{aligned}
$$

In order to prove the inverse inequality, we construct a new bipartite multigraph $H$ with bipartition $(A, B \cup D)$ from $G$ by adding a new vertex set $D$ of $d$ vertices and by joining every vertex of $D$ to all the vertices of $A$ (Figure 1.8). Then for every $\emptyset \neq S \subseteq A$, since $d \geq|S|-\left|N_{G}(S)\right|$, we have

$$
\left|N_{H}(S)\right|=\left|N_{G}(S)\right|+|D|=\left|N_{G}(S)\right|+d \geq|S| \text {. }
$$

Hence $H$ has a matching $M_{H}$ saturating $A$. Then $M_{H} \cap E(G)$ is a matching in $G$ that contains at least $|A|-d$ edges. Therefore ||$M|| \geq|A|-d$, and the theorem is proved.

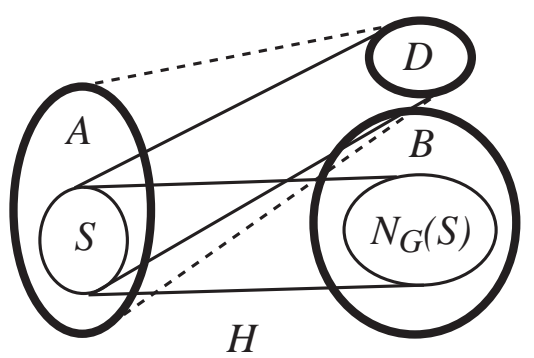

Figure 1.8: A new bipartite multigraph $H$.

An induced matching of a general graph $G$ is a matching that is an induced subgraph of $G$, that is, an induced matching can be expressed as $\langle U\rangle_{G}$ for some vertex set $U \subseteq V(G)$.

Theorem 1.1.12 (Liu and Xhou, [98]) Let $G$ be a connected bipartite graph with bipartition $(A, B)$. Then the size of a maximum induced matching $M$ of $G$ is given by

$$
\|M\|=\max \left\{|X| \mid X \subseteq A \text { such that } N_{G}(Y) \neq N_{G}(X) \text { for all } Y \subset X\right\} .
$$

Proof. We say that a subset $X \subset A$ has the property $\mathcal{P}$ if $N_{G}(Y) \neq N_{G}(X)$ for all $Y \subset X$. Let

$$
k=\max \{|X| \mid X \subseteq A, X \text { has the property } \mathcal{P}\} .
$$


Let $M$ be a maximum induced matching in $G$, and let $A_{M}$ and $B_{M}$ be the sets of vertices of $A$ and $B$, respectively, which are saturated by $M$. Since $M$ is an induced matching, we have $E_{G}\left(A_{M}, B_{M}\right)=M$. Then for every $Y \subset A_{M}$, we have $N_{G}(Y) \neq N_{G}\left(A_{M}\right)$ since

$$
\left|N_{G}(Y) \cap B_{M}\right|=\left|N_{M}(Y)\right|=|Y|<\left|A_{M}\right|=\left|N_{G}\left(A_{M}\right) \cap B_{M}\right| .
$$

Hence $\|M\|=\left|A_{M}\right| \leq k$.

We now show that $\|M\| \geq k$. We may assume $k \geq 2$. Let $S=$ $\left\{a_{1}, a_{2}, \ldots, a_{k}\right\}$ be a maximum subset of $A$ that has the property $\mathcal{P}$ and size $k$. Then $N_{G}(Y) \neq N_{G}(S)$ for all $Y \subset S$. For every $1 \leq i \leq k$, we have $N_{G}\left(a_{i}\right) \nsubseteq \cup_{j \neq i} N_{G}\left(a_{j}\right)$ by $N_{G}\left(S \backslash\left\{a_{i}\right\}\right) \neq N_{G}(S)$, and so we can choose $b_{i} \in N_{G}\left(a_{i}\right) \backslash \cup_{j \neq i} N_{G}\left(a_{j}\right)$. Then $\left\{a_{i} b_{i} \mid 1 \leq i \leq k\right\}$ is an induced matching with $k$ edges, which implies $\|M\| \geq k$. Consequently the theorem is proved.

It is remarkable that the following problem is NP-complete [26]: Is there an induced matching of size $k$ in a bipartite graph? For other results, we have polynomial time algorithms, and some of them will be given in Section 1.3.

Exercises Recall that a bipartite graph means a bipartite simple graph.

Exercise 1.1.1 In the proof of Theorem 1.1.1, show that $H$ satisfies condition (1.1).

Exercise 1.1.2 Prove that if a tree, which is a bipartite graph, has a 1factor, then it has the unique 1-factor.

Exercise 1.1.3 Prove that if a tree has a 1-factor, then it consists of the edges e such that the two components of $T-e$ are of odd order.

Exercise 1.1.4 Prove that for every bipartite graph $G$ with maximum degree $\Delta$, there exists a $\Delta$-regular bipartite graph which contains $G$ as a subgraph. Note that (i) both $G$ and $H$ have no multiple edges and (ii) the two bipartite sets of $H$ might be bigger than those of $G$.

Exercise 1.1.5 Show that a bipartite graph $G$ has at least $\Delta(G)$ distinct maximum matchings, where two matchings $M_{1}$ and $M_{2}$ are said to be distinct if $M_{1} \neq M_{2}$. 
Exercise 1.1.6 Prove the following theorem: Let $G$ be a bipartite graph with bipartition $(A, B)$, and $k \geq 1$ be an integer. Then $G$ has a spanning subgraph $H$ such that

$$
\begin{aligned}
& \operatorname{deg}_{H}(a)=1 \quad \text { for all } a \in A, \quad \text { and } \\
& \operatorname{deg}_{H}(b) \leq k \quad \text { for all } b \in B
\end{aligned}
$$

if and only if

$$
\left|N_{G}(S)\right| \geq \frac{|S|}{k} \quad \text { for all } S \subseteq A
$$

Exercise 1.1.7 Prove that a bipartite graph $G$ has a matching having at least $\|G\| / \Delta(G)$ edges.

\subsection{Covers and Transversals}

In this section we discuss covers of bipartite graphs and transversals of family of subsets. Some of the results are called min-max theorems because they say that the minimum value of some invariant is equal to the maximum value of another invariant.

When a vertex $v$ is incident with an edge $e$, we say that $v$ covers $e$ and vice versa. A vertex cover of a graph $G$ is a set of vertices that cover all the edges of $G$. A vertex cover of minimum cardinality is called a minimum vertex cover, and its cardinality is denoted by $\beta(G)$ (Figure 1.9). Similarly an edge cover of a graph $G$ is defined to be a set of edges that cover all the vertices of $G$, and an edge cover with minimum cardinality is called a minimum edge cover, and its cardinality is denoted by $\beta^{\prime}(G)$.

Recall that a set of vertices of a graph $G$ is said to be independent if no two of its vertices are adjacent, and a set of edges of $G$ is said to be independent if no two of its edges have an endvertex in common, that is, a set of edges is independent if and only if it forms a matching. We analogously define a maximum independent vertex subset and a maximum independent edge subset of $G$, and denote their cardinalities by $\alpha(G)$ and $\alpha^{\prime}(G)$, respectively.

$\beta(G)=$ the cardinality of a minimum vertex cover

$\beta^{\prime}(G)=$ the cardinality of a minimum edge cover

$\alpha(G)=$ the cardinality of a maximum independent vertex subset

$\alpha^{\prime}(G)=$ the cardinality of a maximum independent edge subset 


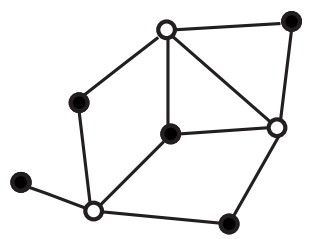

(1)

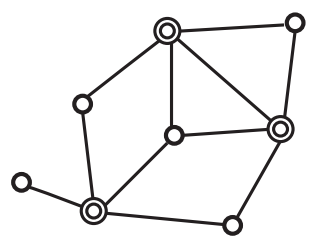

(2) $W=\{\bigcirc\}$

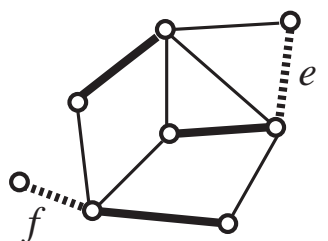

(3) $M=\{-\}$

Figure 1.9: (1) A maximum independent vertex subset $U$; (2) A minimum vertex cover $W=V(G)-U$; (3) A maximum matching $M$ and a minimum edge cover $M+\{e, f\}$.

Lemma 1.2.1 A vertex subset $S$ of a simple graph $G$ is independent if and only if $\bar{S}=V(G)-S$ is a vertex cover. Moreover,

$$
\alpha(G)+\beta(G)=|G|
$$

Proof. Suppose that $S$ is independent. Then no edge of $G$ joins two vertices of $S$, that is, for each edge $e$ of $G$, at least one endvertex of $e$ is contained in $\bar{S}$. Hence $\bar{S}$ is a vertex cover.

Conversely, if $\bar{S}$ is a vertex cover, then every edge is incident with a vertex in $\bar{S}$, which implies that no edge joins two vertices in $S=V(G)-\bar{S}$, and thus $S$ is an independent vertex subset.

Moreover, from the above arguments, it follows that if $S$ is a maximum independent vertex subset, then $\bar{S}$ is a minimum vertex cover. Thus $\alpha(G)+$ $\beta(G)=|S|+|\bar{S}|=|G|$.

The next theorem shows that a similar equality also holds for edges.

Theorem 1.2.2 (Gallai [51]) If a simple graph $G$ has no isolated vertices, then $\alpha^{\prime}(G)+\beta^{\prime}(G)=|G|$.

Proof. Let $M$ be a maximum matching of $G$. For every vertex $v$ of $G$ unsaturated by $M$, by adding one edge incident with $v$ to $M$ we can obtain an edge cover which contains

$$
\|M\|+|G|-2|| M||=|G|-|| M||=|G|-\alpha^{\prime}(G) \text { edges. }
$$

Hence $\beta^{\prime}(G) \leq|G|-\alpha^{\prime}(G)$.

Conversely, if $L$ is a minimum edge cover of $G$, then $\langle L\rangle$ is a spanning subgraph of $G$ and each component of $\langle L\rangle$ is a tree. Thus the number of components of $\langle L\rangle$ is $|G|-\|L\|$. By choosing one edge from each component 
of $\langle L\rangle$, we can obtain a matching, which contains $|G|-\|L\|$ edges. Hence $\alpha^{\prime}(G) \geq|G|-\beta^{\prime}(G)$. Consequently we have $\alpha^{\prime}(G)+\beta^{\prime}(G)=|G|$.

It follows that for each edge $e$ of a maximum matching in a graph $G$, at least one endvertex of $e$ must be contained in a vertex cover of $G$. Hence $\alpha^{\prime}(G) \leq \beta(G)$. However, the equality $\alpha^{\prime}(G)=\beta(G)$ does not hold in general except for bipartite graphs.
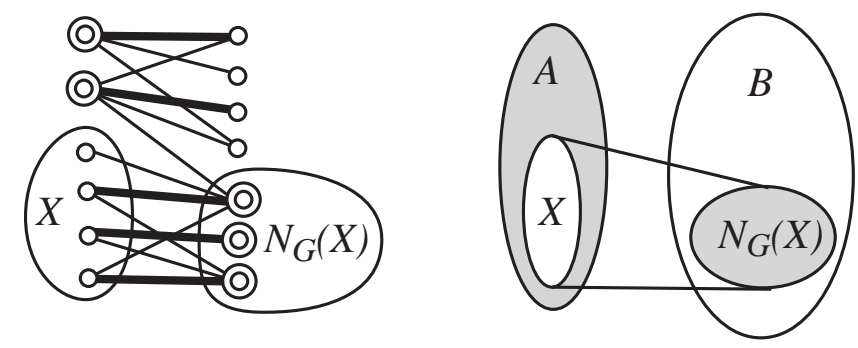

A maximum matching $M=\{-\}$

A vertex cover $\mathrm{W}=\{\bigcirc\}=\bigcirc$

Figure 1.10: A maximum matching and a minimum vertex cover in a bipartite graph.

Theorem 1.2.3 (König [90]) In a bipartite graph $G$ without isolated vertices, we have $\alpha^{\prime}(G)=\beta(G)$. Moreover this implies $\alpha(G)=\beta^{\prime}(G)$.

Proof. Since $\alpha^{\prime}(G) \leq \beta(G)$ as shown above, it suffices to prove $\alpha^{\prime}(G) \geq$ $\beta(G)$.

Let $(A, B)$ be a bipartition of $G$, and $M$ a maximum matching in $G$. Let $X$ be a subset of $A$ such that

$$
|X|-\left|N_{G}(X)\right|=\max _{S \subseteq A}\left\{|S|-\left|N_{G}(S)\right|\right\}
$$

and $W=(A-X) \cup N_{G}(X)$. Then $W$ is a vertex cover of $G$ since $G$ has no edge joining $X$ to $B-N_{G}(X)$ (Figure 1.10). By Theorem 1.1.11, we have

$$
\beta(G) \leq|W|=|A|-|X|+\left|N_{G}(X)\right|=|| M||=\alpha^{\prime}(G) .
$$

Therefore the theorem is proved.

A $(0,1)$-matrix is a matrix all of whose entries are 0 or 1 . The preceding theorem leads to the following interesting property of $(0,1)$-matrices. 


$$
M=\left(\begin{array}{rrrrr}
1 & 1 & 0 & 1 & 1 \\
0 & 1 & 0 & 0 & 0 \\
0 & 1 & 0 & 0 & 0 \\
1 & 0 & 0 & 1 & 1
\end{array}\right) \quad \begin{aligned}
& |\{\bigcirc\}|=3 \\
& \{1 \text { st row, 4th row, 2nd column }\} \\
& \text { contains all the 1-entries. }
\end{aligned}
$$

Figure 1.11: A $(0,1)$-matrix $M$.

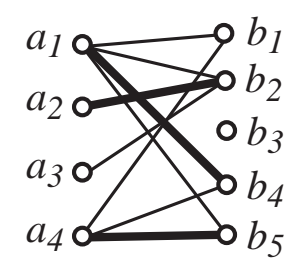

Figure 1.12: The bipartite graph corresponding to the $(0,1)$-matrix in Figure 1.11, where $\alpha^{\prime}(G)=\left|\left\{a_{1} b_{4}, a_{2} b_{2}, a_{4} b_{5}\right\}\right|=3$ and $\beta(G)=\left|\left\{a_{1}, b_{2}, a_{4}\right\}\right|=3$.

Theorem 1.2.4 (König-Egerváry [89], [44]) Let $M=\left(m_{i j}\right)$ be a $(0,1)$-matrix. Then the maximum number of 1-entries of $M$ no two of which lie on the same row or column is equal to the minimum number of rows and columns which contains all the 1-entries of $M$ (Figure 1.11).

Proof. From the given $n \times m(0,1)$-matrix $M=\left(m_{i j}\right)$, we construct a bipartite graph $G$ with bipartite sets $A=\left\{a_{1}, a_{2}, \ldots, a_{n}\right\}$ and $B=\left\{b_{1}, b_{2}, \ldots, b_{m}\right\}$ as follows:

$$
a_{i} b_{j} \in E(G) \quad \text { if and only if } \quad m_{i j}=1 . \quad \text { (Figure 1.12) }
$$

Then a set of 1-entries, no two of which lie on the same row or column, corresponds to a matching of $G$, and a set of rows and columns which contains all the 1-entries corresponds to a vertex cover of $G$. Therefore the theorem follows from the previous Theorem 1.1.11.

Let $X$ be a finite set and $\mathcal{F}=\left\{S_{1}, S_{2}, \ldots, S_{n}\right\}$ a family of subsets of $X$, where $S_{i}^{\prime}$ s are not necessarily distinct. We say that $\mathcal{F}$ has a transversal if there exists a set of $n$ distinct elements of $X$, one from each $S_{i}$. For example, a family $\{\{a, b, e\},\{b\},\{a, c, d\},\{b, c\}\}$ of subsets of $\{a, b, c, d, e\}$ has a transversal $\{a, b, d, c\}$. On the other hand, a family $\{\{a, b, e\},\{b, c\},\{c\},\{b, c\}\}$ has no transversal.

Theorem 1.2.5 (Hall [55]) A family $\mathcal{F}=\left\{S_{1}, S_{2}, \ldots, S_{n}\right\}$ of subsets of $X$ 
has a transversal if and only if

$$
\left|\bigcup_{i \in I} S_{i}\right| \geq|I| \quad \text { for all } \quad I \subseteq\{1,2, \ldots, n\} .
$$

Proof. We prove only sufficiency since necessity is immediate. We construct a bipartite graph $G$ with partite sets $\left\{S_{1}, S_{2}, \ldots, S_{n}\right\}$ and $X$ as follows: A vertex $S_{i}$ is adjacent to a vertex $x \in X$ if and only if $x \in S_{i}$ (Figure 1.13). Then for every subset $\left\{S_{i} \mid i \in I\right\}$ of $\left\{S_{1}, S_{2}, \ldots, S_{n}\right\}$, we have by (1.6)

$$
\left|N_{G}\left(\left\{S_{i} \mid i \in I\right\}\right)\right|=\left|\bigcup_{i \in I} S_{i}\right| \geq|I|
$$

Hence $G$ has a matching $M$ saturating $\left\{S_{1}, S_{2}, \ldots, S_{n}\right\}$ by the Marriage Theorem. Then we can obtain a transversal from $M$ by taking the set of vertices of $X$ saturated by $M$.

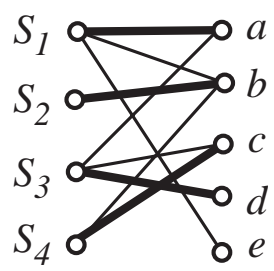

Figure 1.13: The bipartite graph corresponding to $\left\{S_{1}=\{a, b, e\}, S_{2}=\{b\}, S_{3}=\right.$ $\left.\{a, c, d\}, S_{4}=\{b, c\}\right\}$, and its matching saturating $\left\{S_{1}, S_{2}, S_{3}, S_{4}\right\}$.

Let $X$ be a finite set and $\mathcal{F}=\left\{S_{1}, S_{2}, \ldots, S_{n}\right\}$ and $\mathcal{H}=\left\{T_{1}, T_{2}, \ldots, T_{n}\right\}$ be two families of subsets of $X$. Then we say that $\mathcal{F}$ and $\mathcal{H}$ have a common transversal if there exist a set of $n$ distinct elements of $X$ that is a transversal of both $\mathcal{F}$ and $\mathcal{H}$, i.e., there exists a set $\left\{x_{1}, x_{2}, \ldots, x_{n}\right\}$ of $n$ distinct elements of $X$ such that $x_{i} \in S_{i} \cap T_{(i)}$ for all $1 \leq i \leq n$, where $\left\{T_{(1)}, T_{(2)}, \ldots, T_{(n)}\right\}$ is a rearrangement of $\mathcal{H}=\left\{T_{1}, T_{2}, \ldots, T_{n}\right\}$.

For example, $\left\{S_{1}^{\prime}=S_{2}^{\prime}=\{a, b\}, S_{3}^{\prime}=\{a, c, e\}\right\}$ and $\left\{T_{1}^{\prime}=\{b, c\}, T_{2}^{\prime}=\right.$ $\left.\{e\}, T_{3}^{\prime}=\{a, b, e\}\right\}$ have a common transversal $\{b, a, e\}$, where $b \in S_{1}^{\prime} \cap$ $T_{1}^{\prime}, a \in S_{2}^{\prime} \cap T_{3}^{\prime}$ and $e \in S_{3}^{\prime} \cap T_{2}^{\prime}$. On the other hand, $\left\{S_{1}=S_{2}=\{a, b\}, S_{3}=\right.$ $\{a, c, e\}\}$ and $\left\{T_{1}=\{b, c\}, T_{2}=\{b, d\}, T_{3}=\{c, d, e\}\right\}$ have transversals, respectively, but do not have a common transversal. Let $n=3, I=\{1,2\}$ and $J=\{1,2,3\}$, and substitute these into the left and right sides of (1.7) given in the following theorem, then

$$
\left|\left(S_{1} \cup S_{2}\right) \cap\left(T_{1} \cup T_{2} \cup T_{3}\right)\right|=|\{b\}|=1<|I|+|J|-n=2+3-3=2 .
$$

Hence the inequality (1.7) does not hold. 
Theorem 1.2.6 Let $\mathcal{F}$ and $\mathcal{H}$ be two families of subsets of a set $X$. Then $\mathcal{F}$ and $\mathcal{H}$ have a common transversal if and only if for all subsets $I$ and $J$ of $\{1,2, \ldots, n\}$, it follows that

$$
\left|\left(\bigcup_{i \in I} S_{i}\right) \bigcap\left(\bigcup_{j \in J} T_{j}\right)\right| \geq|I|+|J|-n .
$$

Proof. Let $\mathcal{F}=\left\{S_{1}, S_{2}, \ldots, S_{n}\right\}$ and $\mathcal{H}=\left\{T_{1}, T_{2}, \ldots, T_{n}\right\}$. Assume that $\mathcal{F}$ and $\mathcal{H}$ have a common transversal $\left\{x_{1}, x_{2}, \cdots, x_{n}\right\}$. Let $I$ and $J$ be subsets of $\{1,2, \ldots, n\}$. We may assume that $|I|+|J|>n$ since otherwise (1.7) trivially holds. Then two sets $\left\{x_{r} \mid x_{r} \in S_{i}, i \in I\right\}$ and $\left\{x_{r} \mid x_{r} \in T_{j}, j \in J\right\}$ must have at least $|I|+|J|-n$ elements in common, and these elements are contained in

$$
\left(\bigcup_{i \in I} S_{i}\right) \bigcap\left(\bigcup_{j \in J} T_{j}\right)
$$

Hence (1.7) holds.

In order to prove sufficiency, we construct a bipartite graph $G$ with bipartite sets

$$
A=\left\{S_{1}, S_{2}, \ldots, S_{n}\right\} \cup X \quad \text { and } \quad B=\left\{T_{1}, T_{2}, \ldots, T_{n}\right\} \cup X^{\prime},
$$

where $X^{\prime}=\left\{x^{\prime} \mid x \in X\right\}$, as follows. Two vertices $S_{i} \in A$ and $x^{\prime} \in X^{\prime}$ are joined by an edge if $x \in S_{i}$. Similarly two vertices $x \in X$ and $T_{j} \in B$ are joined by an edge if $x \in T_{j}$. Moreover, $x \in X$ and $x^{\prime} \in X^{\prime}$ are joined by an edge, and $G$ has no more edges.

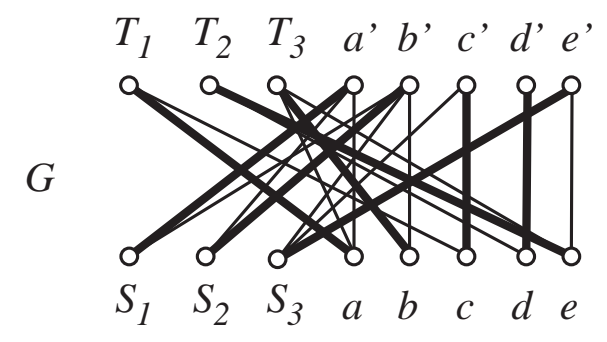

Figure 1.14: The bipartite graph corresponding to two families of subsets.

For example, the bipartite graph $G$ given in Figure 1.14 corresponds to $X=\{a, b, c, d, e\}, \mathcal{F}=\left\{S_{1}=S_{2}=\{a, b\}, S_{3}=\{b, c, e\}\right\}$ and $\mathcal{H}=\left\{T_{1}=\right.$ $\left.\{a, c\}, T_{2}=\{d, e\}, T_{3}=\{a, b, e\}\right\}$. Then $G$ has a perfect matching, and these two families have a common transversal $\{a, b, e\}$ such that $a \in S_{1} \cap T_{1}, b \in$ $S_{2} \cap T_{3}$ and $e \in S_{3} \cap T_{2}$. 
We first show that if the bipartite graph $G$ has a perfect matching $M$, then $\mathcal{F}$ and $\mathcal{H}$ have a common transversal. If $M$ contains an edge $S_{i} a_{k}^{\prime}\left(a_{k}^{\prime} \in\right.$ $\left.X^{\prime}\right)$, then $M$ must contain an edge $a_{k} T_{j}\left(a_{k} \in X\right)$ for some $T_{j}$, and thus $a_{k} \in S_{i} \cap T_{j}$. Hence it is easy to see that $\left\{a_{k} \mid S_{i} a_{k}^{\prime} \in M\right\}$ forms a common transversal of $\mathcal{F}$ and $\mathcal{H}$. Note that if $M$ contains an edge joining $a_{t} \in X$ to $a_{t}^{\prime} \in X^{\prime}$, then this fact implies that $a_{t}$ is not chosen to be an element of the common transversal.

Let

$$
P \subseteq\left\{S_{1}, S_{2}, \ldots, S_{n}\right\} \quad \text { and } \quad Q \subseteq X .
$$

Then $P \cup X \subseteq A$. Put $I=\left\{i \mid S_{i} \in P\right\}, J=\left\{j \mid T_{j} \cap Q=\emptyset\right\}$ and $Q^{\prime}=\left\{x^{\prime} \mid x \in Q\right\} \subseteq X^{\prime}$. Hence $Q^{\prime} \subset B$ and

$$
\left(\bigcup_{i \in I} S_{i}\right)^{\prime} \backslash Q^{\prime} \supseteq\left(\bigcup_{i \in I} S_{i}\right)^{\prime} \cap\left(X^{\prime}-Q^{\prime}\right) \supseteq\left(\bigcup_{i \in I} S_{i}\right)^{\prime} \cap\left(\bigcup_{j \in J} T_{j}\right)^{\prime},
$$

where $\left(\bigcup_{i \in I} S_{i}\right)^{\prime}$ and $\left(\bigcup_{j \in J} T_{j}\right)^{\prime}$ denote the subsets of $X^{\prime}$ (Figure 1.15). Therefore

$$
\begin{aligned}
\left|N_{G}(P \cup Q)\right| & =\left|N_{G}(P) \cup\left(N_{G}(Q) \cap X^{\prime}\right)\right|+\left|N_{G}(Q) \cap\left\{T_{1}, T_{2}, \ldots, T_{n}\right\}\right| \\
& =\left|\left(\bigcup_{i \in I} S_{i}\right)^{\prime} \cup Q^{\prime}\right|+\left|\left\{T_{j} \mid T_{j} \cap Q \neq \emptyset\right\}\right| \\
& \geq\left|\left(\bigcup_{i \in I} S_{i}\right)^{\prime} \backslash Q^{\prime}\right|+\left|Q^{\prime}\right|+n-|J| \\
& \geq\left|\left(\bigcup_{i \in I} S_{i}\right)^{\prime} \cap\left(\bigcup_{j \in J} T_{j}\right)^{\prime}\right|+\left|Q^{\prime}\right|+n-|J| \\
& \geq|I|+|J|-n+\left|Q^{\prime}\right|+n-|J| \quad \quad \text { by (1.7)) } \\
& =|I|+\left|Q^{\prime}\right|=|P|+|Q|=|P \cup Q| .
\end{aligned}
$$

Consequently, $G$ has a matching $M$ saturating $A$ by the Marriage Theorem. Since $|A|=|B|, M$ is a perfect matching, and thus the proof is complete.

\section{Exercises}

Exercise 1.2.1 Let $G$ be a simple graph without isolated vertices and with maximum degree $\Delta(G)$. Show that

$$
\alpha(G) \geq \frac{|G|}{\Delta(G)+1} .
$$




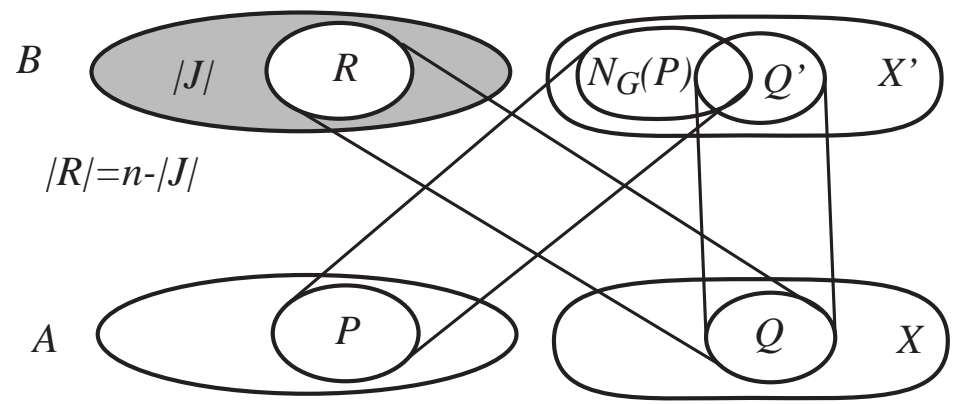

Figure 1.15: Illustration of Theorem 1.2.6; $P=\left\{X_{i} \mid i \in I\right\}, J=\left\{j \mid T_{j} \cap Q=\emptyset\right\}$, and $R=\left\{T_{j} \mid T_{j} \cap Q \neq \emptyset\right\}$.

Exercise 1.2.2 Prove that a bipartite graph $G$ has a 1-factor if and only if $\alpha(G)=|G| / 2$.

Exercise 1.2.3 Verify Proposition 1.2.4 by the following matrix:

$$
M=\left(\begin{array}{lllll}
0 & 1 & 0 & 1 & 1 \\
0 & 1 & 1 & 0 & 0 \\
0 & 0 & 0 & 0 & 0 \\
1 & 0 & 0 & 1 & 1
\end{array}\right)
$$

Exercise 1.2.4 Let $X=\{a, b, c, d, e\}, \mathcal{F}=\{\{a, b, d\},\{c, d\},\{e\}\}$, and $\mathcal{H}=$ $\{\{d\},\{a, e\},\{c, d\}\}$. Then (i) Find a transversal of $\mathcal{F}$. (ii) Find a transversal of $\mathcal{H}$. (iii) Show that $\mathcal{F}$ and $\mathcal{H}$ have common transversal.

\subsection{Augmenting Paths and Algorithms}

In this section we consider matchings in bipartite graphs by using alternating paths instead of neighborhoods, which is a new approach to matchings and useful for algorithms. For two edge subsets $X$ and $Y$ of a graph $G$, we define

$$
X \triangle Y=(X \cup Y)-(X \cap Y) .
$$

Lemma 1.3.1 Let $M_{1}$ and $M_{2}$ be matchings in a simple graph. Then each component of $\left\langle M_{1} \triangle M_{2}\right\rangle$ is either (i) a path whose edges are alternately in $M_{1}$ and in $M_{2}$, or (ii) an even cycle whose edges are alternately in $M_{1}$ and $M_{2}$ (Figure 1.16). 
Proof. Let $H=\left\langle M_{1} \triangle M_{2}\right\rangle$ and $v$ a vertex of $H$. Then $1 \leq \operatorname{deg}_{H}(v) \leq 2$, and $\operatorname{deg}_{H}(v)=2$ implies that exactly one edge of $M_{1}$ and one edge of $M_{2}$ are incident with $v$. Hence each component $C$ of $H$ is a path or cycle, whose edges are alternately in $M_{1}$ and $M_{2}$. In particular, if $C$ is a cycle, it must be an even cycle (see Figure 1.16).
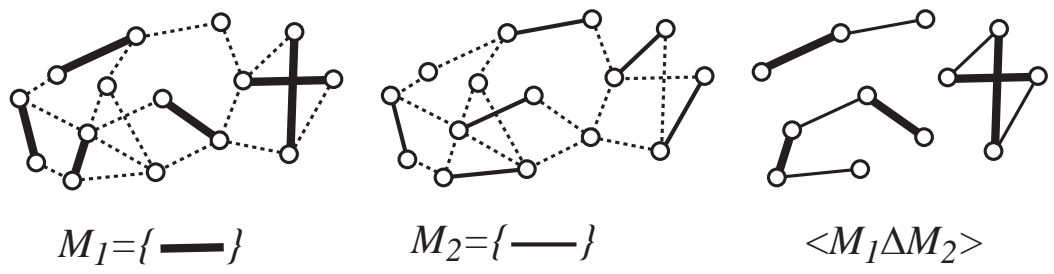

Figure 1.16: Matchings $M_{1}$ and $M_{2}$; and $\left\langle M_{1} \triangle M_{2}\right\rangle$.

Let $M$ be a matching in a simple graph $G$. Then a path of $G$ is called an $M$-alternating path if its edges are alternately in $M$ and not in $M$. If both endvertices of an $M$-alternating path are unsaturated by $M$, then such an $M$-alternating path is called an $M$-augmenting path.

For example, in the graph $G$ shown in Figure 1.17, $M=\{a, b, c\}$ is a matching, $(d, b, f, a)$ and $(g, c, e)$ are $M$-alternating paths, and $P=(d, b, e, c, g)$ is an $M$-augmenting path, whose endvertices $u$ and $v$ are not saturated by $M$. Furthermore, it is immediate that $M \triangle E(P)=\{a, d, e, g\}$ is a matching that contains $\|M\|+1$ edges and is larger than $M$. The next theorem states a characterization of maximum matchings by using augmenting path.

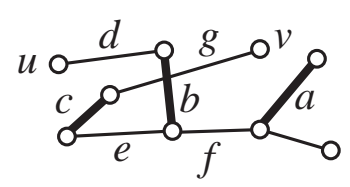

(1)

$$
M=\{-\}
$$

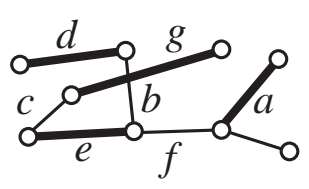

(2) $M \Delta E(P)$

Figure 1.17: (1) A matching $M=\{a, b, c\}$ and an $M$-augmenting path $P=$ $(d, b, e, c, g) ;(2) M \triangle E(P)$.

Theorem 1.3.2 (Berge [17]) A matching $M$ in a simple graph $G$ is maximum if and only if there exists no $M$-augmenting path in $G$.

Proof. The contraposition of the statement, which we shall prove, is the following: A matching $M$ is not maximum if and only if there exists an $M$-augmenting path in $G$. 
Suppose that there is an $M$-augmenting path $P$ in $G$. Then $M \triangle E(P)$ is a matching which contains $\|M\|+1$ edges, and so $M$ is not a maximum matching.

We next assume that $M$ is not a maximum matching in $G$. Let $M^{\prime}$ be a maximum matching in $G$. Then by Lemma 1.3.1, $\left\langle M \triangle M^{\prime}\right\rangle$ contains a path $P$ in which the number of edges in $M^{\prime}$ is greater than the number of edges in $M$. Hence both end-edges of $P$ are contained in $M^{\prime}$, which implies that $P$ is an $M$-augmenting path of $G$.

By making use of $M$-alternating paths and $M$-augmenting paths, we can obtain some properties of matchings in a graph and and an algorithm for finding a maximum matching in a bipartite graph. We begins one basic theorem and one result on a game played on a graph.

Theorem 1.3.3 For any matching $M$ in a simple graph $G, G$ has a maximum matching that saturates all vertices saturated by $M$.

Proof. Suppose $M$ is not a maximum matching. By Theorem 1.3.2, $G$ has an $M$-augmenting path $P$. Then $M \triangle E(P)$ is a matching which contains $\|M\|+1$ edges and saturates all the vertices saturated by $M$. Since a maximum matching can be obtained by repeating this procedure, we can find a maximum matching that saturates all the vertices saturated by $M$.

Proposition 1.3.4 Two players play a game on a connected simple graph $G$ by alternately selecting distinct vertices $v_{1}, v_{2}, v_{3}, \ldots$ so that $\left(v_{1} v_{2} v_{3} \cdots\right)$ forms a path. The player who cannot select a vertex loses, that is, the player who selects the last vertex wins (Figure 1.18). The second player has a winning strategy if $G$ has a perfect matching; otherwise the first player has a winning strategy.

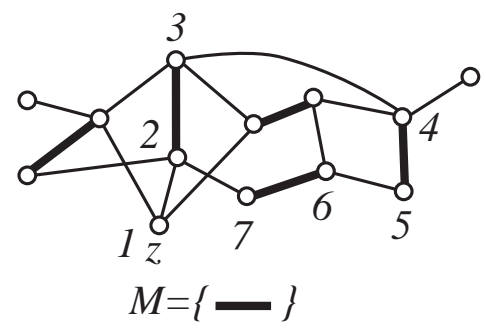

Figure 1.18: A graph for which the first player can win, and its maximum matching $M$. 
Proof. Assume that $G$ has a perfect matching $M$. If the first player selects a vertex $x$ in his turn, then the second player selects a vertex which is joined to $x$ by an edge of $M$. Since $M$ is a perfect matching, the second player can always select such a vertex. Therefore he wins since the game is over in a finite number of moves.

Next suppose that $G$ has no perfect matching. Let $M$ be a maximum matching and $z$ be a vertex unsaturated by $M$. The first player selects $z$ in the first turn, and if the second player selects a vertex $y$ in his turn, then the first player selects a vertex which is joined to $y$ by an edge of $M$. Then the vertices selected by the players form an $M$-alternating path $P$ starting with $z$. Since $G$ has no $M$-augmenting paths by Theorem 1.3.2, the path $P$ does not pass through any other $M$-unsaturated vertices, which implies that the first player can always select a vertex. Therefore the first player wins.

If a matching $M$ in a graph $G$ saturates no vertex in a subset $X \subset V(G)$, then we say that $M$ avoids $X$.

Theorem 1.3.5 Let $A$ and $B$ be vertex subsets of a simple graph $G$ such that $|A|<|B|$. Then

(i) If there exist two matchings, one saturating $A$ and the other saturating $B$, then there exists a matching that saturates $A$ and at least one vertex in $B \backslash A$.

(ii) If there exist two maximum matchings, one avoiding $A$ and the other avoiding $B$, then there exists a maximum matching that avoids $A$ and at least one vertex in $B \backslash A$.

Proof. We first prove (i). Let $M_{A}$ and $M_{B}$ be matchings in $G$ which saturate $A$ and $B$ respectively. If $M_{A}$ saturates one vertex in $B \backslash A$, then $M_{A}$ itself is the desired matching. Hence we may assume that $M_{A}$ avoids $B \backslash A$.

Consider $\left\langle M_{A} \triangle M_{B}\right\rangle$. Then for every vertex $b$ in $B \backslash A$, there exists a path in $\left\langle M_{A} \triangle M_{B}\right\rangle$ starting with $b$, which may end with a vertex in $A \backslash B$. Since $|A|<|B|$ and no path of $\left\langle M_{A} \triangle M_{B}\right\rangle$ ends with a vertex in $A \cap B$, there exists a path $P$ in $\left\langle M_{A} \triangle M_{B}\right\rangle$ that starts with $b_{1} \in B \backslash A$ and ends with $x \notin A$ (Figure 1.19). Since $P$ is an $M_{A}$-alternating path connecting $b_{1}$ and $x, M_{A} \triangle E(P)$ is the desired matching in $G$, which saturates $A$ and $b_{1} \in B \backslash A$.

We next prove (ii). Let $N_{A}$ and $N_{b}$ be maximum matchings in $G$ which avoid $A$ and $B$ respectively. We may assume that $N_{A}$ saturates $B \backslash A$ since otherwise $N_{A}$ itself is the desired matching. Then by the same argument as above, there exists a $N_{A}$-alternating path $P$ in $\left\langle N_{A} \triangle M_{B}\right\rangle$ starting with a vertex $b \in B \backslash A$ and ending with a vertex not contained in $A$. If both end-edges of $P$ are contained in $N_{A}$, then $N_{B} \triangle E(P)$ is a matching with 
$\left\|N_{B}\right\|+1$ edges, contrary to the maximality of $N_{B}$. Thus one end-edge of $P$ belongs to $N_{B}$, and thus $N_{A} \triangle E(P)$ is the desired maximum matching in $G$, which avoids $A$ and $b \in B \backslash A$.

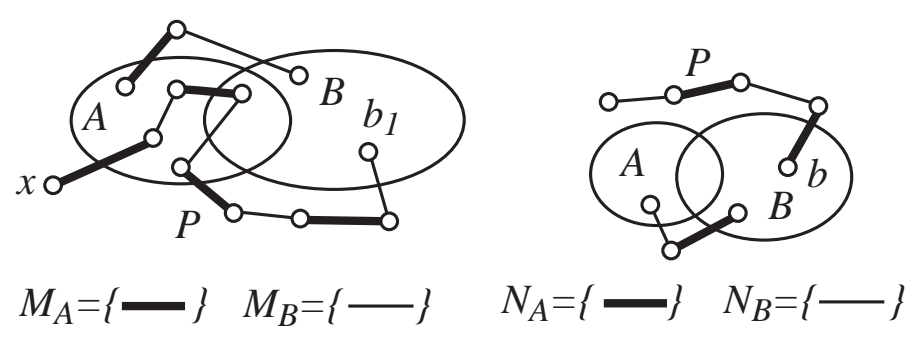

Figure 1.19: $M_{A}$-alternating path $P$ and $N_{A}$-alternating path $P$.

Theorem 1.3.6 Let $G$ be a bipartite graph with bipartition $(A, B)$, and $X \subseteq$ $A$ and $Y \subseteq B$. If $X$ and $Y$ are saturated by matchings in $G$, respectively, then $G$ has a matching that saturates $X \cup Y$.

Proof. Let $M_{X}$ and $M_{Y}$ be matchings in $G$ saturating $X$ and $Y$, respectively. We may assume that $M_{X}$ does not saturate $Y$ since otherwise $M_{X}$ is the desired matching.

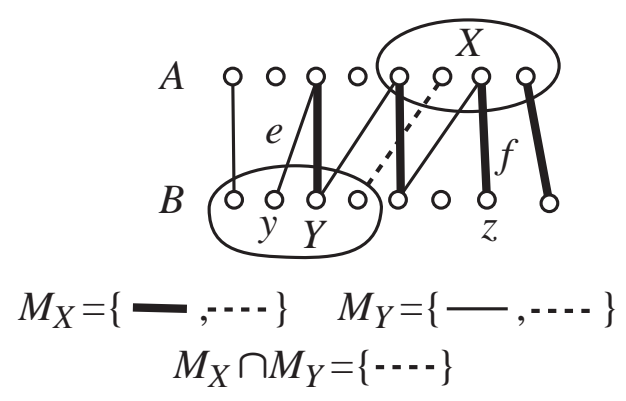

Figure 1.20: A path $P=(y, e, \ldots, f, z)$ in $H=\left\langle M_{X} \triangle M_{Y}\right\rangle$.

Let $H=\left\langle M_{X} \triangle M_{Y}\right\rangle$, and choose a vertex $y \in Y$ unsaturated by $M_{X}$. Then there exists a path $P$ in $H$ starting with $y$, which can be expressed as

$$
P=(y, e, \ldots, f, z), \quad y, \ldots, z \in V(H), \quad e, \cdots, f \in E(H),
$$

where $z$ is the other endvertex of $P$ and $e \in M_{Y}$. It is obvious that the vertices of $P$ are alternately in $B$ and $A$, and the edges of $P$ are alternately 
in $M_{Y}$ and $M_{X}$. If $z \in X$, then the end-edge $f$ of $P$ must belong to $M_{Y}$, which contradicts the fact that $X$ is saturated by $M_{X}$ (Figure 1.20). Hence $z \notin X$. Similarly, if $z \in Y$, then $f \in M_{X}$, which contradicts the fact that $Y$ is saturated by $M_{Y}$. Therefore $z \notin X \cup Y$.

Thus $M_{X} \triangle E(P)$ is a matching that saturates $V\left(\left\langle M_{X}\right\rangle\right)$ and $y \in Y \backslash$ $V\left(M_{X}\right)$. By repeating this procedure, we can obtain the desired matching in $G$, which saturates $X \cup Y$.

We now give an algorithm for finding a maximum matching in a bipartite graph, which is often called the Hungarian Method.

Algorithm 1.3.7 (Hungarian Method) Let $G$ be a bipartite graph with bipartition $(A, B)$. Then a maximum matching of $G$ can be obtained by the following procedure: Let $M$ be any matching of $G$, and $A_{0}=A \backslash V(\langle M\rangle)$ be the set of vertices in $A$ unsaturated by $M$. Let $B_{1}=N_{G}\left(A_{0}\right) \subseteq B$ and define

$$
A_{i}=N_{M}\left(B_{i}\right), \quad B_{i+1}=N_{G}\left(A_{i}\right) \backslash\left(B_{1} \cup B_{2} \cup \cdots \cup B_{i}\right)
$$

for every $1 \leq i \leq k$ until $B_{k+1}$ contains an $M$-unsaturated vertex or $B_{k+1}=$ $\emptyset$. If $B_{k+1}$ contains an $M$-unsaturated vertex, say $b_{k+1}$, then we find an $M$-augmenting path $P$ joining $b_{k+1} \in B_{k+1}$ to $a \in A_{0}$, and obtain a larger matching $M \triangle E(P)$ containing $\|M\|+1$ edges. We apply the above procedure to $M \triangle E(P)$. If $B_{k+1}=\emptyset$, then $M$ is the desired maximum matching.
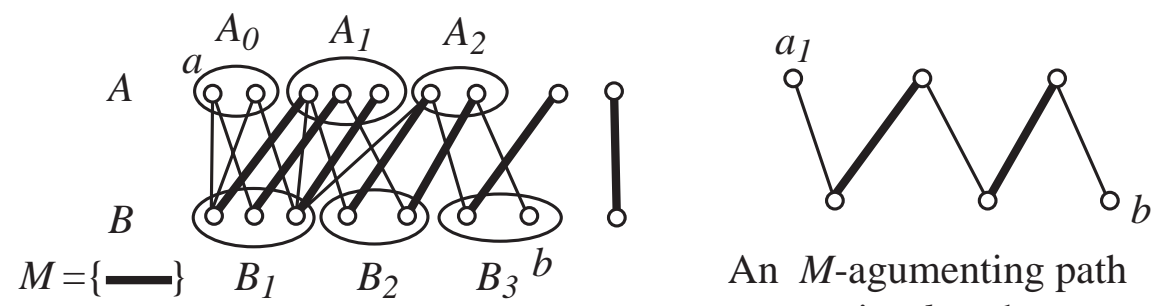

An $M$-agumenting path connecting $b$ and $a$.

Figure 1.21: An Algorithm for finding a maximum matching in a bipartite graph; and an improved algorithm.

Proof. It is easy to see that if $B_{k+1}$ contains a $M$-unsaturated vertex, then we can find an $M$-augmenting path $P$, and get a larger matching $M \triangle E(P)$ (Figure 1.21). Hence it suffices to show that if $B_{k+1}=\emptyset$, then $M$ is a maximum matching. 
Suppose $B_{k+1}=\emptyset$. Let $X=A_{0} \cup A_{1} \cup \cdots \cup A_{k}$ and $Y=B_{1} \cup B_{2} \cup \cdots \cup B_{k}$. Then $N_{G}(X)=Y$ and $N_{M}(Y)=X-A_{0}$, and so $|Y|=\left|N_{M}(Y)\right|=\left|X-A_{0}\right|=$ $|X|-\left|A_{0}\right|$. Hence

$$
\begin{aligned}
\|M\| & =|A|-\left|A_{0}\right|=|A|-(|X|-|Y|) \\
& =|A|-\left(|X|-\left|N_{G}(X)\right|\right) \geq|A|-\max _{S \subseteq A}\left\{|S|-\left|N_{G}(S)\right|\right\} .
\end{aligned}
$$

By Theorem 1.1.11, this implies that $M$ is a maximum matching of $G$.

Of course, we can do the procedure given in the above Algorithm 1.3.7 for each vertex $a \in A_{0}$ individually, that is, put $B_{1}(a)=N_{G}(a) \subseteq B$ and obtain

$$
A_{i}(a)=N_{M}\left(B_{i}(a)\right), \quad B_{i+1}(a)=N_{G}\left(A_{i}(a)\right) \backslash\left(B_{1}(a) \cup \cdots \cup B_{i}(a)\right)
$$

for every $1 \leq i \leq k$ until $B_{k+1}(a)$ contains an $M$-unsaturated vertex or $B_{k+1}(a)=\emptyset$. If $B_{k+1}(a)$ contains an $M$-unsaturated vertex, then we can get a larger matching than $M$. If $B_{k+1}(a)=\emptyset$, then we remove $X=\{a\} \cup A_{1}(a) \cup$ $\cdots \cup A_{k}(a)$ and $Y=B_{1}(a) \cup \cdots \cup B_{k}(a)$ from $G$, and obtain $G-(X \cup Y)$, and then apply the same procedure in $G-(X \cup Y)$ for another vertex $a^{\prime} \in A-\{a\}$.

\subsection{1-Factor Theorems}

In this section we investigate matchings and 1-factors of graphs. Since a 1-factor contains neither loops nor multiple edges, we can restrict ourselves to simple graphs when we consider 1-factors. So in this section, we mainly consider simple graphs. However some results hold for multigraphs or general graphs, and these generalization are useful and interesting. For example, every 2-connected cubic simple graph has a 1-factor, but also every 2-connected cubic multigraph has a 1 -factor. Thus we occasionally consider multigraphs and general graphs.

A criterion for a graph to have a 1-factor was obtained by Tutte [138] in 1947 and is one of most important results in factor theory. It is called the 1-Factor Theorem. We begin with this theorem. The proof presented here is due to Anderson [11] and Mader [110]. Tutte's original proof uses the Pfaffian of a matrix. Other proofs of 1-Factor Theorem are found in Hetyei [58] and Lovász [106].

After the 1-Factor Theorem, we discuss some other criteria for graphs to have 1-factors with given properties or for some special graphs to have 1-factors, for example, a criterion for a graph to have a 1-factor containing 
any given edge and a criterion for a tree to have a 1-factor, which is much simpler than the criterion given in the 1-Factor Theorem.

For a vertex subset $X$ of $G$ and a subgraph $R$ of $G-X$, we simplify notation by denoting $E_{G}(V(R), X)$ and $e_{G}(V(R), X)$ by $E_{G}(R, X)$ and $e_{G}(R, X)$, respectively. A component of a graph is said to be odd or even according to whether its order is odd or even. For a graph $G, \operatorname{Odd}(G)$ denotes the set of odd components of $G$, and $\operatorname{odd}(G)$ denotes the number of odd components of $G$, that is,

$$
\operatorname{odd}(G)=|\operatorname{Odd}(G)|=\text { the number of odd components of } G \text {. }
$$

Lemma 1.4.1 Let $G$ be a general graph and $S \subseteq V(G)$. Then

$$
\operatorname{odd}(G-S)+|S| \equiv|G|(\bmod 2) \text {. }
$$

In particular, if $G$ is of even order, then

$$
\operatorname{odd}(G-S) \equiv|S|(\bmod 2),
$$

and odd $(G-v) \geq 1$ for every vertex $v$.

Proof. Let $C_{1}, C_{2}, \ldots, C_{m}$ be the odd components of $G-S$, and $D_{1}, D_{2}$, $\ldots, D_{r}$ the even components of $G-S$, where $m=\operatorname{odd}(G-S)$. Then

$$
|G|=|S|+\left|C_{1}\right|+\cdots+\left|C_{m}\right|+\left|D_{1}\right|+\cdots+\left|D_{r}\right| \equiv|S|+m(\bmod 2) .
$$

Hence (1.8) holds. (1.9) is an immediate consequence of (1.8).

Before giving the 1-factor Theorem, we give a remark. A matching of a general graph contains no loops, and so does a 1-factor. Then a 1-factor of a general graph is a spanning subgraph with all vertices degree one. Thus it is obvious that a general $G$ has a 1 -factor if and only if its underlying graph has a 1-factor, where the underlying graph of $G$ is a simple graph obtained from $G$ by removing all the loops and, for every pair of adjacent vertices, by replacing the multiple edges joining them by a single edge. Therefore essential part of the following 1-factor Theorem is that the theorem holds for simple graphs.

Theorem 1.4.2 (1-Factor Theorem, Tutte [138]) A general graph G has a 1-factor if and only if

$$
\operatorname{odd}(G-S) \leq|S| \quad \text { for all } S \subset V(G) \text {. }
$$


Proof. As we remark above, we may assume that a given general graph $G$ is a simple graph. Assume that $G$ has a 1 -factor $F$. Let $\emptyset \neq S \subseteq V(G)$, and $C_{1}, C_{2}, \ldots, C_{m}$ be the odd components of $G-S$, where $m=\operatorname{odd}(G-S)$. Then for every odd component $C_{i}$ of $G-S$, there exists at least one edge in $F$ that joins $C_{i}$ to $S$ (Figure 1.22). Hence

$$
\operatorname{odd}(G-S)=m \leq e_{F}\left(C_{1} \cup C_{2} \cup \cdots \cup C_{r}, S\right) \leq|S| .
$$

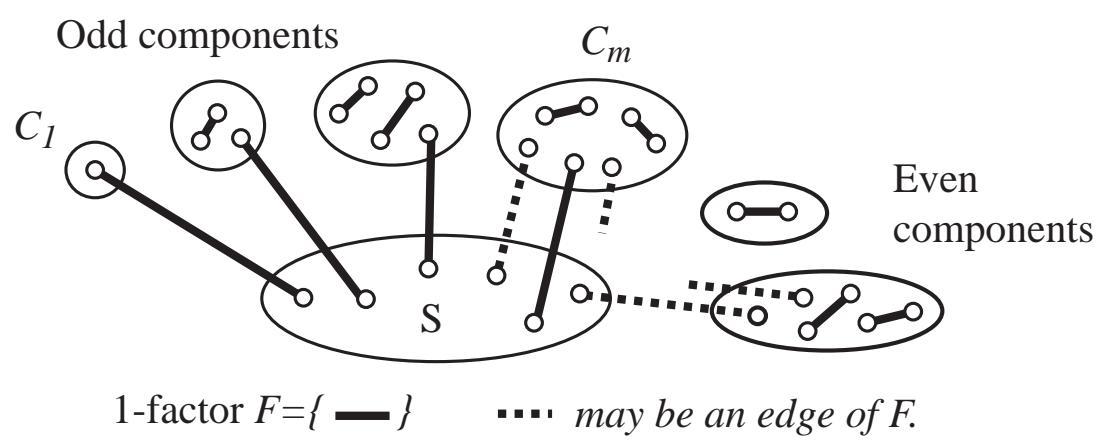

Figure 1.22: For every odd component $C_{i}$ of $G-S$, at least one edge of $F$ joins $C_{i}$ to $S$.

We now prove sufficiency by induction on $|G|$. By setting $S=\emptyset$, we have $\operatorname{odd}(G)=0$, which implies that each component of $G$ is even. If $G$ is not connected, then every component of $G$ satisfies (1.10), and so it has a 1 -factor by the inductive hypothesis. Hence $G$ itself has a 1 -factor. Therefore we may assume that $G$ is connected and has even order.

It follows from the above Lemma 1.4.1 and (1.10) that odd $(G-\{v\})=$ $|\{v\}|=1$. Let $S$ be a maximal subset of $V(G)$ with the property that $\operatorname{odd}(G-S)=|S|$. Then $\emptyset \neq S \subset V(G)$ and

$$
\operatorname{odd}(G-R)<|R| \quad \text { for all } \quad S \subset R \subseteq V(G) .
$$

Claim 1. Every component of $G-S$ is of odd order.

Suppose that $G-S$ has an even component $D$. Let $v$ be a vertex of $D$. Then by Lemma 1.4.1, $D-v$ has at least one odd component, and so

$$
\operatorname{odd}(G-(S \cup\{v\}))=\operatorname{odd}(G-S)+\operatorname{odd}(D-v) \geq|S|+1,
$$

which implies odd $(G-(S \cup\{v\}))=|S \cup\{v\}|$ by (1.10). This contradicts the maximality of $S$. Hence Claim 1 holds. 
Claim 2. For any vertex $v$ of each odd component $C$ of $G-S, C-v$ has a 1-factor.

Let $C$ be an odd component of $G-S$, and $v$ a vertex of $C$. Then for every subset $T \subseteq V(C-v)$, we have by (1.11)

$$
\begin{aligned}
|S|+1+|T| & >\operatorname{odd}(G-(S \cup\{v\} \cup T)) \\
& =\operatorname{odd}(G-S)-1+\operatorname{odd}(C-(\{v\} \cup T)) \\
& =|S|-1+\operatorname{odd}((C-v)-T) .
\end{aligned}
$$

Thus odd $((C-v)-T)<|T|+2$, which implies odd $((C-v)-T) \leq|T|$ by (1.9). Hence $C-v$ has a 1 -factor by the induction hypothesis, and thus Claim 2 is proved.

Let $C_{1}, C_{2}, \ldots, C_{m}$ be the odd components of $G-S$, where $m=\operatorname{odd}(G-$ $S)=|S|$. We construct a bipartite graph $B$ with partite sets $\left\{C_{1}, C_{2}, \ldots\right.$ ,$C_{m}$ \} and $S$ as follows: a vertex $x$ of $S$ and $C_{i}$ are joined by an edge of $B$ if and only if $x$ and $C_{i}$ are joined by an edge of $G$ (Figure 1.23).
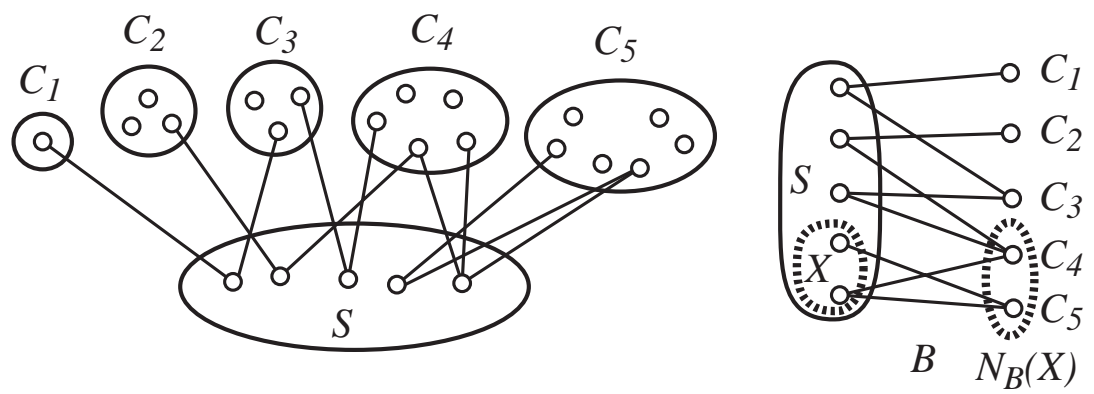

Figure 1.23: $G-S$ and the bipartite graph $B$.

Claim 3. The bipartite graph $B$ has a 1-factor.

It follows that $\left|N_{B}(S)\right|=\left|\left\{C_{1}, C_{2}, \ldots, C_{m}\right\}\right|=|S|$ since $G$ is connected. Assume that $\left|N_{B}(X)\right|<|X|$ for some $\emptyset \neq X \subset S$. Then every vertex $C_{i} \in\left\{C_{1}, C_{2}, \ldots, C_{m}\right\}-N_{B}(X)$ is an isolated vertex of $B-(S-X)$, which implies $C_{i}$ is an odd component of $G-(S-X)$, and thus we obtain

$$
\operatorname{odd}(G-(S-X)) \geq\left|\left\{C_{1}, C_{2}, \ldots, C_{m}\right\}-N_{B}(X)\right|>m-|X|=|S-X| .
$$

This contradicts (1.10). Therefore $\left|N_{B}(X)\right| \geq|X|$ for all $X \subseteq S$, and so by the Marriage Theorem, $B$ has a matching $K$ saturating $S$. Since $m=|S|$, $K$ must saturate $\left\{C_{1}, C_{2}, \ldots, C_{m}\right\}$, and thus $K$ is a 1 -factor of $B$. 
For every edge $x_{i} C_{i}$ of $K$, choose a vertex $v_{i} \in V\left(C_{i}\right)$ that is adjacent to $x_{i}$ in $G$, and take a 1-factor $F\left(C_{i}\right)$ of $C_{i}-v_{i}$, whose existence is guaranteed by Claim 2. Therefore we obtain the following desired 1-factor of $G$ :

$$
\begin{aligned}
& \left(F\left(C_{1}\right) \cup \cdots \cup F\left(C_{m}\right)\right) \\
\cup & \left\{x_{i} v_{i} \mid x_{i} C_{i} \in K, x_{i} \in S, v_{i} \in V\left(C_{i}\right), 1 \leq i \leq m\right\} .
\end{aligned}
$$

Consequently the theorem is proved.

A graph $G$ is said to be factor-critical if $G-v$ has a 1-factor for every vertex $v$ of $G$ (Figure 1.24). It is easy to see that if $G$ is factor-critical, then $G$ is of odd order, connected and not a bipartite graph. The proof of this statement is left to the reader (Exercise 1.4.3).
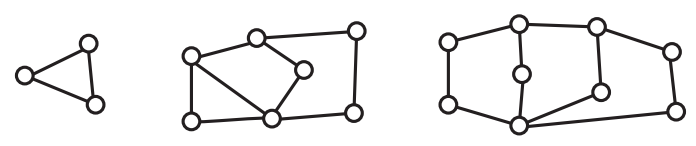

Figure 1.24: Factor-critical graphs.

Theorem 1.4.3 A simple graph $G$ of even order has a 1-factor if and only if for every subset $S$ of $V(G)$, the number of factor-critical components of $G-S$ is less than or equal to $|S|$.

Proof. Necessity follows immediately from the 1-Factor Theorem since every factor-critical component of $G-S$ is an odd component of $G-S$.

We now prove sufficiency. Suppose that $G$ satisfies the condition in the theorem, but has no 1-factor. Then by the 1-Factor Theorem, there exists a subset $\emptyset \neq X \subseteq V(G)$ such that $\operatorname{odd}(G-X)>|X|$, which implies $X \neq V(G)$. Take a maximal subset $\emptyset \neq S \subset V(G)$ such that $\operatorname{odd}(G-S)>|S|$. Then

$$
\operatorname{odd}(G-Y) \leq|Y| \quad \text { for all } \quad S \subset Y \subseteq V(G) .
$$

We first show that $G-S$ has no even component, since otherwise for a vertex $v$ of an even component of $G-S$, we have by Lemma 1.4.1

$$
\operatorname{odd}(G-(S \cup\{v\})) \geq \operatorname{odd}(G-S)+1>|S|+1=|S \cup\{v\}|,
$$

contrary to (1.12). We shall next show that every odd component of $G-S$ is factor-critical. Let $C$ be an odd component of $G-S$, and $v$ any vertex of 
$C$. Then for every $T \subseteq V(C-v),(1.12)$ implies

$$
\begin{aligned}
|S|+1+|T| & \geq \operatorname{odd}(G-(S \cup\{v\} \cup T)) \\
& =\operatorname{odd}(G-S)-1+\operatorname{odd}(C-(\{v\} \cup T)) \\
& >|S|-1+\operatorname{odd}((C-v)-T) .
\end{aligned}
$$

Hence $\operatorname{odd}((C-v)-T)<|T|+2$, which implies $\operatorname{odd}((C-v)-T) \leq|T|$ by (1.9). Therefore $C-v$ has a 1 -factor by the 1 -Factor Theorem, and so $C$ is factor-critical. Consequently we obtain

the number of factor-critical components of $G-S=\operatorname{odd}(G-S)>|S|$.

This contradicts the assumption, and thus the theorem is proved.

Theorem 1.4.4 (Amahashi [9]) A tree $T$ of even order has a 1-factor if and only if odd $(T-v)=1$ for every vertex $v$ of $T$.

Proof. Suppose that $T$ has a 1-factor $F$. Then for every vertex $v$ of $T$, let $w$ be the vertex of $T$ joined to $v$ by an edge of $F$. Then the component of $T-v$ containing $w$ is odd, and all the other components of $T-v$ are even (Figure 1.25. Hence $\operatorname{odd}(T-v)=1$.

Suppose that $\operatorname{odd}(T-v)=1$ for every $v \in V(T)$. It is obvious that for each edge $e$ of $T, T-e$ has exactly two components, and both of them are simultaneously odd or even. Define the edge-induced subgraph $F$ of $T$ as follows:

$$
F=\langle\{e \in E(T) \mid \operatorname{odd}(T-e)=2\}\rangle_{T} .
$$

For every vertex $v$ of $T$, there exists exactly one edge $e$ that is incident with $v$ and satisfies $o d d(T-e)=2$ since $T-v$ has exactly one odd component, where $e$ is the edge joining $v$ to this odd component (Figure 1.25). Therefore $e$ is an edge of $F$, and thus $F$ is a 1-factor of $G$.
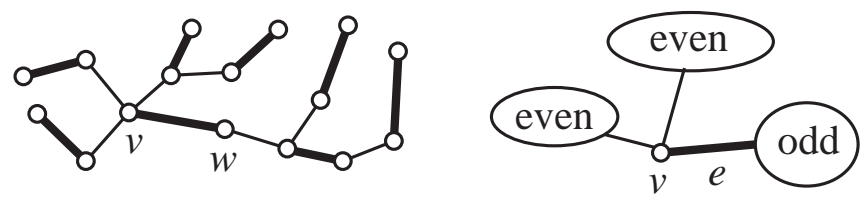

Figure 1.25: A tree having a 1-factor, and a tree $T$ satisfying $\operatorname{odd}(T-v)=1$.

We now give a variety of criteria for graphs to have 1-factors possessing some given properties. The following theorem was obtained by Little, Grant and Holton [96]. A graph $G$ is said to be 1-factor covered if for every edge $e$, $G$ has a 1 -factor containing $e[95]$. 
Theorem 1.4.5 Let $G$ be a simple graph. Then for every edge e of $G, G$ has a 1-factor containing e if and only if

$$
\operatorname{odd}(G-S) \leq|S|-\epsilon_{1} \quad \text { for all } S \subset V(G),
$$

where $\epsilon_{1}=2$ if $\langle S\rangle_{G}$ contains an edge; otherwise $\epsilon_{1}=0$.

Proof. We first prove necessity. Let $S \subseteq V(G)$. Then $\operatorname{odd}(G-S) \leq|S|$ by the 1-Factor Theorem. Assume that $\langle S\rangle_{G}$ contains an edge, say $e=$ $x y(x, y \in S)$. Since $G$ has a 1 -factor $F$ containing $e$, for each odd component $C$ of $G-S$, there exists an edge in $F$ joining $C$ to a vertex in $S-\{x, y\}$, and hence

$$
\operatorname{odd}(G-S) \leq e_{F}(G-S, S-\{x, y\}) \leq|S-\{x, y\}|=|S|-2 .
$$

We next prove sufficiency. Let $e=x y(x, y \in V(G))$ be any edge of $G$. We shall show that $G-\{x, y\}$ has a 1-factor, which obviously implies that $G$ has a 1-factor containing $e=x y$.

Let $S \subseteq V(G)-\{x, y\}$. Since $\langle S \cup\{x, y\}\rangle_{G}$ contains an edge $e$, we have

$$
\operatorname{odd}((G-\{x, y\})-S)=\operatorname{odd}(G-(S \cup\{x, y\})) \leq|S \cup\{x, y\}|-2=|S| .
$$

Therefore $G-\{x, y\}$ has a 1 -factor by the 1-Factor Theorem.

An edge $e$ of a connected graph $G$ is called an odd-bridge if $e$ is a bridge of $G$ and $G-e$ consists of two odd components, in particular, such a graph $G$ has even order. The next theorem was obtained by C. Chen [27], [29].

Theorem 1.4.6 Let $G$ be a connected graph. Then for every edge e of $G, G$ has a 1-factor excluding $e$ if and only if

$$
\operatorname{odd}(G-S) \leq|S|-\epsilon_{2} \quad \text { for all } S \subset V(G),
$$

where $\epsilon_{2}=2$ if $G-S$ has a component containing an odd-bridge; otherwise $\epsilon_{2}=0$.

Proof. We first prove necessity. Let $S \subseteq V(G)$. Then $\operatorname{odd}(G-S) \leq|S|$ by the 1-Factor Theorem. Assume that $G-S$ has a component $D$ containing an odd-bridge $e$. Consider a 1-factor $F$ of $G$ excluding $e$. Then for each odd component $C$ of $G-S, F$ contains at least one edge joining $C$ to $S$. Furthermore, for each odd component $C^{\prime}$ of $D-e$, at least one edge of $F$ joins $C^{\prime}$ to $S$. Hence

$$
\operatorname{odd}(G-S)+2 \leq e_{F}(G-S, S) \leq|S| .
$$


Consequently, (1.14) holds.

Conversely, assume that $G$ satisfies (1.14). We shall show that for any edge $e$ of $G, G-e$ has a 1-factor, which is of course a 1-factor of $G$ excluding $e$. Let $S \subseteq V(G-e)=V(G)$. If $e$ is an odd-bridge of an even component of $G-S$, then $\operatorname{odd}(G-e-S)=\operatorname{odd}(G-S)+2$; otherwise $\operatorname{odd}(G-e-S)=$ $\operatorname{odd}(G-S)$. For example, if $e$ joins $S$ to $V(G)-S$, then $\operatorname{odd}(G-e-S)=$ $\operatorname{odd}(G-S)$, and if $e$ joins two vertices of an odd component $C$ of $G-S$, then $C-e$ has exactly one odd component, which may be $C-e$, and so $\operatorname{odd}(G-S-e)=\operatorname{odd}(G-S)$. Therefore

$$
\operatorname{odd}((G-e)-S)=\operatorname{odd}(G-S-e)=\operatorname{odd}(G-S)+\epsilon_{2} \leq|S| .
$$

Hence $G-e$ has a 1 -factor.

Theorem 1.4.7 (Corollary 1.6 of [20]) Let $G$ be a simple graph and $W$ a subset of $V(G)$. Then $G$ has a matching that saturates $W$ if and only if

$$
\operatorname{odd}(G-S \mid W) \leq|S| \quad \text { for all } S \subseteq V(G),
$$

where odd $(G-S \mid W)$ denotes the number of those odd components of $G-S$ whose all vertices are contained in $W$ (Figure 1.26).
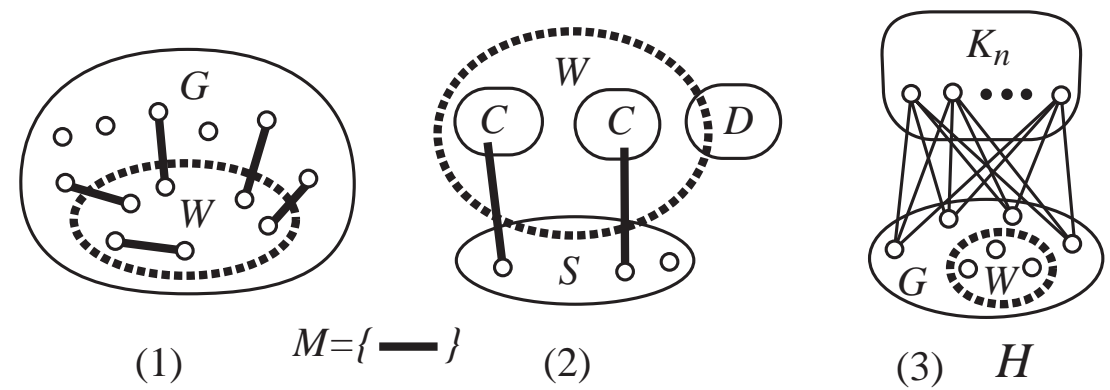

Figure 1.26: (1) A matching $M$ saturating $W$; (2) Each odd component $C$ of $G-S$ is counted in $\operatorname{odd}(G-S \mid W)$ but not an odd component $D$ of $G-S$; and (3) the graph $H$.

Proof. Assume that $G$ has a matching $M$ which saturates $W$. Then for every odd component $C$ of $G-S$ such that $V(C) \subseteq W$, at least one edge of $M$ joins $C$ to $S$. Thus odd $(G-S \mid W) \leq e_{M}(V(G)-S, S) \leq|S|$.

We next prove sufficiency. By the 1-Factor Theorem, we may assume that $W$ is a proper subset of $V(G)$, and so $V(G)-W \neq \emptyset$. Let $n=|G|$. We 
construct a new graph $H$ from $G$ by adding the complete graph $K_{n}$ and by joining every vertex in $V(G)-W$ to every vertex of $K_{n}$ (Figure 1.26). Then $H$ has even order, and it is easy to see that $G$ has a matching saturating $W$ if and only if $H$ has a 1-factor.

Let $X \subseteq V(H)$. If $V\left(K_{n}\right) \subseteq X$, then $\operatorname{odd}(H-X) \leq|G| \leq|X|$. If $V\left(K_{n}\right) \nsubseteq X$, then since $V(H)-(X \cup W)$ is contained in a component of $H-X$, we have by (1.15) that

$$
\operatorname{odd}(H-X) \leq \operatorname{odd}(G-(V(G) \cap X) \mid W)+1 \leq|V(G) \cap X|+1 \leq|X|+1,
$$

which implies odd $(H-X) \leq|X|$ by (1.9). Therefore $H$ has a 1 -factor, and thus $G$ has the desired matching, which saturates $W$.

For a graph $G, \max _{S \subseteq V(G)}\{\operatorname{odd}(G-S)-|S|\}$ is called the deficiency of $G$. This concept is introduced in the next theorem.

Theorem 1.4.8 (Berge [18]) Let $M$ be a maximum matching in a simple graph $G$. Then the number $|M|$ of vertices saturated by $M$ is given by

$$
|M|=|G|-\max _{S \subseteq V(G)}\{\operatorname{odd}(G-S)-|S|\} .
$$

Proof. The proof given here is due to Bollobás [20]. Let

$$
d=\max _{S \subseteq V(G)}\{\operatorname{odd}(G-S)-|S|\},
$$

and $T$ a subset of $V(G)$ such that odd $(G-T)-|T|=d$. Then odd $(G-X)+d \leq$ $|X|$ for every $X \subset V(G)$.

We first show $|M| \leq|G|-d$. Let $M$ be a maximum matching of $G$. Then for any odd component $C$ of $G-T$, if $V(C)$ is saturated by $M$, then at least one edge of $M$ joins $C$ to $T$ (Figure 1.27). Thus at least odd $(G-T)-|T|=d$ odd components of $G-T$ are not saturated by $M$, which implies $|M| \leq$ $|G|-d$.

In order to prove the inverse inequality, we construct the join $H=G+K_{d}$, where $K_{d}$ is the complete graph of order $d$. Then for every $\emptyset \neq Y \subseteq V(H)$, if $V\left(K_{d}\right) \nsubseteq Y Y$, then $\operatorname{odd}(H-Y) \leq 1 \leq|Y|$; and if $V\left(K_{d}\right) \subseteq Y$, then

$$
\operatorname{odd}(H-Y)=\operatorname{odd}(G-(V(G) \cap Y)) \leq|V(G) \cap Y|+d=|Y| .
$$

Hence $H$ has a 1-factor $F$ by the 1-Factor Theorem. Then $F \cap E(G)=$ $F-V\left(K_{d}\right)$ is a matching of $G$ and saturates at least $|G|-d$ vertices. This which implies $|M| \geq|F \cap E(G)| \geq|G|-d$. Consequently the theorem is proved.

\section{Exercises}



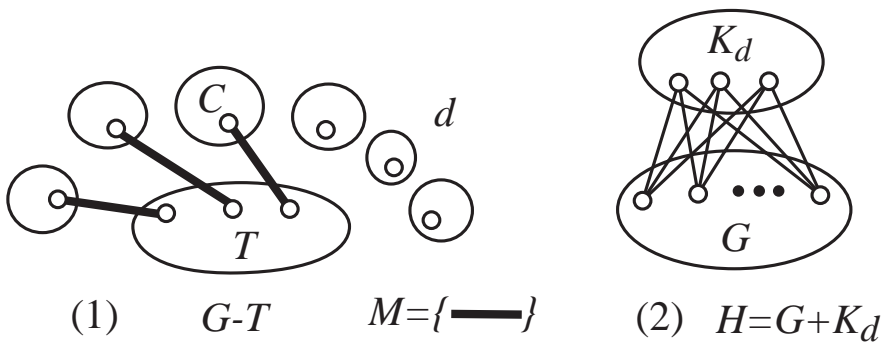

Figure 1.27: (1) At least $\operatorname{odd}(G-T)-|T|=d$ vertices are not saturated by $M$; (2) The graph $H=G+K_{d}$.

Exercise 1.4.1 Describe infinitely many of non-bipartite graphs that have no 1-factors and satisfy $\left|N_{G}(S)\right| \geq|S|$ for all $S \subset V(G)$.

Exercise 1.4.2 Deduce the Marriage Theorem (Theorem 1.1.1) from the 1Factor Theorem (Theorem 1.4.2).

Exercise 1.4.3 Prove that a factor-critical graph is connected, is of odd order and is not a bipartite graph.

Exercise 1.4.4 Let $S$ be a maximal vertex subset of a graph $G$ such that $\operatorname{odd}(G-S)-|S|=\max _{X \subseteq V(G)}\{\operatorname{odd}(G-X)-|X|\}$. Then prove that every component of $G-S$ is factor-critical.

\subsection{Graphs Having 1-Factors}

We shall show some classes of graphs that have 1-factors, and give some results on the sizes of maximum matchings. Among the results, the following is well-known: every $(r-1)$-edge connected $r$-regular graph of even order has a 1-factor that contains any given edge.

When we consider $r$-regular graphs or $n$-edge connected graphs, $r$ and $n$ always denote positive integers. The next lemma is useful when we prove the existence of 1 -factors in regular graphs.

Lemma 1.5.1 Let $r \geq 2$. Let $G$ be an $r$-regular general graph, and $S$ a vertex subset of $G$. Then for every odd component $C$ of $G-S$,

$$
e_{G}(C, S) \equiv r(\bmod 2),
$$

that is, $e_{G}(C, S)$ and $r$ have the same parity. In particular, if $G$ is an $(r-1)$ edge connected $r$-regular multigraph, then $e_{G}(C, S) \geq r$ (Figure 1.28). 
Proof. Since $|C|$ is odd, the congruence (1.17) follows from

$$
r \equiv r|C|=\sum_{x \in V(C)} \operatorname{deg}_{G}(x)=e_{G}(C, S)+2\|C\| \equiv e_{G}(C, S)(\bmod 2),
$$

where $\|C\|$ denotes the size of $C$ (Figure 1.28). If $G$ is an $(r-1)$-edge connected $r$-regular multigraph, then $e_{G}(C, S) \geq r-1$, and so by combining this inequality and (1.17), we have $e_{G}(C, S) \geq r$.

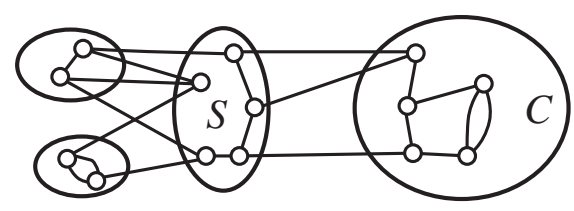

Figure 1.28: A 2-edge connected 3-regular multigraph $G$ and an odd component $C$ of $G-S$.

The next theorem, obtained by Petersen in 1891, is one of the oldest theorems on factors of graphs, and many researches were undertaken to generalize this result.

Theorem 1.5.2 (Petersen [126]) Let $G$ be a connected 3-regular general graph such that all the bridges of $G$, if any, are contained in a path of $G$. Then $G$ has a 1-factor (Figure 1.29). In particular, every 2-edge connected 3-regular multigraph has a 1-factor (Figure 1.29).

(1)

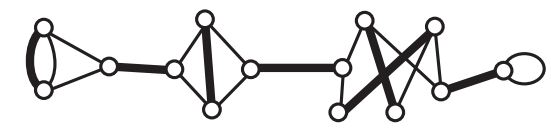

$(2)$
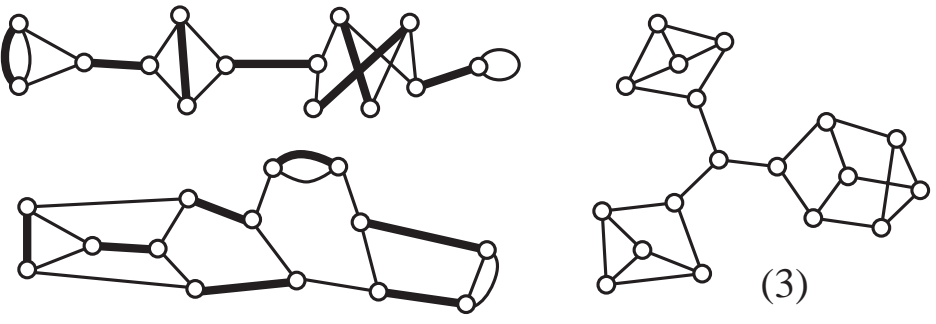

Figure 1.29: (1) A 3-regular general graph having a 1-factor; (2) A 2-edge connected 3-regular multigraph having a 1-factor; and (3) A 3-regular simple graph having no 1-factor.

Proof. We prove only the first statement because the second statement follows immediately since a 2-edge connected graph has no bridges. Let 
$\emptyset \neq S \subset V(G)$, and $C_{1}, C_{2}, \cdots, C_{m}$ be the odd components of $G-S$, where $m=\operatorname{odd}(G-S)$, such that

$$
e_{G}\left(C_{i}, S\right)=1 \quad \text { for } 1 \leq i \leq t, \text { and } \quad e_{G}\left(C_{j}, S\right) \geq 2 \quad \text { for } t+1 \leq j \leq m .
$$

An edge joining $C_{i}(1 \leq i \leq t)$ to $S$ is a bridge of $G$ and is contained in a path of $G$, and thus $t \leq 2$ since otherwise $e_{G}\left(C_{i}, S\right) \geq 2$ for some $i$. By Lemma 1.5.1,

$$
e_{G}\left(C_{j}, S\right) \geq 3 \quad \text { for all } \quad t+1 \leq j \leq m .
$$

Therefore

$$
3|S| \geq e_{G}\left(C_{1} \cup C_{2} \cup \cdots \cup C_{m}, S\right) \geq t+3(m-t)=3 m-2 t \geq 3 m-4 .
$$

Hence $m \leq|S|+4 / 3<|S|+2$, which implies odd $(G-S)=m \leq|S|$ by (1.9). Therefore $G$ has a 1 -factor by the 1-Factor Theorem.

The above theorem can be extended to $r$-regular graphs as follows. Note that an $(r-1)$-edge connected $r$-regular general graph contains no loops, and so it must be a multigraph.

Theorem 1.5.3 Let $r \geq 2$, and $G$ be an $(r-1)$-edge connected $r$-regular multigraph of even order. Then for every edge e of $G, G$ has a 1-factor containing e. In particular, $G$ has a 1-factor ([14]).

Proof. We use Theorem 1.4.5, which also gives a necessary and sufficient condition for a multigraph to have a 1-factor containing any given edge. Let $\emptyset \neq S \subset V(G)$. Let $C_{1}, C_{2}, \cdots, C_{m}$ be the odd components of $G-S$, where $m=\operatorname{odd}(G-S)$. Then by Lemma 1.5.1, we have $e_{G}\left(C_{i}, S\right) \geq r$. Hence

$$
r|S|=\sum_{x \in S} \operatorname{deg}_{G}(x) \geq e_{G}\left(C_{1} \cup C_{2} \cup \cdots \cup C_{m}, S\right) \geq r m .
$$

Thus odd $(G-S)=m \leq|S|$.

Suppose that $\langle S\rangle_{G}$ contains an edge. Then

$$
r|S|=\sum_{x \in S} \operatorname{deg}_{G}(x) \geq e_{G}\left(C_{1} \cup C_{2} \cup \cdots \cup C_{m}, S\right)+2 \geq r m+2 .
$$

Hence $m \leq|S|-2 / r<|S|$, which implies odd $(G-S)=m \leq|S|-2$ by (1.9). Therefore the theorem follows by Theorem 1.4.5.

Theorem 1.5.4 Let $r \geq 2$ be an even integer, and $G$ be an $(r-1)$-edge connected $r$-regular multigraph of odd order. Then for every vertex $v, G-v$ has a 1-factor, that is, $G$ is factor-critical. 
Proof. Let $\emptyset \neq S \subset V(G-v)=V(G)-v$. Let $C_{1}, C_{2}, \cdots, C_{m}$ be the odd components of $(G-v)-S=G-(S \cup\{v\})$, where $m=\operatorname{odd}((G-v)-S)$. Then by Lemma 1.5.1, we have $e_{G}\left(C_{i}, S \cup\{v\}\right) \geq r$. Thus

$$
r(|S|+1)=\sum_{x \in S \cup\{v\}} \operatorname{deg}_{G}(x) \geq e_{G}\left(C_{1} \cup C_{2} \cup \cdots \cup C_{m}, S \cup\{v\}\right) \geq r m .
$$

Hence $m \leq|S|+1$, which implies $m \leq|S|$ by (1.9). Consequently $G-v$ has a 1-factor by the 1-Factor Theorem.

Theorem 1.5.5 (Plesník [128]) Let $r \geq 2$ be an integer, and $G$ an $(r-1)$ edge connected $r$-regular multigraph of even order. Then for any $r-1$ edges $e_{1}, e_{2}, \ldots, e_{r-1}$ of $G, G$ has a 1-factor excluding $\left\{e_{1}, e_{2}, \ldots, e_{r-1}\right\}$.

Proof. Let $H=G-\left\{e_{1}, \ldots, e_{r-1}\right\}$. It suffices to show that $H$ has a 1 -factor. Let $\emptyset \neq S \subset V(H)=V(G)$, and $C_{1}, C_{2}, \cdots, C_{m}$ be the odd components of $H-S$. Then by the same argument as in the proof of Lemma 1.5.1, we have $e_{G}\left(C_{i}, V(G)-V\left(C_{i}\right)\right) \geq r-1 \quad$ and $\quad e_{G}\left(C_{i}, V(G)-V\left(C_{i}\right)\right) \equiv r \quad(\bmod 2)$.

Hence

$$
r \leq e_{G}\left(C_{i}, V(G)-V\left(C_{i}\right)\right)=e_{G}\left(C_{i}, S\right)+e_{G}\left(C_{i}, V(G)-S-V\left(C_{i}\right)\right) .
$$

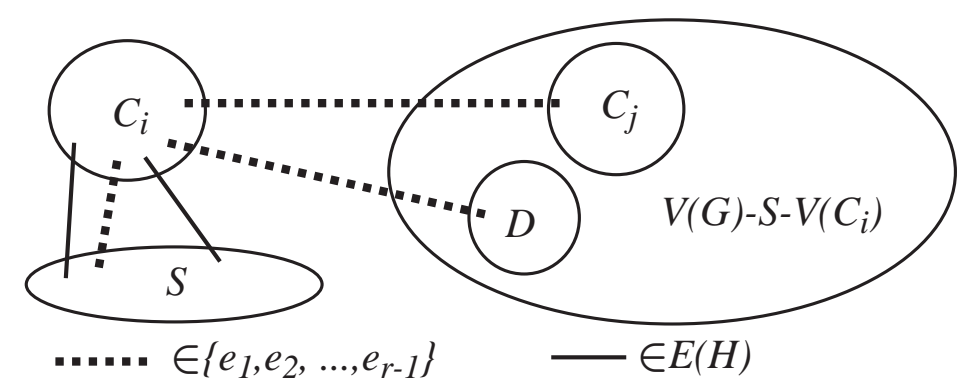

Figure 1.30: $e_{G}\left(C_{i}, S\right)$ and $e_{G}\left(C_{i}, V(G)-S-V\left(C_{i}\right)\right)$, where $D$ denotes an even component of $G-S$.

It is obvious that $E_{G}\left(C_{i}, V(G)-S-V\left(C_{i}\right)\right) \subseteq\left\{e_{1}, \ldots, e_{r-1}\right\}$ and each $e_{j}$ is contained in at most two such edge subsets, and thus

$$
\sum_{i=1}^{m} e_{G}\left(C_{i}, V(G)-S-V\left(C_{i}\right)\right) \leq 2\left|\left\{e_{1}, e_{2}, \ldots, e_{r-1}\right\}\right|=2(r-1) .
$$


Therefore by (1.18),

$$
\begin{aligned}
r m & \leq \sum_{i=1}^{m}\left(e_{G}\left(C_{i}, S\right)+e_{G}\left(C_{i}, V(G)-S-V\left(C_{i}\right)\right)\right) \\
& \leq \sum_{x \in S} \operatorname{deg}_{G}(x)+\sum_{i=1}^{m} e_{G}\left(C_{i}, V(G)-S-V\left(C_{i}\right)\right) \\
& \leq r|S|+2(r-1)
\end{aligned}
$$

Hence $m \leq|S|+2(r-1) / r<|S|+2$, which implies $m \leq|S|$ by (1.9). Consequently $H$ has a 1 -factor.

By Theorem 1.5.3, every $(r-1)$-edge connected $r$-regular multigraph of even order has a 1-factor. We can say that this result is best possible in the sense that there exist infinitely many $(r-2)$-edge connected $r$-regular multigraphs of even order that have no 1-factor. An example is given below.

Example Let $r \geq 3$ be an odd integer. Let $\overline{K_{r-2}}=(r-2) K_{1}$ be the totally disconnected graph of order $r-2$ and $R$ an $(r-2)$-edge connected simple graph of odd order such that $r-2$ vertices of $R$ have degree $r-1$ and all the other vertices have degree $r$. Such an $R$ can be obtained from the complete graph $K_{r+2}$ by deleting one cycle with $r-2$ edges and two independent edges. It is easy to see that there exist infinitely many such graphs $R$ for any given $r$.

We construct an $(r-2)$-edge connected $r$-regular simple graph $G$ from $\overline{K_{r-2}}$ and $r$ copies of $R$ : Join each vertex of $\overline{K_{r-2}}$ to one vertex of degree $r-1$ in every copy of $R$. The resulting graph is an $(r-2)$-edge connected $r$-regular simple graph $G$ (Figure 1.31). Then $G$ has no 1-factor since

$$
\begin{aligned}
\operatorname{odd}\left(G-V\left(\overline{K_{r-2}}\right)\right) & =\text { the number of copies of } R \\
& =r>\left|V\left(\overline{K_{r-2}}\right)\right|=r-2 .
\end{aligned}
$$

We can similarly construct such graphs for even integers $r$.

The next theorem shows that an $r$-regular simple graph with small order has a 1-factor even if it is not $(r-1)$-edge connected.

Theorem 1.5.6 Let $r \geq 2$ be an integer, and $G$ be an $n$-edge connected $r$ regular simple graph of even order. Define an integer $n^{\prime}$ in $\{n, n+1\}$ such that $n^{\prime} \equiv n \quad(\bmod 2)$. Then the following two statements holds:

(i) If $r$ is odd and

$$
\left\lfloor\frac{|G|-1}{r+2}\right\rfloor<\frac{2 r}{r-n^{\prime}}
$$



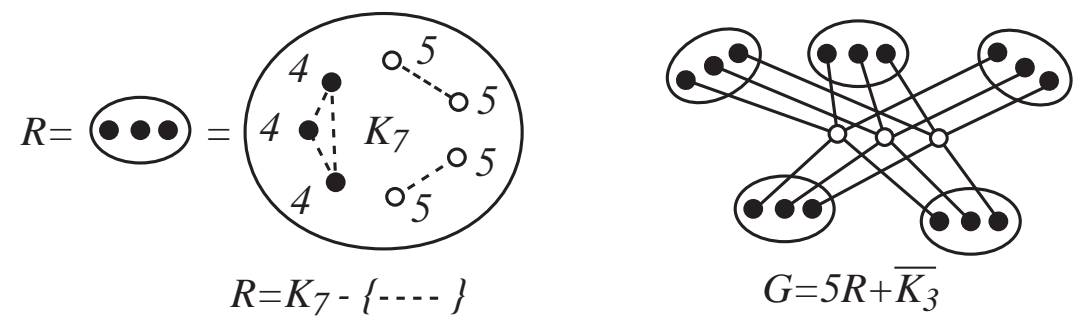

Figure 1.31: A 3-edge connected 5-regular graph $G$ having no 1-factors; numbers denote the degrees of vertices in $R$.

then $G$ has a 1-factor. In particular, every connected $r$-regular simple graph with order at most $3(r+2)$ has a 1-factor.

(ii) If $r$ is even and

$$
\left\lfloor\frac{|G|-1}{r+1}\right\rfloor<\frac{2 r}{r-n^{\prime}},
$$

then $G$ has a 1-factor. In particular, every connected $r$-regular simple graph with order at most $3(r+1)$ has a 1-factor.

Proof. We shall prove only (i). The proof of (ii), which is similar to that of (i), is left to the reader. Let $r \geq 3$ be an odd integer. Let $\emptyset \neq S \subset V(G)$, and $C_{1}, C_{2}, \ldots, C_{m}$ the odd components of $G-S$, where $m=\operatorname{odd}(G-S)$. If $\left|C_{i}\right| \leq r$, then $e_{G}\left(S, C_{i}\right) \geq r$ since

$$
\begin{aligned}
r\left|C_{i}\right| & =\sum_{x \in V\left(C_{i}\right)} \operatorname{deg}_{G}(x)=e_{G}\left(C_{i}, S\right)+2|| C_{i}|| \\
& \leq e_{G}\left(C_{i}, S\right)+\left|C_{i}\right|\left(\left|C_{i}\right|-1\right) \leq e_{G}\left(C_{i}, S\right)+r\left(\left|C_{i}\right|-1\right)
\end{aligned}
$$

Since $r+1$ is an even integer, no $C_{i}$ has order $r+1$. Let us define

$m_{1}=$ the number of $C_{i}$ with $\left|C_{i}\right| \leq r$; and

$m_{2}=$ the number of $C_{i}$ with $\left|C_{i}\right| \geq r+2$.

Then $m=m_{1}+m_{2}$, and $e_{G}\left(S, C_{i}\right) \geq r$ if $\left|C_{i}\right| \leq r$, and by Lemma 1.5.1, $e_{G}\left(S, C_{i}\right) \geq n^{\prime}$ if $\left|C_{i}\right| \geq r+2$. Therefore we have

$$
r|S| \geq e_{G}\left(S, C_{1} \cup C_{2} \cup \cdots \cup C_{m}\right) \geq r m_{1}+n^{\prime} m_{2}=r m+\left(n^{\prime}-r\right) m_{2},
$$

which implies $m \leq|S|+\left(r-n^{\prime}\right) m_{2} / r$.

Therefore the inequality $m=\operatorname{odd}(G-S)<|S|+2$ holds if

$$
m_{2}<\frac{2 r}{r-n^{\prime}}
$$


On the other hand, it follows that

$$
|G| \geq|S|+\left|C_{1}\right|+\cdots+\left|C_{m}\right| \geq|S|+(r+2) m_{2}
$$

which implies

$$
m_{2} \leq\left\lfloor\frac{|G|-|S|}{r+2}\right\rfloor \leq\left\lfloor\frac{|G|-1}{r+2}\right\rfloor .
$$

Consequently, $m<|S|+2$, which implies that $G$ has a 1 -factor if the following inequality holds:

$$
\left\lfloor\frac{|G|-1}{r+2}\right\rfloor<\frac{2 r}{r-n^{\prime}} .
$$

Therefore the first part of (i) is proved.

We next prove the second part of (i). If $|G| \leq 3(r+2)$ and $n^{\prime}=1$, then

$$
\left\lfloor\frac{|G|-1}{r+2}\right\rfloor=2<\frac{2 r}{r-1}=\frac{2 r}{r-n^{\prime}} .
$$

Hence $G$ has a 1 -factor by the first part of (i).

We next identify some non-regular graphs which have 1-factors. The binding number $\operatorname{bind}(G)$ of a graph $G$, which was introduced by Anderson [12], is defined by

$$
\operatorname{bind}(G)=\min \left\{\frac{\left|N_{G}(X)\right|}{|X|} \mid \emptyset \neq X \subseteq V(G), N_{G}(X) \neq V(G)\right\} .
$$

Thus for every subset $\emptyset \neq S \subset V(G)$ with $N_{G}(S) \neq V(G)$, it follows that $\left|N_{G}(S)\right| \geq \operatorname{bind}(G)|S|$.

Theorem 1.5.7 (Woodall [151]) Let $G$ be a connected simple graph of even order. If for every $\emptyset \neq S \subseteq V(G)$,

$$
N_{G}(S)=V(G) \quad \text { or } \quad\left|N_{G}(S)\right|>\frac{4}{3}|S|-1,
$$

then $G$ has a 1-factor. In particular, a connected simple graph $H$ with $\operatorname{bind}(H) \geq 4 / 3$ has a 1-factor.

Proof. The proof is by contradiction. Assume that $G$ has no 1-factor. Then by the 1-Factor Theorem and by (1.9), there exists a subset $\emptyset \neq S \subset V(G)$ such that $\operatorname{odd}(G-S) \geq|S|+2$.

Let $V=V(G)$ and $n=|V|$. Let $X$ be the set of isolated vertices of $G-S$, and $C_{1}, C_{2}, \ldots, C_{k}$ the odd components of $G-S$ with order at least three. Let $Y=V\left(C_{1}\right) \cup V\left(C_{2}\right) \cup \cdots \cup V\left(C_{k}\right)$ (Figure 1.32). Then

$$
\operatorname{odd}(G-S)=|X|+k \geq|S|+2, \quad|Y| \geq 3 k \text { and }|V| \geq|S|+|X|+|Y| \text {. }
$$




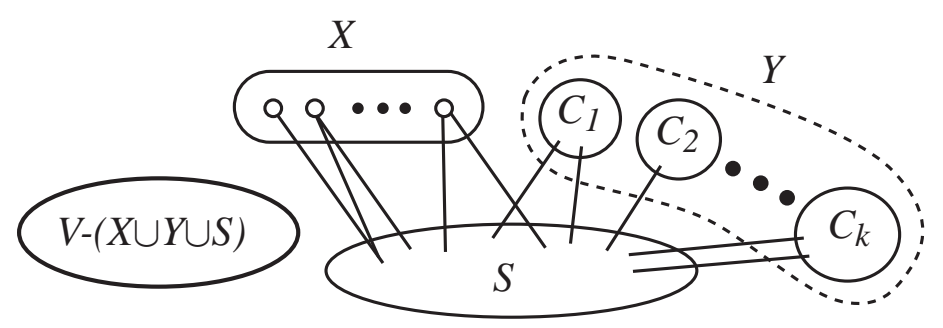

Figure 1.32: The graph $G$ with a subset $S$.

We consider two cases:

Case $1 \quad X \neq \emptyset$.

Since $N_{G}(V-S) \subseteq V-X \neq V$, it follows from (1.19) that

$$
\left|N_{G}(V-S)\right|>\frac{4}{3}|V-S|-1=\frac{4 n}{3}-\frac{4}{3}|S|-1
$$

and

$$
\left|N_{G}(V-S)\right| \leq|V-X|=n-|X|
$$

By the previous two inequalities, we obtain

$$
n<4|S|+3-3|X|
$$

On the other hand, it follows from (1.20) that

$$
\begin{aligned}
|V-S| & \geq|X|+|Y| \geq|X|+3 k \\
& \geq|X|+3(|S|+2-|X|)=3|S|-2|X|+6
\end{aligned}
$$

Hence $n=|V| \geq 4|S|-2|X|+6$. By this inequality and (1.21), we have

$$
4|S|-2|X|+6 \leq n<4|S|+3-3|X|,
$$

which is a contradiction.

Case $2 \quad X=\emptyset$.

In this case $\operatorname{odd}(G-S)=k$. Let $Z=V\left(C_{2}\right) \cup \cdots \cup V\left(C_{k}\right)=Y-V\left(C_{1}\right)$. Since $N_{G}(Z) \subseteq Z \cup S \neq V$, it follows from (1.19) that

$$
|Z|+|S| \geq\left|N_{G}(Z)\right|>\frac{4}{3}|Z|-1
$$

Thus $|Z|<3|S|+3$. On the other hand, by (1.20) we have that

$$
|Z| \geq 3(k-1)=3(\operatorname{odd}(G-S)-1) \geq 3(|S|+2-1) .
$$




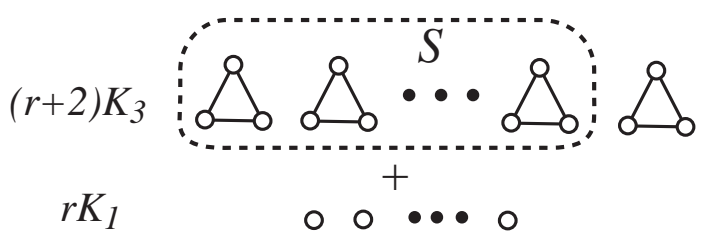

Figure 1.33: A graph $G=(r+2) K_{3}+r K_{1}$, which has no 1-factor and whose binding number is $(4 / 3)-(1 / 3 r)$.

Therefore

$$
3(|S|+1) \leq|Z|<3|S|+3 .
$$

This is again a contradiction. Consequently the theorem is proved.

Consider a graph $G=(r+2) K_{3}+r K_{1}$, where $r \geq 1$ is an integer (Figure 1.33). Then we can easily show that $G$ has no 1 -factor, and setting $S=V\left((r+1) K_{3}\right)$, we have

$$
\operatorname{bind}(G)=\frac{\left|N_{G}(S)\right|}{|S|}=\frac{\left|V(G)-V\left(K_{3}\right)\right|}{|S|}=\frac{4(r+1)-1}{3(r+1)}=\frac{4}{3}-\frac{1}{3(r+1)} .
$$

Hence the condition of Theorem 1.5.7 is best possible.

Recall that the toughness $\operatorname{tough}(G)$ of a connected non-complete graph $G$ is defined as

$$
\operatorname{tough}(G)=\min \left\{\frac{|X|}{\omega(G-X)} \mid \omega(G-X) \geq 2, \emptyset \neq X \subseteq V(G)\right\},
$$

where $\omega(G-X)$ denotes the number of components of $G-X$. A graph $G$ is said to be $t$-tough if $\operatorname{tough}(G) \geq t$. Hence, if $G$ is $t$-tough, then for a subset $S \subset V(G)$ with $\omega(G-S) \geq 2$, it follows that

$$
\omega(G-S) \leq \frac{|S|}{\operatorname{tough}(G)} \leq \frac{|S|}{t} .
$$

Theorem 1.5.8 Every 1-tough connected simple graph of even order has a 1-factor. Moreover, for every real number $\epsilon>0$, there exist graphs $G$ of even order that have no 1-factors and satisfy tough $(G)>1-\epsilon$.

Proof. Let $G$ be a 1-tough connected graph of even order, and $\emptyset \neq S \subset$ $V(G)$. If $\omega(G-S) \geq 2$, then

$$
\operatorname{odd}(G-S) \leq \omega(G-S) \leq \frac{|S|}{\operatorname{tough}(G)} \leq|S|
$$


If $\omega(G-S)=1$, then $\operatorname{odd}(G-S) \leq \omega(G-S)=1 \leq|S|$. Hence $\operatorname{odd}(G-S) \leq$ $|S|$ always holds, and thus $G$ has a 1-factor by the 1-Factor Theorem.

Let $G=K_{m}+t K_{n}$, which is a join of the complete graph $K_{m}$ and the $t$ copies of the complete graph $K_{n}$, where $n$ is an odd integer (Figure $1.34(1)$ ). Moreover, we can choose $m, n$ and $t$ so that

$$
t>m, \quad \frac{m}{t}>1-\epsilon \text { and } m+n t \equiv 0 \quad(\bmod 2)
$$

Then $G$ has no 1-factor since $\operatorname{odd}\left(G-V\left(K_{m}\right)\right)=t>\left|V\left(K_{m}\right)\right|=m$, and

$$
\operatorname{tough}(G)=\frac{\left|V\left(K_{m}\right)\right|}{\omega\left(G-V\left(K_{m}\right)\right)}=\frac{m}{t}>1-\epsilon
$$

Therefore the theorem is proved.

Recall that a graph $G$ is said to be claw-free if $G$ contains no induced subgraph isomorphic to the claw $K_{1,3}$ (Figure $1.34(2)$ ).

(1)

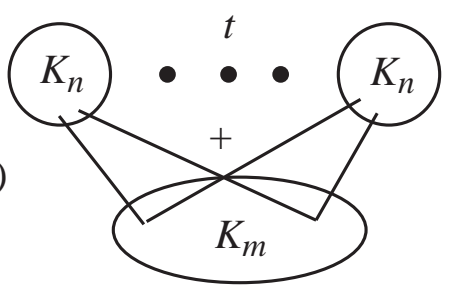

(2)

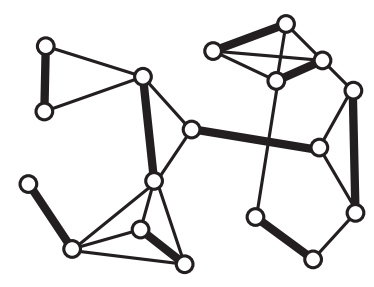

Figure 1.34: (1) $K_{m}+t K_{n}$; (2) A claw-free graph and its 1-factor.

Theorem 1.5.9 (Sumner [133], Las Vergnas [91]) Every connected claw-free simple graph of even order has a 1-factor.

Proof. We prove the theorem by induction on $|G|$. Let $P=\left(v_{1}, v_{2}, \ldots, v_{k}\right)$, $\left(v_{i} \in V(G)\right)$ be a longest path of $G$. Then $k \geq 3$. It is immediate that $N_{G}\left(v_{1}\right) \subseteq V(P)-\left\{v_{1}\right\}$.

We shall show that $G-\left\{v_{1}, v_{2}\right\}$ is connected. If $\operatorname{deg}_{G}\left(v_{2}\right)=2$, then $G-\left\{v_{1}, v_{2}\right\}$ is connected since $N_{G}\left(v_{1}\right) \subseteq V(P)-\left\{v_{1}\right\}$. Thus we may assume that $\operatorname{deg}_{G}\left(v_{2}\right) \geq 3$. For every $x \in N_{G}\left(v_{2}\right)-V(P)$, $x v_{3}$ must be an edge of $G$ since otherwise $v_{1} x$ or $v_{1} v_{3}$ is an edge of $G$ as $\left\langle\left\{v_{2}, v_{1}, v_{3}, x\right\}\right\rangle_{G} \neq K_{1,3}$; but this implies $G$ contains a path longer than $P$, a contradiction. Therefore $G-\left\{v_{1}, v_{2}\right\}$ is connected, and is of course claw-free, and has even order. By the induction hypothesis, $G-\left\{v_{1}, v_{2}\right\}$ has a 1-factor, and so does $G$. 
It is known that every 4-connected planar graph has a Hamiltonian cycle [141], which implies that it has a 1-factor, but, there are infinitely many 3 -connected planar graphs without 1 -factors. The next theorem gives the lower bound for the order of a maximum matching of a planar graph. The proof given here is slightly different from that given in [121].

Theorem 1.5.10 (Nishizeki and Baybars [121]) Let $G$ be a connected planar simple graph with $\delta(G) \geq 3$. Then the number $|M|$ of vertices saturated by a maximum matching $M$ in $G$ is

$$
|M| \geq \frac{2|G|+4}{3}
$$

If $G$ is 2-connected, then

$$
|M| \geq \frac{2|G|+8}{3}
$$

Proof. Let $G$ be a 2-connected planar simple graph. We may assume that $G$ is drawn in the plane as a plane graph. Let $\emptyset \neq S \subset V(G)$. Let us denote by $X$ the set of isolated vertices of $G-S$, and by $C_{1}, C_{2}, \ldots, C_{m}$ the odd components of $G-S$ of order at least three. Then

$$
\operatorname{odd}(G-S)=|X|+m, \quad \text { and } \quad|G| \geq|S|+|X|+3 m .
$$

If $|S|=1$, then $\operatorname{odd}(G-S) \leq 1=|S|$ as $G$ is 2-connected. Thus we may assume that $|S| \geq 2$. We now construct a planar bipartite graph $B$ with bipartite sets $S$ and $X \cup\left\{v_{1}, v_{2}, \ldots, v_{m}\right\}$ from $G$ by contracting $C_{1}, C_{2}, \ldots, C_{m}$ into single vertices $v_{1}, v_{2}, \ldots, v_{m}$ and by replacing all multiple edges in the resulting graph by single edges, that is, $s \in S$ and $v_{i}$ are joined by an edge of $B$ if and only if $s$ and a vertex of $C_{i}$ are joined by an edge of $G$ (Figure 1.35) .

Since $G$ is a 2 -connected graph with $\delta(G) \geq 3$, it follows that

$$
\operatorname{deg}_{B}(x) \geq 3 \text { for all } x \in X, \quad \text { and } \quad \operatorname{deg}_{B}\left(v_{i}\right) \geq 2 \text { for all } 1 \leq i \leq m \text {. }
$$

Since $B$ is a planar bipartite graph, it follows from Theorem 05 that

$$
\|B\| \leq 2|B|-4
$$

By combining this inequality and (1.25), we obtain

$$
3|X|+2 m \leq|| B|| \leq 2|B|-4=2(|S|+|X|+m)-4 .
$$




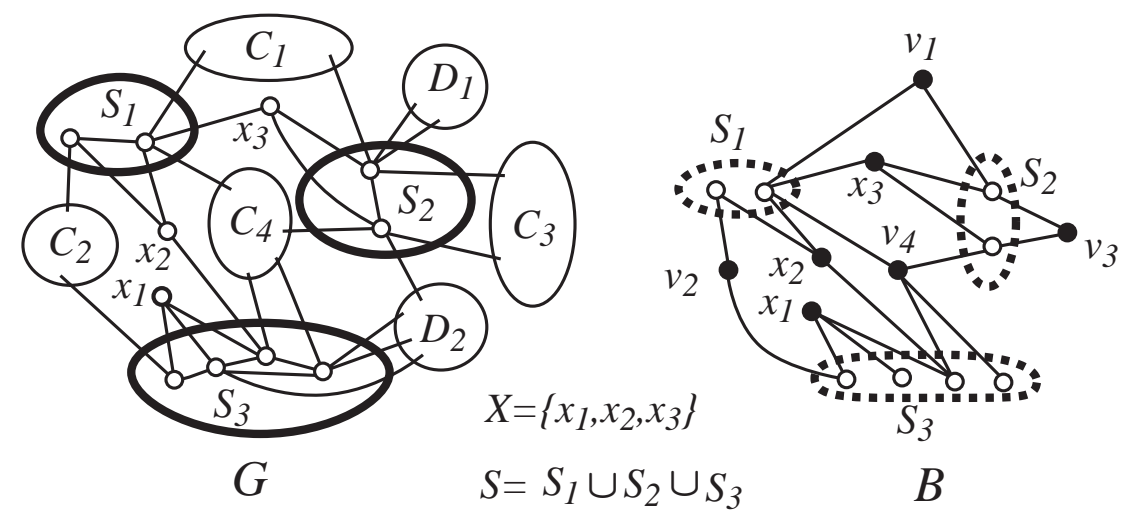

Figure 1.35: A 2-connected planar graph $G$ with odd components $\left\{x_{1}, \ldots, x_{3}\right.$, $\left.C_{1}, \ldots, C_{4}\right\}$ and even components $\left\{D_{1}, D_{2}\right\}$ of $G-S$; and the corresponding planar bipartite graph $B$.

Hence $|X| \leq 2|S|-4$. By this inequality and (1.24), we get

$$
\begin{aligned}
& \operatorname{odd}(G-S)-|S|=|X|+m-|S| \\
& \leq|X|+\frac{|G|-|S|-|X|}{3}-|S|=\frac{|G|+2|X|-4|S|}{3} \leq \frac{|G|-8}{3} .
\end{aligned}
$$

Consequently by (1.16) in Theorem 1.4.8, we have

$$
|M|=|G|-\max _{S}(\operatorname{odd}(G-S)-|S|) \geq|G|-\frac{|G|-8}{3}=\frac{2|G|+8}{3},
$$

which implies the desired inequality (1.22).
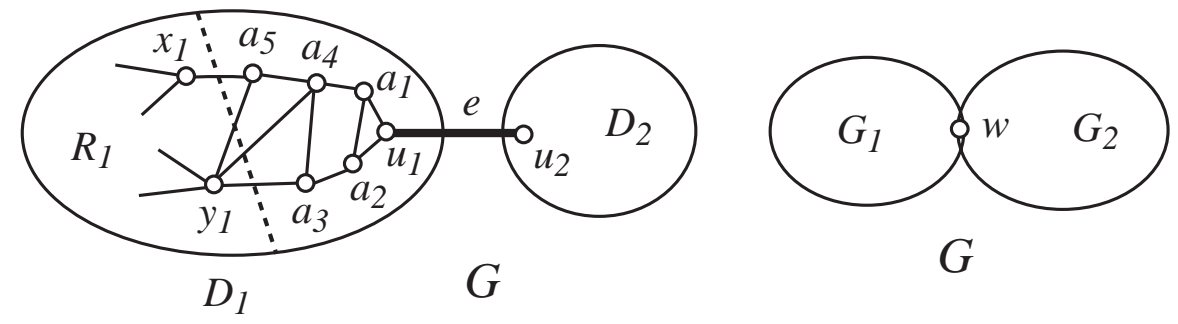

Figure 1.36: A connected planar graph $G$ with bridge $e$; and a connected planar graph $G$ with cut vertex $w$.

We next prove (1.22) in the case where $G$ is connected by induction on $|G|$. Since (1.22) holds for a small graph $G$, we may assume that the order 
of $G$ is not small (for example, $|G| \geq 10$ ). Suppose first $G$ has a bridge $e=u_{1} u_{2}$. Let $D_{1}$ and $D_{2}$ be the two components of $G-e$ containing $u_{1}$ and $u_{2}$, respectively. We shall latter show that each $D_{i}$ has a matching that covers at least $\left(2\left|D_{i}\right|+2\right) / 3$ vertices of $D_{i}$. If the above statement holds, then $G$ has a matching that covers at least

$$
\frac{2\left|D_{1}\right|+2}{3}+\frac{2\left|D_{2}\right|+2}{3}=\frac{2|G|+4}{3} .
$$

Hence the statement holds.

If $G$ has no bridges and has a cut vertex $w$, then by letting $G=G_{1} \cup$ $G_{2}, V\left(G_{1}\right) \cap V\left(G_{2}\right)=\{w\}$, we shall show that each $G_{i}$ has a matching that covers at least $\left(2\left|G_{i}\right|+4\right) / 3$ vertices of $G_{i}$. Then $G$ has a matching that covers at least the following number of vertices.

$$
\frac{2\left|G_{1}\right|+4}{3}-2+\frac{2\left|G_{2}\right|+4}{3}=\frac{2\left(\left|G_{1}\right|+\left|G_{2}\right|-1\right)+4}{3}=\frac{2|G|+4}{3} .
$$

Hence the statement holds.

We first show that each $D_{i}$ has a matching that covers at least $\left(2\left|D_{i}\right|+2\right) / 3$ vertices of $D_{i}$, and latter show that each $G_{i}$ has a matching that covers at least $\left(2\left|G_{i}\right|+4\right) / 3$ vertices of $G_{i}$. If $\delta\left(D_{i}\right) \geq 3$, then the above statement holds by induction. Without loss of generality, we may assume $\delta\left(D_{1}\right)=2$, which implies $\operatorname{deg}_{D_{1}}\left(u_{1}\right)=2$ (see Figure 1.36). Let $a_{1}$ and $a_{2}$ be the two vertices adjacent to $u_{1}$. If $\delta\left(D_{1}-u_{1}\right) \geq 3$, then by induction, $D_{1}-u_{1}$ has a matching covering at least $\left(2\left(\left|D_{1}\right|-1\right)+4\right) / 3$ vertices of $D_{1}-u_{1}$. Hence this is the desired matching of $D_{1}$. So we may assume that $\delta\left(D_{1}-u_{1}\right)=2$. If $a_{1}$ and $a_{2}$ are non-adjacent, then $D_{1}-u_{1}+a_{1} a_{2}$ has minimum degree three, and so by the induction it has a matching $M_{1}$ covering at least $\left(2\left(\left|D_{1}\right|-1\right)+4\right) / 3$ vertices. If $M_{1}$ contains $a_{1} a_{2}$, then by considering $M_{1}-a_{1} a_{2}+a_{1} u_{1}$ we can also get the desired matching of $D_{1}$. If $a_{1}$ and $a_{2}$ are adjacent, then we may assume that $\operatorname{deg}_{D_{1}-u_{1}}\left(a_{1}\right)=2$. If $\delta\left(D_{1}-u_{1}-a_{1}\right) \geq 3$, then by induction it has a matching $M_{2}$ covering at least $\left(2\left(\left|D_{1}\right|-2\right)+4\right) / 3$ vertices of $D_{1}-u_{1}-a_{1}$. Hence $M_{2}+a_{1} u_{1}$ is the desired matching of $D_{1}$. By repeating this argument, we can finally find the desired matching of $D_{1}$. As an example, consider the matching of $D_{1}$ obtained from a matching of $R_{1}$ and edges $a_{5} a_{4}, a_{3} a_{2}, a_{1} u_{1}$ (see $R_{1}$ of $D_{1}$ in Figure 1.36).

By a similar argument, we can show that each $G_{i}$ has a matching that covers at least $\left(2\left|G_{i}\right|+4\right) / 3$ vertices of $G_{i}$. Consequently the proof is complete.

A surface (a compact orientable 2-manifold) is a sphere on which a number of handles has been placed. The number of handles is referred to as the genus 
of the surface (Figure 1.37). A surface with genus one is called a torus, and, of course, a plane is a surface with genus zero. A graph $G$ is said to be embedded on a surface if $G$ can be drawn on the surface in such a way that edges intersect only at their common end-vertices. The genus $\gamma(G)$ of a simple graph $G$ is defined to be smallest genus of all surfaces on which $G$ has be embedded.
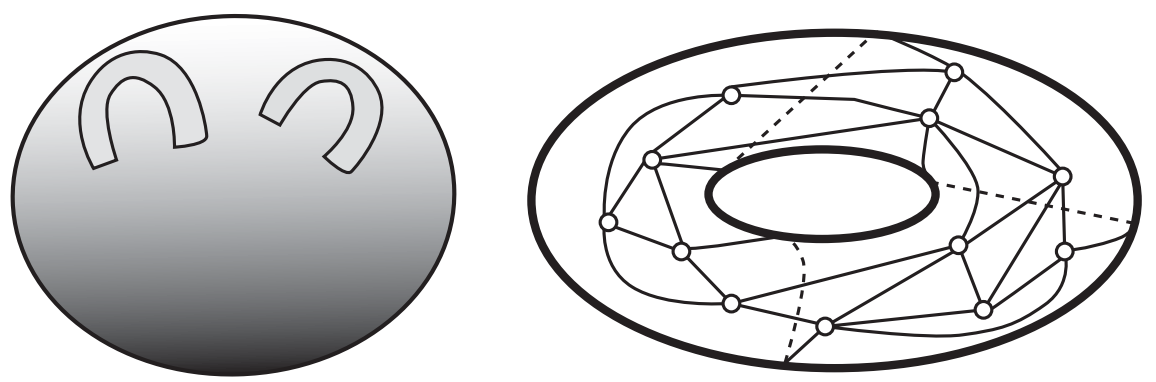

Figure 1.37: A surface with genus two, and an embedding of a graph on the torus.

Theorem 1.5.11 (Nishizeki [120]) Let $G$ be a n-connected simple graph of even order. If $\gamma(G)<n(n-2) / 4$, then $G$ has a 1-factor. In particular, every 4-connected simple graph of even order which is embeddable on the torus has a 1-factor.

\section{Exercises}

Exercise 1.5.1 For every even integer $r \geq 4$, find an $(r-2)$-edge connected $r$-regular simple graph of even order that has no 1-factor.

Exercise 1.5.2 Prove statement (ii) of Theorem 1.5.6.

Exercise 1.5.3 Prove Theorem 1.5.5 by making use of the 1-factor Theorem.

\subsection{Structure Theorem}

In this section we consider the structure of a graph having no 1-factors, and characterize such a graph by using vertex-decomposition together with their properties. These results are contained in the Gallai-Edmonds Structure Theorem and give us much information about maximum matchings. 
Let $G$ be a graph. Recall that for a subset $S \subseteq V(G)$, odd $(G-S)-|S|$ is the deficiency of $S$. A subset $X \subseteq V(G)$ is called a barrier in $G$ if

$$
\operatorname{odd}(G-X)-|X|=\operatorname{def}(G)=\max _{S \subseteq V(G)}\{\operatorname{odd}(G-S)-|S|\} .
$$

That is, $X$ is a barrier if its deficiency is equal to that of $G$. A barrier $X$ is said to be minimal if no proper subset of $X$ is a barrier.

Theorem 1.6.1 Suppose a simple connected graph $G$ with even order has no 1-factor. Let $X$ be a minimal barrier of $G$. Then every vertex $x \in X$ is joined to at least three odd components of $G-X$, in particular, $x$ is the center of certain induced claw subgraph of $G$.

Proof. Since $G$ has no 1 -factor and has even order, we have $\operatorname{def}(G) \geq 2$ by the 1-factor Theorem and Lemma 1.4.1. If $X=\{x\}$, then $\operatorname{odd}(G-X) \geq 3$ as $\operatorname{odd}(G-X) \equiv|X| \quad(\bmod 2)$ by Lemma 1.4.1. Hence $x \in X$ is joined to at least three odd components of $G-X$ and the theorem holds. Thus we may assume that $|X| \geq 2$.

Suppose that a vertex $x \in X$ is joined to at most two odd components of $G-X$ (Figure 1.38). Then $\operatorname{odd}(G-(X-x)) \geq \operatorname{odd}(G-X)-2$, and thus

$$
\operatorname{odd}(G-(X-x))-|X-x| \geq \operatorname{odd}(G-X)-|X|-1 \text {. }
$$

Since $\operatorname{odd}(G-(X-x))-|X-x| \equiv \operatorname{odd}(G-X)-|X| \equiv|G|(\bmod 2)$ by Lemma 1.4.1, we have

$$
\operatorname{odd}(G-(X-x))-|X-x| \geq \operatorname{odd}(G-X)-|X|
$$

which implies that $X-x$ is also a barrier. This contradicts the minimality of $X$. Therefore every $x \in X$ is joined to at least three odd components of $G-X$.

By taking three vertices adjacent to $x$ from each of the three odd components of $G-X$ joined to $x$, we can obtain an induced subgraph $K_{1,3}$ with center $x$.

It is clear that Theorem 1.5.9, which says that every connected claw-free graph of even order has a 1-factor, is an immediate consequence of the above Theorem 1.6.1.

Consider a simple graph $G$. Let $D(G)$ denote the set of all vertices $v$ of $G$ such that $v$ is not saturated by at least one maximum matching of $G$. Let $A(G)$ be the set of vertices of $V(G)-D(G)$ that are adjacent to at least one 

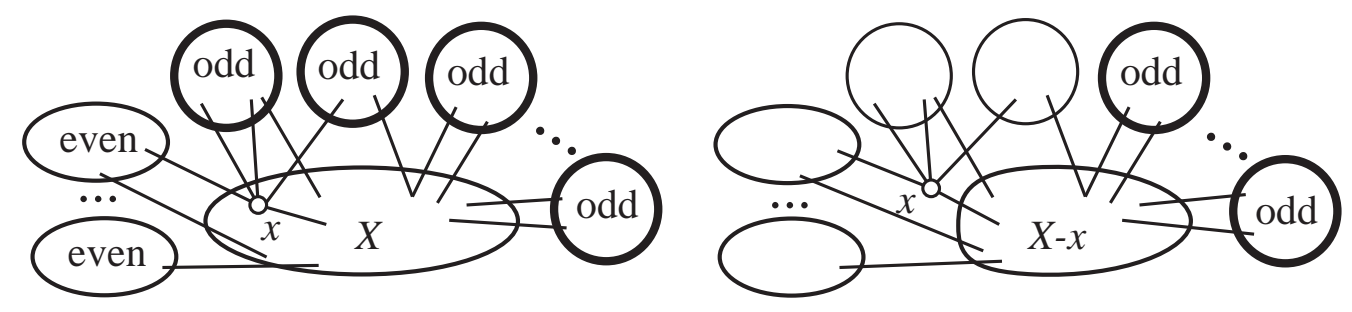

Figure 1.38: Components of $G-X$ and those of $G-(X-x)$.

vertex in $D(G)$. Finally define $C(G)=V(G)-D(G)-A(G)$. Then $V(G)$ is decomposed into three disjoint subsets

$$
V(G)=D(G) \cup A(G) \cup C(G),
$$

where

$$
\begin{aligned}
& D(G)=\left\{x \in V(G) \mid{ }^{\exists} \text { a maximum matching unsaturating } x\right\} \\
& A(G)=N_{G}(D(G)) \backslash D(G) \\
& C(G)=V(G)-(A(G) \cup D(G))
\end{aligned}
$$

Some properties of the above decomposition are given in the GallaiEdmonds Structure Theorem. This theorem was obtained by Gallai [52], [53] and Edmonds [36] independently and in different ways. The proof presented here is based on [71].

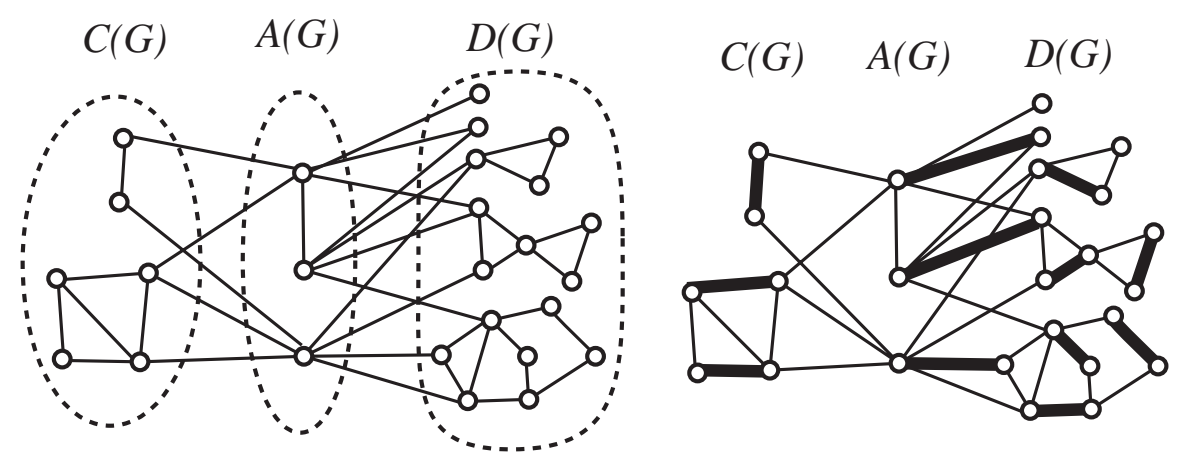

Figure 1.39: The decomposition $V(G)=D(G) \cup A(G) \cup C(G)$ and a maximum matching of $G$.

Theorem 1.6.2 (Gallai-Edmonds Structure Theorem [52], [53], [36] ) Let $G$ be a simple graph, and $V(G)=D(G) \cup A(G) \cup C(G)$ the decomposition 
defined in (1.23). Then the following statements hold (Figure 1.39):

(i) Every component of $\langle D(G)\rangle_{G}$ is factor-critical.

(ii) $\langle C(G)\rangle_{G}$ has a 1-factor.

(iii) Every maximum matching $M$ in $G$ saturates $C(G) \cup A(G)$, and every edge of $M$ incident with $A(G)$ joins a vertex in $A(G)$ to a vertex in $D(G)$.

(iv) The number $|M|$ of vertices saturated by a maximum matching $M$ is given by

$$
|M|=|G|+\omega\left(\langle D(G)\rangle_{G}\right)-|A(G)|,
$$

where $\omega\left(\langle D(G)\rangle_{G}\right)$ denote the number of components of $\langle D(G)\rangle_{G}$.

Proof. The proof of the theorem is by induction on $|G|$. We may assume that $G$ is connected since otherwise each component of $G$ satisfies the statements and so does $G$. Moreover we may assume that $G$ has no 1 -factor since otherwise $D(G)=\emptyset, A(G)=\emptyset$ and $C(G)=V(G)$, and thus the theorem holds.

Let $S$ be a maximal barrier of $G$, that is, $S$ is a subset of $V(G)$ such that

$$
\operatorname{odd}(G-S)-|S|=\max _{X \subset V(G)}\{\operatorname{odd}(G-X)-|X|\}=\operatorname{def}(G)>0
$$

and

$$
\operatorname{odd}(G-Y)-|Y|<\operatorname{odd}(G-S)-|S| \text { for all } S \subset Y \subseteq V(G)
$$

Claim 1. Every component of $G-S$ has odd order.

Assume that $G-S$ has an even component $D$. Then for a vertex $v$ of $D$, odd $(D-v) \geq 1$, and thus

$$
\begin{aligned}
& \operatorname{odd}(G-(S \cup\{v\}))-|S \cup\{v\}| \\
= & \operatorname{odd}(G-S)-|S|+\operatorname{odd}(D-v)-|v| \\
\geq & \operatorname{odd}(G-S)-|S|,
\end{aligned}
$$

contrary to (1.30). Hence Claim 1 is proved.

Claim 2. Every odd component of $G-S$ is factor-critical.

Let $C$ be an odd component of $G-S, v$ any vertex of $C$ and $T \subseteq V(G)-v$. Then by (1.30), we have

$$
\operatorname{odd}(G-(S \cup\{v\} \cup T))-|S \cup\{v\} \cup T|<\operatorname{odd}(G-S)-|S| .
$$

Since $\operatorname{odd}(G-(S \cup\{v\} \cup T))=\operatorname{odd}(G-S)-1+\operatorname{odd}((C-v)-T)$, we obtain $\operatorname{odd}((C-v)-T)<|T|+2$, which implies odd $((C-v)-T) \leq|T|$ 
by Lemma 1.4.1. Hence $C-v$ has a 1 -factor by the 1-Factor Theorem, and thus Claim 2 is proved.

Let $\left\{C_{1}, C_{2}, \ldots, C_{m}\right\}$ be the set of odd components of $G-S$, where $m=$ $\operatorname{odd}(G-S)$. We define the bipartite graph $B$ with bipartite sets $S$ and $\left\{C_{1}, C_{2}, \ldots, C_{m}\right\}$ as follows: a vertex $x \in S$ and $C_{i}$ is joined by an edge of $B$ if and only if $x$ and $C_{i}$ are joined by at least one edge of $G$ (Figure 1.40).

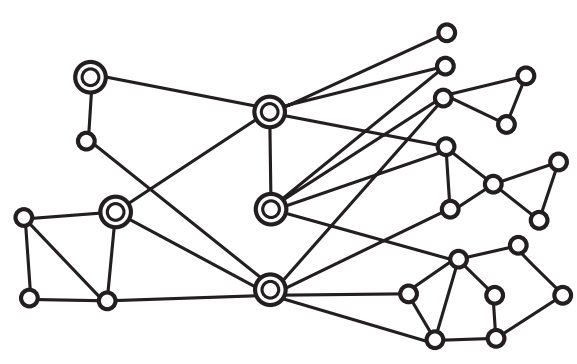

$S=\{\bigcirc\} \quad G$

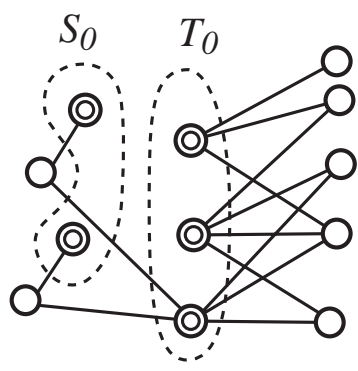

$B$

Figure 1.40: A graph $G$ with a maximal barrier $S$, and the bipartite graph $B$ with subsets $S_{0}$ and $T_{0}$.

Claim 3. $\left|N_{B}(X)\right| \geq|X|$ for all $X \subseteq S$.

Assume that $\left|N_{B}(Y)\right|<|Y|$ for some $\emptyset \neq Y \subseteq S$. Then

$$
\begin{aligned}
& \operatorname{odd}(G-(S-Y))-|S-Y| \geq\left|\left\{C_{1}, C_{2}, \ldots, C_{m}\right\}-N_{B}(Y)\right|-|S-Y| \\
> & m-|Y|-|S-Y|=m-|S|=\operatorname{odd}(G-S)-|S|,
\end{aligned}
$$

contrary to (1.29). Hence Claim 3 holds.

Claim 4. There exists a unique maximum proper subset $S_{0} \subset S$ such that $\left|N_{B}\left(S_{0}\right)\right|=\left|S_{0}\right|$. Furthermore, $\left|N_{B}(Y) \backslash N_{B}\left(S_{0}\right)\right|>|Y|$ for every $\emptyset \neq Y \subseteq$ $S-S_{0}$.

By $\left|N_{B}(S)\right|=m>|S|$, Claim 3 and by Proposition 1.1.9, there exists a unique maximum proper subset $S_{0} \subset S$ such that $\left|N_{B}\left(S_{0}\right)\right|=\left|S_{0}\right|$.

Let $\emptyset \neq Y \subseteq S-S_{0}$. Then it follows from the maximality of $S_{0}$ and $S_{0} \subset Y \cup S_{0}$ that

$$
\left|N_{B}(Y) \backslash N_{B}\left(S_{0}\right)\right|=\left|N_{B}\left(Y \cup S_{0}\right)-N_{B}\left(S_{0}\right)\right|>\left|Y \cup S_{0}\right|-\left|S_{0}\right|=|Y| .
$$

Therefore the claim is proved. 
Let $T_{0}=S-S_{0}$. Then

$$
\begin{aligned}
\operatorname{odd}\left(G-T_{0}\right) & =\operatorname{odd}(G-S)-\left|N_{B}\left(S_{0}\right)\right|=\operatorname{odd}(G-S)-\left|S_{0}\right| \\
& =\operatorname{odd}(G-S)-\left(|S|-\left|T_{0}\right|\right),
\end{aligned}
$$

and so $\operatorname{odd}\left(G-T_{0}\right)-\left|T_{0}\right|=\operatorname{odd}(G-S)-|S|=\operatorname{def}(G)$.

Let $\left\{C_{1}, C_{2}, \ldots, C_{k}\right\}$ be the set of odd components of $G-T_{0}$, where $k=\operatorname{odd}\left(G-T_{0}\right)$, and let $\left\{C_{1}^{\prime}, C_{2}^{\prime}, \ldots, C_{r}^{\prime}\right\}$ be the set of odd components of $G-S$ corresponding to $N_{B}\left(S_{0}\right)$, where $r=\left|N_{B}\left(S_{0}\right)\right|=\left|S_{0}\right|$. Then by the Marriage Theorem and Claim 4, it follows that

(i) $B$ has a matching saturating $S$;

(ii) every matching in $B$ saturating $S$ saturates $\left\{C_{1}^{\prime}, C_{2}^{\prime}, \ldots, C_{r}^{\prime}\right\}$; and

(iii) for each $C_{i}(1 \leq i \leq k)$, there exists a matching in $B$ that saturates $S$ but not $C_{i}$.

Let $H$ be a matching in $B$ saturating $S$. Then for every odd component $C_{j}^{\prime}(1 \leq j \leq r)$, there exists an edge in $H$ joining $C_{j}^{\prime}$ to a vertex $x_{j} \in S_{0}$. Take an edge $e_{j}$ of $G$ joining $x_{j}$ to a vertex $v_{j}$ in $C_{j}^{\prime}$. By Claim 4 , we can get a 1-factor $R_{j}^{\prime}$ of $C_{j}^{\prime}-v_{j}$.

Similarly, for an odd component $C_{i}(1 \leq i \leq k)$, if $H$ has an edge joining $C_{i}$ to $x_{i} \in S$, then $x_{i} \in T_{0}$ and we can find an edge $e_{i}$ of $G$ joining $x_{i}$ to a vertex $w_{i}$ of $C_{i}$ and a 1 -factor $R_{i}$ of $C_{i}-w_{i}$. If $H$ has no edge joining $C_{i}$ to $S$, then take a maximum matching $R_{i}$ in $C_{i}$. Define

$$
M=\bigcup_{1 \leq j \leq r}\left(R_{j}^{\prime}+e_{j}\right)+\bigcup_{1 \leq i \leq k}\left\{\left(R_{i}+e_{i}\right) \text { or } R_{i}\right\} .
$$

Then by Theorem 1.4.8, $M$ is a maximum matching of $G$ since the number of unsaturated vertices in $M$ is $k-\left|T_{0}\right|=\operatorname{odd}\left(G-T_{0}\right)-\left|T_{0}\right|=\operatorname{def}(G)$.

Conversely, every maximum matching in $G$ is obtained in this way since for any maximum matching $M^{\prime}$ in $G, M^{\prime}$ cannot saturate at least $k-\left|T_{0}\right|$ odd components in $\left\{C_{1}, C_{2}, \ldots, C_{k}\right\}$. Since $M^{\prime}$ is a maximum matching, $M^{\prime}$ does not saturate exactly $k-\left|T_{0}\right|=\operatorname{def}(G)$ components of $\left\{C_{1}, C_{2}, \ldots, C_{k}\right\}$. Therefore $M^{\prime}$ induces a matching $H^{\prime}$ in $B$ that saturates $S$ and $\left\{C_{1}^{\prime}, C_{2}^{\prime}, \ldots, C_{r}^{\prime}\right\}$, and thus $M^{\prime}$ can be constructed from $H^{\prime}$ as above.

Claim 5. $D(G)=V\left(C_{1}\right) \cup V\left(C_{2}\right) \cup \cdots \cup V\left(C_{k}\right)$ and $A(G)=T_{0}$.

It is clear that for every vertex $v_{i}$ of any $C_{i}(1 \leq i \leq k), B$ has a matching that saturates $S$ but not $C_{i}$, and $C_{i}-v_{i}$ has a 1 -factor by Claim 2 . Hence by (1.31), we can find a maximum matching in $G$ that does not saturate $v_{i}$. Thus $V\left(C_{1}\right) \cup V\left(C_{2}\right) \cup \cdots \cup V\left(C_{k}\right) \subseteq D(G)$. Since every maximum matching in $G$ is obtained in the way mentioned above, $D(G) \subseteq V\left(C_{1}\right) \cup V\left(C_{2}\right) \cup \cdots \cup V\left(C_{k}\right)$. Consequently, $D(G)=V\left(C_{1}\right) \cup V\left(C_{2}\right) \cup \cdots \cup V\left(C_{k}\right)$. 
Since $N_{B}\left(S_{0}\right)=\left\{C_{1}^{\prime}, C_{2}^{\prime}, \ldots, C_{r}^{\prime}\right\}$, it follows that $N_{B}\left(\left\{C_{1}, C_{2}, \ldots, C_{k}\right\}\right)=$ $S-S_{0}=T_{0}$. Therefore

$$
A(D(G))=N_{G}\left(V\left(C_{1}\right) \cup \cdots \cup V\left(C_{k}\right)\right) \backslash\left(V\left(C_{1}\right) \cup \cdots \cup V\left(C_{k}\right)\right)=T_{0} .
$$

It is easy to see that $\left\langle V\left(C_{1}^{\prime}\right) \cup \cdots \cup V\left(C_{r}^{\prime}\right) \cup S_{0}\right\rangle$ has a 1-factor, and forms the even components of $G-T_{0}$.

Since every component of $\langle D(G)\rangle_{G}$ is factor-critical, Theorem 1.4.3 is an easy consequence of the Gallai-Edmonds Structure Theorem.

The following lemma is interesting its own right, but it is also useful to prove Gallai-Edmonds Structure Theorem, that is, we can first prove the following Stability Lemma without using Gallai-Edmonds Structure Theorem, and then apply the lemma to prove the Structure Theorem ([108] section 3.2). However, we shall prove the lemma using Gallai-Edmonds Structure Theorem because it is shorter.

Lemma 1.6.3 (The Stability Lemma) Let $G$ be a simple graph and $V(G)=$ $C(G) \cup A(G) \cup D(G)$. Then for every vertex $u \in A(G)$, we have $A(G-u)=$ $A(G)-u, C(G-u)=C(G)$ and $D(G-u)=D(G)$.

Proof. Let $u \in A(G)$ and $M$ be any maximum matching in $G$. Then by the definition of $A(G), M$ has an edge $e$ incident with $u$. Then $M-e$ is a maximum matching in $G-u$ since the size of a maximum matching in $G-u$ must be less than or equal to $\|M\|-1$ and $\|M-e\|=\|M\|-1$. Therefore $D(G) \subseteq D(G-u)$, and the size of a maximum matching in $G-u$ is $\|M\|-1$.

If a maximum matching $H$ in $G-u$ does not saturate a vertex $x \in$ $A(G)-u$, then $H$ cannot saturate at least $\omega\left(\langle D(G)\rangle_{G}\right)-|A(G)|+1$ vertices in $D(G)$ and two more vertices $x, u \in A(G)$ since $H$ can cover at most $|A(G)|-1$ components in $\langle D(G)\rangle_{G}$ by Theorem 1.6.2. Therefore $\|H\|<\|M\|-1$, contrary to the fact that a maximum matching in $G-u$ has size $\|M\|-1$. Hence $(A(G)-u) \cap D(G-u)=\emptyset$. We can similarly show that $H$ saturates $C(G)$, which implies $C(G) \cap D(G-u)=\emptyset$. Consequently, $D(G-u)=D(G)$.

The other equalities $A(G-u)=A(G)-u$ and $C(G-u)=C(G)$ follow immediately from $D(G-u)=D(G)$.

Since a factor-critical graph with order at least three is not a bipartite graph (Exercise 1.6.2), every component of $\langle D(G)\rangle_{G}$ in a bipartite graph $G$ must be a single vertex. Thus the following theorem holds.

Theorem 1.6.4 Let $G$ be a connected bipartite graph with bipartition $X \cup Y$, and let $C_{X}=C(G) \cap X$ and $C_{Y}=C(G) \cap Y$. Then the following statements hold (Figure 1.41): 


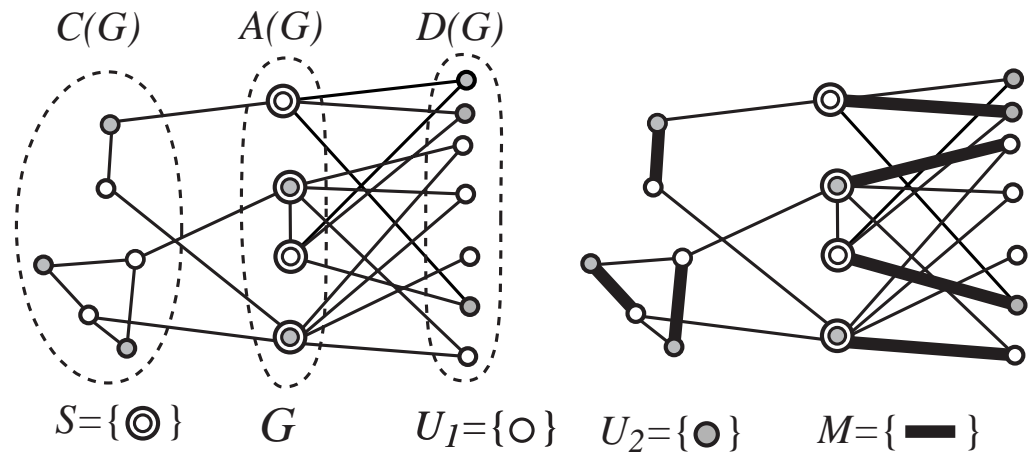

Figure 1.41: The decomposition $V(G)=D(G) \cup A(G) \cup C(G)$ of a bipartite graph $G$ with bipartition $U_{1} \cup U_{2}$; and it maximum matching $M$ x.

(i) $\langle D(G)\rangle_{G}$ is a set of independent vertices of $G$.

(ii) $\langle C(G)\rangle_{G}$ has a 1-factor, and $\left|C_{X}\right|=\left|C_{Y}\right|$.

(iii) Every maximum matching $M$ in $G$ consists of a 1-factor of $\langle C(G)\rangle_{G}$ and a matching in $\langle A(G) \cup D(G)\rangle_{G}$ saturating $A(G)$.

(iv) Both $A(G) \cup C_{X}$ and $A(G) \cup C_{Y}$ are minimum vertex covers of $G$.

(v) Both $D(G) \cup C_{X}$ and $D(G) \cup C_{Y}$ are maximum independent vertex subsets of $G$.

\section{Exercises}

Exercise 1.6.1 Let $v$ be a vertex of a graph $G$ and $M$ a maximum matching of a graph $G$, and let $M^{\prime}$ be a maximum matching of $G-v$. Prove that $\|M\|-1 \leq\left\|M^{\prime}\right\| \leq\|M\|$ and $\left\|M^{\prime}\right\|=\|M\|$ hold if and only if $v \in D(G)$.

Exercise 1.6.2 Show that a factor-critical graph of order at least three is not a bipartite graph.

Exercise 1.6.3 Let $G$ be a connected bipartite graph. Prove that Statements $(i v)$ and $(v)$ in Theorem 1.6.4 hold.

\subsection{Algorithms for Maximum Matchings}

We gave an algorithm for finding a maximum matching in a bipartite graph in section 1.3. In this section we shall give an algorithm for finding a maximum matching in a graph, which was obtained by Edmonds [36]. 
Before stating the algorithm, let us recall Theorem 1.3.2, which says that "a matching $M$ in a graph $G$ is maximum if and only if $G$ has no $M$ augmenting path". Therefore, to find a maximum matching, we should find $M$-augmenting paths or determine the non-existence of such paths. In order to effectively explore $M$-augmenting paths in a graph, we introduce some new concepts and notation.

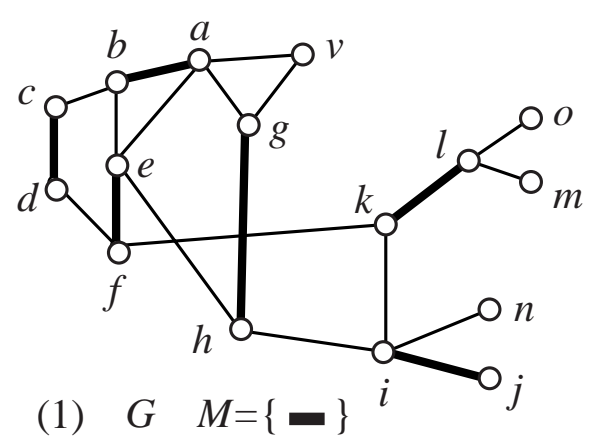

(1)

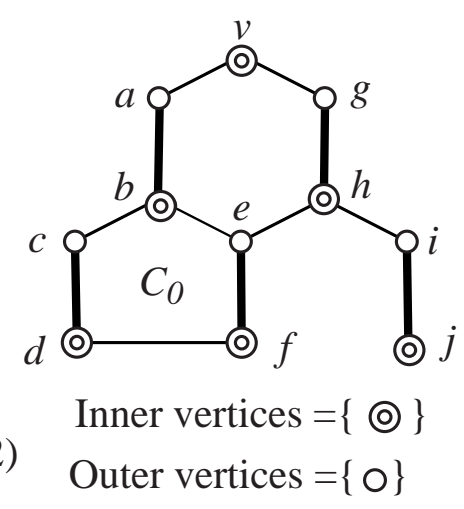

(2)

Figure 1.42: (1) A matching $M$ and an $M$-unsaturated vertex $v$; (2) The root $v$, inner vertices and outer vertices.

We shall first explain the algorithm and new definitions by using examples. Let $G$ be a graph and $M$ a matching in $G$, and let $v$ be a vertex unsaturated by $M$. We call $v$ a root, and explore all the $M$-alternating path starting with $v$. If $P=\left(v, x_{1}, x_{2}, x_{3}, x_{4}, \ldots, x_{k}\right)$ is an $M$-alternating path, where each $x_{i}$ is a vertex of $G$, then we call $x_{1}, x_{3}, \ldots$ inner vertices and $v, x_{2}, x_{4}, \ldots$ outer vertices (Figure 1.42)

Consider the graph $G$ and the matching $M$ given in Figure 1.42. We try to find all the $M$-alternating paths starting with $v$ as follows:

$$
\{v\}\{a, g\},\{b, h\},\{c, e, i\},\{d, f, j\} \text { and }\{f, k\} \text {. }
$$

In the last step, we find that $f$ is simultaneously an outer and inner vertex since $(u, a, b, e, f)$ and $(u, a, b, c, d, f)$ are both $M$-alternating paths. Then we find an odd cycle $C_{0}=(b, c, d, f, e, b)$ containing $f$, i.e., if we find a vertex which is simultaneously outer and inner, then there exists an odd cycle containing it, and we can easily find it.

Next we contract $C_{0}$ into a single vertex $v_{1}$, which also implies that we delete loops and replace every multiple edge by a single edge, and denote the resulting graph by $G_{1}$. The matching of $G_{1}$ corresponding to $M$ is obtained 
by deleting the edges in $C_{0} \cap M$ (Figure 1.43), i.e., we obtain

$$
G_{1}=G / C_{0}, \quad M_{1}=M-\left(E\left(C_{0}\right) \cap M\right)=M \cap E\left(G_{1}\right), \quad v_{1}=C_{0} .
$$
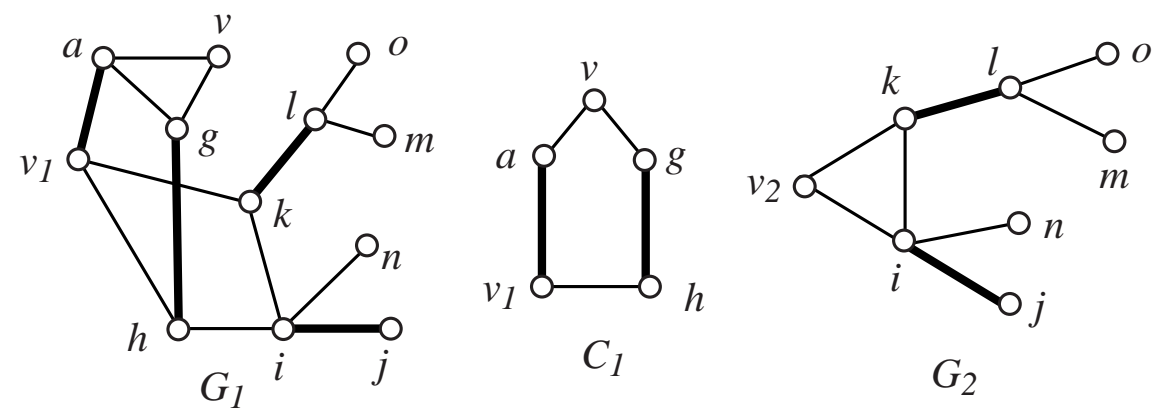

Figure 1.43: The graph $G_{1}=G / C_{0}$ with the matching $M_{1}$; an odd cycle $C_{1}=$ $\left(v, a, v_{1}, h, g, a\right)$; and the graph $G_{2}=G_{1} / C_{1}$ and the matching $M_{2}$.

In Figure 1.43, we find an odd cycle $C_{1}=\left(v, a, v_{1}, h, g\right)$ since $h$ is simultaneously an outer and inner vertex in $G_{1}$. Then we obtain the new graph $G_{2}$ from $G_{1}$ by contracting $C_{1}$, where $v_{2}$ is the new root since $C_{1}$ contains the root $v$. In general, if the odd cycle $C_{i}$ contains the root, then $C_{i}$ corresponds to the new root in $G_{i} / C_{i}$, otherwise, $C_{i}$ corresponds to a saturated vertex in $G_{i} / C_{i}$.

$$
G_{2}=G_{1} / C_{1}, \quad M_{2}=M_{1} \cap E\left(G_{2}\right), \quad v_{2}=C_{1}
$$

Then we find an $M_{2}$-augmenting path

$$
P_{2}=\left(v_{2}, k, l, m\right) \text { in } G_{2} .
$$

¿From this path $P_{2}$, we can obtain an $M_{1}$-augmenting path

$$
P_{1}=\left(v, a, v_{1}, k, l, m\right) \text { in } G_{1} .
$$

Since $v_{2}$ corresponds to the odd cycle $C_{1}$ in $G_{1}, k$ and $v_{1} \in V\left(C_{1}\right)$ are joined by an edge in $G_{1}$. There are two alternating paths in $C_{1}$ joining $v_{1}$ to $v$ and one of them can be added to $(k, l, m)$. Since $v_{1}$ corresponds to $C_{0}$ in $G$ and $v_{1}$ can be replaced by the alternating path $(a, b, e, f)$ in $C_{0}$, we obtain the desired $M$-augmenting path

$$
P=(v, a, b, e, f, k, l, m) \text { in } G \text {. }
$$



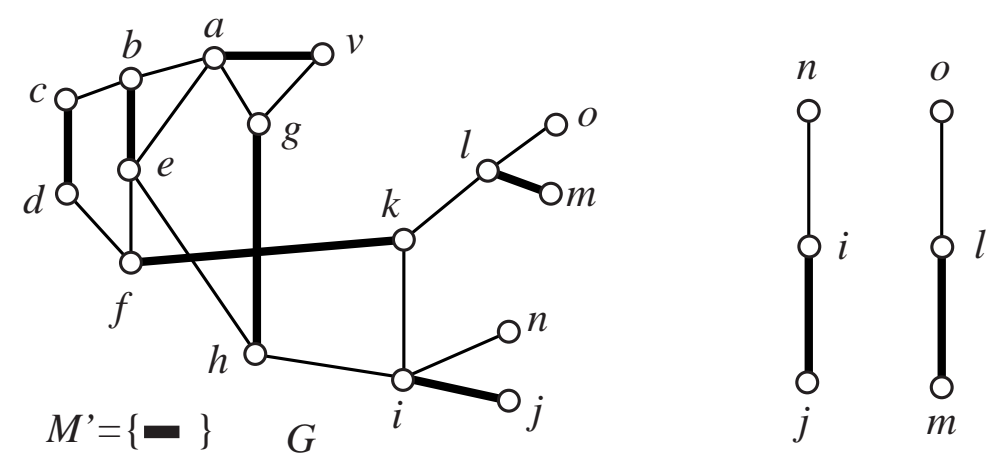

Figure 1.44: A matching $M^{\prime}$ in $G$; and non-existence of $M^{\prime}$-augmenting path in $G$.

Therefore we obtain a larger matching $M^{\prime}=M \triangle E(P)$ in $G$ (Figure 1.44).

There are two $M^{\prime}$-unsaturated vertices $n$ and $o$, and by the same argument as above, we can easily determine that $G$ has no $M^{\prime}$-augmenting paths starting with $n$ or $o$, which implies that $M^{\prime}$ is a maximum matching in $G$.

We conclude this section by giving the following algorithm for finding a maximum matching in a graph.

Algorithm 1.7.1 Let $G$ be a connected graph. Then a maximum matching of $G$ can be obtained by repeating the following procedure: Let $i=0, G_{0}=G$, $M_{0}$ be any matching of $G_{0}, v_{0}$ be any $M_{0}$-unsaturated vertex of $G_{0}$. Initially, $v_{0}$ is the root.

We explore all the $M_{i}$-alternating paths starting with $v_{i}$ as explained above. If we find a vertex $x_{i}$ that is both inner and outer, then we can find an odd cycle $C_{i}$ containing $x_{i}$, and obtain a graph $G_{i+1}$ from $G_{i}$ by contracting $C_{i}$. The contraction of $C_{i}$ is the vertex $v_{i+1}$ of $G_{i+1}$. If $C_{i}$ contains the root of $G_{i}$, then $v_{i+1}$ is the new root of $G_{i+1}$ and is unsaturated by a matching $M_{i+1}=M_{i}-\left(E\left(C_{i}\right) \cap M_{i}\right)$. Otherwise, the vertex $v_{i+1}$ of $G_{i+1}$ is a vertex saturated by $M_{i+1}$. Set $i=i+1$, and repeat the procedure.

If we find an $M_{i}$-augmenting path in $G_{i}$ connecting the root and another $M_{i}$-unsaturated vertex $y$, then we can find an $M_{i-1}$-augmenting path in $G_{i-1}$ connecting the root of $G_{i-1}$ and another $M_{i-1}$-unsaturated vertex. Set $i=$ $i-1$, and repeating the above procedure until $i=1$, we get the desired $M$ augmenting path starting with $v_{0}$. Moreover, if $G_{i}$ has no $M_{i}$-augmenting path starting with the root, then $G$ has no $M$-augmenting path starting with the root $v_{0}$.

Some improvements on Algorithm 1.7.1 results in an algorithm that finds 
a maximum matching in $O\left(|G|^{3}\right)$ time ([108], section 9).

\section{Exercises}

Exercise 1.7.1 Prove some parts in Algorithm 1.7.1, that is, show that if $G_{1}$ has an $M_{1}$-augmenting path starting with the root in $G_{1}$, then $G$ has an $M$-augmenting path starting with the root $v$.

Exercise 1.7.2 Let $M$ be a matching in a connected simple graph $G$ and $C$ be a odd cycle of $G$ containing $(|C|-1) / 2$ edges of $M$, and let $G^{\prime}=G / C$ be the graph obtained from $G$ by contracting $C$. Prove that $M$ is a maximum matching in $G$ if and only if $M^{\prime}=M \cap E\left(G^{\prime}\right)$ is a maximum matching in $G^{\prime}$. 


\section{Chapter 2}

\section{Regular Factors and $f$-Factors}

\subsection{The $f$-Factor Theorem}

In this chapter we consider regular factors and $f$-factors mainly in general graphs. For a positive integer $k$, a regular spanning subgraph each of whose vertices has degree $k$ is called a $k$-regular factor or briefly a $k$-factor (Figure 2.1). In order to avoid confusion, we use " $k$-regular factors" in theorems, but often use " $k$-factors" in proofs. For a general graph $G$ and an integervalued function

$$
f: V(G) \rightarrow \mathbb{Z}^{+}=\{0,1,2,3, \ldots\},
$$

a spanning subgraph $F$ such that

$$
\operatorname{deg}_{F}(x)=f(x) \quad \text { for all } \quad x \in V(G)
$$

is called an $f$-factor (Figure 2.1). For an integer $k$, if the function $f$ is defined as $f(x)=k$ for all $x \in V(G)$, then an $f$-factor is equivalent to a $k$-regular factor, and so an $f$-factor is a natural generalization of a regular factor. Moreover, a $k$-regular factor or an $f$-factor $F$ usually means a subset of $E(G)$ since the vertex set of $F$ is always $V(G)$ and so the factor $F$ is determined by its edge set.

We shall give criteria for a graph to have a $k$-regular factor and an $f$ factor, and show results on regular factors and $f$-factors by using these criteria.

If the edge set $E(G)$ of a graph $G$ is decomposed into edge disjoint $k$ regular factors $\left\{F_{i}\right\}$ as

$$
E(G)=F_{1} \cup F_{2} \cup \cdots \cup F_{m}, \quad F_{i} \cap F_{j}=\emptyset(i \neq j)
$$

then $G$ is said to be $k$-factorable and the decomposition is called a $k$ factorization of $G$. 


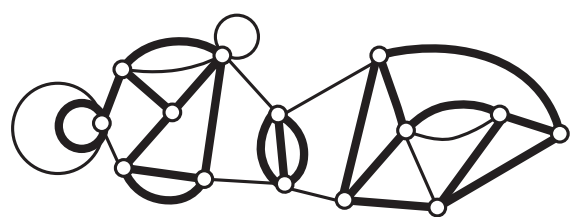

(1)

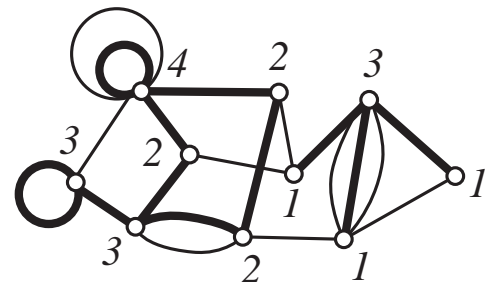

(2)

Figure 2.1: (1) A general graph $G$ with a 3-regular factor; (2) A general graph $G$ with an $f$-factor, where numbers denote $f(v)$.

The following theorem, which was obtained by Petersen in 1891, is one of the oldest results on regular factors.

Theorem 2.1.1 (The 2-Factorable Theorem Petersen [126]) For every integer $r \geq 1$, every $2 r$-regular general graph is 2-factorable. In particular, for every integer $k, 1 \leq k \leq r$, every $2 r$-regular general graph has a $2 k$ regular factor.

Proof. Let $G$ be a $2 r$-regular general graph. We shall prove that $G$ is 2 -factorable by induction on $r$. If we prove that $G$ has a 2 -factor $F$, then the theorem is proved because $G-F$ is a $2(r-1)$-regular graph and has a 2 -factorization by induction. Moreover, the union of $k$ edge-disjoint 2-factors of $G$ forms a $2 k$-factor of $G$, and so the latter part also follows immediately from the existence of a 2 -factorization.

We may assume $r \geq 2$ and the graph $G$ is connected. Since every vertex of $G$ has even degree, $G$ has an Euler circuit $C$. Traversing this Euler circuit, we get an orientation of $G$ (Figure 2.2). Then it is clear that in the oriented graph $\vec{G}$, every vertex has indegree $r$ and outdegree $r$.

We construct an $r$-regular bipartite multigraph $B$ with bipartition $(X, Y)$ as follows: two vertices $x \in X=V(G)$ and $y \in Y=V(G)$ are joined by an edge of $B$ if and only if $G$ has a directed edge (xy) (Figure 2.2). By Theorem 1.1.2, $B$ can be decomposed into $r$ 1-factors $H_{1}, H_{2}, \ldots, H_{r}$. From each $H_{i}$, we obtain a spanning subgraph $F_{i}=\left(V(G), H_{i}\right)$ of $G$ by regarding each edge of $H_{i}$ as an edge of $G$. Since for every vertex $v$ of the oriented graph $\vec{G}, H_{i}$ has two directed edges expressed as $(v x)$ and $(y v), F_{i}$ is a 2-factor of the given graph $G$. Consequently the theorem is proved.

We now give the most important theorem in this chapter. This is called the $f$-factor Theorem obtained by Tutte in 1952. His original proof uses alternating trails and is interesting, but rather long. In 1954 he gave a new 


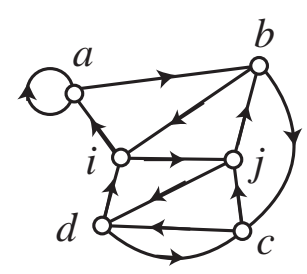

(1) $G$

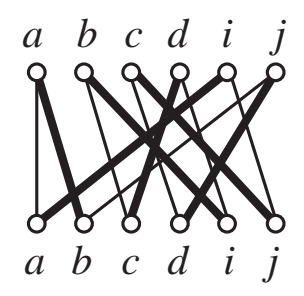

(2) $B$

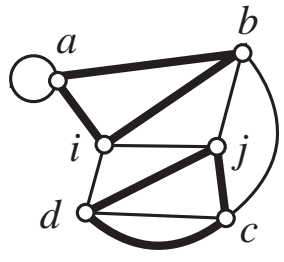

(3) $F=\{-\}$

Figure 2.2: (1) A graph $G$ with Euler circuit $(a, a, b, c, d, c, j, b, i, j, d, i, a)$; (2) The bipartite graph $B$ with a 1-factor $H_{1}$; and (3) The 2-factor $F_{1}$ corresponding to $H_{1}$.

considerably simpler proof by making use of the 1-factor Theorem. Here we adopt this new proof. Note that a different form of the criterion for a graph to have a $k$-regular factor was obtained by Ore $([124])$.

Theorem 2.1.2 (The $f$-factor Theorem Tutte [139], [140]) Let $G$ be a general graph and $f: V(G) \rightarrow \mathbb{Z}^{+}=\{0,1,2,3, \ldots\}$. Then $G$ has an $f$-factor if and only if for all disjoint subsets $S$ and $T$ of $V(G)$,

$$
\delta(S, T)=\sum_{x \in S} f(x)+\sum_{x \in T}\left(\operatorname{deg}_{G}(x)-f(x)\right)-e_{G}(S, T)-q(S, T) \geq 0,
$$

where $q(S, T)$ denotes the number of components $C$ of $G-(S \cup T)$ such that

$$
\sum_{x \in V(C)} f(x)+e_{G}(C, T) \equiv 1(\bmod 2),
$$

and the function $\delta(S, T)$ is defined by (2.1).

Some remarks are in order. For convenience, we call a component $C$ of $G-(S \cup T)$ satisfying $(2.2)$ an $f$-odd component of $G-(S \cup T)$. It is easy to see that

$$
\sum_{x \in T} \operatorname{deg}_{G}(x)-e_{G}(S, T)=\sum_{x \in T} \operatorname{deg}_{G-S}(x)
$$

and thus (2.1) can be expressed as

$$
\delta(S, T)=\sum_{x \in S} f(x)+\sum_{x \in T}\left(\operatorname{deg}_{G-S}(x)-f(x)\right)-q(S, T) \geq 0 .
$$

Another useful relation, due to Tutte, is the following: 


$$
\delta(S, T) \equiv \sum_{x \in V(G)} f(x)(\bmod 2) .
$$

Thus if $\sum_{x \in V(G)} f(x)$ is even, then

$$
\delta(S, T) \equiv 0(\bmod 2)
$$

Note that the $f$-factor theorem holds even if $f(v)>\operatorname{deg}_{G}(v)$ or $f(v)<0$ for some vertices $v$ of $G$, since the inequality (2.1) includes the condition that $0 \leq f(x) \leq \operatorname{deg}_{G}(x)$ for every $x \in V(G)$. This will be shown in the proof of sufficiency of the $f$-factor Theorem.

For a constant integer $k \geq 1$, by setting $f(x)=k$ for all $x \in V(G)$, we have the following $k$-regular factor theorem, which were obtained by Belck [16] and Tutte [139] independently.

Theorem 2.1.3 (The Regular Factor Theorem, Belck and Tutte) Let $k \geq$ 1 be an integer and $G$ be a general graph. Then $G$ has a $k$-regular factor if and only if for all disjoint subsets $S$ and $T$ of $V(G)$,

$$
\begin{aligned}
\delta(S, T) & =k|S|+\sum_{x \in T} \operatorname{deg}_{G}(x)-k|T|-e_{G}(S, T)-q(S, T) \\
& =k|S|+\sum_{x \in T} \operatorname{deg}_{G-S}(x)-k|T|-q(S, T) \geq 0
\end{aligned}
$$

where $q(S, T)$ denotes the number of components $C$, called $k$-odd components, of $G-(S \cup T)$ such that

$$
k|C|+e_{G}(C, T) \equiv 1(\bmod 2) .
$$

Proof of necessity of the $f$-factor Theorem. Suppose that a general graph $G$ has an $f$-factor $F$. We may assume that $G$ is connected since if each component of $G$ satisfies (2.1), then $G$ itself satisfies (2.1). Then we have

$$
\sum_{x \in V(G)} f(x)=\sum_{x \in V(G)} \operatorname{deg}_{F}(x)=2\|F\|,
$$

and so $G$ itself is not an $f$-odd component of $G=G-(\emptyset \cup \emptyset)$. Thus we have $\delta(\emptyset, \emptyset)=-q(\emptyset, \emptyset)=0$.

Let $S$ and $T$ be two disjoint subsets of $V(G)$ such that $S \cup T \neq \emptyset$. Let $G-F$ denote the spanning subgraph of $G$ with edge set $E(G)-E(F)$, and let 


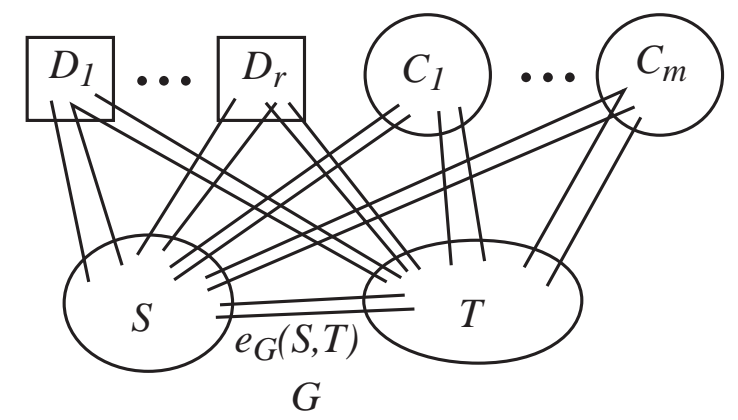

Figure 2.3: A graph $G$ with disjoint subsets $S \cup T$, the $f$-odd components $C_{1}, \ldots, C_{m}$ and the other components $D_{1}, \ldots, D_{r}$.

$C_{1}, C_{2}, \ldots, C_{m}$, where $m=q(S, T)$, be the $f$-odd components of $G-(S \cup T)$ (Figure 2.3). By the equation

$$
\operatorname{deg}_{G}(x)-f(x)=\operatorname{deg}_{G}(x)-\operatorname{deg}_{F}(x)=\operatorname{deg}_{G-F}(x),
$$

we have

$$
\begin{aligned}
\sum_{x \in T}\left(\operatorname{deg}_{G}(x)-f(x)\right) & =\sum_{x \in T} \operatorname{deg}_{G-F}(x) \\
& \geq e_{G-F}(T, S)+\sum_{i=1}^{m} e_{G-F}\left(T, C_{i}\right) .
\end{aligned}
$$

Furthermore, it follows that

$$
\sum_{x \in S} f(x)=\sum_{x \in S} \operatorname{deg}_{F}(x) \geq e_{F}(S, T)+\sum_{i=1}^{m} e_{F}\left(S, C_{i}\right) .
$$

By the two previous inequalities, we have

$$
\begin{aligned}
\delta(S, T) \geq & e_{F}(S, T)+\sum_{i=1}^{m} e_{F}\left(S, C_{i}\right)+e_{G-F}(T, S)+\sum_{i=1}^{m} e_{G-F}\left(T, C_{i}\right) \\
& -e_{G}(S, T)-m \\
= & \sum_{i=1}^{m}\left(e_{F}\left(S, C_{i}\right)+e_{G-F}\left(T, C_{i}\right)-1\right) .
\end{aligned}
$$

Therefore, in order to prove (2.1), it is sufficient to show that for every $C=C_{i}$

$$
e_{F}(S, C)+e_{G-F}(T, C)-1 \geq 0 \text {. }
$$


If $e_{G-F}(T, C) \geq 1$, then $(2.9)$ holds, and so we may assume that $e_{G-F}(T, C)$ $=0$, which implies

$$
e_{G}(T, C)=e_{F}(T, C)
$$

Hence

$$
\begin{aligned}
\sum_{x \in V(C)} f(x) & +e_{G}(C, T)=\sum_{x \in V(C)} \operatorname{deg}_{F}(x)+e_{F}(C, T) \\
& =2\left\|\langle V(C)\rangle_{F}\right\|+e_{F}(C, S \cup T)+e_{F}(C, T) \\
& \equiv e_{F}(C, S)(\bmod 2) .
\end{aligned}
$$

By (2.2) and the above equation, we have $e_{F}(C, S) \geq 1$, which implies (2.9) holds. Consequently necessity is proved.

Proof of (2.4). Let $C_{1}, C_{2}, \ldots, C_{m}, m=q(S, T)$, be the $f$-odd components of $G-(S \cup T)$, and $D_{1}, D_{2}, \ldots, D_{r}$ be the other components of $G-(S \cup T)$ (Figure 2.3). Then

$$
\sum_{x \in V\left(D_{i}\right)} f(x)+e_{G}\left(D_{i}, T\right) \equiv 0(\bmod 2) \quad \text { for every } 1 \leq i \leq r .
$$

Hence

$$
\begin{aligned}
m & \equiv \sum_{i=1}^{m}\left(\sum_{x \in V\left(C_{i}\right)} f(x)+e_{G}\left(C_{i}, T\right)\right) \\
& \equiv \sum_{i=1}^{m}\left(\sum_{x \in V\left(C_{i}\right)} f(x)+e_{G}\left(C_{i}, T\right)\right)+\sum_{i=1}^{r}\left(\sum_{x \in V\left(D_{i}\right)} f(x)+e_{G}\left(D_{i}, T\right)\right) \\
& =\sum_{x \in V(G)-(S \cup T)} f(x)+e_{G}(V(G)-(S \cup T), T)(\bmod 2) .
\end{aligned}
$$

Therefore

$$
\begin{aligned}
\delta(S, T) \equiv & \sum_{x \in S} f(x)+\sum_{x \in T}\left(\operatorname{deg}_{G}(x)+f(x)\right)+e_{G}(S, T)+m \\
\equiv & \sum_{x \in S} f(x)+\sum_{x \in T}\left(\operatorname{deg}_{G}(x)+f(x)\right)+e_{G}(S, T) \\
& \quad+\sum_{x \in V(G)-(S \cup T)} f(x)+e_{G}(V(G)-(S \cup T), T) \\
= & \sum_{x \in V(G)} f(x)+2\left\|\langle T\rangle_{G}\right\|+e_{G}(T, V(G)-T)+e_{G}(S, T)
\end{aligned}
$$




$$
\equiv \sum_{x \in V(G)} f(x)(\bmod 2) .
$$

Consequently (2.4) holds.

We now prove the sufficiency of the $f$-factor Theorem based on Tutte [140], ([20], Section 2.3).

Proof of the sufficiency of the $f$-factor Theorem. If a general graph $G$ satisfies the condition (2.1), then each of its components satisfies (2.1), and if each component of $G$ has the desired $f$-factor, then their union is the desired $f$-factor of $G$. Hence we may assume that $G$ is connected.

For each vertex $v$ of $G$, we have by (2.1)

$$
\begin{aligned}
& \delta(\emptyset,\{v\})=\operatorname{deg}_{G}(v)-f(v)-q(\emptyset,\{v\}) \geq 0, \quad \text { and } \\
& \delta(\{v\}, \emptyset)=f(v)-q(\{v\}, \emptyset) \geq 0,
\end{aligned}
$$

and so $0 \leq f(x) \leq \operatorname{deg}_{G}(x)$. Since $\delta(\emptyset, \emptyset)=-q(\emptyset, \emptyset) \geq 0$, we have $q(\emptyset, \emptyset)=0$, which implies

$$
\sum_{x \in V(G)} f(x) \equiv 0(\bmod 2) .
$$

We now construct a new simple graph $G^{*}$ from $G$ as follows: For every edge $e=x y \in E(G)$, where we allow $x=y$, we introduce two vertices $e(x)$ and $e(y)$ of $G^{*}$, and join them by an edge $e$ of $G^{*}$. Thus for every vertex $v$ of $G$, if $\left\{e_{1}, e_{2}, \ldots, e_{r}\right\}$ is the set of edges incident with $v$ in $G$, then $G^{*}$ has a set

$$
S(v)=\left\{e_{1}(v), e_{2}(v), \ldots, e_{r}(v)\right\}
$$

of vertices corresponding to $v$ (Figure 2.4).

Next for every vertex $v$ of $G$, we add a set

$$
T(v)=\left\{v_{1}, \ldots, v_{p}\right\}, \quad p=\operatorname{deg}_{G}(v)-f(v)
$$

of vertices to $G^{*}$, and join every vertex of $T(v)$ to every vertex of $S(v)$ (Figure 2.4). Of course, if $f(v)=\operatorname{deg}_{G}(v)$, then $T(v)=\emptyset$. Hence we have

$$
\begin{aligned}
& V\left(G^{*}\right)=S^{*} \cup T^{*}, \quad \text { where } \\
& S^{*}=\bigcup_{v \in V(G)} S(v) \text { and } T^{*}=\bigcup_{v \in V(G)} T(v) ; \text { and } \\
& E\left(G^{*}\right)=E(G) \cup\{x y \mid x \in S(v) \text { and } y \in T(v)\} .
\end{aligned}
$$

We next show that $G$ has an $f$-factor if and only if $G^{*}$ has a 1 -factor. Assume that $G$ has an $f$-factor $F$. Then by regarding $E(F)$ as a subset of 

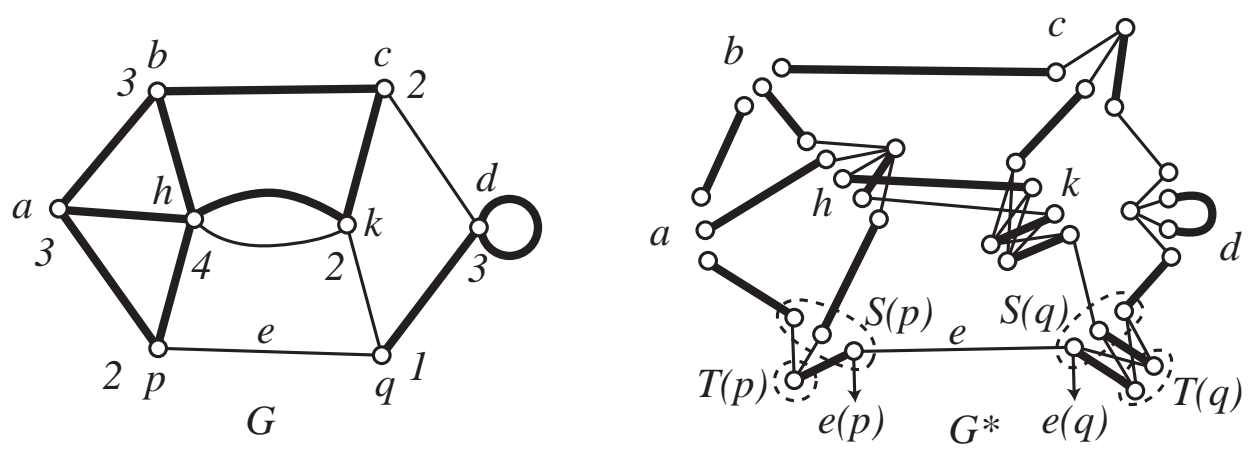

Figure 2.4: A general graph $G$ with an $f$-factor; and the corresponding simple graph $G^{*}$ with a 1 -factor.

$E\left(G^{*}\right)$, we can get a matching which covers $f(v)$ vertices of $S(v)$ for every $v \in V(G)$. The remaining uncovered vertices of $S(v)$ can be covered by a matching of $G^{*}$ which joins the remaining uncovered vertices of $S(v)$ to all the vertices of $T(v)$. Therefore $G^{*}$ has a 1-factor. Conversely, if $G^{*}$ has a 1factor $K$, then $E(G) \cap E(K) \subseteq E\left(G^{*}\right)$ induces an $f$-factor of $G$ by regarding it as a edge subset of $G$. Therefore $G$ has an $f$-factor if and only if $G^{*}$ has a 1-factor (Figure 2.4).

Suppose that $G$ satisfies the condition (2.1) but has no $f$-factor. Then $G^{*}$ has no 1-factor. By the 1-Factor Theorem (Theorem 1.4.2), there exists a subset $Y \subset V\left(G^{*}\right)$ such that $\operatorname{odd}\left(G^{*}-Y\right)>|Y|$. Choose a minimal set $X$ among such subsets $Y$. Then $X \neq \emptyset$. By Lemma 1.4.1, we have

$$
\operatorname{odd}\left(G^{*}-X\right) \geq|X|+2
$$

Then for each $v \in V(G)$, the following claim holds.

Claim 1. (1) If $T(v) \cap X \neq \emptyset$, then $T(v) \subseteq X$.

(2) If $S(v) \cap X \neq \emptyset$, then $S(v) \subseteq X$.

(3) $S(v) \cup T(v) \nsubseteq X$. In particular, if $T(v)=\emptyset$ then $S(v) \cap X=\emptyset$.

Proof. (1) Suppose that $X \cap T(v) \neq \emptyset$ but $T(v) \nsubseteq X$ (Figure $2.5(1)$ ). Then $T(v) \neq \emptyset$, and

$$
\operatorname{odd}\left(G^{*}-(X \backslash T(v))\right) \geq \operatorname{odd}\left(G^{*}-X\right)-1 \geq|X|+1>|X \backslash T(v)| .
$$

This contradicts the minimality of $X$.

(2) Suppose that $X \cap S(v) \neq \emptyset$ but $S(v) \nsubseteq X$ (Figure 2.5 (2)). Let $e(v) \in S(v) \cap X$, where $e=v u \in E(G)$. Then $e(v)$ is adjacent to at most 
two components of $G^{*}-X$ since $T(v) \backslash X$ is empty set or is contained in a component as $S(v) \nsubseteq X$. Hence

$$
o\left(G^{*}-(X \backslash\{e(v)\})\right) \geq \operatorname{odd}\left(G^{*}-X\right)-2 \geq|X|>|X \backslash\{e(v)\}| .
$$

This contradicts the choice of $X$.

(3) Assume $S(v) \cup T(v) \subseteq X$ for some $v \in V(G)$. Choose a vertex $e(v) \in S(v)$. Then

$$
\operatorname{odd}\left(G^{*}-(X \backslash\{e(v)\})\right) \geq \operatorname{odd}\left(G^{*}-X\right)-1 \geq|X|+1>|X \backslash\{e(v)\}|,
$$

a contradiction.

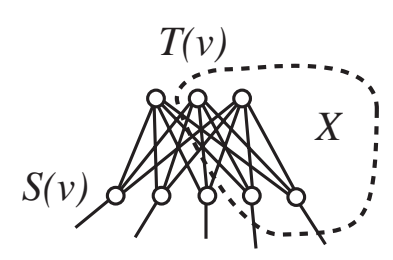

(1)

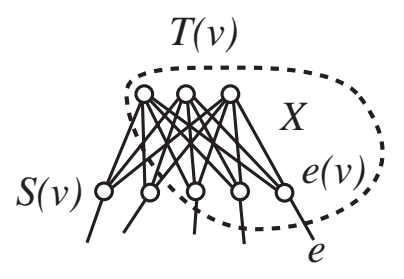

(2)

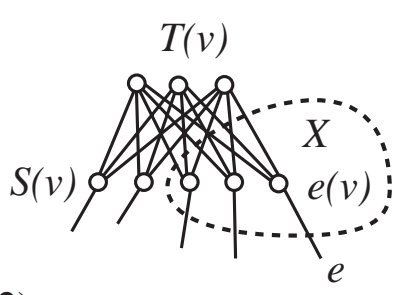

)

Figure 2.5: (1) $X \cap T(v) \neq \emptyset$ but $T(v) \nsubseteq X$; (2) $X \cap S(v) \neq \emptyset$ but $S(v) \nsubseteq X$.

We define

$$
\begin{aligned}
& X(S)=\{v \in V(G) \mid S(v) \subseteq X\} \\
& X(T)=\{v \in V(G) \mid T(v) \subseteq X\}
\end{aligned}
$$

Then if $T(v)=\emptyset$, then $v \in X(T)$, and the next claim holds.

Claim 2. (4) The number of isolated vertices of $G^{*}-X$ contained in $S^{*}$ is $e_{G}(X(T), X(S)$ ) (Figure 2.6).

(5) The number of isolated vertices of $G^{*}-X$ contained in $T^{*}$ is $\sum_{x \in X(S)}\left(\operatorname{deg}_{G}(x)-f(x)\right)$ (Figure 2.6).

(6) $|X|-\operatorname{odd}\left(G^{*}-X\right)=\delta(X(S), X(T))$.

Proof. (4) Let $e(v)$ be an isolated vertex of $G^{*}-X$ contained in $S^{*}$, and let $e=v w \in E(G) \subset E\left(G^{*}\right)$. Then $T(v) \subseteq X$ and $S(w) \subseteq X$, and thus $v \in X(T)$ and $w \in X(S)$, which implies $e \in E_{G}(X(T), X(S))$. Conversely, if $e=v w \in E_{G}(X(T), X(S))$, then $T(v) \subseteq X$ and $S(w) \subseteq X$, and so $e(v)$ is an isolated vertex of $G^{*}-X$ contained in $S^{*}$. Hence (4) holds.

(5) This follows immediately from (2) and the definition of $G^{*}$. 


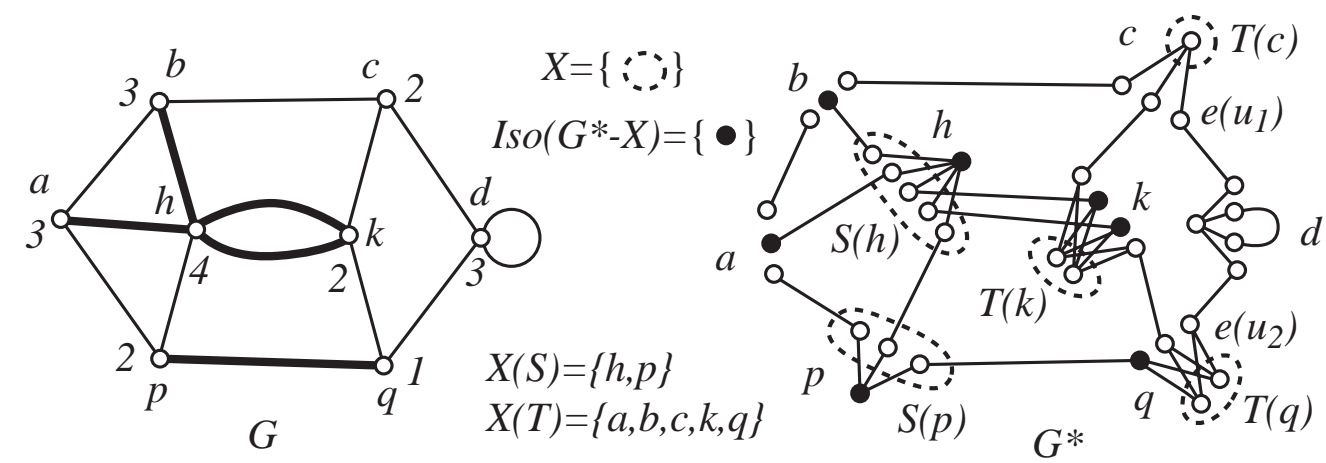

Figure 2.6: $G$ with $X(S)$ and $X(T)$, and $G^{*}$ with $X$. The isolated vertices of $G^{*}-X$ are denoted by black circles. $C^{*}$ is an odd component of $G^{*}-X$, $V\left(C^{*}\right)=S(d) \cup T(d) \cup\left\{e\left(u_{1}\right), e\left(u_{2}\right)\right\}$, and $V(C)=\{d\}$.

(6) Let $C^{*}$ be an odd component of $G^{*}-X$ with order at least three (Figure 2.6). It is clear that if $C^{*}$ contains an edge joining a vertex of $S(x)$ to a vertex of $T(x)$, then $C^{*}$ must contain $S(x) \cup T(x)$. Hence we can write

$$
V\left(C^{*}\right)=\bigcup_{x}(S(x) \cup T(x)) \cup\left\{e\left(u_{1}\right), \ldots, e\left(u_{r}\right)\right\}
$$

where $e\left(u_{i}\right)$ are the endvertices of $C^{*}$ such that $T\left(u_{i}\right) \subseteq X$. Let

$$
C=\left\langle\left\{x \in V(G) \mid S(x) \cup T(x) \subseteq V\left(C^{*}\right)\right\}\right\rangle_{G} .
$$

Then $C$ is a component $G-(X(S) \cup X(T))$, and

$$
\begin{aligned}
\left|C^{*}\right| & =\sum_{x \in V(C)}\left(2 \operatorname{deg}_{G}(x)-f(x)\right)+\left|\left\{e\left(u_{1}\right), \ldots, e\left(u_{r}\right)\right\}\right| \\
& \equiv \sum_{x \in V(C)} f(x)+e_{G}(C, X(T)) \\
& \equiv 1(\bmod 2) \quad\left(\text { since } C^{*} \text { is an odd component of } G^{*}-X .\right)
\end{aligned}
$$

By (2.13), $C$ is an $f$-odd component of $G-(X(S) \cup X(T))$. Therefore the number of those odd components of $G^{*}-X$ whose order is at least three is equal to $q(X(S), X(T))$.

It is obvious that

$$
|X|=\sum_{x \in X(S)} \operatorname{deg}_{G}(x)+\sum_{x \in X(T)}\left(\operatorname{deg}_{G}(x)-f(x)\right) .
$$


By combining (4), (5), (6) and the above equation, we get

$$
\begin{aligned}
& |X|-\operatorname{odd}\left(G^{*}-X\right) \\
= & \sum_{x \in X(S)} \operatorname{deg}_{G}(x)+\sum_{x \in X(T)}\left(\operatorname{deg}_{G}(x)-f(x)\right)-e_{G}(X(S), X(T)) \\
& \quad-\sum_{x \in X(S)}\left(\operatorname{deg}_{G}(x)-f(x)\right)-q(X(S), X(T)) \\
= & \sum_{x \in X(S)} f(x)+\sum_{x \in X(T)}\left(\operatorname{deg}_{G}(x)-f(x)\right) \\
& \quad-e_{G}(X(S), X(T))-q(X(S), X(T) \\
= & \delta(X(S), X(T)) .
\end{aligned}
$$

By (6) and by (2.1), we can derive a contradiction since $0>|X|-o d d\left(G^{*}-\right.$ $X)=\delta(X(S), X(T)) \geq 0$. Consequently the proof is complete.

We have now proved the $f$-Factor Theorem and $k$-Regular Factor Theorem. It is not so easy to apply these theorems because $\delta(S, T)$ may be complex and the number $q(S, T)$ of $k$-odd components of $G-(S \cup T)$ may be difficult to estimate. So many results in the following sections were only recently obtained in the 1980s. The $k$-Regular Factor Theorem, however, was proved in the early 1950s.

We conclude this section by giving the $f$-Factor Theorem in Bipartite Graphs, which is simpler than the $f$-factor theorem.

Theorem 2.1.4 (The f-Factor Theorem in a Bipartite Graph) Let $G$ be a bipartite multigraph and $f: V(G) \rightarrow \mathbb{Z}^{+}$. Then $G$ has an $f$-factor if and only if for all disjoint subsets $S$ and $T$ of $V(G)$,

$$
\delta(S, T)=\sum_{x \in S} f(x)+\sum_{x \in T}\left(\operatorname{deg}_{G}(x)-f(x)\right)-e_{G}(S, T) \geq 0 .
$$

The proof of Theorem 2.1.4 is shown in Section 2.4.

\subsection{Regular Factors in Regular Graphs}

In this section, we first consider regular factors in regular graphs. We already showed that every $2 r$-regular graph has a $2 k$-regular factor for every $k, 1 \leq$ $k \leq r$. Furthermore, some results on 1-factors in an $r$-regular graph show the existence of $(r-1)$-regular factors in the $r$-regular graph by taking the 
complement of the 1-factor in the given graph. For example, the existence of a 1-factor in every 2-edge connected cubic graph means the existence of a 2 -factor in such a graph. We begin with the next theorem, whose proof is based on [68] and give another proofs to the theorems mentioned above.

Theorem 2.2.1 (Regular Factors in Regular Graphs) Let $r$ and $k$ be integers such that $1 \leq k<r$, and $G$ be a $\lambda$-edge connected $r$-regular general graph, where $\lambda \geq 1$. If one of the following conditions holds, then $G$ has a $k$-regular factor.

(1) $r$ and $k$ are both even. (Petersen [126] (1891))

(2) $r$ is even, $k$ is odd, $|G|$ is even, and $\frac{r}{\lambda} \leq k \leq r\left(1-\frac{1}{\lambda}\right)$. (Gallai [51] (1950))

(3) $r$ is odd, $k$ is even and $2 \leq k \leq r\left(1-\frac{1}{\lambda}\right)$. (Gallai [51] (1950))

(4) $r$ and $k$ are both odd and $\frac{r}{\lambda} \leq k$. (Gallai [51], Bäbler [14])

(5) Let $\lambda$ be an integer such that $\lambda^{*} \in\{\lambda, \lambda+1\}$ and $\lambda^{*} \equiv 1(\bmod 2)$. Either $r$ is odd, $k$ is even and $k \leq r\left(1-\frac{1}{\lambda^{*}}\right)$; or both $r$ and $k$ are odd and $\frac{r}{\lambda^{*}} \leq k$. (Bollobás, Saito and Wormald [22])

Proof. We use the Regular Factor Theorem 2.1.3. It is immediate that $\delta(\emptyset, \emptyset)=0$ since $k|G|$ is even. Let $S$ and $T$ be disjoint subsets of $V(G)$ such that $S \cup T \neq \emptyset$, and let $C_{1}, C_{2}, \ldots, C_{m}$ be the $k$-odd components of $G-(S \cup T)$. Set $\theta=\frac{k}{r}$. Then $0<\theta<1$, and we have

$$
\begin{aligned}
\delta(S, T)= & k|S|+\sum_{x \in T} \operatorname{deg}_{G}(x)-k|T|-e_{G}(S, T)-q(S, T) \\
= & k|S|+(r-k)|T|-e_{G}(S, T)-q(S, T) \\
= & \theta r|S|+(1-\theta) r|T|-e_{G}(S, T)-m \\
= & \theta \sum_{x \in S} \operatorname{deg}_{G}(x)+(1-\theta) \sum_{x \in T} \operatorname{deg}_{G}(x)-e_{G}(S, T)-m \\
\geq & \theta\left(e_{G}(S, T)+\sum_{i=1}^{m} e_{G}\left(S, C_{i}\right)\right)+(1-\theta)\left(e_{G}(T, S)\right. \\
& \left.+\sum_{i=1}^{m} e_{G}\left(T, C_{i}\right)\right)-e_{G}(S, T)-m \\
= & \sum_{i=1}^{m}\left(\theta e_{G}\left(S, C_{i}\right)+(1-\theta) e_{G}\left(T, C_{i}\right)-1\right) .
\end{aligned}
$$

Hence it suffices to show that for every $C=C_{i}, 1 \leq i \leq m$,

$$
\theta e_{G}(S, C)+(1-\theta) e_{G}(T, C) \geq 1 .
$$


Since $C$ is a $k$-odd component of $G-(S \cup T)$, we have

$$
k|C|+e_{G}(T, C) \equiv 1(\bmod 2) .
$$

Moreover, since $G$ is $\lambda$-edge connected and

$$
r|C|=\sum_{x \in V(C)} \operatorname{deg}_{G}(x)=e_{G}(S \cup T, C)+2\|C\|,
$$

we have

$$
r|C| \equiv e_{G}(S \cup T, C)(\bmod 2) \quad \text { and } \quad e_{G}(S \cup T, C) \geq \lambda .
$$

It is obvious that the two inequalities $e_{G}(S, C) \geq 1$ and $e_{G}(T, C) \geq 1$ imply

$$
\theta e_{G}(S, C)+(1-\theta) e_{G}(T, C) \geq \theta+(1-\theta)=1 .
$$

Hence we may assume $e_{G}(S, C)=0$ or $e_{G}(T, C)=0$. We consider the following two cases under the assumption that one of conditions (1)-(4) is satisfied. Condition (5) will be considered later.

Case 1. $e_{G}(S, C)=0$.

If both $r$ and $k$ are even, then by (2.16) and (2.17), we have

$$
e_{G}(T, C) \equiv 1(\bmod 2) \text { and } 0 \equiv e_{G}(T, C)(\bmod 2) .
$$

This is a contradiction. If $k \leq r\left(1-\frac{1}{\lambda}\right)$, then $\theta \leq 1-\frac{1}{\lambda}$ and so $1 \leq(1-\theta) \lambda$. By substituting (2.17) and $e_{G}(S, C)=0$ into (2.15), we have

$$
(1-\theta) e_{G}(T, C) \geq(1-\theta) \lambda \geq 1 .
$$

If both $r$ and $k$ are odd, then by (2.16) and (2.17), we have

$$
|C|+e_{G}(T, C) \equiv 1(\bmod 2) \text { and }|C| \equiv e_{G}(T, C)(\bmod 2) .
$$

This is again a contradiction.

Case 2. $e_{G}(T, C)=0$.

By (2.16) we have $k|C| \equiv 1(\bmod 2)$, which implies that $k$ must be odd. Hence we may assume that $k$ is odd. Then $\frac{r}{\lambda} \leq k$ by (2) and (4). Hence $\theta \lambda \geq 1$, and so by (2.17) we obtain

$$
\theta e_{G}(S, C) \geq \theta \lambda \geq 1 .
$$

Consequently, every condition (1)-(4) guarantees the existence of $k$-factors. 
In order to prove (5), it suffices to show that (2.15) holds under the assumption that $e_{G}(S, C)=0$ or $e_{G}(T, C)=0$. We first consider the first part of (5), i.e., we assume that $r$ is odd, $k$ is even and $k \leq r\left(1-\frac{1}{\lambda^{*}}\right)$. If $e_{G}(S, C)=0$, then by (2.16) and (2.17), we have

$$
e_{G}(T, C) \equiv 1(\bmod 2) \text { and } e_{G}(T, C) \geq \lambda .
$$

Hence $e_{G}(T, C) \geq \lambda^{*}$, and thus

$$
(1-\theta) e_{G}(T, C) \geq(1-\theta) \lambda^{*} \geq 1 .
$$

If $e_{G}(T, C)=0$, then by $(2.16)$, we have $k|C| \equiv 1(\bmod 2)$, which contradicts the assumption that $k$ is even.

We next consider the second part of (5), i.e., we assume that both $r$ and $k$ are odd and $\frac{r}{\lambda^{*}} \leq k$. If $e_{G}(S, C)=0$, then by (2.16) and (2.17), we have

$$
|C|+e_{G}(T, C) \equiv 1(\bmod 2) \text { and }|C| \equiv e_{G}(T, C)(\bmod 2) \text {. }
$$

This is a contradiction. If $e_{G}(T, C)=0$, then by (2.16) and (2.17), we have

$$
|C| \equiv 1(\bmod 2) \text { and }|C| \equiv e_{G}(S, C)(\bmod 2) \text {, }
$$

which implies $e_{G}(S, C) \geq \lambda^{*}$. Thus

$$
\theta e_{G}(S, C) \geq \theta \lambda^{*} \geq 1
$$

Consequently the proof is complete.

We now show that every $(r-1)$-edge connected $r$-regular multigraph $G$ has a 1-factor by making use of the above Theorem 2.2.1. First assume $r$ is even. Then an $(r-1)$-edge connected $r$-regular graph must be $r$-edge connected because $G$ is an Euler graph. Hence

$$
\frac{r}{\lambda}=\frac{r}{r}=1=k \leq r\left(1-\frac{1}{r}\right)=r-1,
$$

and so $G$ has a 1-factor by (2) of Theorem 2.2.1. We next assume that $r$ is odd. Then by (5) of Theorem 2.2.1, $\lambda^{*}=r$ since $\lambda=r-1$ is even. Hence

$$
\frac{r}{\lambda^{*}}=\frac{r}{r}=1=k,
$$

which implies $G$ has a 1 -factor.

The following corollary is an immediate consequence of Theorem 2.2.1, but is a nice generalization of Petersen's Theorem, which says that every 2-connected cubic graph has a 2-factor. 
Corollary 2.2.2 (Baebler [14] (1938)) (1) Let $r \geq 3$ be an odd integer. Then every 2-edge connected $r$-regular general graph has a 2-regular factor. (2) Let $r \geq 3$ be an odd integer and $k$ an even integer such that $2 \leq k<r$. Then every $k$-edge connected $r$-regular general graph has a $k$-regular factor.

Proof. We shall prove only (2). We apply (5) of Theorem 2.2.1 to an odd integer $r$ and an even integer $k$ such that $2 \leq k \leq r-1$. Since $\lambda=k$ and $\lambda^{*}=k+1$ in (5), we have

$$
r\left(1-\frac{1}{\lambda^{*}}\right)=r\left(1-\frac{1}{k+1}\right) \geq(k+1)\left(1-\frac{1}{k+1}\right)=k .
$$

Hence a $k$-edge connected $r$-regular general graph described in (2) has a $k$-factor.

We now show that some conditions in Theorem 2.2.1 are sharp (Bollobás, Saito and Wormald, [22]). Let $r \geq 2$ be an even integer, $k \geq 1$ an odd integer and $\lambda \geq 1$ an even integer such that $k<\frac{r}{\lambda}$ or $r\left(1-\frac{1}{\lambda}\right)<k$.

For integers $r, t, \lambda$ such that $1 \leq \lambda \leq t \leq r$, let $H(r, t ; \lambda)$ be a $\lambda$-edge connected bipartite simple graph with bipartition $(U, W)$ such that every vertex of $U$ has degree $r$ and every vertex of $W$ has degree $t$, and let $J(r, t ; \lambda)$ be a $\lambda$-edge connected simple graph of even order in which one vertex, say $v_{0}$, has degree $t$ and all the other vertices have degree $r$ (Figure 2.7). We now construct our graph $G(r, \lambda)$ from $H=H(r, \lambda ; \lambda)$ and $|W|$ copies of $J=J(r, \lambda ; \lambda)$ 's as follows: for every vertex $w \in W$ of $H$, replace $w$ by $J$, i.e., remove $w$ from $H$, remove $v_{0}$ from $J$ and join every $u \in U$ adjacent to $w$ in $H$ to a vertex of $J$ adjacent to $v_{0}$ in such a way that the resulting graph is $r$-regular (Figure 2.7). It is immediate that $G(r, \lambda)$ is a $\lambda$-edge connected $r$-regular simple graph.
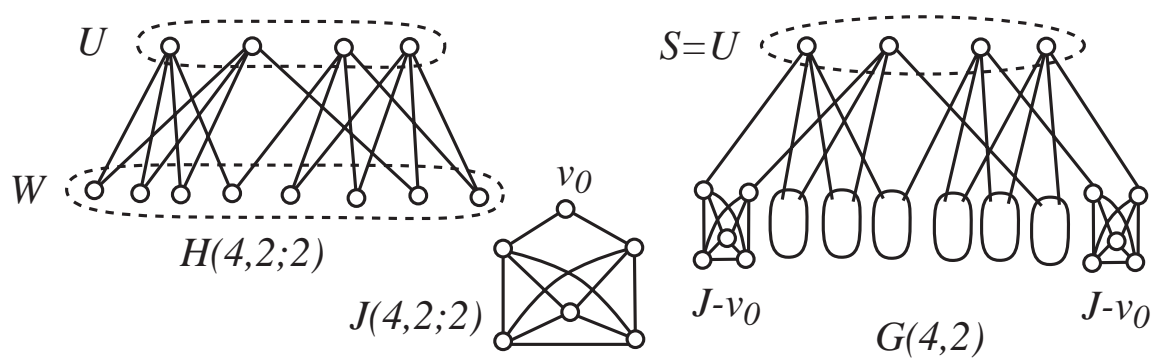

Figure 2.7: Graphs $H(4,2, ; 2), J(4,2)$ and $G(4,2)$.

We now show that the graph $G=G(r, \lambda)$ has no $k$-regular factor for every odd integer $k$ such that $k<\frac{r}{\lambda}$ or $r\left(1-\frac{1}{\lambda}\right)<k$. Let $S=U$ and $T=\emptyset$. Then 
each component $J-v_{0}$ of $G-S$ is a $k$-odd component since

$$
k\left|J-v_{0}\right|+e_{G}\left(V\left(J-v_{0}\right), \emptyset\right)=k\left|J-v_{0}\right| \equiv 1(\bmod 2) .
$$

Thus if $k<\frac{r}{\lambda}$, then

$$
\delta(U, \emptyset)=k|U|-q(U, \emptyset)<\frac{r}{\lambda}|U|-|W|=0 .
$$

Hence $G$ has no $k$-factor by the Regular Factor Theorem. Moreover, $G$ has no $(r-k)$-factor, which implies $k^{\prime}=r-k$ satisfies $r\left(1-\frac{1}{\lambda}\right)<k^{\prime}$ and an $r$-regular graph $G$ has no $k^{\prime}$-factor.

We consider graphs $H\left(r, \lambda^{*} ; \lambda\right)$ and $J\left(r, \lambda^{*} ; \lambda\right)$ when $r$ is odd and $\lambda \geq 1$ and obtain a graph $G(r, \lambda)$. Then we can show that $G=G(r, \lambda)$ has no $k$-regular factor if either $k$ is even and $k>r\left(1-\frac{1}{\lambda^{*}}\right)$, or $k$ is odd and $k<\frac{r}{\lambda^{*}}$. The proof is left to the reader (Exercise 2.2.1).

The next theorem says that every regular graph of small order has a regular factor. Of course, a connected 2-regular simple graph is a cycle, and so if $r=2$ and $k=1$, the statement (i) in the following theorem holds for every $G$. Except for this trivial case, the conditions in the theorem are sharp.

Theorem 2.2.3 (Niessen and Randerath [116]) Let $G$ be a connected $r$-regular simple graph with $r \geq 3$, and let $k$ be an integer such that $1 \leq k<r$. If $k|G|$ is even and one of the following conditions holds, then $G$ has a $k$-regular factor.

(i) $r$ is even, $k$ is odd, and $|G| \leq(r+1) 3 r+2$.

(ii) $r$ and $k$ are both odd, and $|G| \leq(r+2)(k+1)$.

(iii) $r$ is odd and $k$ is even and $|G| \leq(r+2)(r-k+1)$.

Proof. We shall first prove (i). We may assume that $k \leq r / 2$ since if $G$ has a $k$-factor $F$ for an odd integer $1 \leq k \leq r / 2$, then $G-F$ is an $(r-k)$-factor and $r-k$ is an odd integer with $r-k \geq r / 2$. Let $S$ and $T$ be disjoint subsets of $V(G)$ such that $S \cup T \neq \emptyset$, and let $C_{1}, C_{2}, \ldots, C_{m}$ be the $k$-odd components of $G-(S \cup T)$. Set $\theta=k / r$. Then $0<\theta \leq 1 / 2$, and we have

$$
\begin{aligned}
\delta(S, T) & =k|S|+\sum_{x \in T} \operatorname{deg}_{G}(x)-k|T|-e_{G}(S, T)-q(S, T) \\
& =k|S|+(r-k)|T|-e_{G}(S, T)-q(S, T) \\
& =\theta r|S|+(1-\theta) r|T|-e_{G}(S, T)-m \\
& \geq \theta \sum_{x \in S} \operatorname{deg}_{G}(x)+(1-\theta) \sum_{x \in T} \operatorname{deg}_{G}(x)-e_{G}(S, T)-m \\
& \geq \theta\left(e_{G}(S, T)+\sum_{i=1}^{m} e_{G}\left(S, C_{i}\right)\right)+(1-\theta)\left(e_{G}(T, S)+\sum_{i=1}^{m} e_{G}\left(T, C_{i}\right)\right)
\end{aligned}
$$




$$
\begin{aligned}
& -e_{G}(S, T)-m \\
= & \sum_{i=1}^{m}\left(\theta e_{G}\left(S, C_{i}\right)+(1-\theta) e_{G}\left(T, C_{i}\right)-1\right) \\
\geq & \sum_{i=1}^{m}\left(\theta e_{G}\left(S \cup T, C_{i}\right)-1\right) \quad(\text { by } 1-\theta \geq \theta)
\end{aligned}
$$

Let $C=C_{i}, 1 \leq i \leq m$. Hereafter we show that $\theta e_{G}(S \cup T, C)-1 \geq 0$ or $\sum_{i=1}^{m}\left(\theta e_{G}\left(S \cup T, C_{i}\right)-1\right) \geq 0$ which completes the proof.

Case 1. $1 \leq|C| \leq r$.

Since $G$ is a simple graph, for every vertex $x \in V(C),\left|N_{G}(x) \cap(S \cup T)\right| \geq$ $r-|C|+1$, which implies $e_{G}(C, S \cup T) \geq|C|(r-|C|+1) \geq r$. Hence

$$
\theta e_{G}(S \cup T, C)-1 \geq \theta r-1=k-1 \geq 0 .
$$

Case 2. $|C| \geq r+1$.

There exist at most $P$ components $C_{i}$ with $\left|C_{i}\right| \geq r+1$ as $|G| \leq 3 r+2$. So

$$
\delta(S, T) \geq \sum_{i=1}^{2}\left(\theta e_{G}\left(S \cup T, C_{i}\right)-1\right) \geq(\theta-1)>-2,
$$

which implies $\delta(S, T) \geq 0$ by $\delta(S, T) \equiv 0(\bmod 2)(2.5)$. Consequently (i) is proved.

We next prove (ii). Let $S$ and $T$ be disjoint subsets of $V(G)$ such that $S \cup T \neq \emptyset$, and let $C_{1}, C_{2}, \ldots, C_{m}$ be the $k$-odd components of $G-(S \cup T)$ such that

(i) $\left|C_{i}\right| \leq r$ for $1 \leq i \leq a$;

(ii) $\left|C_{i}\right| \geq r+1$ and $e_{G}\left(T, C_{i}\right) \geq 1$ for $a<i \leq a+b$; and

(iii) $\left|C_{i}\right| \geq r+1$ and $e_{G}\left(T, C_{i}\right)=0$ for $a+b<i \leq a+b+c=m$, where some of $a, b, c$ can be 0 .

Since $C_{i}$ is a $k$-odd component, we have

$$
k\left|C_{i}\right|+e_{G}\left(T, C_{i}\right) \equiv\left|C_{i}\right|+e_{G}\left(T, C_{i}\right) \equiv 1(\bmod 2) .
$$

If $e_{G}\left(S, C_{i}\right)=0$, then

$$
\left|C_{i}\right| \equiv r\left|C_{i}\right|=\sum_{x \in V\left(X_{i}\right)} \operatorname{deg}_{G}(x)=e_{G}\left(S \cup T, C_{i}\right)+2|| C_{i}|| \equiv e_{G}\left(T, C_{i}\right)(\bmod 2) .
$$

The previous two equations result in a contradiction, and so

$$
e_{G}\left(S, C_{i}\right) \geq 1 \quad \text { for all } 1 \leq i \leq m .
$$


If $a \geq 1$, then for every $C_{i}, 1 \leq i \leq a$, by the same argument as before, we have $e_{G}\left(S \cup T, C_{i}\right) \geq r$, and so

$$
\begin{aligned}
\theta e_{G}\left(S, C_{i}\right)+(1-\theta) e_{G}\left(T, C_{i}\right) & \geq \min \{\theta, 1-\theta\} e_{G}\left(S \cup T, C_{i}\right) \\
& \geq \min \{k, r-k\} .
\end{aligned}
$$

For every $C_{i}, a<i \leq a+b$, we have

$$
\theta e_{G}\left(S, C_{i}\right)+(1-\theta) e_{G}\left(T, C_{i}\right)-1 \geq \theta+1-\theta-1=0 .
$$

If $a+b<i \leq a+b+c$, then $k\left|C_{i}\right|+e_{G}\left(T, C_{i}\right) \equiv\left|C_{i}\right| \equiv 1(\bmod 2)$, and so $\left|C_{i}\right| \geq r+2$, which implies $c \leq k$ as $|G| \leq(r+2)(k+1)$.

Claim. If $a \geq 1$, then $\delta(S, T) \geq 0$. Hence we may assume $a=0$.

Suppose $a \geq 1$. If $k \leq r / 2$, then

$$
\begin{aligned}
\delta(S, T) \geq & \sum_{i=1}^{a}\left(\theta e_{G}\left(S, C_{i}\right)+(1-\theta) e_{G}\left(T, C_{i}\right)-1\right) \\
& \quad+\sum_{i=a+b+1}^{a+b+c}\left(\theta e_{G}\left(S, C_{i}\right)-1\right) \\
\geq & (k-1) a+(\theta-1) c \quad(\text { by }(2.19)) \\
\geq & (k-1)+(\theta-1) k=k \theta-1>-2 . \quad(\text { by } c \leq k)
\end{aligned}
$$

This implies $\delta(S, T) \geq 0$. If $k>r / 2$, then

$$
\begin{aligned}
\delta(S, T) \geq & \sum_{i=1}^{a}\left(\theta e_{G}\left(S, C_{i}\right)+(1-\theta) e_{G}\left(T, C_{i}\right)-1\right) \\
& +\sum_{i=a+b+1}^{a+b+c}\left(\theta e_{G}\left(S, C_{i}\right)-1\right) \\
\geq & (r-k-1) a+(\theta-1) c \geq r-k-1+(\theta-1) k \\
= & r-2 k-1+\frac{k^{2}}{r}>r-2 r-1+\frac{r^{2}}{r}=-1 . \quad(\text { by } r / 2<k<r)
\end{aligned}
$$

This implies $\delta(S, T) \geq 0$. Therefore we may assume that $a=0$. We consider the following two cases.

Case 1. $|S|>|T|$.

In this case, $m=b+c \leq k+1$ as $|G| \leq(r+2)(k+1)<(r+1)(k+2)$, and

$$
\sum_{x \in S} \operatorname{deg}_{G}(x)=r|S| \geq r(|T|+1)=\sum_{x \in T} \operatorname{deg}_{G}(x)+r .
$$


Hence

$$
\begin{aligned}
\delta(S, T) & =\theta \sum_{x \in S} \operatorname{deg}_{G}(x)+(1-\theta) \sum_{x \in T} \operatorname{deg}_{G}(x)-e_{G}(S, T)-m \\
& \geq \theta \sum_{x \in T} \operatorname{deg}_{G}(x)+\theta r+(1-\theta) \sum_{x \in T} \operatorname{deg}_{G}(x)-e_{G}(S, T)-m \\
& \geq \sum_{x \in T} \operatorname{deg}_{G}(x)-e_{G}(S, T)+k-(k+1) \geq-1 .
\end{aligned}
$$

Thus $\delta(S, T) \geq 0$.

Case 2. $|S| \leq|T|$.

It follows that

$$
\sum_{x \in T} \operatorname{deg}_{G}(x)=r|T| \geq r|S|=\sum_{x \in S} \operatorname{deg}_{G}(x) .
$$

Then

$$
\begin{aligned}
\delta(S, T) & =\theta \sum_{x \in S} \operatorname{deg}_{G}(x)+(1-\theta) \sum_{x \in T} \operatorname{deg}_{G}(x)-e_{G}(S, T)-m \\
& \geq \theta \sum_{x \in S} \operatorname{deg}_{G}(x)+(1-\theta) \sum_{x \in S} \operatorname{deg}_{G}(x)-e_{G}(S, T)-m \\
& =\sum_{x \in S} \operatorname{deg}_{G}(x)-e_{G}(S, T)-m \\
& \geq e_{G}(S, V(G)-(S \cup T))+e_{G}(S, T)-e_{G}(S, T)-m \geq 0 .
\end{aligned}
$$

Therefore $\delta(S, T) \geq 0$. Consequently (ii) is proved.

(iii) is an easy corollary of (ii) since if $r$ is odd and $k$ is even, then $r-k$ is odd and so $G$ has an $(r-k)$-factor $F$ by (ii), and then $G-F$ is a $k$-factor.

Theorem 2.2.4 (Katerinis [83] (1985)) Let $a, b, k$ be odd integers such that $1 \leq a \leq k \leq b$. If a general graph $G$ has both an a-regular factor and $a$ $b$-regular factor, then $G$ has a k-regular factor. In particular, if an r-regular general graph $H$ has a 1-factor, then for every integer $h, 1 \leq h \leq r$, G has an $h$-regular factor.

Proof. We first prove that $G$ has a $k$-factor. Assume that $G$ has no $k$-factor. Then by the Regular Factor Theorem, there exist two disjoint subsets $S$ and $T$ of $V(G)$ such that

$$
\delta(S, T)=k|S|-\sum_{x \in T} \operatorname{deg}_{G}(x)-k|T|+e_{G}(S, T)-q(S, T ; k)<0,
$$


where $q(S, T ; k)$ denote the number of $k$-odd components $C$ of $G-(S \cup T)$, which satisfy

$$
k|C|+e_{G}(C, T) \equiv 1(\bmod 2) .
$$

Since $G$ has both an $a$-factor and a $b$-factor, we have

$$
\begin{aligned}
& a|S|-\sum_{x \in T} \operatorname{deg}_{G}(x)-a|T|+e_{G}(S, T)-q(S, T ; a) \geq 0 \\
& b|S|-\sum_{x \in T} \operatorname{deg}_{G}(x)-b|T|+e_{G}(S, T)-q(S, T ; b) \geq 0 .
\end{aligned}
$$

Since $a, b$ and $k$ are all odd integers, by $(2.21)$ we can easily see that $q(S, T ; k)=$ $q(S, T ; a)=q(S, T ; b)$. By the two previous inequalities and (2.20), we obtain

$$
(a-k)(|S|-|T|)>0 \text { and }(b-k)(|S|-|T|)>0 .
$$

If $|S| \geq|T|$, then $(a-k)(|S|-|T|) \leq 0$ as $a \leq k$, a contradiction. Similarly, if $|S|<|T|$, then $(b-k)(|S|-|T|) \leq 0$ as $k \leq b$, which is again a contradiction. Consequently $G$ has a $k$-factor.

Suppose that and an $r$-regular general graph $H$ has a 1-factor $F_{1}$. If $r$ is odd, then by the statement proved above, for every odd integer $c, 1 \leq c \leq r$, $H$ has a $c$-factor $F_{c}$, and also an $(r-c)$-factor $H-E\left(F_{c}\right)$. Hence for every integer $h, 1 \leq h \leq r, H$ has a $h$-factor.

If $r$ is even, then $H-F_{1}$ is an $(r-1)$-factor. Hence for every odd integer $c, 1 \leq c \leq r-1, H$ has a $c$-factor by the statement proved above. By the 2-Factorable Theorem 2.1.1, it is obvious that for every even integer $d, 2 \leq d \leq r, H$ has a $d$-factor. Hence the latter part of this theorem is proved.

The next theorem shows the existence a $k$-regular factor that contains a given edge or excludes such an edge, and its proof is based on [67].

Theorem 2.2.5 Let $r$ and $k$ be integers such that $1 \leq k<r$, and $G$ be a $\lambda$-edge connected $r$-regular general graph, where $\lambda \geq 2$. If $G$ satisfies one the following conditions, which are the same as in Theorem 2.2.1, then for any given edge $e, G$ has a $k$-regular factor containing $e$ and another $k$-regular factor excluding e.

(1) $r$ and $k$ are both even.

(2) $r$ is even, $k$ is odd, $|G|$ is even, and $\frac{r}{\lambda} \leq k \leq r\left(1-\frac{1}{\lambda}\right)$.

(3) $r$ is odd, $k$ is even and $2 \leq k \leq r\left(1-\frac{1}{\lambda}\right)$.

(4) $r$ and $k$ are both odd and $\frac{r}{\lambda} \leq k$.

(5) Let $\lambda$ be an integer such that $\lambda^{*} \in\{\lambda, \lambda+1\}$ and $\lambda^{*} \equiv 1(\bmod 2)$. Either $r$ is odd, $k$ is even and $k \leq r\left(1-\frac{1}{\lambda^{*}}\right)$; or $r$ is odd, $k$ is odd and $\frac{r}{\lambda^{*}} \leq k$. 

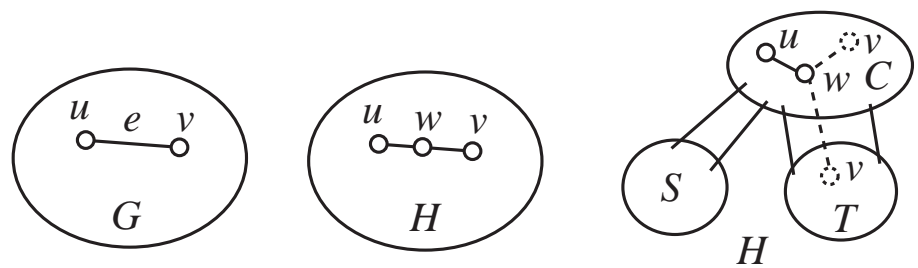

Figure 2.8: A graph $G$ with an edge $e$; a new graph $H$ with a new vertex $w$ of degree two; and an $f$-odd component $C$ of $H-(S \cup T)$.

Proof. Let $e=u v$ be a given edge of $G$. Here we assume that $e$ is not a loop since otherwise we can prove the theorem as well. Moreover, if $k=r-1$, then $G$ must be $(r-1)$-edge connected and so $G$ has no loops, and thus $e$ is a standard edge. Let $H$ be the graph obtained from $G$ by inserting a new vertex $w$ of degree two into $e$ (Figure 2.8). Then $V(H)=V(G) \cup\{w\}$, and define two functions $f_{1}, f_{2}: V(H) \rightarrow \mathbb{Z}^{+}$as

$$
f_{1}(x)=\left\{\begin{array}{cc}
0 & \text { if } x=w \\
k & \text { otherwise }
\end{array} \text { and } f_{2}(x)=\left\{\begin{array}{cc}
2 & \text { if } x=w \\
k & \text { otherwise }
\end{array}\right.\right.
$$

Then it is obvious that $G$ has a $k$-factor excluding $e$ or containing $e$ if and only if $H$ has an $f_{1}$-factor or an $f_{2}$-factor, respectively. So we use the $f$-factor Theorem 2.1.2. For convenience, we write $f$ for $f_{1}$ or $f_{2}$ because most parts of the proof of the existence of an $f_{i}$-factor, $i \in\{1,2\}$, are the same.

Since both $\sum_{x \in V(H)} f_{1}(x)=k|G|$ and $\sum_{x \in V(H)} f_{2}(x)=k|G|+2$ are even numbers and $G$ is connected, $\delta_{H}(\emptyset, \emptyset)=0$. Let $S$ and $T$ be disjoint subsets of $V(H)$ such that $S \cup T \neq \emptyset$. We shall show that $\delta(S, T) \geq 0$, which guarantees the existence of the desired $f$-factor. Set $\theta=k / r$. Then $0<\theta<1$, and

$$
\begin{aligned}
& f(x)=k=\theta \operatorname{deg}_{H}(x) \quad \text { for all } x \in V(H)-w, \\
& f_{1}(w)=\theta \operatorname{deg}_{H}(w)-2 \theta, \\
& -f_{2}(w)=-\theta \operatorname{deg}_{H}(w)-2(1-\theta) .
\end{aligned}
$$

Hence we have

$$
\begin{aligned}
\delta(S, T)= & \sum_{x \in S} f(x)+\sum_{x \in T}\left(\operatorname{deg}_{H}(x)-f(x)\right)-e_{H}(S, T)-q(S, T) \\
\geq & \theta \sum_{x \in S} \operatorname{deg}_{H}(x)+(1-\theta) \sum_{x \in T} \operatorname{deg}_{H}(x) \\
& -e_{H}(S, T)-q(S, T)+\epsilon,
\end{aligned}
$$


where

$$
\epsilon= \begin{cases}-2 \theta & \text { if } w \in S \text { and } f=f_{1} \\ -2(1-\theta) & \text { if } w \in T \text { and } f=f_{2} \\ 0 & \text { otherwise. }\end{cases}
$$

Since $\delta(S, T) \equiv 0(\bmod 2)$ by $(2.5)$, it suffices to show that $\delta(S, T) \geq \epsilon$ as $\epsilon>-2$.

Let $C_{1}, C_{2}, \ldots, C_{m}$ be the $f$-odd components of $H-(S \cup T)$, where $m=$ $q(S, T)$. Then

$$
\begin{aligned}
\delta(S, T) \geq & \theta \sum_{x \in S} \operatorname{deg}_{H}(x)+(1-\theta) \sum_{x \in T} \operatorname{deg}_{H}(x)-e_{H}(S, T)-m+\epsilon \\
\geq & \theta\left(e_{H}(S, T)+\sum_{i=1}^{m} e_{H}\left(S, C_{i}\right)\right) \\
& +(1-\theta)\left(e_{H}(T, S)+\sum_{i=1}^{m} e_{H}\left(T, C_{i}\right)\right)-e_{H}(S, T)-m+\epsilon \\
= & \sum_{i=1}^{m}\left(\theta e_{H}\left(S, C_{i}\right)+(1-\theta) e_{H}\left(T, C_{i}\right)-1\right)+\epsilon .
\end{aligned}
$$

We shall show that for every $C=C_{i}, 1 \leq i \leq m$,

$$
\theta e_{H}(S, C)+(1-\theta) e_{H}(T, C) \geq 1
$$

The proof is complete.

Since $C$ is an $f$-odd component of $H-(S \cup T)$, by the $f$-Factor Theorem we have

$$
\sum_{x \in V(C)} f(x)+e_{H}(T, C) \equiv 1(\bmod 2) .
$$

Moreover,

$$
\sum_{x \in V(C)} \operatorname{deg}_{H}(x)=e_{H}(S \cup T, C)+2\|C\|,
$$

follows and so

$$
r|C| \equiv \sum_{x \in V(C)} \operatorname{deg}_{H}(x) \equiv e_{H}(S \cup T, C)(\bmod 2) \quad \text { if } \quad w \notin C .
$$

Suppose $V(C)=\{w\}$. Then $f(w)+e_{H}(\{w\}, T) \equiv 1(\bmod 2)$ by $(2.25)$. Hence $e_{H}(\{w\}, T)=1,|\{u, v\} \cap T|=1$ and $|\{u, v\} \cap S|=1$. Therefore

$$
\theta e_{H}(S, C)+(1-\theta) e_{H}(T, C) \geq \theta+(1-\theta)=1 \text {. }
$$


Hence we may assume that $C \neq\{w\}$, so, even if $C$ contains $w$, at least one of $\{u, v\}$ is contained in $C$, and thus $e_{H}(C, S \cup T) \geq \lambda$ (Figure 2.8). Therefore we may assume that

$$
e_{H}(S \cup T, C) \geq \lambda
$$
imply

It is obvious that the two inequalities $e_{H}(S, C) \geq 1$ and $e_{H}(T, C) \geq 1$

$$
\theta e_{H}(S, C)+(1-\theta) e_{H}(T, C) \geq \theta+(1-\theta)=1 .
$$

Hence we may assume $e_{H}(S, C)=0$ or $e_{H}(T, C)=0$. We consider two cases under the assumption that one of conditions (1)-(4) holds. Condition (5) will be considered later.

Case 1. $e_{H}(S, C)=0$.

If both $r$ and $k$ are even, then by (2.25) and (2.26), we have

$$
e_{H}(T, C) \equiv 1(\bmod 2) \text { and } 0 \equiv e_{H}(T, C)(\bmod 2) \text {, }
$$

which is a contradiction. If $k \leq r\left(1-\frac{1}{\lambda}\right)$, then $\theta \leq 1-\frac{1}{\lambda}$ and so $1 \leq(1-\theta) \lambda$. By (2.28) and $e_{H}(S, C)=0$, we have $e_{H}(T, C) \geq \lambda$, and hence

$$
(1-\theta) e_{H}(T, C) \geq(1-\theta) \lambda \geq 1 \text {. }
$$

Therefore we may assume that both $r$ and $k$ are odd.

If $w \notin C$, then by $(2.25)$ and (2.26), we have

$$
|C|+e_{G}(T, C) \equiv 1(\bmod 2) \text { and }|C| \equiv e_{G}(T, C)(\bmod 2) .
$$

This is a contradiction. If $w \in C$, then (2.25) and (2.26), we have

$$
|C|-1+e_{G}(T, C) \equiv 1(\bmod 2) \text { and }|C|-1 \equiv e_{G}(T, C)(\bmod 2) .
$$

This is again a contradiction.

Case 2. $e_{H}(T, C)=0$.

By $(2.25)$ we have $\sum_{x \in V(C)} f(x) \equiv 1(\bmod 2)$, which implies that $k$ must be odd. Hence we may assume that $k$ is odd. Then $\frac{r}{\lambda} \leq k$ by (2) and (4). Hence $\theta \lambda \geq 1$, and so by (2.28) we obtain

$$
\theta e_{H}(S, C) \geq \theta \lambda \geq 1 \text {. }
$$

Consequently, statements (1)-(4) in the theorem are proved.

We next prove the first part of (5), i.e., we assume that $r$ is odd, $k$ is even and $k \leq r\left(1-\frac{1}{\lambda^{*}}\right)$. Suppose first $e_{H}(S, C)=0$. Then by (2.25) and (2.26), we have

$$
e_{H}(T, C) \equiv 1(\bmod 2) \text { and } e_{H}(T, C) \geq \lambda
$$


Hence $e_{H}(T, C) \geq \lambda^{*}$, and thus

$$
(1-\theta) e_{H}(T, C) \geq(1-\theta) \lambda^{*} \geq 1 .
$$

Next assume $e_{H}(T, C)=0$. Then by $(2.25)$, we have $k|C| \equiv 1(\bmod 2)$, which is a contradiction.

The remaining part of (5) can be similarly proved (Exercise 2.2.2).

Theorem 2.2.6 (Katerinis [86]) Let $1 \leq k \leq r-1$ be integers, and $G$ be an $(r-1)$-edge connected $r$-regular multigraph of even order. Then for any $r-k$ given edges of $G, G$ has a k-regular factor excluding these edges. Moreover, for any given $k$ edges of $G, G$ has a $k$-regular factor containing these edges.

Note that the latter part of the above theorem is an immediate consequence of the first part because for given $k$ edges, where $k=r-(r-k)$, if $G$ has a $(r-k)$-regular factor $F$ excluding these $k$ edges, then $G$ has a $k$-factor $G-E(F)$ that contains the given $k$ edges.

\section{Exercises}

Exercise 2.2.1 Consider graphs $H\left(r, \lambda^{*} ; \lambda\right)$ and $J\left(r, \lambda^{*} ; \lambda\right)$ when $r$ is odd and $\lambda \geq 1$ and obtain a graph $G(r, \lambda)$. Then show that $G=G(r, \lambda)$ has no $k$-regular factor if either $k$ is even and $k>r\left(1-\frac{1}{\lambda^{*}}\right)$, or $k$ is odd and $k<\frac{r}{\lambda^{*}}$.

Exercise 2.2.2 Show that (5) of Theorem 2.2.5.

\subsection{Regular Factors and $f$-Factors in Graphs}

In this section we shall give some sufficient conditions for simple graphs to have regular factors, where regular factors include 1-factors; but we mainly deal with $k$-regular factors with $k \geq 2$, since the 1 -factor cases have already been proved earlier using shorter arguments. For a general graph $G$, it is clear that $G$ is $t$-tough if and only if its underlying graph is $t$-tough, where the underlying graph of $G$ is obtained from $G$ by deleting loops and replacing multiple edges by a single edge. Thus some results in this section can be applied to general graphs though most results are applicable to simple graphs.

Recall that a graph $G$ is said to be $t$-tough if for every subset $S \subset V(G)$ such that $G-S$ has at least two components, it follows $|S| \geq t \cdot \omega(G-S)$, where $\omega(G-S)$ denotes the number of components of $G-S$. In particular, every $t$-tough graph with $t>0$ is connected. We showed that every 1tough graph of even order has a 1-factor (Theorem 1.5.8). Here we consider 
regular factors and $f$-factors in $t$-tough graphs. The following theorem was conjectured by Chvátal in 1973, and proved by Enomoto, Jackson, Katerinis and Saito in 1985. Chvátal also proposed the conjecture that every 2-tough graph has an Hamiltonian cycle. By the following theorem, such a graph has a 2-factor, however a counterexample to this conjecture was found [15].

Theorem 2.3.1 (Enomoto, Jackson, Katerinis and Saito [45]) Let $k \geq 2$ be an integer. Then every $k$-tough simple graph with $|G| \geq k+1$ and $k|G|$ even has a k-regular factor.

Suppose that a simple graph $G$ has no $k$-factor. Then there exists a pair $(S, T)$ of disjoint subsets of $V(G)$ such that $\delta(S, T)<0$. We say that $(S, T)$ is a minimal pair of $G$ if $\delta(S, T)<0$ and either $T=\emptyset$ or $\delta\left(S, T^{\prime}\right) \geq 0$ for every proper subset $T^{\prime} \subset T$. The following lemma will be used in the proof of Theorem 2.3.1.

Lemma 2.3.2 [45] Let $k \geq 2$ be an integer, and $G$ be a simple graph with $k|G|$ even and having no k-regular factor. Let $(S, T)$ be a minimal pair of $G$. If $T \neq \emptyset$, then the maximum degree of $\langle T\rangle_{G}$ is at most $k-2$.

Proof. Let $v \in T$ and $V=V(G)$. Since $\delta(S, T)<0$ and $k|G|$ is even, we have $\delta(S, T) \leq-2$ by $\delta(S, T) \equiv 0(\bmod 2)(2.5)$. It follows from $\delta(S, T-v) \geq$ 2 that

$$
\begin{aligned}
-2 \geq & \delta(S, T)-\delta(S, T-v) \\
= & k|S|+\sum_{x \in T} \operatorname{deg}_{G}(x)-k|T|-e_{G}(S, T)-q(S, T) \\
& -\left(k|S|+\sum_{x \in T-v} \operatorname{deg}_{G}(x)-k|T-v|-e_{G}(S, T-v)-q(S, T-v)\right) \\
= & \operatorname{deg}_{G}(v)-k-e_{G}(S, v)-q(S, T)+q(S, T-v) .
\end{aligned}
$$

Since $q(S, T-v) \geq q(S, T)-e_{G}(v, V-(S \cup T))$, we have

$$
-2 \geq \operatorname{deg}_{G}(v)-k-e_{G}(S, v)-e_{G}(v, V-(S \cup T)),
$$

which implies

$$
e_{G}(v, T-v)=\operatorname{deg}_{G}(v)-e_{G}(v, S)-e_{G}(v, V-(S \cup T)) \leq k-2 .
$$

Consequently $\Delta\left(\langle T\rangle_{G}\right) \leq k-2$.

Proof of Theorem 2.3.1. Suppose that $G$ has no $k$-factor. Then $G$ is not a complete graph since a complete graph with order at least $k+1$ and $k|G|$ even 
has a $k$-factor. Since $k|G|$ is even and $G$ is connected, $\delta(\emptyset, \emptyset)=-q(\emptyset, \emptyset)=0$, and thus every minimal pair $(S, T)$ of $G$ satisfies $S \cup T \neq \emptyset$.

Let $(S, T)$ be a minimal pair of $G$, and let $C_{1}, C_{2}, \ldots, C_{m}$ be the components of $G-(S \cup T)$, where $m=\omega(G-(S \cup T)) \geq q(S, T)$. For any two non-adjacent vertices $u \in S$ and $x \in V(G)-u, \delta(S, T)$ does not change if a new edge $u x$ is added to $G$, and so the resulting graph still does not have a $k$-factor. Hence we may assume that for every $u \in S, N_{G}(u)=V(G)-u$ (Figure 2.9). Similarly, joining two non-adjacent vertices in any component $C_{i}, i=1, \ldots, m$ of $G-(S \cup T)$ results in a graph that has no $k$-factor. Therefore the following Claim holds.

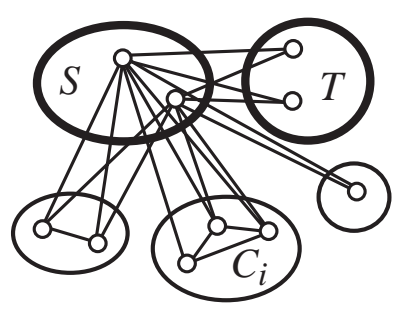

Figure 2.9: $G-(S \cup T)$, where $N_{G}(u)=V(G)-u$ for all $u \in S$ and every $\left\langle V\left(C_{i}\right)\right\rangle_{G}$ is a complete graph.

Claim 1. $N_{G}(u)=V(G)-u$ for all $u \in S$, and every $\left\langle V\left(C_{i}\right)\right\rangle_{G}$ is a complete graph (Figure 2.9).

For convenience, set $U=V(G)-(S \cup T)$. Then

$$
\begin{aligned}
0 & >\delta(S, T)=k|S|+\sum_{x \in T} \operatorname{deg}_{G}(x)-k|T|-e_{G}(S, T)-q(S, T) \\
& \geq k|S|+e_{G}(T, U)+e_{G}(T, S)+2||\langle T\rangle_{G} \|-k|T|-e_{G}(S, T)-m \\
& =k|S|+e_{G}(T, U)+2||\langle T\rangle_{G} \|-k|T|-m .
\end{aligned}
$$

Let $B_{1}$ be a maximal independent set of $\langle T\rangle_{G}$ and $T_{1}=T-B_{1}$. For every $i, 2 \leq i \leq k-1$, we recursively define $B_{i}$ and $T_{i}$ as follows: let $B_{i}$ be a maximal independent set of $\left\langle T_{i-1}\right\rangle_{G}$ and $T_{i}=T_{i-1}-B_{i}$ (Figure 2.10). By Lemma 2.3.2 and $N_{G}\left(B_{i}\right) \supseteq T_{i}$, it follows that $\left\langle T_{k-2}\right\rangle_{G}$ is an independent set, if exists, and thus $B_{k-1}=T_{k-2}$ and $T_{k-1}=\emptyset$.

Claim 2. $|S|+\left|T_{1}\right|+e_{G}\left(U, B_{1}\right)-m \geq k\left|B_{1}\right|$.

Proof. Let

$$
\begin{aligned}
& U=V(G)-(S \cup T), \quad U_{1}=\left\{x \in U \mid e_{G}\left(x, B_{1}\right)>0\right\} \\
& L_{1}=\left\{C_{i} \mid e_{G}\left(C_{i}, B_{1}\right) \geq 1\right\}=\left\{C_{1}, \ldots, C_{m_{1}}\right\}
\end{aligned}
$$




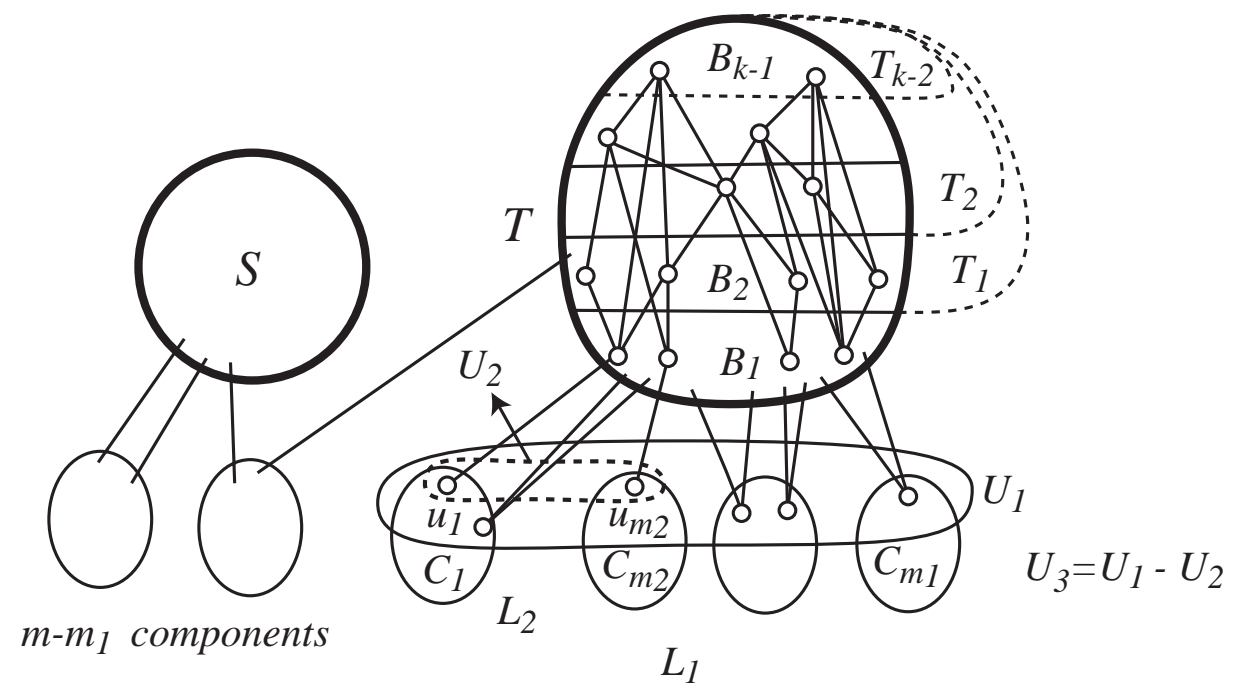

Figure 2.10: A minimal pair $(S, T)$ and the structure of $G$.

$$
\begin{aligned}
& L_{2}=\left\{C_{i} \mid e_{G}\left(u, B_{1}\right)=1 \text { for some } u \in V\left(C_{i}\right)\right\}=\left\{C_{1}, \ldots, C_{m_{2}}\right\}, \\
& U_{2}=\left\{u_{i} \mid \text { For each } C_{i}, 1 \leq i \leq m_{2}\right. \text {, choose exactly one vertex } \\
& \left.\qquad u_{i} \in V\left(C_{i}\right) \text { such that } e_{G}\left(u_{i}, B_{1}\right)=1\right\} \text {, and } \\
& U_{3}=U_{1}-U_{2},
\end{aligned}
$$

where $m_{2} \leq m_{1} \leq m$. Then $\left|U_{2}\right|=m_{2}$, and

$$
\begin{aligned}
& L_{1}-L_{2}=\left\{C_{i} \mid e_{G}\left(u, B_{1}\right) \geq 2 \text { for all } u \in V\left(C_{i}\right)\right\}, \text { and } \\
& \left|U_{1}\right| \leq e_{G}\left(U_{1}, B_{1}\right)-\left(m_{1}-m_{2}\right)=e_{G}\left(U, B_{1}\right)-\left(m_{1}-m_{2}\right) .
\end{aligned}
$$

Hence

$$
\left|U_{3}\right| \leq e_{G}\left(U, B_{1}\right)-m_{1} .
$$

Let $G_{1}=G-\left(S \cup T_{1} \cup U_{3}\right)$. Since $B_{1}$ is an independent set of $\langle T\rangle_{G}$ and $e_{G}\left(B_{1}, U-U_{1}\right)=0$, each vertex of $B_{1}$ belong to different component of $G_{1}$. Hence $\omega\left(G_{1}\right) \geq\left|B_{1}\right|+m-m_{1}$.

We first assume $w\left(G_{1}\right) \geq 2$. Then since $G$ is $k$-tough, we have

$$
\left|S \cup T_{1} \cup U_{3}\right| \geq k \cdot \omega\left(G_{1}\right) \geq k\left(\left|B_{1}\right|+m-m_{1}\right) .
$$

On the other hand, $\left|S \cup T_{1} \cup U_{3}\right| \leq|S|+\left|T_{1}\right|+e_{G}\left(U, B_{1}\right)-m_{1}$. Therefore

$$
\begin{aligned}
& |S|+\left|T_{1}\right|+e_{G}\left(U, B_{1}\right)-m_{1} \geq k \cdot \omega\left(G_{1}\right) \\
& \geq k\left(\left|B_{1}\right|+m-m_{1}\right) \geq k\left|B_{1}\right|+m-m_{1}
\end{aligned}
$$


and the claim follows.

We next consider the case $\omega\left(G_{1}\right)=1$. Then $\left|B_{1}\right|=0$ or 1 since $\left|B_{1}\right|+$ $m-m_{1} \leq \omega\left(G_{1}\right)$. Suppose that the claim does not hold. Then

$$
|S|+\left|T_{1}\right|+e_{G}\left(U, B_{1}\right)-m<k\left|B_{1}\right|
$$

If $\left|B_{1}\right|=0$, then $T=\emptyset$, and so $T_{1}=\emptyset$ and $|S|-m<0$ by (2.29). If $\omega(G-S)=m \geq 2$, then $|S| \geq k m$, which contradicts $|S|-m<0$. Therefore $\omega(G-S)=m \leq 1$. This implies $S=\emptyset$ as $|S|-m<0$, and contradicts $S \cup T \neq \emptyset$.

Hence we may assume that $\left|B_{1}\right|=1$ and this holds for any choice of $B_{1}$. Therefore $\langle T\rangle_{G}$ is a complete graph. Furthermore, $\omega\left(G_{1}\right)=1$ means that for every component $C_{i}$, there exists at least one edge joining $C_{i}$ to $B_{1}$, and thus $e_{G}\left(U, B_{1}\right) \geq m$. Then by $(2.30)$, we have $|S|+\left|T_{1}\right|<k\left|B_{1}\right|=k$. Hence $|S \cup T|=|S|+\left|T_{1}\right|+\left|B_{1}\right|<k+1$. On the other hand, we shall show that $m \geq 2$ and $|S \cup T| \geq 2 k$, which contradicts the above inequality $|S \cup T|<k+1$. Assume $m=1$. Since $G$ is not a complete graph, by Claim 1 there exist two non-adjacent vertices $x_{1} \in V\left(C_{1}\right)$ and $y_{1} \in T$. Setting $B_{1}=\left\{y_{1}\right\}$, then $V\left(C_{1}\right)-U_{3}$ contains $x_{1}$ and so $\omega\left(G_{1}\right) \geq 2$, which gives us a contradiction as in the previous case $\omega\left(G_{1}\right) \geq 2$. Hence $m \geq 2$, which implies

$$
|S \cup T| \geq k \cdot \omega(G-(S \cup T))=k m \geq 2 k .
$$

Therefore the claim is proved.

Claim 3. For every $i, 2 \leq i \leq k-1$, it follows that

$$
|S|+e_{G}\left(T-T_{i-1}, B_{i}\right)+\left|T_{i}\right|+e_{G}\left(U, B_{i}\right) \geq k\left|B_{i}\right| .
$$

Proof. If $B_{i}=\emptyset$, then $T_{i}=\emptyset$ and the claim holds. Thus we may assume $B_{i} \neq$ $\emptyset$. Since $B_{i}$ is a set of isolated vertices of $G-N_{G}\left(B_{i}\right)$, if $\omega\left(G-N_{G}\left(B_{i}\right)\right) \geq 2$, then

$$
\left|N_{G}\left(B_{i}\right)\right| \geq k \cdot \omega\left(G-N_{G}\left(B_{i}\right)\right) \geq k\left|B_{i}\right|
$$

It is clear that

$$
\left|N_{G}\left(B_{i}\right)\right| \leq|S|+e_{G}\left(T-T_{i-1}, B_{i}\right)+\left|T_{i}\right|+e_{G}\left(U, B_{i}\right)
$$

Hence (2.31) holds. Suppose $\omega\left(G-N_{G}\left(B_{i}\right)\right)=1$, which implies $\left|B_{i}\right|=1$ and $V(G)=N_{G}\left(B_{i}\right) \cup B_{i}$. Then

$$
\begin{aligned}
k\left|B_{i}\right|+1 & =k+1 \leq|V(G)|=\left|N_{G}\left(B_{i}\right) \cup B_{i}\right| \\
& \leq|S|+e_{G}\left(T-T_{i-1}, B_{i}\right)+\left|T_{i}\right|+e_{G}\left(U, B_{i}\right)+1
\end{aligned}
$$


Consequently (2.31) follows.

By Claim 2 and (2.31), we have

$$
\begin{aligned}
k|T|= & k \sum_{i=1}^{k-1}\left|B_{i}\right| \quad\left(\text { by } T=\bigcup_{i=1}^{k-1} B_{i}\right) \\
\leq & |S|+\left|T_{1}\right|+e_{G}\left(U, B_{1}\right)-m \\
& \sum_{i=2}^{k-1}\left(|S|+e_{G}\left(T-T_{i-1}, B_{i}\right)+\left|T_{i}\right|+e_{G}\left(U, B_{i}\right)\right) \\
\leq & (k-1)|S|+\sum_{i=1}^{k-1}\left|T_{i}\right|+\sum_{i=2}^{k-1} e_{G}\left(T-T_{i-1}, B_{i}\right)+\sum_{i=1}^{k-1} e_{G}\left(U, B_{i}\right)-m \\
\leq & (k-1)|S|+\sum_{i=1}^{k-2}\left|T_{i}\right|+||\langle T\rangle_{G}||+e_{G}(U, T)-m,
\end{aligned}
$$

where $T_{k-1}=\emptyset$.

By (2.29) and (2.32), we have

$$
\begin{aligned}
& (k-1)|S|+\sum_{i=1}^{k-2}\left|T_{i}\right|+\left\|\langle T\rangle_{G}\right\|+e_{G}(T, U)-m \\
\geq & k|T|>k|S|+e_{G}(T, U)+2||\langle T\rangle_{G} \|-m,
\end{aligned}
$$

and thus

$$
\sum_{i=1}^{k-2}\left|T_{i}\right|>|S|+\left\|\langle T\rangle_{G}|| \geq\right\|\langle T\rangle_{G}||
$$

On the other hand, since $B_{i}$ is a maximal independent set of $\left\langle T_{i-1}\right\rangle_{G}$ and $T_{i}=T_{i-1}-B_{i}, e_{G}\left(x, B_{i}\right) \geq 1$ for every vertex $x \in T_{i}$, and thus we have

$$
\left\|\langle T\rangle_{G}\right\|=\sum_{i=1}^{k-2} e_{G}\left(B_{i}, T_{i}\right) \geq \sum_{i=1}^{k-2}\left|T_{i}\right| .
$$

This contradicts (2.33). Consequently the theorem is proved.

Theorem 2.3.3 ([45]) Let $k \geq 1$ be integer. For every real number $\epsilon>0$, there exists a $(k-\epsilon)$-tough simple graph $G$ with $k|G|$ even and $|G| \geq k+1$ that has no $k$-factor. 
Proof. We construct the desired graph $G=G(m, k)$ as follows (Figure 2.11).

$$
V(G)=A \cup B \cup C=A \cup\left(\bigcup_{i=1}^{k+2} B_{i} \cup \bigcup_{i=1}^{k+2} B_{i}^{\prime}\right) \cup \bigcup_{i=1}^{k(k+2)} C_{i}
$$

where

$$
\begin{aligned}
& A=\left\{a_{1}, a_{2}, \ldots, a_{k}\right\} \\
& B_{i}=\left\{b_{i, 1}, b_{i, 2}, \ldots, b_{i, m}\right\} \quad(1 \leq i \leq k+2) \\
& B_{i}^{\prime}=\left\{b_{i, 1}^{\prime}, b_{i, 2}^{\prime}, \ldots, b_{i, m}^{\prime}, b_{i, m+1}^{\prime}\right\} \quad(1 \leq i \leq k+2) \\
& C_{i}=\left\{x_{i, 1}, \ldots, x_{i, m}, y_{i, 1}, \ldots, y_{i, m+1}, z_{i}\right\} \quad(1 \leq i \leq k(k+2))
\end{aligned}
$$

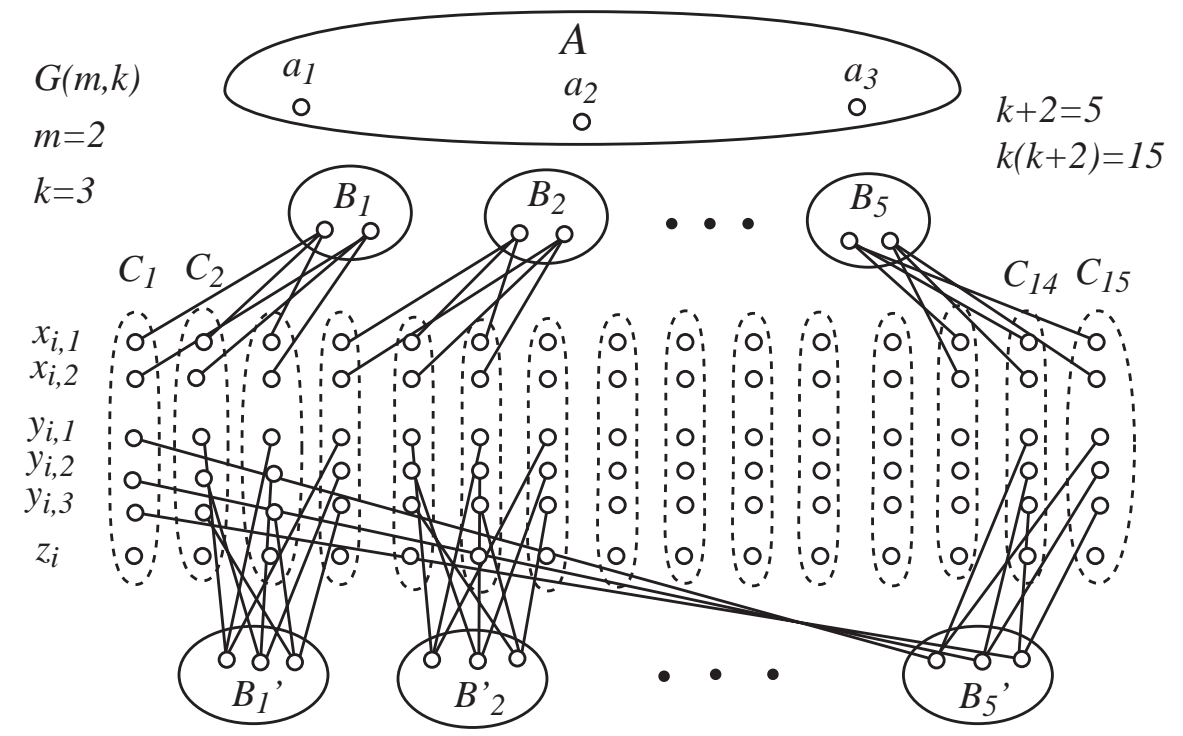

Figure 2.11: The graph $G(m, k)$ with $k=3$ and $m=2$, where $|A|=k,\left|B_{i}\right|=$ $m,\left|B_{i}^{\prime}\right|=m+1,\left|C_{i}\right|=2 m+2, N_{G}(x)=V(G)-x$ for $x \in A$, and $\left\langle C_{i}\right\rangle_{G}$ is a complete graph.

The adjacency of $G$ is defined as follows:

1. $N_{G}\left(a_{i}\right)=V(G)-a_{i}$,

2. $\left\langle C_{i}\right\rangle_{G}=K_{2 m+2}$.

3. $N_{G}\left(b_{i, j}\right)=A \cup\left\{x_{(i-1) k+1, j}, \ldots, x_{i k, j}\right\} \quad(1 \leq i \leq k+2,1 \leq j \leq m)$,

4. $N_{G}\left(b_{i, j}^{\prime}\right)=A \cup\left\{y_{(i-1) k+2, j}, \ldots, x_{i k+1, j}\right\} \quad(1 \leq i \leq k+1,1 \leq j \leq m)$, 
5. $N_{G}\left(b_{k+2, j}^{\prime}\right)=A \cup\left\{y_{k(k+1)+2, j}, \ldots, y_{k(k+2), j}, y_{1, j}\right\}$,

6. $e_{G}\left(C_{i}, C_{j}\right)=0 \quad(1 \leq i<j \leq m+1)$.

Sketch of the proof of Theorem 2.3.3 Let $G=G(m, k)$ be the graph defined above. It is clear that

$$
\begin{aligned}
|G| & =|A|+(k+2)\left|B_{i}\right|+(k+2)\left|B_{i}^{\prime}\right|+k(k+2)\left|C_{i}\right| \\
& =k+(k+2) m+(k+2)(m+1)+k(k+2)(2 m+2) \\
& \equiv k+k m+k(m+1) \equiv 0(\bmod 2) .
\end{aligned}
$$

Hence $|G| \geq k+1$ and $k|G|$ is even.

Let $S=A$ and $T=B$. Then every $C=C_{i}$ is a component of $G-(S \cup T)$, and

$$
k|C|+e_{G}(C, T)=k(2 m+2)+2 m+1 \equiv 1(\bmod 2) .
$$

Hence $C$ is a $k$-odd component of $G-(S, T)$. Thus we have

$$
\begin{aligned}
\delta(S, T) & =k|S|+\sum_{x \in T} \operatorname{deg}_{G-S}(x)-k|T|-q(S, T) \\
& =k^{2}+\sum_{x \in T}\left(\operatorname{deg}_{G-S}(x)-k\right)-k(k+2) \\
& =k^{2}-k(k+2)=-2 k .
\end{aligned}
$$

Hence $G$ has no $k$-factor.

We can show that

$$
k-1<\operatorname{tough}(G)<k .
$$

Let

$$
X=\bigcup_{i=1}^{k(k+2)}\left(C_{i}-z_{i}\right) \quad \cup A .
$$

Then $|X|=k(k+2)(2 m+1)+k$ and

$$
\omega\left(G-S_{0}\right)=\left|\bigcup_{i=1}^{k+2}\left(B_{i} \cup B_{i}^{\prime}\right)\right|+\left|\bigcup_{i=1}^{k(k+2)}\left\{z_{i}\right\}\right|=(2 m+k+1)(k+2) .
$$

and if $m \geq k(k+1)$, then we can show that

$$
\operatorname{tough}(G)=\frac{\omega(G-X)}{|X|}=\frac{k(k+2)(2 m+1)+k}{(2 m+k+1)(k+2)}<k,
$$

and so for sufficiently large $m, k-\epsilon<\operatorname{tough}(G)$, and the theorem is proved.

The next theorem gives a sufficient condition for a tough graph to have an $f$-factor for some function $f$. 
Theorem 2.3.4 (Katerinis [85]) (1) Let $G$ be a 2-tough graph. Then for every function $f: V(G) \rightarrow\{1,2\}$ such that $\sum_{x \in V(G)} f(x)$ is even, $G$ has an $f$-factor.

(2) Let $1 \leq a \leq b$ be integers. If

$$
\operatorname{tough}(G) \geq \begin{cases}\frac{(a+b)^{2}+2(b-a)}{4 a} & \text { if } b \equiv a(\bmod 2) \\ \frac{(a+b)^{2}+2(b-a)+1}{4 a} & \text { otherwise, }\end{cases}
$$

then for every function $f: V(G) \rightarrow\{a, a+1, \ldots, b\}$ such that $\sum_{x \in V(G)} f(x)$ is even, $G$ has an $f$-factor.

The binding number $\operatorname{bind}(G)$ of a simple graph $G$ was defined as

$$
\operatorname{bind}(G)=\min _{X \subset V(G)}\left\{\frac{\left|N_{G}(X)\right|}{|X|} ; \quad \text { where } N_{G}(X) \neq V(G)\right\} \text {. }
$$

We proved that if $G$ is of even order and $\operatorname{bind}(G) \geq \frac{4}{3}$, then $G$ has a 1 factor (Theorem 1.5.7). We now give a sufficient condition for a graph to have a regular factor by using binding number. This result was obtained by Katerinis and Woodall [88] and by Egawa and Enomoto [38], independently.

Theorem 2.3.5 ([88], [38]) Let $k \geq 2$ be an integer, and $G$ be a simple connected graph of order $n$. If $n \geq 4 k-6, k n$ is even and

$$
\operatorname{bind}(G)>\frac{(2 k-1)(n-1)}{k(n-2)+3},
$$

then $G$ has a k-regular factor. In particular, (i) if bind $(G) \geq \frac{3}{2}$, then $G$ has a 2-factor; (ii) if bind $(G) \geq \frac{5}{3}$, then $G$ has a 3-factor; and (iii) if bind $(G) \geq 2$, then $G$ has a $k$-factor for every $k \geq 2$ with $n \geq 4 k-6$.

Note that Woodall [151] proved that every graph $G$ with $\operatorname{bind}(G) \geq \frac{3}{2}$ has a Hamiltonian cycle, and so does 2-factor.

In order to prove the above theorem, we need to introduce a new concept, which will be used in the proofs of other theorems too. Suppose that a connected graph $G$ has no $k$-factor. Then by the regular factor theorem, there exists a pair $(X, Y)$ of disjoint subsets $V(G)$ such that $\delta(X, Y)<0$. We say that $(S, T)$ is a maximal pair if $|S \cup T|$ is maximal among all pairs $(X, Y)$ with $\delta(X, Y)<0$.

Lemma 2.3.6 Let $k \geq 1$ be an integer and $G$ be a connected simple graph with $k|G|$ even. Suppose $G$ has no k-factor. Let $(S, T)$ be a maximal pair of $G$. Then the following statements hold.

(1) For every $u \in V(G)-(S \cup T), \operatorname{deg}_{G-S}(u) \geq k+1$ and $\left|N_{G}(u) \cap T\right| \leq k-1$

(2) For every component $C$ of $G-(S \cup T)$, we have $|V(C)| \geq 3$. 
Proof. (1) Let $u \in V(G)-(S \cup T)$. Since $\delta(S, T \cup\{u\}) \geq 0$ and $\delta(S, T) \leq-2$ by $\delta(S, T) \equiv 0(\bmod 2)$, we have

$$
\begin{aligned}
2 \leq & \delta(S, T \cup\{u\})-\delta(S, T) \\
= & k|S|+\sum_{x \in T \cup\{u\}} \operatorname{deg}_{G-S}(x)-k|T \cup\{u\}|-q(S, T \cup\{u\}) \\
& -\left(k|S|+\sum_{x \in T} \operatorname{deg}_{G-S}(x)-k|T|-q(S, T)\right) \\
\leq & \operatorname{deg}_{G-S}(u)-k-h_{G}(S, T \cup\{u\})+q(S, T) \\
\leq & \operatorname{deg}_{G-S}(u)-k+1 . \quad(\text { by } q(S, T \cup\{u\}) \geq q(S, T)-1)
\end{aligned}
$$

Hence

$$
k+1 \leq \operatorname{deg}_{G-S}(u) .
$$

Similarly, it follows that

$$
\begin{aligned}
2 \leq & \delta(S \cup\{u\}, T)-\delta(S, T) \\
= & k|S \cup\{u\}|+\sum_{x \in T} \operatorname{deg}_{G}(x)-k|T| \\
& -e_{G}(S \cup\{u\}, T)-q(S \cup\{u\}, T) \\
& -\left(k|S|+\sum_{x \in T} \operatorname{deg}_{G}(x)-k|T|-e_{G}(S, T)-q(S, T)\right) \\
\leq & k-e_{G}(u, T)-h_{G}(S \cup\{u\}, T)+q(S, T) \\
\leq & k-e_{G}(u, T)+1 . \quad(\operatorname{by} q(S \cup\{u\}, T) \geq q(S, T)-1)
\end{aligned}
$$

since $q(S \cup\{u\}, T) \geq q(S, T)-1$. Hence

$$
\left|N_{G}(u) \cap T\right|=e_{G}(u, T) \leq k-1 .
$$

(2) Let $C$ be a component of $G-(S \cup T)$ and $u \in V(C)$ (Figure 2.12). By (1), we have

$$
\begin{aligned}
|V(C)-\{u\}| & \geq\left|N_{G}(u) \cap V(C)\right|=\operatorname{deg}_{G-S}(u)-\left|N_{G}(u) \cap T\right| \\
& \geq k+1-(k-1)=2 .
\end{aligned}
$$

Hence $|V(C)| \geq 3$. Consequently the lemma is proved.

Lemma 2.3.7 Let $G$ be a connected simple graph of order $n$ and $\operatorname{bind}(G)>$ $b$. Then the minimum degree $\delta(G)$ satisfies

$$
\delta(G)>\frac{(b-1) n+1}{b} .
$$




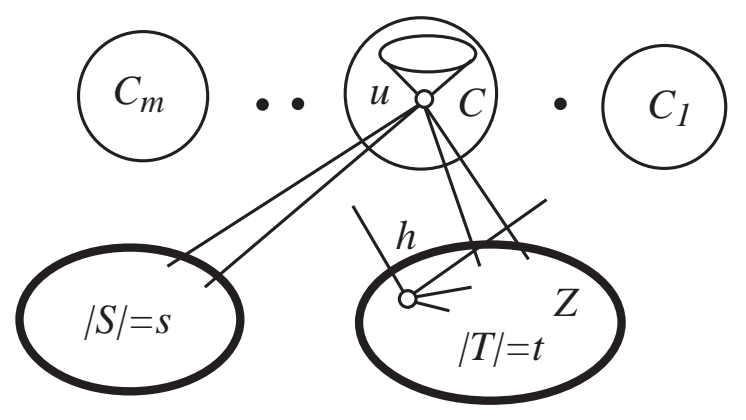

Figure 2.12: $G-(S \cup T), u \in V(C)$ and $M=V(G)-(S \cup T)-V(D)$.

Moreover, if $X$ is a non-empty independent set of $G$, then

$$
\left|N_{G}(X)\right|>\frac{(b-1) n+|X|}{b} .
$$

Proof. Let $X$ be a non-empty independent set of $G$, and let $Y=V(G)-$ $N_{G}(X)$. Then $X \subseteq Y$ and $N_{G}(Y) \subseteq V(G)-X$, and thus

$$
n-|X| \geq\left|N_{G}(Y)\right|>b|Y|=b\left(n-\left|N_{G}(X)\right|\right) .
$$

Hence

$$
\left|N_{G}(X)\right|>\frac{(b-1) n+|X|}{b} .
$$

For any vertex $v$ of $G$, since $\{v\}$ is an independent set of $G$, we have

$$
\operatorname{deg}_{G}(v)=\left|N_{G}(\{v\})\right|>\frac{(b-1) n+1}{b},
$$

and the lemma is proved.

In the proof of [88], the authors assume $k \geq 3$ since they use a result of [151] for $k=2$. Our proof includes the case $k=2$ and is based on [38].

Proof of Theorem 2.3.5. Let $b$ be the right-hand-side of (2.34). By Lemma 2.3.7,

$$
\begin{aligned}
\delta(G) & >\frac{(b-1) n+1}{b}=\frac{\left(\frac{(2 k-1)(n-1)}{k(n-2)+3}-1\right) n+1}{\frac{(2 k-1)(n-1)}{k(n-2)+3}} \\
& =\frac{(k-1) n+2 k-3}{(2 k-1)}=\frac{(k-1) n-2}{(2 k-1)}+1 .
\end{aligned}
$$


Hence

$$
\delta(G) \geq \frac{(k-1) n-1}{(2 k-1)}+1>\frac{(k-1) n}{(2 k-1)} .
$$

Assume that $G$ has no $k$-factor. Let $(S, T)$ be a maximal pair of $G$, where $S \cup T \neq \emptyset$ since $\delta(\emptyset, \emptyset)=0$ by $k n \equiv 0(\bmod 2)$. Put

$$
s=|S| \quad \text { and } \quad t=|T| .
$$

Let $m$ be the number of components of $G-(S \cup T)$ and $c$ denote the minimum order of components of $G-(S \cup T)$. Then by the Regular Factor Theorem and Lemma 2.3.6, we have

$$
\begin{aligned}
-2 & \geq \delta(S, T)=k|S|+\sum_{x \in T} \operatorname{deg}_{G-S}(x)-k|T|-q(S, T) \\
& \geq k s+\sum_{x \in T} \operatorname{deg}_{G-S}(x)-k t-m, \\
m & \leq n-s-t, \\
3 & \left.\leq c \leq \frac{n-s-t}{m} \quad \quad \text { (when } m \geq 1\right), \text { and } \\
\delta(G) & \leq c-1+s+t .
\end{aligned}
$$

Claim. $T \neq \emptyset$.

Suppose $T=\emptyset$. Then $t=0$ and $s \geq 1$, and by (2.36) and (2.37),

$$
k s+2 \leq m \leq n-s .
$$

Hence by (2.35), (2.39), (2.38) and by (2.40), we have

$$
\begin{aligned}
& \frac{(k-1) n}{(2 k-1)}<\delta(G) \leq c-1+s \leq \frac{n-s}{m}-1+s \\
& \leq \frac{n-s}{k s+2}-1+s=\frac{n-2}{k+1}-\frac{(n-2-k s-s)(k s-k+1)}{(k+1)(k s+2)} .
\end{aligned}
$$

Since $n-2-k s-s \geq 0$ by (2.40), it follows that

$$
\frac{(k-1) n}{2 k-1}<\frac{n-2}{k+1}
$$

This is equivalent to $\left(k^{2}-1\right) n<(2 k-1)(n-2)$, which is a contradiction since $k^{2}-1>2 k-1$ when $k \geq 3$ and $3 n>3(n-2)$ when $k=2$. Therefore the claim is proved. 
By the above claim, we define

$$
h=\min _{x \in T} \operatorname{deg}_{G-S}(x) .
$$

Clearly

$$
\delta(G) \leq h+s
$$

We shall consider the three cases.

Case 1. $h=0$.

Let $Z=\left\{x \in T \mid \operatorname{deg}_{G-S}(x)=0\right\} \neq \emptyset$. Since $Z$ is an independent set, we have by Lemma 2.3.7,

$$
\begin{aligned}
s & =|S| \geq\left|N_{G}(Z)\right|>\frac{(b-1) n+|Z|}{b} \\
& =\frac{\left(\frac{(2 k-1)(n-1)}{k(n-2)+3}-1\right) n+|Z|}{\frac{(2 k-1)(n-1)}{k(n-2)+3}}=\frac{(k-1) n+k|Z|+\frac{(k-3)(n-|Z|)}{n-1}}{2 k-1} \\
& \geq \frac{(k-1) n+|Z|-1}{2 k-1} .
\end{aligned}
$$

On the other hand, by (2.36) and (2.37), we obtain

$$
\begin{aligned}
-2 & \geq k s-k|Z|+\sum_{x \in T-Z}\left(\operatorname{deg}_{G-S}(x)-k\right)-m \\
& \geq k s-k|Z|+(1-k)(t-|Z|)-(k-1)(n-s-t),
\end{aligned}
$$

where $m \leq(k-1)(n-s-t)$. Hence

$$
s \leq \frac{(k-1) n+|Z|-2}{2 k-1} .
$$

This contradicts $(2.42)$.

Case 2. $1 \leq h \leq k-1$.

By (2.36), (2.37) and by the assumption $k-h \geq 1$ of this case, we have

$$
k s+(h-k) t-(k-h)(n-s-t) \leq-2 .
$$

Thus

$$
s \leq \frac{(k-h) n-2}{2 k-h} .
$$

On the other hand, we obtain by (2.35) and (2.41)

$$
\frac{(k-1) n-1}{2 k-1}+1 \leq \delta(G) \leq s+h .
$$


By combining the above inequality with (2.43), we have

$$
\frac{(k-1) n-1}{2 k-1} \leq h-1+\frac{(k-h) n-2}{2 k-h} .
$$

Hence

$$
(h-1) k n \leq(h-1)(2 k-1)(2 k-h)-(2 k+h-2) .
$$

This implies $h \geq 2$, and thus

$$
\begin{aligned}
k n & \leq(2 k-1)(2 k-h)-\frac{2 k+h-2}{h-1} \\
& =4 k^{2}-2(h+1) k-\frac{2 k+h-2}{h-1}<4 k^{2}-6 k .
\end{aligned}
$$

This contradicts the assumption $n \geq 4 k-6$.

Case 3. $h=k$.

It is clear from (2.36) that $m \geq k s+2$. So by (2.38),

$$
3 \leq c \leq \frac{n-s-t}{m} \leq \frac{n-s-1}{k s+2},
$$

and thus

$$
k s+2 \leq \frac{n-s-t}{3} \leq \frac{n-1}{3} .
$$

If $k \geq 3$, then by (2.35) and (2.41), we have

$$
\frac{(k-1) n}{2 k}<\frac{(k-1) n+2 k-3}{2 k-1} \leq \delta(G) \leq h+s=k+s .
$$

Since $k+s \leq s k+2$, we have

$$
\frac{(k-1) n}{2 k}<\frac{n-1}{3}
$$

which is equal to $(k-3) n+2 k<0$, a contradiction. If $k=2$, then by $(2.35)$, (2.41) and (2.44), we have

$$
\frac{n-1}{3}<\frac{(k-1) n-1}{2 k-1}+1 \leq \delta(G) \leq h+s=k+s \leq s k+2 \leq \frac{n-1}{3} .
$$

This is a contradiction.

Case 4. $h>k$.

By (2.36), we have $-2 \geq k s+(h-k) t-m$, and so

$$
m \geq k s+t+2 \geq s+t+2
$$


Then $m \geq 3$, and by (2.45), (2.38) and (2.39), we obtain

$$
\begin{aligned}
\delta(G) & \leq c-1+s+t \leq c+m-3 \\
& \leq c+m-3+\frac{(c-3)(m-3)}{3}=\frac{c m}{3} \leq \frac{n}{3} .
\end{aligned}
$$

This contradicts (2.35). Consequently the theorem is proved.

We now show that the conditions in Theorem 2.3.5 are sharp. Let us define the graph $G_{n, k}$ as follows:

$$
G_{n, k}=K_{m}+(n k-1) K_{2}, \quad \text { where } m=2(n k-n-1) .
$$

Then we can show that (i) $G_{n, k}$ has no $k$-factor, $k n$ is even, and

$$
\operatorname{bind}\left(G_{n, k}\right)=2-\frac{2 n-1}{2 n k-3}=\frac{(2 k-1)(n-1)}{k(n-2)+3} .
$$

Note that (ii) when $k=2, \operatorname{bind}(G) \rightarrow \frac{3}{2}$ as $n \rightarrow \infty$; (iii) when $k=3$, $\operatorname{bind}(G) \rightarrow \frac{5}{3}$ as $n \rightarrow \infty$; and (iv) when $k \geq 4$, $\operatorname{bind}(G) \rightarrow 2-\frac{1}{k}$ as $n \rightarrow \infty$.

Theorem 2.3.8 (Kano and Tokushige [79]) Let a and $b$ be integers such that $1 \leq a \leq b$ and $2 \leq b$, and let $G$ be a connected simple graph with order $n, n \geq(a+b)^{2} / 2$. Let $f: V(G) \rightarrow\{a, a+1, \ldots, b\}$ be a function such that $\sum_{x \in V(G)} f(x) \equiv 0(\bmod 2)$. If one of the following two conditions is satisfied, then $G$ has an $f$-factor.

$$
\begin{aligned}
& \operatorname{bind}(G) \geq \frac{(a+b-1)(n-1)}{a n-(a+b)+3} . \\
& \delta(G) \geq \frac{b n-2}{a+b} .
\end{aligned}
$$

In order to prove the above theorem, by the $f$-Factor Theorem 2.1.2 we should show that

$$
\delta(S, T)=\sum_{x \in S} f(x)+\sum_{x \in T}\left(\operatorname{deg}_{G-S}(x)-f(x)\right)-q(S, T) \geq 0 .
$$

Since $a \leq f(x) \leq b$, it suffices to show that

$$
a|S|+\sum_{x \in T} \operatorname{deg}_{G}(x)-b|T|-q(S, T) \geq 0 .
$$

The proof of this inequality is similar to that of Theorem 2.3.5 
It is well-known that if $\alpha(G) \leq \kappa(G)$, then a graph $G$ has a Hamiltonian cycle (Theorem 11 in Basic Terminology). The next theorem gives a sufficient condition for a graph to have a regular factor using independence number $\alpha(G)$ and connectivity $\kappa(G)$. A similar result was obtained by Katerinis [82], which says that if $k \geq 2, k|G|$ is even, $|G| \geq k+1$ and if

$$
\frac{(k+1)^{2}}{4 k} \alpha(G)+\frac{5 k-4}{8}-\frac{2}{k} \leq \kappa(G),
$$

then $G$ has a $k$-regular factor.

Theorem 2.3.9 (Nishimura [117]) Let $k \geq 2$ be an integer, and $G$ be a connected simple graph. If $k|G|$ is even and one of the following two conditions holds, then $G$ has a k-regular factor.

(1) $k$ is odd, $(k+1)^{2} / 2 \leq \kappa(G)$ and $\left((k+1)^{2} / 4 k\right) \alpha(G) \leq \kappa(G)$.

(2) $k$ is even, $k(k+2) / 2 \leq \kappa(G)$ and $((k+2) / 4) \alpha(G) \leq \kappa(G)$.

Proof. Assume that $k$ is odd and $G$ has no $k$-factor. Let $(S, T)$ be a maximal pair of $G$. Since $k|G|$ is even and $G$ is connected, $S \cup T \neq \emptyset$. Set $U=V(G)-(S \cup T)$.

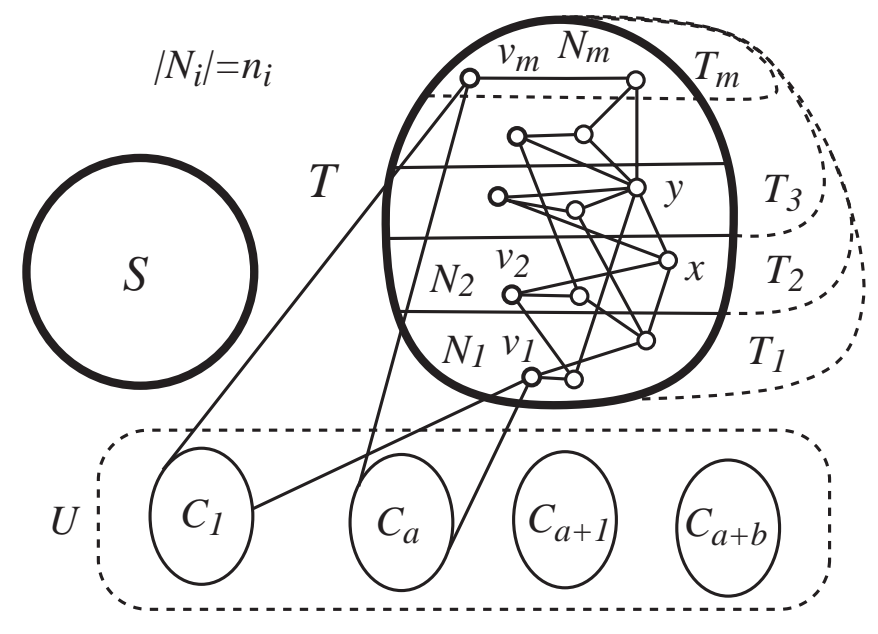

Figure 2.13: A maximal pair $(S, T)$, vertices $v_{i}$, closed neighborhoods $N_{i}$ and components $C_{i}$ of $G-(S \cup T)$.

Let $T_{1}=\langle T\rangle_{G}$, and $v_{1}$ be a vertex of $T_{1}$ with minimum degree, and let $N_{1}=N_{T_{1}}\left[v_{1}\right]=N_{T_{1}}\left(v_{1}\right) \cup\left\{v_{1}\right\}$ and $T_{2}=T_{1}-N_{1}$. For $i \geq 2$, we inductively define $v_{i}, T_{i}$ and $N_{i}$ until $T_{i+1}=\emptyset$ as follows (Figure 2.13): 
Let $v_{i}$ be a vertex of $T_{i}$ with minimum degree, and

$$
N_{i}=N_{T_{i}}\left[v_{i}\right] \quad \text { and } \quad T_{i+1}=T_{i}-N_{i} .
$$

Assume $T_{m+1}=\emptyset$, and let $n_{i}=\left|N_{i}\right|$ for every $1 \leq i \leq m$.

Then the following three statements hold, and their proofs are given below.

$$
\begin{aligned}
& \alpha\left(\langle T\rangle_{G}\right) \geq m, \\
& |T|=n_{1}+n_{2}+\cdots+n_{m} \\
& \sum_{1 \leq i \leq m}\left(\sum_{x \in N_{i}} \operatorname{deg}_{T_{i}}(x)\right) \geq \sum_{1 \leq i \leq m}\left(n_{i}^{2}-n_{i}\right) .
\end{aligned}
$$

(2.46) is an immediate consequence of the fact that $\left\{v_{1}, \ldots, v_{m}\right\}$ is an independent set of $\langle T\rangle_{G}$. (2.47) follows from $T_{m+1}=\emptyset$. Since $\operatorname{deg}_{T_{i}}(x) \geq n_{i}-1$ for every $x \in V\left(T_{i}\right)$ and $\left|N_{i}\right|=n_{i}$, (2.48) holds.

An edge $x y$ joining $x \in N_{i}$ and $y \in N_{j}(1 \leq i<j \leq m)$ is counted in $\operatorname{deg}_{G-S}(x), \operatorname{deg}_{G-S}(y)$ and $\operatorname{deg}_{T_{i}}(x)$ but not in $\operatorname{deg}_{T_{j}}(y)$, and thus by (2.48), we have

$$
\sum_{x \in T} \operatorname{deg}_{G-S}(x) \geq \sum_{1 \leq i \leq m}\left(n_{i}^{2}-n_{i}\right)+\sum_{1 \leq i<j \leq m} e_{G}\left(N_{i}, N_{j}\right)+e_{G}(T, U) .
$$

Let $c=\kappa(G-S)$. Then we have

$$
e_{G}\left(N_{i}, \bigcup_{j \neq i} N_{j}\right)+e_{G}\left(N_{i}, U\right) \geq c \quad \text { for every } 1 \leq i \leq m .
$$

Note that the above inequality does not hold when $m=1$ and $U=\emptyset$. However, in this case, by the choice of $v_{1}$, we have

$$
\begin{aligned}
0>\delta(S, T) & =k|S|+\sum_{x \in T} \operatorname{deg}_{G-S}(x)-k|T|-q(S, T) \\
& \geq k|S|+n_{1}\left(n_{1}-1\right)-k n_{1},
\end{aligned}
$$

which implies

$$
\begin{aligned}
|G|= & |S|+n_{1}<n_{1}-\frac{n_{1}\left(n_{1}-1\right)}{k}+n_{1}=\frac{n_{1}\left(2 k+1-n_{1}\right)}{k} \\
\leq & \frac{k(k+1)}{k}=k+1, \\
& \quad\left(\text { since } n_{1}\left(2 k+1-n_{1}\right) \text { takes a maximum value when } n_{1}=k\right) .
\end{aligned}
$$

This contradicts the assumption that $|G| \geq \kappa(G) \geq \frac{(k+1)^{2}}{2}$. 
By summing up (2.50), we have

$$
\begin{aligned}
& \sum_{1 \leq i \leq m}\left(e_{G}\left(N_{i}, \bigcup_{j \neq j} N_{j}\right)+e_{G}\left(N_{i}, U\right)\right) \\
= & 2 \sum_{1 \leq i<j \leq m} e_{G}\left(N_{i}, N_{j}\right)+e_{G}(T, U) \geq \mathrm{cm} .
\end{aligned}
$$

By (2.49) and (2.51), we get

$$
\sum_{x \in T} \operatorname{deg}_{G-S}(x) \geq \sum_{1 \leq i \leq m} n_{i}\left(n_{i}-1\right)+\frac{c m+e_{G}(T, U)}{2}
$$

By applying (2.49) and (2.52) to $\delta(S, T) \leq-2$, we have

$$
\begin{aligned}
-2 & \geq \delta(S, T)=k|S|+\sum_{x \in T} \operatorname{deg}_{G-S}(x)-k|T|-q(S, T) \\
& \geq k|S|+\sum_{1 \leq i \leq m} n_{i}\left(n_{i}-1\right)+\frac{m c+e_{G}(T, U)}{2}-\sum_{1 \leq i \leq m} k n_{i}-\omega(U) \\
& =k|S|+\sum_{1 \leq i \leq m} n_{i}\left(n_{i}-k-1\right)+\frac{m c+e_{G}(T, U)}{2}-\omega(U) \\
& \geq k|S|-\frac{(k+1)^{2} m}{4}+\frac{m c+e_{G}(T, U)}{2}-\omega(U)
\end{aligned}
$$

(since $n_{i}\left(n_{i}-k-1\right)$ takes a minimum value when $n_{i}=\frac{k+1}{2}$ ).

Let $C_{1}, \ldots, C_{a}$ be the components of $G-(S \cup T)$ such that $V\left(C_{i}\right) \subseteq$ $N_{G}\left(\left\{v_{1}, v_{2}, \ldots, v_{m}\right\}\right)$, and let $C_{a+1}, \ldots, C_{a+b}$ be the other components of $G-(S \cup T)$. We estimate $e_{G}(T, U)$. If $c \geq 1$ and $T=\emptyset$, then $\omega(U)=1$ and so $q(S, \emptyset) \leq 1$ and $\delta(S, \emptyset)=k|S|-q(S, \emptyset) \geq k-1 \geq 0$, a contradiction. Therefore if $c \geq 1$, then $T \neq \emptyset$, and thus

$$
e_{G}\left(C_{i}, T\right) \geq c \quad \text { for all } \quad 1 \leq i \leq a+b .
$$

By Lemma 2.3.6, $e_{G}\left(C_{i}, T\right) \geq\left|C_{i}\right| \geq 3$ for every $1 \leq i \leq a$. Hence

$$
e_{G}(T, U) \geq \sum_{1 \leq i \leq m} e_{G}\left(C_{i}, T\right) \geq 3 a+c b
$$

Since

$$
\omega(U)=a+b, \quad \alpha(G) \geq\left|\left\{v_{1}, \ldots, v_{m}\right\}\right|+b=m+b, \quad \text { and } \quad \kappa(G) \leq|S|+c,
$$


by (2.53) we have

$$
\begin{aligned}
-2 \geq & k|S|-\frac{(k+1)^{2} m}{4}+\frac{m c+e_{G}(T, U)}{2}-\omega(U) \\
\geq & k(\kappa(G)-c)-\frac{(k+1)^{2} m}{4}+\frac{m c+3 a+c b}{2}-a-b \\
\geq & k(\kappa(G)-c)-\left(\frac{(k+1)^{2}}{4}-\frac{c}{2}\right)(m+b) \quad\left(\text { by } \frac{(k+1)^{2}}{4}>1\right) \\
\geq & k(\kappa(G)-c)-\left(\frac{(k+1)^{2}}{4}-\frac{c}{2}\right) \alpha(G) \\
\geq & k(\kappa(G)-c)-\left(\frac{(k+1)^{2}}{4}-\frac{c}{2}\right) \frac{4 k \kappa(G)}{(k+1)^{2}} \\
& \left.\quad \text { by the assumption } \frac{(k+1)^{2} \alpha(G)}{4 k} \leq \kappa(G)\right)
\end{aligned}
$$$$
\left.\geq k c\left(\frac{2 \kappa(G)}{(k+1)^{2}}-1\right) \geq 0 \text {. (by the assumption } \frac{(k+1)^{2}}{2} \leq \kappa(G)\right)
$$

This contradiction completes the proof of the case where $k$ is odd.

When $k$ is even, we have the following instead of (2.53) since $n_{i}\left(n_{i}-k-\right.$ 1) $\geq-\frac{k(k+2)}{4}$.

$$
-2 \geq k|S|-\frac{k(k+2)^{2} m}{4}+\frac{m t+e_{G}(T, U)}{2}-\omega(U) .
$$

Then we can similarly derive a contradiction. Consequently the theorem is proved.

We now show that the condition $\frac{(k+1)^{2}}{4 k} \alpha(G) \leq \kappa(G)$ and $\frac{k+2}{4} \alpha(G) \leq \kappa(G)$ are best possible. Let $k \geq 2$ be integer, and

$$
G=K_{s}+t K_{(k+1) / 2} \quad \text { or } \quad G=K_{s}+t K_{k / 2}
$$

according to the parity of $k$. If $s$ and $t$ satisfy

$$
k s<\frac{t(k+1)^{2}}{4} \quad \text { or } \quad k s<\frac{t k(k+2)}{4},
$$

then $G$ has no $k$-factor. Also, the above inequalities are equivalent to

$$
\frac{(k+1)^{2}}{4 k} \alpha(G)>\kappa(G) \quad \text { or } \quad \frac{k(k+1)}{4 k} \alpha(G)>\kappa(G) .
$$

Hence we can choose $s$ and $t$ so that the difference between $\frac{(k+1)^{2}}{4 k} \alpha(G)$ and $\kappa(G)$ is as small as we want. Therefore the conditions are sharp. Note that 
we can easily choose $s$ and $t$ so that they satisfy the above conditions and $k|G|$ is even.

We next consider sufficient conditions for a graph to have a regular factor using neighborhoods.

Theorem 2.3.10 Let $k \geq 2$ be an integer, and $G$ be a connected simple graph of order $n$ such that $\delta(G) \geq k$ and $k n$ is even. If one of the following three conditions holds, then $G$ has a k-regular factor.

(1) $\delta(G) \geq n / 2$ and $n \geq 4 k-5$. (Egawa and Enomoto [38]; Katerinis [82])

(2) $\operatorname{deg}_{G}(x)+\operatorname{deg}_{G}(y) \geq n$ for every pair of non-adjacent vertices $x$ and $y$ of $G$ and $n \geq 4 k-5$. (Iida and Nishimura [63])

(3) $\max \left\{\operatorname{deg}_{G}(x), \operatorname{deg}_{G}(y)\right\} \geq n / 2$ for every pair of non-adjacent vertices $x$ and $y$ of $G$ and $n \geq 4 k-3$. (Nishimura [119]).

For a graph $G$, we define $N C(G)$ as

$$
N C(G)=\min \left\{\left|N_{G}(x) \cup N_{G}(y)\right| \mid x, y \in V(G), x y \notin E(G)\right\},
$$

that is, $N C(G)$ is the minimum size of $N_{G}(x) \cup N_{G}(y)$ taken over all pairs of non-adjacent vertices $x$ and $y$ of $G$. For a graph $G$ with sufficiently large order, Niessen generalizes the above theorem by using $N C(G)$ as follows:

Theorem 2.3.11 (Niessen [113] (1995)) Let $k \geq 2$ be an integer, and $G$ be a connected simple graph of order $n$ such that $k n$ is even and $n \geq 8 k-7$. If one of the following two conditions holds, then $G$ has a $k$-regular factor.

(1) $N C(G) \geq n / 2$ and $\delta(G) \geq k+1$.

(2) $N C(G) \geq(n+k-2) / 2$ and $\delta(G) \geq k$.

Note that Niessen obtained a more general result, that is, he proved that if $\delta(G) \geq k, n \geq 8 k-7$ and $N C \geq n / 2$, then $G$ has a $k$-regular factor or $G$ belongs to two families of exceptional graphs ([113]), which have no $k$ factors. The above theorem is an easy consequence of this result; but we shall prove the above theorem because of simplicity. Furthermore, when $k=2$, Broersma, van den Heuvel and Veldman obtained the next theorem, and so we assume $k \geq 3$, which makes the proof shorter.

Theorem 2.3.12 [23] Let $G$ be a 2-connected graph. If $N C(G) \geq|G| / 2$, then $G$ has a Hamiltonian cycle, $G$ is the Petersen graph, or $G$ belongs to one of three families of exceptional graphs.

We prove only (1) of Theorem 2.3.11, but the proof is almost the same as the original one. In order to do so, we need the next two lemmas. 
Lemma 2.3.13 Let $G$ be a connected simple graph of order $n$, and let $\emptyset \neq$ $X \subset V(G)$. If $N C(G) \geq n / 2$, then the following statements hold.

(1) If $|X| \geq 3$, then $\omega(G-X) \leq|X|$.

(2) If $|X| \leq 3$, then $\omega(G-X) \leq 3$.

(3) If $4 \leq|X|<(n+20) / 6$, then $\omega(G-X) \leq 4$.

Proof. (1) Let $m=\omega(G-X)$. If $m \leq 3$, then the lemma holds, and so we may assume $m \geq 4$. Let $C_{1}, C_{2}, \ldots, C_{m}$ be the components of $G-X$, where $\left|C_{1}\right| \leq\left|C_{2}\right| \leq \cdots \leq\left|C_{m}\right|$. For two vertices $x \in V\left(C_{1}\right)$ and $y \in V\left(C_{2}\right)$, we have

$$
\frac{n}{2} \leq\left|N_{G}(x) \cup N_{G}(y)\right| \leq\left|C_{1}\right|-1+\left|C_{2}\right|-1+|X|
$$

Thus

$$
\frac{n}{2}+2-|X| \leq\left|C_{1}\right|+\left|C_{2}\right| \leq 2 \cdot \frac{n-|X|}{m}
$$

This is equivalent to

$$
m(n+4-2|X|) \leq 4(n-|X|)
$$

If $|X| \geq \frac{n}{2}$, then $m \leq|V(G)-X| \leq \frac{n}{2} \leq|X|$, and (1) holds. Thus we may assume $3 \leq|X|<\frac{n}{2}$. Suppose $m \geq|X|+1$. Then by (2.55),

$$
(|X|+1)(n+4-2|X|) \leq 4(n-|X|)
$$

which implies

$$
(2|X|-n)(|X|-3) \geq 4 \text {. }
$$

This is a contradiction. Therefore $m \leq|X|$, which implies (1).

(2) We may assume $1 \leq|X| \leq 3$ since if $X=\emptyset$, (2) clearly holds. Assume $m \geq 4$. By (2.55), we have

$$
4(n+4-2|X|) \leq 4(n-|X|)
$$

which implies $4 \leq|X|$, a contradiction. Thus (2) follows.

(3) Assume that $4 \leq|X|<(n+20) / 6$ and $m \geq 5$. Then $n+4-2|X|>$ $(2 n-8) / 3 \geq 0$. Thus, by $(2.55)$ we have

$$
5(n+4-2|X|) \leq 4(n-|X|),
$$

which implies $n+20 \leq 6|X|$. This contradicts $|X|<(n+20) / 6$, and thus (3) holds. 
Lemma 2.3.14 Let $k \geq 2$ be an integer. Suppose that a connected simple graph $G$ with $k|G|$ even has no k-regular factor. Let $(S, T)$ be a maximal pair of $G$. Assume $T \neq \emptyset$. Choose a subset $T^{*} \subseteq T$ so that $\delta\left(S, T^{*}\right)<0$ but $\delta\left(S, T^{\prime}\right) \geq 0$ for every proper subset $T^{\prime} \subset T^{*}$. If $T^{*} \neq \emptyset$, then for every $u \in T^{*}$ we have

$$
\operatorname{deg}_{G-S}(u) \leq k-2+c(u) \leq k-2+q\left(S, T^{*}\right),
$$

where $c(u)$ denotes the number of $k$-odd components of $G-\left(S \cup T^{*}\right)$ joined to $u$ by edges of $G$, and $q\left(S, T^{*}\right)$ is the number of $k$-odd components of $G-\left(S \cup T^{*}\right)$. In particular, $\left|N_{G}(u) \cap T^{*}\right| \leq k-2$ for every $u \in T^{*}$.

Proof. Since $\delta\left(S, T^{*}\right) \leq-2$ and $\delta\left(S, T^{*} \backslash\{u\}\right) \geq 0$, we have

$$
\begin{aligned}
2 \leq & \delta\left(S, T^{*} \backslash\{u\}\right)-\delta\left(S, T^{*}\right) \\
= & k|S|+\sum_{x \in T^{*} \backslash\{u\}} \operatorname{deg}_{G-S}(x)-k\left|T^{*} \backslash\{u\}\right|-q\left(S, T^{*} \backslash\{u\}\right) \\
& -\left(k|S|+\sum_{x \in T^{*}} \operatorname{deg}_{G-S}(x)-k\left|T^{*}\right|-q\left(S, T^{*}\right)\right) \\
= & -\operatorname{deg}_{G-S}(u)+k-h_{G}\left(S, T^{*} \backslash\{u\}\right)+q\left(S, T^{*}\right) .
\end{aligned}
$$

Since $q\left(S, T^{*} \backslash\{u\}\right) \geq q\left(S, T^{*}\right)-c(u)$, we obtain

$$
\operatorname{deg}_{G-S}(u) \leq k-2+c(u) \leq k-2+q\left(S, T^{*}\right) .
$$

Proof of (1) of Theorem 2.3.11 Suppose that $k \geq 3$ and $G$ satisfies the conditions in (1), but has no $k$-factor. Let $(S, T)$ be a maximal pair, and if $T \neq \emptyset$, choose a subset $T^{*} \subseteq T$ as Lemma 2.3.14. Then $\delta(S, T) \leq-2$ by the Regular Factor Theorem, which is equivalent to

$$
q(S, T) \geq k|S|+2-k|T|+\sum_{x \in T} \operatorname{deg}_{G-S}(x) .
$$

Since $k n$ is even, $S \cup T \neq \emptyset$. Let

$$
s=|S|, \quad t=|T| \quad \text { and } \quad U=V(G)-(S \cup T) .
$$

Claim 1. $T \neq \emptyset$ and $T^{*} \neq \emptyset$.

Assume $T=\emptyset$. Then $S \neq \emptyset$. By (2.56), we have $q(S, \emptyset) \geq k|S|+2 \geq 5$. This implies $\omega(G-S) \geq 5$, which contradicts Lemma 2.3.13. Hence $T \neq \emptyset$. If $T^{*}=\emptyset$, then $\delta(S, \emptyset) \leq-2$, and so $(2.56)$ holds, and we can derive a contradiction as above. Therefore $T^{*} \neq \emptyset$. 
Claim 2. $\left|T^{*}\right| \leq k-1$.

Since the proof of Claim 2 is difficult and long, we shall give it after the proof of the theorem, i.e., we continue the proof under the assumption that Claim 2 holds.

Claim 3. $T^{*}$ induces a complete graph of $G$.

Suppose that there exist two non-adjacent vertices $v, w \in T^{*}$. Then $\left|T^{*}\right| \geq 2$. By $N C(G) \geq n / 2 \geq 4 k-(7 / 2)$, we have

$$
\operatorname{deg}_{G-S}(v)+\operatorname{deg}_{G-S}(w) \geq\left|N_{G}(v) \cup N_{G}(w)\right|-|S| \geq 4 k-3-|S| .
$$

Furthermore, for every $x \in T^{*}-\{v, w\}$, it follows that $\operatorname{deg}_{G-S}(x) \geq \delta(G)-$ $|S| \geq k+1-|S|$. Hence setting $\left|T^{*}\right|=t^{*}$, we have by (2.56) that

$$
\begin{aligned}
q\left(S, T^{*}\right) & \geq k|S|+2-k\left|T^{*}\right|+\sum_{x \in T^{*}} \operatorname{deg}_{G-S}(x) \\
& \geq k s+2-k t^{*}+\left(t^{*}-2\right)(k+1-s)+4 k-3-s \\
& =s\left(k+1-t^{*}\right)+t^{*}+2 k-3 \\
& \geq 2 s+t^{*}+2 k-3 \geq 2+6-3=5 . \quad \text { (by Claim 2) }
\end{aligned}
$$

We can derive a contradiction from Lemma 2.3.13 as $q\left(S, T^{*}\right)>\left|S \cup T^{*}\right|=$ $s+t^{*}$ (see the above inequality). Therefore Claim 3 is proved.

By the previous claims, we know that

$$
1 \leq\left|T^{*}\right| \leq k-1 \quad \text { and } \quad T^{*} \text { induces a complete graph. }
$$

Let $C_{1}, C_{2}, \ldots, C_{m}$ be the $k$-odd components of $G-\left(S \cup T^{*}\right)$, where $m=$ $q\left(S, T^{*}\right)$ and $\left|C_{1}\right| \leq\left|C_{2}\right| \leq \cdots \leq\left|C_{m}\right|$.

If $\omega\left(G-\left(S \cup T^{*}\right)\right) \geq 2$, then the edge-maximality of $G$ implies $m=$ $\omega\left(G-S \cup T^{*}\right)$, that is, if there is an even component, then we can add an edge joining an even component to a $k$-odd component because the resulting graph still has no $k$-factor since $\delta\left(S, T^{*}\right)$ does not change. By the same reasons, we may assume that each component $C_{i}$ induces a complete graph. We consider two cases.

Case 1. $\left|T^{*}\right|=1$.

Let $T^{*}=\{u\}$. If $S=\emptyset$, then by (2.56) we have

$$
q(\emptyset,\{u\}) \geq 2-k+\operatorname{deg}_{G}(u) \geq 2-k+\delta(G) \geq 3 .
$$

By Lemma 2.3.13 we have $q(\emptyset,\{u\}) \leq \omega(G-\{u\}) \leq 3$, and so $q(\emptyset,\{u\})=3$. By substituting $q(\emptyset,\{u\})=3$ into (2.58), we have

$$
\operatorname{deg}_{G}(u)=\delta(G)=k+1 .
$$


Let $C_{1}, C_{2}, C_{3}$ be the components of $G-T^{*}=G-\{u\}$. Since $1 \leq e_{G}\left(u, C_{i}\right) \leq$ $\operatorname{deg}_{G}(u)-2=k-1$ for every $1 \leq i \leq 3$ and $\left|C_{i}\right| \geq \delta(G) \geq k+1$, there exists a vertex $y_{i} \in V\left(C_{i}\right)$ such that $x y_{i} \notin E(G)$. It follows that

$$
\sum_{i=1}^{3}\left(\left|C_{i}\right|+e_{G}\left(u, V(G)-V\left(C_{i}\right)\right)\right)=n-1+2 \operatorname{deg}_{G}(u)=n+2 k+1 .
$$

We may assume that $C_{1}$ takes the minimum value among all $\left|C_{i}\right|+e_{G}(u, V(G)-$ $V\left(C_{i}\right), 1 \leq i \leq 3$. For two vertices $u$ and $y_{1} \in V\left(C_{1}\right)$, we have

$$
\frac{n}{2} \leq\left|N_{G}(u) \cup N_{G}\left(y_{1}\right)\right| \leq\left|C_{1}\right|+e_{G}\left(u, V(G)-V\left(C_{1}\right)\right)-1 \leq \frac{n+2 k-2}{3} .
$$

This is a contradiction. Therefore $S \neq \emptyset$.

It is clear that $\operatorname{deg}_{G-S}(u) \geq \delta(G)-|S| \geq k+1-s$. By (2.56), we have

$$
q(S,\{u\}) \geq k|S|+2-k+\operatorname{deg}_{G-S}(u) \geq(k-1) s+3 \geq 5 .
$$

This inequality contradicts Lemma 2.3.13 as $q(S,\{u\}) \geq s+1$.

Case 2. $\left|T^{*}\right| \geq 2$.

It follows from Claim 2 that $2 \leq\left|T^{*}\right| \leq k-1$. We first assume $S=\emptyset$. Since $\delta\left(S, T^{*}\right) \leq-2$ and $\delta(G) \geq k+1$, we have

$$
q\left(\emptyset, T^{*}\right) \geq 2-k\left|T^{*}\right|+\sum_{x \in T^{*}} \operatorname{deg}_{G}(x) \geq 2+\left|T^{*}\right| \geq 4 .
$$

This contradicts Lemma 2.3 .13 as $q\left(\emptyset, T^{*}\right) \geq\left|T^{*}\right|+1$. So we may assume that $S \neq \emptyset$. Let

$$
U^{*}=V(G)-\left(S \cup T^{*}\right) .
$$

Since $|S|+\left|T^{*}\right| \geq 1+2=3$, by Lemma 2.3 .13 we have

$$
q\left(S, T^{*}\right) \leq s+t^{*} .
$$

By Claim 3 and $\delta(G) \geq k+1$, we have

$$
\begin{aligned}
\sum_{x \in T^{*}} \operatorname{deg}_{G-S}(x) & =t^{*}\left(t^{*}-1\right)+e_{G}\left(T^{*}, U^{*}\right) \\
& \geq t^{*}\left(t^{*}-1\right)+t^{*}\left(k+1-s-\left(t^{*}-1\right)\right)
\end{aligned}
$$

Combining (2.56), (2.59) and (2.60), we obtain

$$
\begin{aligned}
s+t^{*} \geq q\left(S, T^{*}\right) & \geq k s+2-k t^{*}+t^{*}\left(t^{*}-1\right)+t^{*}\left(k+1-s-\left(t^{*}-1\right)\right) \\
& =\left(k-t^{*}\right) s+2+t^{*} \geq s+2+t^{*} . \quad \text { (by Claim 2). }
\end{aligned}
$$




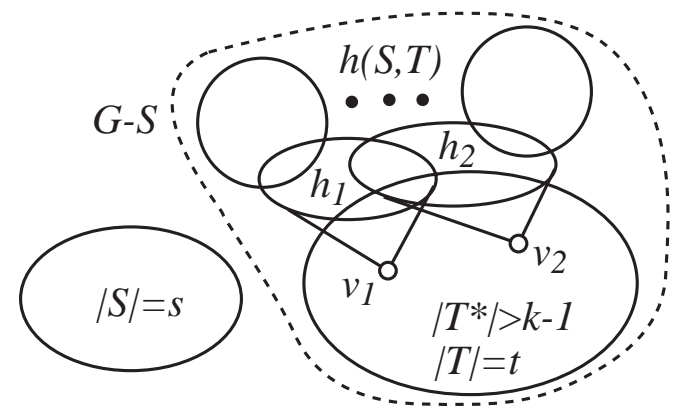

Figure 2.14: Proof of Claim 2; $\left|T^{*}\right| \geq k, v_{1}, v_{2}, G-S, S$ and $T$.

This is a contradiction. Consequently Theorem 2.3.11 is proved.

We now prove the Claim 2.

Claim 2. $\left|T^{*}\right| \leq k-1$.

Proof. Suppose

$$
\left|T^{*}\right| \geq k
$$

Then $3 \leq k \leq\left|T^{*}\right| \leq|T|=t$. Define

$$
h_{1}=\min _{x \in T} \operatorname{deg}_{G-S}(x),
$$

and choose a vertex $v_{1} \in T$ so that $\operatorname{deg}_{G-S}\left(v_{1}\right)=h_{1}$, and $v_{1} \in T^{*}$, if possible (see Figure 2.14).

Sub-Claim 2A. $h_{1} \leq k$.

Assume $h_{1} \geq k+1$. Then by (2.56) we have

$$
\omega(G-(S \cup T)) \geq q(S, T) \geq k s+2+t \geq 4 .
$$

This contradicts Lemma 2.3.13 as $|S \cup T|=s+t<k s+t+2 \leq \omega(G-(S \cup T))$. Hence the sub-claim is proved.

Sub-Claim 2B. $T \backslash N_{G}\left[v_{1}\right] \neq \emptyset$.

Suppose $T \subseteq N_{G}\left[v_{1}\right]$, which implies $T \subseteq N_{G-S}\left[v_{1}\right]$. If $v_{1} \in T^{*}$, then $\left|N_{G}\left(v_{1}\right) \cap T^{*}\right|=\left|T^{*}-v_{1}\right| \geq k-1$ as $\left|T^{*}\right| \geq k$, which contradicts Lemma 2.3.14. Hence $v_{1} \notin T^{*}$. Thus

$$
|T| \geq\left|T^{*}\right|+1 \geq k+1 \quad \text { and } \quad h_{1} \geq\left|T-v_{1}\right| \geq\left|T^{*}\right| \geq k .
$$

On the other hand, $|T| \leq\left|N_{G-S}\left[v_{1}\right]\right| \leq h_{1}+1 \leq k+1$ by Sub-Claim 2A. Hence

$$
t=|T|=k+1, h_{1}=k, T=T^{*} \cup\left\{v_{1}\right\} \text { and } t^{*}=k .
$$


By the choice of $v_{1}, v_{1} \notin T^{*}$ implies $\operatorname{deg}_{G-S}(x) \geq h_{1}+1$ for every $x \in T^{*}$. By (2.56) we have

$$
q(S, T) \geq k s+2-k t+h_{1}+t^{*}\left(h_{1}+1\right)=k s+2+k>s+t,
$$

which contradicts Lemma 2.3.13. Thus $T \backslash N_{G}\left[v_{1}\right] \neq \emptyset$, and Sub-Claim 2B holds.

Choose a vertex $v_{2} \in T \backslash N_{G}\left(v_{1}\right)$ so that $\operatorname{deg}_{G-S}\left(v_{2}\right)$ is minimum among all vertices in $T \backslash N_{G}\left(v_{1}\right)$. Let $h_{2}=\operatorname{deg}_{G-S}\left(v_{2}\right)$. Then

$$
\begin{aligned}
& \sum_{x \in T} \operatorname{deg}_{G-S}(x) \geq \begin{cases}h_{1}\left(h_{1}+1\right)+h_{2}\left(|T|-h_{1}-1\right) & \text { if }|T| \geq h_{1}+2, \\
h_{1}^{2}+h_{2} & \text { if }|T|=h_{1}+1, \\
h_{1}\left(h_{1}-1\right)+h_{2} & \text { if }|T|=h_{1},\end{cases} \\
& \geq h_{1}\left(h_{1}+1\right)+h_{2}\left(t-h_{1}-1\right) . \quad\left(\text { by } h_{1} \leq h_{2}\right)(2.61)
\end{aligned}
$$

Hereafter we often use the above inequality without specifically mentioning it. By $N C(G) \geq \frac{n}{2}$, we have $\frac{n}{2} \leq\left|N_{G}\left(v_{1}\right) \cup N_{G}\left(v_{2}\right)\right| \leq|S|+h_{1}+h_{2}$, and so

$$
s=|S| \geq\left\lceil\frac{n}{2}\right\rceil-h_{1}-h_{2} \geq 4 k-3-h_{1}-h_{2} . \quad(\text { by } n \geq 8 k-7)
$$

We consider three cases.

Case A. $0 \leq h_{2} \leq k-1$.

Using (2.56), we have

$$
\begin{aligned}
q(S, T) & \geq k|S|+2-k|T|+\sum_{x \in T} \operatorname{deg}_{G-S}(x) \\
& \geq k s+2-k t+h_{1}\left(h_{1}+1\right)+h_{2}\left(t-h_{1}-1\right) \\
& =k s+2+\left(h_{2}-k\right) t+\left(h_{1}+1\right)\left(h_{1}-h_{2}\right)
\end{aligned}
$$

Since $q(S, T) \leq \omega(S, T) \leq|U|$, by the above inequality we have

$$
\begin{aligned}
\left(k-h_{2}\right) t+|U| & \geq k s+2+\left(h_{1}+1\right)\left(h_{1}-h_{2}\right) \\
& =k(n-t-|U|)+2+\left(h_{1}+1\right)\left(h_{1}-h_{2}\right)
\end{aligned}
$$

and thus

$$
\begin{aligned}
t & \geq \frac{k n+2+\left(h_{1}+1\right)\left(h_{1}-h_{2}\right)}{2 k-h_{2}}-\frac{k+1}{2 k-h_{2}}|U| \\
& \geq \frac{k n+2+\left(h_{1}+1\right)\left(h_{1}-h_{2}\right)}{2 k-h_{2}}-|U| \quad\left(\text { by } 2 k-h_{2} \geq k+1\right)
\end{aligned}
$$


By (2.62) we can obtain

$$
\begin{aligned}
n=t+|U|+s & \geq \frac{k n+2+\left(h_{1}+1\right)\left(h_{1}-h_{2}\right)}{2 k-h_{2}}+\frac{n}{2}-h_{1}-h_{2} \\
& =\frac{h_{1}^{2}-(2 k-1) h_{1}-h_{2}}{2 k-h_{2}}+\frac{k n+2}{2 k-h_{2}}+\frac{n}{2}-h_{2}
\end{aligned}
$$

Here the right-hand-side takes its minimum value when $h_{1}=h_{2}$ since $0 \leq$ $h_{1} \leq h_{2} \leq k-1$, and thus

$$
n \geq \frac{k n+2}{2 k-h_{2}}+\frac{n}{2}-2 h_{2}
$$

which is equivalent to $8 h_{2} k-4 h_{2}^{2}-4 \geq n h_{2}$. Therefore $h_{2} \geq 1$ and hence $n \leq 8 k-4 h_{2}-\frac{4}{h_{2}} \leq 8 k-8$. This contradicts the assumption $n \geq 8 k-7$.

Case B. $h_{2}=k$.

By Lemma 2.3.6, we have $|U| \geq 3 q(S, T)$. Combining this with (2.56), we have

$$
\begin{aligned}
\frac{|U|}{3} & \geq q(S, T) \geq k|S|+2+\sum_{x \in T} \operatorname{deg}_{G-S}(x)-k|T| \\
& =k s+2+h_{1}\left(h_{1}+1\right)+k\left(t-h_{1}-1\right)-k t \\
& =k(n-t-|U|)+2-\left(k-h_{1}\right)\left(h_{1}+1\right),
\end{aligned}
$$

and thus

$$
\begin{aligned}
\left(k+\frac{1}{3}\right)(n-s) & =\left(k+\frac{1}{3}\right)(|U|+t) \\
& \geq k n+2-\left(k-h_{1}\right)\left(h_{1}+1\right)+\frac{t}{3} . \\
\left(k+\frac{1}{3}\right) n-k n-2 & \geq\left(k+\frac{1}{3}\right) s-\left(k-h_{1}\right)\left(h_{1}+1\right) .
\end{aligned}
$$

Therefore we have

$$
\begin{aligned}
\frac{n}{3}-2 & =\left(k+\frac{1}{3}\right) n-k n-2 \geq\left(k+\frac{1}{3}\right) s-\left(k-h_{1}\right)\left(h_{1}+1\right) \\
& \geq\left(k+\frac{1}{3}\right)\left(\frac{n}{2}-h_{1}-k\right)-\left(k-h_{1}\right)\left(h_{1}+1\right) \quad(\text { by }(2 . \\
& =h_{1}^{2}-\left(2 k+\frac{1}{3}-1\right) h_{1}+\left(k+\frac{1}{3}\right)\left(\frac{n}{2}-k\right)-k
\end{aligned}
$$

Since the right-hand-side takes the minimum value when $h_{1}=k$ since $0 \leq$ $h_{1} \leq h_{2}=k$, 


$$
\frac{n}{3}-2 \geq\left(k+\frac{1}{3}\right)\left(\frac{n}{2}-2 k\right)
$$

Thus

$$
12 k^{2}+4 k-12 \geq(3 k-1) n \geq(3 k-1)(8 k-7),
$$

i.e., we have

$$
0 \geq 12 k^{2}-33 k+19 k=(k-2)(12 k-9)+1 .
$$

This is a contradiction since $k \geq 3$.

Case C. $h_{2} \geq k+1$.

We shall consider six subcases.

Subcase C1. $h_{1}=k$ and $t=k$.

In this case $T^{*}=T$ as $k=|T| \geq\left|T^{*}\right| \geq k$. By (2.56), we have

$$
q(S, T) \geq k s+2-k^{2}+k(k-1)+k+1=k s+3 \geq 3 .
$$

If $s+t \geq q(S, T)$, then $s+t \geq q(S, t) \geq k s+3$, which implies $s=0$ (if $s \geq 1$, then $s+k=s+t \geq k s+3 \geq k+s+2$, a contradiction.) Therefore

$$
k+1 \leq \delta(G) \leq \operatorname{deg}_{G}\left(v_{1}\right)=\operatorname{deg}_{G-S}\left(v_{1}\right)=h_{1}=k,
$$

a contradiction. Hence $s+t=|S \cup T|<q(S, T)$.

By Lemma 2.3.13 and $t=k \geq 3$, we have

$$
|S \cup T|<q(S, T) \leq \omega(G-(S \cup T)) \leq|S \cup T| .
$$

This is a contradiction.

Subcase C2. $h_{1}=k$ and $t=k+1$.

Since $|T|=k+1 \geq 4$, we can apply Lemma 2.3.13 to $S \cup T$, and so by

$$
\begin{aligned}
k+1+|S| & =|T|+|S| \geq q(S, T) \geq k|S|+2-k|T|+h_{1}^{2}+h_{2} \\
& =k|S|+2-k+h_{2} .
\end{aligned}
$$

Hence

$$
2 k-1 \geq(k-1)|S|+h_{2} .
$$

Since $h_{2} \geq k+1$, we have $S=\emptyset$ and $h_{2} \leq 2 k-1$. By (2.62) we obtain

$$
4 k-3 \leq\left\lceil\frac{n}{2}\right\rceil \leq h_{1}+h_{2} \leq 3 k-1 .
$$


Thus $k \leq 2$, a contradiction.

Subcase C3. $h_{1}=k$ and $t \geq k+2$.

Since $|S|+|T| \geq k+2 \geq 5$, we can apply Lemma 2.3.13 to $S \cup T$, and so by $(2.56)$

$$
\begin{aligned}
s+t & \geq q(S, T) \geq k|S|+2-k|T|+k(k+1)+h_{2}(t-k-1) \\
0 & \geq(k-1) s+2+t\left(h_{2}-k-1\right)+(k+1)\left(k-h_{2}\right) \\
& \geq(k-1) s+h_{2}-2 k
\end{aligned}
$$

Hence $h_{2} \leq 2 k-(k-1) s$. By $(2.62)$

$$
h_{2} \geq\left\lceil\frac{n}{2}\right\rceil-s-h_{1} \geq 3 k-3-s,
$$

and so we have $3 \geq k+(k-2) s$. This is a contradiction if $k \geq 4$. So we may assume $k=3$. Then $s=0$ and the equality holds in all the above inequalities, in particular, $t=q(S, T)=5 \geq \omega(G-(S \cup T))$. By Lemma 2.3.13 (3), we have $5=t=|S \cup T| \geq \frac{n+20}{6}$. This contradicts $n \geq 8 k-7=17$.

Subcase C4. $h_{1}=k-1$ and $t \geq k$.

Clearly

$$
s=|S| \geq \operatorname{deg}_{G}\left(v_{1}\right)-\operatorname{deg}_{G-S}\left(v_{1}\right) \geq \delta(G)-h_{1} \geq k+1-h_{1}=2 .
$$

Since $s+t \geq 2+k \geq 4$, we can apply Lemma 2.3.13 to $S \cup T$. If $t=k$, then by (2.61) and (2.63) we have

$$
\begin{aligned}
s+t & \geq q(S, T) \geq k|S|+2-k t+h_{1}^{2}+h_{2} \\
& \geq s+t+(k-1) s-2 k+h_{2}+3>s+t, \quad\left(\text { by } h_{2} \geq k+1\right)
\end{aligned}
$$

a contradiction.

Thus we may assume $t \geq k+1$. By (2.56) and (2.61) we have

$$
s+t \geq q(S, T) \geq k|S|+2-k t+(k-1) k+h_{2}(t-k) .
$$

Thus

$$
\begin{aligned}
0 & \geq(k-1) s+2+t\left(h_{2}-k-1\right)+k(k-1)-h_{2} k \\
& \geq(k-1) s+2+(k+1)\left(h_{2}-k-1\right)+k(k-1)-h_{2} k \\
& =(k-1) s+h_{2}-3 k+1 \\
& =(k-2) s+s+h_{2}-3 k+1 \\
& \left.\geq(k-2) s+k-2-h_{1}=(k-2) s-1 \quad \text { (by }(2.62)\right)
\end{aligned}
$$


This is a contradiction since $k \geq 3$ and $s \geq 2$.

Subcase C5. $h_{1} \leq k-2$ and $t \geq k$.

It follows that

$$
s=|S| \geq \operatorname{deg}_{G}\left(v_{1}\right)-\operatorname{deg}_{G-S}\left(v_{1}\right) \geq \delta(G)-h_{1} \geq k+1-h_{1} \geq 3 .
$$

By applying Lemma 2.3.13 to $S \cup T$, and by (2.56) and (2.61) we have

$$
s+t \geq q(S, T) \geq k|S|+2-k t+h_{1}\left(h_{1}+1\right)+h_{2}\left(t-h_{1}-1\right) .
$$

Thus by $h_{2} \geq k+1$ and $s \geq k+1-h_{1}$ (by 2.64), we have

$$
\begin{aligned}
0 \geq & (k-1) s+2+t\left(h_{2}-k-1\right)-\left(h_{2}-h_{1}\right)\left(h_{1}+1\right) \\
\geq & (k-1) s+2+k\left(h_{2}-k-1\right)-\left(h_{2}-h_{1}\right)\left(h_{1}+1\right) \\
\geq & (k-1)\left(4 k-3-h_{1}-h_{2}\right)+2+k\left(h_{2}-k-1\right) \\
& -h_{2} h_{1}-h_{2}+h_{1}^{2}+h_{1} \quad \text { (by (2.62)) }
\end{aligned}
$$

Then we have

$$
\begin{aligned}
h_{1}\left(k-2+h_{2}\right)-h_{1}^{2} & \geq(k-1)(4 k-3)-k(k+1)+2 \\
& =(3 k-2)(k-2)+1 .
\end{aligned}
$$

Since left-hand-side takes its maximum when $h_{1}=k-2$ as $0 \leq h_{1} \leq k-2$, we have

$$
(k-2) h_{2} \geq(3 k-2)(k-2)+1 .
$$

Hence $h_{2} \geq 3 k-1$. By substituting $s \geq k+1-h_{1}$ (by 2.64) in (2.65), we have

$$
\begin{aligned}
0 & \geq(k-1)\left(k+1-h_{1}\right)+2+k\left(h_{2}-k-1\right)-\left(h_{2}-h_{1}\right)\left(h_{1}+1\right) \\
& =1-k+h_{2}\left(k-h_{1}-1\right)+h_{1}\left(h_{1}+2-k\right) \\
& =\left(k-h_{1}-2\right)\left(h_{2}-h_{1}\right)+h_{2}+1-k \\
& \geq 2 k>0 \quad\left(\text { by } h_{1} \leq k-2, h_{2} \geq h_{1} \text { and } h_{2} \geq 3 k-1 .\right)
\end{aligned}
$$

This is a contradiction. Consequently Claim 2 is proved, and the proof of Theorem 2.3.11 is complete.

It is well-known that a claw-free graph, i.e., a graph with no $K_{1,3}$ as an induced subgraph, has good properties (Figure 2.15). For example, we showed that a connected claw-free graph of even order has a 1-factor. Here we consider regular factors in such graphs. It is easy to see that every line graph is claw-free, and Nishimura [118] showed that for an integer $k \geq 2$, if a 
connected graph $G$ such that $k|E(G)|$ is even satisfies $\delta(L(G)) \geq(9 k+12) / 8$, then the line graph $L(G)$ of $G$ has a $k$-factor. This result was extended to the following Theorem 2.3.15 by Egawa and Ota. Moreover, a similar but weaker result, which says that every claw-free graph with $k|G|$ even and minimum degree at least $2 k$ has a $k$-regular factor, was obtained by Choudum and Paulraj [32] independently.
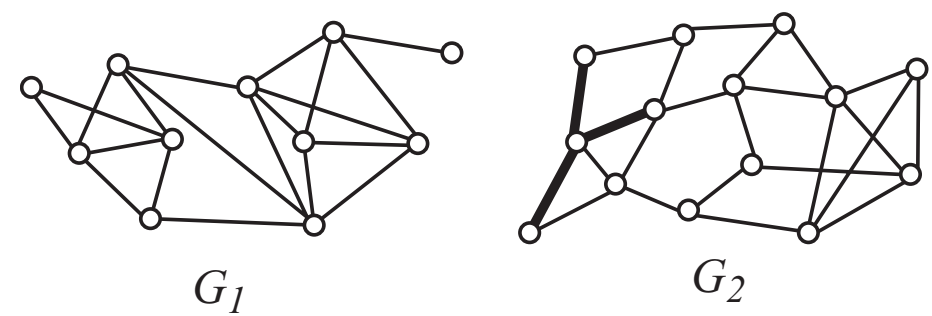

Figure 2.15: A claw-free graph $G_{1}$ and a $K_{1,4}$-free graph $G_{2}$, which is not clawfree.

Theorem 2.3.15 ([40]) Let $k \geq 1$ be an integer and $G$ be a connected clawfree simple graph with $k|G|$ even. If the minimum degree $\delta(G)$ of $G$ satisfies

$$
\delta(G) \geq \frac{9 k+12}{8},
$$

then $G$ has a k-regular factor.

In the same paper, Egawa and Ota obtained the following theorem, and showed that the condition on minimum degree is almost best possible. By substituting $n=3$ into the inequality (2.67) below, we obtain $\delta(G) \geq(9 / 8) k+(3 / 2)+(1 / 2 k)$, which is slightly worse than $(2.66)$.

Theorem 2.3.16 (Egawa and Ota [40]) Let $n \geq 3$ and $k$ be integers such that $k \geq n-1$ if $k$ is odd, and $k \geq 2$ if $k$ is even. Then every $K_{1, n}$-free connected simple graph $G$ satisfying

$$
\delta(G) \geq \frac{n^{2}}{4(n-1)} k+\frac{3 n-6}{2}+\frac{n-1}{4 k}
$$

has a k-regular factor.

Note that the condition $k \geq n-1$ for an odd integer $k$ cannot be removed, i.e., for every $n \geq 4$ and every odd integer $k$ such that $k<n-1$, there exists 
a $K_{1, n}$-free connected graph with even order and minimum degree $2 k$ that has no $k$-factor. We shall prove only Theorem 2.3.16, however, Theorem 2.3.15 can be proved in the same way with some careful estimation.

Proof of Theorem 2.3.16 Suppose that $G$ has no $k$-factor. Then there exists a pair $(S, T)$ of disjoint vertex subsets $S$ and $T$ of $G$ such that

$$
\delta(S, T)=k|S|+\sum_{x \in T} \operatorname{deg}_{G-S}(x)-k|T|-q(S, T)<0 .
$$

It is obvious that $S \cup T \neq \emptyset$ since $\delta(\emptyset, \emptyset)=-q(\emptyset, \emptyset)=0$ as $G$ is connected and $k|G|$ is even. Choose such a pair $(S, T)$ so that $T$ is minimal. Then for every proper subset $T^{\prime} \subset T$, if any, $\delta\left(S, T^{\prime}\right) \geq 0$. This implies $\delta\left(S, T^{\prime}\right) \geq \delta(S, T)+2$ by $\delta(X, Y) \equiv 0(\bmod 2)$.

Claim 1. For every vertex $u \in T$, $\operatorname{deg}_{G-S}(u) \leq k+n-3$.

For a vertex $u \in T$, let $T^{\prime}=T-\{u\}$. Then $\delta\left(S, T^{\prime}\right) \geq \delta(S, T)+2$. Since $G$ is $K_{1, n}$-free, $u$ is adjacent to at most $n-1$ components of $G-(S \cup T)$, and so $q(S, T)-(n-1) \leq q\left(S, T^{\prime}\right)$. Thus

$$
\begin{aligned}
2 \leq & \delta\left(S, T^{\prime}\right)-\delta(S, T) \\
= & k|S|+\sum_{x \in T^{\prime}} \operatorname{deg}_{G-S}(x)-k\left|T^{\prime}\right|-q\left(S, T^{\prime}\right) \\
& -\left(k|S|+\sum_{x \in T} \operatorname{deg}_{G-S}(x)-k|T|-q(S, T)\right) \\
\leq & -\operatorname{deg}_{G-S}(u)+k+n-1
\end{aligned}
$$

Hence $\operatorname{deg}_{G-S}(u) \leq k+n-3$.

For a subset $Y \subseteq T$, we write $\alpha(Y)$ for the number of $k$-odd components of $G-(S \cup T)$ that are joined to $Y$ by edges of $G$. For $y \in T$, let $\alpha(y)=\alpha(\{y\})$. Then $\alpha(y) \leq n-1$ since $G$ is $K_{1, n}$-free. Assume $T \neq \emptyset$. Then we define a set $\left\{y_{1}, y_{2}, \ldots, y_{r}\right\}$ of vertices of $T$ as follows: Let $y_{1}$ be a vertex of $T$ such that $\operatorname{deg}_{G-S}(y)-\alpha(y)$ is minimum among all $y \in T$. Let $T_{1}=N_{G}\left[y_{1}\right] \cap T$, where $N_{G}\left[y_{1}\right]$ is the closed neighborhood of $y_{1}$. Then for $j \geq 2$, if $T-\left(T_{1} \cup \cdots \cup\right.$ $\left.T_{j-1}\right) \neq \emptyset$, then choose $y_{j}$ so that $\operatorname{deg}_{G-S}(y)-\alpha(y)$ is minimum among all $y \in T-\left(T_{1} \cup \cdots \cup T_{j-1}\right)$, and assume $T-\left(T_{1} \cup \cdots \cup T_{r}\right)=\emptyset$ (see Figure 2.16). When $T=\emptyset$, we define $r=0$. Then $\left\{y_{1}, y_{2}, \ldots, y_{r}\right\}$ is an independent set of G.

Claim 2. $|S| \geq \frac{1}{n-1} \sum_{i=1}^{r} e_{G}\left(v_{i}, S\right)+\frac{1}{k}(q(S, T)-\alpha(T))$. 


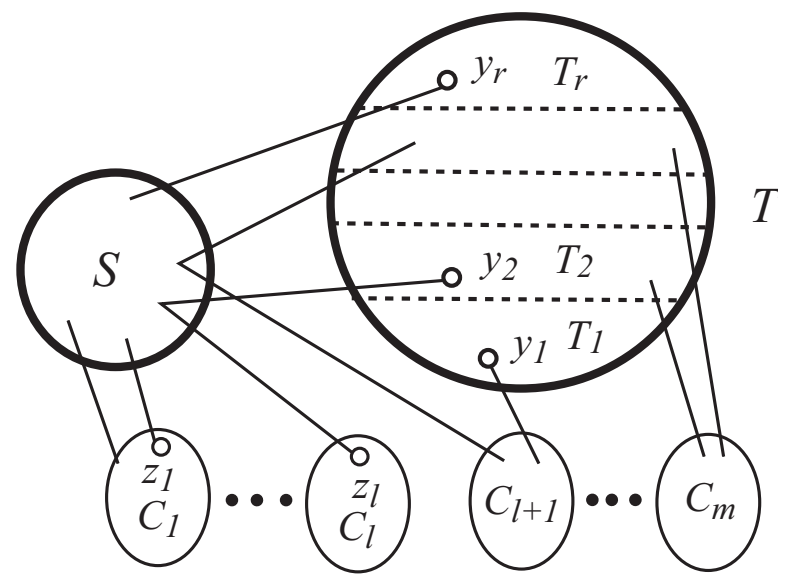

Figure 2.16: The graph $G$ and vertices $y_{i}$ and $z_{j}$.

Let $\ell=q(S, T)-\alpha(T)$. Then there exist $\ell k$-odd components $C_{1}, C_{2}, \ldots, C_{\ell}$ such that $e_{G}\left(T, C_{i}\right)=0$ for all $1 \leq i \leq \ell$.

Suppose $S=\emptyset$. Then $\ell=0$ by $T \neq \emptyset$, and so the claim holds. Hence we may assume $S \neq \emptyset$. Since $G$ is connected, $e_{G}\left(S, C_{i}\right) \geq 1$ for all $1 \leq i \leq \ell$, and thus we can choose a vertex $z_{i} \in V\left(C_{i}\right)$ so that $z_{i}$ and $S$ are adjacent. Let $X=\left\{y_{1}, \ldots, y_{r}, z_{1}, \ldots, z_{\ell}\right\}$ (see Figure 2.16). Since $X$ is an independent set and $G$ is $K_{1, n}$-free, every $x \in S$ is adjacent to at most $n-1$ vertices of $X$. Therefore

$$
\begin{aligned}
(n-1)|S| & \geq e_{G}(X, S)=\sum_{i=1}^{r} e_{G}\left(y_{i}, S\right)+\sum_{j=1}^{\ell} e_{G}\left(z_{j}, S\right) \\
& \geq \sum_{i=1}^{r} e_{G}\left(y_{i}, S\right)+\ell .
\end{aligned}
$$

On the other hand, if $k$ is even then $\ell=0$ since for any $k$-odd component $C_{i}$ of $G-(S \cup T)$, it follows that $k\left|C_{i}\right|+e_{G}\left(T, C_{i}\right) \equiv e_{G}\left(T, C_{i}\right) \equiv 1(\bmod 2)$. If $k$ is odd, then $k \geq n-1$ by the assumption of the theorem. Hence

$$
(n-1)|S| \geq \sum_{i=1}^{r} e_{G}\left(y_{i}, S\right)+\ell \geq \sum_{i=1}^{r} e_{G}\left(y_{i}, S\right)+\frac{n-1}{k} \ell .
$$

Therefore Claim 2 holds.

By Claim 2, we have

$$
\delta(S, T) \geq \frac{k}{n-1} \sum_{i=1}^{r} e_{G}\left(y_{i}, S\right)+\sum_{x \in T}\left(\operatorname{deg}_{G-S}(x)-k\right)-\alpha(T)
$$




$$
\geq \sum_{i=1}^{r}\left(\frac{k}{n-1} e_{G}\left(y_{i}, S\right)+\sum_{x \in T_{i}}\left(\operatorname{deg}_{G-S}(x)-k\right)-\alpha\left(T_{i}\right)\right) .
$$

Let

$$
\beta_{i}=\sum_{x \in T_{i}}\left(\operatorname{deg}_{G-S}(x)-k\right)-\alpha\left(T_{i}\right) .
$$

Then in order to prove the theorem, it suffices to show that

$$
\frac{k}{n-1} e_{G}(y, S)+\beta \geq 0, \quad \text { where } y=y_{j} \text { and } \beta=\beta_{j},
$$

for all $1 \leq j \leq r$ because this implies $\delta(S, T) \geq 0$, a contradiction.

Case 1. $\alpha(y) \leq n-2$.

It follows from $\alpha\left(T_{j}\right) \leq \sum_{x \in T_{j}} \alpha(x)$ and the choice of $y$ that

$$
\begin{aligned}
\beta & \geq \sum_{x \in T_{j}}\left(\operatorname{deg}_{G-S}(x)-k\right)-\sum_{x \in T_{j}} \alpha(x) \\
& \geq\left|T_{j}\right|\left(\operatorname{deg}_{G-S}(y)-\alpha(y)-k\right) .
\end{aligned}
$$

If $\operatorname{deg}_{G-S}(y)-\alpha(y)-k \geq 0$ then $\beta \geq 0$, and thus (2.68) holds. So we may assume that $\operatorname{deg}_{G-S}(y)-\alpha(y)-k<0$. On the other hand, since $N_{G-S}[y] \supseteq T_{j}$, we have

$$
\left|T_{j}\right| \leq \operatorname{deg}_{G-S}(y)-\alpha(y)+1 .
$$

Therefore

$$
\begin{aligned}
\beta & \geq\left(\operatorname{deg}_{G-S}(y)-\alpha(y)+1\right)\left(\operatorname{deg}_{G-S}(y)-\alpha(y)-k\right) \\
& =\left(\operatorname{deg}_{G-S}(y)-\alpha(y)-\frac{k-1}{2}\right)^{2}-\frac{(k+1)^{2}}{4} .
\end{aligned}
$$

Subcase $1.1 \operatorname{deg}_{G-S}(y)-\frac{k-1}{2} \leq n-2$.

By (2.70) we have $\beta \geq-(k+1)^{2} / 4$. Since

$$
e_{G}(y, S)=\operatorname{deg}_{G}(y)-\operatorname{deg}_{G-S}(y) \geq \operatorname{deg}_{G}(y)-\left(n-2+\frac{k-1}{2}\right),
$$

it follows that

$$
\frac{k}{n-1} e_{G}(y, S)+\beta \geq \frac{k}{n-1}\left(\operatorname{deg}_{G}(y)-n+2-\frac{k-1}{2}\right)-\frac{(k+2)^{2}}{4} .
$$


By the condition of the minimum degree of $G$, we have

$$
\begin{aligned}
& \frac{k}{n-1} e_{G}(y, S)+\beta \\
\geq & \frac{k}{n-1}\left(\frac{n^{2}}{4(n-1)} k+\frac{3 n-6}{2}+\frac{n-1}{4 k}-n+2-\frac{k-1}{2}\right)-\frac{(k+1)^{2}}{4} \\
= & \frac{k}{n-1}\left(\frac{k n^{2}}{4(n-1)}+\frac{n-1-k}{2}+\frac{n-1}{4 k}\right)-\frac{(k+1)^{2}}{4} \\
= & \frac{k^{2} n^{2}+2 k(n-1)(n-1-k)+(n-1)^{2}-(k+1)^{2}(n-1)^{2}}{4(n-1)^{2}} \\
= & \frac{k^{2}}{4(n-1)^{2}} \geq 0
\end{aligned}
$$

Hence (2.68) holds.

Subcase $1.2 \operatorname{deg}_{G-S}(y)-\frac{k-1}{2}>n-2$.

Since $\alpha(y) \leq n-2$, the right-hand-side of (2.70) takes the minimum value when $\alpha(y)=n-2$. Hence it follows from (2.69) that

$$
\beta \geq\left(\operatorname{deg}_{G-S}(y)-n+3\right)\left(\operatorname{deg}_{G-S}(y)-n-k+2\right) .
$$

Then

$$
\begin{aligned}
& \frac{k}{n-1} e_{G}(y, S)+\beta \\
= & \frac{k}{n-1}\left(\operatorname{deg}_{G}(y)-\operatorname{deg}_{G-S}(y)\right)+\beta \\
\geq & \frac{k}{n-1}\left(\frac{n^{2}}{4(n-1)} k+\frac{3 n-6}{2}+\frac{n-1}{4 k}-\operatorname{deg}_{G-S}(y)\right) \\
& +\left(\operatorname{deg}_{G-S}(y)-n+3\right)\left(\operatorname{deg}_{G-S}(y)-n-k+2\right) \\
= & \left(\operatorname{deg}_{G-S}(y)-\frac{n k+(n-1)(2 n-5)}{2(n-1)}\right)^{2} \geq 0
\end{aligned}
$$

Consequently (2.68) holds.

Case 2. $\alpha(y)=n-1$.

In this case, there exist $n-1 k$-odd components $C_{1}, C_{2}, \ldots, C_{n-1}$ of $G-$ $(S \cup T)$ such that every $C_{i}, 1 \leq i \leq n-1$, contains a vertex $z_{i}$ adjacent to $y$. Since $G$ is $K_{1, n}$-free, every $x \in T_{j}-\{y\}$ must be adjacent to some $z_{i}$. In particular, $e_{G}\left(x, C_{i}\right) \geq 1$ for some $i$ and hence $C_{i}$ is counted in $\alpha(x)$. 
Therefore

$$
\begin{aligned}
\alpha\left(T_{j}\right) & \leq \alpha(y)+\sum_{x \in T_{j}-\{y\}}(\alpha(x)-1) \\
& =\sum_{x \in T_{j}} \alpha(x)-\left|T_{j}\right|+1 .
\end{aligned}
$$

Thus

$$
\begin{aligned}
\beta & =\sum_{x \in T_{j}}\left(\operatorname{deg}_{G-S}(x)-k\right)-\alpha\left(T_{j}\right) \\
& \geq \sum_{x \in T_{j}}\left(\operatorname{deg}_{G-S}(x)-\alpha(x)-k\right)+\left|T_{j}\right|-1 \\
& \geq\left|T_{j}\right|\left(\operatorname{deg}_{G-S}(y)-\alpha(y)-k\right)+\left|T_{j}\right|-1 \\
& =\left|T_{j}\right|\left(\operatorname{deg}_{G-S}(y)-n-k+2\right)-1 .
\end{aligned}
$$

By Claim 1, $\operatorname{deg}_{G-S}(y) \leq n+k-3$, and so $\operatorname{deg}_{G-S}(y)-n-k+2 \leq-1<0$. On the other hand, $\left|T_{j}\right| \leq \operatorname{deg}_{G-S}(y)-\alpha(y)+1=\operatorname{deg}_{G-S}(y)-n+2$. Therefore

$$
\begin{aligned}
\beta & \geq\left(\operatorname{deg}_{G-S}(y)-n+2\right)\left(\operatorname{deg}_{G-S}(y)-n-k+2\right)-1 \\
& \geq\left(\operatorname{deg}_{G-S}(y)-n+3\right)\left(\operatorname{deg}_{G-S}(y)-n-k+2\right)
\end{aligned}
$$

This inequality is equal to (2.71). Hence by the same argument given above, we can show that (2.68) holds. Consequently, in each case we can derive a contradiction, and thus the proof of the theorem is complete.

We now give some variety of sufficient conditions for a graph to have regular factor without proofs.

Theorem 2.3.17 (Saito [131]) Let $G$ be a connected simple graph with order at least four and having a 1-factor $F$. Let $k$ be a positive integer. If $G-\{x, y\}$ has a $k$-regular factor for every edge $x y \in E(F)$, then $G$ itself has a $k$-regular factor.

Theorem 2.3.18 (Katerinis [87]) Let $r \geq 2$ be an even integer and $1 \leq k \leq$ $r / 2$ be an integer. Let $G$ be a $r$-edge connected $r$-regular multigraph of odd order. Then for every vertex $v$ of $G, G-v$ has a $k$-regular factor.

Theorem 2.3.19 (Niessen [113]) Let $k \geq 4$ be an even integer and let $G$ be a simple graph of order at least $k+1$. If

$$
\delta(G)>\frac{k+2}{4} \alpha(G)+\frac{5 k-3}{8}-\frac{2}{k},
$$

then $G$ has a $k$-regular factor, where $\alpha(G)$ denotes the independence number of $G$. 
Note that the situation of the above Theorem 2.3.19 is changed a lot when we consider an odd regular factor. Namely, for every integer $\alpha \geq 2$ and any arbitrary large number $\delta$, there exists graphs with independence number $\alpha$ and minimum degree $\delta$ that have no $k$-factor. For example, such graphs are $K_{b}+\alpha K_{d}$, where $\alpha \geq k d+2, d$ is odd and $b+\alpha d$ is even.

For positive integers $n$ and $k$ with $n k$ even, let $\rho(n, k)$ denote the maximal number of edges in a graph of order $n$ that has a unique $k$-factor. Some studies on $\rho(n, k)$ were done as given below, and for some $k, n, \rho(n, k)$ is settled.

Theorem 2.3.20 (Hetyei [59] (1972)) If $n$ is an even positive integer, then

$$
\rho(n, 1)=\frac{n^{2}}{4}
$$

Proof. ([60]) Assume that a simple graph $G$ has the unique 1-factor $F$. Let $H_{1}, H_{2}, \ldots, H_{n / 2}$ be the components of $F$. There can be at most two edges joining $H_{i}$ to $H_{j}$ in $G$ since otherwise $\left\langle V\left(H_{i}\right) \cup V\left(H_{j}\right)\right\rangle_{G}$ contains two 1 -factors and so does $G$. Hence

$$
|E(G)| \leq \frac{n}{2}+2\left(\begin{array}{c}
n / 2 \\
2
\end{array}\right)=\frac{n^{2}}{4} .
$$

Theorem 2.3.21 (Hendry [57] (1984)) If $n$ is a positive integer, then

$$
\rho(n, 2)=\left\lceil\frac{n(n+1)}{4}\right\rceil .
$$

Hendry [57] determined $\rho(n, 2)$ as above. Moreover for other $\rho(n, k)$, he constructed a class of graphs $G$ that have a unique $k$-factor and satisfy

$$
\begin{array}{ll}
\|G\|=\frac{n^{2}}{4}+\frac{(k-1) n}{4} & \text { if } k \leq \frac{n}{2}, \text { and } \\
\|G\|=\frac{n k}{2}+\left(\begin{array}{c}
n-k \\
2
\end{array}\right) & \text { if } k>\frac{n}{2} .
\end{array}
$$

He conjectured that these two values give $\rho(n, k)$, that is, he conjectured that a graph in the class has a unique $k$-factor and has the maximum number of edges. This conjecture was partially proved by Johann in the following theorem. 
Theorem 2.3.22 (Johann [65]) Let $n$ and $k$ with $n k$ even and $k<n$. Then

$$
\rho(n, k) \leq \frac{n^{2}}{4}+\frac{(k-1) n}{4} \quad \text { if } k \leq \frac{n}{2},
$$

where equality holds for $n=2 k l, l \geq 1$, and

$$
\rho(n, k)=\frac{n k}{2}+\left(\begin{array}{c}
n-k \\
2
\end{array}\right) \quad \text { if } k>\frac{n}{2} .
$$

Recently $\rho(n, 3)$ and $\rho(n, k)$ for many $k$ were determined by Volkmann as follows:

Theorem 2.3.23 (Volkmann [148]) Let $n$ and $k$ be positive integers such that $k n$ or $3 n$ is even. Then

$$
\rho(n, k)=k^{2}+2\left(\begin{array}{c}
n-k \\
2
\end{array}\right) \quad \text { for } \frac{n}{3} \leq k \leq \frac{n}{2},
$$

and

$$
\begin{array}{ll}
\rho(n, 3)=\frac{n^{2}}{4}+\frac{n}{2} & \text { for } \quad n \equiv 0,4 \quad(\bmod 6), \\
\rho(n, 3)=\frac{n^{2}}{4}+\frac{n}{2}-1 & \text { for } \quad n \equiv 2 \quad(\bmod 6) .
\end{array}
$$

\subsection{Regular Factors and f-Factors in Bipar- tite Graphs}

The criterion for a graph to have an $f$-factor or a regular factor becomes much simpler when we consider bipartite graphs, i.e., the most complicated term $q(S, T)$ in $\delta(S, T)$ disappears and also it may be assumed that $S$ and $T$ are contained in distinct partite sets. We begin with this simpler criterion, which was obtained by Ore [124], and Folkman and Fulkerson [49].

Theorem 2.4.1 (The $f$-Factor Theorem for Bipartite Graphs) Let $G$ be a bipartite multigraph with bipartition $(A, B)$ and $f: V(G) \rightarrow \mathbb{Z}^{+}$. Then $G$ has an $f$-factor if and only if $\sum_{x \in A} f(x)=\sum_{x \in B} f(x)$ and

$$
\delta^{*}(S, T)=\sum_{x \in S} f(x)+\sum_{x \in T}\left(\operatorname{deg}_{G-S}(x)-f(x)\right) \geq 0
$$

for all subsets $S \subseteq A$ and $T \subseteq B$. 
It is clear that $\delta^{*}(S, T)$ can be expressed as

$$
\delta^{*}(S, T)=\sum_{x \in S} f(x)+\sum_{x \in T}\left(\operatorname{deg}_{G}(x)-f(x)\right)-e_{G}(S, T) .
$$

We first show that under the assumption $\sum_{x \in A} f(x)=\sum_{x \in B} f(x),(2.72)$ is equivalent to the following symmetric condition

$$
\delta^{*}(T, S)=\sum_{x \in T} f(x)+\sum_{x \in S}\left(\operatorname{deg}_{G-T}(x)-f(x)\right) \geq 0
$$

for all subsets $S \subseteq A$ and $T \subseteq B$.

Proof. Assume that (2.72) holds for all $S \subseteq A$ and $T \subseteq B$. Then

$$
\begin{aligned}
0 \leq & \delta^{*}(A-S, B-T) \\
= & \sum_{x \in A-S} f(x)+\sum_{x \in B-T}\left(\operatorname{deg}_{G}(x)-f(x)\right)-e_{G}(A-S, B-T) \\
= & \sum_{x \in A} f(x)-\sum_{x \in S} f(x)+\sum_{x \in B} \operatorname{deg}_{G}(x)-\sum_{x \in T} \operatorname{deg}_{G}(x) \\
& -\sum_{x \in B} f(x)+\sum_{x \in T} f(x)-e_{G}(A-S, B-T) \\
= & -\sum_{x \in S} f(x)+\sum_{x \in T} f(x)+\sum_{x \in A} \operatorname{deg}_{G}(x)-\sum_{x \in T} \operatorname{deg}_{G}(x) \\
& -e_{G}(A-S, B-T) \\
= & -\sum_{x \in S} f(x)+\sum_{x \in T} f(x)+\sum_{x \in S} \operatorname{deg}_{G}(x)+e_{G}(A-S, B) \\
& -e_{G}(S, T)-e_{G}(A-S, T)-e_{G}(A-S, B-T) \\
= & -\sum_{x \in S} f(x)+\sum_{x \in T} f(x)+\sum_{x \in S} \operatorname{deg}_{G}(x)-e_{G}(S, T) \\
= & \delta^{*}(T, S) .
\end{aligned}
$$

Therefore (2.72) and (2.73) are equivalent.

An elementary direct proof of Theorem 2.4.1 will be given in the next chapter. We now give some results on regular factors and $f$-factors in bipartite graphs, which are proved using Theorem 2.4.1 or (2.73).

Theorem 2.4.2 Let $G$ be a bipartite multigraph and $k \geq 1$ be an integer. Suppose that the degree of every vertex of $G$ is divisible by $k$. Then for a function $f$ defined by

$$
f(x)=\frac{\operatorname{deg}_{G}(x)}{k} \quad \text { for all } \quad x \in V(G),
$$

$G$ has an $f$-factor. 
Proof. Let $S$ and $T$ be disjoint subsets of $V(G)$. It is clear that $\delta^{*}(\emptyset, \emptyset)=0$, and so we may assume that $S \cup T \neq \emptyset$. Then

$$
\begin{aligned}
\delta^{*}(S, T) & =\sum_{x \in S} f(x)+\sum_{x \in T}\left(\operatorname{deg}_{G}(x)-f(x)\right)-e_{G}(S, T) \\
& =\frac{1}{k} \sum_{x \in S} \operatorname{deg}_{G}(x)+\left(1-\frac{1}{k}\right) \sum_{x \in T} \operatorname{deg}_{G}(x)-e_{G}(S, T) \\
& \geq \frac{1}{k} e_{G}(S, T)+\left(1-\frac{1}{k}\right) e_{G}(T, S)-e_{G}(S, T) \\
& =0 .
\end{aligned}
$$

Hence $\delta^{*}(S, T) \geq 0$, and thus $G$ has an $f$-factor.

Theorem 2.4.3 (Katerinis [84]) Let $G$ be a bipartite simple graph of order at least four. If tough $(G) \geq 1$, then $G$ has a 2-regular factor.

Lemma 2.4.4 Let $G$ be a bipartite simple graph of order at least three and with bipartition $(A, B)$. Then tough $(G) \leq 1$, and tough $(G)=1$ implies $|A|=|B|$.

Proof. We may assume $|A| \geq|B|$. Then $|A|=\omega(G-B) \geq 2$ by $|G| \geq 3$, and so

$$
\operatorname{tough}(G) \leq \frac{|B|}{\omega(G-B)}=\frac{|B|}{|A|} \leq 1 .
$$

In particular, $\operatorname{tough}(G)=1$ implies $|A|=|B|$.

Proof of Theorem 2.4.3 By the above lemma, $|A|=|B|$. Hence $2|A|=$ $2|B|$. Suppose that $G$ has no 2-factor. Then by Theorem 2.4.1 there exist subsets $S \subseteq A$ and $T \subseteq B$ such that

$$
\delta^{*}(S, T)=2|S|+\sum_{x \in T} \operatorname{deg}_{G-S}(x)-2|T|<0 .
$$

Choose such a pair $(S, T)$ so that $T$ is minimal. Since $\delta^{*}(S, \emptyset)=2|S| \geq 0$ and $\delta^{*}(A, T)=2|A|-2|T| \geq 0$, we have $T \neq \emptyset$ and $S \neq A$.

Claim For every $v \in T$, it follows that $\operatorname{deg}_{G-S}(v) \leq 1$.

Let $T^{\prime}=T \backslash\{v\}$. Then

$$
\begin{aligned}
1 \leq & \delta^{*}\left(S, T^{\prime}\right)-\delta^{*}(S, T) \\
= & 2|S|+\sum_{x \in T^{\prime}} \operatorname{deg}_{G-S}(x)-2\left|T^{\prime}\right| \\
& -\left(2|S|+\sum_{x \in T} \operatorname{deg}_{G-S}(x)-2|T|\right) \\
= & -\operatorname{deg}_{G-S}(v)+2
\end{aligned}
$$


Hence $\operatorname{deg}_{G-S}(v) \leq 1$.

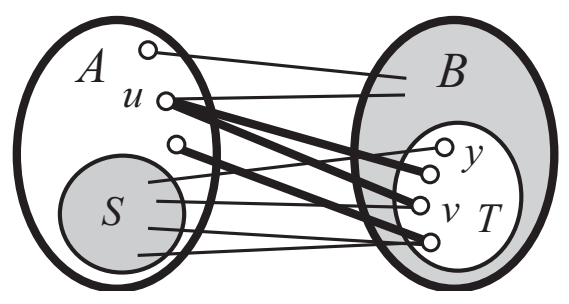

Figure 2.17: A bipartite graph $G$ with $\operatorname{tough}(G)=1$.

Let $R=\left\{y \in T \mid \operatorname{deg}_{G-S}(y)=0\right\}$ and $X=S \cup(B-T)$. By the Claim, if $u \in A-S$ and $v \in T$ are adjacent, then the component $D$ of $G-X$ containing $u$ satisfies $V(D) \cap(S-A)=\{u\}$ (see Figure 2.17). Thus

$$
\omega(G-X)=|A|-|S|+|R| .
$$

Assume first $\omega(G-X) \geq 2$. By $\operatorname{tough}(G)=1$, we have

$$
|A|-|S|+|R|=\omega(G-X) \leq|X|=|S|+|B|-|T|
$$

which implies $0 \leq 2|S|-|T|-|R|$. On the other hand, by the Claim we obtain

$$
\delta^{*}(S, T)=2|S|+|T-R|-2|T|=2|S|-|R|-|T|<0 .
$$

This contradicts the above inequality $0 \leq 2|S|-|T|-|R|$. Therefore we may assume $\omega(G-X)=1$. Then $|A-S|=1$ and $R=\emptyset$, and so by $|A| \geq 2$ and Claim, we have

$$
\delta^{*}(S, T)=2(|A|-1)+|T|-2|T| \geq 2|A|-2-|B| \geq 0,
$$

which is a contradiction. Consequently the proof is complete.

Katerinis showed that a similar result does not hold for a 3-factor. Namely, he showed that for any large integer $\lambda \geq 2$, there exist 1-tough $\lambda$-edge connected bipartite graphs that have no 3 -factor. In the same paper, he obtained another sufficient condition for a bipartite graph to have a 2 -factor, which is given in the next theorem.

Theorem 2.4.5 (Katerinis [84]) Let $G$ be a bipartite simple graph with bipartition $(A, B)$. If the following two conditions hold, then $G$ has a 2-regular factor. 
(i) $|A|=|B|$, and

(ii) for every subset $S \subseteq A$, it follows that

$$
\begin{array}{rlrl}
\left|N_{G}(S)\right| & \geq \frac{3}{2}|S| & & \text { if }|S|<\left\lfloor\frac{2}{3}|A|\right\rfloor, \\
N_{G}(S) & =B & & \text { and } \\
\text { otherwise. } & &
\end{array}
$$

Proof. Suppose that $G$ has no 2-factor. Since $2|A|=2|B|$ by (i), there exists a pair $(S, T)$ of subsets $S \subseteq A$ and $T \subseteq B$ such that

$$
\delta^{*}(T, S)=2|T|+\sum_{x \in S} \operatorname{deg}_{G-T}(x)-2|S|<0 . \quad(\text { see } \quad(2.73))
$$

Choose such a pair $(S, T)$ so that $S$ is minimal. Since $\delta^{*}(T, S) \geq 2(|T|-|S|) \geq$ 0 if $|T| \geq|S|$, we have $|T|<|S|$. By the same arguments used in the proof of Theorem 2.4.3, we have

$$
\begin{aligned}
& |T|<|S|, \quad T \neq A \quad \text { and } \quad S \neq \emptyset, \text { and } \\
& \operatorname{deg}_{G-T}(v) \leq 1 \quad \text { for every } v \in S .
\end{aligned}
$$

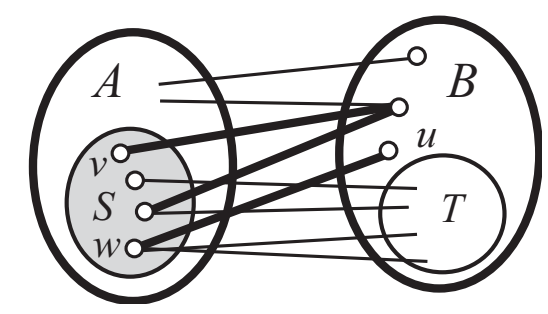

Figure 2.18: A bipartite graph $G$ of Theorem 2.4.5.

Let $R_{0}=\left\{x \in S \mid \operatorname{deg}_{G-T}(x)=0\right\}$ and $R_{1}=\left\{x \in S \mid \operatorname{deg}_{G-T}(x)=1\right\}$ Figure 2.18). Then $S=R_{0} \cup R_{1}$. Since

$$
\delta^{*}(T, S)=2|T|+\left|R_{1}\right|-2|S|<0 \quad \text { and } \quad\left|N_{G}(S)\right| \leq|T|+\left|R_{1}\right|,
$$

we have

$$
2\left|N_{G}(S)\right| \leq 2|T|+2\left|R_{1}\right|<2|S|+\left|R_{1}\right| \leq 3|S| .
$$

Hence

$$
|S|>\frac{2}{3}\left|N_{G}(S)\right|
$$


Therefore it follows from (i) and (ii) that

$$
N_{G}(S)=B \quad \text { and } \quad|S| \geq\lfloor(2 / 3)|A|\rfloor+1,
$$

which implies $e_{G}(S, y) \geq 1$ for all $y \in B$. If $e_{G}(S, y) \geq 2$ for all $y \in B-T$, then

$$
\begin{aligned}
\delta^{*}(S, T) & =2|T|+\sum_{x \in S} \operatorname{deg}_{G-T}(x)-2|S| \\
& \geq 2|T|+2|B-T|-2|S| \geq 2|B|-2|S| \geq 0,
\end{aligned}
$$

a contradiction. Hence there exists a vertex $u \in B-T$ with $e_{G}(S, u)=1$. Let $w \in S$ be a vertex adjacent to $u$. Then

$$
u \notin N_{G}(S-\{w\}), \text { but }|S-\{w\}| \geq\left\lfloor\frac{2}{3}|A|\right\rfloor .
$$

This contradicts (ii). Consequently the theorem is proved.

Note that Theorem 2.4.5 is best possible in the sense that the condition $|S| \geq(3 / 2)|S|$ in (ii) cannot be replaced by $|S| \geq\lfloor(3 / 2)|S|\rfloor$, i.e., there exist bipartite graphs $G$ that have no 2-factor and satisfy that for every subset $S \subseteq A,\left|N_{G}(S)\right| \geq\lfloor(3 / 2)|S|\rfloor$ if $|S|<\lfloor(2 / 3)|A|\rfloor$, and $N_{G}(S)=B$ otherwise.

The above Theorem 2.4.5 was generalized as follows.

Theorem 2.4.6 (Enomoto, Ota and Kano [46]) Let $a \geq 2$ and $b \geq 2$ be integers, and let $\lambda=a-1+(1 / b)$. Let $G$ be a bipartite simple graph with bipartition $(A, B)$. Suppose that $G$ satisfies the following two conditions:

(i) $a|A| \leq b|B|$ and $|B| \geq a$, and

(ii) for every $S \subseteq A$, it follows that

$$
\begin{aligned}
\left|N_{G}(S)\right| & \geq \lambda|S| & & \text { if }|S|<\left\lfloor\frac{|B|}{\lambda}\right\rfloor, \text { and } \\
N_{G}(S) & =B & & \text { otherwise. }
\end{aligned}
$$

Then $G$ has a spanning subgraph $F$ such that

$$
\begin{array}{ll}
\operatorname{deg}_{F}(x)=a & \text { for all } x \in A, \text { and } \\
\operatorname{deg}_{F}(y) \leq b & \text { for all } y \in B .
\end{array}
$$

If $a|A|=b|B|$, then equality holds in (2.77), and furthermore if additionally $a=b=2$ holds, then $\lambda=3 / 2$ and the theorem implies Theorem 2.4.6. Hence Theorem 2.4.6 is a generalization of Theorem 2.4.5. In order to prove the above theorem, we need the next theorem, which will be proved in the next chapter. 
Theorem 2.4.7 Let $a \geq 2$ and $b \geq 2$ be integers, and $G$ be a bipartite simple graph with bipartition $(A, B)$. Then $G$ has a spanning subgraph $F$ such that

$$
\begin{array}{ll}
\operatorname{deg}_{F}(x)=a & \text { for all } \quad x \in A, \text { and } \\
\operatorname{deg}_{F}(y) \leq b & \text { for all } y \in B .
\end{array}
$$

if and only if

$$
\delta^{*}(T, S)=b|T|+\sum_{x \in S} \operatorname{deg}_{G-T}(x)-a|S| \geq 0
$$

for all subsets $S \subseteq A$ and $T \subseteq B$ (see (2.73)).

Proof of Theorem 2.4.6 Suppose that $G$ has no spanning subgraph satisfying (2.76) and (2.77). Then by Theorem 2.4.7, there exists a pair $(S, T)$ of subsets $S \subseteq A$ and $T \subseteq B$ such that

$$
\begin{aligned}
\delta^{*}(T, S) & =b|T|+\sum_{x \in S} \operatorname{deg}_{G-T}(x)-a|S| \\
& =b|T|+e_{G}(S, B-T)-a|S|<0 .
\end{aligned}
$$

Choose such a pair $(S, T)$ so that $S \cup(B-T)$ is minimal. Then it is easy to see that $S \neq \emptyset$ and $T \neq B$.

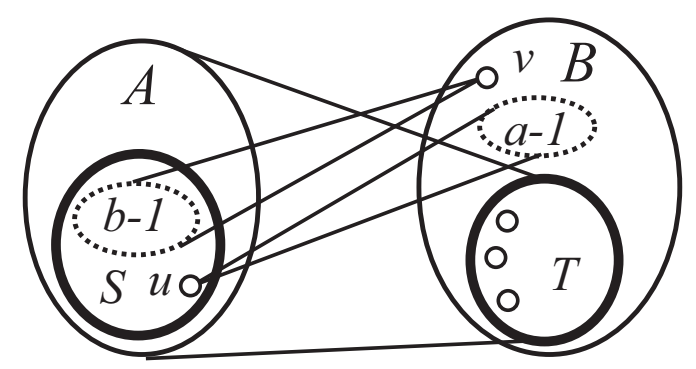

Figure 2.19: A bipartite graph $G$ of Theorem 2.4.6.

Claim 1. For every $u \in S$, it follows that $\operatorname{deg}_{G-T}(u) \leq a-1$, in particular, $e_{G}(S, B-T) \leq(a-1)|S|$ (Figure 2.19).

Let $S^{\prime}=S-\{u\}$. Then

$$
\begin{aligned}
1 & \leq \delta^{*}\left(T, S^{\prime}\right)-\delta^{*}(T, S) \\
& =b|T|+\sum_{x \in S^{\prime}} \operatorname{deg}_{G-T}(x)-a\left|S^{\prime}\right|-\left(b|T|+\sum_{x \in S} \operatorname{deg}_{G-T}(x)-a|S|\right) \\
& =-\operatorname{deg}_{G-T}(u)+a
\end{aligned}
$$


Hence $\operatorname{deg}_{G-T}(u) \leq a-1$. The second inequality follows immediately from this.

Claim 2. For every $v \in B-T$, it follows that $e_{G}(S, v) \leq b-1$ (Figure 2.19).

Let $T^{\prime}=T \cup\{v\}$. Then

$$
\begin{aligned}
1 \leq & \delta^{*}\left(T^{\prime}, S\right)-\delta^{*}(T, S) \\
= & b\left|T^{\prime}\right|+\sum_{x \in S} \operatorname{deg}_{G}(x)-e_{G}\left(T^{\prime}, S\right)-a|S| \\
& -\left(b|T|+\sum_{x \in S} \operatorname{deg}_{G}(x)-e_{G}(T, S)-a|S|\right) \\
= & b-e_{G}(v, S)
\end{aligned}
$$

Hence $e_{G}(v, S) \leq b-1$.

Claim 3. $\left|N_{G}(S)\right|<\lambda|S|$.

Since $G$ is a bipartite simple graph, we have $\left|N_{G}(S)\right| \leq|T|+e_{G}(S, B-T)$, and by (2.79) we obtain

$$
\begin{aligned}
\left|N_{G}(S)\right| & \leq|T|+e_{G}(S, B-T) \\
& <\frac{a}{b}|S|+\left(1-\frac{1}{b}\right) e_{G}(S, B-T) \\
& \leq \frac{a}{b}|S|+\left(1-\frac{1}{b}\right)(a-1)|S| \quad \text { (by Claim 1) } \\
& =\left(a-1+\frac{1}{b}\right)|S|=\lambda|S| .
\end{aligned}
$$

Thus Claim 3 holds.

By Claim 3 and the condition (2.75), we have $N_{G}(S)=B$. Note that we have shown that $B-T \neq \emptyset$. Let

$$
h=\min \left\{e_{G}(S, v) \mid v \in B-T\right\} .
$$

By Claim 2 and the fact that $N_{G}(S)=B$, we have

$$
1 \leq h \leq b-1 .
$$

By the definition of $h$ and $N_{G}(S)=B$, we have

$$
|B|=\left|N_{G}(S)\right| \leq|T|+\frac{1}{h} e_{G}(S, B-T)
$$




$$
\begin{aligned}
& <\frac{a}{b}|S|+\left(\frac{1}{h}-\frac{1}{b}\right) e_{G}(S, B-T) \quad(\text { by }(2.79)) \\
& \leq \frac{a}{b}|S|+\left(\frac{1}{h}-\frac{1}{b}\right)(a-1)|S| \quad(\text { by Claim } 1) \\
& =\left(\frac{1}{b}+\frac{a-1}{h}\right)|S| .
\end{aligned}
$$

Hence

$$
|B|<\left(\frac{1}{b}+\frac{a-1}{h}\right)|S| .
$$

Let $v_{0} \in B-T$ such that $e_{G}\left(S, v_{0}\right)=h$, and let $S_{0}=S-N_{G}\left(v_{0}\right)$. Since $N_{G}\left(S_{0}\right) \subseteq B-\left\{v_{0}\right\}$, we have by (2.75) that

$$
\left|S_{0}\right|=|S|-h \leq \frac{|B|}{\lambda}-1
$$

By (2.82) and (2.81), we obtain

$$
\begin{gathered}
\lambda(|S|-h+1) \leq|B|<\left(\frac{1}{b}+\frac{a-1}{h}\right)|S|, \\
\left(\lambda-\frac{1}{b}-\frac{a-1}{h}\right)|S|=(a-1)\left(1-\frac{1}{h}\right)|S|<\lambda(h-1), \\
\left.|S|<\frac{\lambda h}{a-1}=\left(a-1+\frac{1}{b}\right) \frac{h}{a-1} \quad\left(b y-1 \leq \frac{b-1}{b(a-1)}\right)<b . \quad 1 \leq b-1\right)
\end{gathered}
$$

Hence $|S|<b$. Choose $u_{0} \in S$ so that

$$
\rho=e_{G}\left(u_{0}, B-T\right)=\min \left\{e_{G}(u, B-T) \mid u \in S\right\} .
$$

Then by Claim 1, $\rho \leq a-1$ and thus

$$
\begin{aligned}
\left|N_{G}\left(u_{0}\right)\right| & \leq|T|+e_{G}\left(u_{0}, B-T\right) \\
& <\frac{a}{b}|S|-\frac{1}{b} e_{G}(S, B-T)+e_{G}\left(u_{0}, B-T\right) \quad(\text { by }(2.79)) \\
& \leq\left(\frac{a}{b}-\frac{\rho}{b}\right)|S|+\rho \\
& \leq\left(\frac{a}{b}-\frac{a-1}{b}\right)(b-1)+a-1 \quad(\text { by }|S|<b \text { and } \rho \leq a-1) \\
& =1-\frac{1}{b}+a-1 .
\end{aligned}
$$


Hence $\left|N_{G}\left(u_{0}\right)\right| \leq a-1<\lambda$ by $b \geq 2$, which implies $N_{G}\left(u_{0}\right)=B$ by (2.75). However this contradicts $a \leq|B|=\left|N_{G}\left(u_{0}\right)\right| \leq a-1$ (see Condition (i)). Consequently the theorem is proved.

Theorem 2.4.6 is best possible in the sense that there exist bipartite graphs that have no spanning subgraph satisfying (2.77) and (2.77) but which satisfy $\left|N_{G}(S)\right| \geq\lceil\lambda|S|\rceil-1$ if $|S| \leq|S|<\lfloor\lambda|B| / \lambda\rfloor$, and $N_{G}(S)=B$ otherwise. 


\section{Chapter 3}

\section{$(g, f)$-Factors and $[a, b]$-Factors}

\subsection{The $(g, f)$-Factor Theorem}

In this chapter we investigate $(g, f)$-factors and $[a, b]$-factors, which are natural generalizations of $f$-factors and regular factors, respectively. In this section we begin with a criterion for a graph to have a $(g, f)$-factor, which is given in the $(g, f)$-factor Theorem. In the following sections we obtain some sufficient conditions for graphs to have $(g, f)$-factors and $[a, b]$-factors.

For a general graph $G$ and two functions $g, f: V(G) \rightarrow \mathbb{Z}=\{\ldots,-1,0$, $1,2, \ldots\}$ with $g(x) \leq f(x)$ for all $x \in V(G)$, a $(g, f)$-factor is a spanning subgraph $F$ of $G$ that satisfies

$$
g(x) \leq \operatorname{deg}_{F}(x) \leq f(x) \quad \text { for all } x \in V(G) .
$$

Note that we allow $g$ and $f$ to satisfy $g(x)<0$ for some vertices $x$ and $\operatorname{deg}_{G}(y)<f(y)$ for some vertices $y$. For two integers $a$ and $b$ such that $0 \leq a \leq b$, a spanning subgraph $H$ of $G$ is called an $[a, b]$-factor if

$$
a \leq \operatorname{deg}_{H}(x) \leq b \quad \text { for all } x \in V(G) .
$$

Of course, an $[a, b]$-factor is nothing but a $(g, f)$-factor with $g(x)=a$ and $f(x)=b$ for all vertices $x$.

A necessary and sufficient condition for a graph to have a $(g, f)$-factor was obtained by Lovász [101] in 1970, and is contained the $(g, f)$-Factor Theorem. The original proof is difficult and long, however, in 1981, Tutte [143] gave an elegant short proof to this theorem. This is the one we will use here.

Theorem 3.1.1 (The $(g, f)$-Factor Theorem, Lovász [101]) Let $G$ be a general graph and $g, f: V(G) \rightarrow \mathbb{Z}$ such that $g(x) \leq f(x)$ for all $x \in V(G)$. 
Then $G$ has a $(g, f)$-factor if and only if for all disjoint subsets $S$ and $T$ of $V(G)$,

$$
\gamma(S, T)=\sum_{x \in S} f(x)+\sum_{x \in T}\left(\operatorname{deg}_{G}(x)-g(x)\right)-e_{G}(S, T)-q^{*}(S, T) \geq 0,
$$

where $q^{*}(S, T)$ denotes the number of components $C$ of $G-(S \cup T)$ such that $g(x)=f(x)$ for all $x \in V(C)$ and

$$
\sum_{x \in V(C)} f(x)+e_{G}(C, T) \equiv 1(\bmod 2) .
$$

The function $\gamma(S, T)$ is defined by (3.1).

For convenience, we call a component $C$ of $G-(S \cup T)$ satisfying $g(x)=$ $f(x)$ for all $x \in V(C)$ and $(3.2)$ a $(g, f)$-odd component of $G-(S \cup T)$. It is immediate that

$$
\sum_{x \in T} \operatorname{deg}_{G}(x)-e_{G}(S, T)=\sum_{x \in T} \operatorname{deg}_{G-S}(x),
$$

and thus (3.1) can be expressed as

$$
\gamma(S, T)=\sum_{x \in S} f(x)+\sum_{x \in T}\left(\operatorname{deg}_{G-S}(x)-g(x)\right)-q^{*}(S, T) \geq 0 .
$$

Notice that the inequality (3.1) includes the following condition. This will be shown later.

$$
g(x) \leq \operatorname{deg}_{G}(x) \quad \text { and } \quad 0 \leq f(x) \quad \text { for every } x \in V(G) .
$$

Moreover, as we mentioned before, the $(g, f)$-factor Theorem holds even if

$$
g(x)<0 \quad \text { and } \quad \operatorname{deg}_{G}(y)<f(y) \quad \text { for some } x, y \in V(G) .
$$

This small improvement will play a useful role later, though the following natural condition was assumed in the preceding papers [101] and [143]: $0 \leq$ $g(x) \leq f(x) \leq \operatorname{deg}_{G}(x)$ for all $x \in V(G)$.

Proof of necessity of the $(g, f)$-Factor Theorem. Suppose that $G$ has a $(g, f)$-factor $F$. We shall show that $\gamma(S, T) \geq 0$. It is clear that we may assume $G$ is connected.

Consider first $\gamma(\emptyset, \emptyset)$. By the definition of $(g, f)$-odd component, if $g(v)<$ $f(v)$ for some vertex $v$ of $G$, then $q^{*}(\emptyset, \emptyset)=0$. If $g(x)=f(x)$ for all $x \in V(G)$, then

$$
\sum_{x \in V(G)} f(x)=\sum_{x \in V(G)} \operatorname{deg}_{F}(x)=2\|F\|,
$$


and so $G$ itself is not a $(g, f)$-odd component of $G$, which implies $q^{*}(\emptyset, \emptyset)=0$. Thus we have $\gamma(\emptyset, \emptyset)=-q^{*}(\emptyset, \emptyset)=0$.

Let $S$ and $T$ be two disjoint subsets of $V(G)$ such that $S \cup T \neq \emptyset$. Let $G-F$ denote the spanning subgraph $G-E(F)$, and let $C_{1}, C_{2}, \ldots, C_{m}$ be the $(g, f)$-odd components of $G-(S \cup T)$, where $m=q^{*}(S, T)$. Since

$$
\operatorname{deg}_{G}(x)-g(x) \geq \operatorname{deg}_{G}(x)-\operatorname{deg}_{F}(x)=\operatorname{deg}_{G-F}(x),
$$

we have

$$
\begin{aligned}
\sum_{x \in T}\left(\operatorname{deg}_{G}(x)-g(x)\right) & \geq \sum_{x \in T} \operatorname{deg}_{G-F}(x) \\
& \geq e_{G-F}(T, S)+\sum_{i=1}^{m} e_{G-F}\left(T, C_{i}\right) .
\end{aligned}
$$

Furthermore, it follows that

$$
\sum_{x \in S} f(x) \geq \sum_{x \in S} \operatorname{deg}_{F}(x) \geq e_{F}(S, T)+\sum_{i=1}^{m} e_{F}\left(S, C_{i}\right) .
$$

By the previous inequalities, we have

$$
\begin{aligned}
\gamma(S, T) \geq & e_{F}(S, T)+\sum_{i=1}^{m} e_{F}\left(S, C_{i}\right) \\
& +e_{G-F}(T, S)+\sum_{i=1}^{m} e_{G-F}\left(T, C_{i}\right)-e_{G}(S, T)-m \\
= & \sum_{i=1}^{m}\left(e_{F}\left(S, C_{i}\right)+e_{G-F}\left(T, C_{i}\right)-1\right) .
\end{aligned}
$$

Therefore, in order to prove (3.1), it is sufficient to show that for every $C=C_{i}$,

$$
e_{F}(S, C)+e_{G-F}(T, C)-1 \geq 0 .
$$

If $e_{G-F}(T, C) \geq 1$, then (3.4) holds, and so we may assume that $e_{G-F}(T, C)$ $=0$. This implies

$$
e_{G}(T, C)=e_{F}(T, C) .
$$

Since $g(x)=f(x)=\operatorname{deg}_{F}(x)$ for every $x \in V(C)$, we obtain

$$
\begin{aligned}
& \sum_{x \in V(C)} f(x)+e_{G}(C, T)=\sum_{x \in V(C)} \operatorname{deg}_{F}(x)+e_{F}(C, T) \\
& =2\left\|\langle V(C)\rangle_{F}\right\|+e_{F}(C, S \cup T)+e_{F}(C, T) \\
& \equiv e_{F}(C, S)(\bmod 2) .
\end{aligned}
$$


By (3.2) and by the above equation, we have $e_{F}(C, S) \geq 1$, which implies (3.4). Consequently necessity is proved.

As we said before, the present proof of sufficiency is based on Tutte [143].

Proof of the sufficiency of the $(g, f)$-Factor Theorem. If a general graph $G$ satisfying the condition (3.1) is not connected, then each of its components satisfies (3.1). Hence it suffices to prove that a general connected graph $G$ satisfying $(3.1)$ has a $(g, f)$-factor, and so we assume that $G$ is connected.

For each vertex $v$ of $G$, it follows from (3.1) that

$$
\begin{aligned}
& \gamma(\emptyset,\{v\})=\operatorname{deg}_{G}(v)-g(v)-q^{*}(\emptyset,\{v\}) \geq 0, \quad \text { and } \\
& \gamma(\{v\}, \emptyset)=f(v)-q^{*}(\{v\}, \emptyset) \geq 0
\end{aligned}
$$

Thus

$$
g(v) \leq \operatorname{deg}_{G}(v) \quad \text { and } \quad 0 \leq f(v) \quad \text { for every } \quad v \in V(G) .
$$

If $g(x)=f(x)$ for all $x \in V(G)$, then a $(g, f)$-factor is an $f$-factor, and $\delta(S, T)=\gamma(S, T)$. So the $(g, f)$-Factor Theorem holds by the $f$-Factor Theorem 2.1.2. Therefore we may assume that $G$ has at least one vertex $u$ such that $g(u)<f(u)$.

We now construct a new connected graph $G^{*}$ from $G$ as follows (see Figure 3.1): Add a new vertex $w$ to $G$, and join $w$ to every vertex $x$ of $G$ by $f(x)-g(x)$ multiple edges, and add $M$ loops to $w$, where $M$ is a sufficiently large integer expressed as

$$
M=\sum_{x \in V(G)} f(x)+2 N
$$

Note that $N$ can be defined as $\sum_{x \in V(G)}|g(x)|+|G|$ (see Exercises). We next define the function $f^{*}: V\left(G^{*}\right) \rightarrow\{0,1,2,3, \ldots\}$ as

$$
f^{*}(x)= \begin{cases}f(x) & \text { if } x \in V(G) \\ M & \text { if } x=w .\end{cases}
$$

We first show that $G$ has a $(g, f)$-factor if and only if $G^{*}$ has an $f^{*}$-factor. Assume $G^{*}$ has an $f^{*}$-factor $F^{*}$. Then $F=F^{*}-\{w\}$ becomes a $(g, f)$-factor of $G$ since for every vertex $x \in V(G)$, we have

$$
\begin{aligned}
\operatorname{deg}_{F}(x) & =\operatorname{deg}_{F^{*}}(x)-e_{F^{*}}(x, w) \\
& \geq \operatorname{deg}_{F^{*}}(x)-e_{G^{*}}(x, w) \\
& =f(x)-(f(x)-g(x))=g(x) ; \quad \text { and } \\
\operatorname{deg}_{F}(x) & \leq \operatorname{deg}_{F^{*}}(x)=f(x) .
\end{aligned}
$$




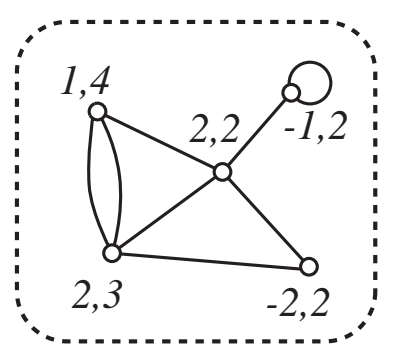

G

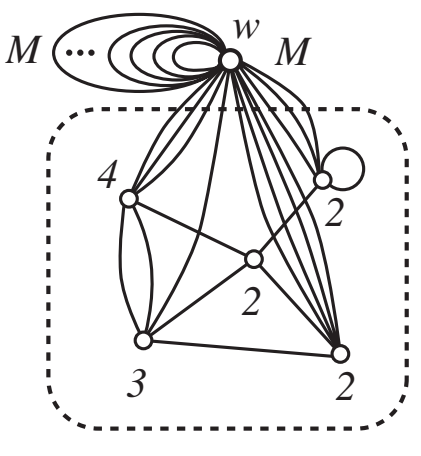

$G^{*}$

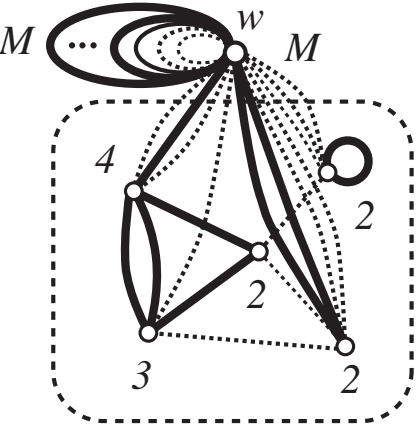

$G^{*}$

Figure 3.1: Connected general graphs $G$ and $G^{*}$, and an $f^{*}$-factor of $G^{*}$; numbers denote $g(x)$ and $f(x)$.

Conversely, assume that $G$ has a $(g, f)$-factor $F$. For every vertex $x$ of $G$, add $f(x)-\operatorname{deg}_{F}(x)$ multiple edges connecting $x$ and $w$ to $F$, and add $N+\left(\sum_{x \in V(G)} \operatorname{deg}_{F}(x)\right) / 2$ loops incident with $w$. Then the degree of each vertex $x$ of $G$ is $f(x)$ and that of $w$ is

$$
\left.\sum_{x \in V(G)}\left(f(x)-\operatorname{deg}_{F}(x)\right)+2 N+\sum_{x \in V(G)} \operatorname{deg}_{F}(x)\right)=M .
$$

Hence the resulting subgraph is an $f^{*}$-factor of $G^{*}$.

We now prove that $G^{*}$ has an $f^{*}$-factor by making use of the $f$-Factor Theorem 2.1.2. Since $G^{*}$ is connected and

$$
\sum_{x \in V\left(G^{*}\right)} f^{*}(x)=\sum_{x \in V(G)} f(x)+M \equiv 0(\bmod 2),
$$

we have $\delta_{G^{*}}(\emptyset, \emptyset)=-q_{G^{*}}(\emptyset, \emptyset)=0$.

Let $S$ and $T$ be two disjoint subsets of $V\left(G^{*}\right)$ such that $S \cup T \neq \emptyset$. If $w \in S$ then since $M$ is sufficiently large, we have

$$
\delta_{G^{*}}(S, T)=\sum_{x \in S \backslash\{w\}} f(x)+M+\sum_{x \in T}\left(\operatorname{deg}_{G^{*}}(x)-f(x)\right) \geq 0 .
$$

If $w \in T$, then $\operatorname{deg}_{G^{*}}(w)-f^{*}(w) \geq 2 M-M=M$, and thus

$$
\begin{aligned}
\delta_{G^{*}}(S, T) \geq \sum_{x \in S} f(x)+\sum_{x \in T \backslash\{w\}}\left(\operatorname{deg}_{G^{*}}(x)-f(x)\right)+M \\
\\
-e_{G^{*}}(S, T)-q_{G^{*}}(S, T) \geq 0 .
\end{aligned}
$$


Therefore we may assume that $w \notin S \cup T$. Then

$$
\begin{aligned}
\delta_{G^{*}}(S, T)= & \sum_{x \in S} f^{*}(x)+\sum_{x \in T}\left(\operatorname{deg}_{G^{*}}(x)-f^{*}(x)\right) \\
& -e_{G^{*}}(S, T)-q_{G^{*}}(S, T) \\
= & \sum_{x \in S} f(x)+\sum_{x \in T}\left(\operatorname{deg}_{G}(x)-g(x)\right) \\
& -e_{G}(S, T)-q_{G^{*}}(S, T) .
\end{aligned}
$$

Let $C$ be an $f^{*}$-odd component of $G^{*}-(S \cup T)$ not containing $w$. Then since $e_{G^{*}}(C, w)=0$, we have $g(x)=f(x)$ for all $x \in V(C)$. Moreover,

$$
\sum_{x \in V(C)} f^{*}(x)+e_{G^{*}}(C, T)=\sum_{x \in V(C)} f(x)+e_{G}(C, T) \equiv 1(\bmod 2) .
$$

Hence $C$ is a $(g, f)$-odd component of $G-(S \cup T)$. Therefore $q_{G^{*}}(S, T) \leq$ $q_{G}^{*}(S, T)+1$, where +1 corresponds to a component of $G-(S \cup T)$ containing $w$, which might be an $f^{*}$-odd component. Consequently

$$
\begin{aligned}
\delta_{G^{*}}(S, T) & \geq \sum_{x \in S} f(x)+\sum_{x \in T}\left(\operatorname{deg}_{G}(x)-g(x)\right)-e_{G}(S, T)-q_{G}^{*}(S, T)-1 \\
& =\gamma(S, T)-1 \geq-1
\end{aligned}
$$

which implies $\delta_{G^{*}}(S, T) \geq 0$ by $\delta_{G^{*}}(S, T) \equiv 0(\bmod 2)(2.4)$. Consequently $G^{*}$ has an $f^{*}$-factor by the $f$-Factor Theorem, which implies that $G$ has the desired $(g, f)$-factor, and the proof is complete.

We conclude this section by giving a direct simple proof for the $(g, f)$ Factor Theorem with $g<f$, which does not use the $f$-Factor Theorem. This proof was found independently by Kano and Saito [78],

Theorem 3.1.2 (The $(g, f)$-Factor Theorem with $g<f$ ) Let $G$ be a general graph and $g, f: V(G) \rightarrow \mathbb{Z}$ such that $g(x)<f(x)$ for all $x \in V(G)$. Then $G$ has a $(g, f)$-factor if and only if for all disjoint subsets $S$ and $T$ of $V(G)$, it follows that

$$
\gamma^{*}(S, T)=\sum_{x \in S} f(x)+\sum_{x \in T}\left(\operatorname{deg}_{G}(x)-g(x)\right)-e_{G}(S, T) \geq 0 .
$$

Proof of sufficiency. Since necessity can be proved in the same way as the $(g, f)$-Factor Theorem, we prove only sufficiency. Suppose that a general graph $G$ satisfies $(3.7)$ but has no $(g, f)$-factor. Since for every $x \in V(G)$, 
$\gamma^{*}(\{x\}, \emptyset) \geq 0$ and $\gamma^{*}(\emptyset,\{x\}) \geq 0$, we have $g(x) \leq \operatorname{deg}_{G}(x)$ and $0 \leq f(x)$. We define a $(0, f)$-factor of $G$ as a spanning subgraph $K$ satisfying

$$
0 \leq \operatorname{deg}_{K}(x) \leq f(x) \quad \text { for all } x \in V(G) .
$$

Then a spanning subgraph with no edge is a $(0, f)$-factor of $G$. Choose a $(0, f)$-factor $H$ among all $(0, f)$-factors of $G$ so that

$$
\begin{aligned}
& \rho(H)=\sum_{x \in U}\left(g(x)-\operatorname{deg}_{H}(x)\right) \quad \text { is minimum, } \\
& \text { where } U=\left\{x \in V(G) \mid \operatorname{deg}_{H}(x)<g(x)\right\} .
\end{aligned}
$$

Note that if $U=\emptyset$, then $H$ is a $(g, f)$-factor and so we may assume $U \neq \emptyset$. An $H$-alternating trail is a trail whose edges alternately in and not in $H$. Take one vertex $u$ from $U$, and define

$$
\begin{aligned}
O V= & \{x \in V(G) \mid \text { There exists an } H \text {-alternating trail of odd } \\
& \text { length connecting } u \text { to } x\}, \\
E V= & \{x \in V(G) \mid \text { There exists an } H \text {-alternating trail of even } \\
& \text { length connecting } u \text { to } x\} \cup\{u\} .
\end{aligned}
$$

Then the following four statements hold (see Figure 3.2):

(1) If $\left(u x_{1} y_{1} x_{2} y_{2} \cdots x_{r}\left(\right.\right.$ or $\left.\left.y_{r}\right)\right), x_{i}, y_{i} \in V(G)$, is an $H$-alternating trail, then $\operatorname{deg}_{H}\left(x_{i}\right)=f\left(x_{i}\right)$ and $\operatorname{deg}_{H}\left(y_{i}\right) \leq g\left(y_{i}\right) \quad$ for every $i$.

(2) $O V \cap E V=\emptyset$.

(3) $\operatorname{deg}_{G-O V}(y)=\operatorname{deg}_{H-O V}(y)$ for all $y \in E V$.

(4) $e_{H}(O V, E V)=\sum_{x \in O V} \operatorname{deg}_{H}(x)$.

(1) If $\operatorname{deg}_{H}\left(x_{1}\right)<f\left(x_{1}\right)$, then $H+u x_{1}$ is a $(0, f)$-factor and $\rho(H+$ $\left.u x_{1}\right)<\rho(H)$ (see Figure $3.2(1)$ ). This contradicts the choice of $H$, and thus $\operatorname{deg}_{H}\left(x_{1}\right)=f\left(x_{1}\right)$. If $\operatorname{deg}_{H}\left(y_{1}\right)>g\left(y_{1}\right)$, then we can get a contradiction by considering $H+u x_{1}-x_{1} y_{1}$. Thus $\operatorname{deg}_{H}\left(y_{1}\right) \leq g\left(y_{1}\right)$. If $\operatorname{deg}_{H}\left(x_{2}\right)<f\left(x_{2}\right)$, then we can derive a contradiction by considering $H+u x_{1}-x_{1} y_{1}+y_{1} x_{2}$. Hence $\operatorname{deg}_{H}\left(x_{2}\right)=f\left(x_{2}\right)$. we can prove (1) by repeating this argument.

(2) If $v \in E V \cap O V$, then we obtain by (1) $\operatorname{deg}_{H}(v)=f(v)$ as $v \in O V$ and $\operatorname{deg}_{H}(v) \leq g(v)$ as $v \in E V$. However this is impossible since $g(v)<f(v)$. Hence (2) holds.

(3) We show that if $y \in E V$ and $y x \in E(G) \backslash E(H)$, then $x \in O V$, which implies the desired property

$$
\operatorname{deg}_{G-O V}(y)=\operatorname{deg}_{H-O V}(y) .
$$


(1)

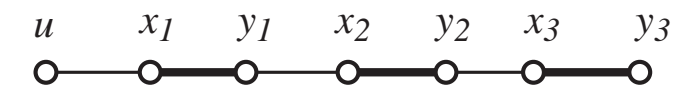

$H=\{-\} ; \quad E V=\left\{x_{i}\right\}, O V=\left\{y_{i}\right\}$

(3)
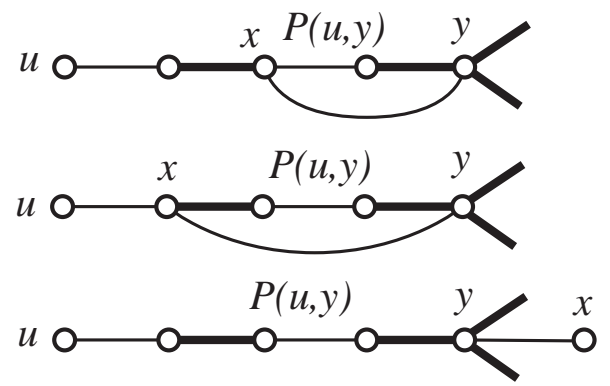

Figure 3.2: $H$-alternating trails to illustrate the proofs of (1) and (3), where $\operatorname{deg}_{H}(u)<g(u)$.

Let $y \in E V, y x \in E(G) \backslash E(H)$ and $P(u, y)$ denote a shortest $H$ alternating trail connecting $u$ and $y$ (see Figure $3.2(3)$ ). Then $P(u, y)$ terminates at $y$ and does not pass through $y$ on the way. If $x \in P(u, y) \cap E V$, then we can find an $H$-alternating trail $P(u, x)+x y$ of odd length connecting $u$ and $y$, which contradicts $y \in E V$ and (2). If $x \in P(u, y) \cap O V$, then of course $x \in O V$. Thus we may assume that $x \notin P(u, y)$. Again $P(u, y)+y x$ is an $H$-alternating trail of odd length connecting $u$ and $x$, and so $x \in O V$. Therefore (3) is proved.

(4) We can similarly show that if $x \in O V$ and $x y \in E(H)$, then $y \in E V$, which implies (4).

By (1), (2), (3), (4) and by $g(u)>\operatorname{deg}_{H}(u)$, we obtain

$$
\begin{aligned}
\gamma^{*}(O V, E V) & =\sum_{x \in O V} f(x)+\sum_{x \in E V}\left(\operatorname{deg}_{G-O V}(x)-g(x)\right) \\
& <\sum_{x \in O V} \operatorname{deg}_{H}(x)+\sum_{x \in E V}\left(\operatorname{deg}_{H-O V}(x)-\operatorname{deg}_{H}(x)\right) \\
& =e_{H}(O V, E V)-e_{H}(E V, O V)=0 .
\end{aligned}
$$

This contradicts (3.7). Consequently sufficiency is proved.

When we consider a $(g, f)$-factor in a bipartite graph, the criterion becomes simpler. This result was obtained by Folkman and Fulkerson [49]. 
Theorem 3.1.3 (The $(g, f)$-Factor Theorem for Bipartite Graphs) Let $G$ be a bipartite multigraph with bipartition $(A, B)$ and $g, f: V(G) \rightarrow \mathbb{Z}$ such that $g(x) \leq f(x)$ for all $x \in V(G)$. Then $G$ has a $(g, f)$-factor if and only if

$$
\begin{aligned}
& \gamma^{*}(X, Y)=\sum_{x \in X} f(x)+\sum_{x \in Y}\left(\operatorname{deg}_{G}(x)-g(x)\right)-e_{G}(X, Y) \geq 0, \\
& \text { and } \\
& \gamma^{*}(Y, X)=\sum_{x \in Y} f(x)+\sum_{x \in X}\left(\operatorname{deg}_{G}(x)-g(x)\right)-e_{G}(Y, X) \geq 0
\end{aligned}
$$

for all subsets $X \subseteq A$ and $Y \subset B$.

Proof of sufficiency. Since necessity can be proved in the same way as the $(g, f)$-Factor Theorem, we here prove only sufficiency. Suppose that a bipartite multigraph $G$ satisfies (3.8) and (3.9). We first show that for any disjoint subsets $S$ and $T$ of $V(G)$, we have

$$
\gamma^{*}(S, T)=\sum_{x \in S} f(x)+\sum_{x \in T}\left(\operatorname{deg}_{G}(x)-g(x)\right)-e_{G}(S, T) \geq 0 .
$$

Let $S_{A}=S \cap A, S_{B}=S \cap B, T_{A}=T \cap A$ and $T_{B}=T \cap B$. Then by the two inequalities (3.8) and (3.9), we have

$$
\begin{aligned}
\gamma^{*}(S, T) & =\sum_{x \in S} f(x)+\sum_{x \in T}\left(\operatorname{deg}_{G}(x)-g(x)\right)-e_{G}(S, T) \\
& =\sum_{x \in S_{A}} f(x)+\sum_{x \in T_{B}}\left(\operatorname{deg}_{G}(x)-g(x)\right)-e_{G}\left(S_{A}, T_{B}\right) \\
& +\sum_{x \in S_{B}} f(x)+\sum_{x \in T_{A}}\left(\operatorname{deg}_{G}(x)-g(x)\right)-e_{G}\left(S_{B}, T_{A}\right) \geq 0 .
\end{aligned}
$$

Hence $\gamma^{*}(S, T) \geq 0$.

We now apply the same argument as in the proof of Theorem 3.1.2 since the statements (2), (3) and (4) in the proof hold even if $g(v)=f(v)$ for some vertices $v$ of $G$. This is because $O A$ and $E V$ are subsets of $A$ and $B$, respectively, or vice versa. Therefore we can prove the theorem in exactly the same way as Theorem 3.1.2.

We next give a criterion for a graph to have a special $(g, f)$-factor, which satisfies $0 \leq g(x) \leq 1$ for all vertices $x$. This criterion, obtained by Las Vergnas [92], is a natural extension of the 1-factor Theorem.

Theorem 3.1.4 (The $(g, f)$-Factor Theorem with $g \leq 1$, Las Vergnas) Let $G$ be a simple graph and $g, f: V(G) \rightarrow \mathbb{Z}^{+}$such that $0 \leq g(x) \leq 1$ and 
$g(x) \leq f(x)$ for all $x \in V(G)$. Then $G$ has a $(g, f)$-factor if and only if for every subset $S \subset V(G)$, we have

$$
\operatorname{odd}(g ; G-S) \leq \sum_{x \in S} f(x)
$$

where odd $(g ; G-S)$ denotes the number of components $C$ of $G-S$ such that either (i) $C=\{x\}$ and $g(x)=1$ or (ii) $|C|$ is odd, $|C| \geq 3$ and $g(x)=f(x)=1$ for all $x \in V(C)$.

Las Vergnas gave two proofs for Theorem 3.1.4. In one proof, he considered a graph $G$ that satisfies (3.11) but has no $(g, f)$-factor and for any new edge $e$ not in $G, G+e$ has a $(g, f)$-factor. That is, if $G$ satisfies (3.11) then for any new edge $e^{\prime}$ not in $G, G+e^{\prime}$ also satisfies the same condition. So we can take as a counterexample $G$ having a maximal number of edges and having some nice properties. This proof technique is used for other theorems. In the second proof, he used the $(g, f)$-Factor Theorem. We give another proof, which uses a standard technique found in many other theorems including the 1-Factor Theorem.

Proof of Theorem 3.1.4. Let $C$ be a component of $G-S$ satisfying the condition (i) or (ii) in the theorem. It is convenient to refer to $C$ as $g$ odd component, and to any other components of $G-S$ not satisfying the conditions (i) and (ii) $g$-even components.

We first prove necessity. Assume that $G$ has a $(g, f)$-factor $F$. Then it is obvious that $\operatorname{odd}(g ; G)=0$, and so (3.11) holds for $S=\emptyset$. Let $S$ be an non-empty vertex subset of $G$, and let $C$ be a $g$-odd component of $G-S$. Then we can easily show that

$$
e_{F}(S, C) \geq 1
$$

that is, there exists at least one edge of $F$ joining $C$ to $S$. Hence

$$
\operatorname{odd}(g ; G-S) \leq e_{F}(S, V(G)-S) \leq \sum_{x \in S} \operatorname{deg}_{F}(x) \leq \sum_{x \in S} f(x) .
$$

We next prove sufficiency by induction on the order $|G|$ of $G$. We may assume that $G$ is connected since otherwise we can apply the inductive hypothesis to each component, and get a $(g, f)$-factor of $G$. If $G$ has a vertex $v$ with $g(v)=f(v)=0$, then $G-v$ satisfies the condition (3.9) since for every subset $S \subset V(G)-\{v\}$, we have

$$
\operatorname{odd}(g ;(G-v)-S)=\operatorname{odd}(g ; G-(S \cup\{v\})) \leq \sum_{x \in S \cup\{v\}} f(x)=\sum_{x \in S} f(x) .
$$


Hence by the inductive hypothesis, $G-v$ has a $(g, f)$-factor, which is the desired $(g, f)$-factor of $G$ as $g(v)=f(v)=0$. Therefore we may assume that $G$ has no vertex $v$ with $g(v)=f(v)=0$, in particular, $f(x) \geq 1$ for very vertex $x$.

Claim 1. There exists $\emptyset \neq X \subset V(G)$ for which odd $(g ; G-X) \geq \sum_{x \in X} f(x)$ -1 .

Assume that $\operatorname{odd}(g ; G-S) \leq \sum_{x \in S} f(x)-2$ for all $\emptyset \neq S \subset V(G)$. Then for any chosen edge $e$ of $G$ and for any subset $S \subset V(G)$, we have

$$
\operatorname{odd}(g ; G-e-S) \leq \operatorname{odd}(g ; G-S)+2 \leq \sum_{x \in S} f(x) .
$$

Thus by the inductive hypothesis, $G-e$ has a $(g, f)$-factor, which is the desired $(g, f)$-factor of $G$. Hence we may assume that Claim 1 holds.

Let us define the integer $\tau$ as

$$
\tau=\max _{\emptyset \neq X \subseteq V(G)}\left\{o d d(g ; G-X)-\sum_{x \in X} f(x)\right\} .
$$

By the condition (3.11) and Claim 1, we have $\tau \in\{-1,0\}$. Choose a maximal vertex subset $S$ of $G$ subject to

$$
o d d(g ; G-S)-\sum_{x \in S} f(x)=\tau .
$$

Then $S \neq \emptyset$ and

$$
\operatorname{odd}(g ; G-X)-\sum_{x \in X} f(x)<\tau \quad \text { for all } \quad S \subset X \subseteq V(G) .
$$

Claim 2. For every g-odd component $C$ of $G-S$ and any vertex $v$ of $C$, $C-v$ has a 1-factor, which is a $(g, f)$-factor of $C-v$.

We may assume $|C| \geq 3$. Let $T \subset V(C) \backslash\{v\}$. It is clear that every odd component of $(C-v)-T$ is a $g$-odd component since $g(x)=f(x)=1$ for all $x \in V(C)$. Then since $S \subset S \cup\{v\} \cup T$, we have by (3.13)

$$
\begin{aligned}
& o d d(g ; G-(S \cup\{v\} \cup T))=\operatorname{odd}(g ; G-S)-1+\operatorname{odd}(C-v-T) \\
& \leq \sum_{x \in S \cup\{v\} \cup T} f(x)+\tau-1=\sum_{x \in S} f(x)+\tau+|T| .
\end{aligned}
$$

Hence $\operatorname{odd}(C-v-T) \leq|T|+1$, which implies odd $(C-v-T) \leq|T|$ as $\operatorname{odd}(C-v-T) \equiv|T|(\bmod 2)$. Therefore $C-v$ has a 1 -factor by the 1-factor Theorem. 
Claim 3. Every g-even component $D$ of $G-S$ has a $(g, f)$-factor. Moreover, if $g(v)<f(v)$ for some vertex $v$ of $D$, then for every edge uw of $G$ joining $u \in S$ to $w \in V(D), D+u w$ also has a $(g, f)$-factor, where we define $g(u)=f(u)=1$.

Let $\emptyset \neq T \subset V(D)$. Then by (3.13), we have

$$
\begin{aligned}
\operatorname{odd}(g ; G-S)+\operatorname{odd}(g ; D-T) & =\operatorname{odd}(g ; G-(S \cup T)) \\
& \leq \sum_{x \in S \cup T} f(x)+\tau-1
\end{aligned}
$$

By (3.12) we have

$$
\operatorname{odd}(g ; D-T) \leq \sum_{x \in T} f(x)-1,
$$

and so $D$ has a $(g, f)$-factor by induction. Moreover, by the above inequality, we have

$$
\operatorname{odd}(g ;(D+u w)-T) \leq \operatorname{odd}(g ; D-T)+1 \leq \sum_{x \in T} f(x) .
$$

It is clear that

$$
\operatorname{odd}(g ;(D+u w)-(T \cup\{u\}))=\operatorname{odd}(g ; D-T) \leq \sum_{x \in T \cup\{u\}} f(x)-2 .
$$

Furthermore the existence of a vertex $v$ with $g(v)<f(v)$ guarantees odd $(g ; D+$ $u w)=0$. Hence $D+u w$ has a $(g, f)$-factor by induction when $D+u w \neq G$.

Note that if $G=D+u w$, then $S=\{u\}, f(u)=1, \tau=-1$ and $\operatorname{odd}(g ; G-$ $S)=0$. In such a case $D$ has a $\left(g, f^{\prime}\right)$-factor with $f^{\prime}(x)=f(x), x \in$ $V(D) \backslash\{w\}$, and $f^{\prime}(w)=f(w)-1$ by (3.14). Hence by adding $u w$ to this factor, we can get the desired $(g, f)$-factor of $G=D+u w$.

Let $\left\{C_{1}, C_{2}, \ldots, C_{m}\right\}$ be the set of $g$-odd components of $G-S$, where $m=\operatorname{odd}(g ; G-S)$. We construct a bipartite graph $B$ with bipartition $\left(S,\left\{C_{1}, C_{2}, \ldots, C_{m}\right\}\right)$ as follows: $x \in S$ and $C_{i}$ are joined by an edge of $B$ if and only if $x$ and $C_{i}$ are joined by at least one edge of $G$ (see Figure 3.3). Then $B$ has the following properties.

Claim 4. If $\tau=0$, then $B$ has a factor $F$ such that (i) $\operatorname{deg}_{F}(x)=f(x)$ for all $x \in S$ and (ii) $\operatorname{deg}_{F}\left(C_{i}\right)=1$ for all $1 \leq i \leq m$. If $\tau=-1$, then for any given vertex $u \in S, B$ has a factor $F$ such that (iii) $\operatorname{deg}_{F}(x)=f(x)$ for all $x \in S-u, \operatorname{deg}_{F}(u)=f(u)-1$, and (iv) $\operatorname{deg}_{F}\left(C_{i}\right)=1$ for all $1 \leq i \leq m$. 

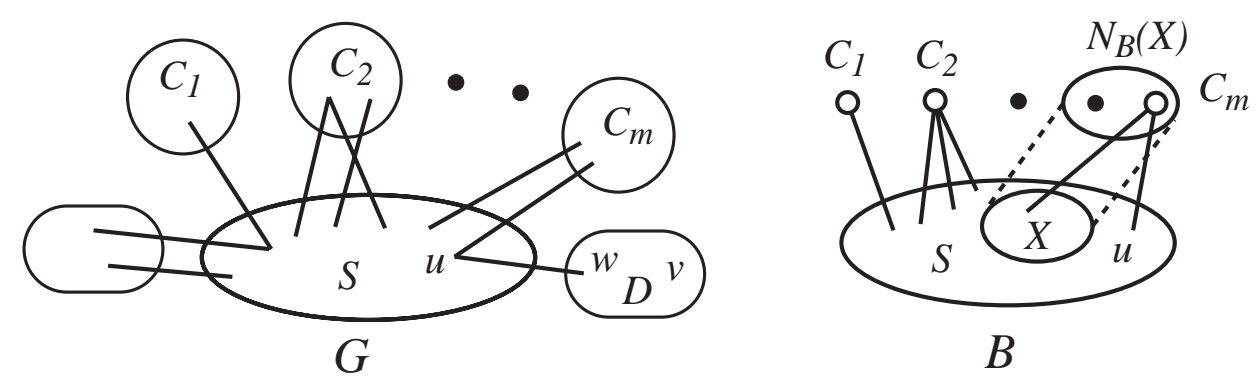

Figure 3.3: The graph $G$ with $S$, and the bipartite graph $B$.

We shall prove only the case $\tau=-1$ since the proof of the case $\tau=0$ is similar. Let $\emptyset \neq X \subset S$. Assume that $\left|N_{B}(X)\right|<\sum_{x \in X} f(x)$. Then

$$
\begin{aligned}
\operatorname{odd}(g ; G-(S \backslash X)) & \geq \operatorname{odd}(g ; G-S)-\left|N_{B}(X)\right| \\
& >\sum_{x \in S} f(x)+\tau-\sum_{x \in X} f(x)=\sum_{x \in S \backslash X} f(x)+\tau .
\end{aligned}
$$

This contradicts the definition of $\tau$. Hence $\left|N_{B}(X)\right| \geq \sum_{x \in X} f(x)$. Moreover,

$$
\left|N_{B}(S)\right|=m=\operatorname{odd}(g ; G-S)=\sum_{x \in S} f(x)-1 .
$$

Consequently by Theorem 1.1.10, $B$ has a factor $F$ such that (i) $\operatorname{deg}_{F}(x)=$ $f(x)$ for every $x \in S-u$, $\operatorname{deg}_{F}(u)=f(u)-1$, and (ii) $\operatorname{deg}_{F}\left(C_{i}\right)=1$ for every $1 \leq i \leq m$.

Claim 5. If $G-S$ has a g-even component $D$ such that $g(v)<f(v)$ for some vertex $v$ of $D$, then $G$ has a $(g, f)$-factor.

We may assume that $\tau=-1$ since if $\tau=0$, then we can similarly prove the claim. Suppose that $G-S$ has a $g$-even component $D$ that contains a vertex $v$ with $g(v)<f(v)$. Let $u w$ be an edge of $G$ joining $u \in S$ to $w \in D$. Let $\left\{C_{1}, \ldots, C_{m}\right\}$ be the set of $g$-odd component of $G-S$. Then by Claim 4 , the bipartite graph $B$ with bipartition $\left(S,\left\{C_{1}, C_{2}, \ldots, C_{m}\right\}\right)$ has a factor $F$ such that $\operatorname{deg}_{F}(x)=f(x)$ for all $x \in S-u, \operatorname{deg}_{F}(u)=f(u)-1$ and $\operatorname{deg}_{F}\left(C_{i}\right)=1$ for all $1 \leq i \leq m$. For every edge $x C_{i}$ of $F$, we can choose an edge of $G$ joining $x$ to a vertex $v_{i} \in V\left(C_{i}\right)$, and obtain a subgraph $H$ of $G$ by collecting these edges. Since $D+u w$, the other $g$-even components and $C_{i}-v_{i}$ have $(g, f)$-factors by Claims 3 and 4 . By combining these factors and $H$, we can obtain the desired $(g, f)$-factor of $G$ (see Figure 3.4). 


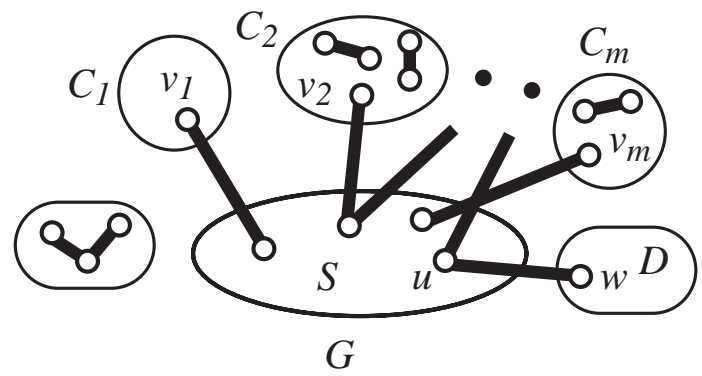

Figure 3.4: A $(g, f)$-factor, denoted by bold edges, of $G$ with $\tau=-1$.

By Claim 5, we may assume that no $g$-even component $D$ of $G-S$ satisfies the condition of Claim 5 . Thus every $g$-even component $D$ satisfies $g(x)=f(x)=1$ for all $x \in V(D)$, and has a 1-factor by Claim 3.

If $\tau=0$, then we can get the desired $(g, f)$-factor of $G$ in the same way as in the proof of Claim 5, i.e., by considering the factor $F$ given in Claim 4 with $\tau=0$ and by the fact of Claim 2 .

So we may assume that $\tau=-1$. If $g(u)<f(u)$ for some vertex $u \in S$, then by considering the $(g, f)$-factor $F$ in Claim 4 with $\tau=-1$ and by Claim 2, we can get the desired $(g, f)$-factor of $G$. So we may assume $g(x)=$ $f(x)$ for all $x \in S$. In this case, if $G-S$ has an isolated vertex $v$, then $v$ and $S$ must be joined by at least two edges as $\tau=-1$. If an isolated vertex $v_{1}$ of $G-S$ satisfies $f\left(v_{1}\right) \geq 2$, then we can find the desired $(g, f)$-factor of $G$. Its two edges join $v_{1}$ to $S$. If every isolated vertex $v$ of $G-S$ satisfies $f(v)=1$, then $g(x)=f(x)=1$ for all $x \in V(G)$, and thus

$$
|G| \equiv|S|+m \equiv \tau \equiv 1(\bmod 2)
$$

Hence $G$ itself is a $g$-odd component, which contradicts (3.9) with $S-\emptyset$. Consequently the proof is complete.

We now consider factors combining $f$-factors and $(g, f)$-factors. Let $g, f$ : $V(G) \rightarrow \mathbb{Z}^{+}$such that $g(x) \leq f(x)$ for all $x \in V(G)$. Then we say that $G$ has all $(g, f)$-factors if for every function $h: V(G) \rightarrow \mathbb{Z}^{+}$such that $g(x) \leq h(x) \leq f(x)$ for all $x \in V(G)$ and $\sum_{x \in V(G)} h(x) \equiv 0(\bmod 2), G$ has an $h$-factor. A necessary and sufficient condition for a graph to have all $(g, f)$-factors is given in the next theorem.

Theorem 3.1.5 (Niessen [115]) Let $G$ be a general graph and $g, f: V(G) \rightarrow$ $\mathbb{Z}^{+}$with $g(x) \leq f(x)$ for all $x \in V(G)$ and $g(w)<f(w)$ for at least one vertex $w$, which guarantees the existence of a function $h$ defined above. Then $G$ has 
all $(g, f)$-factors if and only if for all disjoint subsets $S$ and $T$ of $V(G)$,

$$
\sum_{x \in S} g(x)+\sum_{x \in T}\left(\operatorname{deg}_{G}(x)-f(x)\right)-e_{G}(S, T)-\hat{q}(S, T) \geq-1,
$$

where $\hat{q}(S, T)$ denotes the number of components $C$ of $G-(S \cup T)$ such that either (i) $g(u)<f(u)$ for some vertex $u \in V(C)$ or (ii) $g(x)=f(x)$ for all $x \in V(C)$ and

$$
\sum_{x \in V(C)} f(x)+e_{G}(C, T) \equiv 1(\bmod 2) .
$$

Proof. We first prove sufficiency. Let $h: V(G) \rightarrow \mathbb{Z}^{+}$such that $g(x) \leq$ $h(x) \leq f(x)$ for all $x \in V(G)$ and $\sum_{x \in V(G)} h(x) \equiv 0(\bmod 2)$. Let $S$ and $T$ be disjoint subsets of $V(G)$. Then by the $f$-factor Theorem, it suffices to show that

$$
\delta(h ; S, T)=\sum_{x \in S} h(x)+\sum_{x \in T}\left(\operatorname{deg}_{G}(x)-h(x)\right)-e_{G}(S, T)-q(h ; S, T) \geq 0,
$$

where $q(h ; S, T)$ denotes the number of components $C$ of $G-(S \cup T)$ satisfying

$$
\sum_{x \in V(C)} h(x)+e_{G}(C, T) \equiv 1(\bmod 2) .
$$

Since every component $C$ of $G-(S \cup T)$ counted in $q(h ; S, T)$ satisfies the condition (i) or (ii) in the theorem, we have $q(h ; S, T) \leq \hat{q}(S, T)$. Hence by the inequality $g(x) \leq h(x) \leq f(x)$, we have

$$
\begin{aligned}
\delta(h ; S, T) & =\sum_{x \in S} h(x)+\sum_{x \in T}\left(\operatorname{deg}_{G}(x)-h(x)\right)-e_{G}(S, T)-q(S, T) \\
& \geq \sum_{x \in S} g(x)+\sum_{x \in T}\left(\operatorname{deg}_{G}(x)-f(x)\right)-e_{G}(S, T)-\hat{q}(S, T) \\
& \geq-1 .
\end{aligned}
$$

The following congruent expression holds by the $f$-Factor Theorem.

$$
\delta(h ; S, T) \equiv \sum_{x \in V(G)} h(x) \equiv 0(\bmod 2) .
$$

Hence $\delta(h ; S, T) \geq 0$. Therefore $G$ has an $h$-factor.

We next prove necessity. Let $S$ and $T$ be disjoint subsets of $V(G)$. We define a function $h: V(G) \rightarrow \mathbb{Z}^{+}$as follows: 
(1) $h(x)=f(x)$ for $x \in S$,

(2) $h(x)=g(x)$ for $x \in T$,

(3) for every component $C$ of $G-(S \cup T)$ having a vertex $u$ with $g(u)<f(u)$, define $h(x)=f(x)$ for all $x \in V(C)-u$ and $h(u)=f(u)$ or $f(u)-1$ so that $\sum_{x \in V(C)} h(x)+e_{G}(C, T) \equiv 1$ $(\bmod 2) ;$ and

(4) for every component $C$ of $G-(S \cup T)$ with $g(x)=f(x)$ for all $x \in V(C)$, define $h(x)=f(x)$ for all $x \in V(C)$.

If $\sum_{x \in V(G)} h(x)$ is even, then $G$ has an $h$-factor, and so by the $f$-Factor Theorem, we have

$$
\begin{aligned}
0 & \leq \delta(h ; S, T) \\
& =\sum_{x \in S} h(x)+\sum_{x \in T}\left(\operatorname{deg}_{G}(x)-h(x)\right)-e_{G}(S, T)-q(h ; S, T) \\
& =\sum_{x \in S} g(x)+\sum_{x \in T}\left(\operatorname{deg}_{G}(x)-f(x)\right)-e_{G}(S, T)-\hat{q}(S, T) .
\end{aligned}
$$

If $\sum_{x \in V(G)} h(x)$ is odd, then since $g \neq f, G$ has a vertex $w$ with $g(w)<$ $f(w)$. We change exactly one value $h(w)$ so that $g(w) \leq h(w) \leq f(w)$ and $\sum_{x \in V(G)} h(x)$ is even. If $w \in S \cup T$ then $q(h ; S, T)=\hat{q}(S, T)$ and $h(w)=f(w)-1$ or $h(w)=g(w)+1$; otherwise $q(h ; S, T) \geq \hat{q}(S, T)-1$. Then $G$ has an $h$-factor, and so by the $f$-factor Theorem, we have

$$
\begin{aligned}
0 & \leq \delta(h ; S, T) \\
& =\sum_{x \in S} h(x)+\sum_{x \in T}\left(\operatorname{deg}_{G}(x)-h(x)\right)-e_{G}(S, T)-q(h ; S, T) \\
& \leq \sum_{x \in S} g(x)+\sum_{x \in T}\left(\operatorname{deg}_{G}(x)-f(x)\right)-e_{G}(S, T)-\hat{q}(S, T)+1 .
\end{aligned}
$$

Consequently (3.15) holds in this case, and the proof is complete.

Theorem 3.1.6 (Katerinis [85]) Let $G$ be a 2-tough simple graph. Then for every function $f: V(G) \rightarrow\{1,2\}$ such that $\sum_{x \in V(G)} f(x) \equiv 0(\bmod 2), G$ has an $f$-factor.

Proof. Since $G$ is 2-tough, $G$ is connected. Suppose that the theorem does not hold. Then $G$ is not a complete graph, and so

$$
|G| \geq 6 \quad \text { and } \quad \delta(G) \geq 4
$$


by $\operatorname{tough}(G) \geq 2$ (see Exercises). By Theorem 3.1.5, there exists a pair $(S, T)$ of disjoint subsets $S$ and $T$ of $V(G)$ such that

$$
\rho(S, T)=|S|+\sum_{x \in T} \operatorname{deg}_{G-S}(x)-2|T|-\omega(G-(S \cup T)) \leq-2,
$$

where $\omega(G-(S \cup T))=\hat{q}(S, T)$ denotes the number of components of $G-$ $(S \cup T)$. We choose such a pair $(S, T)$ so that $T$ is minimal. Then $S \cup T \neq \emptyset$. It follows that $T \neq \emptyset$ since if $\omega(G-S)=1$ then $\rho(S, \emptyset)=|S|-\omega(G-S) \geq 0$; otherwise, $|S| \geq 2 \omega(G-S)$ and thus $\rho(S, \emptyset)=|S|-\omega(G-S) \geq \omega(G-S) \geq 2$, which are contradictions.

Claim 1. For every $x \in T$, it follows that $e_{G}(x, T)=0$ and $\operatorname{deg}_{G-S}(x) \leq 2$. In particular, $|S| \geq 2$.

Let $v \in T$. By the choice of $(S, T)$, it follows that

$$
\begin{aligned}
1 \leq & \rho(S, T-v)-\rho(S, T) \\
= & |S|+\sum_{x \in T-v} \operatorname{deg}_{G-S}(x)-2|T-v|-\omega(G-(S \cup(T-v))) \\
& -\left(|S|+\sum_{x \in T} \operatorname{deg}_{G-S}(x)-2|T|-\omega(G-(S \cup T))\right) \\
\leq & -\operatorname{deg}_{G-S}(v)+2+e_{G}(v, V(G)-(S \cup T))-1 \\
= & -e_{G}(v, T)+1 .
\end{aligned}
$$

Hence $e_{G}(v, T)=0$.

Similarly, it follows from $\omega(G-(S \cup T))=\omega(G-((S+v) \cup(T-v)))$ that

$$
\begin{aligned}
1 \leq & \rho(S+v, T-v)-\rho(S, T) \\
= & |S|+1+\sum_{x \in T-v} \operatorname{deg}_{G}(x)-e_{G}(S+v, T-v)-2|T-v| \\
& -\left(|S|+\sum_{x \in T} \operatorname{deg}_{G}(x)-e_{G}(S, T)-2|T|\right) \\
= & 1-\operatorname{deg}_{G}(v)+e_{G}(v, S)-e_{G}(v, T-v)+2 \\
= & -\operatorname{deg}_{G-S}(v)+3 . \quad\left(\text { by } e_{G}(v, T)=0\right)
\end{aligned}
$$

Hence $\operatorname{deg}_{G-S}(v) \leq 2$. Since $\delta(G) \geq 4$, we have

$$
4 \leq \operatorname{deg}_{G}(v) \leq|S|+\operatorname{deg}_{G-S}(v) \leq|S|+2,
$$

which implies $|S| \geq 2$. 
Claim 2. $|T| \geq 2$.

Assume $T=\{v\}$. If $\omega(G-(S \cup\{v\}))=1$, then it follows from $|S| \geq 2$ that

$$
\rho(S,\{v\}) \geq|S|-2|\{v\}|-\omega(G-(S \cup\{v\})) \geq-1,
$$

which contradicts (3.18). If $\omega(G-(S \cup\{v\})) \geq 2$, then $|S \cup\{v\}| \geq 2 \omega(G-$ $(S \cup\{v\}))$, and so

$\rho(S,\{v\}) \geq|S|-2|\{v\}|-\omega(G-(S \cup\{v\})) \geq \omega(G-(S \cup\{v\}))-1-2|\{v\}| \geq-1$,

a contradiction. Therefore the claim holds.

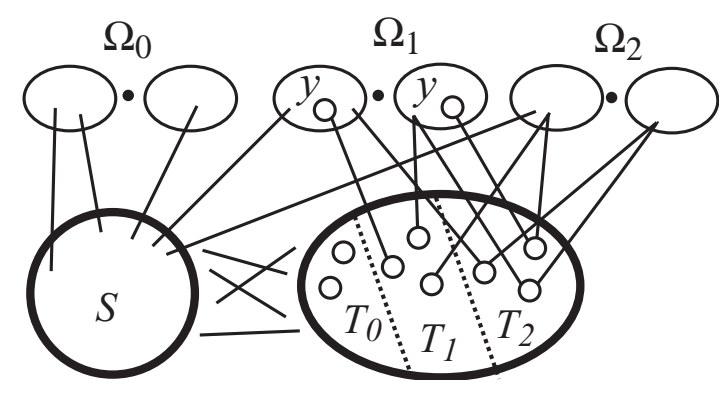

Figure 3.5: A graphs $G$ with a pair $(S, T)$.

By Claim 1, we can partition $T$ into the following three disjoint subsets.

$$
\begin{aligned}
& T_{0}=\left\{x \in T \mid \operatorname{deg}_{G-S}(x)=0\right\} ; \quad T_{1}=\left\{x \in T \mid \operatorname{deg}_{G-S}(x)=1\right\} ; \\
& T_{2}=\left\{x \in T \mid \operatorname{deg}_{G-S}(x)=2\right\} .
\end{aligned}
$$

We divide the set $\Omega$ of components of $G-(S \cup T)$ into the following three subsets.

$$
\begin{aligned}
& \Omega_{0}=\left\{C \in \Omega \mid e_{G}(C, T)=0\right\}, \\
& \Omega_{1}=\left\{C \in \Omega \mid e_{G}(C, T) \geq 1 \text { and } e_{G}(x, T)=1 \text { for some vertex } x \in V(C)\right\}, \\
& \Omega_{2}=\left\{C \in \Omega \mid e_{G}(C, T) \geq 1 \text { and } e_{G}(x, T) \geq 2 \text { for all } x \in V(C)\right\} .
\end{aligned}
$$

For each $C \in \Omega_{1}$, choose one vertex $y$ of $C$ such that $e_{G}(y, T)=1$, and let $Y$ denote the set of these vertices, that is,

$$
Y=\left\{y \in V(C)\left|e_{G}(y, T)=1, C \in \Omega_{1},\right| Y \cap V(C) \mid=1\right\}
$$


Then by Claim 2, $G-\left(S \cup\left(N_{G}(T)-Y\right)\right)$ has at least $|T| \geq 2$ components, and so

$$
\begin{aligned}
& \left.|S|+\left|T_{1}\right|+2\left|T_{2}\right|-\left|\Omega_{1}\right|-\left|\Omega_{2}\right| \geq \mid S \cup\left(N_{G}(T)-Y\right)\right) \mid \\
\geq & 2 \omega\left(G-\left(S \cup\left(N_{G}(T)-Y\right)\right)\right. \\
\geq & 2\left(\left|\Omega_{0}\right|+\left|T_{0}\right|+\left|T_{1}\right|+\left|T_{2}\right|\right) .
\end{aligned}
$$

Hence

$$
|S| \geq 2\left|\Omega_{0}\right|+\left|\Omega_{1}\right|+\left|\Omega_{2}\right|+2\left|T_{0}\right|+\left|T_{1}\right| .
$$

Therefore

$$
\begin{aligned}
\rho(S, T) & =|S|+\sum_{x \in T} \operatorname{deg}_{G-S}(x)-2|T|-\omega(G-(S \cup T)) \\
& \geq|S|+\left|T_{1}\right|+2\left|T_{2}\right|-2\left(\left|T_{0}\right|+\left|T_{1}\right|+\left|T_{2}\right|\right)-\left(\left|\Omega_{0}\right|+\left|\Omega_{1}\right|+\left|\Omega_{2}\right|\right) \\
& \geq|S|-\left(2\left|T_{0}\right|+\left|T_{1}\right|+\left|\Omega_{0}\right|+\left|\Omega_{1}\right|+\left|\Omega_{2}\right|\right) \geq 0 .
\end{aligned}
$$

This contradicts (3.18). Consequently the theorem is proved.

\section{Exercises}

Exercise 3.1.1 Show that $N$ in (3.6) can be defined as $N=\sum_{x \in V(G)}|g(x)|+$ $|G|$. In order to show this, it suffices to prove that $\delta_{G^{*}}(S, T) \geq 0$ when $w \in S$ or $w \in T$.

Hint + Answer If $w \in S$, then

$$
\begin{aligned}
\delta_{G^{*}}(S, T) \geq & \sum_{x \in S} f(x)+\sum_{x \in T}\left(\operatorname{deg}_{G^{*}}(x)-f(x)\right) \\
\geq & \sum_{x \in S-\{w\}} f(x)+M+\sum_{G^{*}}(S, T)-q_{G^{*}}(S, T) \\
& -\sum_{x \in T}(f(x)-g(x))-e_{G}(S-\{w\}, T)-|G| \\
\geq & M+\sum_{x \in T} \operatorname{deg}_{G}(x)-e_{G}(S-\{w\}, T)-|G| \geq 0 .
\end{aligned}
$$

Assume $w \in T$, then

$$
\delta_{G^{*}}(S, T) \geq \sum_{x \in S} f(x)+\sum_{x \in T-\{w\}}\left(\operatorname{deg}_{G^{*}}(x)-f(x)\right)+M
$$




$$
\begin{aligned}
& -e_{G^{*}}(S, T)-q_{G^{*}}(S, T) \\
\geq & \sum_{x \in S} f(x)+\sum_{x \in T-\{w\}}\left(\operatorname{deg}_{G}(x)-g(x)\right) \\
& -\sum_{x \in S}(f(x)-g(x))-e_{G}(S, T-\{w\})-|G|+M \\
\geq & \sum_{x \in S} g(x)-\sum_{x \in T-\{w\}} g(x)-|G|+M \\
& +\sum_{x \in T-\{w\}} \operatorname{deg}_{G}(x)-e_{G}(S, T-\{w\}) \geq 0 .
\end{aligned}
$$

Exercise 3.1.2 Let $G$ be a connected non-complete graph with tough $(G) \geq$ 2. Show that the order of $G$ is at least six and $\delta(G) \geq 4$.

\subsection{Graphs Having the Odd-Cycle Property}

Before going to the next stage of $(g, f)$-factors and $[a, b]$-factors, we investigate factor theory in a special class of graphs which possess the odd-cycle property. Many results on factors in bipartite graphs can be extended to factors in graphs of this class. A graph is said to have the odd-cycle property if any two odd cycles either have a vertex in common or are joined by an edge. Of course, every bipartite graph has the old-cycle property, and so the class of graphs having the odd-cycle property is larger than the class of bipartite graphs. For example, the graph $G$ given in Figure 3.6 has the odd-cycle property because each of its odd cycles passes through $u$ or $w$ and two vertices $u$ and $w$ are adjacent.

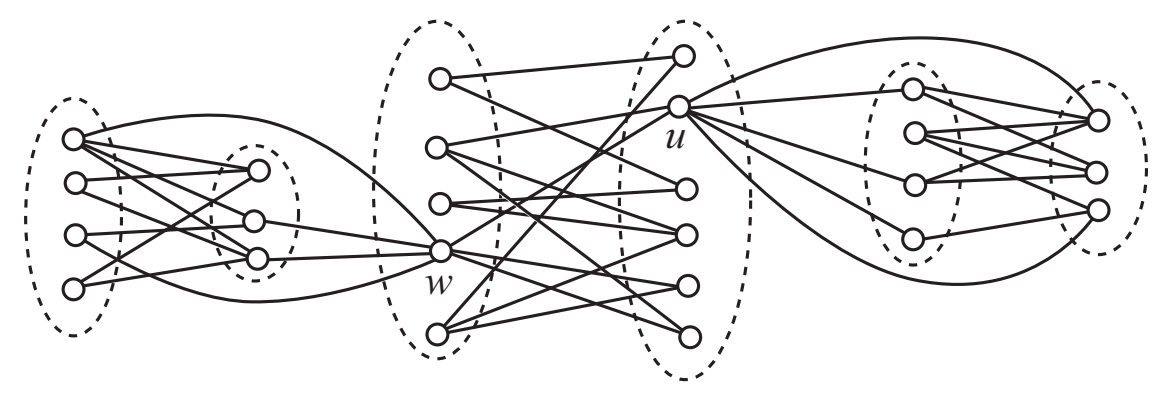

Figure 3.6: A graph possessing the odd-cycle property.

We begin with the next theorem, which is similar to Hall's theorem in bipartite graphs. 
Theorem 3.2.1 (Berge [19]) Let $G$ be a connected simple graph of even order possessing the odd-cycle property. Then $G$ has a 1-factor if and only if

$$
\left|N_{G}(S)\right| \geq|S| \quad \text { for all } S \subset V(G) .
$$

Moreover, the above theorem is equivalent to the following theorem, which is similar to Tutte's 1-Factor Theorem but counts only isolated vertices. Note that the two conditions (3.19) and (3.20) are equivalent only in special graphs. They are not equivalent in general graphs though (3.19) implies (3.20).

Theorem 3.2.2 Let $G$ be a connected simple graph of even order possessing the odd-cycle property. Then $G$ has a 1-factor if and only if

$$
\text { iso }(G-S) \leq|S| \quad \text { for all } S \subset V(G) \text {. }
$$

Proof of Theorems 3.2.1 and 3.2.2. Suppose that $G$ has a 1-factor $F$. Let $\emptyset \neq S \subset V(G)$. Then $\left|N_{G}(S)\right| \geq\left|N_{F}(S)\right|=|S|$. Moreover, since iso $(G-S) \leq \operatorname{odd}(G-S)$, necessity follows immediately from the 1-Factor Theorem.

In order to prove sufficiency, we use the same argument as we did in the proof of Theorem 1.4.3, i.e. if for every vertex subset $S$ of a graph $H$, the number of factor critical components of $H-S$ is less than or equal to $|S|$, then $H$ has a 1-factor.

Assume that $G$ has no 1-factor. Then by the 1-Factor Theorem, there exists a subset $S \subset V(G)$ such that $\operatorname{odd}(G-S) \geq|S|+2$. Choose such a maximal subset $S$. Then by the proof of Theorem 1.4.3, every component of $G-S$ is factor critical. Since every factor critical component of order at least three contains an odd cycle, $G-S$ has at most one such component. Hence

$$
\begin{aligned}
i s o(G-S) & \geq \operatorname{odd}(G-S)-1 \geq|S|+1 ; \quad \text { and } \\
\left|N_{G}(I s o(G-S))\right| & \leq|S| \leq|I \operatorname{so}(G-S)|-1,
\end{aligned}
$$

where $I s o(G-S)$ denotes the set of isolated vertices of $G-S$. Therefore neither (3.20) nor (3.19) hold.

We now give a criterion of $f$-factors and $(g, f)$-factors in graphs with the odd-cycle property. The proof presented here was given by Mahmoodian [109]. 
Theorem 3.2.3 (Folkman, Hoffman and McAndrew [50]) Let $G$ be a connected multigraph possessing the odd-cycle property, and $f: V(G) \rightarrow \mathbb{Z}$. Then $G$ has an $f$-factor if and only if $\sum_{x \in V(G)} f(x)$ is even and for all disjoint subsets $S$ and $T$ of $V(G)$,

$$
\delta^{*}(S, T)=\sum_{x \in S} f(x)+\sum_{x \in T}\left(\operatorname{deg}_{G}(x)-f(x)\right)-e_{G}(S, T) \geq 0 .
$$

Proof. Let $S$ and $T$ be two disjoint subsets of $V(G)$ such that $S \cup T \neq \emptyset$. We use the $f$-Factor Theorem 2.1.2.

Assume first $G$ has an $f$-factor $F$. Then

$$
\sum_{x \in V(G)} f(x)=\sum_{x \in V(G)} \operatorname{deg}_{F}(x)=2\|F\|=\text { even }
$$

and $\delta^{*}(\emptyset, \emptyset)=0$. It follows from the $f$-Factor Theorem 2.1.2 that

$$
\delta^{*}(S, T)=\delta(S, T)+q(S, T) \geq \delta(S, T) \geq 0 .
$$

We next prove sufficiency. In order to do so, it suffices to show that $\delta(S, T) \geq 0$ and $\delta(\emptyset, \emptyset)=0$, where $\delta(\emptyset, \emptyset)=0$ follows from $\sum_{x \in V(G)} f(x)$.

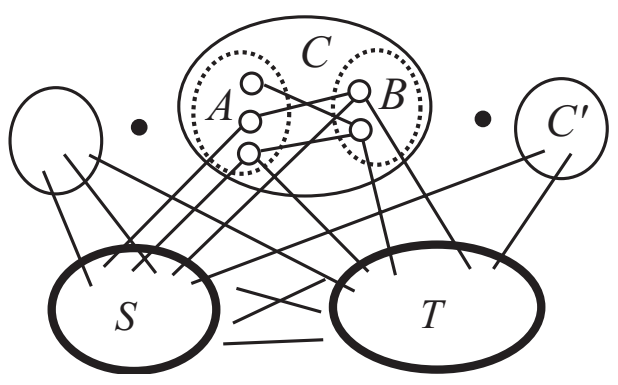

Figure 3.7: A bipartite $f$-odd component $C$ of $G-(S \cup T)$ with bipartition $(A, B)$, and another $f$-odd component $C^{\prime}$.

Let $C^{\prime}$ be an $f$-odd component of $G-(S \cup T)$. Then $C^{\prime}$ satisfies

$$
\sum_{x \in V\left(C^{\prime}\right)} f(x)+e_{G}\left(C^{\prime}, T\right) \equiv 1(\bmod 2) .
$$

If an $f$-odd component $C$ of $G-(S \cup T)$ is a bipartite graph with bipartition $(A, B)$, then

$$
q(S \cup A, T \cup B)=q(S \cup B, T \cup A)=q(S, T)-1 . \quad \text { (see Figure 3.7) }
$$


Thus we obtain

$$
\begin{aligned}
& \delta(S \cup A, T \cup B)+\delta(S \cup B, T \cup A)-2 \delta(S, T) \\
= & \sum_{x \in S \cup A} f(x)+\sum_{x \in S \cup B} f(x)+\sum_{x \in T \cup B}\left(\operatorname{deg}_{G}(x)-f(x)\right) \\
& +\sum_{x \in T \cup A}\left(\operatorname{deg}_{G}(x)-f(x)\right)-e_{G}(S \cup A, T \cup B)-e_{G}(S \cup B, T \cup A) \\
& -q(S \cup A, T \cup B)-q(S \cup B, T \cup A) \\
& -2 \sum_{x \in S} f(x)-2 \sum_{x \in T}\left(\operatorname{deg}_{G}(x)-f(x)\right)+2 e_{G}(S, T)+2 q(S, T) \\
= & \sum_{x \in A \cup B} f(x)+\sum_{x \in A \cup B}\left(\operatorname{deg}_{G}(x)-f(x)\right) \\
& -2 e_{G}(A, B)-e_{G}(A \cup B, S \cup T)+2 \\
= & \sum_{x \in A \cup B} \operatorname{deg}_{G}(x)-\sum_{x \in A \cup B} \operatorname{deg}_{G}(x)+2=2 . \quad(C \text { is a bipartite graph. })
\end{aligned}
$$

Since

$$
\delta(S, T) \equiv \delta(S \cup A, T \cup B) \equiv \delta(S \cup B, T \cup A)(\bmod 2),
$$

it follows that

$$
\delta(S, T) \geq \delta(S \cup A, T \cup B) \text { or } \delta(S \cup B, T \cup A) .
$$

Namely, by setting $\left(S^{\prime}, T^{\prime}\right)=(S \cup A, T \cup B)$ or $(S \cup B, T \cup A)$, we have

$$
\delta(S, T) \geq \delta\left(S^{\prime}, T^{\prime}\right) .
$$

By repeating the same procedure for every bipartite $f$-odd components of $G-(S \cup B)$ one by one, we obtain

$$
\delta(S, T) \geq \delta\left(S^{*}, T^{*}\right),
$$

where $G-\left(S^{*} \cup T^{*}\right)$ has at most one $f$-odd component since $G-(S \cup T)$ has at most one non-bipartite component. Therefore it follows from $\delta^{*}\left(S^{*}, T^{*}\right) \geq 0$ and $q\left(S^{*}, T^{*}\right) \leq 1$ that

$$
\delta(S, T) \geq \delta\left(S^{*}, T^{*}\right)=\delta^{*}\left(S^{*}, T^{*}\right)-q\left(S^{*}, T^{*}\right) \geq-1 .
$$

This implies $\delta(S, T) \geq 0$ by $\delta(S, T) \equiv \sum_{x \in V(G)} f(x) \equiv 0(\bmod 2)$. Consequently, the proof is complete by the $f$-Factor Theorem.

The proof of the $f$-Factor Theorem for a graph having the odd-cycle property used the original $f$-Factor Theorem. On the other hand, we gave an elementary direct proof to the $f$-Factor Theorem for a bipartite graph without 
using the $f$-Factor Theorem. So it might be possible to give an elementary proof for Theorem 3.2.3. Actually Folkman, Hoffman and McAndrew gave another proof using flow theory without using the $f$-Factor Theorem.

A criterion for a graph with the odd-cycle property to have a $(g, f)$-factor is different from the $(g, f)$-Factor Theorem for general graphs. In fact, it gives only a sufficient condition and the sufficient condition cannot be obtained by only removing $q^{*}(S, T)$ from $\gamma(S, T)$. The assumption of the existence of a vertex $u$ with $g(u)<f(u)$ in the following theorem is natural since otherwise a $(g, f)$-factor becomes an $f$-factor.

Theorem 3.2.4 (Chen and Wang [30] (1993)) Let $G$ be a connected multigraph possessing the odd-cycle property. Let $g, f: V(G) \rightarrow \mathbb{Z}^{+}$such that $g(x) \leq f(x)$ for all $x \in V(G)$ and $g(u)<f(u)$ for some vertex $u$. Then $G$ has a $(g, f)$-factor if for all disjoint subsets $S$ and $T$ of $V(G)$ with $S \cup T \neq \emptyset$, we have

$$
\gamma^{*}(S, T)=\sum_{x \in S} f(x)+\sum_{x \in T}\left(\operatorname{deg}_{G}(x)-g(x)\right)-e_{G}(S, T) \geq \epsilon_{0},
$$

where $\epsilon_{0}=1$ if $G$ has an odd cycle with $g(x)=f(x)$ for all its vertices; otherwise $\epsilon_{0}=0$.

The constant $\epsilon_{0}$ cannot be removed, that is, there are graphs that have no $(g, f)$-factors but have the odd-cycle property and satisfy $\gamma^{*}(S, T) \geq 0$ for all disjoint subsets $S$ and $T$ of $V(G)$ with $S \cup T \neq \emptyset$.

Proof. Our proof is similar to that of Theorem 3.2.3 and uses the $(g, f)$ Factor Theorem 3.1.1 instead of the $f$-Factor Theorem.

Let $S$ and $T$ be two disjoint subsets of $V(G)$ such that $S \cup T \neq \emptyset$. In order to prove the theorem, it suffices to show that $\gamma(S, T) \geq 0$ and $\gamma(\emptyset, \emptyset)=0$, where $\gamma(S, T)$ is given in $(g, f)$-Factor Theorem and $\gamma(\emptyset, \emptyset)=0$ holds because of the existence of a vertex $u$ with $g(u)<f(u)$.

Let $C^{\prime}$ be a $(g, f)$-odd component of $G-(S \cup T)$. Then $C^{\prime}$ satisfies

$$
\begin{aligned}
& g(x)=f(x) \quad \text { for all } x \in V\left(C^{\prime}\right), \quad \text { and } \\
& \sum_{x \in V\left(C^{\prime}\right)} f(x)+e_{G}\left(C^{\prime}, T\right) \equiv 1(\bmod 2) .
\end{aligned}
$$

If a $(g, f)$-odd component $C$ of $G-(S \cup T)$ is a bipartite graph with bipartition $(A, B)$, then

$$
q^{*}(S \cup A, T \cup B)=q^{*}(S \cup B, T \cup A)=q^{*}(S, T)-1 .
$$


Thus we obtain

$$
\begin{aligned}
& \gamma(S \cup A, T \cup B)+\gamma(S \cup B, T \cup A)-2 \gamma(S, T) \\
= & \sum_{x \in S \cup A} f(x)+\sum_{x \in S \cup B} f(x)+\sum_{x \in T \cup B}\left(\operatorname{deg}_{G}(x)-g(x)\right) \\
& +\sum_{x \in T \cup A}\left(\operatorname{deg}_{G}(x)-g(x)\right)-e_{G}(S \cup A, T \cup B)-e_{G}(S \cup B, T \cup A) \\
& -q^{*}(S \cup A, T \cup B)-q^{*}(S \cup B, T \cup A) \\
& -2 \sum_{x \in S} f(x)-2 \sum_{x \in T}\left(\operatorname{deg}_{G}(x)-g(x)\right)+2 e_{G}(S, T)+2 q^{*}(S, T) \\
= & \sum_{x \in A \cup B} f(x)+\sum_{x \in A \cup B}\left(\operatorname{deg}_{G}(x)-g(x)\right) \\
& -2 e_{G}(A, B)-e_{G}(A \cup B, S \cup T)+2 \\
= & \sum_{x \in A \cup B} \operatorname{deg}_{G}(x)-\sum_{x \in A \cup B} \operatorname{deg}_{G}(x)+2 \quad(\text { by } g(x)=f(x) \text { for } x \in A \cup B) \\
= & 2 .
\end{aligned}
$$

On the other hand, it follows that

$$
\begin{aligned}
& \gamma(S \cup A, T \cup B)-\gamma(S, T) \\
= & \sum_{x \in A} f(x)+\sum_{x \in B}\left(\operatorname{deg}_{G}(x)-g(x)\right)-e_{G}(S, B)-e_{G}(A, T)-e_{G}(A, B)+1 \\
\equiv & \sum_{x \in A \cup B} f(x)+\sum_{x \in B} \operatorname{deg}_{G}(x) \quad(\operatorname{by} g(x)=f(x) \text { for } x \in A \cup B) \\
& -e_{G}(S, B)-e_{G}(A, T)-e_{G}(A, B)+\sum_{x \in V(C)} f(x)+e_{G}(C, T)(\bmod 2) \\
\equiv & 2 \sum_{x \in V(C)} f(x)+e_{G}(B, A)+e_{G}(B, S)+e_{G}(B, T) \\
& -e_{G}(S, B)-e_{G}(A, T)-e_{G}(A, B)+e_{G}(C, T)(\bmod 2) \\
\equiv & 2 \sum_{x \in V(C)} f(x)+2 e_{G}(B, T) \equiv 0(\bmod 2) .
\end{aligned}
$$

Hence

$$
\gamma(S, T) \equiv \gamma(S \cup A, T \cup B) \equiv \gamma(S \cup B, T \cup A)(\bmod 2)
$$

Therefore we obtain

$$
\gamma(S, T) \geq \gamma(S \cup A, T \cup B) \text { or } \gamma(S \cup B, T \cup A),
$$


i.e., by setting $\left(S^{\prime}, T^{\prime}\right)=(S \cup A, T \cup B)$ or $(S \cup B, T \cup A)$, we have

$$
\gamma(S, T) \geq \gamma\left(S^{\prime}, T^{\prime}\right) .
$$

By repeating the above procedure for every bipartite $(g, f)$-odd components of $G-(S \cup B)$ one by one, we obtain

$$
\gamma(S, T) \geq \gamma\left(S^{*}, T^{*}\right),
$$

where $G-\left(S^{*} \cup T^{*}\right)$ has at most one $(g, f)$-odd component since $G-(S \cup T)$ has at most one non-bipartite component. Define $\epsilon=1$ if $G-(S \cup T)$ has exactly one non-bipartite component and $\epsilon=0$ otherwise. Then if $\epsilon=1$ then $\epsilon_{0}=1$ by the definition of $(g, f)$-odd components, and therefore it follows from $\gamma^{*}\left(S^{*}, T^{*}\right) \geq \epsilon_{0}$ that

$$
\begin{aligned}
\gamma(S, T) & \geq \gamma\left(S^{*}, T^{*}\right)=\gamma^{*}\left(S^{*}, T^{*}\right)-q^{*}\left(S^{*}, T^{*}\right)=\gamma^{*}\left(S^{*}, T^{*}\right)-\epsilon \\
& \geq \epsilon_{0}-\epsilon=0 .
\end{aligned}
$$

This implies $\gamma(S, T) \geq 0$. Consequently, the proof is complete by the $f$ Factor Theorem.

\section{3 $[a, b]$-Factors and $(g, f)$-Factors}

In this section we shall give some sufficient conditions for a graph to have an $[a, b]$-factor, also some sufficient conditions for a graph to have a $(g, f)$-factor. Recall that for two integers $0 \leq a \leq b$, a spanning subgraph $F$ of a graph $G$ is called an $[a, b]$-factor if

$$
a \leq \operatorname{deg}_{F}(x) \leq b \quad \text { for all } \quad x \in V(G) .
$$

Similarly, a graph $G$ is called an $[a, b]$-graph if $a \leq \operatorname{deg}_{G}(x) \leq b$ for all vertices $x$ of $G$.

We begin with a criterion for a graph to have an $[a, b]$-factor, which is easily obtained from the $(g, f)$-Factor Theorem or the $(g, f)$-Factor Theorem with $g<f$ by simply setting $g(x)=a$ and $f(x)=b$ for all vertices $x$. Note that if $a=b$, then an $[a, b]$-factor becomes a regular $a$-factor, and so in this section we always assume $a<b$. If $a=0$, then every graph has an $[a, b]$ factor, which contains no edges, and so we may restrict ourselves to the case of $a \geq 1$. 
Theorem 3.3.1 (The $[a, b]$-Factor Theorem, Lovász) Let $a$ and $b$ be integers such that $1 \leq a<b$. Then a general graph $G$ has an $[a, b]$-factor if and only if for all two disjoint subsets $S$ and $T$ of $V(G)$, we have

$$
\gamma^{*}(S, T)=b|S|+\sum_{x \in T} \operatorname{deg}_{G}(x)-a|T|-e_{G}(S, T) \geq 0 .
$$

It is obvious that $\gamma^{*}(\emptyset, \emptyset)=0$ and so we check only the case $S \cup T \neq \emptyset$. Note that (3.23) is equivalent to

$$
\gamma^{*}(S, T)=b|S|+\sum_{x \in T} \operatorname{deg}_{G-S}(x)-a|T| \geq 0 .
$$

The conditions (3.23) and (3.24) can be replaced by the following inequality, which is sometimes more useful.

Theorem 3.3.2 (Heinrich, Hell, Kirkpatrick and Liu, [56]) Let $G$ be a general graph, and $a$ and $b$ be integers such that $1 \leq a<b$. For any subset $S \subset V(G)$ and any integer $j \geq 0$, let $n_{j}(G-S)$ denote the number of vertices of $G-S$ with degree $j$. Then $G$ has an $[a, b]$-factor if and only if

$$
\sum_{0 \leq j<a}(a-j) \cdot n_{j}(G-S) \leq b|S| \quad \text { for all } S \subset V(G) .
$$

Proof. We first prove sufficiency by using the $[a, b]$-Factor Theorem 3.3.1. Let $S$ and $T$ be two disjoint subsets of $V(G)$. Then

$$
\begin{aligned}
& a|T|-\sum_{x \in T} \operatorname{deg}_{G-S}(x) \\
= & \sum_{0 \leq j}(a-j)\left|\left\{x \in T \mid \operatorname{deg}_{G-S}(x)=j\right\}\right| \\
\leq & \sum_{0 \leq j<a}(a-j)\left|\left\{x \in T \mid \operatorname{deg}_{G-S}(x)=j\right\}\right| \\
\leq & \sum_{0 \leq j<a}(a-j)\left|\left\{x \in V(G)-S \mid \operatorname{deg}_{G-S}(x)=j\right\}\right| \\
= & \sum_{0 \leq j<a}(a-j) n_{j}(G-S) .
\end{aligned}
$$

Hence if $G$ satisfies (3.25), then

$$
b|S| \geq \sum_{0 \leq j<a}(a-j) n_{j}(G-S) \geq a|T|-\sum_{x \in T} \operatorname{deg}_{G-S}(x) .
$$


Therefore (3.24) holds, and so $G$ has an $[a, b]$-factor.

Conversely, assume that $G$ has an $[a, b]$-factor. For any subset $S \subset V(G)$, we define

$$
T=\left\{x \in V(G)-S \mid \operatorname{deg}_{G-S}(x)<a\right\} .
$$

Then all the inequalities between (3.26) and (3.27) become equations, and so

$$
a|T|-\sum_{x \in T} \operatorname{deg}_{G-S}(x)=\sum_{0 \leq j<a}(a-j) n_{j}(G-S) .
$$

On the other hand, by the $[a, b]$-Factor Theorem, we have

$$
b|S|+\sum_{x \in T} \operatorname{deg}_{G-S}(x)-a|T| \geq 0 .
$$

Combining the two inequalities, we have

$$
b|S| \geq \sum_{0 \leq j<a}(a-j) n_{j}(G-S) .
$$

Consequently the theorem is proved.

To show the existence of an $[a, b]$-factor, we may apply Theorem 3.3.1 together with the next lemma instead of Theorem 3.3.2.

Lemma 3.3.3 Let $G$ be a general graph, and $a$ and $b$ be integers such that $1 \leq a<b$. Suppose that $G$ has no $[a, b]$-factors. Then there exist disjoint subsets $S$ and $T$ of $V(G)$ such that

$$
\gamma^{*}(S, T)=b|S|+\sum_{x \in T} \operatorname{deg}_{G-S}(x)-a|T|<0 .
$$

Among such subsets $S$ and $T$, choose $S$ and $T$ so that $T$ is minimal. Then (i) $T \neq \emptyset$ and (ii) $\operatorname{deg}_{G-S}(x) \leq a-1$ for all $x \in T$.

Proof. It is trivial that $T \neq \emptyset$. Let $v \in T$. Then

$$
\begin{aligned}
1 \leq & \gamma^{*}(S, T-v)-\gamma^{*}(S, T) \\
= & b|S|+\sum_{x \in T-v} \operatorname{deg}_{G-S}(x)-a|T-v| \\
& -\left(b|S|+\sum_{x \in T} \operatorname{deg}_{G-S}(x)-a|T|\right) \\
= & -\operatorname{deg}_{G-S}(v)+a .
\end{aligned}
$$

Hence $\operatorname{deg}_{G-S}(v) \leq a-1$. 
By setting $a=1$ in Theorem 3.3.2, we can obtain the following Theorem 3.3.4 since

$$
n_{0}(G-S)=i s o(G-S)=\text { the number of isolated vertices in } G-S .
$$

This theorem is also obtained from Las Vergnas's $(g, f)$-Factor Theorem with $g \leq 1$ (Theorem 3.1.4) because if $g(x)=1$ and $f(x)=b \geq 2$ for all $x \in V(G)$, then $\operatorname{odd}(g ; G-S)$ is equal to the number of isolated vertices of $G-S$ (see (3.9)). We shall give another direct proof for this theorem in the Chapter 5 by regarding a $[1, b]$-factor as a component factor.

Theorem 3.3.4 (Las Vergnas $(b \geq 2)[92])$ Let $b \geq 2$ be an integer. Then a general graph $G$ has a $[1, b]$-factor if and only if

$$
\text { iso }(G-S) \leq b|S| \quad \text { for all } S \subset V(G) .
$$

Furthermore, the following inequality guarantees the existence of $a[1, b]$ factor.

$$
\left|N_{G}(S)\right| \geq \frac{|S|}{b} \quad \text { for all } S \subset V(G) .
$$

We explain only that (3.29) implies (3.28). For every $S \subset C(G)$, (3.28) follows from

$$
|S| \geq\left|N_{G}(I s o(G-S))\right| \geq \frac{|I \operatorname{sso}(G-S)|}{b}=\frac{i s o(G-S)}{b},
$$

where $I s o(G-S)$ denotes the set of isolated vertices of $G-S$.

The following theorem was obtained independently by Heinrich, Hell, Kirkpatrick and Liu [56] and Egawa and Kano ([41], [146] p. 121).

Theorem 3.3.5 Let $G$ be a connected general graph and $g, f: V(G) \rightarrow \mathbb{Z}$ such that $g(x) \leq \operatorname{deg}_{G}(x), 0 \leq f(x)$ and $g(x)<f(x)$ for all $x \in V(G)$. If for every pair of adjacent vertices $x$ and $y$ of $G$, we have

$$
\frac{g(x)}{\operatorname{deg}_{G}(x)} \leq \frac{f(y)}{\operatorname{deg}_{G}(y)}
$$

then $G$ has a $(g, f)$-factor.

Proof. We use the $(g, f)$-Factor Theorem with $g<f$ (Theorem 3.1.2) since $g(x)<f(x)$ for all $x \in V(G)$. Let $S$ and $T$ be disjoint subsets of $V(G)$ such that $S \cup T \neq \emptyset$. Then we have

$$
\begin{aligned}
\gamma^{*}(S, T) & =\sum_{s \in S} f(s)+\sum_{t \in T}\left(\operatorname{deg}_{G}(t)-g(t)\right)-e_{G}(S, T) \\
& =\sum_{s \in S} \operatorname{deg}_{G}(s) \frac{f(s)}{\operatorname{deg}_{G}(s)}+\sum_{t \in T} \operatorname{deg}_{G}(t)\left(1-\frac{g(t)}{\operatorname{deg}_{G}(t)}\right)-e_{G}(S, T) .
\end{aligned}
$$




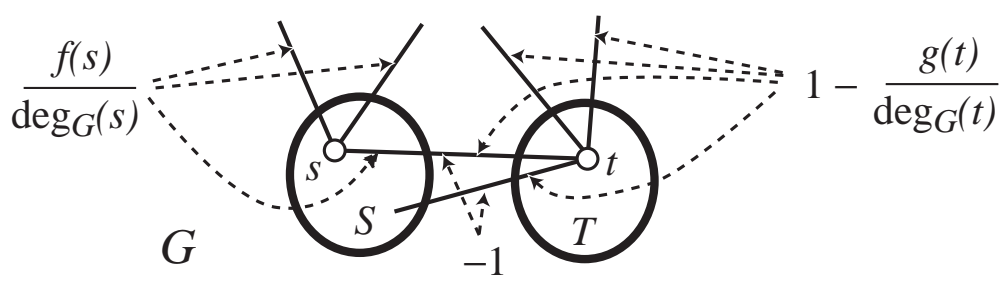

Figure 3.8: The weights of edges of $G$ with $S \cup T$.

For each vertex $s \in S$, add the weight $f(s) / \operatorname{deg}_{G}(s)$ to all the edges incident with $s$, for each vertex $t \in T$, we add the weight $1-g(t) / \operatorname{deg}_{G}(t)$ to all edges incident with $t$, and finally add the weight -1 to all the edges between $S$ and $T$, (see Figure 3.8). Since $f(s) / \operatorname{deg}_{G}(s) \geq 0$ and $1-g(t) / \operatorname{deg}_{G}(t) \geq 0$, we have by the above expression for $\gamma^{*}(S, T)$

$$
\begin{aligned}
\gamma^{*}(S, T) & =\sum_{s \in S} \operatorname{deg}_{G}(s) \frac{f(s)}{\operatorname{deg}_{G}(s)}+\sum_{t \in T} \operatorname{deg}_{G}(t)\left(1-\frac{g(t)}{\operatorname{deg}_{G}(t)}\right)-e_{G}(S, T) \\
& \geq \text { The total sum of weights of edges between } S \text { and } T \\
& \sum_{s t \in E(G), s \in S, t \in T}\left(\frac{f(s)}{\operatorname{deg}_{G}(s)}+1-\frac{g(t)}{\operatorname{deg}_{G}(t)}-1\right) \\
& \geq 0 . \quad \text { by }(3.30)
\end{aligned}
$$

Hence $\gamma^{*}(S, T) \geq 0$ and thus $G$ has a $(g, f)$-factor.

The next theorem follows immediately from the above theorem, but it also can be proved directly by the $(g, f)$-Factor Theorem with $(g<f)$ as we show in the proof.

Theorem 3.3.6 (Kano and Saito [77] (1983)) Let $G$ be connected general graph and $g, f: V(G) \rightarrow \mathbb{Z}$ such that $g(x)<f(x)$ for all $x \in V(G)$. If there exists a real number $0 \leq \theta \leq 1$ such that

$$
g(x) \leq \theta \operatorname{deg}_{G}(x) \leq f(x) \quad \text { for all } \quad x \in V(G),
$$

then $G$ has a $(g, f)$-factor.

Proof. We use the $(g, f)$-Factor Theorem with $g<f$ (Theorem 3.1.2). Let $S$ and $T$ be disjoint subsets of $V(G)$ such that $S \cup T \neq \emptyset$.

$$
\begin{aligned}
\gamma^{*}(S, T) & =\sum_{x \in S} f(x)+\sum_{x \in T}\left(\operatorname{deg}_{G}(x)-g(x)\right)-e_{G}(S, T) \\
& \geq \theta \sum_{x \in S} \operatorname{deg}_{G}(x)+\sum_{x \in T}(1-\theta) \operatorname{deg}_{G}(x)-e_{G}(S, T) \\
& \geq \theta e_{G}(S, T)+(1-\theta) e_{G}(S, T)-e_{G}(S, T)=0 .
\end{aligned}
$$


Therefore $G$ has a $(g, f)$-factor.

Theorem 3.3.7 (Kano and Saito [77] (1983)) Let $m, n$, $a$ and $b$ be integers such that $1 \leq m \leq n$ and $1 \leq a<b \leq n$. If

$$
\frac{a}{b} \leq \frac{m}{n}
$$

then every general $[m, n]$-graph has an $[a, b]$-factor.

Proof. Let $G$ be a $[m, n]$-graph. Define $g(x)=a$ and $f(x)=b$ for all $x \in V(G)$, and $\theta=b / n$. Then

$$
g(x)=a \leq \theta m \leq \theta \operatorname{deg}_{G}(x) \leq \theta n=b=f(x) .
$$

Hence by Theorem 3.3.6, $G$ has an $[a, b]$-factor.

We now show that the condition in Theorem 3.3.7 is sharp, i.e., we can prove that if $a / b>m / n$ then the complete bipartite graph $K(m, n)$, which is an $[m, n]$-graph, does not have an $[a, b]$-factor. Let $G=K(m, n)$, and let $S$ and $T$ be the partite sets of $K(m, n)$ such that $|S|=m$ and $|T|=n$. Then

$$
\begin{aligned}
& b|S|+\sum_{x \in T} \operatorname{deg}_{G}(x)-a|T|-e_{G}(S, T) \\
= & b m+m n-a n-m n=b m-a n<0 \quad(\text { by } a / b>m / n)
\end{aligned}
$$

Hence by Theorem 3.3.1, $G$ has no $[a, b]$-factor.

As was shown in Chapter 3, some $r$-regular simple graphs have no $k$ regular factors, but the next theorem, which was conjectured by Erdős and proved by Tutte, shows that every $r$-regular graph has a $[k, k+1]$-factor. Such a factor is sometimes called a semi-regular factor.

Theorem 3.3.8 (Tutte [142] (1978)) Let $k$ and $r$ be integers such that $1 \leq$ $k<r$. Then every r-regular general graph has $a[k, k+1]$-factor.

The next theorem is a generalization of previous theorem. Thomassen gave an elementary proof for it by using Hall's Marriage Theorem. Note that Theorems 3.3.8 and 3.3.9 follows from Theorem 3.3.7 as $k /(k+1) \leq r / r$ and $k /(k+1) \leq r /(r+1)$. We shall later show that if $1 \leq k \leq 2 r / 3$, then every $r$-regular simple graph has a $[k, k+1]$-factor each of whose components is regular.

Theorem 3.3.9 (Thomassen [136] (1981)) Let $k$ and $r$ be integers such that $1 \leq k<r$. Then every general $[r, r+1]$-graph has a $[k, k+1]$-factor. 
Theorem 3.3.10 (Heinrich, Hell, Kirkpatrick and Liu [56] (1990)) Let a, b, m, $n$ and $k$ be integers such that $1 \leq a<b, 1 \leq m \leq n$ and $1 \leq k$. Then $a$ general $[m, n]$-graph has $k$ edge-disjoint $[a, b]$-factor if

$$
(k-1)\left(b^{2}-a^{2}\right) \leq m b-n a \quad \text { and } \quad a<b \leq n-(k-1) a .
$$

In particular, if $(k-1)(a+b) \leq r$ and $a<b \leq r-(k-1) a$, then every general $r$-regular graph has $k$ edge disjoint $[a, b]$-factors.

Proof. Let $G$ be a general $[m, n]$-graph. We prove the theorem by induction on $k$. If $k=1$, then $0 \leq m b-n a$ by (3.32), which implies $a / b \leq m / n$, and so $G$ has an $[a, b]$-factor by Theorem 3.3.7. Assume $k \geq 2$. Then $0 \leq m b-n a$, and so $G$ has an $[a, b]$-factor $F$ by Theorem 3.3.7. It is clear that $G-E(F)$ is an $[m-b, n-a]$-graph. Then

$$
\begin{aligned}
(k-2)\left(b^{2}-a^{2}\right) & \leq(m-b) b-(n-a) a \\
\Leftrightarrow \quad(k-1)\left(b^{2}-a^{2}\right) & \leq m b-n a,
\end{aligned}
$$

and

$$
b \leq(n-a)-(k-2) a \Leftrightarrow b \leq n-(k-1) a .
$$

Hence by applying the inductive hypothesis to $G-F$ and $k-1$, we can obtain $k-1$ edge disjoint $[a, b]$-factors of $G-F$, and thus $G$ has $k$ edge disjoint $[a, b]$-factors.

A general graph $G$ is said to be locally $s$-almost regular if

$$
\left|\operatorname{deg}_{G}(x)-\operatorname{deg}_{G}(y)\right| \leq s \quad \text { for all two adjacent vertices } x \text { and } y \text {. }
$$

In particular, a locally 0-almost regular connected graph is a regular graph. This notion was introduced by Joentgen and Volkmann [64], and they proved the following theorem.

Theorem 3.3.11 (Joentgen and Volkmann [64] (1991)) Let $k$, $s$ and $t$ be integers such that $1 \leq k, t$ and $0 \leq s$, and let $G$ be a locally s-almost regular general graph. If

$$
k \leq \delta(G) \quad \text { and } \quad \frac{s}{\delta(G)} \leq \frac{t}{k},
$$

then $G$ has a $[k, k+t]$-factor.

Proof. We define $g(x)=k$ and $f(x)=k+t$ for all $x \in V(G)$. Let $x$ and $y$ be two adjacent vertices of $G$. Since $\delta(G) \leq \operatorname{deg}_{G}(x)$, sk $\leq \delta(G) t$ and $\operatorname{deg}_{G}(y) \leq \operatorname{deg}_{G}(x)+s$, we have

$$
\frac{g(x)}{\operatorname{deg}_{G}(x)} \leq \frac{k}{\delta(G)} \leq \frac{k+t}{\delta(G)+s} \leq \frac{f(y)}{\operatorname{deg}_{G}(y)} .
$$


Therefore by Theorem 3.3.5, $G$ has a $(g, f)$-factor, which is a $[k, k+t]$-factor.

Theorem 3.3.12 (Egawa and Kano [41] (1996)) Let $G$ be a connected general graph and $g, f: V(G) \rightarrow \mathbb{Z}$ such that $g(x) \leq \operatorname{deg}_{G}(x), 0 \leq f(x)$ and $g(x) \leq f(x)$ for all vertices $x$ of $G$. If the following three conditions hold, then $G$ has a $(g, f)$-factor.

(i) $G$ has at least one vertex $u$ with $g(u)<f(u)$.

(ii) For any two adjacent vertices $x$ and $y$ of $G$, we have

$$
\frac{g(x)}{\operatorname{deg}_{G}(x)} \leq \frac{f(y)}{\operatorname{deg}_{G}(y)}
$$

(iii) For every non-empty proper subset $X$ of $V(G)$ such that $g(x)=f(x)$ for all $x \in X$ and $\langle X\rangle_{G}$ is connected, we have

$$
\sum_{v \in V(G)-X}\left(e_{G}(v, X) \min \left\{\frac{f(v)}{\operatorname{deg}_{G}(v)}, 1-\frac{g(v)}{\operatorname{deg}_{G}(v)}\right\}\right) \geq 1 .
$$

Proof. We shall show that $\gamma(S, T) \geq 0$ as required in the $(g, f)$-Factor Theorem 3.1.1. Let $S$ and $T$ be two disjoint subsets of $V(G)$. By condition (i), we have $\gamma(\emptyset, \emptyset)=0$, and so we may assume $S \cup T \neq \emptyset$. Let $C_{1}, C_{2}, \ldots, C_{m}$ be the odd components of $G-(S \cup T)$, where $m=q^{*}(S, T)$. Then we have

$$
\begin{aligned}
\gamma(S, T) & =\sum_{s \in S} f(s)+\sum_{t \in T}\left(\operatorname{deg}_{G}(t)-g(t)\right)-e_{G}(S, T)-q^{*}(S, T) \\
& =\sum_{s \in S} \operatorname{deg}_{G}(s) \frac{f(s)}{\operatorname{deg}_{G}(s)}+\sum_{t \in T} \operatorname{deg}_{G}(t)\left(1-\frac{g(t)}{\operatorname{deg}_{G}(t)}\right)-e_{G}(S, T)-m .
\end{aligned}
$$

For each vertex $s \in S$, add the weight $f(s) / \operatorname{deg}_{G}(s)$ to all the edges incident with $s$, for each vertex $t \in T$, add the weight $1-g(t) / \operatorname{deg}_{G}(t)$ to all edges incident with $t$, and add the weight -1 to every edge joining $S$ and $T$ (see Figure 3.9).

Then $V\left(C_{i}\right)=X$ satisfies $(3.35), f(s) / \operatorname{deg}_{G}(s) \geq 0,1-g(t) / \operatorname{deg}_{G}(t) \geq 0$ and

$$
\sum_{v \in V(G)-V\left(C_{i}\right)} e_{G}\left(v, C_{i}\right)=\sum_{v \in S \cup T} e_{G}\left(v, C_{i}\right) .
$$

Hence we have

$$
\gamma(S, T)=\sum_{s \in S} \operatorname{deg}_{G}(s) \frac{f(s)}{\operatorname{deg}_{G}(s)}+\sum_{t \in T} \operatorname{deg}_{G}(t)\left(1-\frac{g(t)}{\operatorname{deg}_{G}(t)}\right)
$$




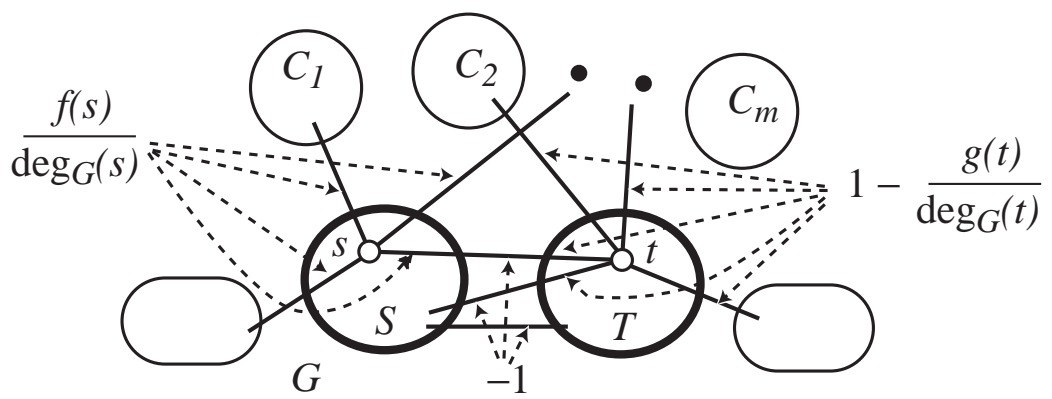

Figure 3.9: The graph $G$ with $S \cup T$, and the components $C_{1}, \ldots, C_{m}$, where $m=q^{*}(S, T)$.

$$
-e_{G}(S, T)-m
$$

$\geq$ (The total sum of weights of edges between $S$ and $T$ )

+ (The total sum of weights of edges joining $S \cup T$ and $\left.V\left(C_{1}\right) \cup \cdots \cup V\left(C_{m}\right)\right) \quad-m$

$$
\begin{aligned}
\geq & \sum_{s t \in E(G), s \in S, t \in T}\left(\frac{f(s)}{\operatorname{deg}_{G}(s)}+1-\frac{g(t)}{\operatorname{deg}_{G}(t)}-1\right) \\
& +\sum_{i=1}^{m}\left(\sum_{v \in S \cup T} e_{G}\left(v, C_{i}\right) \min \left\{\frac{f(v)}{\operatorname{deg}_{G}(v)}, 1-\frac{g(v)}{\operatorname{deg}_{G}(v)}\right\}-1\right) \\
\geq & 0 .
\end{aligned}
$$

Consequently, $G$ has a $(g, f)$-factor.

Theorem 3.3.13 (Egawa and Kano [41] (1996)) Let $G$ be an r-regular simple graph and $g, f: V(G) \rightarrow \mathbb{Z}$ such that $g(x)<r, 0<f(x)$ and $g(x) \leq f(x)$ for all $x \in V(G)$. If the following two conditions hold, then $G$ has a $(g, f)$ factor.

(i) $g(x) \leq f(y)$ for all two adjacent vertices $x$ and $y$; and

(ii) for every vertex $u$ with $g(u)=f(u)$, there exists at least one vertex $w$ that is adjacent to $u$ and satisfies $g(w)<f(w)$ (see Figure 3.10).

Proof. We shall show that the three conditions of Theorem 3.3.12 hold. Since (i) and (ii) of Theorem 3.3.12 follows immediately, it suffices to show that condition (iii) holds. Let $\emptyset \neq X \subset V(G)$ such that $g(x)=f(x)$ for all $x \in X$ and $\langle X\rangle_{G}$ is connected. By condition (ii), for every vertex $x \in X$ (see Figure 3.10), there exists a vertex $y \in V(G)-X$ such that $g(y)<f(y)$ and $y$ is adjacent to $x$. Hence if $|X| \geq r$, then

$$
e_{G}(X, V(G)-X) \geq|X| \geq r .
$$



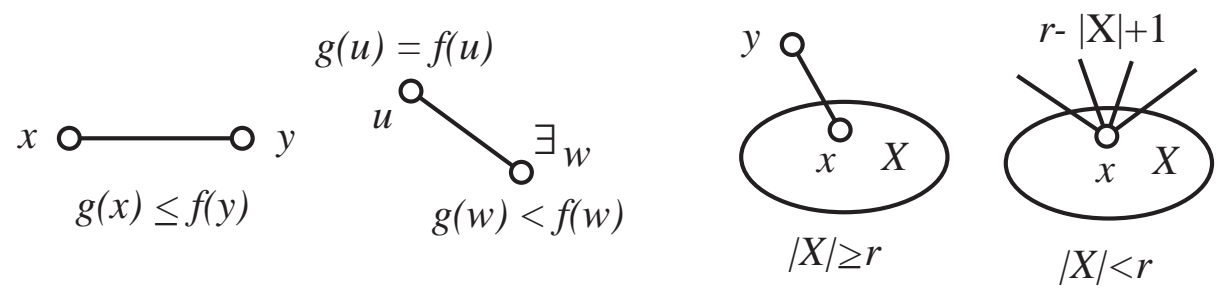

Figure 3.10: The conditions in Theorem 3.3.12, and $X$ in the proof.

If $|X|<r$, then since $G$ is a simple graph, $x \in X$ is adjacent to at least $r-|X|+1$ vertices in $V(G)-X$ (see Figure 3.10), and thus we have

$$
e_{G}(X, V(G)-X) \geq(r-|X|+1)|X| \geq r .
$$

Therefore, by

$$
\frac{1}{r} \leq \frac{f(v)}{\operatorname{deg}_{G}(v)} \quad \text { and } \quad \frac{g(v)}{\operatorname{deg}_{G}(v)} \leq \frac{r-1}{r}
$$

we obtain

$$
\begin{gathered}
\sum_{v \in V(G)-X} e_{G}(v, X) \min \left\{\frac{f(v)}{\operatorname{deg}_{G}(v)}, 1-\frac{g(v)}{\operatorname{deg}_{G}(v)}\right\} \\
\geq e_{G}(X, V(G)-X) \min \left\{\frac{1}{r}, 1-\frac{r-1}{r}\right\} \geq r \cdot \frac{1}{r}=1 .
\end{gathered}
$$

Consequently $G$ has a $(g, f)$-factor by Theorem 3.3.12.

The following theorem is an improvement of Tutte's Theorem 3.3.8, which says that every $r$-regular graph has a $[k, k+1]$-factor, i.e., the following corollary guarantees the existence a $[k, k+1]$-factor that has many vertices of degree $k$ (or $k+1$ ) and a small number of vertices of degree $k+1$ (or $k$ ).

Theorem 3.3.14 (Egawa and Kano [41] (1996)) Let $k$ and $r$ be integers such that $0 \leq k<r$, and $G$ be an $r$-regular simple graph. Let $W$ be a maximal independent vertex subset of $G$. Then $G$ has a $[k, k+1]$-factor $F$ such that $\operatorname{deg}_{F}(x)=k$ for all $x \in V(G)-W$, as well as a $[k, k+1]$-factor $H$ such that $\operatorname{deg}_{H}(x)=k+1$ for all $x \in V(G)-W$.

Proof. This theorem is an easy consequence of Theorem 3.3.13. For any vertex $u \in V(G)-W$, there exists at least one vertex $w$ in $W$ adjacent to $u$ since otherwise $W \cup\{u\}$ becomes an independent set, which contradicts the maximality of $W$. Define $g$ and $f$ as

$$
\begin{aligned}
& g(x)=f(x)=k \quad \text { for all } x \in V(G)-W \text {; and } \\
& g(y)=k \text { and } f(y)=k+1 \text { for all } y \in W \text {. }
\end{aligned}
$$


Then $g$ and $f$ satisfy the conditions of Theorem 3.3.13, which implies that $G$ has the desired $[k, k+1]$-factor $F$. We can similarly prove the existence of a $[k, k+1]$-factor $H$.

The following theorem is proved after the second theorem.

Theorem 3.3.15 (Kano and Saito [77]) Let $l, k, r, s, t, u$ be integers such that $0 \leq l \leq k \leq r, l+u \leq k+t \leq r+s, l \neq r, l+u \neq r+s, 0 \leq s, 0 \leq u$ and $1 \leq t$. Let $G$ be a general $[r, r+s]$-graph and $H$ be an $[l, l+u]$-factor of $G$. If

$$
(l-k) s+(k-r) u+(r-l) t \geq 0,
$$

then $G$ has a $[k, k+t]$-factor that contains the given factor $H$ as a subgraph.

By setting $s=u=t$ in Theorem 3.3.15, we obtain the next theorem.

Theorem 3.3.16 ([77]) Let $l, k, r, t$ be integers such that $0 \leq \ell<k<r$ and $1 \leq t$. Then for any given $[\ell, \ell+t]$-factor $H$ of a general $[r, r+t]$-graph $G$, $G$ has a $[k, k+t]$-factor that contains $H$ as a subgraph.

Proof of Theorem 3.3.15. Let $G$ be a general $[r, r+s]$-graph, $H$ be a $[\ell, \ell+u]$-factor of $G$, and $K=G-E(H)$. Define two functions $g, f$ : $V(K) \rightarrow \mathbb{Z}^{+}$by

$$
g(x)=k-\operatorname{deg}_{H}(x) \text { and } f(x)=k+t-\operatorname{deg}_{H}(x) \quad \text { for all } x \in V(G) .
$$

If $K$ has a $(g, f)$-factor $F$, then $F \cup H$ is the desired $[k, k+t]$-factor. Let

$$
\theta=\frac{k-\ell}{r-\ell} \quad \text { and } \quad \lambda=\frac{k+t-\ell-u}{r+s-\ell-u}
$$

Then $\theta \leq \lambda$ by (3.36). We shall show that $K, g$ and $f$ satisfy condition (3.31) in Theorem 3.3.6. It is clear that $0 \leq \theta \leq 1$ and $g(x)<f(x)$. Since

$$
k=\theta r+(1-\theta) \ell \leq \theta \operatorname{deg}_{G}(x)+(1-\theta) \operatorname{deg}_{H}(x)
$$

and

$$
\operatorname{deg}_{K}(x)=\operatorname{deg}_{G}(x)-\operatorname{deg}_{H}(x)
$$

we have

$$
\begin{aligned}
g(x) & =k-\operatorname{deg}_{H}(x) \leq \theta \operatorname{deg}_{G}(x)+(1-\theta) \operatorname{deg}_{H}(x)-\operatorname{deg}_{H}(x) \\
& =\theta\left(\operatorname{deg}_{G}(x)-\operatorname{deg}_{H}(x)\right)=\theta \operatorname{deg}_{K}(x) .
\end{aligned}
$$


Similarly, since

$$
k+t=\lambda(r+s)+(1-\lambda)(\ell+u) \geq \lambda \operatorname{deg}_{G}(x)+(1-\lambda) \operatorname{deg}_{H}(x),
$$

we have

$$
\begin{aligned}
f(x) & =k+t-\operatorname{deg}_{H}(x) \\
& \geq \lambda \operatorname{deg}_{G}(x)+(1-\lambda) \operatorname{deg}_{H}(x)-\operatorname{deg}_{H}(x) \\
& =\lambda\left(\operatorname{deg}_{G}(x)-\operatorname{deg}_{H}(x)\right)=\lambda \operatorname{deg}_{K}(x) \\
& \geq \theta \operatorname{deg}_{K}(x) . \quad(\operatorname{by} \theta \leq \lambda)
\end{aligned}
$$

Hence $g(x) \leq \theta \operatorname{deg}_{K}(x) \leq f(x)$. Consequently (3.31) holds, and the theorem is proved.

It is shown in Theorem 2.2.4 that for odd integers $a, b, k$ such that $1 \leq$ $a<k<b$, if a graph $G$ has both an $a$-factor and a $b$-factor, then $G$ has a $k$-factor. The following theorem gives a similar result for $[a, b]$-factors. Note that condition (3.37) is equivalent to the condition that the point $(m, n)$ in the plane lies above the straight line passing through the points $(a, b)$ and $(c, d)$. This will be explained after the proof.

Theorem 3.3.17 ([69]) Let $a, b, c, d, m, n$, be integers such that $0 \leq a<$ $m<c$ and $a \leq b, c \leq d$ and $m+1 \leq n$. Suppose that a general graph $G$ has both an $[a, b]$-factor and $[c, d]$-factor. If

$$
\frac{n-b}{m-a} \geq \frac{n-d}{m-c}
$$

then $G$ has an $[m, n]$-factor.

Proof. Let $S$ and $T$ be disjoint subsets of $V(G)$ such that $S \cup T \neq \emptyset$. By the $(g, f)$-Factor Theorem with $g<f$ (Theorem 3.1.2), it suffices to show that $\gamma^{*}(S, T) \geq 0$. Assume that

$$
\gamma^{*}(S, T)=n|S|+\sum_{x \in T} \operatorname{deg}_{G-S}(x)-m|T|<0
$$

for some $S, T \subset V(G), S \cap T=\emptyset$. Since $G$ has an $[a, b]$-factor and a $[c, d]$ factor, if $a<b$ and $c<d$ then

$$
\begin{aligned}
& \gamma_{1}^{*}(S, T)=b|S|+\sum_{x \in T} \operatorname{deg}_{G-S}(x)-a|T| \geq 0, \\
& \gamma_{2}^{*}(S, T)=d|S|+\sum_{x \in T} \operatorname{deg}_{G-S}(x)-c|T| \geq 0 .
\end{aligned}
$$


Note that if $a=b$, then

$$
\gamma(S, T)=b|S|+\sum_{x \in T} \operatorname{deg}_{G-S}(x)-a|T|-q(S, T) \geq 0
$$

and thus the above inequalities hold even if $a=b$ or $c=d$. Hence we obtain

$$
\begin{aligned}
& 0>\gamma^{*}(S, T)-\gamma_{1}^{*}(S, T) \geq(n-b)|S|-(m-a)|T| \\
& 0>\gamma^{*}(S, T)-\gamma_{2}^{*}(S, T) \geq(n-d)|S|-(m-c)|T| .
\end{aligned}
$$

If $S=\emptyset$, then $0>-(m-c)|T|$ by $(3.39)$, which contradicts $m<c$. Thus $S \neq \emptyset$. By (3.38) and (3.39), we obtain

$$
\frac{n-b}{m-a}<\frac{|T|}{|S|} \text { and } \frac{|T|}{|S|}<\frac{n-d}{m-c} .
$$

Thus

$$
\frac{n-b}{m-a}<\frac{n-d}{m-c}
$$

This contradicts condition (3.37). Hence $\gamma^{*}(S, T) \geq 0$, and therefore $G$ has an $[m, n]$-factor.

It is clear that the point $(m, n)$ lies above the line passing through $(a, b)$ and $(c, d)$ if and only if $(c, d)$ lies below the line passing through $(a, b)$ and $(m, n)$. The line passing through $(a, b)$ and $(m, n)$ is

$$
y=\frac{n-b}{m-a}(x-m)+n \text {. }
$$

Hence $(c, d)$ lies below this line if and only if

$$
d \leq \frac{n-b}{m-a}(c-m)+n
$$

This is equivalent to (3.37).

We now turn our attention to other types of sufficient conditions for a graph to have an $[a, b]$-factor. When we consider a general graph with given binding number or given toughness, we may restrict ourselves to a simple graph because a general graph and the simple graph have the same binding number and toughness.

Theorem 3.3.18 (Kano [70] (1990)) Let $a$ and $b$ be integers such that $2 \leq$ $a<b$, and $G$ be a simple graph with $|G| \geq 6 a+b$. Put $\lambda=1+(a-1) / b$. Suppose

$$
\begin{array}{ll}
\left|N_{G}(X)\right| \geq \lambda|X| & \text { if }|X|<\left\lfloor\frac{|G|}{\lambda}\right\rfloor ; \text { and } \\
\left|N_{G}(X)\right|=V(G) \quad \text { otherwise. }
\end{array}
$$

Then $G$ has an $[a, b]$-factor. 
We first note that a similar condition for a graph to have a $[1, b]$-factor with $b \geq 2$ was given in Theorem 3.3.4, which says that if $\left|N_{G}(X)\right| \geq|X| / b$ for all $X \subseteq V(G)$, then $G$ has a $[1, b]$-factor. From this theorem, it follows that if $\operatorname{bind}(G) \geq 1 / b$, then $G$ has a $[1, b]$-factor. On the other hand, if a connected simple graph $G$ with order at least $4 k-6$ satisfies

$$
\operatorname{bind}(G)>\frac{(2 k-1)(|G|-1)}{k(|G|-2)+3}=2-\frac{1}{k}+\epsilon, \quad \epsilon=\frac{2-7 / k+3 / k^{2}}{|G|-2+3 / k},
$$

then $G$ has a $k$-regular factor (see Theorem 2.3.5). If we substitute $a=b=k$ into $\lambda$ in Theorem 3.3.18, then $\lambda=2-1 / k$, which is almost the same as the lower bound of the above $\operatorname{bind}(G)$ though Theorem 3.3.18 holds only in the case $a<b$. We give one more remark on Theorem3.3.18. The conditions (3.40) and (3.41) cannot be replaced by $\left|N_{G}(X)\right| \geq \lambda|X|$ or $N_{G}(X)=V(G)$ for all $X \subset V(G)$.

The proof of Theorem 3.3.18 is fairly long and the proof technique used is similar to that of the next theorem, and so we shall give only the proof to the next theorem.

Theorem 3.3.19 (C. Chen [28] (1993)) Let $a$ and $b$ be integers such that $2 \leq a<b$, and $G$ be a simple graph with $|G| \geq b+3 a$. Put $\lambda=1+(a-1) / b$. If

$$
\operatorname{bind}(G) \geq \lambda \quad \text { and } \quad \delta(G) \geq 1+\frac{(\lambda-1)|G|}{\lambda},
$$

then $G$ has an $[a, b]$-factor.

In the proof of the above theorem, we need the next lemma.

Lemma 3.3.20 (Woodall [151] (1965)) Let $G$ be a simple graph. If bind $(G) \geq$ $t$, then

$$
\left|N_{G}(X)\right| \geq \frac{(t-1)|G|+|X|}{t}
$$

for every independent vertex subset $X$ of $G$.

Proof of Theorem 3.3.19. Suppose that $G$ has no $[a, b]$-factor. Then by the $[a, b]$-Factor Theorem, there exist two disjoint subsets $S$ and $T$ of $V(G)$ such that

$$
b|S|+\sum_{x \in T} \operatorname{deg}_{G-S}(x)-a|T|<0 .
$$

Clearly $T \neq \emptyset$. Define

$$
h=\min \left\{\operatorname{deg}_{G-S}(x) \mid x \in T\right\} .
$$


Then $0 \leq h \leq a-1$. We consider the following two cases.

Case 1. $h=0$.

Let $I=I s o(G-S) \cap T=\left\{x \in T \mid \operatorname{deg}_{G-S}(x)=0\right\}$. Then $I$ is an independent vertex subset of $G$ and $I \neq \emptyset$. By Lemma 3.3.20, we have

$$
\left|N_{G}(I)\right| \geq \frac{(\lambda-1)|G|+|I|}{\lambda} .
$$

On the other hand, by (3.43) we have

$$
\begin{aligned}
0 & >b|S|+\sum_{x \in T} \operatorname{deg}_{G-S}(x)-a|T| \\
& \geq b|S|+|T-I|-a|T| \\
& =b|S|-a|I|+(1-a)|T-I| \\
& \geq b|S|-a|I|+(1-a)(|G|-|S|-|I|) \\
& =(a+b-1)|S|-|I|-(a-1)|G| .
\end{aligned}
$$

Thus

$$
\left|N_{G}(I)\right| \leq|S|<\frac{|I|+(a-1)|G|}{a+b-1}<\frac{|I|+(\lambda-1)|G|}{\lambda} .
$$

This contradicts (3.44).

Case 2. $1 \leq h \leq a-1$.

Since

$$
\begin{aligned}
0 & >b|S|+\sum_{x \in T} \operatorname{deg}_{G-S}-a|T| \geq b|S|+(h-a)|T| \\
& \geq b|S|+(h-a)(|G|-|S|)=(a+b-h)|S|-(a-h)|G|,
\end{aligned}
$$

we have

$$
|S|<\frac{(a-h)|G|}{a+b-h} .
$$

By considering a vertex $v \in T$ with $\operatorname{deg}_{G-S}(v)=h$, we have

$$
\delta(G) \leq \operatorname{deg}_{G}(v) \leq h+|S|<h+\frac{(a-h)|G|}{a+b-h} .
$$

If $h=1$, then we get a contradiction by the assumption that

$$
\delta(G) \geq 1+\frac{(\lambda-1)|G|}{\lambda}=1+\frac{(a-1)|G|}{a+b-1} .
$$


So we may assume $h \geq 2$. Let

$$
f(h)=h+\frac{(a-h)|G|}{a+b-h}=h+|G|-\frac{b|G|}{a+b-h}, \quad 2 \leq h \leq a-1 .
$$

Then $f(h)$ takes its maximum value at $h=2$ since its derivative

$$
\begin{aligned}
f^{\prime}(h) & =1-\frac{b|G|}{(a+b-h)^{2}} \\
& \leq 1-\frac{b(b+3 a)}{(a+b-2)^{2}} \leq 0
\end{aligned}
$$

as $b+3 a \leq|G|$ and $2 \leq h$. Hence

$$
1+\frac{(a-1)|G|}{a+b-1} \leq \delta(G)<f(h) \leq f(2)=2+\frac{(a-2)|G|}{a+b-2} .
$$

This implies $|G|<(a+b-1)(a+b-2) / b<b+3 a$, which contradicts $|G| \geq b+3 a$. Consequently the theorem is proved.

Theorem 3.3.21 (Katerinis [85]) Let $G$ be connected simple graph, and a and $b$ be integers such that $1 \leq a<b$. If

$$
\operatorname{tough}(G) \geq a+\frac{a}{b}-1
$$

then $G$ has an $[a, b]$-factor.

Proof of Theorem 3.3.21 in the case $a=1$. Let $S$ be a non-empty subset of $V(G)$. By the definition of toughness, $\operatorname{tough}(G) \geq 1 / b$ and $\omega(G-S) \geq 2$ implies that

$$
i s o(G-S) \leq \omega(G-S) \leq \frac{|S|}{\operatorname{tough}(G)} \leq b|S| .
$$

If $i s o(G-S)=1$, then obviously iso $(G-S)=1 \leq b|S|$. Hence by Theorem 3.3.4, $G$ has a $[1, b]$-factor. Therefore the theorem holds when $a=1$.

In order to prove the above theorem in the case $a \geq 2$, we need the following lemma. Recall that a vertex subset $D$ of a graph $G$ covers $G$ if $N_{G}[D]=N_{G}(D) \cup D=V(G)$, that is, for every edge of $G$, at least one of its endvertices is contained in $D$. Note that the minimum cardinality of covering vertex subsets of $G$ is called the vertex covering number of $G$ though we will not use it here. 
Lemma 3.3.22 (Katerinis [85]) Let $a \geq 2$ be an integer, $G$ be a simple graph, and $T_{1}, T_{2}, \ldots, T_{a-1}$ be a partition of $V(G)$ such that $\operatorname{deg}_{G}(x) \leq j$ for all $x \in T_{j}$ and every $1 \leq j \leq a-1$, where some $T_{j}$ 's may be empty sets. Then there exist a covering vertex subset $D$ and an independent vertex subset $I$ of $G$ such that $V(G)=D \cup I$ and

$$
\sum_{j=1}^{a-1}(a-j)\left|D \cap T_{j}\right| \leq \sum_{j=1}^{a-1} j(a-j)\left|I \cap T_{j}\right| .
$$

Proof. We prove the lemma by induction on the order $|G|$ of $G$. If $|G|=1$, then $D=I=V(G)$ satisfies (3.46). So we assume $|G| \geq 2$. Let $m=$ $\min \left\{j \mid T_{j} \neq \emptyset\right\}$. Choose a vertex $y \in T_{m}$, and let

$$
\left.G^{\prime}=G-\left(\{y\} \cup N_{G}(y)\right) \quad \text { (see Figure } 3.11\right) .
$$

Then by the induction hypothesis, there exist a covering vertex subset $D^{\prime}$ and an independent vertex subset $I^{\prime}$ of $G^{\prime}$ such that

$$
\sum_{j=1}^{a-1}(a-j)\left|D^{\prime} \cap T_{j}\right| \leq \sum_{j=1}^{a-1} j(a-j)\left|I^{\prime} \cap T_{j}\right| .
$$

Let $I=I^{\prime} \cup\{y\}$ and $D=D^{\prime} \cup N_{G}(y)$. Then

$$
\begin{aligned}
& \sum_{j=1}^{a-1} j(a-j)\left|I \cap T_{j}\right| \\
= & \left.\left.\sum_{j=1}^{a-1} j(a-j)\left|I^{\prime} \cap T_{j}\right|+m(a-m) \quad \text { (by } y \in T_{m}\right)\right) \\
\geq & \sum_{j=1}^{a-1}(a-j)\left|D^{\prime} \cap T_{j}\right|+m(a-m) . \quad \text { (by induction) }
\end{aligned}
$$

Since $\operatorname{deg}_{G}(y) \leq m$ and $m=\min \left\{j \mid T_{j} \neq \emptyset\right\}$, we have

$$
\sum_{j=1}^{a-1}(a-j)\left|N_{G}(y) \cap T_{j}\right| \leq(a-m)\left|N_{G}(y)\right| \leq(a-m) m
$$

and so

$$
\sum_{j=1}^{a-1}(a-j)\left|D \cap T_{j}\right| \leq \sum_{j=1}^{a-1}(a-j)\left|D^{\prime} \cap T_{j}\right|+(a-m) m
$$




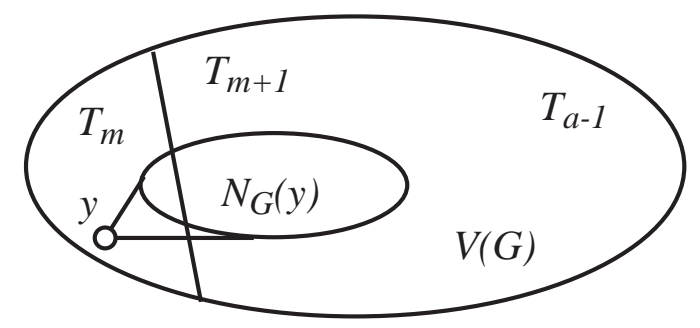

Figure 3.11: The vertex $y$ and $N_{G}(y)$ of $G$.

Therefore

$$
\sum_{j=1}^{a-1}(a-j)\left|D \cap T_{j}\right| \leq \sum_{j=1}^{a-1} j(a-j)\left|I \cap T_{j}\right| .
$$

Lemma 3.3.23 If $G$ is a connected non-complete graph, then $2 \cdot \operatorname{tough}(G) \leq$ $\delta(G)$, in particular, if tough $(G) \geq 1$, then tough $(G)+1 \leq \delta(G)$.

Proof. Let $v$ be a vertex of $G$ with $\operatorname{deg}_{G}(v)=\delta(G)$. Since $G$ is not a complete graph, we have $N_{G}(v) \neq V(G)-v$. Then $\omega\left(G-N_{G}(v)\right) \geq 2$, and so

$$
2 \operatorname{tough}(G) \leq \omega\left(G-N_{G}(v)\right) \operatorname{tough}(G) \leq\left|N_{G}(v)\right|=\operatorname{deg}_{G}(v)=\delta(G) .
$$

Hence $2 \operatorname{tough}(G) \leq \delta(G)$. The latter part is immediate by this inequality.

Proof of Theorem 3.3.21 in the case $a \geq 2$. By Lemma 3.3.23, a complete graph trivially has the desired $[a, b]$-factor as $\delta(G) \geq a+1$. Hence we may assume that $G$ is not a complete graph. Let $\emptyset \neq S \subset V(G)$. By Theorem 3.3.2, it suffices to show that

$$
\sum_{j=0}^{a-1}(a-j) n_{j}(G-S) \leq b|S|
$$

where $n_{j}(G-S)$ denotes the number of vertices in $G-S$ with degree $j$. Let

$$
\begin{aligned}
T & =\left\{x \mid x \in V(G)-S \text { and } 1 \leq \operatorname{deg}_{G-S}(x) \leq a-1\right\}, \\
T_{j} & =\left\{x \mid x \in T \text { and } \operatorname{deg}_{G-S}(x)=j\right\} \quad(1 \leq j \leq a-1), \text { and } \\
H & =\langle T\rangle_{G} .
\end{aligned}
$$


Then $T_{1} \cup T_{2} \cup \cdots \cup T_{a-1}$ is a partition of $T=V(H)$ such that $\operatorname{deg}_{H}(x) \leq j$ for all $x \in T_{j} \quad(1 \leq j \leq a-1)$. Note that we may assume $T \neq \emptyset$ since otherwise (3.47) holds as $a \cdot n_{0}(G-S) \leq a \leq b|S|$ if $n_{0}(G-S) \leq 1$, and

$$
a \cdot n_{0}(G-S) \leq a \omega(G-S) \leq \frac{a|S|}{\operatorname{tough}(G)} \leq b|S| \quad \text { if } n_{0}(G-S) \geq 2 .
$$

By Lemma 3.3.22, there exist a covering vertex subset $D$ and an independent vertex subset $I$ of $H$ such that $V(T)=D \cup I$ and

$$
\sum_{j=1}^{a-1}(a-j)\left|D \cap T_{j}\right| \leq \sum_{j=1}^{a-1} j(a-j)\left|I \cap T_{j}\right| .
$$

It is obvious that $I \neq \emptyset$, and it follows that

$$
\begin{aligned}
& \sum_{j=0}^{a-1}(a-j) n_{j}(G-S)=a \cdot n_{0}(G-S)+\sum_{j=1}^{a-1}(a-j)\left|T_{j}\right| \\
& =a \cdot n_{0}(G-S)+\sum_{j=1}^{a-1}(a-j)\left(\left|I \cap T_{j}\right|+\left|D \cap T_{j}\right|\right) \\
& \leq a \cdot n_{0}(G-S)+\sum_{j=1}^{a-1}(a-j)\left|I \cap T_{j}\right| \\
& +\sum_{j=1}^{a-1} j(a-j)\left|I \cap T_{j}\right| \quad(\text { by }(3.48)) \\
& =a \cdot n_{0}(G-S)+\sum_{j=1}^{a-1}(a-j)(1+j)\left|I \cap T_{j}\right| .
\end{aligned}
$$

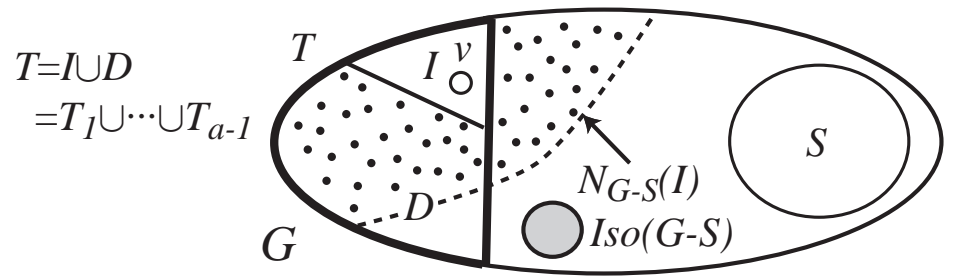

Figure 3.12: $T=I \cup D, I \cap D=\emptyset$ and $X=S \cup N_{G-S}(I)$.

Let $X=S \cup N_{G-S}(I)$ (see Figure 3.12). Then

$$
|X| \leq|S|+\sum_{x \in I} \operatorname{deg}_{G-S}(x)=|S|+\sum_{j=1}^{a-1} j\left|I \cap T_{j}\right|,
$$


and

$$
\omega(G-X) \geq|I \cup I s o(G-S)|=\sum_{j=1}^{a-1}\left|I \cap T_{j}\right|+n_{0}(G-S) .
$$

If $\omega(G-X) \geq 2$, then we have

$$
|X| \geq \operatorname{tough}(G) \omega(G-X) .
$$

If $\omega(G-X)=1$, then for a vertex $v \in I$, we have by Lemma 3.3.23 that

$$
|X| \geq|S|+\left|N_{G-S}(v)\right| \geq \operatorname{deg}_{G}(v) \geq \delta(G) \geq \operatorname{tough}(G) \omega(G-X) .
$$

Therefore in any case, by (3.50), (3.51) and by $|X| \geq \operatorname{tough}(G) \omega(G-X)$, we obtain

$$
|S|+\sum_{j=1}^{a-1} j\left|I \cap T_{j}\right| \geq|X| \geq\left(a+\frac{a}{b}-1\right)\left(\sum_{j=1}^{a-1}\left|I \cap T_{j}\right|+n_{0}(G-S)\right) .
$$

Thus

$$
b|S| \geq(a b+a-b) n_{0}(G-S)+\sum_{j=1}^{a-1}(a b+a-b-b j)\left|I \cap T_{j}\right| .
$$

Hence in order to prove (3.47), by the above inequality and (3.49), it suffices to show that

$$
\begin{aligned}
& a \cdot n_{0}(G-S)+\sum_{j=1}^{a-1}(a-j)(1+j)\left|I \cap T_{j}\right| \\
\leq & (a b+a-b) n_{0}(G-S)+\sum_{j=1}^{a-1}(a b+a-b-b j)\left|I \cap T_{j}\right| .
\end{aligned}
$$

It is clear that $a \leq a b+a-b$ as $2 \leq a<b$, and the desired inequality

$$
(a-j)(1+j) \leq a b+a-b-b j
$$

follows from

$$
(a b+a-b-b j)-(a-j)(1+j)=(a-j-1)(b-j) \geq 0
$$

as $1 \leq j \leq a-1$. Consequently (3.51) holds, and thus the theorem is proved. 
Theorem 3.3.24 (Y. Li and M. Cai [94]) Let $G$ be a simple connected graph, and $a$ and $b$ be integers such that $1 \leq a<b$. If $\delta(G) \geq a,|G| \geq 3 a+b$ and

$$
\max \left\{\operatorname{deg}_{G}(x), \operatorname{deg}_{G}(y)\right\} \geq \frac{a|G|}{a+b}
$$

for any two non-adjacent vertices $x$ and $y$ of $G$, then $G$ has an $[a, b]$-factor.

Proof. Suppose that a simple $G$ satisfies the conditions of the theorem, but has no $[a, b]$-factors. By the $[a, b]$-Factor Theorem and by Lemma 3.3.3, there exist disjoint subsets $S$ and $T \neq \emptyset$ of $V(G)$ such that

$$
\begin{aligned}
& b|S|+\sum_{x \in T} \operatorname{deg}_{G-S}(x)-a|T|<0, \text { and } \\
& 0 \leq \operatorname{deg}_{G-S}(x) \leq a-1 \quad \text { for all } x \in T .
\end{aligned}
$$

Define

$$
h_{1}=\min \left\{\operatorname{deg}_{G-S}(x) \mid x \in T\right\},
$$

and choose a vertex $v_{1} \in T$ such that $\operatorname{deg}_{G-S}\left(v_{1}\right)=h_{1}$. Then, if $T \neq N_{T}\left[v_{1}\right]$, where $N_{T}\left[v_{1}\right]=\left(N_{G}\left(v_{1}\right) \cap T\right) \cup\left\{v_{1}\right\}$, define

$$
h_{2}=\min \left\{\operatorname{deg}_{G-S}(x) \mid x \in T-N_{T}\left[v_{1}\right]\right\},
$$

and choose a vertex $v_{2} \in T-N_{T}\left[v_{1}\right]$ satisfying $\operatorname{deg}_{G-S}\left(v_{2}\right)=h_{2}$. Then

$$
h_{1} \leq h_{2} \leq a-1 \quad \text { and } \quad \operatorname{deg}_{G}\left(v_{i}\right) \leq|S|+h_{i} \quad \text { for } \quad i=1,2 .
$$

If $T \neq N_{T}\left[v_{1}\right]$, then since $v_{1}$ and $v_{2}$ are not adjacent in $G$, we have

$$
|S|+h_{2} \geq \max \left\{\operatorname{deg}_{G}\left(v_{1}\right), \operatorname{deg}_{G}\left(v_{2}\right)\right\} \geq \frac{a|G|}{a+b} .
$$

We consider the following two cases.

Case 1. $T=N_{T}\left[v_{1}\right]$.

In this case, we obtain $|T|=\left|N_{T}\left[v_{1}\right]\right| \leq \operatorname{deg}_{G-S}\left(v_{1}\right)+1 \leq h_{1}+1 \leq a<b$. Then we have

$$
\begin{aligned}
0 & >b|S|+\sum_{x \in T} \operatorname{deg}_{G-S}(x)-a|T| \\
& \geq b|S|+\left(h_{1}-a\right)|T| \\
& \geq b\left(a-h_{1}\right)+\left(h_{1}-a\right)|T| \quad\left(\text { by } a \leq \delta(G) \leq \operatorname{deg}_{G}\left(v_{1}\right) \leq|S|+h_{1}\right) \\
& =\left(a-h_{1}\right)(b-|T|) \\
& \geq 1 .
\end{aligned}
$$


This is a contradiction.

Case 2. $T \neq N_{T}\left[v_{1}\right]$.

Let $p=\left|N_{T}\left[v_{1}\right]\right|$. Then $1 \leq p<|T|$ and $p \leq h_{1}+1$. It follows that

$$
(|G|-|S|-|T|)\left(a-h_{2}\right) \geq 0 \text {. }
$$

Moreover, we have

$$
\begin{aligned}
0 & >b|S|+\sum_{x \in T} \operatorname{deg}_{G-S}(x)-a|T| \\
& \geq b|S|+h_{1} p+h_{2}(|T|-p)-a|T| \\
& =b|S|+\left(h_{1}-h_{2}\right) p+\left(h_{2}-a\right)|T| \\
& \geq b|S|+\left(h_{1}-h_{2}\right)\left(h_{1}+1\right)+\left(h_{2}-a\right)|T| . \quad\left(\text { by } h_{1} \leq h_{2}\right)
\end{aligned}
$$

By the previous two inequalities, we obtain

$$
\begin{aligned}
& (|G|-|S|-|T|)\left(a-h_{2}\right)>b|S|+\left(h_{1}-h_{2}\right)\left(h_{1}+1\right)+\left(h_{2}-a\right)|T| \\
& \left(a-h_{2}\right)|G|-\left(a+b-h_{2}\right)|S|>\left(h_{1}-h_{2}\right)\left(h_{1}+1\right) .
\end{aligned}
$$

By (3.56), we have $|S|-a|G| /(a+b) \geq-h_{2}$. Combining this inequality and $a+b-h_{2} \geq 1$, we have

$$
\left(|S|-\frac{a|G|}{a+b}\right)\left(a+b-h_{2}\right) \geq-h_{2}\left(a+b-h_{2}\right) .
$$

Since $|G| \geq 3 a+b$, we have

$$
\frac{b|G|}{a+b} \geq \frac{b(3 a+b)}{a+b} \geq a+b
$$

and hence

$$
h_{2} \frac{b|G|}{a+b} \geq h_{2}(a+b)
$$

If we add the three inequalities (3.57), (3.58) and (3.59), then the left handside becomes 0 , and therefore

$$
\begin{aligned}
0 & >\left(h_{1}-h_{2}\right)\left(h_{1}+1\right)-h_{2}\left(a+b-h_{2}\right)+h_{2}(a+b) . \\
& =h_{1}^{2}-\left(h_{2}-1\right) h_{1}+h_{2}^{2}-h_{2} .
\end{aligned}
$$

The right hand-side of the above inequality is a function of $h_{1}$, and takes the minimum value at $h_{1}=\left(h_{2}-1\right) / 2$. Substituting $h_{1}=\left(h_{2}-1\right) / 2$ into the 
function, we have

$$
\begin{aligned}
-1 & \geq h_{1}^{2}-\left(h_{2}-1\right) h_{1}+h_{2}^{2}-h_{2} \\
& \geq-\frac{1}{4}\left(h_{2}-1\right)^{2}+h_{2}^{2}-h_{2} \\
& =\frac{1}{4}\left(3 h_{2}^{2}-2 h_{2}-1\right)=\frac{1}{4}\left(3\left(h_{2}-\frac{1}{3}\right)^{2}-\frac{2}{3}\right)>-1 .
\end{aligned}
$$

This is a contradiction, and consequently the theorem is proved.

We now show some other results on $[a, b]$-factors without proof.

Theorem 3.3.25 (C. Chen and G. Liu and [31]) Let $G$ be connected simple graph, and $a$ and $b$ be integers such that $2 \leq a<b$. If

$$
\operatorname{tough}(G) \geq a+\frac{a}{b}-1,
$$

then for any edge e of $G, G$ has an $[a, b]$-factor including $e$ and another $[a, b]$-factor excluding $e$.

We say that a graph $G$ is $(a, b ; k)$-critical if for every vertex subset $X \subset G$ with $|X|=k, G-X$ has an $[a, b]$-factor.

Theorem 3.3.26 (G. Liu and J. Wang [93] (1998)) Let G be connected simple graph, and $a, b$ and $k$ be integers such that $1 \leq a<b$ and $1 \leq k$. Then $G$ is $(a, b ; k)$-critical if and only if

$$
\sum_{j=0}^{a-1}(a-j) n_{j}(G-S) \leq b|S|-b k
$$

for all subset $S \subset V(G)$ with $|S| \geq k$.

Corollary 3.3.27 (G. Liu and J. Wang [93] (1998)) Let G be connected simple graph, and let $k, a, b, m, n$ be integers such that $1 \leq a<b \leq n$ and $1 \leq k$. If

$$
\frac{a}{b} \leq \frac{m-k}{n}
$$

then every $[m, n]$-graph is $(a, b ; k)$-critical.

Theorem 3.3.28 (G. Liu and J. Wang [93] (1998)) Let G be connected simple graph, and $a, b$ and $k$ be integers such that $1 \leq a<b, m \leq n$ and $1 \leq k$. Then $G$ is $(a, b ; k)$-critical if and only if

$$
\sum_{j=0}^{a-1}(a-j) n_{j}(G-S) \leq b|S|-b k
$$

for all subsets $S \subset V(G)$ with $|S| \geq k$. 
We conclude this section with the following theorem on semi-regular factors of regular graphs, which gives an additional property to Theorem 3.3.8 saying that every $r$-regular graph has a $[k-1, k]$-factor. Notice that by Petersen's 2-factorable Theorem 2.1.1, for every integer $1 \leq k<2 r$, every $2 r$-regular graph has a $k$ or $(k-1)$-regular factor, which is obviously a $[k-1, k]$-factor each of whose components is regular. So we may consider only odd regular graphs for such factors in regular graphs.

Theorem 3.3.29 (Kano [69] (1986)) Let $r \geq 3$ be an odd integer and $k$ an integer such that $0<k \leq 2 r / 3$. Then every $r$-regular multigraph has a $[k-1, k]$-factor each of whose components are regular.

In order to prove the above theorem, we need the following theorem.

Theorem 3.3.30 (Kano [68] (1985)) Let $G$ be a $n$-connected multigraph $(n \geq$ $1)$, $\theta$ be a real number such that $0 \leq \theta \leq 1$, and $f: V(G) \rightarrow\{0,2,4,6, \ldots\}$. If the following two conditions hold, then $G$ has an $f$-factor.

(i) $\epsilon=\sum_{x \in V(G)}\left|f(x)-\theta \operatorname{deg}_{G}(x)\right|<2$.

(ii) $m(1-\theta) \geq 1$, where $m \in\{n, n+1\}$ and $m \equiv 1(\bmod 2)$.

Proof. Let $S$ and $T$ be disjoint subsets of $V(G)$. By the $f$-Factor Theorem 2.1.2, it suffices to show that $\delta(S, T) \geq 0$. Since $G$ is connected and $\sum_{x \in V(G)} f(x) \equiv 0(\bmod 2)$, we have $\delta(\emptyset, \emptyset)=0$. So we may assume that $S \cup T \neq \emptyset$. Let $\ell=q(S, T)$, and $C_{1}, C_{2}, \ldots, C_{\ell}$ be the $f$-odd components of $G-(S \cup T)$. Then for every $f$-odd component $C_{i}$, we have

$$
\sum_{x \in V\left(C_{i}\right)} f(x)+e_{G}\left(C_{i}, T\right) \equiv e_{G}\left(C_{i}, T\right) \equiv 1(\bmod 2) .
$$

Hence $e_{G}\left(C_{i}, T\right) \geq 1$. If $e_{G}\left(S, C_{i}\right)=0$, then $n \leq e_{G}\left(C_{i}, S \cup T\right)=e_{G}\left(C_{i}, T\right)$, and so $e_{G}\left(C_{i}, T\right) \geq m$, where $m$ is defined in the condition (ii). It follows that

$$
\begin{aligned}
\delta(S, T)= & \sum_{x \in S} f(x)+\sum_{x \in T}\left(\operatorname{deg}_{G}(x)-f(x)\right)-e_{G}(S, T)-q(S, T) \\
= & \theta \sum_{x \in S} \operatorname{deg}_{G}(x)+(1-\theta) \sum_{x \in T} \operatorname{deg}_{G}(x)-e_{G}(S, T)-\ell \\
& +\sum_{x \in S}\left(f(x)-\theta \operatorname{deg}_{G}(x)\right)-\sum_{x \in T}\left(f(x)-\theta \operatorname{deg}_{G}(x)\right) \\
\geq & \theta\left(e_{G}(S, T)+\sum_{i=1}^{\ell} e_{G}\left(S, C_{i}\right)\right)
\end{aligned}
$$




$$
\begin{aligned}
& +(1-\theta)\left(e_{G}(T, S)+\sum_{i=1}^{\ell} e_{G}\left(T, C_{i}\right)\right) \\
& -e_{G}(S, T)-\ell-\sum_{x \in S \cup T}\left|f(x)-\theta \operatorname{deg}_{G}(x)\right| \\
\geq & \left.\sum_{i=1}^{\ell}\left(\theta e_{G}\left(S, C_{i}\right)+(1-\theta)\left(T, C_{i}\right)\right)-1\right)-\epsilon .
\end{aligned}
$$

If $e_{G}\left(S, C_{i}\right) \geq 1$, then the following holds since $e_{G}\left(T, C_{i}\right) \geq 1$.

$$
\left.\theta e_{G}\left(S, C_{i}\right)+(1-\theta) e_{G}\left(T, C_{i}\right)\right)-1 \geq \theta+(1-\theta)-1=0 .
$$

If $e_{G}\left(S, C_{i}\right)=0$, then $\left.e_{G}\left(T, C_{i}\right)\right) \geq m$ as shown above, and so by condition (ii), we have

$$
\left.\theta e_{G}\left(S, C_{i}\right)+(1-\theta) e_{G}\left(T, C_{i}\right)\right)-1 \geq(1-\theta) m-1 \geq 0 .
$$

Therefore, $\delta(S, T) \geq-\epsilon>-2$ by (i), which implies $\delta(S, T) \geq 0$ as $\delta(S, T) \equiv 0$ $(\bmod 2)$ (see $(2.4))$. Therefore $G$ has an $f$-factor.

For a set $\mathcal{I}$ of integers, an $\mathcal{I}$-factor of a graph $G$ is a spanning subgraph $F$ of $G$ such that

$$
\operatorname{deg}_{F}(x) \in \mathcal{I} \quad \text { for all } x \in V(G) .
$$

Analogously, an $\mathcal{I}$-graph can be defined as a graph $G$ with $\operatorname{deg}_{F}(x) \in \mathcal{I}$ for every $x \in V(G)$.

Lemma 3.3.31 Let $r \geq 3$ be an odd integer, and $G$ be a 2-edge connected $r$-regular multigraph. Then for every even integer $h, 2 \leq h \leq 2 r / 3, G$ has a h-regular factor. Moreover, for every odd integer $h^{\prime}, r / 3 \leq h^{\prime} \leq r, G$ has a $h^{\prime}$-regular factor. In particular, for every integer $k, 1 \leq k \leq r, G$ has a $[k-1, k]$-factor each of whose components is regular.

Proof. This lemma follows immediately from (5) of Theorem 2.2.1. This can also be proved by using the above Theorem 3.3.30. Define $\theta=h / r$ and $f(x)=h$ for all $x \in V(G)$. Then $\epsilon=0, m=3$ and $m(1-\theta)=3(1-h / r) \geq 1$ as $h \leq 2 r / 3$. Hence $G$ has an $h$-factor, which is the desired $h$-regular factor $F$. It is clear that $G-F$ is a $(r-h)$-regular factor, which is the desired $h^{\prime}$-regular factor of $G$. 
Lemma 3.3.32 Let $r \geq 3$ be an odd integer, and $G$ be a 2-edge connected $[r-1, r]$-multigraph having exactly one vertex $w$ of degree $r-1$. Then

(i) for every even integer $h, 2 \leq h \leq 2 r / 3, G$ has a $h$-regular factor; and

(ii) for every odd integer $h^{\prime}, r / 3 \leq h^{\prime} \leq r, G$ has a $\left[h^{\prime}-1, h^{\prime}\right]$-factor $F$ such that $\operatorname{deg}_{F}(w)=h^{\prime}-1$ and $\operatorname{deg}_{F}(x)=h^{\prime}$ for every $x \in V(G)-\{w\}$.

Proof. We prove the lemma by using Theorem 3.3.30. We first prove (i). Define $\theta=h / r$ and a function $f$ by $f(x)=h$ for every $x \in V(G)$. Then

$$
\begin{aligned}
\epsilon & =\sum_{x \in V(G)}\left|f(x)-\theta \operatorname{deg}_{G}(x)\right|=\left|f(w)-\theta \operatorname{deg}_{G}(w)\right| \\
& =h-\theta(r-1)=\frac{h}{r}<1 .
\end{aligned}
$$

Moreover, $m=3$, as is defined in (ii) in Theorem 3.3.30, and so

$$
m(1-\theta)=3\left(1-\frac{h}{r}\right) \geq 3\left(1-\frac{2}{3}\right)=1 .
$$

Hence $G$ has an $f$-factor, which is a $h$-regular factor.

We next prove (ii). Let $F$ be a $h$-regular factor obtained in (i). Then $G-F$ is the desired factor of $G$.

Lemma 3.3.33 Let $r \geq 3$ be an odd integer, and $G$ be a 2-edge connected $r$-regular multigraph having at least two bridges. Then for every integer $k,(r / 3)+1 \leq k \leq 2 r / 3, G$ has a $[k-1, k]$-factor each of whose components is regular.

Proof. We first prove that for every odd integer $k, r / 3 \leq k \leq 2 r / 3, G$ has a $[k-1, k]$-factor with regular components, that is, we first show that the lemma holds for an odd integer $k$.

Since $G$ has at least two bridges, for any bridge $v w \in E(G), G-v w$ has at most one 2-edge connected component. Let $v_{1} w_{1}, v_{2} w_{2}, \ldots, v_{s} w_{s}$ be the bridges of $G$ such that $G-v_{i} w_{i}$ has exactly one 2-edge connected component $D_{i}$ (see Figure 3.13). We may assume $w_{i}$ is contained in $D_{i}$ for every $1 \leq$ $i \leq s$. Let

$$
H=G-\bigcup_{i=1}^{s}\left(V\left(D_{i}\right)-\left\{w_{i}\right\}\right)
$$

Then $H$ is a $\{1, r\}$-graph whose endvertices are $w_{1}, w_{2}, \ldots, w_{s}$. 

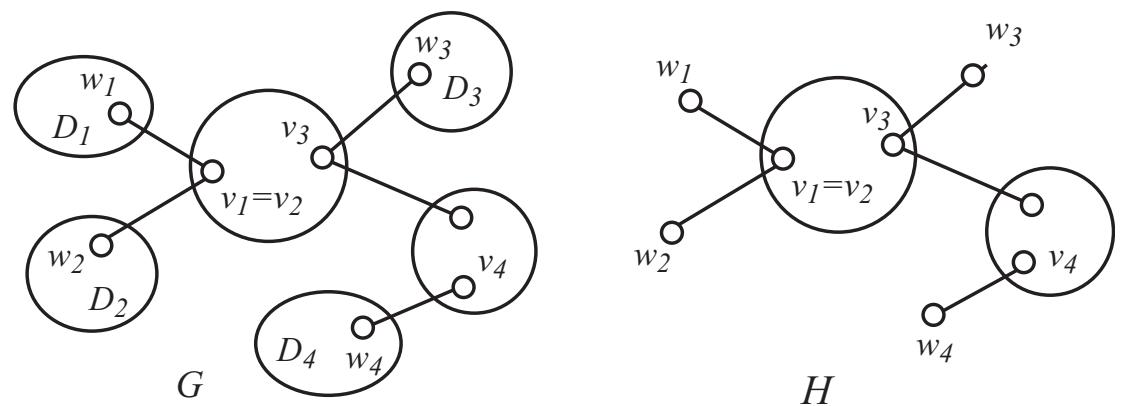

Figure 3.13: A 2-edge connected $r$-regular multigraph $G$ with at least two bridges; and the subgraph $H$.

We now show that $H$ has $\{0,1, k\}$-factor $F$ such that

$$
\begin{aligned}
\operatorname{deg}_{F}(x) & =k & & \text { if } x \in V(H)-\left\{w_{1}, \ldots, w_{s}\right\} ; \text { and } \\
\operatorname{deg}_{F}\left(w_{i}\right) & \in\{0,1\} & & \text { for every } 1 \leq i \leq s .
\end{aligned}
$$

Define two functions $g$ and $f$ on $V(H)$ by

$$
\begin{aligned}
& g(x)= \begin{cases}k & \text { if } x \in V(H)-\left\{w_{1}, \ldots, w_{s}\right\} \\
0 & \text { otherwise, }\end{cases} \\
& f(x)= \begin{cases}k & \text { if } x \in V(H)-\left\{w_{1}, \ldots, w_{s}\right\} \\
1 & \text { otherwise. }\end{cases}
\end{aligned}
$$

Then a $(g, f)$-factor is the desired factor $F$ of $H$.

We apply the $(g, f)$-Factor Theorem 3.1.1. It suffices to show that $\gamma(S, T) \geq$ 0 for all disjoint subsets $S$ and $T$ of $V(H)$. By the existence of $w_{1}$, we have $\gamma(\emptyset, \emptyset)=0$. Suppose that $\gamma(S, T)<0$ for some $S$ and $T$. Choose such a pair $(S, T)$ so that $|S \cup T|$ is minimum. Let $C_{1}, C_{2}, \ldots, C_{m}$ be the $(g, f)$-odd components of $H-(S \cup T)$, where $m=q^{*}(S, T)$. Then $g(x)=f(x)=k$ for all $x \in V\left(C_{i}\right)$ and

$$
\begin{gathered}
\sum_{x \in V\left(C_{i}\right)} f(x)+e_{H}\left(C_{i}, T\right) \\
\equiv\left|C_{i}\right|+e_{H}\left(C_{i}, T\right) \equiv 1(\bmod 2) .
\end{gathered}
$$

In particular, $C_{i}$ contains no endvertices $w_{j}, 1 \leq j \leq s$.

Assume that $S$ contains an endvertex $w=w_{j}$. Let $v=v_{j}$ be the vertex adjacent to $w$ in $H$. Then

$$
\gamma(S-w, T)=\sum_{x \in S-w} f(x)+\sum_{x \in T}\left(\operatorname{deg}_{H}(x)-g(x)\right)
$$




$$
\begin{aligned}
& -e_{H}(S-w, T)-q^{*}(S-w, T) \\
= & \gamma(S, T)-f(w)+e_{H}(w, T)+q^{*}(S, T)-q^{*}(S-w, T) \\
\leq & \gamma(S, T)-1+e_{H}(w, T)+q^{*}(S, T)-q^{*}(S-w, T) .
\end{aligned}
$$

If $v \in T$, then $e_{H}(w, T)=1$ and $q^{*}(S-w, T)=q^{*}(S, T)$. Thus $\gamma(S-w, T)$ $=\gamma(S, T)<0$, which contradicts the choice of $(S, T)$. If $v \in S$, then $e_{H}(w, T)$ $=0$ and $q^{*}(S-w, T)=q^{*}(S, T)$, and so $\gamma(S-w, T)=\gamma(S, T)-1<0$, a contradiction. If $v$ is not contained in $S \cup T$, then $q^{*}(S, T)-q^{*}(S-w, T) \leq 1$, and so $\gamma(S-w, T)<0$, a contradiction. Therefore $S$ contains no $w_{i}, 1 \leq$ $i \leq s$.

Assume that $T$ contains an endvertex $w=w_{j}$. Then

$$
\gamma(S, T-w)=\gamma(S, T)-1+e_{H}(S, w)+q^{*}(S, T)-q^{*}(S, T-w) .
$$

If $v=v_{j} \in T$, then $\gamma(S, T-w)=\gamma(S, T)-1$, a contradiction. If $v \in S$, then $\gamma(S, T-w)=\gamma(S, T)$, a contradiction. If $v \notin S \cup T$, then $\gamma(S, T-w) \leq$ $\gamma(S, T)$ as $q^{*}(S, T)-q^{*}(S, T-w) \leq 1$, which is again a contradiction. Hence $T$ contains no $w_{i}, 1 \leq i \leq s$.

We now consider a $(g, f)$-odd component $C_{i}$ of $H-(S \cup T)$. It is clear that $e_{H}\left(C_{i}, S \cup T\right) \geq 1$. If $e_{H}\left(C_{i}, S \cup T\right)=1$, then $C_{i}$ contains at least one vertex in $\left\{w_{1}, w_{2}, \ldots, w_{s}\right\}$, which contradicts the fact that $g(x)=f(x)$ for all $x \in V\left(C_{i}\right)$. Hence

$$
e_{G}\left(C_{i}, S \cup T\right) \geq 2 \text {. }
$$

Let $\theta=k / r$. Then $0<\theta<1$, and since $S \cup T$ contains no endvertices $w_{j}$, we have

$$
\begin{aligned}
\gamma(S, T)= & \sum_{x \in S} f(x)+\sum_{x \in T}\left(\operatorname{deg}_{H}(x)-g(x)\right)-e_{H}(S, T)-q^{*}(S, T) \\
= & k|S|+(r-k)|T|-e_{H}(S, T)-m \\
= & \theta \sum_{x \in S} \operatorname{deg}_{H}(x)+(1-\theta) \sum_{x \in T} \operatorname{deg}_{H}(x)-e_{H}(S, T)-m \\
\geq & \theta\left(e_{H}(S, T)+\sum_{i=1}^{m} e_{H}\left(S, C_{i}\right)\right) \\
& +(1-\theta)\left(e_{H}(T, S)+\sum_{i=1}^{m} e_{H}\left(T, C_{i}\right)\right)-e_{H}(S, T)-m \\
\geq & \sum_{i=1}^{m}\left(\theta e_{H}\left(S, C_{i}\right)+(1-\theta) e_{H}\left(T, C_{i}\right)-1\right) .
\end{aligned}
$$

Hence it suffices to show that for every $C=C_{i}$,

$$
\theta e_{H}(S, C)+(1-\theta) e_{H}(T, C)-1 \geq 0 .
$$


If $e_{H}(S, C) \geq 1$ and $e_{H}(T, C) \geq 1$, then (3.63) obviously holds. Since $r$ is odd, we have

$$
|C| \equiv r|C|=\sum_{x \in V(C)} \operatorname{deg}_{H}(x)=2|| C||+e_{H}(C, S \cup T)(\bmod 2) .
$$

Assume $e_{H}(S, C)=0$. Then by (3.64) we have $|C| \equiv e_{H}(C, T)(\bmod 2)$. But this contradicts (3.61). Next suppose $e_{H}(T, C)=0$. Then by (3.64) and (3.61), we have

$$
e_{H}(S, C) \equiv|C| \equiv 1(\bmod 2)
$$

Thus $e_{H}(S, C) \geq 3$ by (3.62). Therefore

$$
\theta e_{H}(S, C)-1 \geq \frac{k}{r} \cdot 3-1 \geq 0 . \quad(\text { by } k \geq r / 3)
$$

Consequently $H$ has the desired factor $F$.

We are ready to construct the desired factor of $G$. If $\operatorname{deg}_{F}\left(w_{i}\right)=0$, then by Lemma 3.3.32 we can take a $(k-1)$-regular factor $R(i)$ of $D_{i}$. If $\operatorname{deg}_{F}\left(w_{i}\right)=1$, then by Lemma 3.3 .32 we can take a $[k-1, k]$-factor $R(i)$ of $D_{i}$ such that $\operatorname{deg}_{R(i)}\left(w_{i}\right)=k-1$ and $\operatorname{deg}_{R(i)}(x)=k$ for all $x \in V\left(D_{i}\right)-\left\{w_{i}\right\}$. Then the following set forms the desired $[k-1, k]$-factor of $G$ with regular components:

$$
\bigcup_{i=1}^{s} R_{i} \cup F .
$$

We next prove that for every even integer $k,(r / 3)+1 \leq k \leq 2 r / 3, G$ has a $[k-1, k]$-factor with regular components. Since $k-1$ is an odd integer satisfying $r / 3 \leq k-1 \leq 2 r / 3$, by the preceding result, the graph $H$ defined above has a $\{0,1, k-1\}$-factor $F$ such that

$$
\begin{aligned}
& \operatorname{deg}_{F}(x)=k-1 \quad \text { if } x \in V(H)-\left\{w_{1}, \ldots, w_{s}\right\} \text {; and } \\
& \operatorname{deg}_{F}\left(w_{i}\right) \in\{0,1\} \quad \text { for every } 1 \leq i \leq s \text {. }
\end{aligned}
$$

If $\operatorname{deg}_{F}\left(w_{i}\right)=0$, then by Lemma 3.3 .32 we can take a $k$-regular factor $R(i)$ of $D_{i}$. If $\operatorname{deg}_{F}\left(w_{i}\right)=1$, then by Lemma 3.3 .31 we can take a $[k-2, k-1]$ factor $R_{i}$ of $D_{i}$ such that $\operatorname{deg}_{R(i)}\left(w_{i}\right)=k-2$ and $\operatorname{deg}_{R(i)}(x)=k-1$ for all $x \in V\left(D_{i}\right)-\left\{w_{i}\right\}$. Then $\cup_{i=1}^{s} R(i) \cup F$ is the desired $[k-1, k]$-factor of $G$ with regular components. Consequently the lemma is proved.

Proof of Theorem 3.3.30. We shall prove the theorem by induction on $r$. Let $r \geq 3$ be an odd integer and $k$ be an integer such that $1 \leq k \leq 2 r / 3$. Let $G$ be a connected $r$-regular multigraph. Since every regular graph has a 0 -factor, which is a $[0,1]$-factor with regular components, we may assume 
$k \geq 2$. By Lemma 3.3.31, we may assume that $G$ is not 2-edge connected. Suppose that $G$ has exactly one bridge $v w$. Then each component $C$ of $G-v w$ is 2-edge connected, and so by Lemma 3.3.32, for an even integer $h \in\{k-1, k\}, C$ has an $h$-factor. Thus $G$ itself has an $h$-factor, which is the desired $[k-1, k]$-factor of $G$ with regular components. Hence we may assume that $G$ has at least two bridges.

We first show that if the theorem is true for $3 \leq r \leq 15$ (which will be shown later), then the theorem holds for every odd integer $r$. Let $r \geq 17$ be an odd integer. Let $r=2 s+1$, where $s \geq 8$. If $(r / 3)+1 \leq k \leq 2 r / 3$, then by Lemma 3.3.33, $G$ has a $[k-1, k]$-factor with regular components. So we may assume $2 \leq k<(r / 3)+1=(2 s+4) / 3$, which implies $k \leq(2 s+3) / 3$. Let $h$ be the greatest integer not exceeding $2 r / 3$. Then $(2 r / 3)-(2 / 3)=4 s / 3 \leq h$. Since

$$
\frac{r}{3}+1=\frac{2 s+4}{3} \leq h \leq \frac{2 r}{3}<r,
$$

by Lemma 3.3.33 $G$ has a $[h-1, h]$-factor $F_{1}$ with regular components. Since

$$
k \leq \frac{2 s+3}{3} \leq \frac{2(4 s-3)}{9} \leq \frac{2(h-1)}{3}, \quad(\text { by } s \geq 8)
$$

every odd-regular component $C$ of $F_{1}$, where $C$ is an $(h-1)$ - or $h$-regular graph, has a $[k-1, k]$-factor with regular components by the induction. On the other hand, every even-regular component of $F_{1}$ contains a regular $k$ or $(k-1)$-regular factor by Petersen's 2-Factorable Theorem. By combining these factors, we can obtain the desired $[k-1, k]$-factor of $G$ with regular components ([69]).

We finally show that the theorem holds for every odd integer $3 \leq r \leq 15$ by induction on $r$. We may assume that $G$ is an $r$-regular multigraph having at least two brides. We use Lemma 3.3.33 and the inductive hypothesis without mentioning them.

Case $r=3$. Since $(r / 3)+1 \leq 2 \leq 2 r / 3, G$ has a [1,2]-factor with regular components, and the theorem holds for $r=3$.

Case $r=5$. Since $(r / 3)+1 \leq 3 \leq 2 r / 3, G$ has a $[2,3]$-factor $F(2,3)$ with regular components. Every 3-regular component of $F(2,3)$ has a [1,2]-factor with regular components, and so $F(2,3)$, in particular, $G$ itself, has a [1,2]factor with regular components. Thus the theorem holds for $r=5$.

Case $r=7$. Since $(r / 3)+1 \leq 4 \leq 2 r / 3, G$ has a $[3,4]$-factor $F(3,4)$ with regular components. Every 4-regular component of $F(3,4)$ has a 2-factor, and so $F(3,4)$, in particular, $G$ itself, has a [2,3]-factor $F(2,3)$ with regular components. Since $F(2,3)$ has a $[1,2]$-factor with regular components, the theorem holds. 
Case $r=9$. Since $(r / 3)+1 \leq 4,5,6 \leq 2 r / 3, G$ has a $[k-1, k]$-factor $F(k-1, k)$ with regular components for $k \in\{4,5,6\}$. Since $F(3,4)$ has a $[2,3]$-factor with regular components, $G$ has such a factor. By the same argument as above, the theorem holds for $r=9$.

Case $r=11$. Since $(r / 3)+1 \leq 5,6,7 \leq 2 r / 3, G$ has a $[k-1, k]$-factor $F(k-1, k)$ with regular components for $k \in\{5,6,7\}$. Since every 7 -regular component of $F(6,7)$ has a [3,4]-factor with regular components and every 6-regular component of $F(6,7)$ has a 4-regular factor, $F(6,7)$, in particular, $G$ itself has a [3, 4]-factor with regular components. Since $F(3,4)$ has a $[2,3]$ factor $F(2,3)$ with regular components and $F(2,3)$ has a [1,2]-factor with regular components, $G$ has a $[k-1, k]$-factor with regular components for all $1 \leq k \leq 7$.

Case $r=13,15$. The proof of these case is left to the reader.

Consequently the proof is complete.

The sharpness of the upper bound $k \leq 2 r / 3$ has not yet been established. It is known that if an odd integer $r$ and an integer $k$ satisfy $r+1-\sqrt{r+1}<$ $k<r$, then there exists a simple connected $r$-regular graph that has no $[k-1, k]$-factor with regular components $([69])$. However, for other integers $k$, it is an open problem whether or not every $r$-regular graph has a $[k-1, k]$ factor with regular components. 


\section{Chapter 4}

\section{$[a, b]$-Factorizations}

Let $G$ be a graph, and $g, f: V(G) \rightarrow \mathbb{Z}$ be functions such that $g(x) \leq f(x)$ for all $x \in V(G)$. If the set of edges of $G$ can be decomposed into disjoint subsets

$$
E(G)=F_{1} \cup F_{2} \cup \cdots \cup F_{n}
$$

so that every $F_{i}$ induces a $(g, f)$-factor of $G$, then we say that $G$ is $(g, f)$ factorable, and the above decomposition is called an $(g, f)$-factorization of $G$. As special case of $(g, f)$-factorization, we can define 1-factorization, $k$-regular factorization, $[a, b]$-factorization and $f$-factorization. In this chapter, we mainly investigate $[a, b]$-factorizations of graphs. We begin with some basic results on factorizations of special graphs.

\subsection{Factorizations of Special Graphs}

A graph $G$ is said to be $\mathbf{1}$-factorable if $E(G)$ can be decomposed into disjoint 1-factors

$$
E(G)=F_{1} \cup F_{2} \cup \cdots \cup F_{n}, \quad \text { where every } F_{i} \text { is a } 1 \text {-factor of } G \text {. }
$$

The next theorem shows the existence of 1-factorizations of complete graphs with even order. The readers who are interested in this topics should refer to a survey [111] by Mendelsohn and Rosa.

Theorem 4.1.1 Let $n \geq 2$ be an even integer. Then the complete graph $K_{n}$ is 1-factorable.

Proof. We may assume $n \geq 4$. Let $V(G)=\left\{v_{0}, v_{1}, \cdots, v_{n-2}\right\} \cup\{w\}$. For every integer $0 \leq i \leq n-2$, let

$$
F_{i}=\left\{v_{i-1} v_{i+1}, v_{i-2} v_{i+2}, \ldots, v_{i-(n-2) / 2} v_{i+(n-2) / 2}\right\} \cup\left\{v_{i} w\right\}
$$


where the subscripts are expressed modulo $n-1$ (see Figure 4.1). Then we can obtain the desired 1-factorization $F_{0} \cup F_{1} \cup \cdots \cup F_{n-2}$ of $K_{n}$.
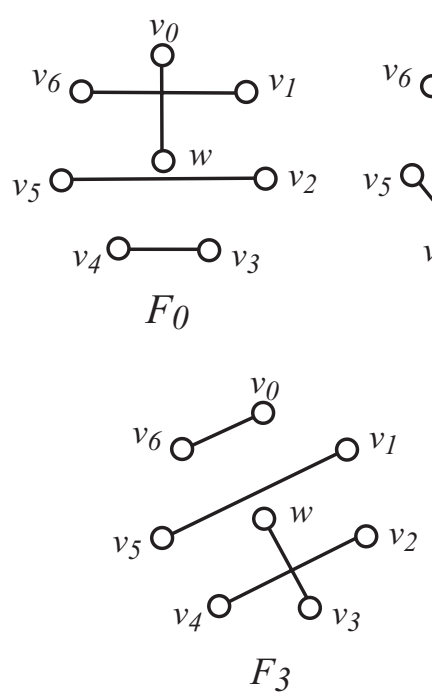
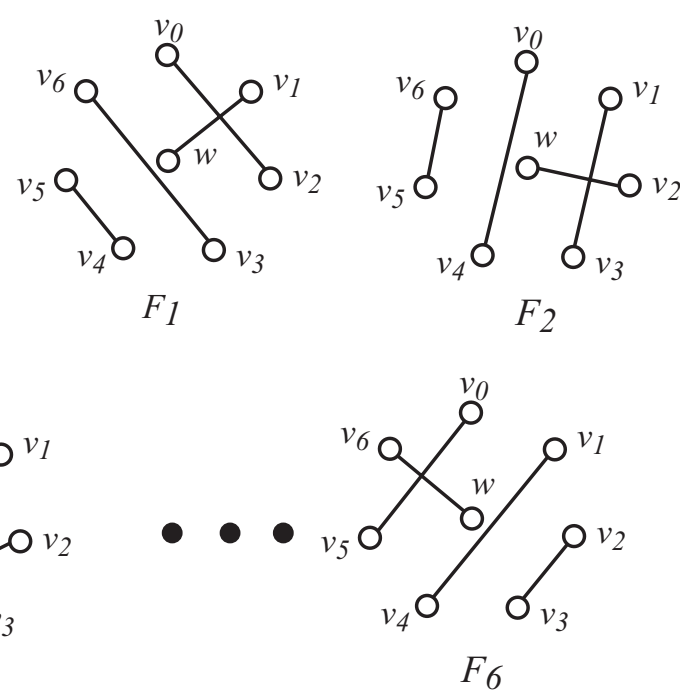

Figure 4.1: A 1-factorization of $K_{8}$.

The following theorem was obtained by König (see Theorem 1.1.2).

Theorem 4.1.2 Every regular bipartite multigraph is 1-factorable.

The following theorem is given in Theorem 2.1.1.

Theorem 4.1.3 (Petersen's 2-Factorable Theorem) Let $r \geq 2$ be an even integer. Then every $r$-regular general graph is 2-factorable.

It is known that the following theorem is equivalent to the Four Color Theorem. But here we prove the theorem by using the Four Color Theorem (Appel and Haken [13]; [129]).

Theorem 4.1.4 Every simple 2-connected planar cubic graph is 1-factorable.

Proof. Let $G$ be a simple 2-connected planar cubic graph, which is drawn in the plane. Then the plane is partitioned into faces, sometimes called regions. By the Four Color Theorem, we can color all the faces with four colors so that any two faces having an boundary edge in common have different colors. We denote these four colors by $00,01,10,11$. From this face-coloring, we can 
get an edge-coloring as follows: If an edge $e$ of $G$ is a boundary edge of two faces with colors $x$ and $y$, then $e$ is colored with $x+y(\bmod 2)$, where $x+y \in$ $\{00,01,10,11\}$ (see Figure 4.2). We shall show that $x+y \in\{01,10,11\}$ and for each color $c \in\{01,10,11\}$, the set of edges colored with $c$ forms a 1-factor of $G$.

Let $v$ be any vertex of $G$. Then there exist three edges $e_{1}, e_{2}, e_{3}$ incident with $v$, and three faces around $v$, which are colored with three distinct colors, say $r, s, t$. Then $r+s, s+t, t+r$ are all distinct and not equal to 00. Hence $e_{1}, e_{2}, e_{3}$ are colored with distinct colors of $\{01,10,11\}$, i.e., for every color $c \in\{01,10,11\}$, exactly one edge colored with $c$ is incident with $v$. Therefore, each color induces a 1 -factor of $G$.
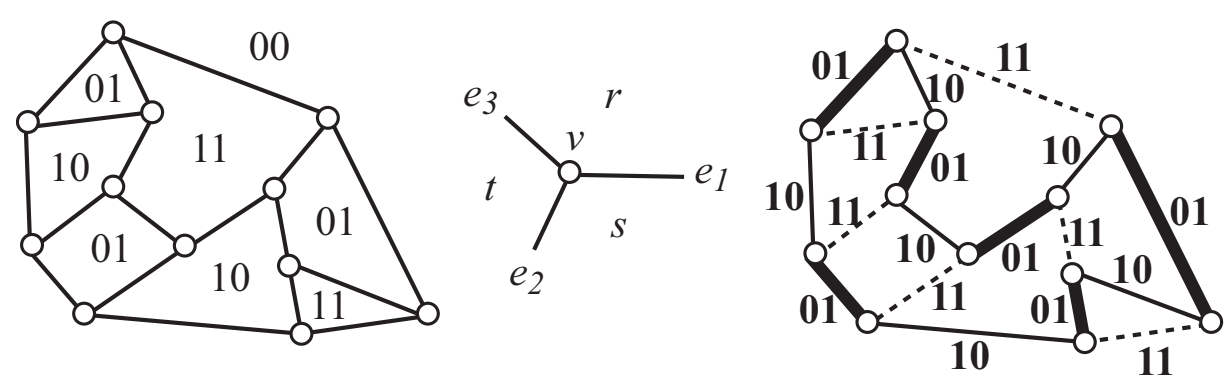

Figure 4.2: A face-coloring of a simple 2-connected cubic planar graph and the edge-coloring induced by it.

The following theorem says that a trivial necessary condition for the existence of a $(g, f)$-factorization is sufficient in bipartite graphs. Thus the $(g, f)$-factorization problem is completely solved for bipartite graphs.

Theorem 4.1.5 (Lovász [107](p.50, Problem 7.11)(1979)) Let $n \geq 1$ be an integer and $G$ be a bipartite multigraph. If two functions $g, f: V(G) \rightarrow \mathbb{Z}$ satisfy

$$
n g(x) \leq \operatorname{deg}_{G}(x) \leq n f(x) \quad \text { for all } \quad x \in V(G),
$$

then $G$ can be decomposed into $n(g, f)$-factors. Moreover, if the degree of every vertex is divisible by $n$, then for a function $h(x)=\operatorname{deg}_{G}(x) / n$ for all $x \in V(G), G$ can be decomposed into $n$ h-factors.

Note that if we define two functions $g, f: V(G) \rightarrow \mathbb{Z}$ as

$$
g(x)=\left\lfloor\frac{\operatorname{deg}_{G}(x)}{n}\right\rfloor, \quad f(x)=\left\lceil\frac{\operatorname{deg}_{G}(x)}{n}\right\rceil,
$$

for all $x \in V(G)$, then $g$ and $f$ always satisfy condition (4.1). 
Lemma 4.1.6 Let $G$ be a bipartite multigraph, and let $\theta$ be a real number such that $0<\theta<1$. If $g, f: V(G) \rightarrow \mathbb{Z}$ satisfy

$$
g(x) \leq \theta \operatorname{deg}_{G}(x) \leq f(x) \quad \text { for all } \quad x \in V(G),
$$

then $G$ has a $(g, f)$-factor.

Proof. Let $S$ and $T$ be disjoint subsets of $V(G)$. Then

$$
\begin{aligned}
\gamma^{*}(S, T) & =\sum_{x \in S} f(x)+\sum_{x \in T}\left(\operatorname{deg}_{G}(x)-g(x)\right)-e_{G}(S, T) \\
& \geq \theta \sum_{x \in S} \operatorname{deg}_{G}(x)+(1-\theta) \sum_{x \in T} \operatorname{deg}_{G}(x)-e_{G}(S, T) \\
& \geq \theta e_{G}(S, T)+(1-\theta) e_{G}(T, S)-e_{G}(S, T)=0 .
\end{aligned}
$$

Hence $\gamma^{*}(S, T) \geq 0$, and thus $G$ has a $(g, f)$-factor by Theorem 3.1.3.

Proof of Theorem 4.1.5 We prove the theorem by induction on $n$. For $n=1$, the theorem holds since $G$ itself is an $h$-factor and thus a $(g, f)$-factor. Hence we may assume $n \geq 2$. We first prove the latter part of the theorem. Assume that the degree of every vertex is divisible by $n$ and a function $h$ is defined by $h(x)=\operatorname{deg}_{G}(x) / n$ for all $x \in V(G)$. By Lemma 4.1.6 with $g(x)=f(x)=h(x), G$ has an $h$-factor $F$. Then every vertex $x$ of $G-F$ has degree $\operatorname{deg}_{G}(x)-h(x)=(n-1) h(x)$, which is a multiple of $n-1$. Hence by applying the inductive hypothesis to $G-F, G$ can decomposed into $n$ disjoint $h$-factors.

We now prove the first part by induction on $n$. For any vertex $x$ of $G$, define $g^{\prime}$ and $f^{\prime}$ as

$$
g^{\prime}(x)=\left\lfloor\frac{\operatorname{deg}_{G}(x)}{n}\right\rfloor, \quad f^{\prime}(x)=\left\lceil\frac{\operatorname{deg}_{G}(x)}{n}\right\rceil .
$$

Then $g^{\prime}(x) \leq \operatorname{deg}_{G}(x) / n \leq f^{\prime}(x)$ for all $x \in V(G)$. So by Lemma 4.1.6, $G$ has a $\left(g^{\prime}, f^{\prime}\right)$-factor $F$, which is of course a $(g, f)$-factor. We shall later show that

$$
(n-1) g^{\prime}(x) \leq \operatorname{deg}_{G-F}(x) \leq(n-1) f^{\prime}(x) \quad \text { for all } x \in V(G) .
$$

If (4.3) holds, then by induction, $G-F$ can be decomposed into $n-1\left(g^{\prime}, f^{\prime}\right)$ factors, and therefore $G$ is decomposed into $n\left(g^{\prime}, f^{\prime}\right)$-factors. Consequently the theorem is proved.

We now prove (4.3). Let

$$
\operatorname{deg}_{G}(x)=m n+t, \quad 0 \leq m \text { and } 0 \leq t<n .
$$


If $t=0$, then $g^{\prime}(x)=f^{\prime}(x)=m$ and so

$$
(n-1) g^{\prime}(x)=(n-1) m=\operatorname{deg}_{G-F}(x)=(n-1) f^{\prime}(x) .
$$

If $t \geq 1$, then $g^{\prime}(x)=m$ and $f^{\prime}(x)=m+1$. If $\operatorname{deg}_{F}(x)=m$, then

$$
(n-1) g^{\prime}(x) \leq \operatorname{deg}_{G-F}(x)=(n-1) m+t \leq(n-1) f^{\prime}(x) .
$$

If $\operatorname{deg}_{F}(x)=m+1$, then

$$
(n-1) g^{\prime}(x) \leq \operatorname{deg}_{G-F}(x)=(n-1) m+t-1 \leq(n-1) f^{\prime}(x) .
$$

Therefore (4.3) holds and the proof is complete.

Theorem 4.1.5 can be proved by using Theorem 4.1.3, which is an easy consequence of Hall's Marriage Theorem 1.1.1, i.e., we can prove Theorem 4.1.5 without using $(g, f)$-Factor Theorem, and we explain this elementary proof below.
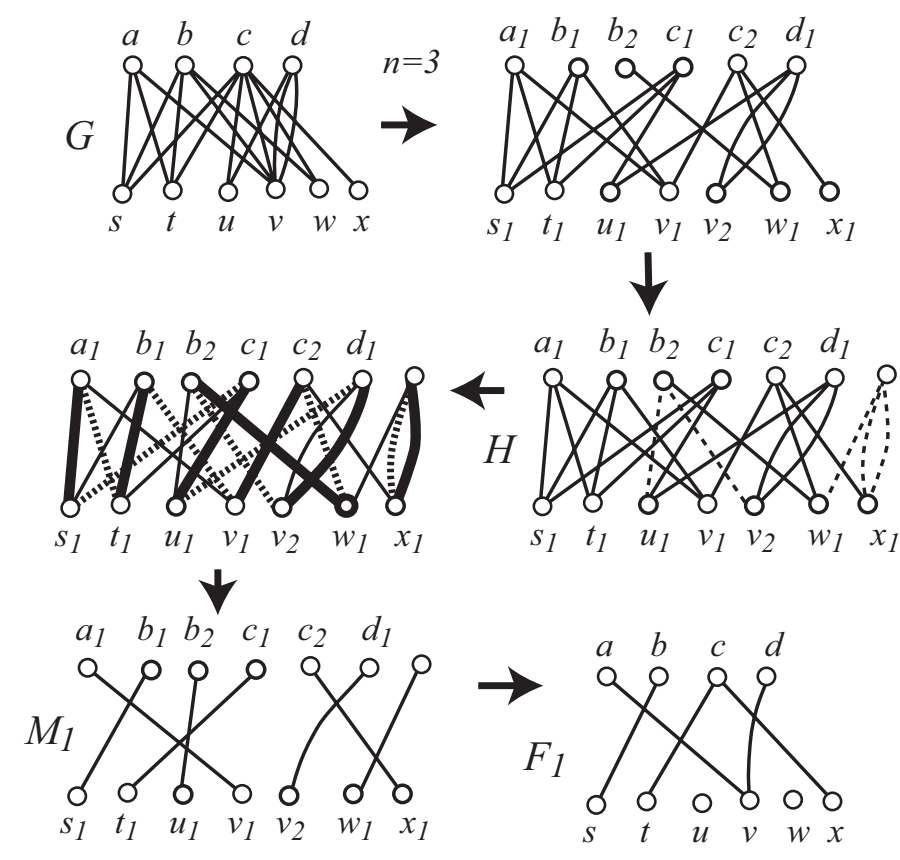

Figure 4.3: A bipartite multigraph $G$, a new 3-regular bipartite multigraph $H$, and a $(g, f)$-factor $F_{1}$ of $G$ obtained from a 1 -factor $M_{1}$, where $n=3$.

Another Proof of Theorem 4.1.5 (Lovász [107], Solution 7.11) If the degree of a vertex $v$ of $G$ is

$$
\operatorname{deg}_{G}(v)=m n+t, \quad \text { where } 0 \leq m \text { and } 0 \leq t<n,
$$


we split $v$ into $m+1$ vertices $v_{1}, v_{2}, \ldots, v_{m+1}$ if $t \geq 1$; otherwise we split $v$ into $m$ vertices $v_{1}, v_{2}, \ldots, v_{m}$ so that all vertices $v_{1}, v_{2}, \ldots, v_{m}$ have degree $n$ and $v_{m+1}$ has degree $t$ if any (Figure 4.3). Then add some new vertices, if necessary, and some new edges between vertices with degree less than $n$ so that the resulting graph $H$ becomes a $n$-regular bipartite multigraph (Figure 4.3).

By Theorem 4.1.2, $H$ can be decomposed into $n$ 1-factors

$$
E(H)=M_{1} \cup M_{2} \cup \cdots \cup M_{n}, \quad \text { where every } M_{i} \text { is a 1-factor of } H \text {. }
$$

Let $F_{j}=M_{j} \cap E(G)$, where $1 \leq j \leq n$ (Figure 4.3). Then for every vertex $v_{i}(v \in V(G), 1 \leq i \leq m), M_{j}$ contains exactly one edge incident with $v_{i}$, which is an edge of the given graph $G$. Hence

$$
m \leq \operatorname{deg}_{F_{j}}(v) \leq m+1
$$

Therefore $F_{j}$ is a $(g, f)$-factor of $G$ since $g(v) \leq m$ and $m+1 \leq f(v)$. Consequently $G$ is decomposed into $n(g, f)$-factors $F_{1} \cup F_{2} \cup \cdots \cup F_{n}$.

\subsection{Semi-Regular Factorization}

Recall that a graph $G$ is said to be $[a, b]$-factorable if $E(G)$ can be decomposed into disjoint $[a, b]$-factors of $G$. The $(g, f)$-factorization problem in bipartite graphs is completely solved as shown in Theorem 4.1.5. However, the situation is very different when we consider the same problem in general graphs. This section deals with $[k, k+1]$-factorization problem in general graphs, and a general $[a, b]$-factorization of usual graphs will be considered in the next section. A $[k, k+1]$-factor is often called an semi-regular factor, and analogously an $[r, r+1]$-graph and a $[k, k+1]$-factorization are called a semi-regular graph and a semi-regular factorization, respectively.

We review some results on semi-regular factorizations. The following result is easy but it is a starting point of the $[a, b]$-factorization problem.

Theorem 4.2.1 (Akiyama, Avis, Era [1] (1980)) Every regular general graph is $[1,2]$-factorable, in particular, if $r$ is an odd integer, then every $r$-regular general graph can be decomposed into $(r+1) / 2$ [1,2]-factors.

Proof. Since every even regular general graph is 2-factorable by Theorem 4.1.3, we may consider only odd regular graphs. Let $r \geq 3$ be an odd integer, and $G$ be an $r$-regular general graph.

Let $G^{\prime}$ be a copy of $G$. We obtain a new $(r+1)$-regular general graph $H$ from $G \cup G^{\prime}$ by joining each pairs of corresponding vertices $v \in V(G)$ 
and $v^{\prime} \in V\left(G^{\prime}\right)$ by a new edge (Figure $4.4(\mathrm{a})$ ). Since $r+1$ is even, $H$ can be decomposed into $m=(r+1) / 2$ 2-factors $F_{1}, F_{2}, \ldots, F_{m}$ by Petersen's 2-Factorable Theorem 4.1.3. Then $G$ is decomposed into $m[1,2]$-factors

$$
\left(G \cap F_{1}\right) \cup\left(G \cap F_{2}\right) \cup \cdots \cup\left(\left(F_{m} \cap G\right) .\right.
$$

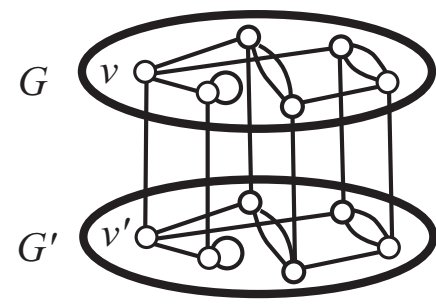

(a) $H$

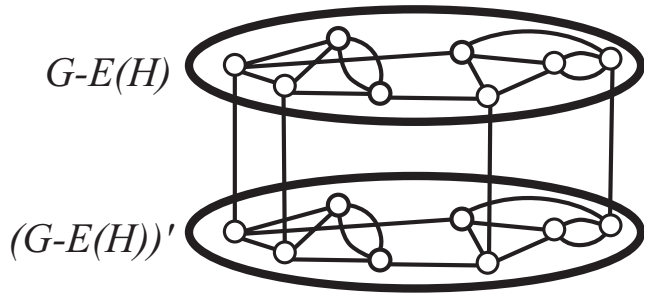

(b) $R$

Figure 4.4: (a) An $r$-regular graphs $G$ and and an $(r+1)$-regular graph $H$ containing $G$ as an induced subgraph; (b) An $[r, r+1]$-graph $G-E(H)$ and an $(r+1)$-regular graph $R$ containing $G-E(H)$ as an induced subgraph.

From Theorem 4.2.1, Akiyama conjectured that for every integer $k \geq 1$, there exists an integer $\Phi(k)$ such that for any integer $r \geq \Phi(k)$, every $r$ regular graph is $[k, k+1]$-factorable. This conjecture was proved by Era [48] in 1985 and the sharp bound for $\Phi(k)$ was obtained by Egawa [37] in the following year. These results are given in the next theorem.

Theorem 4.2.2 (Era [48], [47], Egawa [37]) Let $k \geq 2$ be an integer. Then (i) if $k$ is even and $r \geq k^{2}$, then every simple $r$-regular graph is $[k, k+1]$ factorable;

(ii) if $k$ is odd and $r \geq k^{2}+1$, then every simple $r$-regular graph is $[k, k+1]$ factorable.

(iii) these bounds on $r$ are sharp.

In order to prove this theorem we need some results on edge-coloring. Recall that a proper edge-coloring of a graph $G$ is a coloring of the edges of $G$ so that no two adjacent edges have the same color. The smallest number of colors by which $G$ can be properly edge-colored is called the chromatic index (or edge chromatic number) of $G$ and denoted by $\chi^{\prime}(G)$. It is clear that for each color $c$ of a proper edge-coloring of $G$, the set of edges colored with $c$ forms a matching of $G$. Thus the chromatic index $\chi^{\prime}(G)$ is the smallest integer $k$ for which $E(G)$ can be decomposed into $k$ disjoint matchings. Furthermore, a matching is a nothing but a $[0,1]$-factor. The 
famous Vizing's Theorem 9 says that the chromatic index of a simple graph with maximum degree $\Delta$ satisfies

$$
\Delta \leq \chi^{\prime}(G) \leq \Delta(G)+1
$$

He also proved the next theorem.

Theorem 4.2.3 (Vizing, [145] (1965)) Let $G$ be a simple graph with maximum degree $\Delta$. If the set

$$
W=\left\{x \in V(G) \mid \operatorname{deg}_{G}(x)=\Delta\right\}
$$

is independent, that is, if no two vertices in $W$ are adjacent in $G$, then $\chi^{\prime}(G)=\Delta$. In particular, $G$ can be decomposed into $\Delta[0,1]$-factors.

Lemma 4.2.4 Let $q \geq 2$ be an integer, and $G$ be a simple graph with maximum degree $\Delta$. If the set $W=\left\{x \in V(G) \mid \operatorname{deg}_{G}(x)=\Delta\right\}$ is independent and is decomposed into disjoint subsets

$$
W=W_{1} \cup W_{2} \cup \cdots \cup W_{q} \text { and } \Delta \leq q,
$$

where some $W_{i}$ 's may be empty sets, then $G$ has a [0,1]-factorization $F_{1} \cup$ $F_{2} \cup \cdots \cup F_{q}$ such that

$$
\text { every vertex of } W_{j} \text { has degree one in } F_{j} \text { for all } 1 \leq j \leq q .
$$

Proof. If $W_{\Delta+1} \cup \cdots \cup W_{q}=\emptyset$, then the lemma follows from Theorem 4.2.3 since for a $[0,1]$-factorization $F_{1} \cup \cdots \cup F_{\Delta}$ of $G$, every vertex of $W_{j}$ has degree one in all $F_{i}(1 \leq i \leq \Delta)$. Thus we may assume that $W_{\Delta+1} \cup \cdots \cup W_{q} \neq \emptyset$. Let $\emptyset \neq X \subseteq W_{\Delta+1} \cup \cdots \cup W_{q}$. Then $N_{G}(X) \subseteq V(G)-W$ as $W$ is independent. Since every vertex of $N_{G}(X)$ has degree at most $\Delta-1$, we have

$$
|X| \Delta=\left|\left\{(x, y) \mid x \in X, y \in N_{G}(X)\right\}\right| \leq\left|N_{G}(X)\right|(\Delta-1) .
$$

Hence $|X| \leq\left|N_{G}(X)\right|$. Since $X$ is an arbitrary subset of $W_{\Delta+1} \cup \cdots \cup W_{q}$, by Hall's Marriage Theorem 1.1.1 there exists an injection

$$
h: W_{\Delta+1} \cup \cdots \cup W_{q} \rightarrow V(G)-W \quad \text { such that } h(x) \in N_{G}(x) .
$$

It is obvious that $M=\{x h(x) \mid x \in X\}$ forms a matching of $G$.

Let $H=G-M$. Then $H$ satisfies the condition of Lemma 4.2.4 since $W_{H}=\left\{x \in V(H) \mid \operatorname{deg}_{H}(v)=\Delta\right\}=W_{1} \cup \cdots \cup W_{\Delta} \subset W$ is independent or empty. Hence $H$ has a $[0,1]$-factorization $F_{1} \cup F_{2} \cup \cdots \cup F_{\Delta}$ such that every $j(1 \leq j \leq \Delta)$ satisfies (4.5). Note that if $W_{H}$ is empty, $H$ can be decomposed into $\Delta[0,1]$-factors by $(4.4)$, where some $[0,1]$-factors may be 0 -factors. For every $\Delta<i \leq q$, let us define $F_{i}=\left\{x h(x) \mid x \in W_{i}\right\}$. Then $F_{1} \cup F_{2} \cup \cdots \cup F_{q}$ is the desired [0,1]-factorization of $G$. 
Lemma 4.2.5 Let $r \geq 1$ be an integer, and $G$ be a connected $2 r$-regular general graph. If $G$ has even size, then $G$ can be decomposed into two $r$ regular factors. If $G$ has odd size, then for any vertex $u$ of $G, G$ can be decomposed into an $[r-1, r]$-factor having exactly one vertex $u$ of degree $r-1$ and an $[r, r+1]$-factor having exactly only the vertex $u$ of degree $r+1$.

Proof. Since every vertex of $G$ has even degree, $G$ has an Euler circuit $C$ (Theorem 10). We traverse all the edges of $G$ along $C$ and alternately color the edges red and blue. Then if $G$ is of even size, the sets of red edges and blue edges form $r$-regular factors, respectively. If $G$ is odd size, we start with the vertex $u$, and alternately color the edges red and blue, then the sets of red edges and blue edges form the desired two factors.

Proof of Theorem 4.2.2 Assume first $k \geq 2$ is an even integer. Since $r \geq k^{2}$, we can write

$$
r=k q+s, \quad \text { where } 0 \leq s<k \leq q .
$$

By Theorem 3.3.8, $G$ has an $[s, s+1]$-factor. Choose such a minimal $[s, s+1]$ factor $H$. Then

$$
W=\left\{v \in V(H) \mid \operatorname{deg}_{H}(v)=s+1\right\}
$$

is empty or independent since $H$ has no edge joining two vertices with degree $s+1$ in $H$. Since $G-E(H)$ is a $[k q-1, k q]$-graph, two copies of $G-E(H)$ can be embedded in a $k q$-regular graph $R$ (see Figure 4.4 (b)). Since $k$ is even, by applying Petersen's 2-Factorable Theorem 4.1.3 to $R$, we obtain a $k$-regular factorization of $R$, and so we get a $[k-1, k]$-factorization of $G-E(H)$

$$
G-E(H)=A_{1} \cup A_{2} \cup \cdots \cup A_{q}, \quad \text { where all } A_{i} \text { are }[k-1, k] \text {-factors. }
$$

Let

$$
W_{i}=\left\{x \in V(G) \mid \operatorname{deg}_{A_{i}}(x)=k-1\right\} \quad \text { for } 1 \leq i \leq q .
$$

Since every $v \in W$ has degree $q k-1$ in $G-E(H), v$ has degree $k-1$ in exactly one $A_{j}$. Hence $W$ is decomposed into disjoint subsets

$$
W=W_{1} \cup W_{2} \cup \cdots \cup W_{q} .
$$

Since $s+1 \leq k \leq q$, by Lemma 4.2.4, $H$ has a [0,1]-factorization $F_{1} \cup F_{2} \cup$ $\cdots \cup F_{q}$ such that $\operatorname{deg}_{F_{j}}(x)=1$ for all $x \in W_{j}$ for every $1 \leq j \leq q$. Then

$$
\left(A_{1} \cup F_{1}\right) \cup\left(A_{2} \cup F_{2}\right) \cup \cdots \cup\left(A_{q} \cup F_{q}\right)
$$


is the desired $[k, k+1]$-factorization of $G$.

Next assume that $k \geq 3$ is an odd integer. Here we consider only the case when $r$ can be expressed as $r=k^{2}+2 s+1$, where $s$ is an integer such that $0 \leq s \leq(k-1) / 2$. The proofs of the other cases are similar but fairly long, and so are omitted here. Then $r$ can be expressed as

$$
r=2 k\left(\frac{k-1}{2}-s\right)+2(k+1) s+k+1 .
$$

Since $r$ is even, by Petersen's 2-Factorable Theorem 4.1.3, G can be decomposed into

$$
F_{1} \cup \cdots \cup F_{n} \cup F_{n+1} \cup \cdots \cup F_{n+s} \cup F, \quad n=\frac{k-1}{2}-s,
$$

where $F_{i}(1 \leq i \leq n)$ is a $2 k$-regular factor, $F_{j}(n+1 \leq j \leq n+s)$ is a $2(k+1)$-regular factor and $F$ is a $(k+1)$-regular factor. Since $k+1$ is even, every $F_{j}(n+1 \leq j \leq n+s)$ can be decomposed into two $(k+1)$ regular factors by Lemma 4.2.5. So we shall decompose $F_{1} \cup \cdots \cup F_{n} \cup F$ into $[k, k+1]$-factors of $G$.

Let $H_{i}(1), H_{i}(2), \ldots, H_{i}\left(\ell_{i}\right)$ be the components of $F_{i}, 1 \leq i \leq n$. Then $\left|V\left(H_{i}(t)\right)\right| \geq 2 k+1$ as $G$ is a simple graph. Set

$$
I=\left\{(i, t) \mid 1 \leq i \leq n, 1 \leq t \leq \ell_{i}\right\}
$$

By Theorem 4.2.1, $F$ has a [1,2]-factor. Choose a minimal [1,2]-factor $Q$ of $F$. Then each component of $Q$ is a path of order two or three. Let $Q_{1}, Q_{2}, \ldots, Q_{m}$ be the components of $Q$. For every $(i, t) \in I$, let

$$
\Phi(i, t)=\left\{j \mid V\left(H_{i}(t)\right) \cap V\left(Q_{j}\right) \neq \emptyset\right\} \subseteq\{1,2, \ldots, m\} .
$$

Then for any $X \subseteq I$, we have

$$
\begin{aligned}
& \left|\bigcup_{(i, t) \in X} \Phi(i, t)\right| \cdot n \\
\geq & |\{((i, t), j) \mid(i, t) \in X, j \in \Phi(i, t)\}| \\
\geq & |X|(2 k+1) / 3
\end{aligned}
$$

since for each $j$, there are at most $n(i, t)$ 's (i.e., $F_{1}, \ldots, F_{n}$ ), and for each $(i, t)$ of $X$, there are at least $(2 k+1) / 3 j$ 's as $\left|V\left(H_{i}(t)\right)\right| \geq 2 k+1$. Then $\left|\bigcup_{(i, t) \in X} \Phi(i, t)\right| \geq|X|$. Hence by Hall's Marriage Theorem 1.1.1 there exists an injection

$$
\phi: I \rightarrow\{1,2, \ldots, m\} \quad \text { such that } \phi(i, t) \in \Phi(i, t) .
$$


Let

$$
J=\left\{(i, t) \in I \mid H_{i}(t) \text { has odd size }\right\} .
$$

For every $(i, t) \in J$, choose a vertex

$$
v(i, t) \in V\left(H_{i}(t)\right) \cap V\left(Q_{\phi(i, t)}\right) .
$$

By Lemma 4.2.5, if $H_{i}(t)$ has even size, then $H_{i}(t)$ is decomposed into two $k$-regular factors $A_{i}(t)$ and $B_{i}(t)$, and if $H_{i}(t)$ has odd size, then $H_{i}(t)$ is decomposed into a $[k-1, k]$-factor $A_{i}(t)$ and a $[k, k+1]$-factor $B_{i}(t)$ such that only one vertex $v(i, t)$ has degree $k-1$ in $A_{i}(t)$ and $k+1$ in $B_{i}(t)$. For every $(i, t) \in J$, choose an edge $e(i, t)$ of $Q_{\phi(i, t)}$ that is incident with $v(i, t)$. Then

$$
\bigcup_{t=1}^{m} A_{i}(t) \cup\{e(i, t) \mid(i, t) \in J\} \quad \text { and } \quad \bigcup_{t=1}^{m} B_{i}(t)
$$

are $[k, k+1]$-factors of $G$. Since $F-\{e(i, t) \mid(i, t) \in J\}$ is a $[k, k+1]$-factor of $G, F_{1} \cup \cdots \cup F_{n} \cup F$ is decomposed into $[k, k+1]$-factors of $G$. Consequently the proof of this case is complete.

The proof of (iii) is found in Era [47].

It is plausible that not only regular graphs but also some other graphs might be semi-regular factorable. In fact, the following theorems are obtained. The proof of these results are given in next section.

Theorem 4.2.6 (Akiyama and Kano [5] (1985)) Let $k \geq 2$ be an even integer, and $s \geq 0$ and $t \geq 1$ be integers. Then every $[(6 k+2) t+k s,(6 k+4) t+k s]$ multigraph is $[k, k+1]$-factorable.

Theorem 4.2.7 (Cai [24] (1991)) Let $k \geq 1$ be an odd integer, and $s \geq 0$ and $t \geq 1$ be integers. Then every $[(6 k+2) t+(k+1) s,(6 k+4) t+(k+1) s]$ multigraph is $[k, k+1]$-factorable.

\section{3 $[a, b]$-Factorizations of Graphs}

We begin with the following theorem, which will play an important role in this section and contains some known results as corollaries.

Theorem 4.3.1 (Kano [68]) Let $G$ be an n-edge connected general graph $(n \geq 1), \theta$ be a real number such that $0 \leq \theta \leq 1$, and $g, f: V(G) \rightarrow \mathbb{Z}$ such that $g(x) \leq f(x)$ for all $x \in V(G)$. If one of $\{(\mathrm{ia})$,(ib) $\}$, (ii) and one of $\{$ (iiia), (iiib), (iiic), (iiid), (iiie), (iiif)\} hold, then $G$ has a $(g, f)$-factor. 
(ia) $g(x) \leq \theta \operatorname{deg}_{G}(x) \leq f(x)$ for all $x \in V(G)$.

(ib) $\epsilon=\sum_{x \in V(G)}\left(\max \left\{0, g(x)-\theta \operatorname{deg}_{G}(x)\right\}+\max \left\{0, \theta \operatorname{deg}_{G}(x)-f(x)\right\}\right)<1$.

(ii) $G$ has at least one vertex $v$ such that $g(v)<f(v)$; or $g(x)=f(x)$ for all $x \in V(G)$ and $\sum_{x \in V(G)} f(x) \equiv 0(\bmod 2)$.

(iiia) $n \theta \geq 1$ and $n(1-\theta) \geq 1$.

(iiib) Both $\left\{\operatorname{deg}_{G}(x) \mid g(x)=f(x), x \in V(G)\right\}$ and $\{f(x) \mid g(x)=f(x), x \in$ $V(G)\}$ consist of even numbers.

(iiic) $\left\{\operatorname{deg}_{G}(x) \mid g(x)=f(x), x \in V(G)\right\}$ consists of even numbers, $n$ is odd, $(n+1) \theta \geq 1$ and $(n+1)(1-\theta) \geq 1$.

(iiid) $\{f(x) \mid g(x)=f(x), x \in V(G)\}$ consists of even numbers and $m(1-\theta) \geq$ 1 , where $m \in\{n, n+1\}$ and $m \equiv 1(\bmod 2)$.

(iiie) Both $\left\{\operatorname{deg}_{G}(x) \mid g(x)=f(x), x \in V(G)\right\}$ and $\{f(x) \mid g(x)=f(x), x \in$ $V(G)\}$ consist of odd numbers, and $m \theta \geq 1$, where $m \in\{n, n+1\}$ and $m \equiv 1$ $(\bmod 2)$.

(iiif) $g(x)<f(x)$ for all $x \in V(G)$.

Proof. Let $S$ and $T$ be disjoint subsets of $V(G)$. By the $(g, f)$-Factor Theorem 3.1.1, it suffices to show that $\gamma(S, T) \geq 0$. By (ii), it follows that $\gamma(\emptyset, \emptyset)=0$, and so we may assume that $S \cup T \neq \emptyset$. Since (ia) implies (ib) with $\epsilon=0$, we may assume that (ib), (ii) and one of (iiia)-(iiif) hold. Let $C_{1}, C_{2}, \ldots, C_{m}$ be the $(g, f)$-odd components of $G-(S \cup T)$, where $m=$ $q^{*}(S, T)$. Then

$$
\begin{aligned}
\gamma(S, T)= & \sum_{x \in S} f(x)+\sum_{s \in T}\left(\operatorname{deg}_{G}(x)-g(x)\right)-e_{G}(S, T)-q^{*}(S, T) \\
\geq & \theta \sum_{x \in S} \operatorname{deg}_{G}(x)+(1-\theta) \sum_{x \in T} \operatorname{deg}_{G}(x)-e_{G}(S, T)-m \\
& -\sum_{x \in S}\left(\theta \operatorname{deg}_{G}(x)-f(x)\right)-\sum_{x \in T}\left(g(x)-\theta \operatorname{deg}_{G}(x)\right) \\
\geq & \theta\left(e_{G}(S, T)+\sum_{i=1}^{m} e_{G}\left(S, C_{i}\right)\right) \\
& +(1-\theta)\left(e_{G}(T, S)+\sum_{i=1}^{m} e_{G}\left(T, C_{i}\right)\right)-e_{G}(S, T)-m-\epsilon \\
= & \sum_{i=1}^{m}\left(\theta e_{G}\left(S, C_{i}\right)+(1-\theta) e_{G}\left(T, C_{i}\right)-1\right)-\epsilon .
\end{aligned}
$$


Since $\gamma(S, T)$ is an integer and $\epsilon<1$, in order to prove $\gamma(S, T) \geq 0$ it suffices to show that every $C=C_{i}$ satisfies

$$
\theta e_{G}(S, C)+(1-\theta) e_{G}(T, C)-1 \geq 0 .
$$

It is clear that if $e_{G}(S, C) \geq 1$ and $e_{G}(T, C) \geq 1$, then (4.6) follows. Hence we may assume that

$$
e_{G}(S, C)=0 \quad \text { or } \quad e_{G}(T, C)=0 .
$$

Since $C$ is an $(g, f)$-odd components of $G-(S \cup T)$ and $G$ is $n$-edge connected, we have $g(x)=f(x)$ for all $x \in V(C)$ and

$$
\sum_{x \in V(C)} f(x)+e_{G}(C, T) \equiv 1(\bmod 2) \quad \text { and } \quad e_{G}(S \cup T, C) \geq n .
$$

Moreover, we have

$$
\sum_{x \in V(C)} \operatorname{deg}_{G}(x)=2\|C\|+e_{G}(C, S \cup T)(\bmod 2) .
$$

Assume that (iiia) holds. Then by (4.7) and (4.8), we have either $e_{G}(T, C)=$ 0 and $e_{G}(S, C) \geq n$ or $e_{G}(S, C)=0$ and $e_{G}(T, C) \geq n$, and thus

$$
\theta e_{G}(S, C)-1 \geq \theta n-1 \geq 0 \quad \text { or } \quad(1-\theta) e_{G}(T, C) \geq(1-\theta) n-1 \geq 0 .
$$

Hence (4.6) follows.

Assume (iiib) holds. By $(4.8), e_{G}(C, T) \equiv 1(\bmod 2)$. Hence $e_{G}(C, T) \geq$ 1 , in particular, we may assume $e_{G}(S, C)=0$ by (4.7). So, by (4.9) we have $e_{G}(C, T) \equiv 0(\bmod 2)$, which contradicts the above $e_{G}(C, T) \equiv 1(\bmod 2)$. Thus (4.6) follows.

Assume (iiic) holds. By (4.9), we have $e_{G}(C, S \cup T) \equiv 0(\bmod 2)$, which implies $e_{G}(C, S \cup T) \geq n+1$ as $n$ is odd. So if $e_{G}(C, T)=0$, then $\theta e_{G}(S, C)-$ $1 \geq \theta(n+1)-1 \geq 0$. If $e_{G}(C, S)=0$, then $(1-\theta) e_{G}(S, T)-1 \geq(1-\theta)(n+$ 1) $-1 \geq 0$. Hence (4.6) follows.

Assume (iiid) holds. By $(4.8)$, we have $e_{G}(C, T) \equiv 1(\bmod 2)$, which implies that we may assume $e_{G}(C, S)=0$. Then $e_{G}(T, C)=e_{G}(S \cup T, C) \geq n$ and so $e_{G}(T, C) \geq m$, where $m$ is defined in (iiid). Hence $(1-\theta) e_{G}(T, C)-1 \geq$ $(1-\theta) m-1 \geq 0$. Therefore (4.6) follows.

Assume (iiie) holds. By (4.8), $|C|+e_{G}(C, T) \equiv 1(\bmod 2)$. By (4.9), we have $|C| \equiv e_{G}(C, S \cup T)(\bmod 2)$. Thus we have a contradiction when $e_{G}(S, C)=0$. Hence $e_{G}(T, C)=0$. Then $e_{G}(S, C) \equiv|C| \equiv 1(\bmod 2)$. Therefore $\theta e_{G}(S, C)-1 \geq \theta m-1 \geq 0$, and (4.6) follows. 
Assume (iiif) holds. In this case $m=q^{*}(S, T)=0$ and so $\gamma(S, T) \geq-\epsilon$ from the fist calculation. Consequently the theorem is prove.

As an easy consequence of the above theorem, we can obtain the following theorem, which is an extension of Petersen's 2-Factorable Theorem.

Theorem 4.3.2 (Kano [68] (1985)) Let $a$ and $b$ be even integers such that $0 \leq a \leq b$, and $n \geq 1$ be an integer. Then a general graph $G$ can be decomposed into $n[a, b]$-factors if and only if $G$ is an $[a n, b n]$-graph.

Proof. It is trivial that if a general graph $G$ can be decomposed into $n[a, b]$ factors, then $G$ is $[a n, b n]$-graph. So it suffices to prove that every general $[a n, b n]$-graph can be decomposed into $n[a, b]$-factors by induction on $n$. We may assume $n \geq 2$.

Let $G$ be a general $[a n, b n]$-graph, and $\theta=1 / n$. Define $g, f: V(G) \rightarrow \mathbb{Z}$ as follows:

$$
\begin{aligned}
& g(x)=f(x)=a \quad \text { if } \operatorname{deg}_{G}(x)=a n, \\
& g(x) \leq \theta \operatorname{deg}_{G}(x) \leq f(x) \text { and } f(x)-g(x)=1 \quad \text { if } a n<\operatorname{deg}_{G}(x)<b n, \\
& g(x)=f(x)=b \quad \text { if } \operatorname{deg}_{G}(x)=b n
\end{aligned}
$$

Then $g, f, \theta$ satisfy the conditions (i), (ii) and (iiib) of Theorem 4.2.1. Hence $G$ has a $(g, f)$-factor $F$. For any vertex $x$ with an $<\operatorname{deg}_{G}(x)<b n$, we have

$$
a(n-1)<(1-\theta) \operatorname{deg}_{G}(x)<b(n-1)
$$

as $1-\theta=(n-1) / n$, and thus $a(n-1) \leq \operatorname{deg}_{G-F}(x) \leq b(n-1)$. Hence $G-F$ is an $[a(n-1), b(n-1)]$-graph. By the inductive hypothesis, $G-F$ can be decomposed into $n-1[a, b]$-factors, and therefore $G$ can be decomposed into $n[a, b]$-factors.

Here we give another application of Theorem 4.3.1, that is, we give another proof of the following theorem (Theorem 1.5.4).

Theorem 4.3.3 Let $r \geq 2$ be an even integer, and $G$ be a $(r-1)$-edge connected $r$-regular multigraph of odd order. Then for every vertex $v, G-v$ has a 1-factor.

Proof. Define $\theta=1 / r$, and $g, f: V(G) \rightarrow \mathbb{Z}$ by $g(x)=f(x)=1$ for all $x \in V(G)-v$ and $g(v)=0$ and $f(v)=1$. Then conditions (ia), (ii) and (iiic) are satisfied since $n=r-1$ is odd. Hence $G$ has a $(g, f)$-factor $F$, which is the desired 1-factor of $G-v$ since $\operatorname{deg}_{F}(v)=0$ by the parity of $|G|$.

We now give the main theorem in this section, which was obtained by Cai. 
Theorem 4.3.4 (Cai [24] (1991)) Let $a, b, m, n$ be integers such that $1 \leq$ $a<b, 0 \leq m, n$ and $1 \leq m+n$. Then

(i) If $a$ is even, $b$ is odd and $b \leq 2 a+1$, then every $[(6 a+2) m+a n,(6 b-$ $2) m+(b-1) n]$-multigraph is $[a, b]$-factorable.

(ii) If $a$ is odd, $b$ is even and $b \leq 2 a$, then every $[(6 a+2) m+(a+1) n,(6 b-$ 2) $m+$ bn]-multigraph is $[a, b]$-factorable.

(iii) If both $a$ and $b$ are odd and $b \leq 2 a+1$, then every $[(3 a+1) m+(a+$ 1) $n,(3 b-1) m+(b-1) n]$-multigraph is $[a, b]$-factorable.

The bounds in the above theorem are sharp in the following sense: Consider, for example, the case when $a$ is even and $b$ is odd. Then there exist a $[6 a+1,6 b-2]$-graph and a $[6 a+2,6 b-1]$-graph that are not $[a, b]$-factorable. For other cases, the same situations hold. We now show that the graphs $G_{1}$ and $G_{2}$ of Figure 4.5, which are a $[6 a+1,6 b-2]$-multigraph and a $[6 a+2,6 b-1]$-multigraph, are not $[a, b]$-factorable. Assume that $G_{1}$ is $[a, b]$ factorable. Since $\operatorname{deg}_{G}(v)=6 b-2$ and $\operatorname{deg}_{G}(u)=6 a+1, G_{1}$ must be decomposed into six $[a, b]$-factors. We may assume that an $[a, b]$-factor $F_{1}$ contains the minimum number of edges joining $u$ to $w$. Then $F_{1}$ contains at most $a-(b+1) / 2$ edges joining $u$ to $w$. Since the degree of $u$ in $F_{1}$ is at least $a, F_{1}$ contains at least $(b+1) / 2$ edges joining $u$ to $v$. Similarly $F_{1}$ contains at least $(b+1) / 2$ edges joining $w$ to $v$. Therefore the degree of $v$ in $F_{1}$ is at least $b+1$, which is a contradiction. Therefore $G_{1}$ is not $[a, b]$-factorable.

Assume that $G_{2}$ is $[a, b]$-factorable. Then $G_{2}$ must be decomposed into six $[a, b]$-factors. It is clear that there exist at least three $[a, b]$-factors that contain at least $(b+1) / 2$ edges each joining $y$ and $z$. Similarly, there exist at least two $[a, b]$-factors containing at least $(b+1) / 2$ edges each joining $x$ to $y$, and another two $[a, b]$-factors containing at least $(b+1) / 2$ edges each joining $x$ or $z$. Therefore there exists an $[a, b]$-factor $F_{2}$ that contains at least $(b+1) / 2$ edges joining $y$ and $z$ and also at least $(b+1) / 2$ edges joining either $x$ to $y$ or $x$ to $z$. Then one of $\{y, z\}$ has degree at least $b+1$ in $F_{2}$, which is a contradiction. Therefore $G_{2}$ is not $[a, b]$-factorable.

Some results are corollaries of the above theorem. By setting $a=k$ and $b=k+1$ in (i), we can obtain Theorem 4.2.6. Similarly by setting $a=k$ and $b=k+1$ in (ii) we obtain Theorem 4.2.7. Moreover by taking $a=1$ and $b=2$ in (ii), we obtain the next theorem, which is the main result in [68] and whose proof gives some proof techniques that are used in the proof of Theorem 4.3.4.

Theorem 4.3.5 ([68]) Let $m \geq 1$ and $n \geq 0$ be integers. Then every $[8 m+$ $2 n, 10 m+2 n]$-multigraph is $[1,2]$-factorable. 

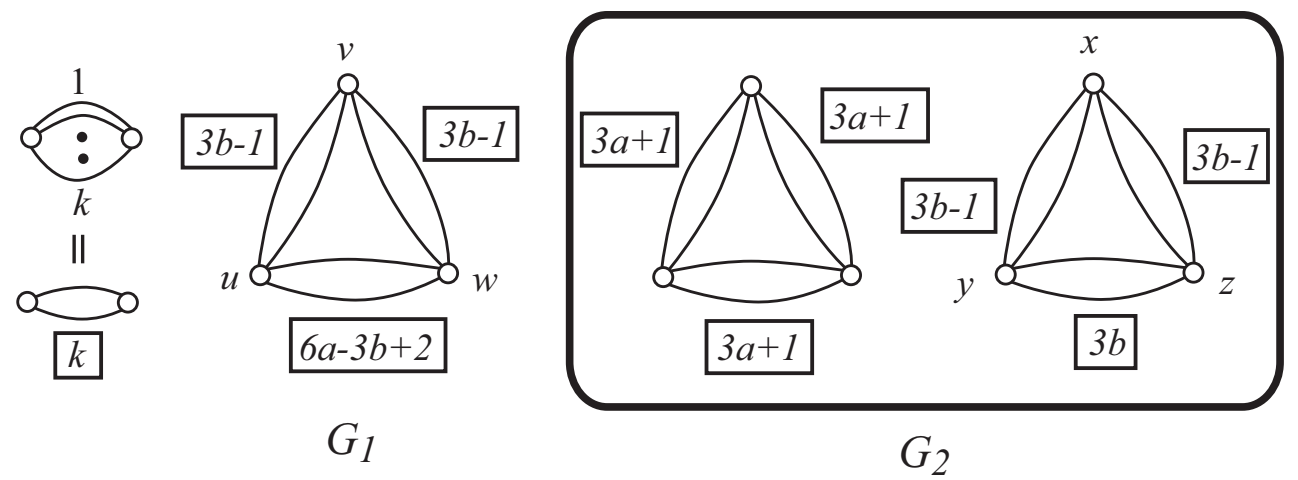

Figure 4.5: (a) An $[6 a+1,6 b-2]$-multigraph $G_{1}$ that is not $[a, b]$-factorable; (b) an $[6 a+2,6 b-1]$-multigraph that is no $[a, b]$-factorable.

We shall prove only (i) and give brief sketch of proofs of (ii) and (iii) which can be proved in a similar way. Let us first consider the case where $a \geq 2$ is an even integer, $b \geq 3$ is an odd integer and $a<b \leq 2 a+1$. We prove (i) of the theorem in several steps, which are given in the following lemmas.

Lemma 4.3.6 Assume that $a$ is even, $b$ is odd and $1 \leq a<b \leq 2 a+1$. Let $m \geq 1$ and $n \geq 1$. Then every $[(6 a+2) m+a n,(6 b-2) m+(b-1) n]$ multigraph $G$ contains an $[a, b]$-factor $F$ such that $G-F$ is a $[(6 a+2) m+$ $a(n-1),(6 b-2) m+(b-1)(n-1)]$-graph.

Proof. Let $G$ be a $[(6 a+2) m+a n,(6 b-2) m+(b-1) n]$-multigraph. Define $g, f: V(G) \rightarrow \mathbb{Z}$ by

$$
\begin{aligned}
& g(x)=\max \left\{a, \operatorname{deg}_{G}(x)-((6 b-2) m+(b-1)(n-1))\right\} \\
& f(x)=\min \left\{b, \operatorname{deg}_{G}(x)-((6 a+2) m+a(n-1))\right\} .
\end{aligned}
$$

Then

$$
\begin{aligned}
& a \leq \operatorname{deg}_{G}(x)-((6 a+2) m+a(n-1)), \\
& \operatorname{deg}_{G}(x)-((6 b-2) m+(b-1)(n-1)) \leq b-1<b, \\
& (6 a+2) m+a(n-1)<(6 b-2) m+(b-1)(n-1) .
\end{aligned}
$$

Hence

$$
\begin{array}{ll}
g(x) \leq f(x) & \text { for all } x \in V(G), \text { and } \\
g(x)=f(x) \quad \text { if and only if } \operatorname{deg}_{G}(x)=(6 a+2) m+a n, & \text { which implies } g(x)=f(x)=a .
\end{array}
$$


Since $a \leq g(x)$ and $f(x) \leq b$, a $(g, f)$-factor is an $[a, b]$-factor. Define

$$
\theta=\frac{a}{(6 a+2) m+a n} \text {. }
$$

Then it follows from $b \leq 2 a+1$ that

$$
\frac{b-1}{(6 b-2) m+(b-1) n} \leq \theta<\frac{b}{(6 b-2) m+(b-1) n} .
$$

In particular,

$$
a \leq \theta \operatorname{deg}_{G}(x)<b .
$$

We obtain

$$
\begin{aligned}
(1-\theta) \operatorname{deg}_{G}(x) & \leq(1-\theta)((6 b-2) m+(b-1) n) \\
& \leq(6 b-2) m+(b-1) n-(b-1) \\
& =(6 b-2) m+(b-1)(n-1),
\end{aligned}
$$

which implies $\operatorname{deg}_{G}(x)-((6 b-2) m+(b-1)(n-1)) \leq \theta \operatorname{deg}_{G}(x)$, and thus

$$
g(x) \leq \theta \operatorname{deg}_{G}(x) .
$$

Similarly,

$$
\begin{aligned}
& (1-\theta) \operatorname{deg}_{G}(x) \geq(1-\theta)((6 a+2) m+a n) \\
= & (6 a+2) m+a n-a=(6 a+2) m+a(n-1),
\end{aligned}
$$

which implies $\operatorname{deg}_{G}(x)-((6 a+2) m+a(n-1)) \geq \theta \operatorname{deg}_{G}(x)$, and so

$$
\theta \operatorname{deg}_{G}(x) \leq f(x) .
$$

Therefore $\theta, n=1, g$ and $f$ satisfy conditions (ia), (ii) and (iiib) of Theorem 4.3.1. Thus $G$ has an $(g, f)$-factor $F$.

It follows from

$$
A-\max \{B, C\} \leq A-B \quad \text { and } \quad A-\min \{B, C\} \geq A-B
$$

that

$$
\begin{aligned}
\operatorname{deg}_{G}(x)-g(x) \leq & \operatorname{deg}_{G}(x) \\
& -\left(\operatorname{deg}_{G}(x)-((6 b-2) m+(b-1)(n-1))\right) \\
= & (6 b-2) m+(b-1)(n-1),
\end{aligned}
$$

and

$$
\begin{aligned}
\operatorname{deg}_{G}(x)-f(x) \geq & \operatorname{deg}_{G}(x) \\
& -\left(\operatorname{deg}_{G}(x)-((6 a+2) m+a(n-1))\right) \\
= & (6 a+2) m+a(n-1) .
\end{aligned}
$$


Hence $G-F$ is a $[(6 a+2) m+a(n-1),(6 b-2) m+(b-1)(n-1)]$-graph, and the lemma is proved.

Lemma 4.3.7 Assume that $a$ is even, $b$ is odd and $2 \leq a<b \leq 2 a+1$. Then every connected $[6 a+2,6 b-2]$-multigraph can be decomposed into a $[3 a, 3 b-1]$-factor having at most one vertex of degree $3 a$ and $a[3 a+1,3 b-1]$ factor.

Proof. Let $G$ be a connected $[6 a+2,6 b-2]$-multigraph. If $G$ has a vertex of degree $6 a+2$, then choose one such vertex $w$. Define $g, f: V(G) \rightarrow \mathbb{Z}$ by

$$
\begin{aligned}
g(x) & =\max \left\{3 a+1, \operatorname{deg}_{G}(x)-(3 b-1)\right\} & & \text { for all } x \in V(G)-w \\
g(w) & =g(x)-1, & & \text { where } x \neq w, \text { and } \\
f(x) & =\min \left\{3 b-1, \operatorname{deg}_{G}(x)-(3 a+1)\right\} & & \text { for all } x \in V(G) .
\end{aligned}
$$

Then

$$
\begin{aligned}
& \operatorname{deg}_{G}(x)-(3 b-1) \leq \operatorname{deg}_{G}(x)-\frac{1}{2} \cdot \operatorname{deg}_{G}(x)=\frac{1}{2} \cdot \operatorname{deg}_{G}(x) \\
& \operatorname{deg}_{G}(x)-(3 a+1) \geq \operatorname{deg}_{G}(x)-\frac{1}{2} \cdot \operatorname{deg}_{G}(x) \geq \frac{1}{2} \cdot \operatorname{deg}_{G}(x)
\end{aligned}
$$

Thus

$$
\begin{aligned}
& g(x) \leq \frac{1}{2} \cdot \operatorname{deg}_{G}(x) \leq f(x) \quad \text { for all } x \in V(G), \text { and } \\
& g(x)=f(x) \quad \text { if and only if } \quad \operatorname{deg}_{G}(x)=6 a+2, \quad x \neq w \\
& \quad \text { or } \operatorname{deg}_{G}(x)=6 b-2 .
\end{aligned}
$$

Then $\theta=1 / 2, n=1, g$ and $f$ satisfy (ia), (ii) and (iiic) of Theorem 4.3.1, and thus $G$ has a $(g, f)$-factor $F$. It is easy to verify that $F$ is the desired $[3 a, 3 b-1]$-factor and $G-F$ is the desired $[3 a+1,3 b-1]$-factor.

Since the proof of the next Lemma 4.3 .8 is long, we postpone it and now prove Theorem 4.3.4 under the assumption that Lemma 4.3.8 is true.

Proof of (i) of Theorem 4.3.4 Let $G$ be a $[(6 a+2) m+a n,(6 b-2) m+(b$ $-1) n]$-multigraph. By considering each component of $G$, we may assume that $G$ is connected. We use this argument below without mentioning it again. If $m=0$, then $G$ is $[a n,(b-1) n]$-graph, and so $G$ can be decomposed into $n[a, b-1]$-factors by Theorem 4.3.2. Hence we may assume $m \geq 1$. By Lemma 4.3.6 $G$ can be decomposed into $n[a, b]$-factors and one $[(6 a+$ $2) m,(6 b-2) m]$-factor. By Theorem 4.3.2 the $[(6 a+2) m,(6 b-2) m]$-factor can be decomposed into $m[(6 a+2),(6 b-2)]$-factors. By Lemma 4.3 .7 and by 
the following Lemma 4.3.8, every [(6a+2), $(6 b-2)]$-factor can be decomposed into six $[a, b]$-factors. Consequently, $G$ is decomposed into $[a, b]$-factors, and the theorem is proved.

Lemma 4.3.8 Assume that $a$ is even, $b$ is odd and $2 \leq a<b \leq 2 a+1$. Then every connected $[3 a, 3 b-1]$-multigraph having at most one vertex of degree $3 a$ can be decomposed into three $[a, b]$-factors.

Proof. Let $G$ be a connected [3a, 3b-1]-graph having at most one vertex of degree $3 a$. We shall prove the lemma by induction on the order $|G|$. Since $G$ has no loops, $|G| \geq 2$. It is easy to verify that the assertion holds if $|G| \leq 3$. So we may assume $|G| \geq 4$. Define two functions $g$ and $f$ on $V(G)$ as

$$
\begin{aligned}
& g(x)=\max \left\{a, \operatorname{deg}_{G}(x)-(2 b-1)\right\}, \\
& f(x)=\min \left\{b, \operatorname{deg}_{G}(x)-2 a\right\} .
\end{aligned}
$$

However, if $G$ has no vertex $x$ with $3 a<\operatorname{deg}_{G}(x)<3 b-1$, then choose one vertex $w$ of degree $3 b-1$ and modify $g$ and $f$ as follows:

$$
\begin{aligned}
& g(x)=\max \left\{a, \operatorname{deg}_{G}(x)-(2 b-1)\right\} \text { for all } x \in V(G)-w, \\
& g(w)=g(x)-1, \quad \text { where } x \neq w, \text { and } \\
& f(x)=\min \left\{b, \operatorname{deg}_{G}(x)-2 a\right\} \quad \text { for all } x \in V(G) .
\end{aligned}
$$

Then

$$
\begin{aligned}
g(x) \leq f(x) \quad \text { for all } x \in V(G), \text { and } \\
g(x)=f(x) \quad \text { if and only if } \quad \operatorname{deg}_{G}(x)=3 a, \\
\text { or } \quad \operatorname{deg}_{G}(x)=3 b-1 \text { and } x \neq w .
\end{aligned}
$$

We consider the following two cases.

Case 1. G has a $(g, f)$-factor $F$.

Let $H=G-F$. Then

$$
\begin{aligned}
\operatorname{deg}_{G}(x)-g(x) & \leq \operatorname{deg}_{G}(x)-\left(\operatorname{deg}_{G}(x)-(2 b-1)\right)=2 b-1, \quad(x \neq w) \\
\operatorname{deg}_{G}(w)-g(w) & =(3 b-1)-(b-1)=2 b, \\
\operatorname{deg}_{G}(x)-f(x) & \geq \operatorname{deg}_{G}(x)-\left(\operatorname{deg}_{G}(x)-2 a\right)=2 a .
\end{aligned}
$$

Hence $H$ is a $[2 a, 2 b]$-graph with at most one vertex of degree $2 b$. Furthermore, if $H$ has a vertex of degree $2 b, G$ has no vertices $x$ with $3 a<\operatorname{deg}_{G}(x)<$ $3 b-1$, in particular, $G$ has at least two vertices of degree $3 b-1$, and thus 
$H$ has a vertex $u$ of degree $2 b-1$, for which the following $g^{\prime}$ and $f^{\prime}$ satisfy $g^{\prime}(u)<f^{\prime}(u)$.

Define $g^{\prime}, f^{\prime}$ on $V(H)$ as

$$
\begin{aligned}
& g^{\prime}(x)=\max \left\{a, \operatorname{deg}_{H}(x)-b\right\}, \\
& f^{\prime}(x)=\min \left\{b, \operatorname{deg}_{H}(x)-a\right\} .
\end{aligned}
$$

Then

$$
\begin{aligned}
& g^{\prime}(x) \leq \frac{1}{2} \cdot \operatorname{deg}_{H}(x) \leq f^{\prime}(x) \quad \text { for all } x \in V(H), \text { and } \\
& g^{\prime}(x)=f^{\prime}(x) \quad \text { if and only if } \quad \operatorname{deg}_{H}(x)=2 a, \text { or } \\
& \quad \operatorname{deg}_{H}(x)=2 b .
\end{aligned}
$$

Then $\theta=1 / 2, n=1, g^{\prime}, f^{\prime}$ satisfies (ia), (ii) and (iiic) of Theorem 4.3.1, and thus $H$ has a $\left(g^{\prime}, f^{\prime}\right)$-factor $F^{\prime}$. It is clear that $F \cup F^{\prime} \cup\left(H-F^{\prime}\right)$ is the required $[a, b]$-factorization of $G$.

Case 2. G has no $(g, f)$-factor.

By Lovász $(g, f)$-Factor Theorem 3.1.1, there exist two disjoint subsets $S$ and $T$ of $V(G)$ such that

$$
\gamma(S, T)=\sum_{x \in S} f(x)+\sum_{x \in T}\left(\operatorname{deg}_{G}(x)-g(x)\right)-e_{G}(S, T)-q^{*}(S, T) \leq-1 .
$$

Let $C_{1}, C_{2}, \ldots, C_{m}$ be the set of $(g, f)$-odd components of $G-(S \cup T)$, where $m=q^{*}(S, T)$. Then every $C=C_{i}$ satisfies $g(x)=f(x)$ for all $x \in V(C)$ and $\sum_{x \in V(C)} f(x)+e_{G}(C, S \cup T) \equiv 1(\bmod 2)$. So every vertex of $C$ has even degree, and thus

$$
e_{G}(C, S \cup T) \geq 2 \quad \text { and } \quad e_{G}(C, S \cup T) \equiv 0(\bmod 2) .
$$

Let

$$
\beta=\#\left\{C_{i} \mid e_{G}\left(C_{i}, S \cup T\right)=2,1 \leq i \leq m\right\} .
$$

Then

$$
\begin{aligned}
\sum_{x \in S \cup T} \operatorname{deg}_{G}(x) & \geq \sum_{i=1}^{m} e_{G}\left(S \cup T, C_{i}\right)+2 e_{G}(S, T) \\
& \geq 4(m-\beta)+2 \beta+2 e_{G}(S, T),
\end{aligned}
$$

and thus

$$
\sum_{x \in S \cup T} \operatorname{deg}_{G}(x)+2 \beta \geq 4 m+2 e_{G}(S, T) .
$$


For any $0 \leq \theta \leq 1 / 2$, we have

$$
\begin{aligned}
\gamma(S, T)= & \sum_{x \in S} f(x)+\sum_{x \in T}\left(\operatorname{deg}_{G}(x)-g(x)\right)-e_{G}(S, T)-m \\
= & \sum_{x \in S} f(x)+\sum_{x \in T}\left(\operatorname{deg}_{G}(x)-g(x)\right)-\theta e_{G}(S, T) \\
& -\frac{1}{4}\left(4 m+2 e_{G}(S, T)\right)-\left(\frac{1}{2}-\theta\right) e_{G}(S, T) \\
\geq & \sum_{x \in S} f(x)+\sum_{x \in T}\left(\operatorname{deg}_{G}(x)-g(x)\right)-\theta \sum_{x \in S} \operatorname{deg}_{G}(x) \\
& -\frac{1}{4}\left(\sum_{x \in S \cup T} \operatorname{deg}_{G}(x)+2 \beta\right)-\left(\frac{1}{2}-\theta\right) \sum_{x \in T} \operatorname{deg}_{G}(x) \\
= & \sum_{x \in S}\left(f(x)-\left(\frac{1}{4}+\theta\right) \operatorname{deg}_{G}(x)\right) \\
& +\sum_{x \in T}\left(\left(\frac{1}{4}+\theta\right) \operatorname{deg}_{G}(x)-g(x)\right)-\frac{\beta}{2} .
\end{aligned}
$$

In particular, taking $\theta=(b+1) / 4(3 b-1)$, we have $1 / 4+\theta=b /(3 b-1)$. For every vertex $x \in S$, it follows that

$$
\begin{aligned}
& f(x)-\left(\frac{1}{4}+\theta\right) \operatorname{deg}_{G}(x)=f(x)-\frac{b}{3 b-1} \operatorname{deg}_{G}(x) \\
= & \frac{1}{3 b-1}\left((3 b-1) f(x)-b \operatorname{deg}_{G}(x)\right) \\
= & \frac{1}{3 b-1} \min \left\{b\left((3 b-1)-\operatorname{deg}_{G}(x)\right),(2 b-1) \operatorname{deg}_{G}(x)-2 a(3 b-1)\right\} \\
\geq & \begin{cases}-a /(3 b-1) & \text { if } \operatorname{deg}_{G}(x)=3 a \\
0 & \text { otherwise. }\end{cases}
\end{aligned}
$$

Similarly, for every $x \in T$, it follows that

$$
\begin{aligned}
& \left(\frac{1}{4}+\theta\right) \operatorname{deg}_{G}(x)-g(x) \\
\geq & \frac{1}{3 b-1} \min \left\{b \operatorname{deg}_{G}(x)-a(3 b-1),(2 b-1)\left((3 b-1)-\operatorname{deg}_{G}(x)\right)\right\} \\
\geq & 0 .
\end{aligned}
$$

Let $S(3 a)=\left\{x \in S \mid \operatorname{deg}_{G}(x)=3 a\right\}$. Then $|S(3 a)| \leq 1$ since $G$ has at most one vertex of degree $3 a$. So it follows that

$$
\begin{aligned}
& \sum_{x \in S}\left(f(x)-\left(\frac{1}{4}+\theta\right) \operatorname{deg}_{G}(x)\right) \\
\geq & -\frac{a}{3 b-1} \cdot|S(3 a)| \geq-\frac{1}{3} \cdot|S(3 a)| .
\end{aligned}
$$


By combining (4.10), (4.12) and (4.13), we have

$$
\frac{|S(3 a)|}{3}+\frac{\beta}{2} \geq 1
$$

From the above inequality and $|S(3 a)| \leq 1$, it follows that $\beta \geq 2$, and thus the next Claim holds.

Claim $G-(S \cup T)$ has a $(g, f)$-odd component, say $C_{t}$, such that

$$
\begin{aligned}
& e_{G}\left(C_{t}, S \cup T\right)=2, \quad \text { and } \\
& \operatorname{deg}_{G}(x)=3 b-1 \quad \text { for all } x \in V\left(C_{t}\right) .
\end{aligned}
$$

Since $G$ has no loops, $\left|C_{t}\right| \geq 2$, and since

$$
\sum_{x \in V\left(C_{t}\right)} f(x)+e_{G}\left(C_{t}, S \cup T\right)=b\left|C_{t}\right|+2 \equiv\left|C_{t}\right| \equiv 1(\bmod 2),
$$

we have $\left|C_{t}\right| \geq 3$. Let $\left\{u_{1} w_{1}, u_{2} w_{2}\right\}$ the edges joining $C_{t}$ and $S \cup T$, where $u_{1}, u_{2} \in S \cup T$ and $w_{1}, w_{2} \in V\left(C_{t}\right)$. Let $K$ be the graph obtained from $C_{t}$ by adding a new edge $w_{1} w_{2}$, which might be a loop of $K$. Let

$$
b=2 s+1, \quad \text { where } \quad s \geq 1 .
$$

Then $3 b-1=6 s+2$ and $K$ is a $(6 s+2)$-regular graph, so by Petersen's 2-Factorable Theorem 4.1.3, $K$ can be decomposed into $3 s+12$-factors $R_{0}, R_{1}, R_{2}, \cdots, R_{3 s}$, where we may assume $w_{1} w_{2} \in R_{1}$. Let

$$
Q_{1}=\bigcup_{i=1}^{s} R_{i}, \quad Q_{2}=\bigcup_{i=s+1}^{2 s} R_{i}, \quad Q_{3}=\bigcup_{i=2 s+1}^{3 s} R_{i}
$$

Then every $Q_{i}$ is a $2 s$-factor of $K$. We can assign the edges of $R_{0}$ one by one to $Q_{1}, Q_{2}, Q_{3}$ so that

$$
\begin{aligned}
& \operatorname{deg}_{Q_{1}}\left(w_{1}\right)=2 s+1, \quad \operatorname{deg}_{Q_{2}}\left(w_{1}\right)=2 s, \quad \text { and } \\
& \operatorname{deg}_{Q_{i}}(x) \leq 2 s+1 \quad \text { for all } \quad x \in V\left(C_{t}\right), \quad i \in\{1,2,3\} \text {. }
\end{aligned}
$$

To see this, first assign an edge of $R_{0}$ incident with $w_{1}$ to $Q_{1}$, and in general, for any unassigned edge $x y$ of $R_{0}$, there exist at most one $Q_{i}$ with $\operatorname{deg}_{Q_{i}}(x)=$ $2 s+1$ and at most one $Q_{j}$ with $\operatorname{deg}_{Q_{j}}(y)=2 s+1$, and so we can assign $x y$ to $Q_{k}$, where $k \notin\{i, j\}$. Since $\operatorname{deg}_{Q_{1}}\left(w_{1}\right)=2 s+1$, we may assume $\operatorname{deg}_{Q_{2}}\left(w_{1}\right)=2 s\left(\right.$ and $\left.\operatorname{deg}_{Q_{3}}\left(w_{1}\right)=2 s+1\right)$.

We consider two subcases according to whether $u_{1}=u_{2}$ or not. 

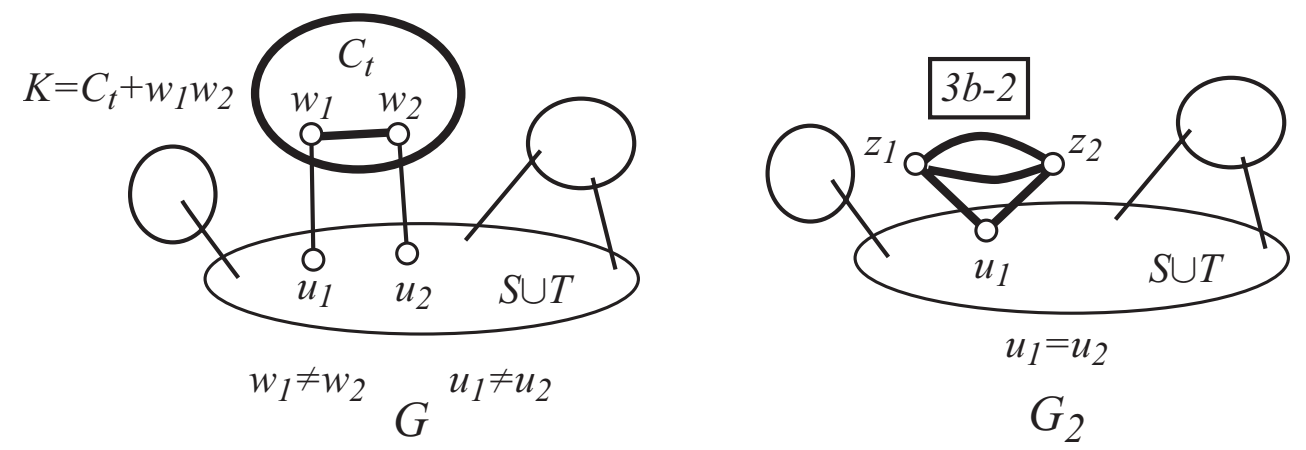

Figure 4.6: A graph $G$ with a component $C_{t}$, where it may occur $w_{1}=w_{2}$; and a $[3 a, 3 b-1]$-graph $G_{2}$, where $u_{1}=u_{2}$.

Subcase $2.1 u_{1} \neq u_{2}$.

By adding a new edge $u_{1} u_{2}$ to $G-V\left(C_{t}\right)$, we obtain a $[3 a, 3 b-1]$ multigraph $G_{1}$, which contains at most one vertex of degree $3 a$ (Figure 4.6). By the induction hypotheses, $G_{1}$ has an $[a, b]$-factorization $A_{1} \cup A_{2} \cup A_{3}$. We may assume $u_{1} u_{2} \in A_{1}$. Let

$$
\begin{aligned}
& F_{1}=\left(A_{1}-u_{1} u_{2}\right) \cup\left(Q_{1}-w_{1} w_{2}\right) \cup\left\{u_{1} w_{1}, u_{2} w_{2}\right\}, \\
& F_{2}=A_{2} \cup Q_{2}, \\
& F_{3}=A_{3} \cup Q_{3} .
\end{aligned}
$$

Then $F_{1} \cup F_{2} \cup F_{3}$ is the desired $[a, b]$-factorization of $G$. Note the above arguments hold when $w_{1}=w_{2}$ without changing anything.

Subcase $2.1 u_{1}=u_{2}$.

In this case, we obtain a new graph $G_{2}$ from $G-V\left(C_{t}\right)$ by adding two new vertices $z_{1}$ and $z_{2}$, by joining them by $3 b-2$ multiple edges, and by adding two new edges $u_{1} z_{1}$ and $u_{1} z_{2}$. Then $G_{2}$ is a $[3 a, 3 b-1]$-multigraph having at most one vertex degree $3 a$. Moreover $\left|G_{2}\right|<|G|$ as $\left|C_{t}\right| \geq 3$. Hence by the induction hypothesis, $G_{2}$ can be decomposed into three $[a, b]$-factors $A_{1}, A_{2}, A_{3}$.

If $u_{1} z_{1}, u_{1} z_{2} \in A_{1}$, then let

$$
\begin{aligned}
& F_{1}=\left(A_{1}-\left\{z_{1}, z_{2}\right\}\right) \cup\left(Q_{1}-w_{1} w_{2}\right) \cup\left\{u_{1} w_{1}, u_{1} w_{2}\right\}, \\
& F_{2}=\left(A_{2}-\left\{z_{1}, z_{2}\right\}\right) \cup Q_{2}, \\
& F_{3}=\left(A_{3}-\left\{z_{1}, z_{2}\right\}\right) \cup Q_{3},
\end{aligned}
$$

where $A_{i}-\left\{z_{1}, z_{2}\right\}$ denotes the graph obtained from $A_{i}$ by removing $z_{1}$ and $z_{2}$ together with all edges incident with them. 
If $u_{1} z_{1} \in A_{1}$ and $u_{1} z_{2} \in A_{2}$, then define

$$
\begin{aligned}
& F_{1}=\left(A_{1}-\left\{z_{1}, z_{2}\right\}\right) \cup\left(Q_{1}-w_{1} w_{2}\right) \cup\left\{u_{1} w_{2}\right\}, \\
& F_{2}=\left(A_{2}-\left\{z_{1}, z_{2}\right\}\right) \cup Q_{2} \cup\left\{u_{1} w_{1}\right\}, \\
& F_{3}=\left(A_{3}-\left\{z_{1}, z_{2}\right\}\right) \cup Q_{3} .
\end{aligned}
$$

Then by (4.14) we can easily show that $F_{1} \cup F_{2} \cup F_{3}$ is the desired $[a, b]$ factorization of $G$ in each case.

It is shown that Lemma 4.3.6 does not hold if $2 a+1<b$. For example, consider the complete bipartite graph $G=K((6 b+2) m+(b-1) n,(6 a+$ $2) m+a n)$. Then $G$ has no $[a, b]$-factor $F$ such that $G-F$ is a $[(6 a+2) m+$ $a(n-1),(6 b+2) m+(b-1)(n-1)]$-graph $([24])$. So it seems to be difficult to remove the condition $b \leq 2 a+1$.

The statement (ii) of Theorem 4.3 .4 can be proved by showing the following three lemmas. We give only a brief sketch of the proof of each lemma.

Lemma 4.3.9 Assume that $a$ is odd, $b$ is even and $1 \leq a<b \leq 2 a$. Let $m \geq 1$ and $n \geq 1$. Then every $[(6 a+2) m+(a+1) n,(6 b-2) m+b n]$-multigraph $G$ contains an $[a, b]$-factor $F$ such that $G-F$ is a $[(6 a+2) m+(a+1)(n-$ 1), $(6 b-2) m+b(n-1)]$-graph.

Proof. Let $G$ be a $[(6 a+2) m+(a+1) n,(6 b-2) m+b n]$-multigraph. Define $g, f: V(G) \rightarrow \mathbb{Z}$ by

$$
\begin{aligned}
& g(x)=\max \left\{a, \operatorname{deg}_{G}(x)-((6 b-2) m+b(n-1))\right\} \\
& f(x)=\min \left\{b, \operatorname{deg}_{G}(x)-((6 a+2) m+(a+1)(n-1))\right\} .
\end{aligned}
$$

Then $G$ has a $(g, f)$-factor $F$, which is an $[a, b]$-factor, and $G-F$ is a $[(6 a+$ $2) m+(a+1)(n-1),(6 b-2) m+b(n-1)]$-graph.

Lemma 4.3.10 Assume that $a$ is odd, $b$ is even and $1 \leq a<b \leq 2 a$. Then every connected $[6 a+2,6 b-2]$-multigraph can be decomposed into a $[3 a+1,3 b]$ factor having at most one vertex of degree $3 b$ and $a[3 a+1,3 b-1]$-factor.

Proof. Let $G$ be a connected $[6 a+2,6 b-2]$-multigraph. If $G$ has a vertex of degree $6 b-2$, then choose one such vertex $w$. Define $g, f: V(G) \rightarrow \mathbb{Z}$ by

$$
\begin{array}{ll}
g(x)=\max \left\{3 a+1, \operatorname{deg}_{G}(x)-(3 b-1)\right\} & \text { for all } x \in V(G), \\
f(x)=\min \left\{3 b-1, \operatorname{deg}_{G}(x)-(3 a+1)\right\} & \text { for all } x \in V(G)-w, \\
f(w)=f(x)+1, & \text { where } x \neq w .
\end{array}
$$


Then $G$ has a $(g, f)$-factor $F$, which is a $[3 a+1,3 b]$-factor having at most one vertex of degree $3 b$, and thus we obtain the desired factorization $F \cup(G-F)$.

Lemma 4.3.11 Assume that $a$ is odd, $b$ is even and $1 \leq a<b \leq 2 a$. Then every connected $[3 a+1,3 b]$-multigraph having at most one vertex of degree $3 b$ can be decomposed into three $[a, b]$-factors.

Proof. Let $G$ be a connected [3a+1,3b]-graph having at most one vertex of degree $3 b$. We shall prove the lemma by induction on the order $|G|$. We may assume $|G| \geq 4$. Define two functions $g$ and $f$ on $V(G)$ as

$$
\begin{aligned}
& g(x)=\max \left\{a, \operatorname{deg}_{G}(x)-2 b\right\}, \\
& f(x)=\min \left\{b, \operatorname{deg}_{G}(x)-(2 a+1)\right\} .
\end{aligned}
$$

However, if $G$ has no vertex $x$ with $3 a+1<\operatorname{deg}_{G}(x)<3 b$, then choose a vertex $w$ of degree $3 a+1$ and modify $g$ and $f$ as follows:

$$
\begin{aligned}
& g(x)=\max \left\{a, \operatorname{deg}_{G}(x)-2 b\right\} \quad \text { for all } x \in V(G), \\
& f(x)=\min \left\{b, \operatorname{deg}_{G}(x)-(2 a+1)\right\} \quad \text { for all } x \in V(G)-w, \\
& f(w)=f(x)+1, \quad \text { where } x \neq w .
\end{aligned}
$$

Then we consider two cases according to whether $G$ has a $(g, f)$-factor or not, and prove the lemma.

Statement (iii) of Theorem 4.3.4 can be proved by showing the following three lemmas. We give only a brief sketch of the proof of each lemma.

Lemma 4.3.12 Assume that both $a$ and $b$ are odd and $1 \leq a<b \leq 2 a+1$. Let $m \geq 1$ and $n \geq 1$. Then every $[(3 a+1) m+(a+1) n,(3 b-1) m+(b-1) n]$ multigraph $G$ contains an $[a, b]$-factor $F$ such that $G-F$ is a $[(3 a+1) m+$ $(a+1)(n-1),(3 b-1) m+(b-1)(n-1)]$-graph.

Proof. Let $G$ be a $[(3 a+1) m+(a+1) n,(3 b-1) m+(b-1) n]$-multigraph. Define $g, f: V(G) \rightarrow \mathbb{Z}$ by

$$
\begin{aligned}
& g(x)=\max \left\{a, \operatorname{deg}_{G}(x)-((3 b-1) m+(b-1)(n-1))\right\} \\
& f(x)=\min \left\{b, \operatorname{deg}_{G}(x)-((3 a+1) m+(a+1)(n-1))\right\} .
\end{aligned}
$$

Then $G$ has a $(g, f)$-factor, which is the required $[a, b]$-factor. 
Lemma 4.3.13 Assume that both $a$ and $b$ are odd and $1 \leq a<b \leq 2 a+1$. Then every connected $[3 a+1,3 b-1]$-multigraph can be decomposed into three $[a, b]$-factors.

Proof. Let $G$ be a connected $[3 a+1,3 b-1]$-multigraph. We shall prove the lemma by induction on the order $|G|$. We may assume $|G| \geq 4$. Define two functions $g$ and $f$ on $V(G)$ as

$$
\begin{aligned}
& g(x)=\max \left\{a, \operatorname{deg}_{G}(x)-2 b\right\}, \\
& f(x)=\min \left\{b, \operatorname{deg}_{G}(x)-(2 a+1)\right\} .
\end{aligned}
$$

We may assume that $G$ has a vertex of degree greater than $3 a+1$ since otherwise the lemma follows from Lemma 4.3.11. Then we consider two cases according to whether $G$ has a $(g, f)$-factor or not, and prove the lemma.

We now turn our attention to $(g, f)$-factorizations. For two functions $h, k: V(G) \rightarrow \mathbb{Z}$ with $h(x) \leq k(x)$ for all $x \in V(G)$ we say that a graph $G$ is $(h, k)$-graph if

$$
h(x) \leq \operatorname{deg}_{G}(x) \leq k(x) \quad \text { for all } x \in V(G) .
$$

Moreover, for integers $s$ and $t, s h+t$ denotes the function defined by

$$
(\operatorname{sh}+t)(x)=\operatorname{sh}(x)+t \quad \text { for all } x \in V(G) .
$$

Some results on $[a, b]$-factorization are generalized to those on $(g, f)$-factorization as follows:

Theorem 4.3.14 (Liu [97](1994)) Let $G$ be a $((g+1) n-1,(f-1) n+1)$ multigraph, where $g, f: V(G) \rightarrow \mathbb{Z}$ such that $1 \leq g(x)<f(x)$ for all $x \in V(G)$, and $n \geq 1$ be an integer. Then $G$ is $(g, f)$-factorable.

Proof. Let $G$ be a $((g+1) n-1,(f-1) n+1)$-multigraph. We shall prove the theorem by induction on $n$. If $n=1$, then $G$ itself is a $(g, f)$-factor. So we may assume $n \geq 2$. Define $h, k: V(G) \rightarrow \mathbb{Z}$ by

$$
\begin{aligned}
h(x) & =\max \left\{g(x), \operatorname{deg}_{G}(x)-((f(x)-1)(n-1)+1)\right\}, \\
k(x) & =\min \left\{f(x), \operatorname{deg}_{G}(x)-((g(x)+1)(n-1)-1)\right\} .
\end{aligned}
$$

Then

$$
\begin{aligned}
& g(x)<\operatorname{deg}_{G}(x)-((g(x)+1)(n-1)-1) \\
& \operatorname{deg}_{G}(x)-((f(x)-1)(n-1)+1)<f(x), \\
& g(x)<f(x)
\end{aligned}
$$


Hence

$$
h(x)<k(x) \text { for all } x \in V(G) .
$$

Since $g(x) \leq h(x)$ and $k(x) \leq f(x)$, a $(h, k)$-factor is an $(g, f)$-factor. Define

$$
\theta=\frac{1}{n}
$$

Then $1-\theta=(n-1) / n$ and so

$$
\begin{aligned}
(1-\theta) \operatorname{deg}_{G}(x) & \leq(1-\theta)((f(x)-1) n+1) \\
& =(f(x)-1)(n-1)+1-\frac{1}{n},
\end{aligned}
$$

which implies $\operatorname{deg}_{G}(x)-((f(x)-1)(n-1)+1)<\theta \operatorname{deg}_{G}(x)$, and since $\theta \operatorname{deg}_{G}(x) \geq(g(x)+1)-1 / n>g(x)$, we have

$$
h(x)<\theta \operatorname{deg}_{G}(x) .
$$

Similarly,

$$
\begin{aligned}
(1-\theta) \operatorname{deg}_{G}(x) & \geq(1-\theta)((g(x)+1) n-1) \\
& =(g(x)+1)(n-1)-1+\frac{1}{n},
\end{aligned}
$$

which implies $\operatorname{deg}_{G}(x)-((g(x)+1)(n-1)-1)>\theta \operatorname{deg}_{G}(x)$, and so

$$
\theta \operatorname{deg}_{G}(x)<k(x) \text {. }
$$

Therefore $\theta, n=1, h$ and $k$ satisfy conditions (ia), (ii) and (iiif) of Theorem 4.3.1. Thus $G$ has an $(h, k)$-factor $F$. It is easy to see that $G-F$ is a $((g+1)(n-1)-1,(f-1)(n-1)+1)$-graph since

$$
\begin{aligned}
& \operatorname{deg}_{G}(x)-h(x) \leq(f(x)-1)(n-1)+1, \\
& \operatorname{deg}_{G}(x)-k(x) \geq(g(x)+1)(n-1)-1 .
\end{aligned}
$$

Consequently the theorem is proved.

The following Theorem 4.3.15 also can be proved by using Theorem 4.3.14 and a proof technique similar to the one used in the proof of Theorem 4.3.4, i.e., show that every $(2 t((g+1) n-1)+(g+1) s, 2 t((f-1) n+1)+(f-1) s)$ multigraph $G$ has a $(g, f)$-factor $F$ such that $G-F$ is $(2 t((g+1) n-1)+$ $(g+1)(s-1), 2 t((f-1) n+1)+(f-1)(s-1))$-multigraph; a $(2 t((g+$ 1) $n-1), 2 t((f-1) n+1)$-multigraph can be decomposed into $t(2((g+1) n-$ $1)),(2((f-1) n+1))$-graph; and every $(2((g+1) n-1)),(2((f-1) n+1))$ multigraph is $(g, f)$-factorable. 
Theorem 4.3.15 (Yan [152](1995)) Let $G$ be a multigraph, $g, f: V(G) \rightarrow$ $\mathbb{Z}$ such that $1 \leq g(x) \leq f(x)$ for all $x \in V(G)$, and $n \geq 2, s \geq 0, t \geq 0$ be integers. Then every $(2 t((g+1) n-1)+(g+1) s, 2 t((f-1) n+1)+(f-1) s)$ graph is $(g, f)$-factorable. 


\section{Chapter 5}

\section{Parity Factors}

In this chapter we consider $(g, f)$-parity factors. In particular, we consider odd factors and even factors, in which every vertex has odd degree or even degree, respectively. Then we consider partial parity factors. These factors are special cases of $\mathcal{H}$-factors. Finally, we give some remarks on $\mathcal{H}$-factors.

\subsection{Parity $(g, f)$-Factors and $(1, f)$-Odd Fac- tors}

Let $G$ be a general graph, and $g, f: V(G) \rightarrow \mathbb{Z}$ be functions such that

$$
g(x) \leq f(x) \quad \text { and } \quad g(x) \equiv f(x)(\bmod 2) \quad \text { for all } x \in V(G) .
$$

Then a spanning subgraph $F$ of $G$ is called a parity $(g, f)$-factor if

$$
g(x) \leq \operatorname{deg}_{F}(x) \leq f(x) \quad \text { and } \quad \operatorname{deg}_{F}(x) \equiv f(x)(\bmod 2)
$$

for all $x \in V(G)$. Note that we allow $g(x)<0$ and $\operatorname{deg}_{G}(y)<f(y)$ for some vertices $x$ and $y$ of $G$. We may restrict ourselves to the case when $0 \leq g(x) \leq$ $f(x) \leq \operatorname{deg}_{G}(x)$ for all $x \in V(G)$. However, when we apply a criterion for the existence of parity $(g, f)$-factors to some problems, this relaxation on $g$ and $f$ plays an important rule, that is, proofs of some theorems become shorter and simpler.

Theorem 5.1.1 (The Parity ( $g, f)$-Factor Theorem, Lovász [101] (1972)) Let $G$ be a general graph and $g, f: V(G) \rightarrow \mathbb{Z}$ such that $g(x) \leq f(x)$ and $g(x) \equiv f(x)(\bmod 2)$ for all $x \in V(G)$. Then $G$ has a parity $(g, f)$-factor if and only if for all disjoint subsets $S$ and $T$ of $V(G)$, it follows that

$$
\eta(S, T)=\sum_{x \in S} f(x)+\sum_{x \in T}\left(\operatorname{deg}_{G}(x)-g(x)\right)-e_{G}(S, T)-q(S, T) \geq 0,
$$


where $q(S, T)$ denotes the number of components $C$ of $G-(S \cup T)$ such that

$$
\sum_{x \in V(C)} f(x)+e_{G}(C, T) \equiv 1(\bmod 2) .
$$

Proof. We first construct a new graph $G^{*}$ from $G$ by adding $(f(x)-g(x)) / 2$ loops to every vertex $x \in V(G)$, and we regard $f$ as a function $f: V\left(G^{*}\right) \rightarrow \mathbb{Z}$ (Figure 5.1).
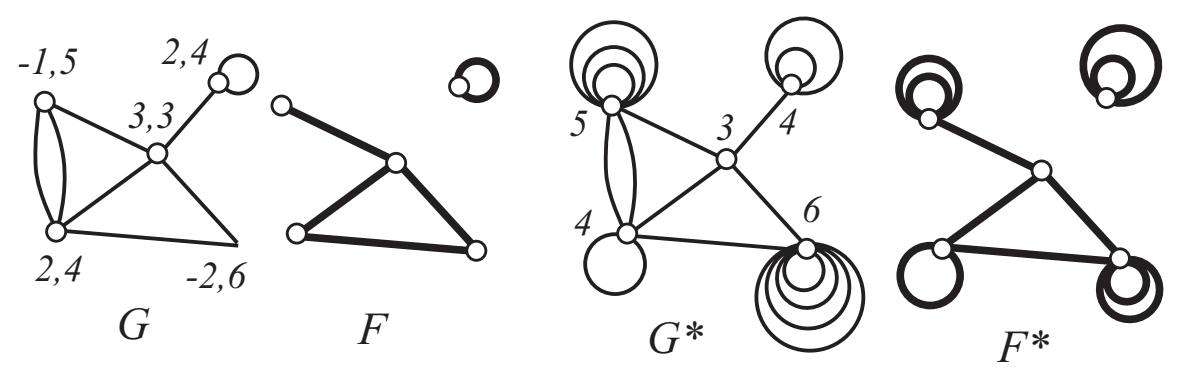

Figure 5.1: A graph $G$, its parity $(g, f)$-factor $F$ and the new graph $G^{*}$ together with an $f$-factor $F^{*}$ denoted by bold edges; numbers denote $g(x)$ and $f(x)$ in $G$ and $f(x)$ in $G^{*}$.

We can easily see that $G$ has a parity $(g, f)$-factor if and only if $G^{*}$ has an $f$-factor since we can get an $f$-factor of $G^{*}$ from a parity $(g, f)$-factor $F$ of $G$ by adding $\left(f(x)-\operatorname{deg}_{F}(x)\right) / 2$ loops to every vertex $x$ of $F$, and conversely, we can obtain a parity $(g, f)$-factor of $G$ from an $f$-factor $F^{*}$ of $G^{*}$ by deleting all the new loops of $F^{*}$ (Figure 5.1). Therefore we can apply the $f$-Factor Theorem 2.1.2 to the new graph $G^{*}$. Since

$$
\begin{aligned}
& \operatorname{deg}_{G^{*}}(x)=\operatorname{deg}_{G}(x)+f(x)-g(x), \\
& e_{G^{*}}(S, T)=e_{G}(S, T), \quad q_{G^{*}}(S, T)=q_{G}(S, T),
\end{aligned}
$$

we have

$$
\begin{aligned}
\delta_{G^{*}}(S, T) & =\sum_{x \in S} f(x)+\sum_{x \in T}\left(\operatorname{deg}_{G^{*}}(x)-f(x)\right)-e_{G^{*}}(S, T)-q_{G^{*}}(S, T) \\
& =\sum_{x \in S} f(x)+\sum_{x \in T}\left(\operatorname{deg}_{G}(x)-g(x)\right)-e_{G}(S, T)-q_{G}(S, T) \\
& =\eta(S, T) .
\end{aligned}
$$

Consequently, the theorem follows from the $(g, f)$-Factor Theorem 2.1.2. 
Later we mainly consider $(1, f)$-odd factors but before moving on to this topic, we first deal with even factors. A spanning subgraph $F$ of a graph $G$ is called an even factor of $G$ if every vertex has a positive even degree in $F$. From the $(g, f)$-Parity Factor Theorem, we can easily obtain the next theorem.

Note that by using a function $f: V(G) \rightarrow\{2,4,6, \cdots\}$ we can also consider an $(2, f)$-even factor, however a criterion for the existence of this factor is not simpler than that for an even factor.

Theorem 5.1.2 A general graph $G$ has an even factor if and only if

$$
\sum_{x \in X}\left(\operatorname{deg}_{G}(x)-2\right)-q(G ; X) \geq 0 \quad \text { for all } X \subset V(G)
$$

where $q(G ; X)$ denotes the number of components $C$ of $G-X$ such that $e_{G}(C, X) \equiv 1(\bmod 2)$.

Proof. Let $N$ be a sufficiently large even integer, and define $g(x)=2$ and $f(x)=N$ for all $x \in V(G)$. Then $G$ has an even factor if and only if $G$ has a parity $(g, f)$-factor. Let $S$ and $T$ be two disjoint subsets of $V(G)$. If $S$ is a nonempty set, then

$$
\begin{aligned}
\eta(S, T) & =\sum_{x \in S} f(x)+\sum_{x \in T}\left(\operatorname{deg}_{G}(x)-2\right)-e_{G}(S, T)-q(S, T) \\
& \geq N+\sum_{x \in T}\left(\operatorname{deg}_{G}(x)-2\right)-e_{G}(S, T)-q(S, T) \geq 0
\end{aligned}
$$

Hence we may assume that $S=\emptyset$. Then

$$
\eta(\emptyset, T)=\sum_{x \in T}\left(\operatorname{deg}_{G}(x)-2\right)-q(\emptyset, T)=\sum_{x \in T}\left(\operatorname{deg}_{G}(x)-2\right)-q(G ; T)
$$

Therefore the theorem is proved by the Parity $(g, f)$-Factor Theorem 5.1.1.

Theorem 5.1.3 (Problem 42, Sec. 7, [107]) Every 2-edge connected multigraph with $\delta(G) \geq 3$ has an even factor.

Proof. Let $X$ be a nonempty vertex subset, and $D_{1}, D_{2}, \ldots, D_{m}$ be the components of $G-X$ such that $e_{G}\left(D_{i}, X\right)$ is odd, where $m=q(G ; X)$. Since $G$ is 2-edge connected, $e_{G}\left(D_{i}, X\right) \geq 3$. Hence

$$
3 m \leq e_{G}\left(D_{1} \cup \cdots \cup D_{m}, X\right) \leq \sum_{x \in X} \operatorname{deg}_{G}(x) .
$$


Thus

$$
\begin{aligned}
& \sum_{x \in X}\left(\operatorname{deg}_{G}(x)-2\right)-m \\
\geq & \sum_{x \in X} \operatorname{deg}_{G}(x)-2|X|-\frac{1}{3} \sum_{x \in X} \operatorname{deg}_{G}(x) \\
\geq & \frac{2}{3} \sum_{x \in X} \operatorname{deg}_{G}(x)-2|X| \geq \frac{2}{3} \cdot 3|X|-2|X|=0 .
\end{aligned}
$$

Therefore $G$ has an even factor by Theorem 5.1.2.

We now consider a special parity factor called an odd factor. For an odd integer-valued function $f: V(G) \rightarrow\{1,3,5, \ldots\}$, a spanning subgraph $F$ of $G$ is called a $(1, f)$-odd factor if

$$
\operatorname{deg}_{F}(x) \in\{1,3, \ldots, f(x)\} \quad \text { for all } \quad x \in V(G) .
$$

Of course, if $f(x)=1$ for all vertices $x$, then $(1, f)$-odd factor is nothing but a 1-factor. For a constant odd integer $n \geq 1$, if $f(x)=n$ for all $x \in V(G)$, then a $(1, f)$-odd factor is called a $[1, n]$-odd factor. So a $[1, n]$-odd factor $F$ satisfies

$$
\operatorname{deg}_{F}(x) \in\{1,3, \ldots, n\} \quad \text { for all } \quad x \in V(F) .
$$

It is interesting that for the existence of an $(1, f)$-odd factor, we have a much simpler criterion than for a parity $(g, f)$-factor. Since a $(1, f)$-odd factor is a special parity $(g, f)$-factor, we can prove the following theorem by making use of the Parity $(g, f)$-Factor Theorem. Also since a $(1, f)$-odd factor is an extension of 1-factor, we can expect that the theorem can also be proved in a manner similar way to the proof of the 1-Factor Theorem. Here we give the two proofs mentioned.

Theorem 5.1.4 ((1,f)-Odd Factor Theorem, Cui and Kano [35]) Let $G$ be a general graph and $f: V(G) \rightarrow\{1,3,5, \ldots\}$. Then $G$ has an $(1, f)$-odd factor if and only if

$$
\operatorname{odd}(G-S) \leq \sum_{x \in S} f(x) \quad \text { for all } \quad S \subseteq V(G)
$$

Proof of necessity. Suppose that $G$ has a $(1, f)$-odd factor $F$. Since every component of $F$ has even order, $G$ has no odd components, and so (5.5) holds for $S=\emptyset$. Let $\emptyset \neq S \subset V(G)$, and $C$ be any odd component of $G-S$. Then 
there exists an edge of $F$ joining $C$ to $S$, since every component of $F$ is of even order. Hence

$$
\operatorname{odd}(G-S) \leq e_{F}(G-S, S) \leq \sum_{x \in S} \operatorname{deg}_{F}(x) \leq \sum_{x \in S} f(x)
$$

Therefore (5.5) holds.

Proof of sufficiency. Suppose that a general graph $G$ satisfies (5.5). Let $N$ be a sufficiently large odd integer. For example, $N$ is an odd integer greater than $|G|+\|G\|$. We define $g: V(G) \rightarrow \mathbb{Z}$ by $g(x)=-N$ for all $x \in V(G)$. Then $(1, f)$-odd factor and parity $(g, f)$-factor are the same. So it suffices to show that $G$ has a parity $(g, f)$-factor.

Let $S$ and $T$ be two disjoint subsets of $V(G)$. If $T \neq \emptyset$, then $\operatorname{deg}_{G}(x)-$ $g(x)=\operatorname{deg}_{G}(x)+N$ for every $x \in T$, where $N$ is sufficiently large, and so

$$
\begin{aligned}
\eta(S, T) & =\sum_{x \in S} f(x)+\sum_{x \in T}\left(\operatorname{deg}_{G}(x)-g(x)\right)-e_{G}(S, T)-h(S, T) \\
& \geq N-e_{G}(S, T)-h(S, T) \geq 0 .
\end{aligned}
$$

Hence we may assume $T=\emptyset$. It is immediate that a component $C$ of $G-S$ satisfying (5.3) has odd order since

$$
\sum_{x \in V(C)} f(x) \equiv|V(C)| \equiv 1(\bmod 2) .
$$

Hence $q(S, \emptyset)=\operatorname{odd}(G-S)$, and therefore it follows from (5.2) and (5.5) that

$$
\eta(S, \emptyset)=\sum_{x \in S} f(x)-q(S, \emptyset)=\sum_{x \in S} f(x)-\operatorname{odd}(G-S) \geq 0 .
$$

Consequently, by the parity $(g, f)$-Factor Theorem 5.1.1, $G$ has a parity $(g, f)$-factor, which is the desired $(1, f)$-odd factor of $G$.

In order to give another proof of the $(1, f)$-Odd Factor Theorem, we need the next theorem, which gives an criterion for a tree to have an $(1, f)$-odd factor.

Theorem 5.1.5 ([35]) Let $T$ be a tree of even order and $f: V(G) \rightarrow$ $\{1,3,5, \ldots\}$. Then $T$ has an $(1, f)$-odd factor if and only if

$$
\text { odd }(T-x) \leq f(x) \quad \text { for all } \quad x \in V(T)
$$


Proof. We shall prove only sufficiency since necessity can be easily shown as in the proof of necessity of Theorem 5.1.4. Let $F$ be a subgraph of $T$ induced by the edge subset

$$
A=\{e \in E(T) \mid \operatorname{odd}(T-e)=2\} . \quad \text { (Figure 5.2) }
$$

Note that for any edge $e$ of $T, T-e$ consists of two components, and those are simultaneously odd or even. Here the set $A$ consists of those edges $e$ for which $T-e$ consists of two odd components. For any vertex $v$ of $T, T-v$ has odd order since $T$ is of even order, and so $T-v$ has at least one odd component, say $C$. Then the edge $e$ of $T$ joining $C$ to $v$ is contained in $A$, which implies that $F$ is a spanning subgraph of $T$. Obviously, an edge of $T$ joining $v$ to an even component of $G-v$ is not contained in $A$. Hence the number of edges in $A$ incident with $v$ is equal to the number of odd components of $G-v$. Furthermore, since $T-v$ has odd order, the number of odd components of $T-v$ is odd, and hence

$$
\operatorname{deg}_{F}(v)=\operatorname{odd}(T-v) \quad \text { and } \quad \operatorname{odd}(T-v) \equiv 1(\bmod 2) .
$$

Consequently, by (5.6), $F$ is the desired $(1, f)$-odd factor of $T$.
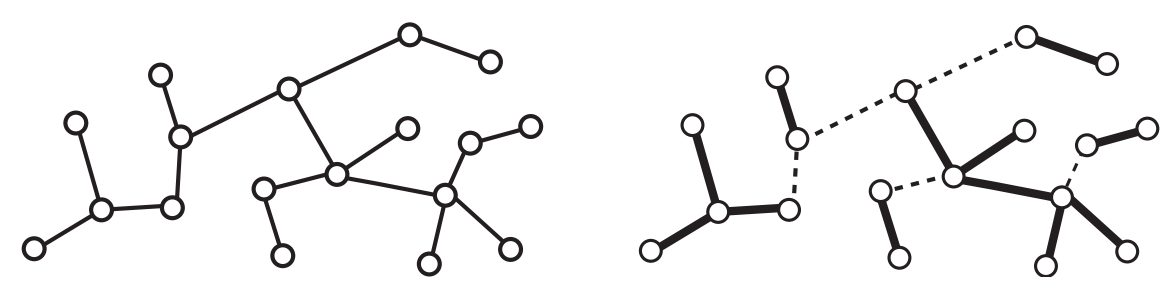

Figure 5.2: A tree $T$ of odd order and its unique odd factor shown by bold edges; Every bold edge $e$ satisfies odd $(T-e)=2$.

The next theorem is a corollary of the previous one, where an odd factor and an odd subgraph mean a spanning subgraph and a subgraph with all degrees odd, respectively. Note that every connected graph $G$ has a vertex $v$ such that $G-v$ is connected.

Theorem 5.1.6 (Problem 42 of Sec. 7 [107]) Let $G$ be a connected general graph. Then the following statements hold.

(i) If $G$ has even order, then $G$ has an odd factor.

(ii) If $G$ has odd order, then $G$ has an odd subgraph of order $|G|-1$. 
We are ready to give another proof to the $(1, f)$-Odd Factor Theorem, which does not use the Parity $(g, f)$-Factor Theorem 5.1.1.

Another proof of necessity of the $(1, f)$-Odd Factor Theorem ([9], [35]). We shall prove the theorem by induction on the size $\|G\|$ of $G$. We may assume that $G$ is connected. Then $G$ is of even order as odd $(G-\emptyset) \leq 0$ by (5.5). Moreover, by Theorem 5.1.5, we may assume that $G$ is not a tree. It is clear that for every non-empty subset $S$ of $V(G)$, we have

$$
\operatorname{odd}(G-S) \equiv|S| \equiv \sum_{x \in S} f(x)(\bmod 2) .
$$

We consider two cases.

Case 1. odd $(G-S)<\sum_{x \in S} f(x)$ for all $\emptyset \neq S \subset V(G)$.

In this case, we have $\operatorname{odd}(G-S) \leq \sum_{x \in S} f(x)-2$ by (5.7). Since $G$ is not a tree, we can take an edge $e_{1}$ of $G$ such that $G-e_{1}$ is connected. For any non-empty subset $S \subset V\left(G-e_{1}\right)=V(G)$, we have

$$
\operatorname{odd}\left(G-e_{1}-S\right) \leq \operatorname{odd}(G-S)+2 \leq \sum_{x \in S} f(x) .
$$

By the inductive hypotheses, $G-e_{1}$ has a $(1, f)$-odd factor, which is of course the desired $(1, f)$-odd factor of $G$.

Case 2. $\operatorname{odd}(G-S)=\sum_{x \in S} f(x)$ for some $\emptyset \neq S \subset V(G)$.

Choose a maximal subset $S \subset V(G)$, which satisfies odd $(G-S)=\sum_{x \in S} f(x)$. Then

$$
\operatorname{odd}(G-X)<\sum_{x \in X} f(x) \quad \text { for all } \quad S \subset X \subseteq V(G) .
$$

Claim 2.1 Every even component of $G-S$ has a $(1, f)$-odd factor.

Let $D$ be an even component of $G-S$, and $\emptyset \neq R \subset V(D)$. Then by (5.8), we have

$$
\operatorname{odd}(G-S)+\operatorname{odd}(D-R)=\operatorname{odd}(G-(S \cup R))<\sum_{x \in S \cup R} f(x),
$$

which implies odd $(D-R)<\sum_{x \in R} f(x)$. Hence the claim holds by induction.

Claim 2.2 For any odd component $C$ of $G-S$ and any edge $e=v w$ joining $v \in V(C)$ to $w \in S, C+e$ has a $(1, f)$-odd factor, where $f$ is defined as $f(w)=1$ (Figure 5.3). 
Let $\emptyset \neq R \subset V(C+e)=V(C) \cup\{w\}$. If $R \neq\{w\}$, then by (5.8), we have

$$
\begin{aligned}
\sum_{x \in S \cup R} f(x) & >\operatorname{odd}(G-(S \cup T)) \\
& =\operatorname{odd}(G-S)-1+\operatorname{odd}((C+e)-(R \cup\{w\})) \\
& \geq \sum_{x \in S} f(x)-1+\operatorname{odd}((C+e)-R)-1 .
\end{aligned}
$$

Thus $\sum_{x \in R} f(x)>\operatorname{odd}((C+e)-R)-2$, which implies $\sum_{x \in T} f(x) \geq \operatorname{odd}((C+$ $e)-R$ ) by (5.7). If $R=\{w\}$, then $C+e-\{w\}=C$ has odd order, and thus odd $((C+e)-\{w\})=1=f(w)$. Hence $C+e$ satisfies (5.5), and has the desired $(1, f)$-odd factor by induction. Therefore Claim 2.2 is proved.

Let $C_{1}, C_{2}, \ldots, C_{m}$ be the odd components of $G-S$, where $m=$ odd $(G-$ $S)=\sum_{x \in S} f(x)$. We construct a bipartite graph $B$ with partition $\left\{C_{1}, C_{2}, \ldots\right.$ ,$\left.C_{m}\right\} \cup S$ as follows: a vertex $x$ of $S$ and $C_{i}$ are joined by an edge of $B$ if and only if $x$ and $C_{i}$ are joined by at least one edge of $G$ (see Figure 5.3).
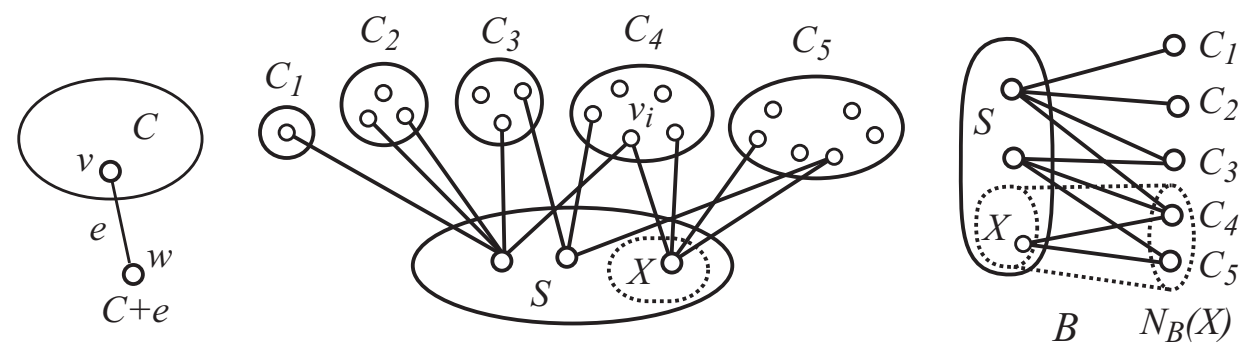

Figure 5.3: A subgraph $C+e$, the odd components of $G-S$ with edges $v_{i} w_{i}$, and the bipartite graph $B$.

Claim 2.3. The bipartite graph $B$ has a factor $K$ such that $\operatorname{deg}_{K}(x)=f(x)$ for $x \in S$ and $\operatorname{deg}_{K}\left(C_{i}\right)=1$ for all $1 \leq i \leq m$.

It follows from the connectedness of $G$ that $\left|N_{B}(S)\right|=\left|\left\{C_{1}, C_{2}, \ldots, C_{m}\right\}\right|=$ $\sum_{x \in S} f(x)$. Assume that $\left|N_{B}(X)\right|<\sum_{x \in X} f(x)$ for some $\emptyset \neq X \subset S$. Then every vertex $C_{i} \in\left\{C_{1}, C_{2}, \ldots, C_{m}\right\}-N_{B}(X)$ is an isolated vertices of $B-(S-X)$, which implies that $C_{i}$ is an odd component of $G-(S-X)$, and thus

$$
\begin{aligned}
& \operatorname{odd}(G-(S-X)) \geq\left|\left\{C_{1}, C_{2}, \ldots, C_{m}\right\}-N_{B}(X)\right| \\
> & \sum_{x \in S} f(x)-\sum_{x \in X} f(x)=\sum_{x \in S-X} f(x) .
\end{aligned}
$$


This contradicts (5.5). Therefore $\left|N_{B}(X)\right| \geq \sum_{x \in X} f(x)$ for all $X \subseteq S$, and so by the Generalized Marriage Theorem 1.1.10, $B$ has the desired factor $K$.

Let $K$ be the factor of $B$ given in Claim 2.3. For every edge $w_{i} C_{i}$ of $K$ with $w_{i} \in S$, choose a vertex $v_{i} \in V\left(C_{i}\right)$ that is adjacent to $w_{i}$ in $G$, and take a $(1, f)$-odd factor $F\left(C_{i}+v_{i} w_{i}\right)$ of $C_{i}+v_{i} w_{i}$, whose existence is guaranteed by Claim 2.2. For every even component $D_{j}$ of $G-S, D_{j}$ has a $(1, f)$-odd factor $F\left(D_{j}\right)$ by Claim 2.1. By combining the above factors, we obtain the following desired $(1, f)$-odd factor $F$ of $G$ :

$$
F=\bigcup_{j} F\left(D_{j}\right) \cup \bigcup_{i} F\left(C_{i}+v_{i} w_{i}\right) .
$$

Note that for any vertex $x \in S$, $\operatorname{deg}_{F}(x)=\operatorname{deg}_{K}(x)=f(x)$ since there exist exactly $f(x)$ odd components $C_{i}$ of $G-S$ for which $w_{i}=x$. Consequently the theorem is proved.

The next theorem is obtained by setting $f(x)=n$ for all vertices $x$ though the second proof of the $(1, f)$-Odd Factor Theorem was first essentially given in the proof of the following theorem.

Theorem 5.1.7 (Amahashi [9] (1985)) Let $G$ be a general graph and $n \geq$ 1 be an odd integer. Then $G$ has an $[1, n]$-odd factor if and only if

$$
\operatorname{odd}(G-S) \leq n|S| \quad \text { for all } \quad S \subseteq V(G) .
$$

\section{$5.2(1, f)$-Odd Subgraphs and Structure The- orem}

Let $f: V(G) \rightarrow\{1,3,5, \ldots\}$. Then a subgraph $H$ of $G$ is called a $(1, f)$-odd subgraph if

$$
\operatorname{deg}_{H}(x) \in\{1,3,5, \ldots, f(x)\} \quad \text { for all } \quad x \in V(H) .
$$

Of course, a spanning $(1, f)$-odd subgraph is a $(1, f)$-odd factor. For a constant odd integer $n \geq 1$, a $[1, n]$-odd subgraph can be defined analogously. A $(1, f)$-odd subgraph $H$ of $G$ is said to be maximum if $G$ has no $(1, f)$-odd subgraph $H^{\prime}$ such that $\left|H^{\prime}\right|>|H|$.

Let $G$ be a general graph, and $H$ be a $(1, f)$-odd subgraph of $G$. Then for any cycle $C$ of $H$, which may be a cycle consisting of two edges, $H-E(C)$ is 
also a $(1, f)$-odd subgraph with vertex set $V(H)$. Hence if we take a minimal $(1, f)$-odd subgraph $H$ with respect to its edge set, then $H$ has no cycles, that is, $H$ is a forest. In particular, a general graph $G$ has a $(1, f)$-odd subgraph with vertex set $W$ if and only if its underlying simple graph has one, with vertex set $W$. Therefore when we consider a $(1, f)$-odd subgraph or $(1, f)$ odd factor, we may restrict ourselves to simple graphs (see, for example, Theorem 5.2.2, 5.2.3).

It is clear that the next theorem is a generalization of Theorem 3.3.2, which gives the formula for the order of a maximum matching.

Theorem 5.2.1 (Kano and Katona [71] (2002)) Let $G$ be a general graph and $f: V(G) \rightarrow\{1,3,5, \ldots\}$. Then the order of a maximum $(1, f)$-odd subgraph $H$ of $G$ is given by

$$
|H|=|G|-\max _{S \subseteq V(G)}\left\{\operatorname{odd}(G-S)-\sum_{x \in S} f(x)\right\} .
$$

Proof. Let $H$ be a maximum $(1, f)$-odd subgraph of $G$, and let

$$
d=\max _{S \subseteq V(G)}\left\{o d d(G-S)-\sum_{x \in S} f(x)\right\} .
$$

Then $d \geq 0$ as odd $(G) \geq 0$, and $|G|+d$ is even, since

$$
\begin{aligned}
|G| & \equiv \operatorname{odd}(G-S)+|S| \equiv \operatorname{odd}(G-S)-|S| \\
& \equiv \operatorname{odd}(G-S)-\sum_{x \in S} f(x)=d(\bmod 2) .
\end{aligned}
$$

We first show that $|H| \leq|G|-d$. Let $S \subset V(G)$ such that

$$
\operatorname{odd}(G-S)-\sum_{x \in S} f(x)=d .
$$

Then for an odd component $C$ of $G-S$, if $V(C)$ is covered by $H$, then there exists at least one edge in $H$ that joins $C$ to $S$. Thus at most $e_{H}(V(G)-S, S)$ odd components of $G-S$ are covered by $H$. Since $e_{H}(V(G)-S, S) \leq$ $\sum_{x \in S} f(x)$, at least $\operatorname{odd}(G-S)-\sum_{x \in S} f(x)$ odd components of $G-S$ are not covered by $H$. This implies $|H| \leq|G|-d$.

We next prove the reverse inequality. Let $G^{*}=G+K_{d}$ be the join of $G$ and the complete graph $K_{d}$, and define $f^{\prime}: V\left(G^{*}\right) \rightarrow\{1,3,5, \ldots\}$ by

$$
f^{\prime}(x)=f(x) \text { for all } x \in V(G) \text {, and } f^{\prime}(x)=1 \text { for all } x \in V\left(K_{d}\right) .
$$




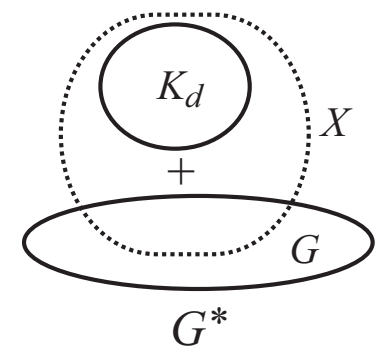

Figure 5.4: $G^{*}=G+K_{d}$ and a subset $X, V\left(K_{d}\right) \subset X \subset V\left(G^{*}\right)$.

Let $\emptyset \neq X \subset V\left(G^{*}\right)$. If $V\left(K_{d}\right) \not X X$, then $\operatorname{odd}\left(G^{*}-X\right) \leq 1 \leq \sum_{x \in X} f^{\prime}(x)$. If $V\left(K_{d}\right) \subseteq X$ (Figure 5.4), then

$$
\operatorname{odd}\left(G^{*}-X\right)=\operatorname{odd}(G-X \cap V(G)) \leq \sum_{x \in X \cap V(G)} f(x)+d \leq \sum_{x \in X} f^{\prime}(x)
$$

Hence by Theorem 5.1.4, $G^{*}$ has a $\left(1, f^{\prime}\right)$-odd factor $F^{\prime}$. Let $H^{\prime}=F^{\prime}-V\left(K_{d}\right)$. Since $\operatorname{deg}_{F^{\prime}}(x)=f^{\prime}(x)=1$ for all $x \in V\left(K_{d}\right), H^{\prime}$ has at most $d$ even vertices. Therefore $H^{\prime}$ has at most $d$ even components.

By applying (i) or (ii) of Theorem 5.1.6 to each component of $H^{\prime}$ according to its parity, we obtain an odd subgraph $M$ of $H^{\prime}$ such that $|M| \geq\left|H^{\prime}\right|-d=$ $|G|-d$. Since $M$ is a $(1, f)$-odd subgraph of $G$, the proof is complete.

We now discuss barriers for $(1, f)$-odd factors. For a graph $G$ and a function $f: V(G) \rightarrow\{1,3,5, \ldots\}$, a non-empty subset $S \subset V(G)$ is called a barrier for $(G, f)$ if $\operatorname{odd}(G-S)>\sum_{x \in S} f(x)$. A barrier $S$ is said to be minimal if no proper subset of $S$ is a barrier. Recall that for a graph $G$, $\alpha(G)$ and $\kappa(G)$ denote the independence number and the connectivity of $G$, respectively.

Theorem 5.2.2 (Topp and Vestergaard [137]) Let $G$ be a connected simple graph of even order, and $f: V(G) \rightarrow\{1,3,5, \ldots\}$. Assume that $G$ has no $(1, f)$-odd factor and let $S$ be a minimal barrier for $(G, f)$. Then

(i) $\operatorname{odd}(G-S) \geq \sum_{x \in S} f(x)+2$;

(ii) each vertex $v$ of $S$ is adjacent to at least $f(v)+2$ distinct odd components of $G-S$;

(iii) each vertex $v$ of $S$ is the center of an induced star $K(1, f(v)+2)$ in $G$; and

(iv) $\kappa(G) \leq|S| \leq \min \left\{(|G|-2) /\left(f_{0}+1\right),(\alpha(G)-2) / f_{0}, \| G||-|G|+2\right\}$, where $f_{0}=\min \{f(x): x \in V(G)\}$. 
Proof. (i) Let $S$ be a minimal barrier for $(G, f)$. Then odd $(G-S)>$ $\sum_{x \in S} f(x)$, which implies (i) by (5.7).

(ii) If $v \in S$ is adjacent to $m$ distinct odd components of $G-S$, then since $S$ is a minimal barrier and by (i), it follows that

$$
\begin{aligned}
& \sum_{x \in S-v} f(x) \geq \operatorname{odd}(G-(S-v)) \\
& \geq \operatorname{odd}(G-S)-m \geq \sum_{x \in S} f(x)+2-m .
\end{aligned}
$$

Hence we have $m \geq f(v)+2$.

(iii) Since each $v \in S$ is adjacent to at least $f(v)+2$ odd components of $G-S$, we can find an induced star $K(1, f(v)+2)$ with center $v$.

(iv) Since $S$ is a cut of $G,|S| \geq \kappa(G)$. By (i), we have

$$
|G| \geq|S|+\operatorname{odd}(G-S) \geq|S|+\sum_{x \in S} f(x)+2 \geq|S|+f_{0}|S|+2 .
$$

Hence $|S| \leq(|G|-2) /\left(f_{0}+1\right)$.

By taking one vertex from each odd component of $G-S$, we get an independent set $I$ of cardinality odd $(G-S)$. Therefore

$$
\alpha(G) \geq|I|=\operatorname{odd}(G-S) \geq \sum_{x \in S} f(x)+2 \geq f_{0}|S|+2,
$$

and thus $|S| \leq(\alpha(G)-2) / f_{0}$. We omit the proof of the last inequality.

Let $n \geq 1$ be an odd integer. By the above Theorem 5.2.2, it follows that if a connected graph $G$ of even order has no induced star $K(1, n+2)$, then $G$ has a $[1, n]$-odd factor. This is a generalization of Theorem 1.5.9, which says that every connected $K(1,3)$-free graph of even order has a 1 -factor. Sumner extended this result to graphs with high connectivity, that is, he showed that if an $n$-connected graph of even order has no induced subgraph isomorphic to $K(1, n+1)$, then $G$ has a 1 -factor. The following theorem is a generalization of this result.

Theorem 5.2.3 (Topp and Vestergaard [137]) Let $G$ be an n-connected simple graph of even order and let $f: V(G) \rightarrow\{1,3,5, \ldots\}$. If no vertex $v$ of $G$ is the center of an induced star $K(1, n f(v)+1)$, then $G$ has a $(1, f)$-odd factor. 
Proof. Suppose that no vertex $v$ of $G$ is the center of an induced star $K(1, n f(v)+1)$ and that $G$ has no $(1, f)$-odd factor. Let $S$ be a minimal barrier for $(G, f)$. Let $D_{1}, D_{2}, \ldots, D_{t}$ be the odd components of $G-S$. Since $G$ is $n$-connected, for every $i \in\{1, \ldots, t\}$, the set $N_{G}\left(D_{i}\right) \cap S$ contains at least $n$ distinct vertices, and so we can take a set $E_{i}$ of $n$ edges joining $D_{i}$ to $n$ distinct vertices of $S$. Then the set $E=\cup_{i=1}^{t} E_{i}$ has exactly $n t$ edges. Since $t=\operatorname{odd}(G-S) \geq \sum_{x \in S} f(x)+2$ by Theorem 5.1.5 and (5.7), we have

$$
|E|=n t \geq n\left(\sum_{x \in S} f(x)+2\right)>\sum_{x \in S} n f(x) .
$$

This implies that some vertex $v \in S$ is incident with at least $n f(v)+1$ edges of $E$. Certainly, if a vertex $v \in S$ is incident with at least $n f(v)+1$ edges of $E$, then $v$ the center of an induced star $K(1, n f(v)+1)$ in $G$. This is a contradiction, and the proof is complete.

It is shown in Theorem 1.5.7 that if a connected simple graph $G$ of even order satisfies

$$
N_{G}(S)=V(G) \quad \text { or } \quad\left|N_{G}(S)\right|>\frac{4}{3}|S|-1 \quad \text { for all } \quad S \subset V(G),
$$

then $G$ has a 1-factor. This result is extended to $[1, n]$-odd factor in the next theorem. However, it is not known whether there exists a similar result for $(1, f)$-odd factor.

Theorem 5.2.4 (Cui and Kano [35]) Let $G$ be a simple graph of even order and $n$ an odd positive integer. If $G$ satisfies

$$
N_{G}(S)=V(G) \quad \text { or } \quad\left|N_{G}(S)\right|>\left(1+\frac{1}{3 n}\right)|S|-\frac{1}{n}
$$

for all $S \subseteq V(G)$, then $G$ has a $[1, n]$-odd factor.

Proof. Suppose that $G$ satisfies (5.12) but has no $[1, n]$-odd factor. Then by Theorem 5.1.7, there exists $\emptyset \neq S \subset V(G)$ such that $\operatorname{odd}(G-S)>n|S|$. Since $\operatorname{odd}(G-S) \equiv|S|(\bmod 2)$, we have $\operatorname{odd}(G-S) \geq n|S|+2$. Let $m$ denote the number of isolated vertices of $G-S$, and let

$$
t=1+\frac{1}{3 n} \quad \text { and } \quad r=\frac{1}{n} .
$$

We consider two cases.

Case 1. $m \geq 1$, i.e., $G-S$ has at least one isolated vertex. 


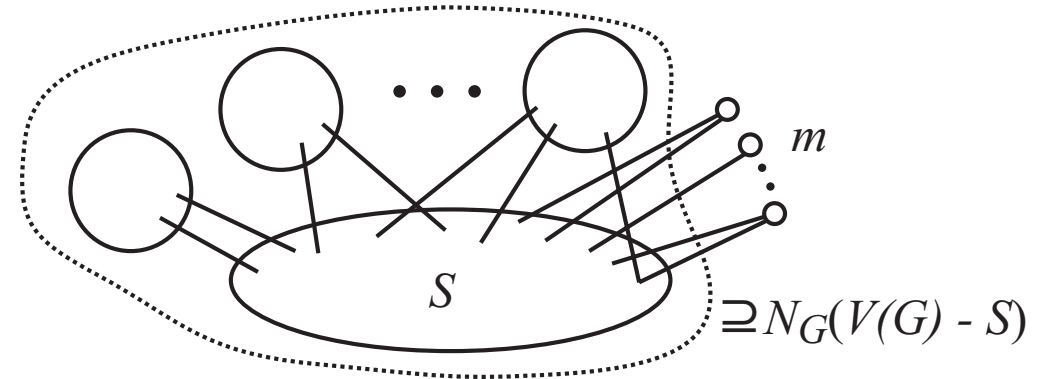

Figure 5.5: A graph $G$ with $S$ and $N_{G}(V(G)-S)$.

Since $N_{G}(V(G)-S) \neq V(G)$ (Figure 5.5), by (5.12) we have

$$
\left|N_{G}(V(G)-S)\right|>t|V(G)-S|-r=t|G|-t|S|-r .
$$

It is clear that $|G|-m \geq\left|N_{G}(V(G)-S)\right|$. From these two inequalities, we obtain

$$
\frac{t|S|+r-m}{t-1}>|G|
$$

On the other hand, $G-S$ has at least $n|S|+2-m$ odd components with order at least three, and thus $m+3(n|S|+2-m) \leq|G|-|S|$. Hence

$$
(3 n+1)|S|+6-2 m \leq|G|
$$

Combining (5.13) and (5.14), we obtain

$$
(3 n+1)|S|+6-2 m<\frac{t|S|+r-m}{t-1} .
$$

Substituting the values of $t$ and $r$ into (5.15), we obtain

$$
\begin{aligned}
(3 n+1)|S|+6-2 m & <\frac{\left(1+\frac{1}{3 n}\right)|S|+\frac{1}{n}-m}{\frac{1}{3 n}} \\
(3 n+1)|S|+6-2 m & <(3 n+1)|S|+3-3 n m \\
3+(3 n-2) m & <0 .
\end{aligned}
$$

This is a contradiction.

Case 2. $\quad m=0$, i.e., $G-S$ has no isolated vertex.

In this case, every odd component has at least three vertices. Take an odd component $C$ of $G-S$, and let $X=V(G)-V(C)$. Then since $N_{G}(X) \neq$ 
$V(G)$, it follows from (5.12) that $\left|N_{G}(X)\right|>t|X|-r$. It is obvious that $\left|N_{G}(X)\right| \leq|X|+|S|$. Thus $|X|+|S|>t|X|-r$ and hence

$$
|X|<\frac{|S|+r}{t-1}
$$

On the other hand, $|X| \geq 3(n|S|+1)$ as well. Thus combining this and (5.16), we obtain

$$
3(n|S|+1)<\frac{|S|+r}{t-1} .
$$

Substituting the values of $t$ and $r$ in the above inequality, we have

$$
\begin{aligned}
3(n|S|+1) & <\frac{|S|+\frac{1}{n}}{\frac{1}{3 n}} \\
3(n|S|+1) & <3 n|S|+3 \\
0 & <0 .
\end{aligned}
$$

This is a contradiction. Consequently the theorem is proved.

Petersen proved that every 2-edge connected cubic graph has a 1-factor (see Theorem 1.5.2) and there are cubic graphs having no 1-factors, where a cubic graph means a 3 -regular graph. If we consider a special $(1, f)$-odd factor instead of a 1-factor, we can prove the existence of such a factor in every cubic graph as shown in the following theorem.

Theorem 5.2.5 ([75]) Let $G$ be a connected 3-regular multigraph, and define a function $f$ as

$$
f(x)=\left\{\begin{array}{l}
3 \quad \text { if } x \text { is a cutvertex } \\
1 \text { otherwise. }
\end{array}\right.
$$

Then $G$ has a $(1, f)$-odd factor (Figure 5.6).

Proof. Let $S \subset V(G)$ with $|S| \geq 2$, and let $D_{1}, D_{2}, \ldots, D_{m}$ be the odd components of $G-S$, where $m=\operatorname{odd}(G-S)$ (Figure 5.6). Note that if $S$ consists of one vertex, then the required inequality (5.5) easily holds. So we may assume that $S$ contains at least two vertices. For every $D=D_{i}$, it follows that

$$
1 \equiv 3|D|=\sum_{x \in V(D)} \operatorname{deg}_{G}(x)=2|| D \|+e_{G}(D, S) \equiv e_{G}(D, S)(\bmod 2) .
$$

Hence $e_{G}(D, S)=1$ or $e_{G}(D, S) \geq 3$, moreover, if $e_{G}(D, S)=1$ then $D$ must be adjacent to a cutvertex of $S$. Let

$$
\Omega=\left\{D_{i} \mid e_{G}\left(D_{i}, S\right)=1,1 \leq i \leq m\right\}, \quad \text { and } \quad t=|\Omega|,
$$



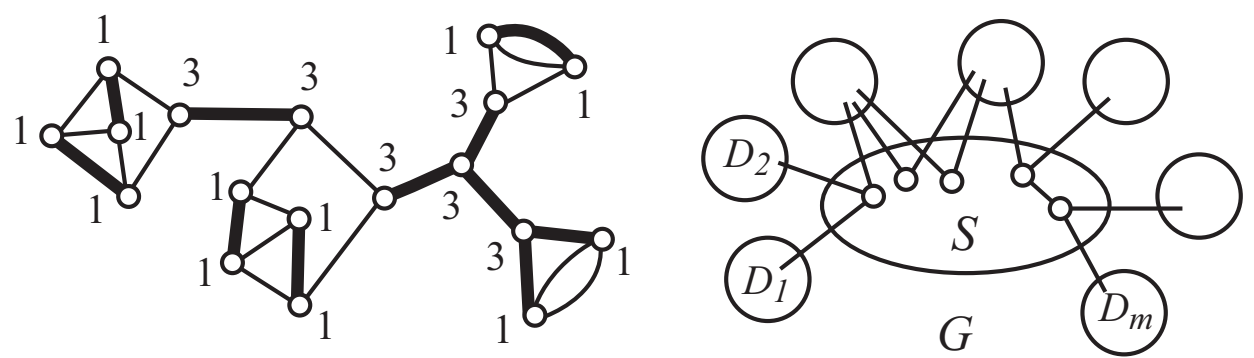

Figure 5.6: (a) A 3-regular multigraph and its $(1, f)$-odd factor; numbers denote $f(x)$. (b) $G-S$ and the odd components of $G-S$.

and let $C u t v(S)$ denote the set of cutvertices of $S$ in $G$. Since $G$ is connected, for a cutvertex $v$ of $S$, at most two components in $\Omega$ are adjacent to $v$ (Figure 5.6), and thus $t \leq 2|C u t v(S)|$. It follows from the above property of $e_{G}(D, S)$ that

$$
\begin{aligned}
& t+3(m-t) \leq e_{G}\left(D_{1} \cup D_{2} \cup \cdots D_{m}, S\right) \\
\leq & \sum_{x \in S} \operatorname{deg}_{G}(x)=3|S|=\sum_{x \in S-C u t v(S)} 3 f(x)+\sum_{x \in C u t v(S)} f(x) .
\end{aligned}
$$

Since $t \leq 2|\operatorname{Cutv}(S)|=(2 / 3) \sum_{x \in C u t v(S)} f(x)$, it follows from the above inequality that

$$
\begin{aligned}
m & \leq \sum_{x \in S-C u t v(S)} f(x)+\frac{1}{3} \sum_{x \in C u t v(S)} f(x)+\frac{2}{3} t \\
& \leq \sum_{x \in S-C u t v(S)} f(x)+\left(\frac{1}{3}+\frac{4}{9}\right) \sum_{x \in C u t v(S)} f(x) \\
& \leq \sum_{x \in S} f(x) .
\end{aligned}
$$

Consequently, by Theorem 5.1.4, $G$ has the desired $(1, f)$-odd factor.

Here we give some remarks on the following problem, which one could easily surmise from Theorems 5.1.4 and 5.1.5. Note that if $G$ satisfies

$$
\operatorname{odd}(G-S) \leq \sum_{x \in S} f(x) \quad \text { for all } S \subset V(G)
$$

then by taking a minimal $(1, f)$-odd factor, which is a forest, and by adding some edges to it, we can obtain a spanning tree $T$. This spanning tree $T$ satisfies $\operatorname{odd}(T-v) \leq f(v)$ for all $v \in V(T)$. 
Problem 5.2.6 Let $G$ be a simple connected graph and $h: V(G) \rightarrow$ $\{2,4,6, \ldots\}$.

(1) If $G$ satisfies

$$
\operatorname{odd}(G-S) \leq 2|S| \quad \text { for all } \emptyset \neq S \subset V(G),
$$

what factor or property does $G$ have?

(2) If $G$ satisfies

$$
\operatorname{odd}(G-S) \leq \sum_{x \in S} h(x) \quad \text { for all } \emptyset \neq S \subset V(G),
$$

what factor or property does $G$ have?

(3) If $G$ satisfies

$$
\operatorname{odd}(G-S) \leq \sum_{x \in S} h(x) \quad \text { for all } \emptyset \neq S \subset V(G),
$$

then does $G$ has a spanning tree $T$ such that

$$
\operatorname{odd}(T-v) \leq h(v) \quad \text { for all } v \in V(T) \text { ? }
$$

For the above problem (3), the following are some negative known results.

Remark 5.2.7 Let $m \geq 2$ be an even integer and $G=K(2,2 m)$. Then $G$ has even order and satisfies odd $(G-S) \leq m|S|$ for all $S \subset V(G)$. However, $G$ has no spanning tree $T$ such that odd $(T-v) \leq m$ for all $v \in(G)$.

Remark 5.2.8 Let $n$ be a positive odd integer and $G$ be the cycle $C_{n}$ of order $n$. Then $G$ has odd order and satisfies odd $(G-S) \leq|S|$ for all non-empty subset $S$ of $V(G)$, but $G$ has no spanning tree $T$ such that odd $(T-v) \leq 1$ for all $v \in V(G)$.

Remark 5.2.9 (Saito [130]) Let $m \geq 2$ be an even integer and $G=K(3,3 m)$. Then $G$ has odd order and satisfies odd $(G-S) \leq m|S|$ for all non-empty subset $S$ of $V(G)$. However, $G$ has no spanning tree $T$ such that odd $(T-S) \leq$ $m|S|$ for all non-empty subsets $S$ of $V(G)$, although $G$ contains a spanning tree $R$ which satisfies odd $(R-v) \leq m$ for all $v \in V(G)$.

We show some other properties of maximum $(1, f)$-odd subgraphs, which are extensions of properties of maximum matchings. In particular, we obtain a structure theorem on $(1, f)$-odd subgraphs. In order to give short proofs 
for these results, we introduce a new method based on Kano, Katona and Szabo [73]. As we stated in the previous section, when we consider $(1, f)$-odd subgraphs we may restrict ourselves to simple graphs. So in order to make the argument short and simple, hereafter we consider only simple graphs though these results can be extended to general graphs.

For a simple graph $G$ and a function $f: V(G) \rightarrow\{1,3,5, \ldots\}$, define the new graph $G^{f}$ as follows: Replace every vertex $v \in V(G)$ by the complete graph $K(f(v))$ on $f(v)$ vertices, and for every edge $x y$ of $G$, join every vertex of $K(f(x))$ to every vertex of $K(f(y))$. In particular, there are $f(x) f(y)$ edges between $K(f(x))$ and $K(f(y))$ in $G^{f}$ (Figure 5.2). Then

$$
\left|G^{f}\right|=\sum_{x \in V(G)} f(x) \quad \text { and } \quad\left\|G^{f}\right\|=\sum_{x y \in E(G)} f(x) f(y) .
$$
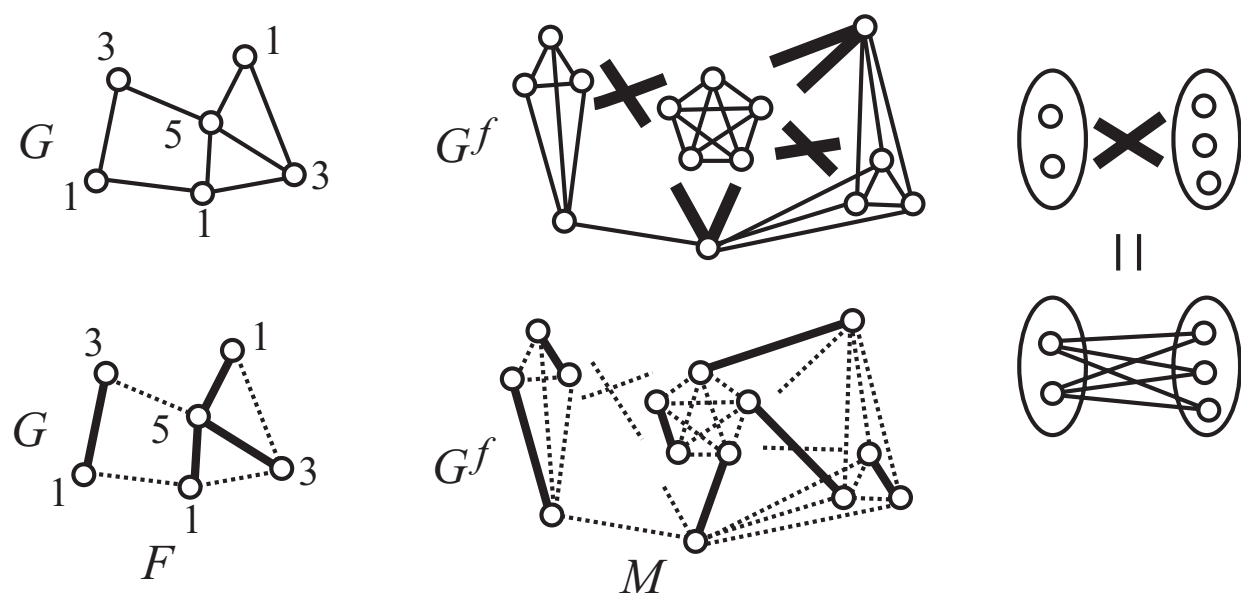

Figure 5.7: A graph $G$ and $G^{f}$; where the numbers denote $f(v)$; a $(1, f)$-odd factor $F$ of $G$ and a perfect matching $M$ of $G^{f}$, where $F=M \cap E(G)$.

There is a strong relationship between a maximum matching of $G^{f}$ and a maximum $(1, f)$-odd subgraph of $G$. Before discussing this, we make a remark on matchings of $G^{f}$. If a matching $M_{1}$ of $G^{f}$ contains two edges joining $K(f(u))$ to $K(f(w))$, then remove these two edges and add two edges, one of $K(f(u))$ and the other of $K(f(w))$, to $M_{1}$ so that the two added edges cover the four endvertices of the two removed edges. By repeating this procedure, we can obtain a matching $M_{2}$ such that $V\left(M_{2}\right)=V\left(M_{1}\right)$ and $M_{2}$ contains at most one edge between $K(f(x))$ and $K(f(y))$ for all edges $x y$ of $G$. Therefore we may assume that 
(a) a matching of $G^{f}$ contains at most one edge joining $K(f(x))$ and $K(f(y))$ for all edges $x y$ of $G$.

For a matching $M$ of $G^{f}$ having property (a), let $M \cap E(G)$ denote the subgraph of $G$ induced by

$$
\{x y \in E(G) \mid M \text { contains an edge joining } K(f(x)) \text { to } K(f(y))\} .
$$

The next proposition relates $(1, f)$-odd factors of $G$ and perfect matchings of $G^{f}$, and provides another proof for the criterion for the existence of a $(1, f)$-odd factor.

Proposition 5.2.10 Let $G$ be a simple graph, $f: V(G) \rightarrow\{1,3,5, \ldots\}$, and $G^{f}$ the graph defined above. Then $G$ has a $(1, f)$-odd factor if and only if $G^{f}$ has a perfect matching (Figure 5.2). Moreover, $G$ has a $(1, f)$-odd factor if and only if

$$
\operatorname{odd}(G-S) \leq \sum_{x \in S} f(x) \quad \text { for all } S \subset V(G)
$$

Proof. Suppose that $G^{f}$ has a perfect matching $M$ with the property (a). Then for every vertex $v$ of $G, \operatorname{deg}_{M \cap E(G)}(v)$ is odd, since an even number of vertices of $K(f(v))$ are saturated by the edges of $M$ contained in $K(f(v))$. It is clear that $\operatorname{deg}_{M \cap E(G)}(v) \leq f(x)$, and thus $M \cap E(G)$ is the desired $(1, f)$-odd factor of $G$.

Conversely, assume that $G$ has a $(1, f)$-odd factor $F$. For each edge $x y$ of $F$, choose one edge joining $K(f(x))$ to $K(f(y))$ so that all the chosen edges form a matching $M_{F}$ of of $G^{f}$. For every vertex $v$ of $G$, an even number $f(v)-\operatorname{deg}_{F}(v)$ of vertices of $K(f(v))$ are not covered by $M_{F}$, and hence we can obtain a perfect matching of $G^{f}$ from $M_{F}$ by adding edges joining two vertices of $K(f(z))$ for every vertex $z$ of $G$. Therefore the first half of the proposition is proved.

We now prove only that if (5.19) holds then $G$ has a $(1, f)$-odd factor, since necessity is easy (Theorem 5.1.5). Let $X$ be a subset of $V\left(G^{f}\right)$, and let

$$
S=\left\{v \in V(G) \mid V(K(f(v)) \subseteq X\} \quad \text { and } \quad T=X-\bigcup_{x \in S} V(K(f(x)) .\right.
$$

Then

$$
\begin{aligned}
\operatorname{odd}\left(G^{f}-X\right) & \leq \operatorname{odd}(G-S)+|T| \\
& \leq \sum_{x \in S} f(x)+|T|=|X|
\end{aligned}
$$




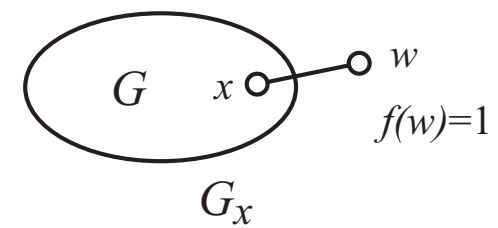

Figure 5.8: A graph $G$ and $G_{x}$ for a vertex $x$.

Therefore $G^{f}$ has a perfect matching by the 1-Factor Theorem 1.4.2, which implies that $G$ has the desired $(1, f)$-odd factor.

For a vertex $x$ of $G$, we denote by $G_{x}$ the graph obtained from $G$ by adding a new vertex $w=w(x)$ together with a new edge $w x$, and define $f(w)=1$ (Figure 5.2). Let

$$
\tau_{f}(G)=\text { the order of a maximum }(1, f) \text {-odd subgraph of } G \text {. }
$$

Note that $\tau(G)=\tau_{1}(G)$ denotes the order of a maximum matching of $G$, where $1: V(G) \rightarrow\{1\}$. Let

$$
D_{f}(G)=\left\{x \in V(G) \mid \tau_{f}\left(G_{x}\right)=\tau_{f}(G)+2\right\} .
$$

Recall that $D(G)$ consists of the vertices $v$ of $G$ such that $v$ is not saturated by some maximum matching of $G$. It is immediate that $D(G)=D_{1}(G)$, where $1: V(G) \rightarrow\{1\}$. Then the following lemma holds.

Lemma 5.2.11 Let $G$ be a simple graph, $f: V(G) \rightarrow\{1,3,5, \ldots\}$, and $G^{f}$ the graph defined above. Then $V(K(f(v))) \cap D\left(G^{f}\right) \neq \emptyset$ implies $V(K(f(v))) \subseteq$ $D\left(G^{f}\right)$. Moreover, it follows that

$$
|G|-\tau_{f}(G)=\left|G^{f}\right|-\tau\left(G^{f}\right)
$$

and

$$
V(K(f(v))) \subseteq D\left(G^{f}\right) \quad \text { if and only if } \quad v \in D_{f}(G)
$$

Proof. By Proposition 5.2.10, we may assume that $G$ has no $(1, f)$-odd factor and $G^{f}$ has no 1-factor. We also assume that maximum matchings of $G^{f}$ considered here possess property (a). It is easy to see that if there exists a maximum matching in $G^{f}$ that does not saturate a vertex in $K(f(v))$, then for any vertex $x$ of $K(f(v)), G^{f}$ has a maximum matching that does not saturate $x$, and so $V(K(f(v))) \subseteq D\left(G^{f}\right)$. For convenience, we say that a matching $M$ of $G^{f}$ saturates $K(f(v))$ if $M$ saturates all the vertices of $K(f(v))$, otherwise, $M$ does not saturate $K(f(v))$. 
Assume that a maximum matching $M$ of $G^{f}$ does not saturate exactly $m \geq 1$ vertices of $G^{f}$.

Then for any vertex $v$ of $G$, $\operatorname{deg}_{M \cap E(G)}(v)$ is even if and only if $M$ does not saturate $K(f(v))$. Thus there are exactly $m$ vertices in $G$ whose degrees in $M \cap E(G)$ are even. Since $M \cap E(G)$ contains at most $m$ odd components, by Theorem 5.1.6 $M \cap E(G)$ contains a $(1, f)$-odd subgraph $H$ with order at least $|G|-m$.

Conversely, let $H^{*}$ be a maximum $(1, f)$-odd subgraph of $G$. Then $G^{f}$ has a maximal matching $M^{*}$ such that $M^{*} \cap E(G)=E\left(H^{*}\right)$, i.e., $M^{*}$ saturates $K(f(v))$ if and only if $H^{*}$ contains $v$. Hence $M^{*}$ satisfies $\left|G^{f}\right|-\left|M^{*}\right|=$ $|G|-\left|H^{*}\right|$. Therefore

$$
|G|-\left|H^{*}\right|=\left|G^{f}\right|-\left|M^{*}\right| \geq\left|G^{f}\right|-|M|=m \geq|G|-|H| \geq|G|-\left|H^{*}\right| .
$$

Hence equality holds in the above inequality, and thus $H$ is a maximum $(1, f)$-odd subgraph and $M^{*}$ is a maximum matching of $G^{f}$. In particular, $|G|-\tau_{f}(G)=\left|G^{f}\right|-\tau\left(G^{f}\right)$, which implies (5.20).

Assume that a vertex $v$ of $G$ is contained in $D_{f}(G)$. Let $H_{v}$ be a maximum $(1, f)$-odd subgraph of $G_{v}=G+v w$, whose order is $\tau_{f}\left(G_{v}\right)$. Since $\tau_{f}\left(G_{v}\right)=$ $\tau_{f}(G)+2, H_{v}$ must contain $w$, and so $H_{v}-w$ has even degree only at $v$ and does not contain exactly $\left|G_{v}\right|-\tau_{f}\left(G_{v}\right)$ vertices of $G$. Thus there exists a maximal matching $M$ in $G^{f}$ such that $M \cap E(G)=E\left(H_{v}-w\right)$. Then $M$ does not saturate the following number of vertices of $G^{f}$ :

$$
\left|G^{f}\right|-|M|=\left|G_{v}\right|-\tau_{f}\left(G_{v}\right)+1=|G|-\tau_{f}(G) .
$$

Hence by (5.20), $M$ is a maximum matching of $G^{f}$ and does not saturate $K(f(v))$. Hence $V\left(K(f(v)) \subseteq D\left(G^{f}\right)\right.$.

Conversely, assume that $V\left(K(f(v)) \subseteq D\left(G^{f}\right)\right.$ for some vertex $v$ of $G$. Then $G^{f}$ has a maximum matching $M$ that does not saturate $K(f(v))$. Then $M \cap E(G)$ has even degree at $v$, and a vertex $u$ of $G$ has odd degree in $M \cap E(G)$ if and only if $V(K(f(u)) \subseteq V(M)$. Thus $M \cap E(G)$ has even degree at exactly $\left|G^{f}\right|-\tau\left(G^{f}\right)$ vertices of $G$ including $v$. Hence a subgraph $(M \cap E(G))+v w$ of $G_{v}=G+v w$ has precisely $\left|G^{f}\right|-\tau\left(G^{f}\right)-1$ vertices of even degree, which implies that $(M \cap E(G))+v w$ contains a $(1, f)$-odd subgraph $H$ with order at least $\left|G_{v}\right|-\left(\left|G^{f}\right|-\tau\left(G^{f}\right)-1\right)$. Hence by (5.20), we have

$$
\left|G_{v}\right|-|H| \leq\left|G^{f}\right|-\left|\tau\left(G^{f}\right)\right|-1=|G|-\tau_{f}(G)-1 .
$$

Since $\left|G_{v}\right|=|G|+1$, we have $\tau_{f}(G)+2 \leq|H| \leq \tau_{f}\left(G_{v}\right)$, and thus $\tau_{f}\left(G_{v}\right)=$ $\tau_{f}(G)+2$ as $\tau_{f}\left(G_{v}\right) \leq \tau_{f}(G)+2$. Therefore $v \in D_{f}(G)$. Consequently the lemma is proved.

The proof of Lemma 5.2.11 includes the proof of the following lemma. 
Lemma 5.2.12 Let $G$ be a simple graph, $f: V(G) \rightarrow\{1,3,5, \ldots\}$, and $G^{f}$ the graph defined above. If $M$ is a maximum matching of $G^{f}$, then $M \cap E(G)$ is a maximum $(1, f)$-odd subgraph of $G$. Conversely, if $H$ is a maximum $(1, f)$-odd subgraph of $G$, then a maximal matching $M^{*}$ such that $M^{*} \cap E(G)=E(H)$ is a maximum matching of $G^{f}$.

A graph $G$ is said to be factor-critical if $G-v$ has a 1-factor for every vertex $v$ of $G$ and, more generally, when $f: V(G) \rightarrow\{1,3,5, \ldots\}$ is given, $G$ is said to be critical with respect to $(1, f)$-odd factors if $G_{v}=G+v w$ has a $(1, f)$-odd factor for all vertices $v$ of $G$.

Lemma 5.2.13 Let $G$ be a simple graph, $f: V(G) \rightarrow\{1,3,5, \ldots\}$, and $G^{f}$ the graph defined above. Then $G$ is critical with respect to $(1, f)$-odd factors if and only if $G^{f}$ is factor-critical.

Proof. Assume that $G$ is critical with respect to $(1, f)$-odd factors. Then for an arbitrary vertex $v$ of $G, G_{v}=G+v w$ has a $(1, f)$-odd factor $F_{v}$. Then $F_{v}-w$ is a spanning subgraph of $G$ in which only one vertex $v$ has even order. Thus there exists a maximal matching $M$ in $G$ with $M \cap E(G)=E\left(F_{v}-w\right)$. Then $M$ does not saturate exactly one vertex of $G^{f}$, which is contained in $K(f(v))$. So for any vertex $x$ of $K(f(v)), G^{f}-x$ has a 1-factor. Hence $G^{f}$ is factor-critical since $v$ is arbitrarily chosen.

Next suppose $G^{f}$ is factor-critical. Let $v$ be arbitrary vertex of $G$, and let $x$ be a vertex of $K(f(v))$. Then $G^{f}-x$ has a 1-factor $M . M \cap E(G)$ is a spanning subgraph of $G$ in which exactly one vertex $v$ has even degree, which is less than $f(v)$. Hence $(M \cap E(G))+v w$ is a $(1, f)$-odd factor of $G_{v}$. Hence $G$ is critical with respect to $(1, f)$-odd factors. Therefore the lemma is proved.

We are now ready to give a structure theorem on $(1, f)$-odd subgraphs. Let $G$ be a simple graph and $f: V(G) \rightarrow\{1,3,5, \ldots\}$. Define

$$
\begin{aligned}
D_{f}(G) & =\left\{x \in V(G) \mid \tau\left(G_{x}\right)=\tau(G)+2\right\}, \\
A_{f}(G) & =N_{G}(D(G)) \backslash D(G), \quad \text { and } \\
C_{f}(G) & =V(G)-D(G)-A(G),
\end{aligned}
$$

i.e., $A_{f}(G)$ be the set of vertices of $V(G)-D(G)$ that are adjacent to at least one vertex in $D_{f}(G)$, and $V(G)$ is decomposed into three disjoint subsets

$$
V(G)=D_{f}(G) \cup A_{f}(G) \cup C_{f}(G) .
$$

Note that if $f(x)=1$ for all vertices $x$ of $G$, then the above decomposition is equivalent to the Gallai-Edmonds decomposition $D(G) \cup A(G) \cup C(G)$. Then the Gallai-Edmonds Structure Theorem 1.6.2 on matchings can be extended to $(1, f)$-odd subgraphs as as follows: 
Theorem 5.2.14 (Structure Theorem on $(1, f)$-odd subgraphs, [72], [135]) Let $G$ be a simple graph, $f: V(G) \rightarrow\{1,3,5, \ldots\}$, and $V(G)=D_{f}(G) \cup$ $A_{f}(G) \cup C_{f}(G)$ be the decomposition defined above. Then the following statements hold (Figure 5.2):

(i) Every component of $\left\langle D_{f}(G)\right\rangle_{G}$ is critical with respect to $(1, f)$-odd factors.

(ii) $\left\langle C_{f}(G)\right\rangle_{G}$ has a $(1, f)$-odd factor.

(iii) Every maximum $(1, f)$-odd subgraph $H$ of $G$ covers $C_{f}(G) \cup A_{f}(G)$, and for every vertex $u \in A_{f}(G), \operatorname{deg}_{H}(u)=f(u)$ and every edge of $H$ incident with $u$ joins $u$ to a vertex in $D_{f}(G)$.

(iv) The order $|H|$ of a maximum $(1, f)$-odd subgraph $H$ is given by

$$
|H|=|G|-\omega\left(\left\langle D_{f}(G)\right\rangle_{G}\right)+\sum_{x \in A_{f}(G)} f(x),
$$

where $\omega\left(\langle D(G)\rangle_{G}\right)$ denotes the number of components of $\langle D(G)\rangle_{G}([71])$.

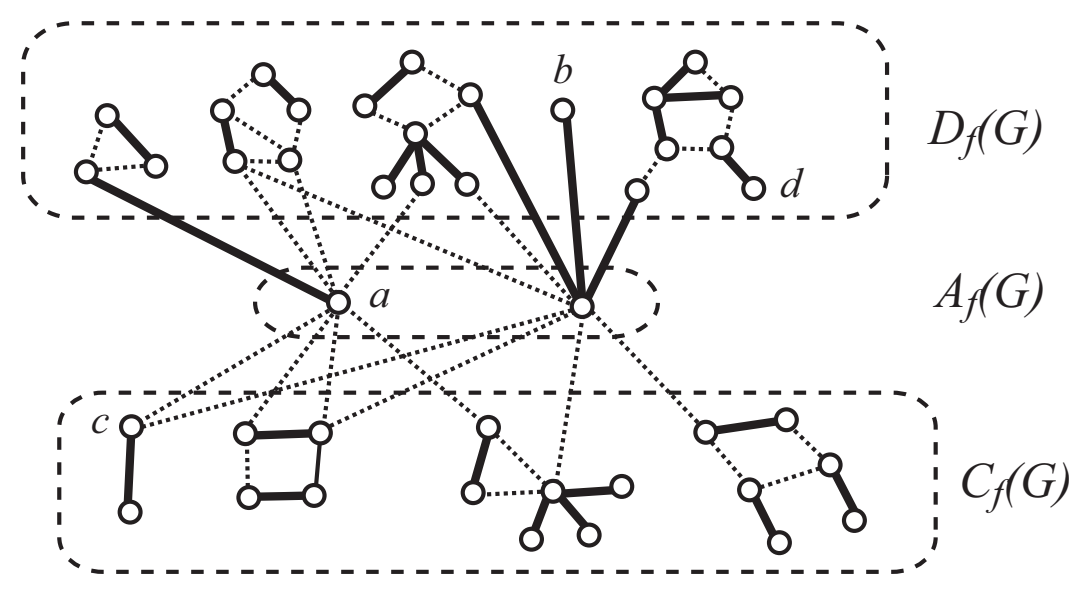

$f(x)=1$ for $x \in\{a, b, c, d\}$, and $f(x)=3$ otherwise.

Figure 5.9: A graph $G$ and its decomposition $D_{f}(G) \cup S_{f}(G) \cup C_{f}(G)$.

Proof. By using the previous lemmas, we shall derive this theorem from the Gallai-Edmonds Structure Theorem 1.6.2, referred to as the Structure Theorem in this proof. 
By Lemma 5.2.11 and the construction of $G^{f}$, it follows that

$$
\begin{aligned}
& V(K(f(v))) \subseteq A\left(G^{f}\right) \text { if and only if } v \in A_{f}(G) ; \text { and } \\
& V(K(f(v))) \subseteq C\left(G^{f}\right) \text { if and only if } v \in C_{f}(G) .
\end{aligned}
$$

Since each component of $C\left(G^{f}\right)$ has a 1-factor by the Structure Theorem, each component of $C_{f}(G)$ has a $(1, f)$-odd factor by Proposition 5.2.10. Thus (ii) holds. Similarly, by the Structure Theorem and by Lemma 5.2.13, the statement (i) holds.

Let $H$ be a maximum $(1, f)$-odd subgraph of $G$. Then $G^{f}$ has a maximum matching $M$ such that $M \cap E(G)=E(H)$ by Lemma 5.2.12. By the Structure Theorem, every edge of $M$ incident with a vertex $u$ of $A\left(G^{f}\right)$ joins $u$ to $D\left(G^{f}\right)$. Hence every edge of $H$ incident with a vertex of $A_{f}(G)$ joins $A_{f}(G)$ to $D_{f}(G)$. Hence the statement (iii) follows.

By the Structure Theorem, it follows that

$$
|M|=\left|G^{f}\right|-\omega\left(\left\langle D\left(G^{f}\right)\right\rangle\right)+\left|A\left(G^{f}\right)\right| .
$$

Since $|G|-|H|=\left|G^{f}\right|-|M|$ by (5.20) and since

$$
\omega\left(\left\langle D\left(G^{f}\right)\right\rangle\right)=\omega\left(\left\langle D_{f}(G)\right\rangle\right), \quad\left|A\left(G^{f}\right)\right|=\sum_{x \in A_{f}(G)} f(x),
$$

we have

$$
|H|=|G|-\omega\left(\left\langle D_{f}(G)\right\rangle\right)+\sum_{x \in A_{f}(G)} f(x) .
$$

Therefore (iv) is proved. Consequently, the proof is complete.

It is known that a matching $M$ of a graph is maximum if and only if there exists no augmenting path connecting two $M$-unsaturated vertices (Theorem 1.3.2). We now generalize this result to a maximum $(1, f)$-odd subgraph.

Let $H$ be a $(1, f)$-odd subgraph of a simple graph $G$. Call the edges of $H$ blue edges and the edges of $E(G)-E(H)$ red edges. For a subgraph $S$ of $G$ and a vertex $x$ of $S$, We denote by $\operatorname{deg}_{S}^{b l u e}(x)$ the number of blue edges of $S$ incident with $x$, and by $\operatorname{deg}_{S}^{r e d}(x)$ the number of red edges of $S$ incident with $x$. In particular, $\operatorname{deg}_{G}^{b l u e}(y)=\operatorname{deg}_{H}(y)$ for every $y \in V(H)$. An $H$-augmenting walk connecting two distinct vertices $u$ and $v$ is a walk $W$ that satisfies

(i) $\operatorname{deg}_{W}^{\text {blue }}(u)=\operatorname{deg}_{W}^{\text {blue }}(v)=0$,

(ii) $\operatorname{deg}_{W}^{r e d}(u)=\operatorname{deg}_{W}^{r e d}(v)=1, \quad$ and

(iii) $\operatorname{deg}_{W}^{\text {red }}(x)-\operatorname{deg}_{W}^{\text {blue }}(x) \leq f(x)-\operatorname{deg}_{H}(x) \quad$ for all $x \in V(W)-\{u, v\}$.

It is easy to see that if $f: V(G) \rightarrow\{1\}$ and $H$ is a matching, then an $H$-augmenting walk is nothing but an augmenting path. 
Theorem 5.2.15 Let $G$ be a simple graph, $f: V(G) \rightarrow\{1,3,5, \ldots\}$, and $H$ a $(1, f)$-odd subgraph of $G$. Then $H$ is a maximum $(1, f)$-odd subgraph of $G$ if and only if $G$ has no $H$-augmenting walk.

Proof. Suppose that $H$ is a $(1, f)$-odd subgraph of $G$ and there is an $H$ augmenting walk $W$ connecting two distinct vertices $u$ and $v$, where $u, v \notin$ $V(H)$. Then $W \triangle H$, which is the subgraph of $G$ induced by $(E(H) \cup E(W))-$ $(E(H) \cap E(W))$, is a $(1, f)$-odd subgraph since for every vertex $x \in V(W)-$ $\{u, v\}$,

$$
\begin{aligned}
\operatorname{deg}_{W \triangle H}(x) & =\operatorname{deg}_{H}(x)-\operatorname{deg}_{W}^{\text {blue }}(x)+\operatorname{deg}_{W}^{\text {red }}(x) \\
& =\operatorname{deg}_{H}(x)+\operatorname{deg}_{W}(x)-2 \operatorname{deg}_{W}^{\text {blue }}(x) \\
& \equiv \operatorname{deg}_{H}(x)(\bmod 2) \quad\left(\text { as } \operatorname{deg}_{W}(x) \text { is even }\right)
\end{aligned}
$$

and $\operatorname{deg}_{W \triangle H}(x) \leq f(x)$ by (iii). Furthermore, $W \triangle H$ covers all the vertices of $H$ and $\{u, v\}$, therefore $H$ is not a maximum $(1, f)$-odd subgraph.

Assume that a $(1, f)$-odd subgraph $H$ of $G$ is not maximum. Call the edges of $H$ red edges and the edges not in $H$ blue edges. Then there exists a maximal matching $M$ in $G^{f}$ with the property that (a) $M \cap E(G)=E(H)$. By Lemma 5.2.12, $M$ is not a maximum matching of $G^{f}$. Hence there exists an augmenting path $P$ connecting two distinct $M$-unsaturated vertices $x_{1}$ of $\left(K(f(u))\right.$ and $y_{1}$ of $K(f(v))$. By taking a shortest augmenting path, we may assume that $P$ passes through $(K(f(u))$ and $K(f(v))$ exactly once.

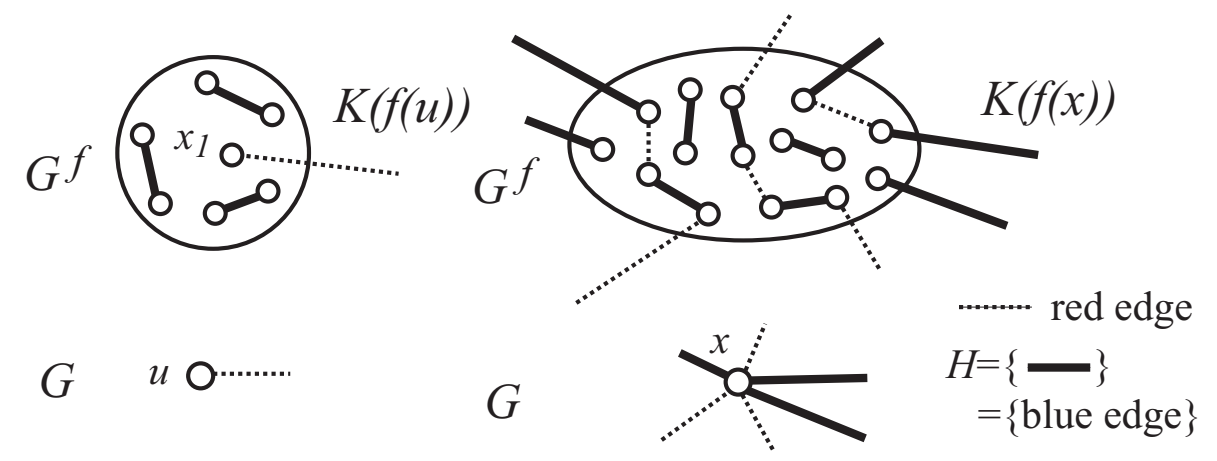

Figure 5.10: An augmenting path $P$ of $G^{f}$ and a walk $W=E(P) \cap E(G)$ of $G$, which is an $H$-augmenting walk.

We now show that $W=E(P) \cap E(G)$ is a $H$-augmenting walk of $G$ (Figure 5.2). Since all the vertices of $V(K(u))-x_{1}$ are covered by $M \cap$ $E(K(f(u)))$, we have

$$
\operatorname{deg}_{W}^{\text {blue }}(u)=\operatorname{deg}_{W}^{\text {blue }}(v)=0 \quad \text { and } \quad \operatorname{deg}_{W}^{r e d}(u)=\operatorname{deg}_{W}^{r e d}(v)=1 .
$$


For any vertex $x \in V(G)-\{u, v\}$, it follows that

$$
\begin{aligned}
& \operatorname{deg}_{W}^{\text {red }}(x)-\operatorname{deg}_{W}^{\text {blue }}(x) \\
\leq & \text { the number of blue edges in } K(f(x)) \\
\leq & f(x)-\operatorname{deg}_{H}(x)
\end{aligned}
$$

Hence $W$ is the desired $H$-augmenting walk.

\section{Exercises}

Exercise 5.2.1 Let $T$ be a tree of even order and $f: V(T) \rightarrow\{1,3,5, \ldots\}$. Without using the $(1, f)$-Odd Factor Theorem, show that if odd $(T-v) \leq f(v)$ for all $v \in V(T)$ then $T$ satisfies

$$
\operatorname{odd}(T-S) \leq \sum_{x \in S} f(x) \quad \text { for all } \quad S \subset V(T) .
$$

Exercise 5.2.2 Let $G$ be a general graph and $f: V(G) \rightarrow\{1,3,5, \ldots\}$. Then a $(1, f)$-odd subgraph is called a maximal $(1, f)$-odd subgraph if $G$ has no $(1, f)$-odd subgraph $H^{\prime}$ such that $V(H) \subset V\left(H^{\prime}\right)$. Show that every maximal $(1, f)$-odd subgraph is a maximum $(1, f)$-odd subgraph.

Exercise 5.2.3 Let $G$ be a simple graph, five $(G) \rightarrow\{1,3,5, \ldots\}$, and $B$ and $R$ vertex subsets of $G$ with $|B|<|R|$. Prove the following two statements by using the Structure Theorem on $(1, f)$-odd subgraphs.

(1) If there exists a $(1, f)$-odd subgraph which covers $B$ and one which covers $R$, then there exists a $(1, f)$-odd subgraph that covers $B$ and at least one vertex of $R \backslash B$ ([71])

(2) If there exists a maximum $(1, f)$-odd subgraph which avoids $B$ and one which avoids $R$, then there exists a maximum $(1, f)$-odd subgraph which avoids $B$ and at least one vertex of $R \backslash B$. Note that a $(1, f)$-odd subgraph $H$ avoids a vertex subset $X$ if $H$ covers no vertex of $X$.

\subsection{Partial parity $(g, f)$-factors and coverings}

For a given vertex subset $W$ of a graph $G$, consider two functions $g, f$ : $V(G) \rightarrow \mathbb{Z}$ such that

$$
\begin{array}{ll}
g(x) \leq f(x) & \text { for all } x \in V(G), \text { and } \\
g(y) \equiv f(y)(\bmod 2) & \text { for all } y \in W .
\end{array}
$$


Then a spanning subgraph $F$ of $G$ is called a partial parity $(g, f)$-factor with respect to $W$ if

$$
\begin{aligned}
& g(x) \leq \operatorname{deg}_{F}(x) \leq f(x) \quad \text { for all } x \in V(G), \text { and } \\
& \operatorname{deg}_{F}(y) \equiv f(y)(\bmod 2) \quad \text { for all } y \in W .
\end{aligned}
$$

Note that if $W=\emptyset$, then a partial parity $(g, f)$-factor is a $(g, f)$-factor and, if $W=V(G)$, then a partial parity $(g, f)$-factor is a parity $(g, f)$-factor. We begin with a necessary and sufficient condition for a graph to have a partial parity $(g, f)$-factor.

Theorem 5.3.1 (The Partial Parity $(g, f)$-Factor Theorem, [76]) Let $G$ be a general graph, and $W$ be a vertex subset of $G$. Let $g, f: V(G) \rightarrow \mathbb{Z}$ be two functions such that

$$
g(x) \leq f(x) \text { for all } x \in V(G), \text { and } g(y) \equiv f(y)(\bmod 2) \text { for all } y \in W \text {. }
$$

Then $G$ has a partial parity $(g, f)$-factor with respect to $W$ if and only if for all disjoint subsets $S$ and $T$ of $V(G)$, it follows that

$$
\eta_{2}(S, T)=\sum_{x \in S} f(x)+\sum_{x \in T}\left(\operatorname{deg}_{G}(x)-g(x)\right)-e_{G}(S, T)-q_{2}(W ; S, T) \geq 0,
$$

where $q_{2}(W ; S, T)$ denotes the number of components $C$ of $G-(S \cup T)$ such that

$$
\begin{aligned}
& g(x)=f(x) \quad \text { for all } x \in V(C) \backslash W, \quad \text { and } \\
& \sum_{x \in V(C)} f(x)+e_{G}(C, T) \equiv 1(\bmod 2) .
\end{aligned}
$$

Proof. We first construct a new graph $G^{*}$ from $G$ by adding $(f(y)-g(y)) / 2$ new loops to every vertex $y \in W$ (see Figure 5.3). Then define

$$
g^{*}(v)= \begin{cases}f(v) & \text { if } v \in W \\ g(v) & \text { otherwise }\end{cases}
$$

It is easy to see that $G^{*}$ has a $\left(g^{*}, f\right)$-factor if and only if $G$ has the desired partial parity $(g, f)$-factor with respect to $W$. By the $(g, f)$-Factor Theorem 3.1.1, $G^{*}$ has a $\left(g^{*}, f\right)$-factor if and only if for all disjoint subsets $S$ and $T$ of $V\left(G^{*}\right)=V(G)$, it follows that

$$
\sum_{x \in S} f(x)+\sum_{x \in T}\left(\operatorname{deg}_{G^{*}}(x)-g^{*}(x)\right)-e_{G^{*}}(S, T)-q_{G^{*}}(S, T) \geq 0 .
$$




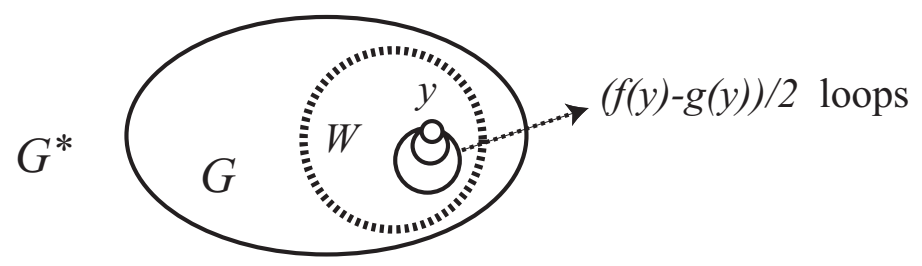

Figure 5.11: The new graph $G^{*}$ constructed from $G$.

The following holds:

$$
\operatorname{deg}_{G^{*}}(x)-g^{*}(x)=\operatorname{deg}_{G}(x)-g(x) \quad \text { for all } x \in V(G) .
$$

Moreover, a component $C$ of $G^{*}-(S \cup T)$ is a $\left(g^{*}, f\right)$-odd component if and only if

$$
\begin{aligned}
& g^{*}(x)=f(x) \quad \text { for all } x \in V(C), \quad \text { and } \\
& \sum_{x \in V(C)} f(x)+e_{G^{*}}(C, T) \equiv 1(\bmod 2) .
\end{aligned}
$$

The above conditions are equivalent to

$$
\begin{aligned}
& g(x)=f(x) \quad \text { for all } x \in V(C) \backslash W, \text { and } \\
& \sum_{x \in V(C)} f(x)+e_{G}(C, T) \equiv 1(\bmod 2) .
\end{aligned}
$$

Hence $q_{G^{*}}(S, T)=q_{2}(W ; S, T)$. Since $e_{G^{*}}(S, T)=e_{G}(S, T),(5.24)$ is equivalent to (5.22). Consequently, the theorem is proved.

Before giving an application of the Partial Parity $(g, f)$-Factor Theorem, we begin with some remark on matchings. We consider a property of matchings that cannot be directly extended to $(1, f)$-odd subgraphs. It is shown in Theorem 1.4.7 that for a graph $G$ and its vertex subset $W, G$ has a matching that covers $W$ if and only if

$$
\operatorname{odd}(G-S \mid W) \leq|S| \quad \text { for all } \quad S \subset V(G),
$$

where $\operatorname{odd}(G-S \mid W)$ denotes the number of odd components of $G-S$ all of whose vertices are contained in $W$. However this result cannot be directly extended to $(1, f)$-odd factors. Consider the tree $T$ given in Figure 5.12. Let $W=\{\bullet\}$, and define the functions $f$ as in Figure 5.12, where numbers denote $f(v)$. Then $T$ satisfies

$$
\operatorname{odd}(T-S \mid W) \leq \sum_{x \in S} f(x) \quad \text { for all } S \subset V(T) .
$$


However, $T$ has no $(1, f)$-odd subgraph that covers $W$. Therefore Theorem 3.3.5 cannot be directly extended to $(1, f)$-odd subgraphs. But we may expect that this condition implies the existence of another subgraph with some other property. The next theorem provides an answer, and it can be proved by using the Partial Parity $(g, f)$-Factor Theorem. Moreover, if $f$ is defined as $f(x)=1$ for all vertices $x$, then the following theorem implies the previous result on matchings.

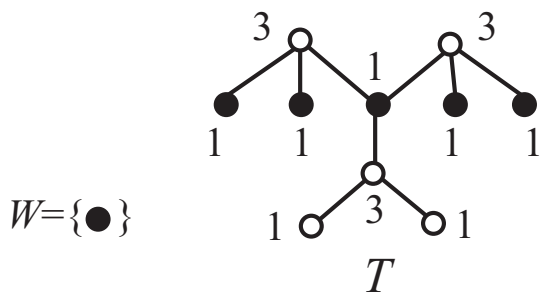

Figure 5.12: A tree that has no $(1, f)$-odd subgraph containing $W$.

Theorem 5.3.2 ([76]) Let $G$ be a general graph, $W$ a nonempty subset $V(G)$, and $f: V(G) \rightarrow\{0,1,2,3, \cdots\}$ such that $f(y)$ is an odd integer for all $y \in W$. Then $G$ has a subgraph $H$ covering $W$ such that

$$
\begin{aligned}
& 1 \leq \operatorname{deg}_{H}(x) \leq f(x) \quad \text { for all } \quad x \in V(H), \quad \text { and } \\
& \operatorname{deg}_{H}(y) \equiv 1(\bmod 2) \quad \text { for all } y \in W
\end{aligned}
$$

if and only if

$$
\operatorname{odd}(G-S \mid W) \leq \sum_{x \in S} f(x) \quad \text { for all } \quad S \subset V(G),
$$

where odd $(G-S \mid W)$ denotes the number of odd components $C$ of $G-S$ with $V(C) \subseteq W$.

Proof. Assume that $G$ has a subgraph $H$ that satisfies (5.25) and (5.26). Then for any odd component $C$ of $G-S$ with $V(C) \subseteq W$, at least one edge of $H$ joins $C$ to $S$ by (5.26). Hence

$$
\operatorname{odd}(G-S \mid W) \leq \sum_{x \in S} \operatorname{deg}_{H}(x) \leq \sum_{x \in S} f(x) .
$$

We next prove sufficiency. Let $N$ be a sufficiently large odd integer. Define a function $g: V(G) \rightarrow \mathbb{Z}$ by

$$
g(x)=-N \quad \text { for all } x \in V(G) .
$$


Then a partial parity $(g, f)$-factor with respect to $W$ is the desired subgraph. So it suffices to show that $G, g$ and $f$ satisfy condition (5.22) in Theorem 5.3.2.

Let $S$ and $T$ be two disjoint subsets of $V(G)$. If $T \neq \emptyset$, then (5.22) holds as

$$
\operatorname{deg}_{G}(x)-g(x)=\operatorname{deg}_{G}(x)+N \quad \text { for every } x \in T .
$$

Thus we may assume that $T=\emptyset$. It is immediate that a component $C$ of $G-S$ satisfying (5.23) is covered by $W$ and has odd order. Therefore, it follows from (5.27) that

$$
\eta_{2}(S, \emptyset)=\sum_{x \in S} f(x)-q_{2}(W ; S, \emptyset)=\sum_{x \in S} f(x)-o d d(G-S \mid W) \geq 0 .
$$

Consequently, $G$ has a partial parity $(g, f)$-factor with respect to $W$, and the theorem is proved.

We now give some results on covering a vertex subset with paths and cycles, which are applications of the Parity $(g, f)$-Factor Theorem with either odd and even numbers.

Theorem 5.3.3 (Kano and Matsuda [76]) Let $G$ be a simple graph and $W$ be a set consisting of an even number of vertices of $G$. Then $G$ has a set of vertex disjoint paths such that the set of their endvertices is precisely equal to $W$ (Figure 5.13) if and only if

$$
\operatorname{odd}(W ; G-S) \leq|S \cap W|+2|S \backslash W| \quad \text { for all } S \subseteq V(G),
$$

where odd $(W ; G-S)$ denotes the number of components $D$ of $G-S$ such that $|V(D) \cap W|$ is odd (Figure 5.13).
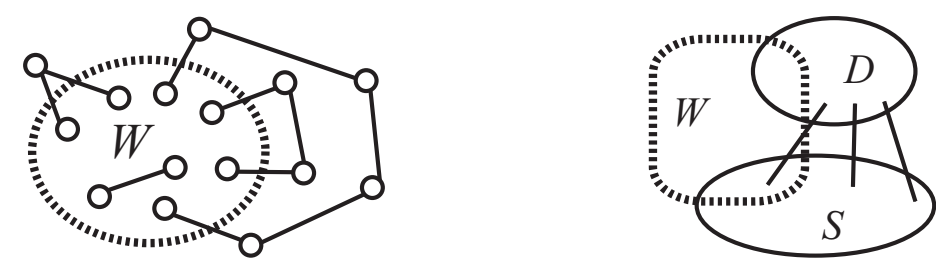

Figure 5.13: A set of vertex disjoint paths whose endvertices are exactly $W ; \quad D$ is a component of $G-S$.

Proof. Let $H$ be a subgraph of $G$ that satisfies

$$
\begin{array}{ll}
\operatorname{deg}_{H}(x)=1 & \text { for all } x \in W, \text { and } \\
\operatorname{deg}_{H}(y)=2 & \text { for all } y \in V(H) \backslash W
\end{array}
$$


Then each component of $H$ is a path or a cycle, and the set of paths in $H$ is the desired set of disjoint paths of $G$. Hence $G$ has the desired set of disjoint paths if and only if $G$ has a subgraph $H$ satisfying (5.29). We show that the existence of such a subgraph $H$ is equivalent to (5.28).

Suppose that $G$ has a subgraph $H$ satisfying (5.29). For each component $D$ of $G-S$ such that $|V(D) \cap W|$ is odd, at least one edge of $H$ joins $D$ to $S$. Thus we obtain

$$
\operatorname{odd}(W ; G-S) \leq \sum_{x \in S} \operatorname{deg}_{H}(x)=|S \cap W|+2|S \backslash W| .
$$

Hence (5.28) follows.

Conversely assume that (5.28) holds. Let $N$ be a sufficiently large integer, and define two functions $g, f: V(G) \rightarrow \mathbb{Z}$ by

$$
g(x)=\left\{\begin{array}{ll}
-2 N-1 & \text { if } x \in W, \\
-2 N & \text { otherwise; }
\end{array} \quad \text { and } \quad f(x)= \begin{cases}1 & \text { if } x \in W \\
2 & \text { otherwise. }\end{cases}\right.
$$

Then the desired subgraph $H$ is a parity $(g, f)$-factor $F$ of $G$, which may have degree 0 for some vertices of $V(G) \backslash W$ but must have degree 1 for every vertex of $W$. More precisely, the subgraph of $G$ induced by $E(F)$ is the desired subgraph $H$. Hence it suffices to show that $G, g$ and $f$ satisfy (5.2) in the Parity $(g, f)$-Factor Theorem.

By $\operatorname{odd}(W ; G)=0$, every component of $G$ contains an even number of vertices in $W$. Thus it follows that $\eta(\emptyset, \emptyset)=-q(\emptyset, \emptyset)=0$. Let $S$ and $T$ be disjoint subsets of $V(G)$ such that $S \cup T \neq \emptyset$. If $T \neq \emptyset$, then since $-g(x) \geq 2 N$ is sufficiently large, we have

$$
\eta(S, T)=\sum_{x \in S} f(x)+\sum_{x \in T}\left(\operatorname{deg}_{G}(x)-g(x)\right)-e_{G}(S, T)-q(S, T) \geq 0 .
$$

Hence we may assume that $T=\emptyset$. Then $q(S, \emptyset)=\operatorname{odd}(W ; G-S)$ and thus

$$
\eta(S, \emptyset)=\sum_{x \in S} f(x)-q(S, \emptyset)=2|S \backslash W|+|S \cap W|-\operatorname{odd}(W ; G-S) \geq 0,
$$

as desired.

Theorem 5.3.4 ([76]) Let $G$ be a simple graph and $W$ be a vertex subset of $G$. Then $G$ has a set of vertex disjoint cycles that cover $W$ (Figure 5.14) if and only if for all disjoint subsets $S \subseteq V(G)$ and $T \subseteq W$, it follows that

$$
2|S|+\sum_{x \in T}\left(\operatorname{deg}_{G}(x)-2\right)-e_{G}(S, T)-q_{3}(S, T) \geq 0,
$$

where $q_{3}(S, T)$ denotes the number of component $C$ of $G-(S \cup T)$ such that $e_{G}(C, T) \equiv 1(\bmod 2)$. 

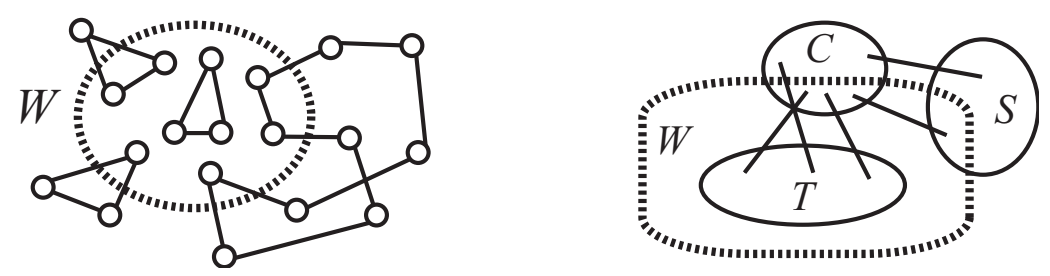

Figure 5.14: A set of vertex disjoint cycles that cover $W$; A component $C$ of $G-(S \cup T)$ counted in $q_{3}(S, T)$.

Proof. Let $N$ be a sufficiently large number, and define two functions $g, f: V(G) \rightarrow \mathbb{Z}$ by

$$
g(x)=\left\{\begin{array}{ll}
2 & \text { if } x \in W, \\
-2 N & \text { otherwise; }
\end{array} \quad \text { and } \quad f(x)=2 \text { for all } x \in V(G) .\right.
$$

Then $G$ has a parity $(g, f)$-factor, (which may have degree 0 for some vertices of $V(G) \backslash W$, ) if and only if $G$ has the desired set of disjoint cycles. Thus it suffices to show that (5.2) and (5.30) are equivalent for the two functions $g$ and $f$.

Suppose that (5.2) holds. Let $S \subseteq V(G)$ and $T \subseteq W$ such that $S \cap T=\emptyset$. Then by (5.2), we have

$$
\eta(S, T)=2|S|+\sum_{x \in T}\left(\operatorname{deg}_{G}(x)-2\right)-e_{G}(S, T)-q(S, T) \geq 0 .
$$

Moreover, every component $D$ of $G-(S \cup T)$ satisfying $(5.3)$ satisfies $e_{G}(T, D) \equiv$ $1(\bmod 2)$. Thus $q(S, T)=q_{3}(S, T)$, and hence $(5.30)$ holds.

Conversely, assume that (5.30) holds. It follows that $\eta(\emptyset, \emptyset)=-q(\emptyset, \emptyset)=$ 0 since $f(x)=2$ for all $x \in V(G)$. Let $S$ and $T$ be disjoint subsets of $V(G)$ such that $S \cup T \neq \emptyset$. If $T \backslash W \neq \emptyset$, then since $-g(x)=2 N$ is sufficiently large for $x \in T \backslash W$, we have $\eta(S, T) \geq 0$. Thus we may assume that $T \subseteq W$. It follows from $f(x)=2$ that $q(S, T)=q_{3}(S, T)$. Hence by (5.30), we obtain

$$
\eta(S, T)=2|S|+\sum_{x \in T}\left(\operatorname{deg}_{G}(x)-2\right)-e_{G}(S, T)-q(S, T) \geq 0
$$

Therefore (5.2) holds. Consequently, the theorem is proved.

Theorem 5.3.5 ([76]) Let $G$ be a graph and $W$ a set of vertices of $G$. Then $G$ has a set of vertex disjoint cycles and paths that cover $W$, all the vertices of the cycles and all the inner vertices of the paths are contained in $W$, and 
all the endvertices of the paths are contained in $V(G) \backslash W$ (Figure 5.15) if and only if for all disjoint subsets $S \subseteq V(G)$ and $T \subseteq W$, it follows that

$$
|S \backslash W|+2|S \cap W|+\sum_{x \in T}\left(\operatorname{deg}_{G}(x)-2\right)-e_{G}(S, T)-q_{4}(W ; S, T) \geq 0,
$$

where $q_{4}(W ; S, T)$ denotes the number of component $C$ of $G-(S \cup T)$ such that $V(C) \subseteq W$ and $e_{G}(T, C) \equiv 1(\bmod 2)$.

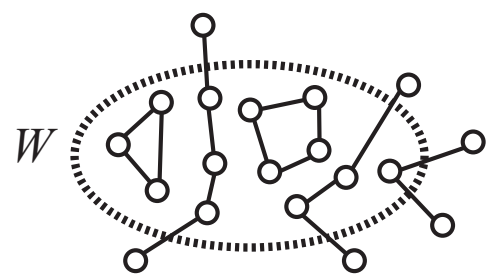

(a)

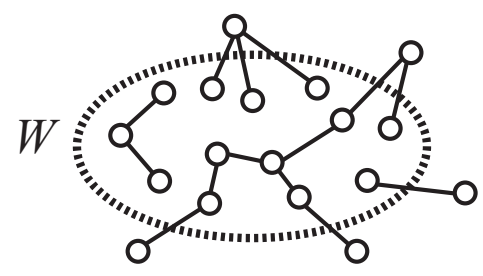

(b)

Figure 5.15: (a) A set of vertex disjoint cycles and paths that have the properties given in Theorem 5.3.5; (b) A [1,3]-subgraph covering $W$.

Proof. Let $N$ be a sufficiently large integer, and define two functions $g, f$ : $V(G) \rightarrow \mathbb{Z}$ by

$$
g(x)=\left\{\begin{array}{ll}
2 & \text { if } x \in W, \\
-N & \text { otherwise; }
\end{array} \quad \text { and } \quad f(x)= \begin{cases}2 & \text { if } x \in W \\
1 & \text { otherwise }\end{cases}\right.
$$

Then $G$ has a $(g, f)$-factor, (which may have degree 0 for some vertices of $V(G) \backslash W$, ) if and only if $G$ has the desired set of cycles and paths. Hence it suffices to show that (5.31) and (3.1) in the $(g, f)$-Factor Theorem 3.1.1 are equivalent.

Suppose that (3.1) holds. Let $S \subseteq V(G)$ and $T \subseteq W$ such that $S \cap T=\emptyset$. Then

$\gamma(S, T)=|S \backslash W|+2|S \cap W|+\sum_{x \in T}\left(\operatorname{deg}_{G}(x)-2\right)-e_{G}(S, T)-q^{*}(S, T) \geq 0$.

Since every component $D$ of $G-(S \cup T)$ counted in $q^{*}(S, T)$ satisfies $f(x)=$ $g(x)$ for all $x \in V(D)$ and $\sum_{x \in V(D)} f(x)+e_{G}(T, D) \equiv 1(\bmod 2)$, we have $V(D) \subseteq W$ and $q^{*}(S, T)=q_{4}(W ; S, T)$. Hence (5.31) follows.

Conversely assume that (5.31) holds. It follows that $\gamma_{G}(\emptyset, \emptyset)=-q^{*}(\emptyset, \emptyset)=$ 0 since $g(x)<f(x)$ for all $x \in V(G) \backslash W$ and $f(y)=2$ for all $y \in W$. Let $S$ and $T$ be disjoint subsets of $V(G)$ such that $S \cup T \neq \emptyset$. If $T \backslash W \neq \emptyset$, then 
$\gamma(S, T) \geq 0$ since $-g(x)=N$ is sufficiently large for $x \in T \backslash W$. Thus we may assume that $T \subseteq W$. It follows immediately that $q^{*}(S, T)=q_{4}(W ; S, T)$, and we obtain the following inequality from (5.31):

$\gamma(S, T)=|S \backslash W|+2|S \cap W|+\sum_{x \in T}\left(\operatorname{deg}_{G}(x)-2\right)-e_{G}(S, T)-q^{*}(S, T) \geq 0$.

Therefore (5.31) and (3.1) are equivalent, and the theorem is proved.

We finally consider the problem of covering a given vertex subset with a $[1, n]$-subgraph, each of whose vertices has degree between 1 and $n$. The condition for the existence of such a subgraph, which is given in the following theorem, is a natural extension of the criterion for the existence of $[1, n]$-factor as the 1-Factor Theorem is generalized to the theorem of a matching which covers a given subset in a graph $G$.

Theorem 5.3.6 Let $G$ be a simple graph, $W$ a subset of $V(G)$ and $n \geq 2$ be an integer. Then $G$ has a $[1, n]$-subgraph covering $W$ (Figure 5.15) if and only if

$$
i s o(G-S \mid W) \leq n|S| \quad \text { for all } S \subseteq V(G),
$$

where iso $(G-S \mid W)$ denotes the number of isolated vertices of $G-S$ contained in $W$.

Proof. Suppose first $G$ has a $[1, n]$-subgraph $H$ which covers $W$. Then for every isolated vertex $v$ of $G-S$ contained in $W, H$ has at least one edge joining $v$ to $S$. Hence

$$
i s o(G-S \mid W) \leq \sum_{x \in S} \operatorname{deg}_{H}(x) \leq n|S| .
$$

Next assume that (5.32) holds. Let $N$ be a sufficiently large integer, and define two functions $g$ and $f$ as follows:

$$
g(x)=\left\{\begin{array}{ll}
1 & \text { if } x \in W, \\
-N & \text { otherwise; }
\end{array} \quad \text { and } \quad f(x)=n \quad \text { for all } x \in V(G) .\right.
$$

Then a $(g, f)$-factor of $G$ is the desired subgraph. So it suffices to show that $G$ satisfies condition (3.1) in the $(g, f)$-Factor Theorem 3.1.1.

Let $S$ and $T$ be two disjoint subsets of $V(G)$. If $T \backslash W \neq \emptyset$, then $\gamma(S, T) \geq$ 0 since $-g(x)=N$ is sufficiently large for every $x \in T \backslash W$. Thus we may assume that $T \subseteq W$. Note that $q^{*}(S, T)=0$ since $g(x)<f(x)$ for all 
$x \in V(G)$. Since $\operatorname{deg}_{G-S}(x)=0$ if and only if $x$ is an isolated vertex of $G-S$, we obtain the following from $T \subseteq W$ and (5.32).

$$
\begin{aligned}
\gamma(S, T) & =n|S|+\sum_{x \in T}\left(\operatorname{deg}_{G}(x)-1\right)-e_{G}(S, T) \\
& =n|S|+\sum_{x \in T}\left(\operatorname{deg}_{G-S}(x)-1\right) \\
& \geq n|S|-i s o(G-S \mid W) \geq 0 .
\end{aligned}
$$

Therefore the theorem is proved.

Exercise 5.3.1 Prove necessity in the Partial Parity $(g, f)$-Factor Theorem 5.3.1 without using the Parity $(g, f)$-Factor Theorem 5.1.1

\section{$5.4 \mathcal{H}$-Factors}

In this chapter we introduce more general degree factor, which is called an $\mathcal{H}$ factor, and give some remarks on it without proofs. For a vertex $v$ of a graph $G$, let $\mathcal{H}_{v}$ denote a non-empty subset of $\{0,1,2,3, \ldots\}$. Then a spanning subgraph $K$ of $G$ is called an $\mathcal{H}$-factor of $G$ if

$$
\operatorname{deg}_{K}(x) \in \mathcal{H}_{x} \quad \text { for all } x \in V(G) .
$$

For a spanning subgraph $F$ of $G$ and for a vertex $v$ of $G$, define

$$
\delta(\mathcal{H} ; F, v)=\min \left\{\left|\operatorname{deg}_{F}(v)-i\right| ; i \in H_{v}\right\},
$$

and let

$$
\delta(\mathcal{H} ; F)=\sum_{x \in V(G)} \delta(\mathcal{H} ; F, x) .
$$

So a spanning subgraph $F$ is an $\mathcal{H}$-factor if and only if $\delta(\mathcal{H} ; F)=0$. The minimum $\delta(\mathcal{H} ; F)$ among the spanning subgraphs $F$ of $G$ is denoted by $\delta_{\mathcal{H}}(G)$, i.e.,

$$
\delta_{\mathcal{H}}(G)=\min \{\delta(\mathcal{H} ; F) \mid F \text { are the spanning subgraphs of } G \text {. }\}
$$

A spanning subgraph $F$ is called $\mathcal{H}$-optimal if

$$
\delta(\mathcal{H} ; F)=\delta_{\mathcal{H}}(G) .
$$

The degree prescribed subgraph problem is to determine the value of $\delta_{\mathcal{H}}(G)$. 
An integer $h$ is called a gap of $\mathcal{H}_{v}$ if $h \notin \mathcal{H}_{v}$ but $\mathcal{H}_{v}$ contains an element less than $h$ and an element greater than $h$.

Lovász [101] gave a structural description on the degree prescribed subgraph problem in case $\mathcal{H}_{v}$ has no two consecutive gaps for all $v \in V(G)$. He showed that the problem is NP-complete without this restriction. The first polynomial algorithm was given by Cornuéjols [34]. It is implicit in Cornuéjols [34] that this algorithm implies a Gallai-Edmonds type structure theorem for the degree prescribed subgraph problem (first stated in [135]), which is similar to, but in some respects much more compact, than that of Lovász.

Of course, for a function $f: V(G) \rightarrow\{1,3,5, \ldots\}$, if we define $\mathcal{H}_{v}=$ $\{1,3, \ldots, f(v)\}$ for every vertex $v$ of $G$, then a $(1, f)$-odd factor is an $\mathcal{H}$-factor. Similarly, a $(g, f)$-factor and a parity $(g, f)$-factor are special $\mathcal{H}$-factors. Thus these results are generalizations of previous obtained results. 


\section{Bibliography}

[1] J.Akiyama, D. Avis and H. Era, On a $\{1,2\}$-factor of a graph, TRU Math. 16 (1980) 97-102.

[2] J. Akiyama, G. Exoo and F. Harary, Covering and packing in graphs III: Cyclic and a cyclic invariants, Math. Slovaca 30 (1980) 405-417.

[3] J. Akiyama and V. Chvatal, A short proof of the linear arboricity for cubic graphs, Bull Liber. Arts \&5 Sci. NMS (Nippon Medical Scholl) 2 (1981) 1-3.

[4] J. Akiyama and M. Kano, Factors and factorizations of graphs - a survey, J. Graph Theory 9 (1985) 1-42.

[5] J. Akiyama and M. Kano, Almost-regular factorization of graphs, $J$. Graph Theory 9 (1985) 123-128

[6] J. Akiyama and M. Kano, Path factors of a graph, Graphs and applications (Boulder, Colo., 1982), (1985) 1-21, Wiley-Intersci. Publ., Wiley, New York, 1985.

[7] J. Akiyama and M. Kano, 1-factors of triangle graphs, Number theory and combinatorics, Japan 1984 (Tokyo, Okayama and Kyoto, 1984) (1985) 21-35, World Sci. Publishing, Singapore.

[8] J. Akiyama and P. Frankl, On the size of graphs with complete factors, J. Graph Theory 9 (1985) 197-201.

[9] A. Amahashi, On factors with all degrees odd, Graphs and Combinatorics, 1 (1985) 1-6.

[10] A. Amahashi and M. Kano, Factors with given components, Discrete math., 42 (1983) 1-6.

[11] I. Anderson, Perfect matchings of a graph, J. Combin. Theory Ser. B 10 (1971) 183-186. 
[12] I. Anderson, Sufficient conditions for matching, Proc. Edinburgh Math. Soc. 18 (1973) 129-136.

[13] K. Appel, W. Haken and J. Koch, Every planar map is four-colorable, Illinois J. Math. 21 (1977) 429-567.

[14] F. Bäbler, Über die Zerlegung regulärer Streckenkomplexe ungerader Ordnung, Comment. Math. Helvetici 10 (1938) 275-287.

[15] D. Bauer. HJ. Broersma and H.J. Veldman, Not every 2-tough graph is hamiltonian, Discrete Appl. Math. 99 (2000) 317-321.

[16] H.B. Belck, Reguläre Faktoren von Graphen, J. Reine Angew. Math. 188 (1950) 228-252.

[17] C. Berge, Two theorems in graph theory, Prc. Nat. Acad. Sci. U.S.A. 43 (1957) 842-844.

[18] C. Berge, Sur le couplage maximum d'un graphe, C.R. Acad. Sci. Paris Ser.I Math. 247, (1958) 258-259.

[19] C. Berge, Graphs and Hypegaphs, North-Holland, (1973)

[20] B. Bollobás Extremal Graph Theory, Academic Press, (1978).

[21] J.A. Bondy and U.S.R. Murty, Graph Theory with Applications, The MacMillian Press (1976).

[22] B. Bollobás, A. Saito and N.C. Wormald, Regular factors of regular graphs, J. Graph Theory 9 (1985) 97-103.

[23] H.J. Broersma, J. von den Heuvel and H.J. Veldman, A generalization of Ore's theorem involving neighborhood unions, Discrete Math. 122 (1993) 37-49.

[24] Mao-cheng Cai, [a,b]-factorizations of graphs, J. Graph Theory 15 (1991) 283-301.

[25] Maocheng Cai, Yangjun Li and M. Kano, Some results on odd factors of graphs, J. Graph Theory 12 (1988) 327-333.

[26] K. Cameron, Induced matchings, Discrete App. Math. 24 (1989) 97102.

[27] Ciping Chen, A note on matching extension (Chinese), J. Beijing Agri. Engin. Univ. 10 (1990) 293-297. 
[28] Ciping Chen, Binding number and minimum degree for $[a, b]$-factors, $J$. Sys. Sci. and Math. Scis. 6 (1993) 179-185.

[29] Ciping Chen, Binding number and toughness for matching extension, Discrete Math. 146 (1995) 303-306.

[30] Ciping Chen, and Jianfang Wang, Factors in graphs with odd-cycle property, Discrete Math. 112 (1993) 29-40.

[31] Ciping Chen and Guizhen Liu, Toughness of Graphs and [a,b]-factors with prescribed properties, J. Combin. Math. Combin. Comput. 12 (1992) 215-221.

[32] S.A. Choudum and M.S. Paulraj, Regular factors in $K_{1,3}$-free graphs. J. Graph Theory 15 (1991), 259-265.

[33] V. Chvátal and P. Erdös, A note on hamiltonian circuits, Discrete Math. 2 (1972) 111-113.

[34] G. Cornuéjols, General factors of graphs. J. Combin. Theory Ser. B 45 (1988) 185-198.

[35] Yuting Cui and M. Kano, Some results on odd factors of graphs, J. Graph Theory 12 (1988) 327-333.

[36] J. Edmonds, Paths, trees and flowers, Canada. J. Math. 17 (1965) 449467.

[37] Y.Egawa, Era's conjecture on $[k, k+1]$-factorizations of regular graphs. Ars Combin. 21 (1986) 217-220.

[38] Y. Egawa and H. Enomoto, Sufficient conditions for the existence of $k$ factors, Recent Studies in Graph Theory (V.R.Kull, Ed) (1989) 97-103.

[39] Y. Egawa and H. Enomoto, Sufficient conditions for the existence of $k$ factors, Recent Studies in Graph Theory (V.R.Kull, ed) (1989) 97-103.

[40] Y. Egawa and K. Ota, Regular factors in $K_{1, n}$-free graphs, J. Graph Theory 15 (1991) 337-344.

[41] Y. Egawa and M. Kano, Sufficient conditions for graphs to have $(g, f)$ factors. Graph theory and combinatorics (Manila, 1991). Discrete Math. 151 (1996) 87-90.

[42] Y. Egawa M. Kano and A. Kelmans, Star partitions of graphs, J. of Graph Theory 25 (1997) 185-190. 
[43] Y. Egawa, M. Kano and K. Ota, Connected $[2, k]$-factors of graphs, unpublished.

[44] E. Egerváry, On combinatorial peoperties of matrices (Hungarian), Mat. Lapok 38 (1931) 16-28.

[45] H. Enomoto, B. Jackson, P. Katerinis and A. Saito, Toughness and the existence of $k$-factors, J. Graph Theory 9 (1985) 87-95.

[46] H. Enomoto, K. Ota and M. Kano, A sufficient condition for a bipartite graph to have a $k$-factor. J. Graph Theory 12 (1988) 141-151.

[47] H. Era, A note on semi-regular factorizations of regular graphs, Proc. Fac. Sci. Tokai Univ. 19 (1984).

[48] H. Era, Semiregular factorizations of regular graphs. Graphs and applications (Boulder, Colo., 1982), 101-116, Wiley-Intersci. Publ., Wiley, New York, 1985.

[49] J. Folkman and D. R. Fulkerson, Flows in infinite graghs, J. Combin. Theory 8 (1970) 30-44.

[50] D.R. Fulkerson, A.J. Hoffman and M.H. McAndrew, Some properties of gaphs with mutiple edges, Canadian J. Math. 17 (1965) 166-177.

[51] T. Gallai, On factorisation of grahs, Acta Math. Acad. Sci. Hung 1 (1950), 133-153.

[52] T. Gallai, Neuer Beweis eines Tutte'schen Satzes, Magyar Tud. Akad. Mat. Kutató Int. Közl 8 (1963) 135-139.

[53] T. Gallai, Köning Dénes (1884-1994), Mat. Lapok 15 (1964) 277-293 (Hungarian).

[54] P.R. Halmos and H.E. Vaughan, The marriage problem, Amer. J. Math. 72 (1950) 214-215.

[55] P. Hall, On representatives of subsets, J. London Math. Soc. 10 (1935) 26-30.

[56] K. Heinrich, P. Hell, D. Kirkpatrick and G. Liu, A simple existence ciriterion for $(g<f)$-factor, Discrete Math. 85(1990) 313-317.

[57] G.R.T. Hendry, Maximum graphs with a unique $k$-factor, J. Combin. Theory Ser. B 37 (1984) 53-63. 
[58] G. Hetyei [ see L. Lovász, On the structure of factorizable graphs Acta Math. Acad. Sci. Hungar. 23 (1972) 179-195; ibid. 23 (1972), 465-478; (Corollary 1.6)]

[59] G. Hetyei, A new proof of a factorization theorem, Acta Acad. Paedagog. Civitate Pécs Ser.6 Math. Phys. Chem. Tech. 16 (1972) 3-6 (Hungarian).

[60] A. Hoffmann and L. Volkmann, On unique $k$-factors and unique $[1, k]$ factors in graphs, Discrete Math. 278 (2004) 127-138.

[61] A. Hoffmann and L. Volkmann, On regular factors in regular graphs with small radius. Electron. J. Combin. 11 (2004), no. 1, Research Paper 7, 7 pp. (electronic).

[62] Hong Wang, Path factors of bipartite graphs, J. Graph Theory, bf 18 (1994) 161-167.

[63] H. Iida and T. Nishimura, An Ore-type condition for the existence of $k$-factors in graphs, Graphs and Combinatorics 7 (1991) 353-361.

[64] A. Joentgen and L. Volkmann, Factors of locally almost regular graphs, Bull. London Math. Soc. 23 (1991) 121-122.

[65] P. Johann, On the structure of graphs with a unique $k$-factor, J. Graph Theory 35 (2000) 227-243.

[66] M. Kano, Connected [2,k]-factors of graphs, in preparation.

[67] M. Kano Graph factors with given properties, Graph theory, Singapore 1983, Springer Lecture Notes in Math. 1073 (1984) 161-168.

[68] M. Kano, [a,b]-factorization of a graph. J. Graph Theory 9 (1985) 129146.

[69] M. Kano, Factors of regular graphs, J. Combin. Theorey, Series B 41 (1986) 27-36.

[70] M. Kano, A sufficient condition for a graph to have $[a, b]$-factors. Graphs Combin. 6 (1990) 245-251.

[71] M. Kano and G. Katona, Odd subgraphs and matchings Discrete Math. 250 (2002) 265-272.

[72] M. Kano and G.Y. Katona, Structure Theorem and Algorithm on $(1, f)$ odd subgraphs Discrete Mathematics, 307 (2007) 1404-1417. 
[73] M. Kano, G.Y. Katona and J. Szabo, Elementary graphs with respect to $(1, f)$-odd factors, submitted.

[74] M. Kano and Li, Spanning trees of $K_{1, n}$-free graphs, unpublished.

[75] M. Kano and H. Matsuda, Some results on (1,f)-odd factros Combinatorics, Graph Theory, and Algorithms, Vol.II (1999) 527-533. (New Issues Press; Kalamazoo, Michigan, ISBN 0-932826-74-8) Proceedings of the eighth quadrennial international conference on graph theory, combinatorics, algorithms and applications

[76] M. Kano and H. Matsuda, Partial parity (g,f)-factors and subgraphs covering given vertex subsets Graphs and Combinatorics, 17 (2001) $501-510$.

[77] M. Kano and A. Saito, [a,b]-factors of graphs. Discrete Math. 47 (1983) 113-116.

[78] M. Kano and A. Saito, A short proof of Lovasz's factor theorem, Memories of Akashi Techonological College 26 (1984) 167-170.

[79] M. Kano and N. Tokushige, Binding numbers and $f$-factors of graphs, J. Combinatorial Theory, Series B 54 (1992) 213-221.

[80] P. Katerinis Toughness of graphs and the existence of factors Discrete Math., 80 (1990) 81-92.

[81] P. Katerinis Some conditions for the existence of $f$-factors, J. Graph Theory, 9 (1985) 513-521.

[82] P. Katerinis A Chvátal-Erdös condition for an $r$-factor in a graph, Ars Combinat. 20-B (1985) 185-191.

[83] P. Katerinis Minimum degree of a graph and the existence of $k$-factors, Proc. Indian. Acad. Sci. Math. Sci. 94 (1985) 123-127.

[84] P. Katerinis Two sufficient conditions for a 2-factor in a bipartite graph. J. Graph Theory 11 (1987) 1-6.

[85] P. Katerinis Toughness of graphs and the existence of factors Discrete Math. 80 (1990) 81-92.

[86] P. Katerinis, Regular factors in regular graphs, Discrete Math. 113 (1993) 269-297. 
[87] P. Katerinis, Regular factors in vertex-deleted subgraphs of regular graphs. Discrete Math. 131 (1994) 357-361.

[88] P. Katerinis and D.R. Woodall, Binding numbers of graphs and the existence of k-factors, Quart. J. Math. Oxford (2), 38 (1987) 221-228.

[89] D. König, Über Graphen und ihre Anwendung auf Determinanttentheorie und Mengenlehre, Math. Ann. 77 (1916) 453-465.

[90] D. König, Graphen und Matrizen, Math. Fiz. Lapok 38 (1931) 116-119.

[91] M. Las Vergnas, A note on matchings in graphs, Cahiers Centre Etudes Reech. Opér. 17 (1975) 257-260.

[92] M. Las Vergnas, An extension of Tutte's 1-factor theorem, Discrete Math. 23 (1978) 241-255.

[93] Guizhen Liu and Jianfang Wang, $(a, b, k)$-critical graphs, Adv. Math. (China) 27 (1998) 536-540.

[94] Yanjun Li and Maocheng Cai, A degree condition for a graph to have [a,b]-factors. J. Graph Theory 27 (1998), no. 1, 1-6

[95] C.H.C. Little, The parity of the number of 1-factors of a graph, Discrete Math., 2 (1972), 179-181.

[96] C.H.C. Little, D.D. Grant and D.A. Holton, On defect-d matching in graphs, (Discrete Math., 13 (1975), 41-54).

[97] Guizhen Liu, (g,f)-factors and (g,f)-factorizations of graphs, Acta. Mathematica Sinica (Chinese Series) 37 (2) (1994) 230-237.

[98] Jiping Liu and Huishan Zhou, Maximum induced matchings in graphs, Discrete Math., 170 (1997) 227-281.

[99] L. Lovász, On graphs not containing independent circuits (in Hungarian), Mat. Lapok 16 (1965) 289-299.

[100] L. Lovász, Subgraphs with prescribed valencies, J. Combin. Theory. 8 (1970), 391-416.

[101] L. Lovász, The factorization of graphs II. Acta Math. Acad. Sci. Hungar. 23 (1972) 223-246.

[102] L. Lovász, On the structure of factorizable graphs, Acta Nath, Acad. Sci. Hungar. 23 (1972) 179-195. 
[103] L. Lovász, On the structure of factorizable graphs. Acta Math. Acad. Sci. Hungar., 23 (1972) 179-195.

[104] L. Lovász, On the structure of factorizable graphs. II. Acta Math. Acad. Sci. Hungar., 23 (1972) 465-478.

[105] Antifactors of graphs. Period. Math. Hungar., 4 (1973) 121-123.

[106] L. Lovász, Three short proofs in graph theory, J. Combin. Theory Ser. B 19 (1975) 269-271.

[107] L. Lovász, Combinatorial Problems and Exercises, North-Holland, Amsterdam (1979).

[108] L. Lovász and M. Plummer, Matching Theory, Annals of Discrete math. 29 North-Holland, (1986).

[109] E. Mahmoodian, On factors of a graph, Canadian J. Math. 29 (1977) $38-440$.

[110] W. Mader, 1-Faktoren von Graphen, Math. Ann. 201 (1973) 269-282.

[111] E. Mendelsohn and A. Rosa, One-factorizations of the complete graph - a survey, J. Graph Theory 9 (1985) 43-65.

[112] D. Miyata, Connected factors of claw-free graphs, Private communication.

[113] T. Niessen, Neighborhood unions and regular factors, J. Graph Theory 19 (1995) 45-64.

[114] T. Niessen, Minimum degree, independence number and regular factors, Graphs Combin. 11 (1995) 367-378.

[115] T. Niessen, A characterization of graphs having all $(g, f)$-factors, $J$. Combin. Theory Ser. B 72 (1998), 152-156.

[116] T. Niessen and B. Randerath, Regular factors of simple regular graphs and factor-spectra. Discrete Math. 185 (1998) 89-103.

[117] T. Nishimura, Independence number, connectivity, and $r$-factors, $J$. Graph Theory, 13 (1989) 63-69.

[118] T. Nishimura, Regular factors of line graphs. II. Math. Japon. 36 (1991) 1033-1040. 
[119] T. Nishimura, A degree condition for the existence of $k$-factors. $J$. Graph Theory 16 (1992), 141-151.

[120] On the relation between the genus and the cardinality of the maximum matchings of a graph, Discrete Math. 25 (1979) 149-156.

[121] T. Nishizeki and I. Baybars, Lower bounds on the cardinality of the maximum matchings of planar graphs, Discrete Math. 28 (1979) 255267.

[122] V. Neumann-Lara and E. Rivera-Campo, Spanning trees with bounded degrees, Combinatorica 11 (1991) 55-61.

[123] O. Ore, Graphs and matching theorems, Duke Math. J. 22 (1955) 625639.

[124] O. Ore, Graphs and subgraphs, Trans. Amer. Math. Soc. 84 (1957) 109-136.

[125] O. Ore, Notes on Hamilton circuits, Amer. Math. Monthly 67 (1960) 55.

[126] J. Petersen, Die Theorie der regulären raphs, Acta Math. 15 (1891) 193-220.

[127] J. Plesník, Connectivity of regular graphs and the existene of 1-factors, Mat. časop. 22 (1972) 310-318.

[128] J. Plesník, Remarks on rgular factors of regular graphs, Czechoslovak Math. J. 24 (1974) 292-300.

[129] N. Robertson, D. Sanders, P. Seymour and R. Thomas, The four-colour theorem, J. Combin. Theory Ser. B 70 (1997) 2-44.

[130] A. Saito, private communication.

[131] A. Saito, One-factors and k-factors. Discrete Math. 91 (1991) 323-326.

[132] A. Saito and M. Watanabe, Partitioning graphs into induced stars, Ars combinatoria, to appear.

[133] D. P. Sumner, Graphs with 1-factors, Proc. Amer. Math. 42 (1974) 8-12.

[134] D. P. Sumner, 1-factors and antifactor sets, J. London Math. Soc. 22 (1976) 351-359. 
[135] J. Szabó, A note on the degree prescribed factor problem, EGRES Technical Reports 2004-19, www.cs.elte.hu/egres

[136] C. Thomassen, A remark on the factor theorems of Lovász and Tutte, J. Graph Theory 5 (1981) 441-442.

[137] J. Topp and P. D. Vestergaard, Odd factors of a graph, Graphs and Combinatorics 9 (1993) 371-381.

[138] W.T. Tutte, The factorization of linear graphs, J. London Math. Soc. 22 (1947) 107-111.

[139] W.T. Tutte, The factors of graphs, Can. J. Math. 4 (1952) 314-328.

[140] W.T. Tutte, A short proof of the factor theorem for finite graphs, Can. J. Math. 6 (1954) 347-352.

[141] W.T. Tutte, A theorem on planar graphs, Trans. Amer. Math. Soc. 82 (1956), 99-116.

[142] W.T. Tutte, The subgraph probelm, Ann. Discrete Math. 3 (1978) 289-295.

[143] W.T. Tutte, Graph factors, Combinatorica 1 (1981) 79-97.

[144] V.G. Vizing, On an estimate of chromatic class of a p-graphs, Diskret. Analiz. 3 (1964) 24-30.

[145] V.G. Vizing, Critical graphs with a given chromatic class, Diskret. Analiz 5 (1965) 9-17.

[146] L. Volkmann, Graphen und Digraphen, Eine Einführung in die Graphentheorie (Springer Verlag), (1991)

[147] L. Volkmann, Regular graphs, regular factors, and the impact of Petersen's Theorems, Jber. d. Dt. Math.-Verein 97 (1995) 19-42.

[148] L. Volkmann, The maximum size of graphs with a unique $k$-factor. Combinatorica 24 (2004) 531-540

[149] S. Winn, Existenz von Gerüsten mit vorgeschriebenem Maximlgrad in Graphen, Abh. Math. Seminar Univ. Humburg 43 (1975) 263-267.

[150] H. Whitney, Congruent graphs and the connectivity of graphs, Amer. J. Math. 54 (1932) 150-168. 
[151] D.R. Woodall, The binding number of a graph and its Andeson number, J. Combin. Theory Ser. B 69B (1965) 91-98.

[152] Yan Guiying, Some new results on $(g, f)$-factorizations of graphs, $J$. Combin. Math. Combin. Comput. 18 (1995), 177-185. 